\title{
STREAMFLOW, TIDAL-WATER-LEVEL, AND \\ WATER-QUALITY DATA FOR \\ THE TIDAL EMBAYMENTS OF \\ THE METEDECONK AND TOMS RIVERS, \\ NEW JERSEY, WATER YEARS 1993-94
}

By R. Edward Hickman

U. S. GEOLOGICAL SURVEY

Open-File Report 96-368

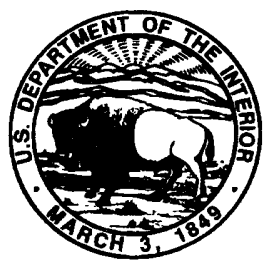

Prepared in cooperation with the NEW JERSEY DEPARTMENT OF ENVIRONMENTAL PROTECTION 


\title{
U.S. DEPARTMENT OF THE INTERIOR \\ BRUCE BABBIT, Secretary
}

\author{
U. S. GEOLOGICAL SURVEY
}

Gordon P. Eaton, Director

For additional information write to:

Copies of this report can be purchased from:

\section{District Chief}

U. S. Geological Survey

Mountain View Office Park

810 Bear Tavern Road

Suite 206

West Trenton, NJ 08628
U. S. Geological Survey

Branch of Information Services

Box 25286

Denver, CO 80225-0286 


\section{CONTENTS}

Page

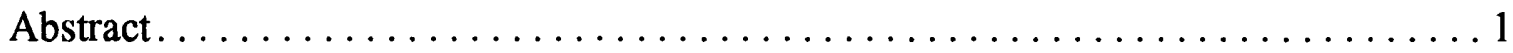

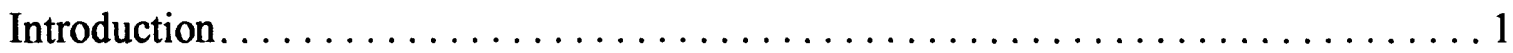

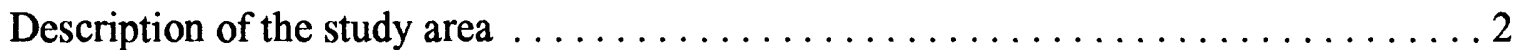

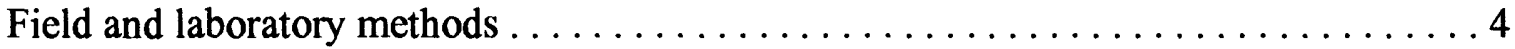

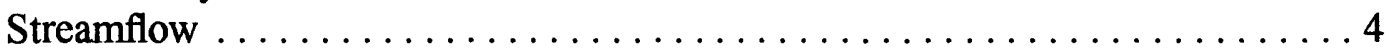

Tidal-water level ...................................... 15

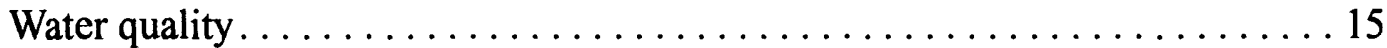

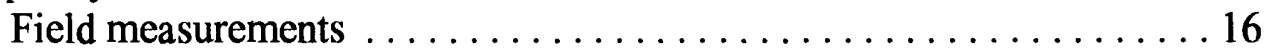

Continuous records from fixed monitors $\ldots \ldots \ldots \ldots \ldots \ldots 16$

Continuous records from movable monitors. . . . . . . . . . 18

Manual measurements at nontidal stations on tributaries...... 18

Manual measurements at tidal stations in and near the tidal embayments ............................. 21

Laboratory measurements of dissolved constituents ..........22

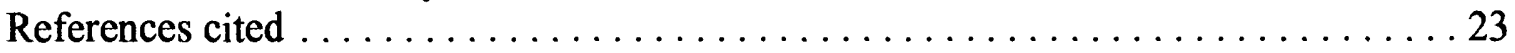

Appendixes 1-2. Measurements at nontidal stations on tributaries to the tidal embayment of the Metedeconk River, N.J.:

1. Daily mean streamflow, October 1992 - September $1994 \ldots \ldots \ldots \ldots \ldots \ldots 25$

2. Water-quality data from manual measurements,

October 1992 - October $1993 \ldots \ldots \ldots \ldots \ldots \ldots \ldots$

3-8. Measurements at tidal stations in and near the tidal embayment of the Metedeconk River, N.J.:

3. Daily minimum and maximum tidal-water level, October 1992 - November 1993. . . . . . . . . . . . . . . . 33

4. Daily minimum and maximum temperature from measurements by fixed monitors, October 1992 - January 1994

5. Daily minimum and maximum specific conductance from measurements by fixed monitors,

October 1992 - January 1994

6. Daily minimum and maximum values of water-quality characteristics from measurements by movable monitors, November 1992 - January 1994 . . . . . . . . . . . . 57

7. Water-quality data from manual measurements, October 1992 - October 1993.

8. Results of laboratory measurements of dissolved constituents, October 1992 - October 1993.

Appendixes 9-12. Measurements at nontidal stations on tributaries to the tidal embayment of the Toms River, N.J.:

9. Daily mean streamflow, October 1992 - September $1994 \ldots 152$ 


\section{CONTENTS--Continuted}

Appendixes 9-12. Measurements at nontidal stations on tributaries to the tidal embayment of the Toms River, N.J.--Continued:

10. Measured values of discrete streamflow,

October 1992 - August $1993 \ldots \ldots \ldots \ldots \ldots . \ldots 155$

11. Daily minimum and maximum values of water-quality

characteristics from measurements by movable monitors,

July 1993 - August 1993 . . . . . . . . . . . . . 157

12. Water-quality data from manual measurements,

October 1992 - October $1993 \ldots \ldots \ldots$. . . . . . . . . 159

Appendixes 13-18. Measurements at tidal stations in and near the tidal embayment of the Toms River, N.J.:

13. Daily minimum and maximum tidal-water level, October 1992 - November 1993............... 163

14. Daily minimum and maximum temperature from measurements by fixed monitors, October 1992 - January 1994

15. Daily minimum and maximum specific conductance from measurements by fixed monitors, October 1992 - January 1994

16. Daily minimum and maximum values of water-quality characteristics from measurements by movable monitors, October 1992 - January 1994 . . . . . . . . . . . . . 187

17. Water-quality data from manual measurements, October 1992 - October 1993

18. Results of laboratory measurements of dissolved constituents, October 1992 - October 1993 275

\section{ILLUSTRATIONS}

Figure 1. Map showing locations of Barnegat Bay and the Metedeconk and Toms Rivers, New Jersey. .

2-3. Maps showing locations of surface-water stations in and near the tidal embayment of the:

2. Metedeconk River, New Jersey. . . . . . . . . . . . . . . 5

3. Toms River, New Jersey..................... 6

4-5. Graphs showing:

4. A relation between salinity and specific conductance......... 17

5. Relations between temperature and dissolved-oxygen concentration at saturation for freshwater and seawater. 


\section{TABLES}

Page

Table 1-2. Surface-water stations in and near the tidal embayment of the:

1. Metedeconk River, New Jersey. . . . . . . . . . . . . . . 7

2. Toms River, New Jersey..................... 9

Tables 3-4. Measurements at surface-water stations in and near the tidal embayment of the:

3. Metedeconk River, New Jersey. . . . . . . . . . . . . . . 11

4. Toms River, New Jersey..................... 13 


\section{CONVERSION FACTORS}

\begin{tabular}{lll}
\multicolumn{1}{c}{ Multiply } & By & \multicolumn{1}{c}{ To obtain } \\
\hline foot & 0.3048 & meter \\
mile & 1.609 & kilometer \\
& & \\
square mile & 2.59 & square kilometer \\
& & \\
cubic foot per second & 0.02832 & cubic meter per second \\
& & \\
degree Celsius $\left({ }^{\circ} \mathrm{C}\right)$ & ${ }^{\circ} \mathrm{F}=(1.8)^{*}\left({ }^{\circ} \mathrm{C}\right)+32$ & degree Fahrenheit $\left({ }^{\circ} \mathrm{F}\right)$ \\
& & \\
\hline
\end{tabular}




\title{
STREAMFLOW, TIDAL-WATER-LEVEL, AND WATER-QUALITY DATA FOR THE TIDAL EMBAYMENTS OF THE METEDECONK AND TOMS RIVERS, NEW JERSEY, WATER YEARS 1993-94
}

\author{
By R. Edward Hickman
}

\begin{abstract}
Streamflow, tidal-water-level, and water-quality measurements made in and near the tidal embayments of the Metedeconk and Toms Rivers during water years 1993-94 (October 1992 September 1994) are presented; most measurements were made during water year 1993 (October 1992 - September 1993). The data were collected as part of a study of the effects of present and future freshwater withdrawals on the quality of the water in the tidal embayments of the Metedeconk and Toms Rivers. Both embayments are part of the Barnegat Bay estuarine system.
\end{abstract}

Streamflow was continuously measured at three nontidal stations on tributaries to the embayments during October 1992 - September 1994; daily mean values are presented. Discrete streamflow values measured or estimated at five nontidal stations are also presented.

Tidal-water level was continuously measured at one station in each embayment during October 1992 - November 1993; daily minimum and maximum values are presented. Some discrete measurements are included.

Temperature, specific conductance, $\mathrm{pH}$, and dissolved-oxygen concentration were continuously measured by fixed and movable monitors; daily minimum and maximum values are presented. Fixed monitors measured temperature and specific conductance at two stations in each embayment during October 1992 - January 1994. Movable monitors were used to make shortterm measurements of temperature, specific conductance, $\mathrm{pH}$, and dissolved-oxygen concentration at selected stations during November 1992 - January 1994.

Discrete measurements of temperature, $\mathrm{pH}$, specific conductance, dissolved-oxygen concentration, and Secchi-disk depth were made in each embayment on 1 or 2 days per month during October 1992 - October 1993. Laboratory analyses for specific conductance, salinity, and concentrations of dissolved chloride and dissolved solids were done on a few water samples.

\section{INTRODUCTION}

Streamflow in the Metedeconk and Toms Rivers is being reduced by ground-water withdrawals and surface-water withdrawals (Robert Nicholson, U.S. Geological Survey, oral commun., 1996). Ground-water withdrawals are made from the unconfined aquifer of both drainage basins; surface-water withdrawals are made from the Metedeconk River.

The water quality in the most downstream reaches of the Metedeconk and Toms Rivers may be affected by a reduction in streamflow. The most downstream reach of each river is tidal 
and contains saline water. A reduction in streamflow may increase the salinity as well as affect other water-quality characteristics of each tidal reach.

The U.S. Geological Survey (USGS), in cooperation with the New Jersey Department of Environmental Protection (NJDEP), conducted a study to determine the effects of reduced streamflow on selected water-quality characteristics of the tidal reaches. The characteristics of interest were the location of the saltwater front and the salinity, temperature, $\mathrm{pH}$, and dissolvedoxygen concentration in the tidal reaches.

This report presents measurements of tributary streamflow to, tidal-water level of, and water quality of the tidal reaches of the Metedeconk and Toms Rivers made during water years 1993-94 (October 1992 - September 1994); most measurements of tidal-water levels and water quality were made during water year 1993 (October 1992 - September 1993). Measurements for the Metedeconk River are presented in appendixes 1 through 8; measurements for the Toms River are presented in appendixes 9 through 18. Field and laboratory methods are discussed.

Streamflow was continuously measured at three nontidal stations on tributaries to the embayments during October 1992 - September 1994; daily mean values are presented. Discrete streamflow values measured or estimated for these and two other nontidal stations also are presented.

Tidal-water level was continuously measured at one station in each embayment during October 1992 - November 1993; daily minimum and maximum values are presented. Some discrete measurements are included.

Temperature, specific conductance, $\mathrm{pH}$, and dissolved-oxygen concentration were continuously measured by fixed and movable monitors; daily minimum and maximum values are presented. Fixed monitors measured temperature and specific conductance at two stations in each embayment during October 1992 - January 1994. Movable monitors were used to make shortterm measurements of temperature, specific conductance, $\mathrm{pH}$, and dissolved-oxygen concentration at selected stations during November 1992 - January 1994.

Discrete measurements of temperature, $\mathrm{pH}$, specific conductance, dissolved-oxygen concentration, and Secchi-disk depth made in each embayment on 1 or 2 days per month during October 1992 - October 1993 are presented, as are results of laboratory analyses of a few water samples for specific conductance, salinity, and concentrations of dissolved chloride and dissolved solids. NJDEP personnel worked with those of the USGS to make the some of the water-quality measurements.

\section{DESCRIPTION OF THE STUDY AREA}

The Toms and Metedeconk Rivers are tributaries to the Barnegat Bay estuarine system in eastern New Jersey (fig. 1). Each river consists of an upland, nontidal reach and a downstream, tidal embayment. 


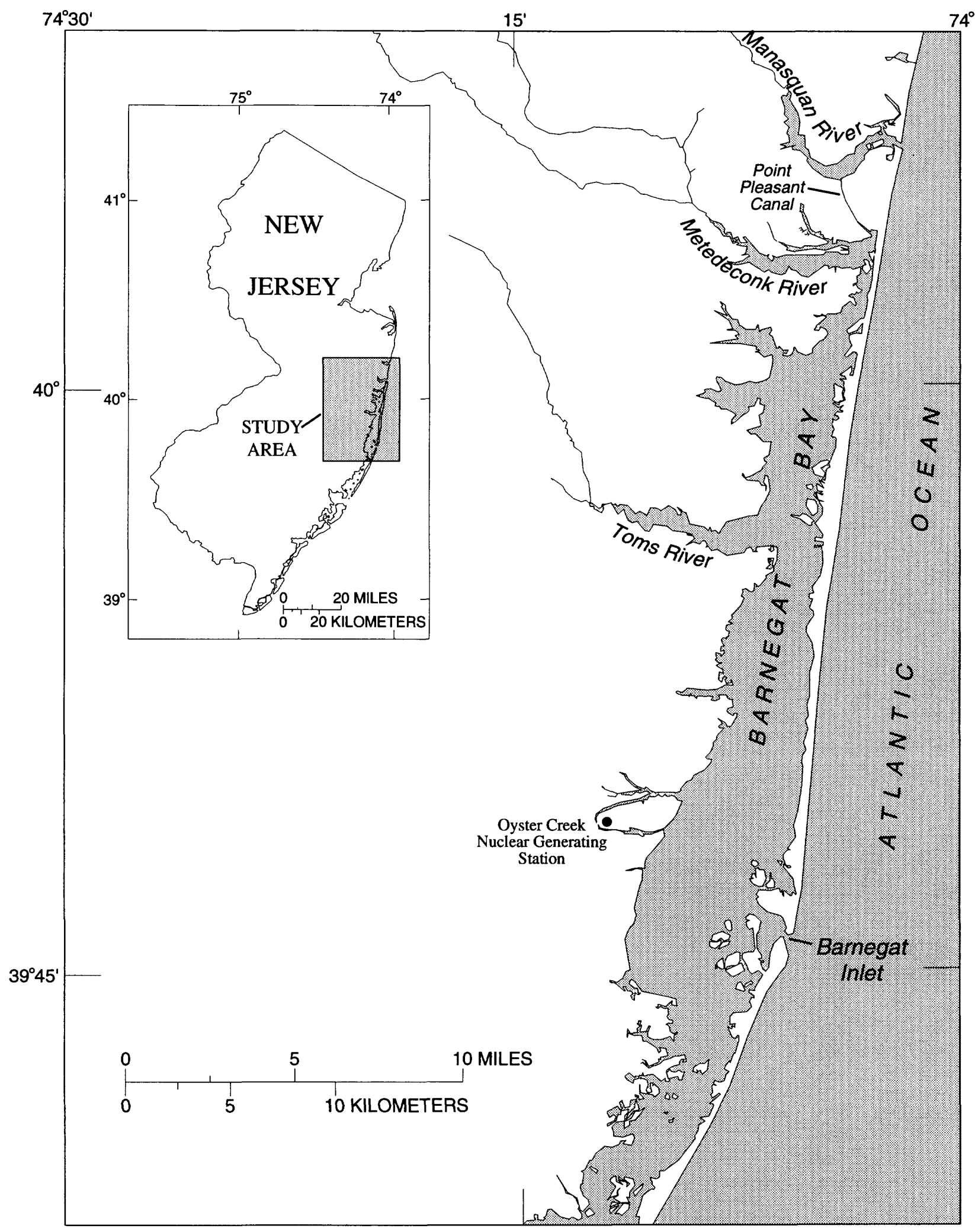

Figure 1. Locations of Barnegat Bay and the Metedeconk and Toms Rivers, New Jersey. 
For the purposes of this report, each tidal embayment extends from the head of tide to the mouth of the river (figs. 2 and 3). Generally, the embayments have an average depth of about 5 feet (U.S. Department of Commerce, 1986) and a mean range of tide of about 0.5 feet (U.S. Department of Commerce, 1988). The heads of tide were taken from New Jersey State tidelands maps (State of New Jersey, undated a; State of New Jersey, undated b).

The tidal embayment of the Metedeconk River is about 6 miles long and has a maximum width of about 0.7 miles (fig. 2). The drainage area at the mouth, 79 square miles, was determined from 1:24,000-scale maps by use of a planimeter; the mouth of the Metedeconk River as designated in this report is different from that in Velnich (1984). Surface water is withdrawn by the Brick Township Municipal Utilities Authority just downstream from the head of tide.

The tidal embayment of the Toms River is 7 miles long and has a maximum width of about 1 mile at its mouth (fig. 3). The drainage area at the mouth is 192 square miles (Velnich, 1984).

\section{FIELD AND LABORATORY METHODS}

Surface-water stations in and near the tidal embayment of the Metedeconk River at which streamflow, tidal-water-level, and water-quality measurements were made are listed in table 1 and shown in figure 2; stations in and near the tidal embayment of the Toms River are listed in table 2 and shown in figure 3 . The types of measurements made at each station and the number of the appendix in which the measurements are shown are given in tables 3 (Metedeconk River) and 4 (Toms River).

All discrete values of time presented in this report are in local time. This is Eastern Standard Time during winter and Eastern Daylight Savings Time during summer.

All discrete and continuous measurements discussed and presented in this report are available in electronic form from computerized data bases at the USGS, New Jersey District, office in West Trenton, N.J. Discrete measurements of water-quality characteristics, stage, and tidal-water level are in the NWIS WATER-QUALITY FILE data base. Continuous measurements of streamflow, tidal-water level, and water-quality characteristics are in the NWIS ADAPS data base.

\section{Streamflow}

Three types of streamflow information are presented in this report: daily mean values, measured discrete values, and estimated discrete values. All streamflow measurements presented in this report were determined at nontidal stations on tributaries to the tidal embayments. The techniques used to measure and determine streamflow are given in Rantz and others (1982).

Daily mean streamflow data for the period October 1992 - September 1994 (water years 1993-94) are presented for three continuous-record stations. For each station, values were calculated from (1) a record of continuous, 15-minute measurements of stage and (2) relations between measured values of streamflow and stage. Stage is the elevation of the surface of the 


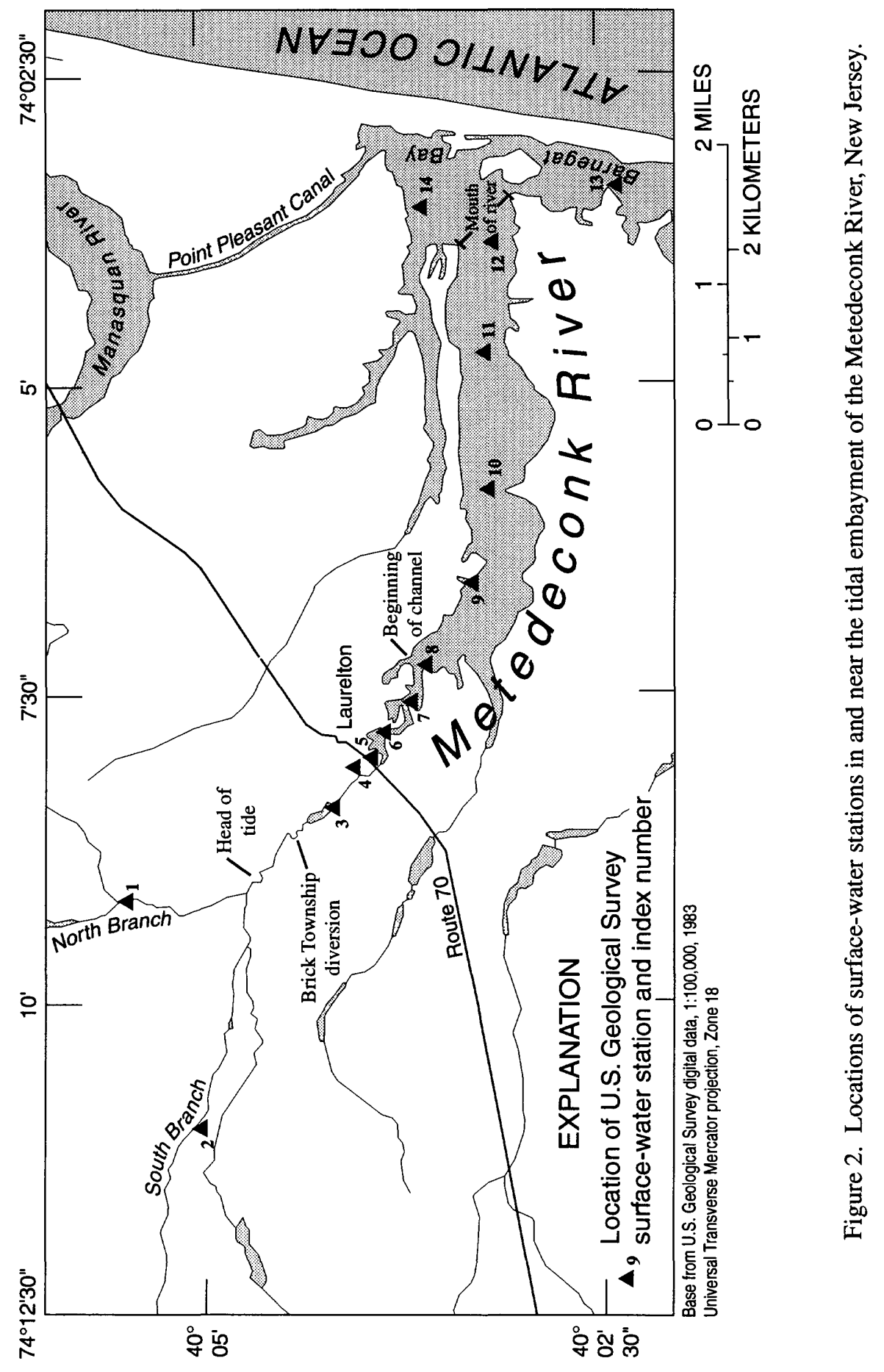




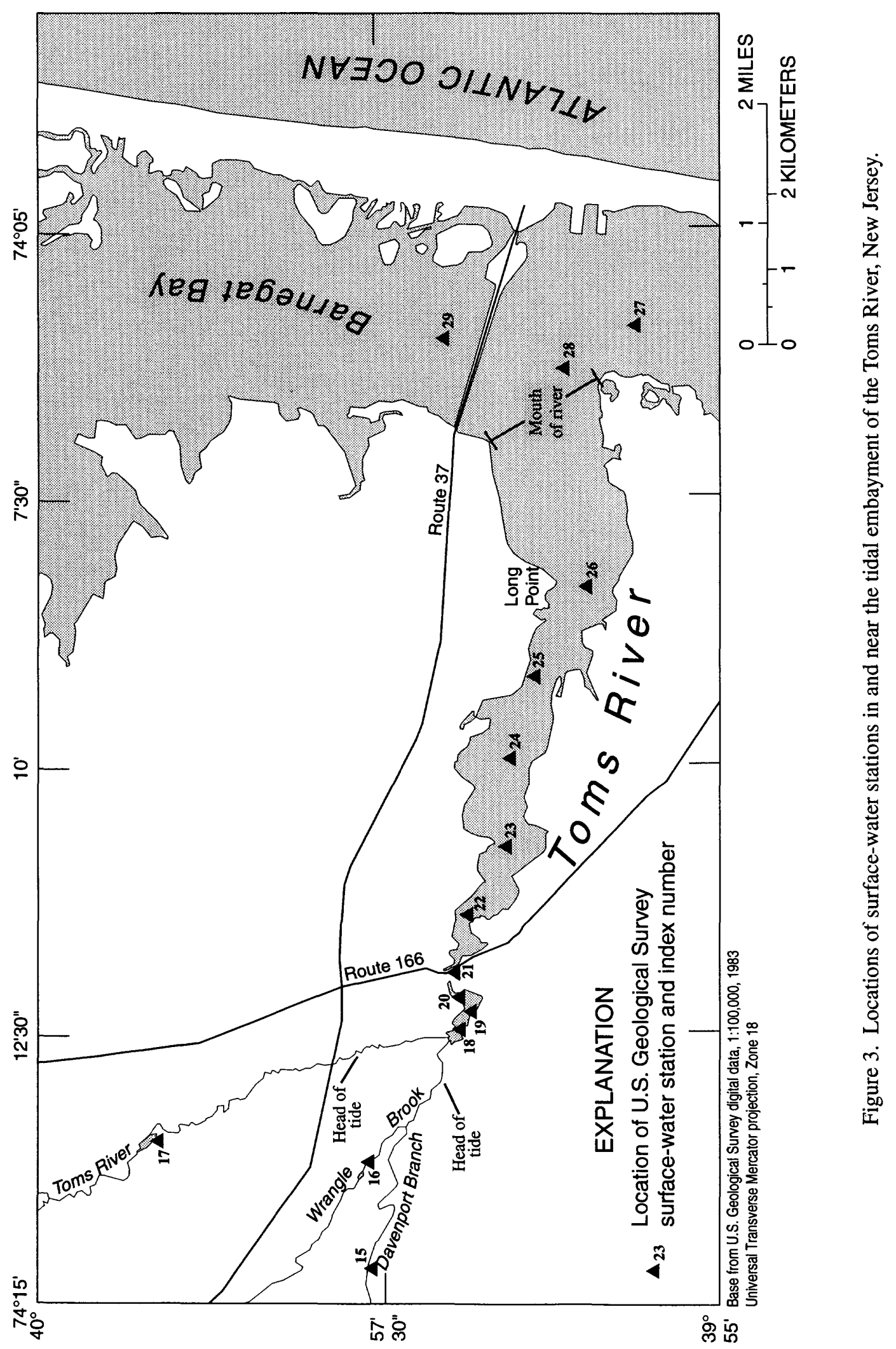




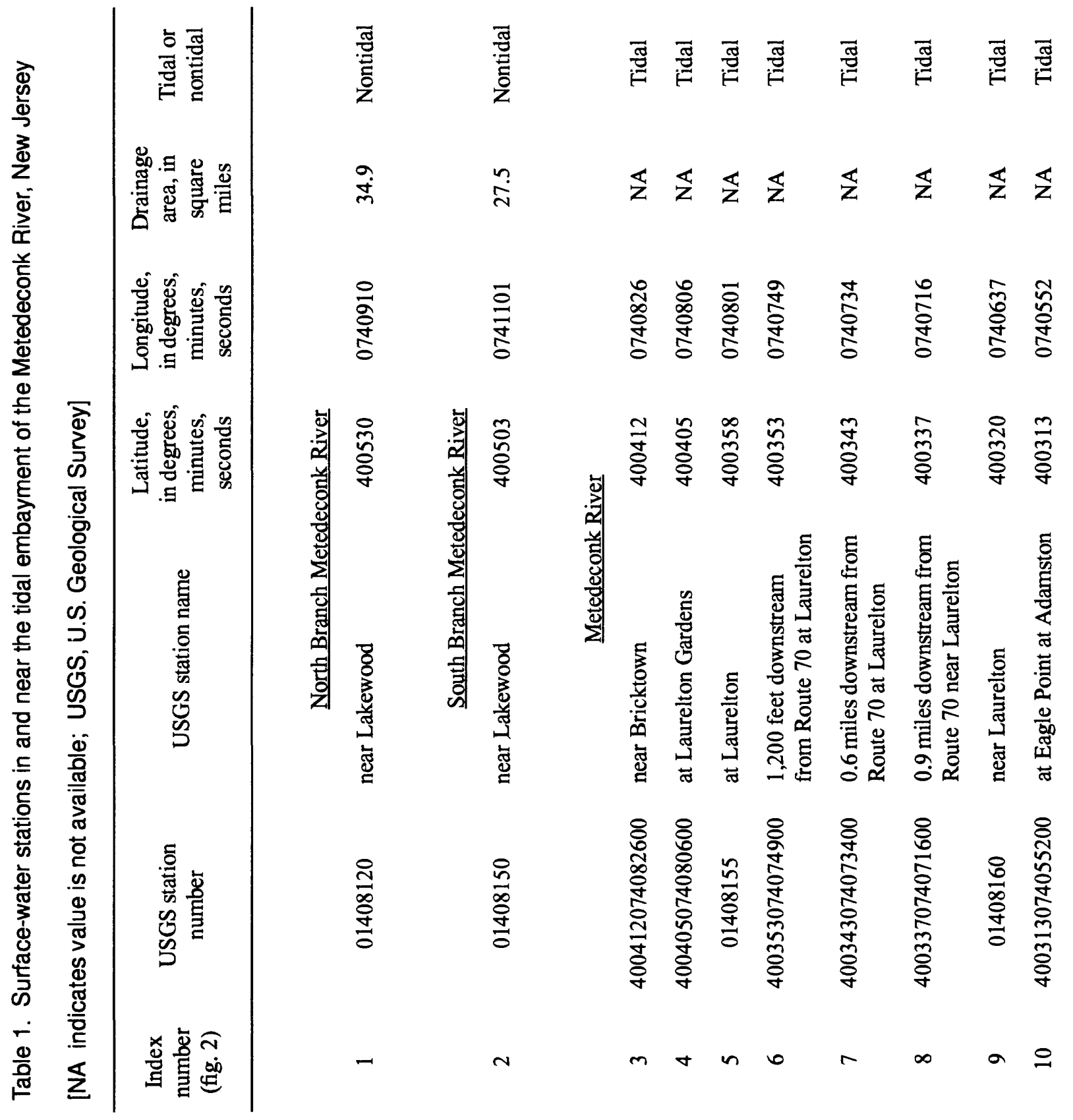




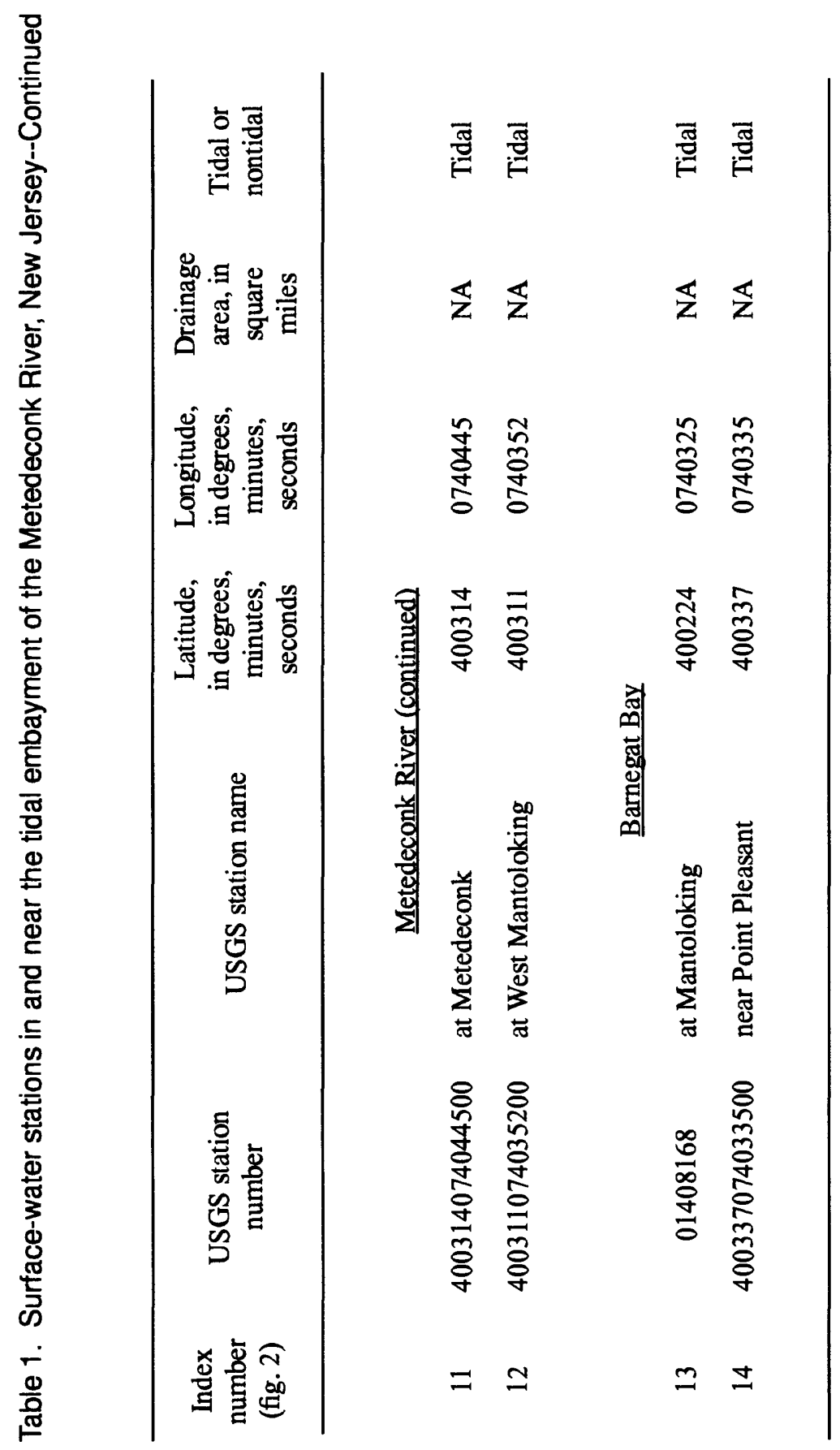




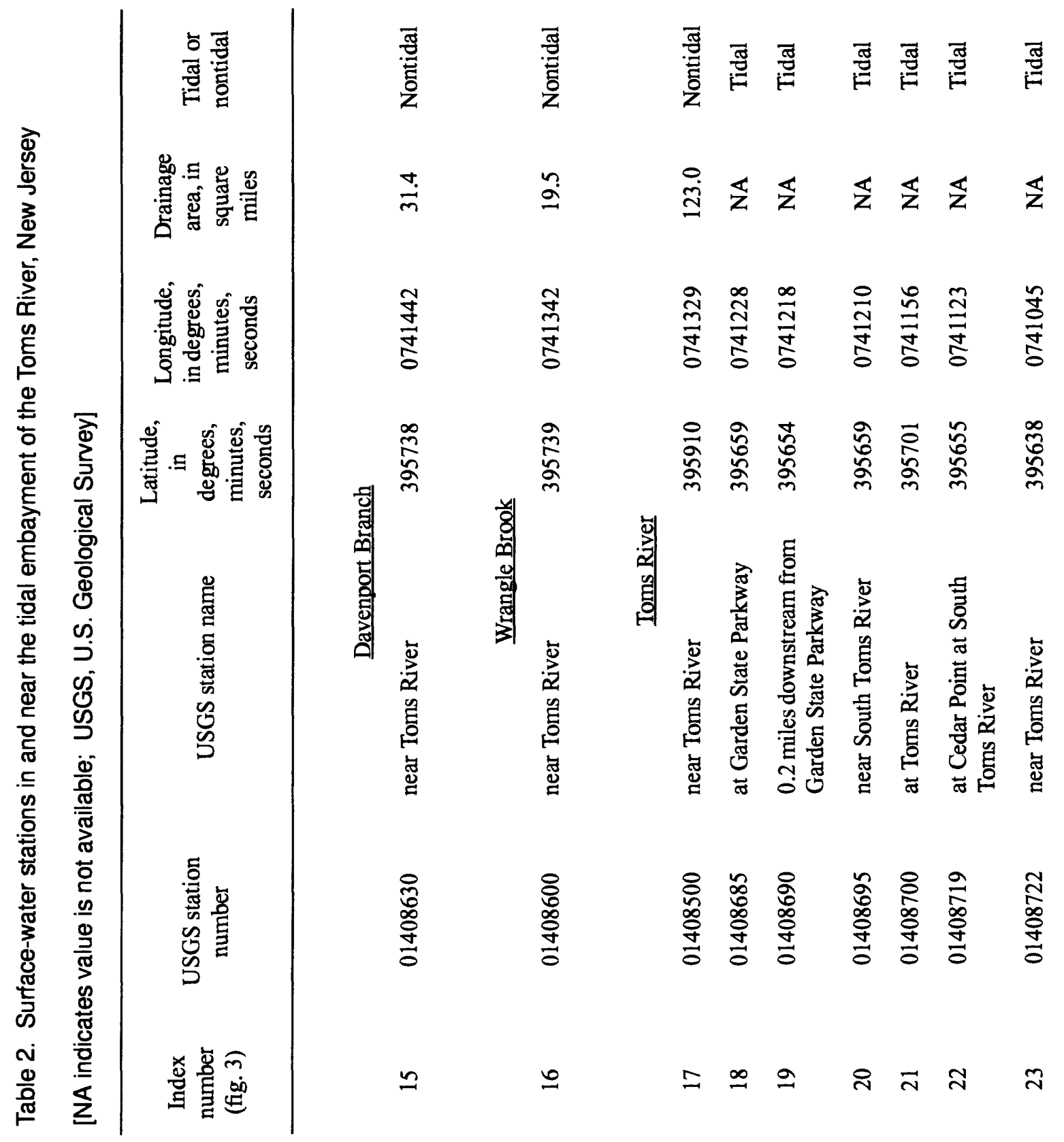




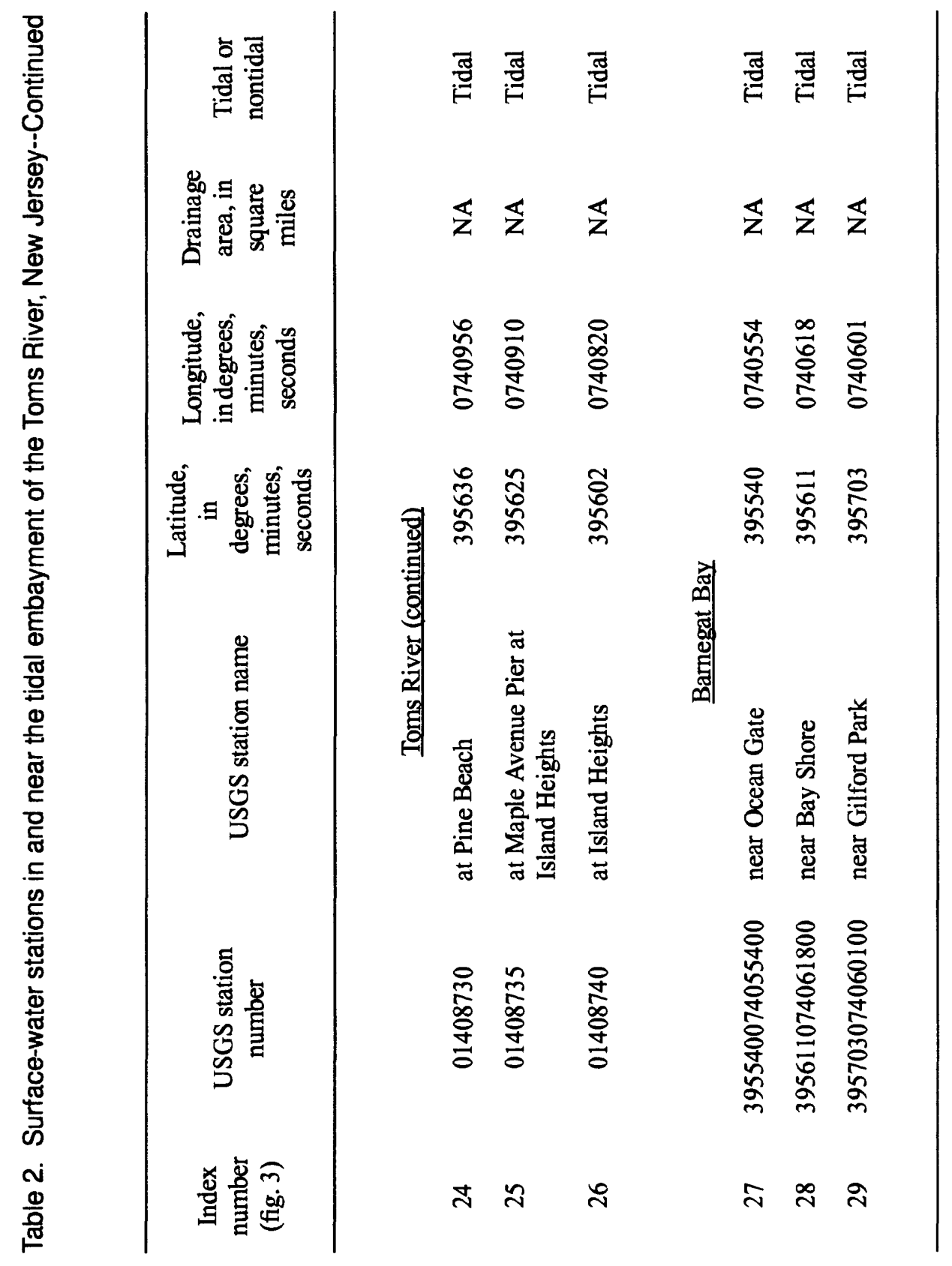




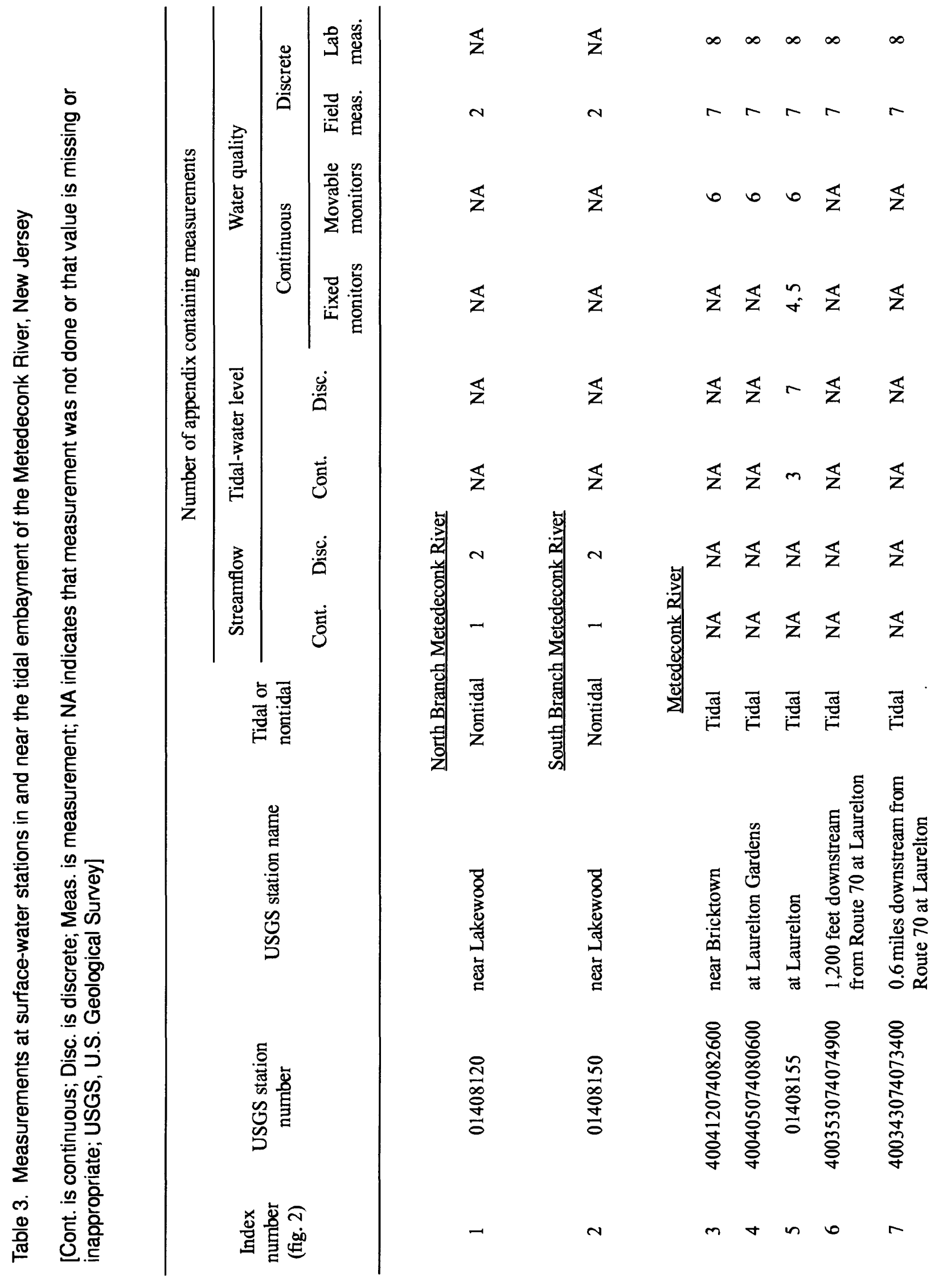




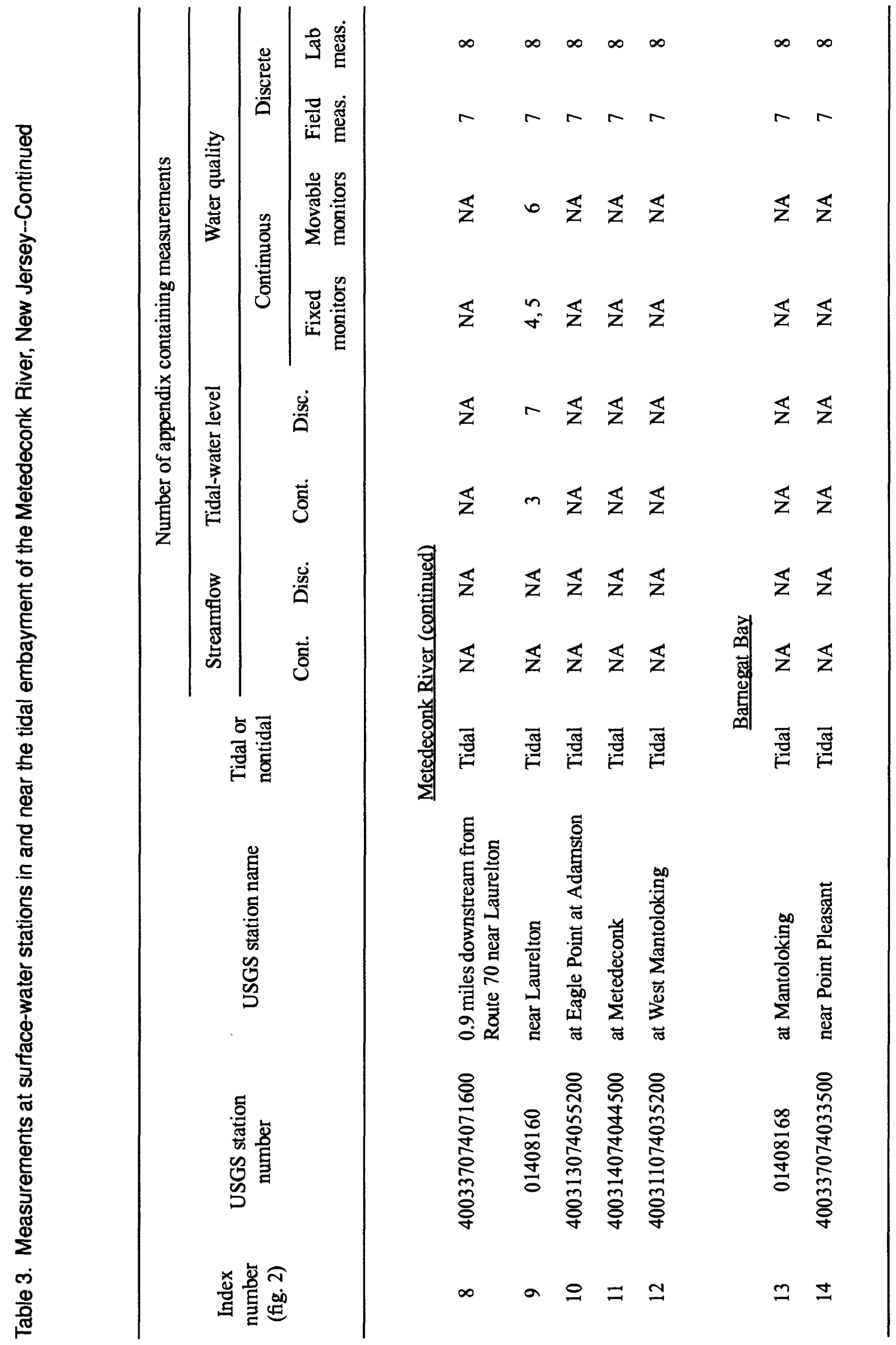




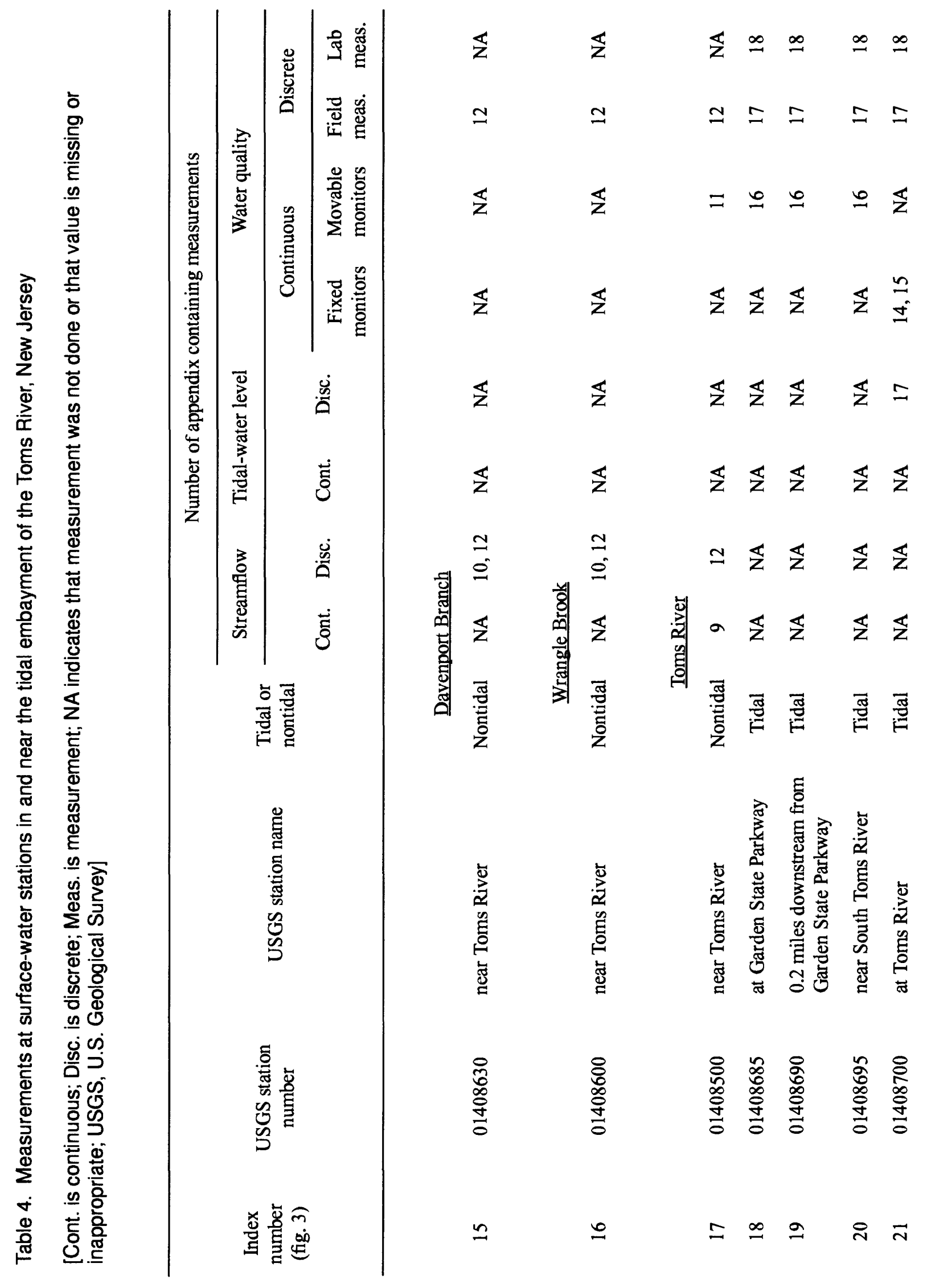




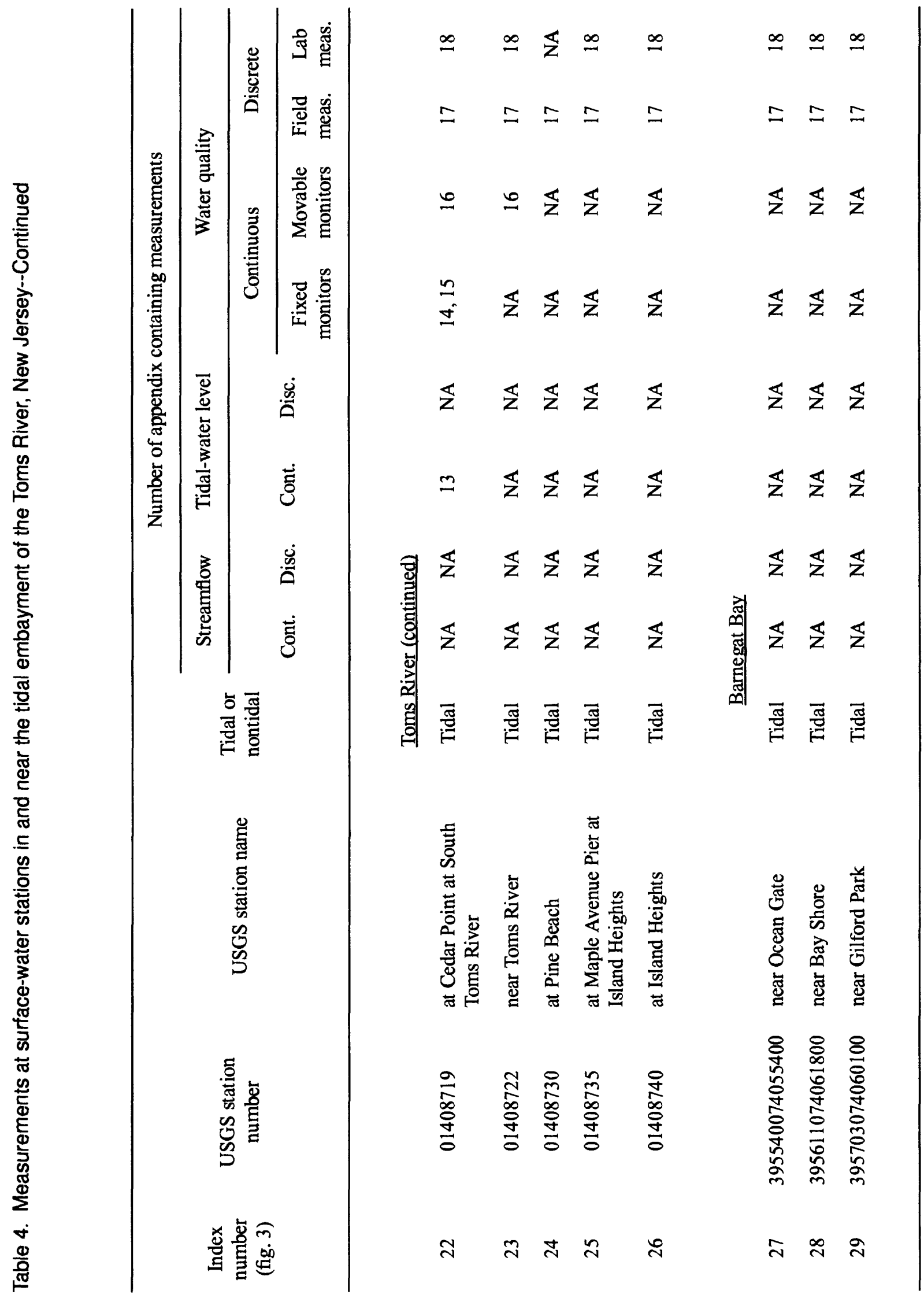


stream above a local datum. Values of streamflow at the two continuous-record stations on tributaries to the tidal embayment of the Metedeconk River, 01408120, North Branch Metedeconk River near Lakewood, and 01408150, South Branch Metedeconk River near Lakewood, are given in appendix 1. Values of streamflow at the one continuous-record station on a tributary to the tidal embayment of the Toms River, 01408500 , Toms River near Toms River, are given in appendix 9.

Discrete streamflow measurements were made during the period October 1992 - August 1993 at the following partial-record stations on tributaries to the tidal embayment of the Toms River: 01408600, Wrangle Brook near Toms River, and 01408630, Davenport Branch near Toms River. These values are given in appendix 10. Partial-record stations are those at which discrete values of streamflow have been measured, but for which a continuous record of streamflow is not available.

Estimated values of discrete streamflow were made at all the above stations for the times of water-quality measurement during the period October 1992 - October 1993. Estimates were calculated from stage at the time of water-quality measurements and relations between measured values of stage and streamflow. The values for 01408120, North Branch Metedeconk River near Lakewood, and 01408150, South Branch Metedeconk River near Lakewood, are given in appendix 2. The values for 01408500, Toms River near Toms River; 01408600, Wrangle Brook near Toms River; and 01408630, Davenport Branch near Toms River, are given in appendix 12.

\section{Tidal-Water Level}

Continuous records of tidal-water level were obtained from measurements at 15-minute intervals during October 1992 - November 1993 at one station on each tidal embayment by using standard USGS methods (Rantz and others, 1982). Only daily maximum and minimum values are presented in this report. Measurements made at 01408160, Metedeconk River near Laurelton, are given in appendix 3; measurements made at 01408719, Toms River at Cedar Point at South Toms River, are given in appendix 13. All values of tidal-water level in the report are given in feet above the National Geodetic Vertical Datum of 1929 (NGVD of 1929).

Discrete measurements of tidal-water level were made at 01408155 , Metedeconk River at Laurelton; 01408160, Metedeconk River near Laurelton; and 01408700, Toms River at Toms River, at the times of water-quality measurements during the period October 1992 - October 1993. The values for 01408155, Metedeconk River at Laurelton, and 01408160, Metedeconk River near Laurelton, are given in appendix 7; the values for 01408700 , Toms River at Toms River, are given in appendix 17.

\section{Water Ouality}

The terms "left" and "right" are used when describing the locations of some water-quality measurements. These are the directions when looking downstream, and are not used when referring to measurements in Barnegat Bay; the downstream direction in the bay is ambiguous. 


\section{Field Measurements}

The results of three types of water-quality measurements are presented. Continuous records of temperature and specific conductance were obtained from measurements by fixed monitors. Continuous records of temperature, specific conductance, $\mathrm{pH}$, and dissolved-oxygen concentration were obtained from measurements by movable water-quality monitors. Discrete, manual measurements were made of temperature, specific conductance, $\mathrm{pH}$, dissolved-oxygen concentration, and Secchi-disk depth, a measure of the transparency of the water.

Although measurements of salinity are not presented in this report, salinity can be calculated from values of specific conductance. One method to do this is presented in Miller and others (1988); other methods exist. The relation presented in Miller and others (1988) is given in equation 1 and shown in figure 4.

$$
\text { Salinity }=\left(\frac{0.4741}{\left.\left((0.001 \times S C)^{-1.07}\right)-\left(0.7464 \times 10^{-3}\right)\right)}\right.
$$

where Salinity is in parts per thousand, and

$S C$ is specific conductance in microsiemens per centimeter at 25 degrees Celsius.

\section{Continuous records from fixed monitors}

On each tidal embayment, temperature and specific conductance were continuously measured by fixed monitors at two stations. Values were recorded every 15 minutes by using USGS minimonitor probes connected to a Campbell Scientific CR-10 data logger ${ }^{1}$.

In the tidal embayment of the Metedeconk River, measurements were made during October 1992 - January 1994 at 01408155 , Metedeconk River at Laurelton (probe at mid-depth), and during October 1992 - October 1993 at 01408160 , Metedeconk River near Laurelton (probes near surface and bottom). In the tidal embayment of the Toms River, measurements were made during October 1992 - January 1994 at 01408700 , Toms River at Toms River (probe at middepth), and during December 1992 - October 1993 at 01408719, Toms River at Cedar Point at South Toms River (probes near surface and bottom).

On each embayment, the upstream monitor measured temperature and specific conductance near the middepth of the water column; the downstream monitor measured temperature and specific conductance near the top and the bottom of the water column. Although the water level varied over time, the depths at all four stations usually were about 5 to 7 feet. Therefore, the mid-depth, near-surface, and near-bottom probes can be assumed to have been about 3 feet, 1 foot, and 6 feet below the surface, respectively.

Daily minimum and maximum values are presented in this report. Temperature and specific-conductance data for the stations in the tidal embayment of the Metedeconk River are

\footnotetext{
${ }^{1}$ The use of brand or company names in this report is for identification purposes only and does not constitute endorsement by the U.S. Geological Survey.
} 


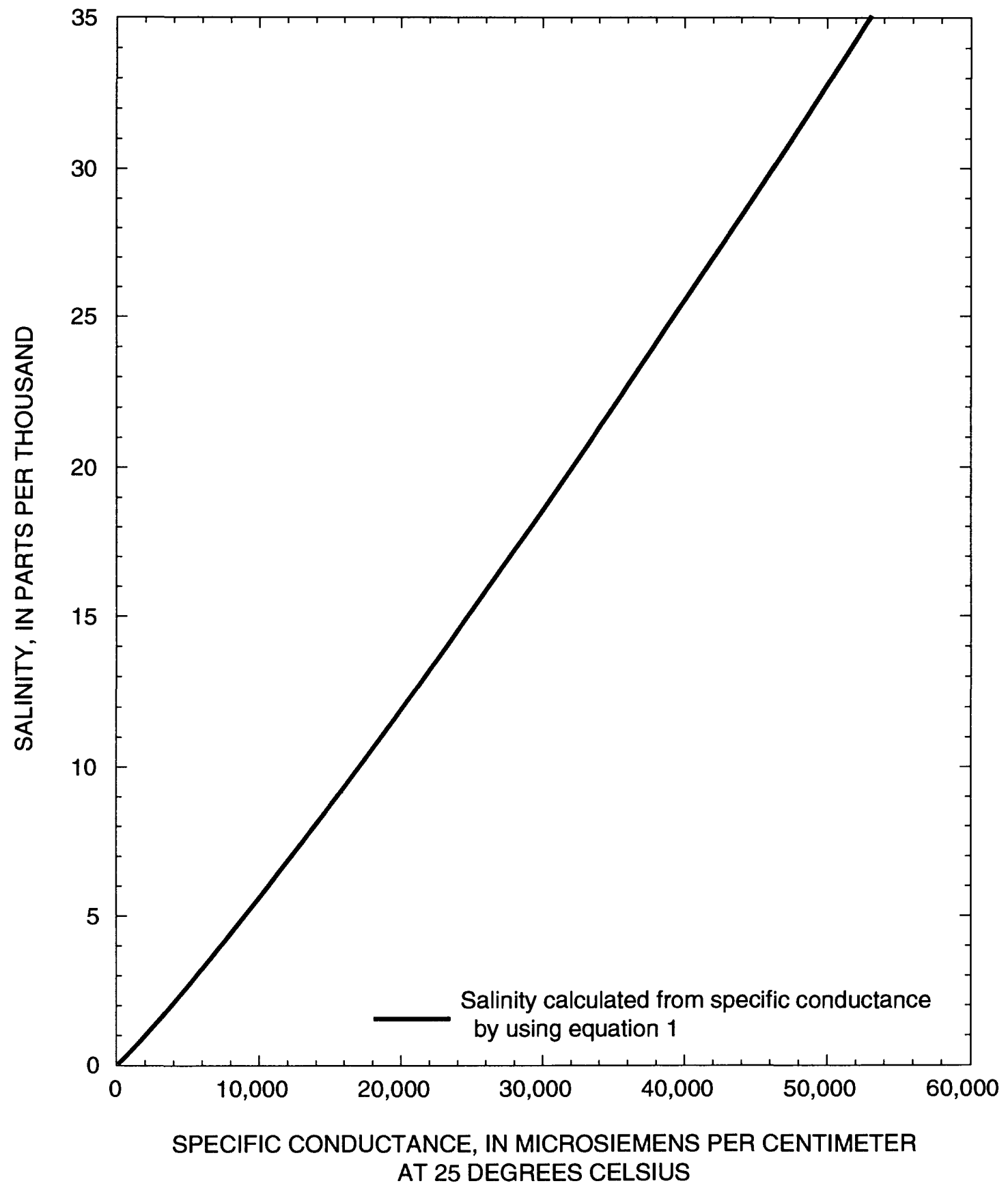

Figure 4. A relation between salinity and specific conductance. 
given in appendixes 4 and 5, respectively. Temperature and specific-conductance data for the stations in the tidal embayment of the Toms River are given in appendixes 14 and 15 , respectively.

\section{Continuous records from movable monitors}

Hydrolab Datasonde II and Datasonde III water-quality monitors were deployed for periods of about a week to about a month to make short-term water-quality measurements at selected stations. The intervals between measurements were 15 to 60 minutes. Both types of monitors were used to measure and record temperature and specific conductance; Datasonde III's were also used to measure and record $\mathrm{pH}$ and dissolved-oxygen concentration. Water-quality measurements were made at middepth, near the surface, and (or) near the bottom of the water column; the maximum depth at which measurements were made was about 6 feet.

In the tidal embayment of the Metedeconk River, monitors were deployed at the following stations: 400412074082600, Metedeconk River near Bricktown; 400405074080600, Metedeconk River at Laurelton Gardens; and 01408160, Metedeconk River near Laurelton. In the tidal embayment of the Toms River, monitors were deployed at the following stations: 01408685, Toms River at Garden State Parkway; 01408690, Toms River 0.2 miles downstream from Garden State Parkway; 01408695, Toms River near South Toms River; and 01408722, Toms River near Toms River. A monitor was also deployed at 01408500, Toms River near Toms River, a nontidal station on a tributary to the tidal embayment of the Toms River.

Only daily minimum and maximum values of water-quality data are presented in this report. Daily minimum and maximum values measured at tidal stations in and near the tidal embayment of the Metedeconk River are given in appendix 6. Daily minimum and maximum values measured in and near the tidal embayment of the Toms River are given in appendixes 11 (the nontidal station) and 17 (tidal stations).

\section{Manual measurements at nontidal stations on tributaries}

Discrete water-quality measurements were made during October 1992 - October 1993. Generally, measurements were made 1 day per month during the winter and 2 days per month during the summer, usually on days on which measurements were also made at stations in the downstream embayment. Measurements on tributaries to the tidal embayment of the Metedeconk River were made at 01408120, North Branch Metedeconk River near Lakewood, and 01408150, South Branch Metedeconk River near Lakewood. Station 01408150, South Branch Metedeconk River near Lakewood, was measured just downstream from the dam at the station. Measurements on tributaries to the tidal embayment of the Toms River were made at 01408500, Toms River near Toms River; 01408600, Wrangle Brook near Toms River; and 01408630, Davenport Branch near Toms River.

The streamflow at each station was well mixed, and the water quality at each station was represented by one measurement made in the middle of the stream. Each water-quality measurement includes the following water-quality characteristics and associated values: (1) stage; (2) streamflow; (3) temperature, specific conductance, $\mathrm{pH}$, and dissolved-oxygen concentration in 
milligrams per liter measured with a Hydrolab Surveyor II; (4) barometric pressure; and (5) calculated dissolved-oxygen concentration as a percent of saturation. Streamflow was estimated from measured stage and relations between stage and streamflow. All values of specific conductance were approximately 100 to 200 microsiemens per centimeter at 25 degrees Celsius; these values are reported as " $<200$ microsiemens per centimeter at 25 degrees Celsius" because the Hydrolab Surveyor II was calibrated for saline water. The barometric pressure on each day of water-quality measurement was either measured with a barometer or obtained from GPU Nuclear Corporation in Forked River, N.J. (Paul Schwartz, GPU Nuclear Corporation, written commun., 1993, 1994); GPU Nuclear Corporation measured barometric pressure at the Oyster Creek Nuclear Generating Station (fig. 1).

Values of dissolved-oxygen concentration as a percent of saturation were calculated by use of the following equation:

$$
D O_{P E R C}=(D O / D O S A T) \times 100
$$

where $D O_{P E R C}$ is dissolved-oxygen concentration as a percent of saturation, $D O$ is dissolved-oxygen concentration in milligrams per liter, and $D O S A T$ is dissolved-oxygen concentration at saturation in milligrams per liter.

Values of dissolved-oxygen concentration at saturation for a barometric pressure of 760 millimeters of mercury were calculated from a method presented by R.J. Pickering (U.S. Geological Survey, written commun., 1981). Figure 5 shows the variation in dissolvedoxygen concentration at saturation with temperature for both freshwater and seawater. The method is presented in equations 3 through 7 .

$$
\begin{aligned}
& \text { DOSAT } T^{760}=1.4276 \times\left(2.7183^{(A+(S \times B))}\right) \\
& A=(-173.4292)+(249.6339 \times K)+(143.3484 \times \ln (1 / K)) \\
& S=\left(\left(5.572 \times 10^{-4}\right) \times S C\right)+\left(\left(\begin{array}{l}
(21.8492 / K) \\
\left.\left.2.02 \times 10^{-9}\right) \times S C^{2}\right)
\end{array}\right.\right. \\
& B=-0.033096+(0.014259 / K)-\left(0.001700 / K^{2}\right) \\
& K=(100 /(T+273.15))
\end{aligned}
$$

where $D O S A T^{760}$ is the dissolved-oxygen concentration at saturation for a barometric pressure of 760 milligrams per liter, in milligrams per liter;

$T$ is temperature in degrees Celsius;

$S C$ is specific conductance in microsiemens per centimeter at 25 degrees Celsius; and $\ln$ is natural $\log$. 


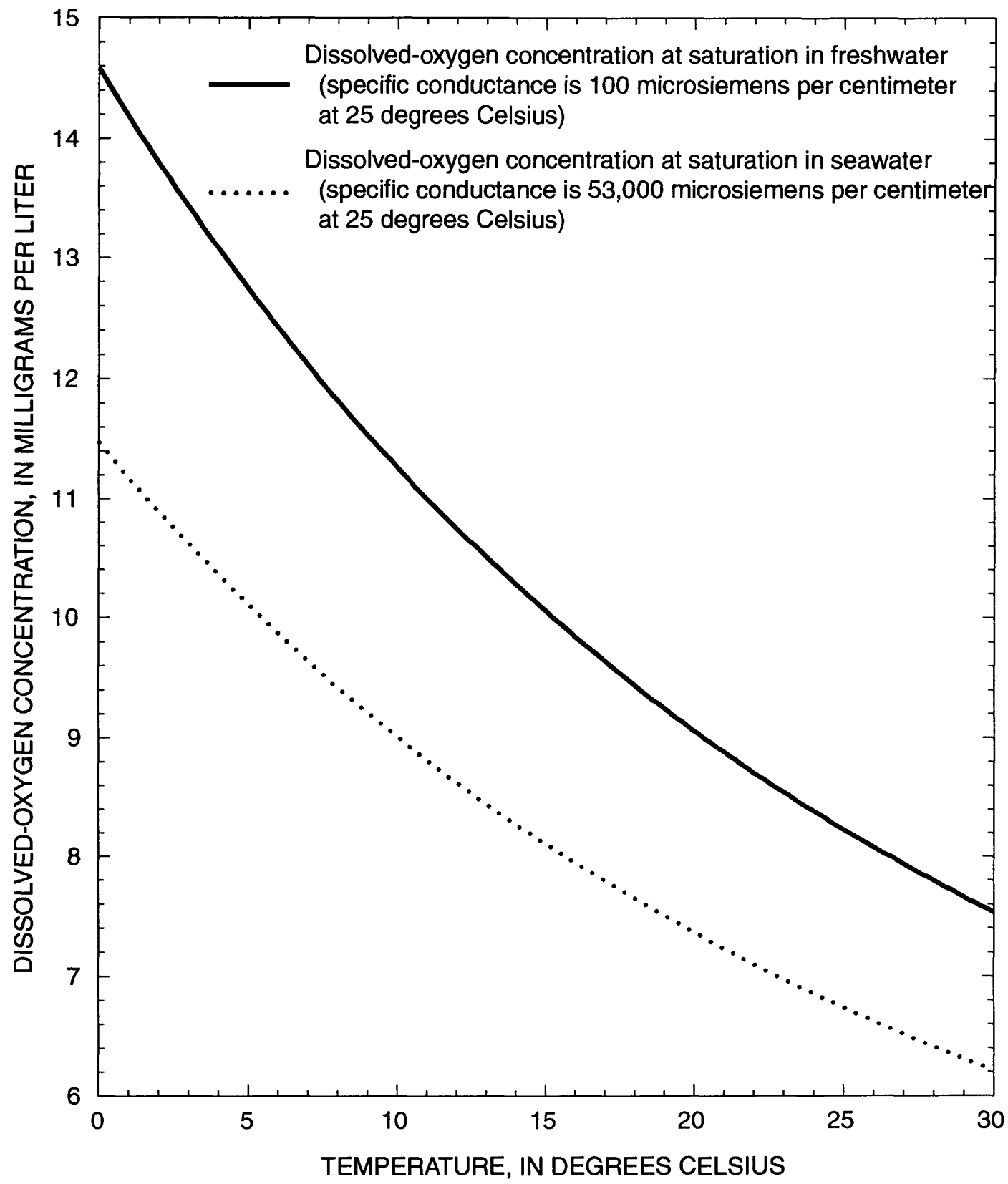

Figure 5. Relations between temperature and dissolved-oxygen concentration at saturation for freshwater and seawater. (Values were calculated for a barometric pressure of 760 millimeters of mercury by using equations 3-7. The specific conductance for seawater was estimated from equation 1 by assuming a salinity of 35 parts per thousand for seawater.) 
The dissolved-oxygen concentration at saturation for barometric pressures other than 760 millimeters of mercury also was determined by use of a method presented by R.J. Pickering (U.S. Geological Survey, written commun., 1981). This method is given in equations 8 and 9.

$$
\text { DOSAT } T^{B P}=\operatorname{DOSAT}^{760} \times((B P-u) /(760-u))
$$

where DOSAT $T^{760}$ is dissolved-oxygen concentration at saturation at a barometric pressure of 760 millimeters of mercury;

$D O S A T^{B P}$ is dissolved-oxygen concentration at saturation at a selected barometric pressure;

$B P$ is barometric pressure, in millimeters of mercury; and

$u$ is vapor pressure of water, in millimeters of mercury.

The vapor pressure, $u$, is calculated from equation 9:

$$
u=2.7183^{(8.10765-(1750.286 /(235+T)))}
$$

where $u$ is vapor pressure of water in millimeters of mercury, and $T$ is temperature in degrees Celsius.

Water-quality measurements made at nontidal stations on tributaries to the tidal embayment of the Metedeconk River are given in appendix 2. Water-quality measurements made at nontidal stations on tributaries to the tidal embayment of the Toms River are given in appendix 12.

\section{Manual measurements at tidal stations in and near the tidal embayments}

Measurements at tidal stations in and near the tidal embayments were generally made on 2 days per month during the summer and lday per month during the winter. Two longitudinal profiles usually were conducted on each day of measurement, one in the morning and one in the afternoon. Some measurements were made at each tidal station in and near the embayment of the Metedeconk River (table 3) and the Toms River (table 4).

Each water-quality measurement included the following water-quality characteristics and associated values: (1) depth of measurement and total depth; (2) distance from left bank; (3) temperature, specific conductance, $\mathrm{pH}$, and dissolved-oxygen concentration; (4) barometric pressure; (5) calculated dissolved-oxygen concentration as a percent of saturation; (6) Secchi-disk depth; and (7) tidal-water level. The distance from left bank is the ratio, in percent, of (1) the distance from left bank to the point of measurement, to (2) the distance from left to right bank. The distance from left bank was estimated from observations in the field and from charts. Most measurements were made in the center of the channel and designated with a value of " 50 ." Some values were measured near the left and right banks; these are designated as "10" and "90," respectively. Values are not given for samples at stations in Barnegat Bay; these stations were located in the navigation channel. Water-quality measurements made at stations in and near the tidal embayments of the Metedeconk and Toms River are given in appendixes 7 and 17, respectively. 
Most values of temperature, specific conductance, $\mathrm{pH}$, and dissolved-oxygen concentration in milligrams per liter were measured with a Hydrolab Surveyor II; some values were measured with either a Hydrolab Datasonde III or a Fisher Scientific digital specificconductance meter. Secchi-disk depth was measured with an 8-inch-diameter limnological disk. The barometric pressure on each day of water-quality measurement was either measured with a barometer or obtained from GPU Nuclear Corporation in Forked River, N.J. (Paul Schwartz, GPU Nuclear Corporation, written commun., 1993, 1994); GPU Nuclear Corporation measured barometric pressure at the Oyster Creek Nuclear Generating Station (fig. 1). Values of dissolvedoxygen concentration as a percent of saturation were calculated by use of methods previously described.

\section{Laboratory measurements of dissolved constituents}

A limited number of water samples was collected at tidal stations in and near the tidal embayments for laboratory determination of selected dissolved constituents. Samples were collected at nearly all tidal stations listed in tables 3 (Metedeconk River) and 4 (Toms River). Most samples were collected in October 1992; additional samples near the saltwater fronts were collected during the period December 1992 - October 1993. Water samples near the surface were collected by using either an open bottle or a Van Dorn-type sampler; samples near the bottom were collected by using a Van Dorn-type sampler.

Samples were analyzed by the State of New Jersey Department of Health and Department of Environmental Protection (Bureau of Marine Water Classification and Analysis) for specific conductance, salinity, and concentrations of dissolved chloride and dissolved solids; methods are given below. The values of salinity are similar to, but not exactly equivalent to, the values of salinity calculated by use of equation 1 . Results of laboratory analyses of water samples for dissolved constituents in and near the tidal embayments of the Metedeconk and Toms River are given in appendixes 8 and 18 , respectively.

\begin{tabular}{lll}
\hline \multicolumn{1}{c}{ Constituent } & \multicolumn{1}{c}{ Method of analysis } & \multicolumn{1}{c}{ Reference for method of analysis } \\
\hline Specific conductance & $\begin{array}{l}\text { U.S. Environmental Protection } \\
\text { Agency Method 120.1 } \\
\text { or }\end{array}$ & Kopp and McKee (1983) \\
& $\begin{array}{l}\text { Standard Methods (16th ed.) } \\
\text { Method 2510 B }\end{array}$ & $\begin{array}{l}\text { American Public Health Association } \\
(1985)\end{array}$ \\
& $\begin{array}{l}\text { American Public Health } \\
\text { Association Method 2520A }\end{array}$ & $\begin{array}{l}\text { American Public Health Association } \\
\text { Salinity }\end{array}$ \\
$\begin{array}{l}\text { American Public Health } \\
\text { Dissolved-chloride } \\
\text { concentration }\end{array}$ & $\begin{array}{l}\text { Association Method 407A } \\
\text { or }\end{array}$ & $\begin{array}{l}\text { American Public Health Association } \\
(1985)\end{array}$ \\
& $\begin{array}{l}\text { Standard Methods (17th ed.) } \\
\text { Method 4500-Cl B }\end{array}$ & $\begin{array}{l}\text { American Public Health Association } \\
(1989)\end{array}$ \\
$\begin{array}{l}\text { Dissolved-solids } \\
\text { concentration }\end{array}$ & U.S. Environmental Protection & Kopp and McKee (1983) \\
\hline
\end{tabular}




\section{REFERENCES CITED}

American Public Health Association, 1989, Standard methods for the examination of water and wastewater, 17th edition: Washington, D.C., p. 1-1 to 10-200 plus plates.

American Public Health Association, 1985, Standard methods for the examination of water and wastewater, 16th edition: Washington, D.C., 1268 p.

Kopp, J.F., and McKee, G.D., 1983, Methods for the chemical analysis of water and waste:

U.S. Environmental Protection Agency, Environmental Monitoring and Support Laboratory, Office of Research and Development, no. EPA-600/4-79-020, $490 \mathrm{p}$.

Miller, R.L., Bradford, W.L., and Peters, N.E., 1988, Specific conductance--theoretical considerations and application to analytical quality control: U.S. Geological Survey WaterSupply Paper 2311, 16 p.

Rantz, S.E., and others, 1982, Measurement and computation of streamflow, volumes 1 and 2:

U.S. Geological Survey Water-Supply Paper 2175, 631 p.

State of New Jersey, undated a, Areas new or formerly below mean high water, Toms River, Toms River: New Jersey Department of Environmental Protection, no. 406-2124, 1 pl.

State of New Jersey, undated b, Areas new or formerly below mean high water, Forge Pond west, Lakewood: New Jersey Department of Environmental Protection, no. 448-2124, 1 pl.

U.S. Department of Commerce, 1986, Nautical chart 12324, intracoastal waterway, Sandy Hook to Little Egg Harbor, New Jersey: Rockville, Maryland, National Oceanic and Atmospheric Administration, National Ocean Service, scale 1:40,000.

U.S. Department of Commerce, 1988, Tide tables, 1989, high and low water predictions, East Coast of North and South America: Rockville, Maryland, National Oceanic and Atmospheric Administration, National Ocean Service, 289 p.

Velnich, A.J., 1984, Drainage areas in New Jersey, Atlantic coastal basins, South Amboy to Cape May: U.S. Geological Survey Open-File Report 84-150, 33 p. 


\section{APPENDIXES}


Appendix 1. Measurements at nontidal stations on tributaries to the tidal embayment of the Metedeconk River, N.J.--Daily mean streamflow, October 1992 - September 1994

[Values of streamflow in cubic feet per second are presented for the continuous-record stations listed below. USGS, U.S. Geological Survey; NA, not applicable]

\begin{tabular}{ccc}
\hline $\begin{array}{c}\text { Index number } \\
\text { (fig. 2) }\end{array}$ & USGS station number & USGS station name \\
\hline 1 & 01408120 & $\begin{array}{c}\text { North Branch Metedeconk River } \\
\text { near Lakewood } \\
2\end{array}$ \\
01408150 & $\begin{array}{c}\text { South Branch Metedeconk River } \\
\text { near Lakewood }\end{array}$ \\
\hline
\end{tabular}




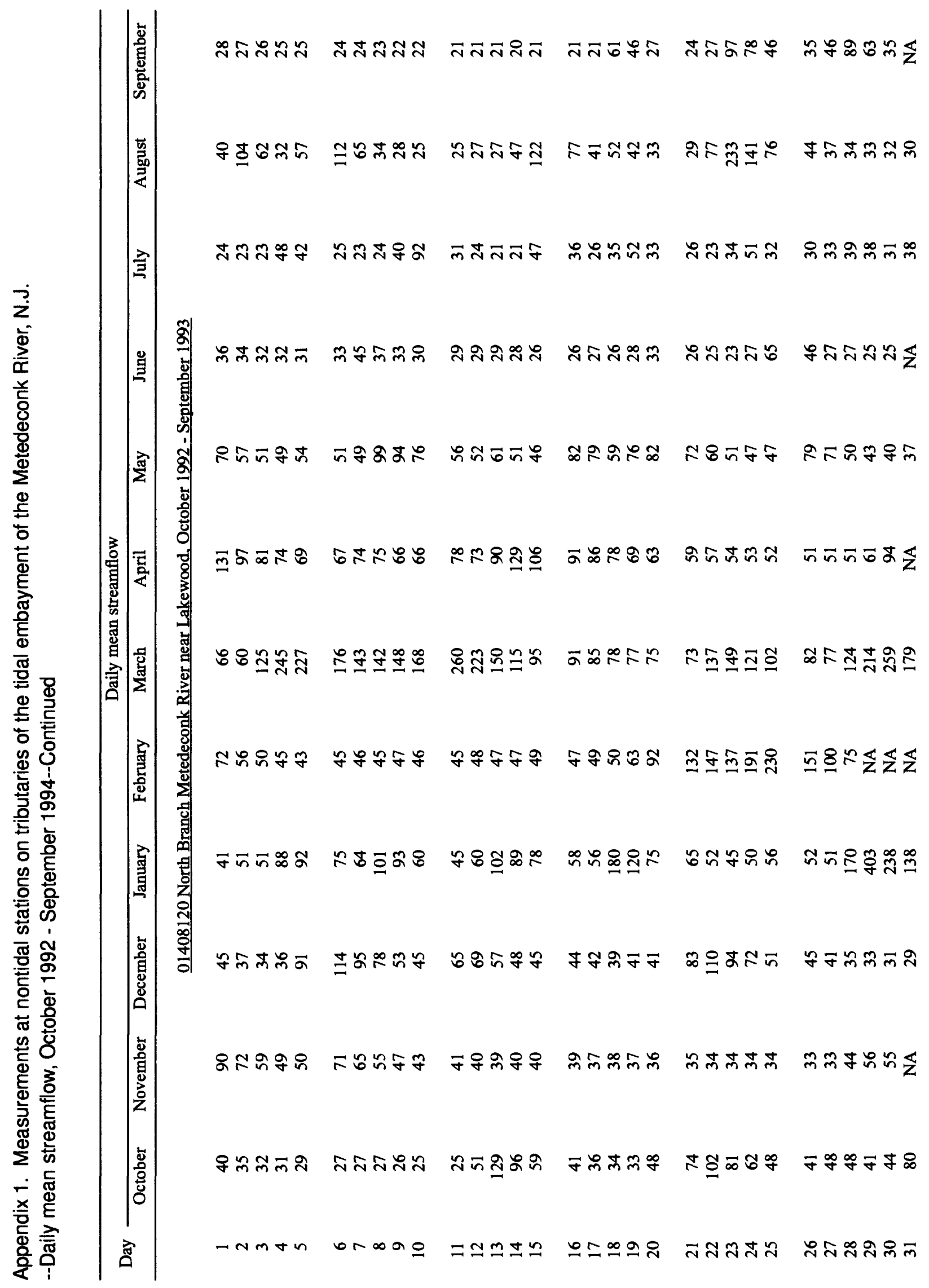




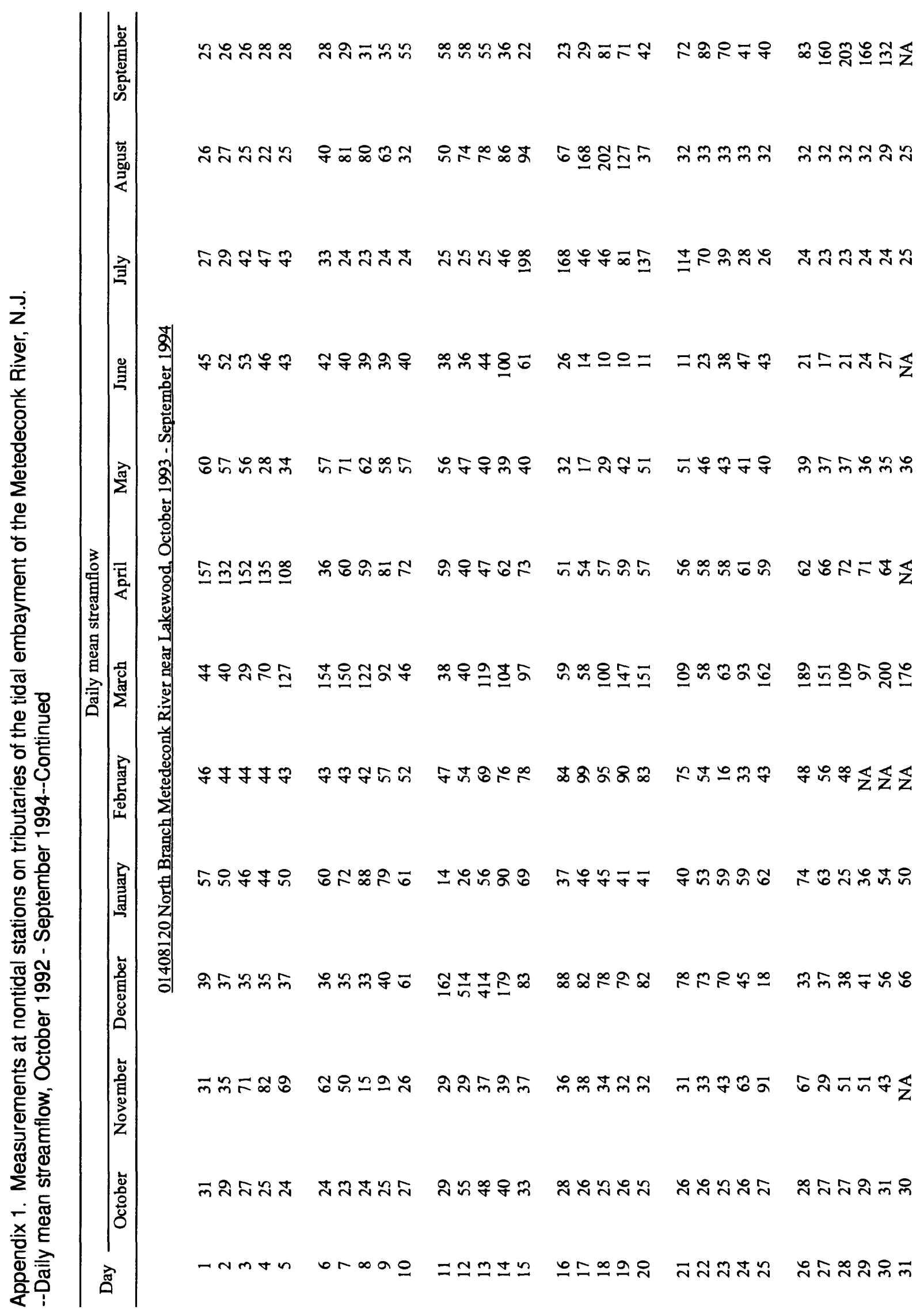




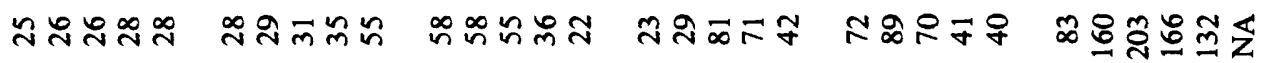

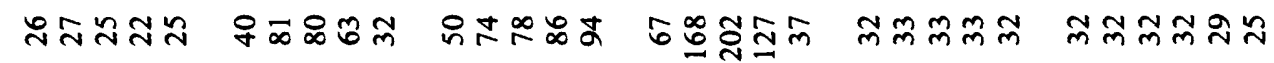

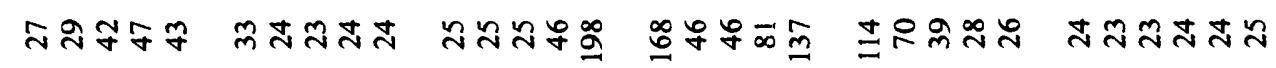

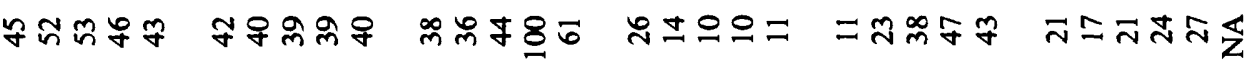

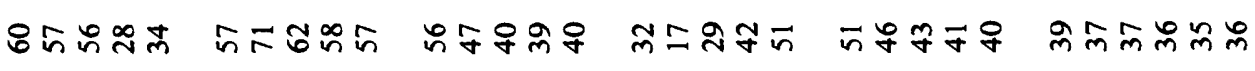

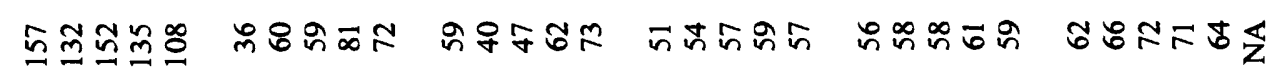

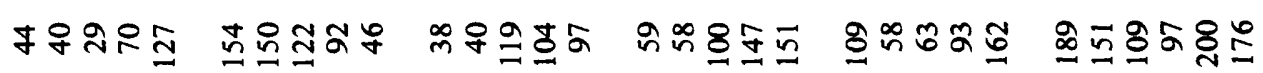

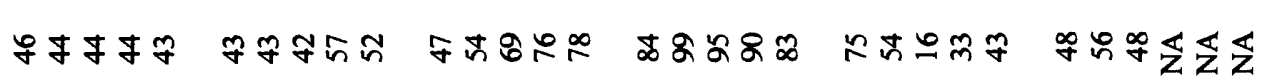

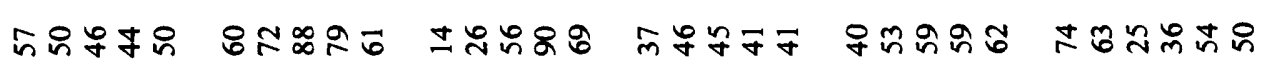

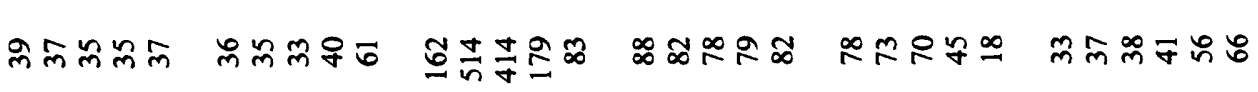

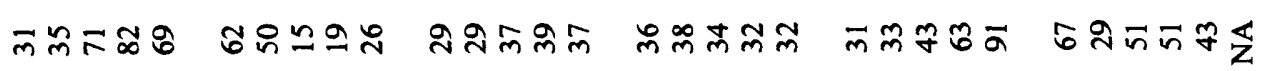

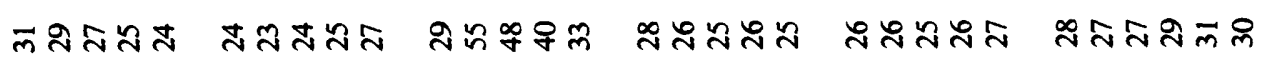

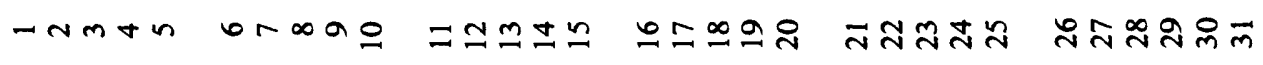




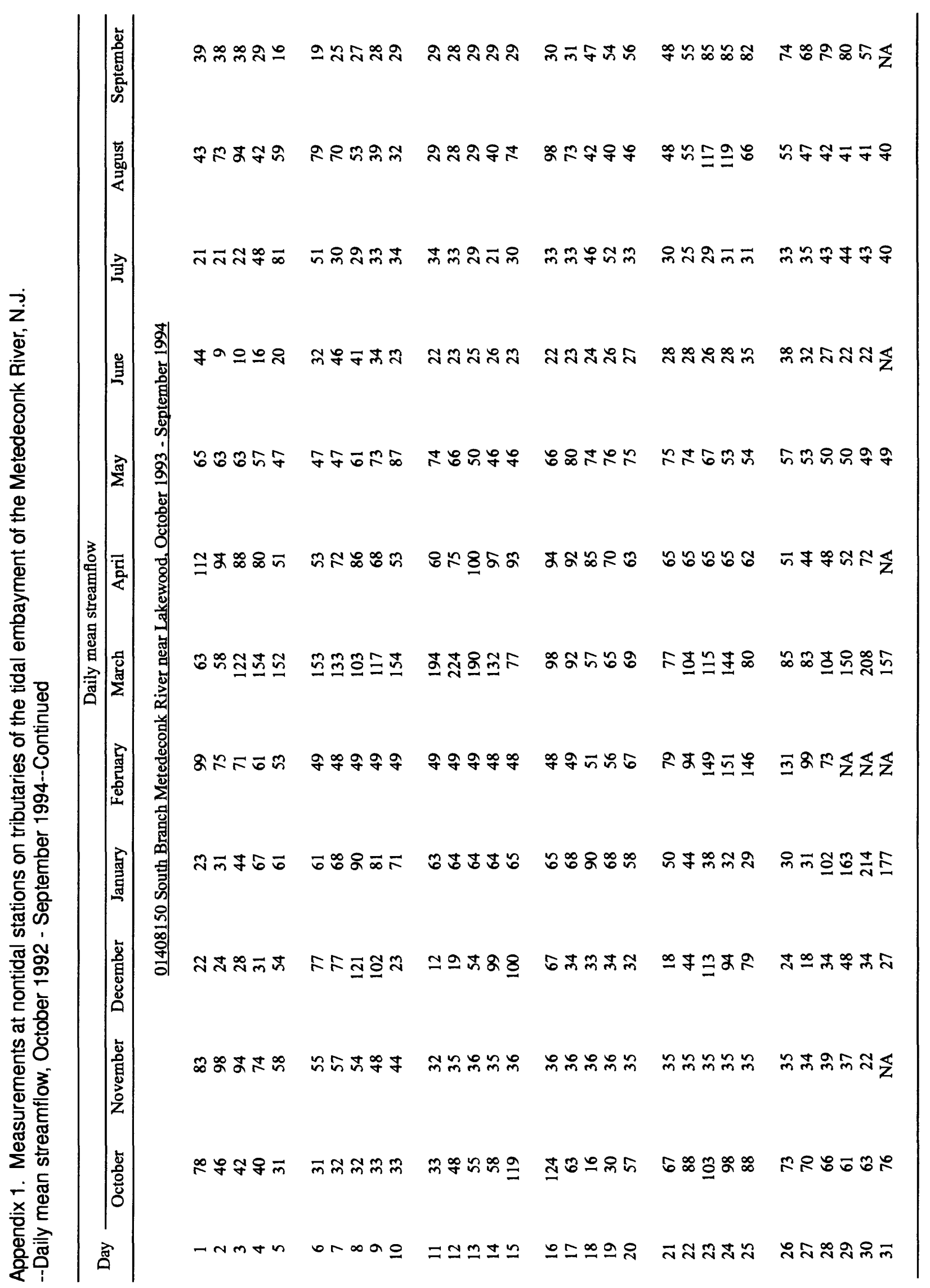


Appendix 2. Measurements at nontidal stations on tributaries to the tidal embayment of the Metedeconk River, N.J.--Water-quality data from manual measurements, October 1992 - October 1993

[Water-quality data are presented for the stations listed below. USGS, U.S. Geological Survey; NA, not applicable or not available; <, less than]

\begin{tabular}{ccc}
\hline $\begin{array}{c}\text { Index number } \\
\text { (fig. 2) }\end{array}$ & USGS station number & USGS station name \\
\hline 1 & 01408120 & $\begin{array}{c}\text { North Branch Metedeconk River } \\
\text { near Lakewood }\end{array}$ \\
2 & 01408150 & $\begin{array}{c}\text { South Branch Metedeconk River } \\
\text { near Lakewood }\end{array}$ \\
\hline
\end{tabular}

The following information is presented:

\begin{tabular}{lll}
\hline \multicolumn{1}{c}{ Characteristic } & \multicolumn{1}{c}{ Unit } & Rounding \\
\hline $\begin{array}{l}\text { Date and time of } \\
\text { measurement }\end{array}$ & NA & NA \\
$\begin{array}{l}\text { Stage } \\
\text { Streamflow }\end{array}$ & Feet above a local datum & 0.01 \\
$\begin{array}{l}\text { Temperature } \\
\text { pH }\end{array}$ & Cubic feet per second & 1 \\
$\begin{array}{l}\text { Dissolved-oxygen } \\
\text { concentration }\end{array}$ & Degrees Celsius & 0.5 \\
$\begin{array}{c}\text { Dissolved-oxygen } \\
\text { concentration as a percent } \\
\text { of saturation }\end{array}$ & Standard units & 0.1 \\
$\begin{array}{l}\text { Barometric pressure } \\
\text { Specific conductance }\end{array}$ & Milligrams per liter & 0.1 \\
& Millimeters of mercury & 1 \\
& Microsiemens per centimeter at & NA \\
\hline
\end{tabular}




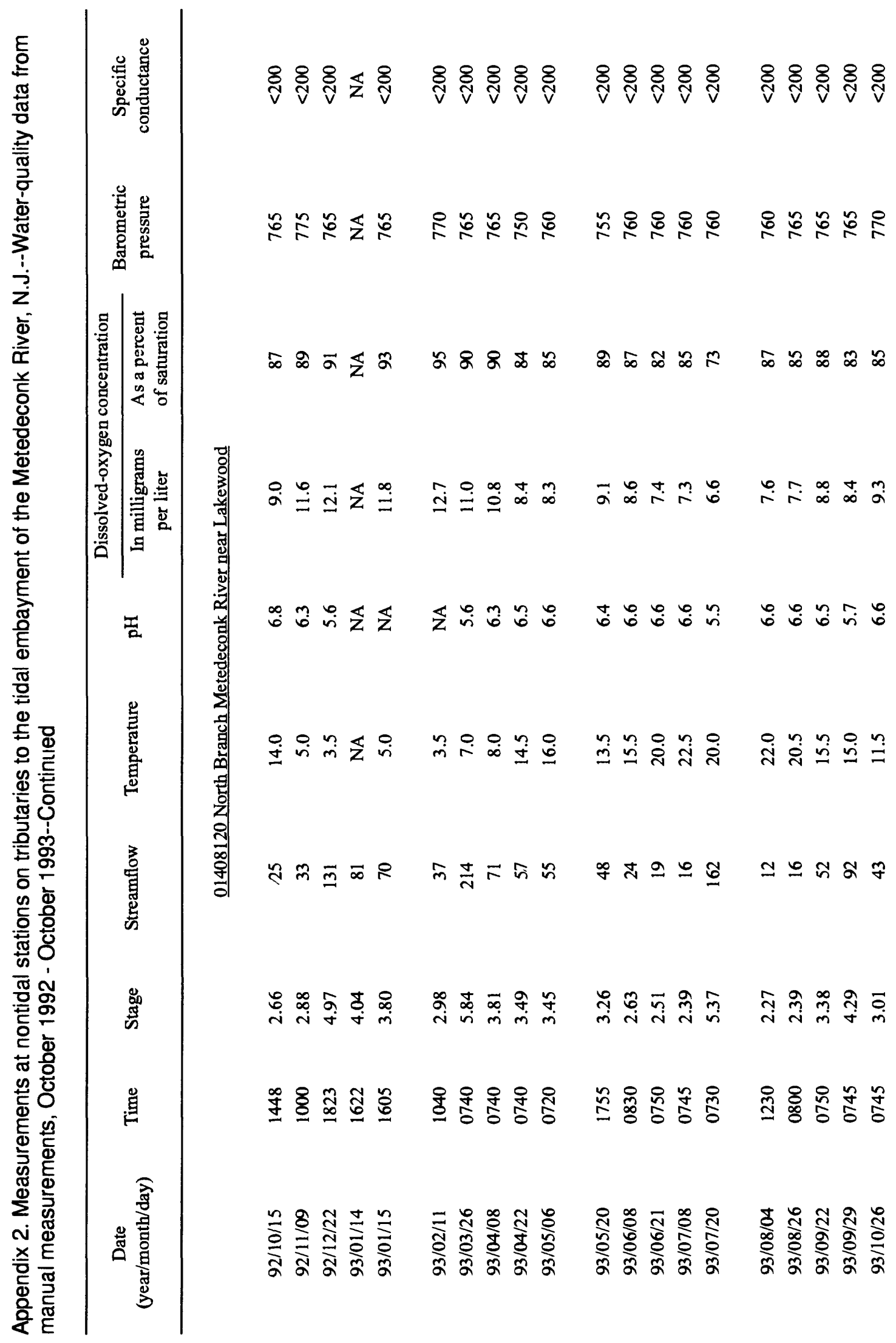




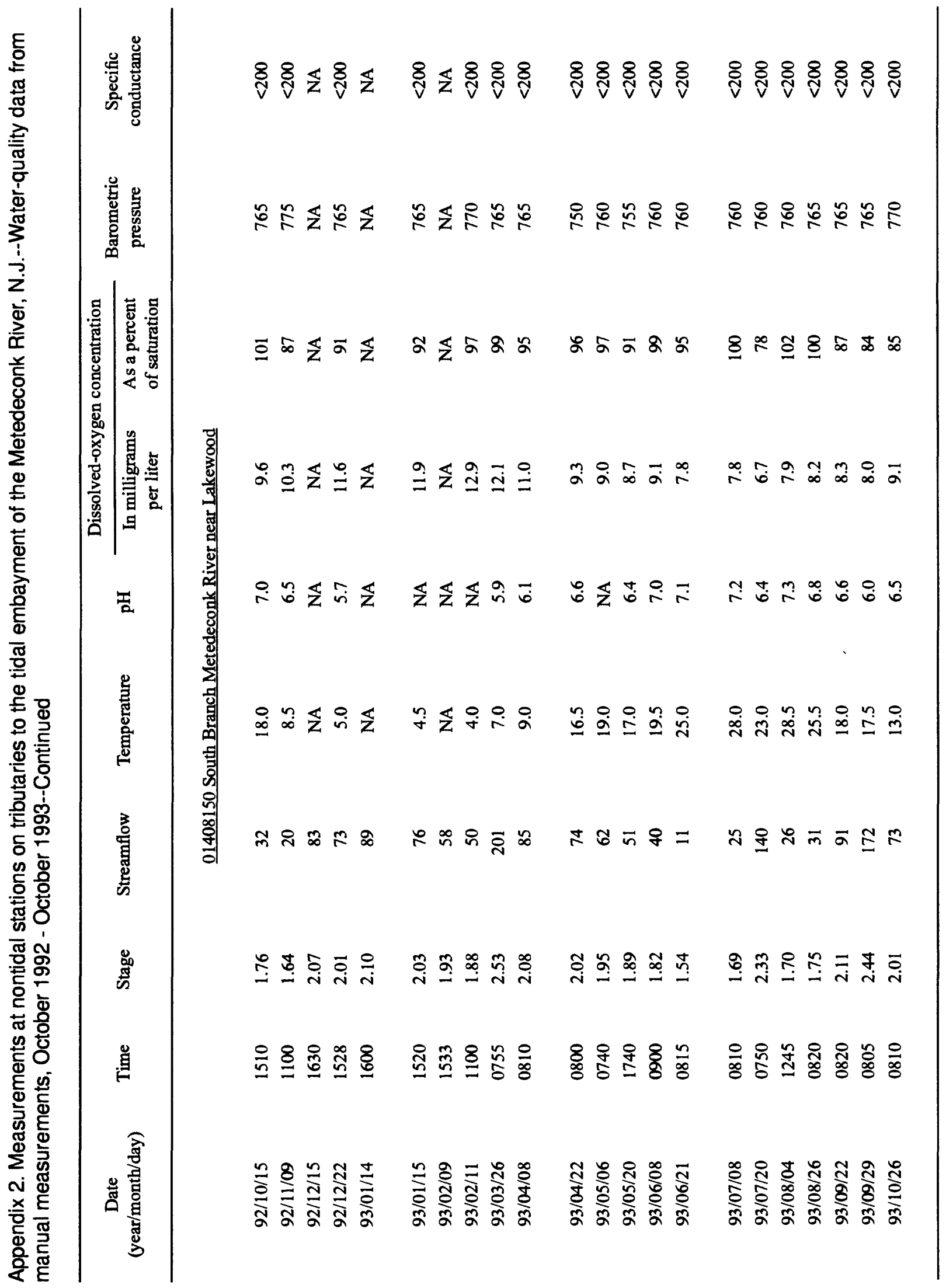


Appendix 3. Measurements at tidal stations in and near the tidal embayment of the Metedeconk River, N.J.--Daily minimum and maximum tidal-water level, October 1992 - November 1993

[Values of tidal-water level are given in feet above the National Geodetic Vertical Datum of 1929 for the station listed below. Min, daily minimum; Max, daily maximum; USGS, U.S. Geological Survey; NA, not available]

\begin{tabular}{ccc}
\hline $\begin{array}{c}\text { Index number } \\
\text { (fig. 2) }\end{array}$ & USGS station number & USGS station name \\
\hline 9 & 01408160 & Metedeconk River near Laurelton \\
\hline
\end{tabular}




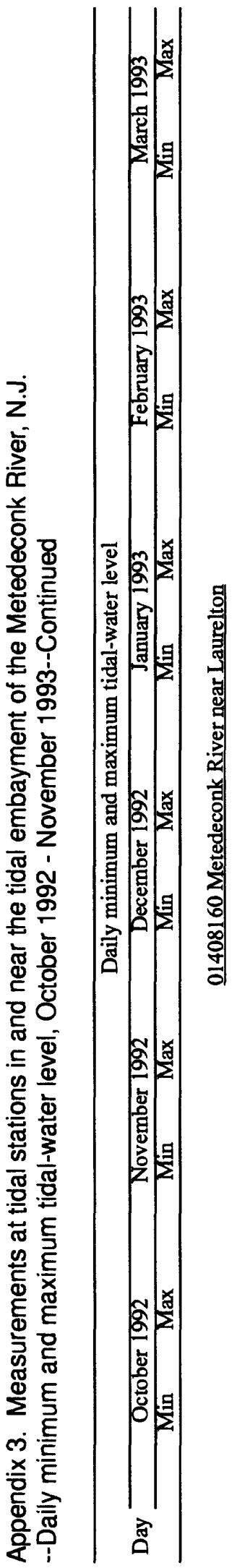

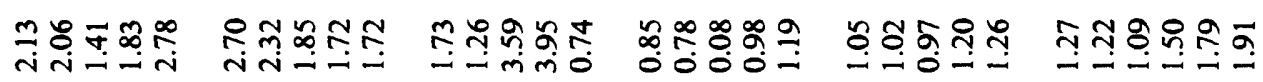

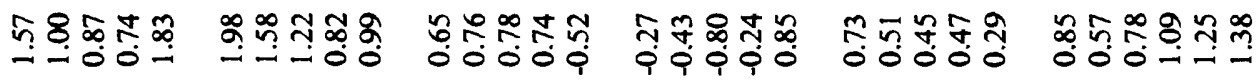

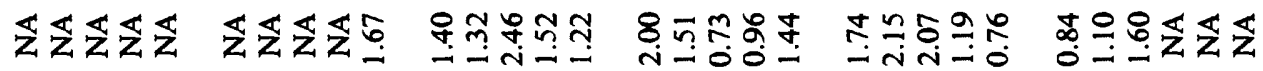

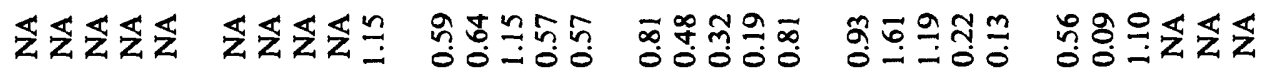

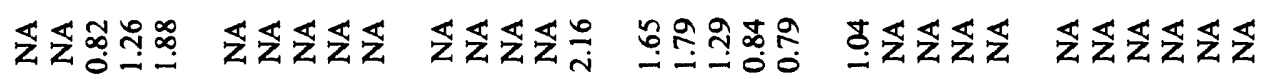

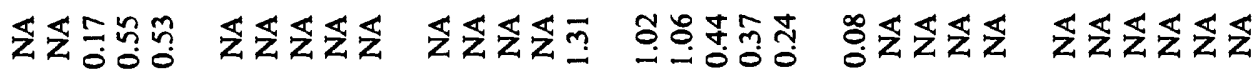

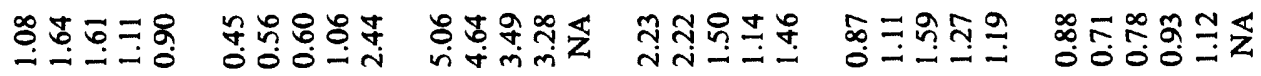

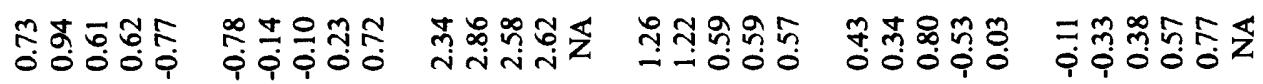

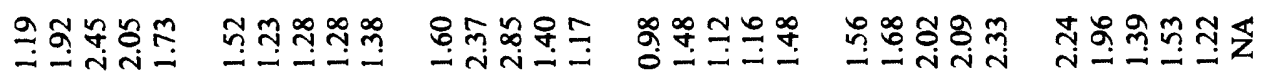

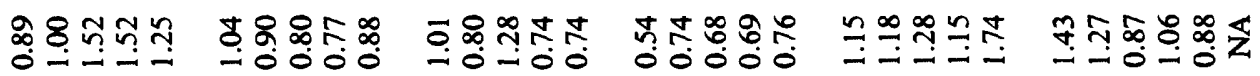

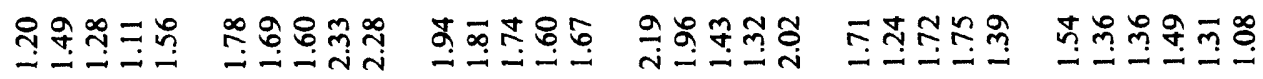

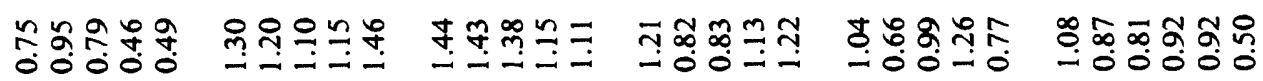

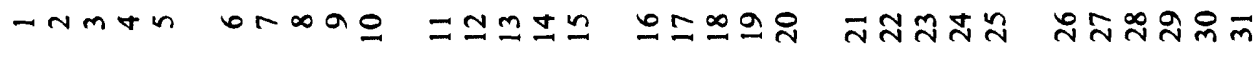




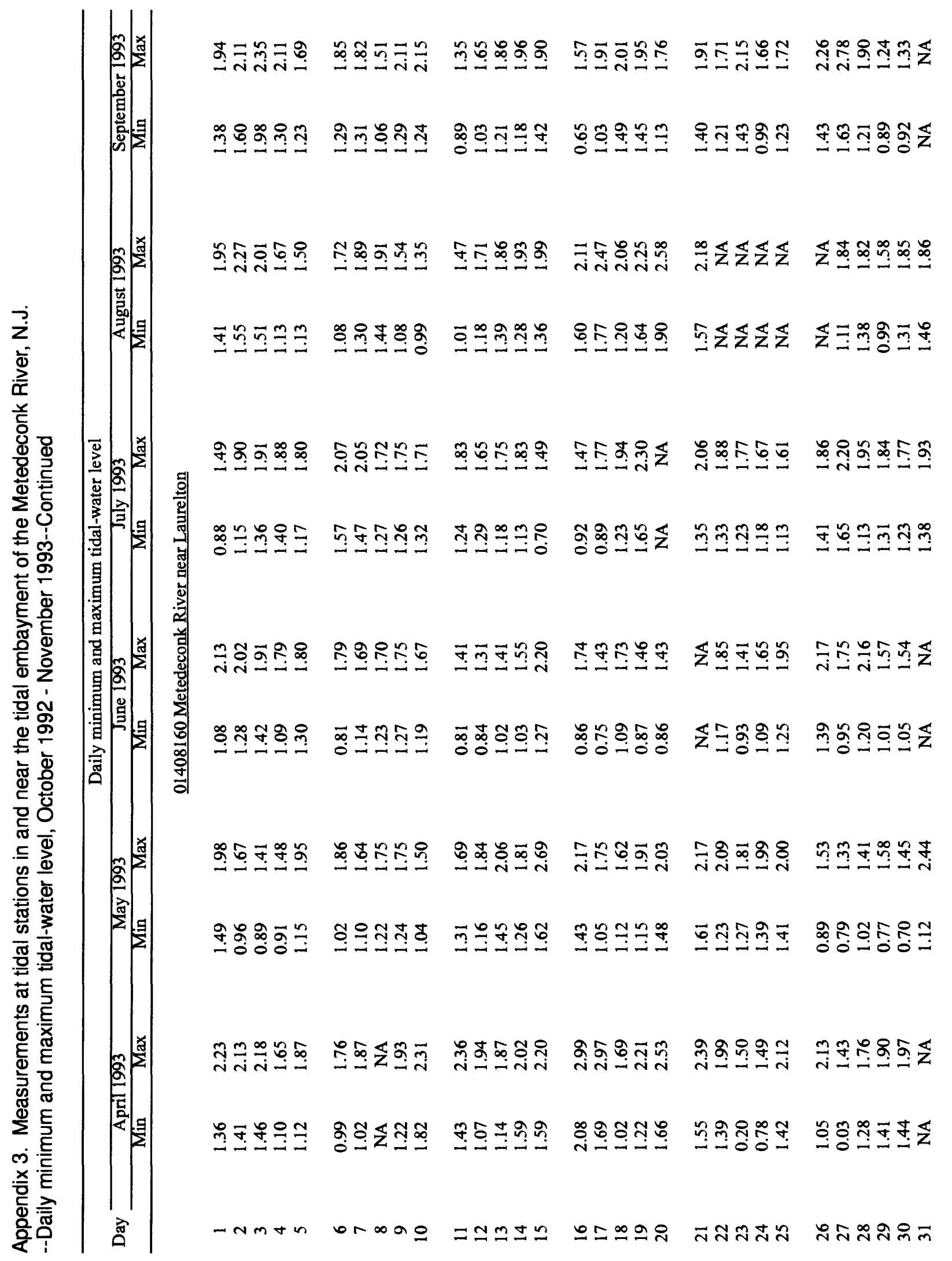




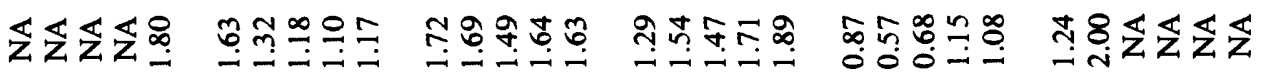

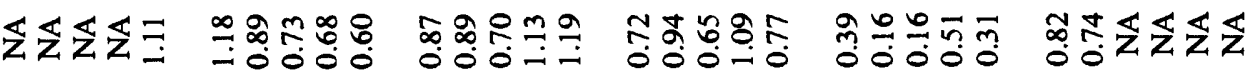

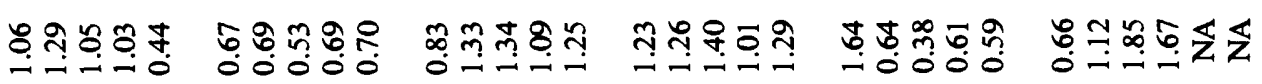

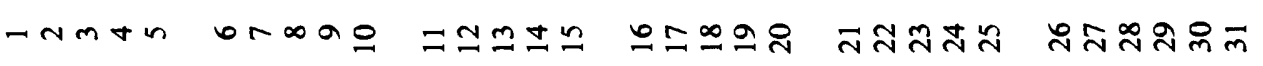
잉 (\$)

\section{돈}


Appendix 4. Measurements at tidal stations in and near the tidal embayment of the Metedeconk River, N.J.--Daily minimum and maximum temperature from measurements by fixed monitors, October 1992 - January 1994

[Values of temperature are presented for the stations and periods listed below. Values are in degrees Celsius and rounded to the nearest 0.5 degree. USGS, U.S. Geological Survey; Min, daily minimum; Max, daily maximum; NA, not available or not applicable]

\begin{tabular}{|c|c|c|c|c|c|}
\hline $\begin{array}{l}\text { Index } \\
\text { number } \\
\text { (fig. 2) }\end{array}$ & $\begin{array}{l}\text { USGS station } \\
\text { number }\end{array}$ & USGS station name & Period of operation & $\begin{array}{l}\text { Location in } \\
\text { cross section }\end{array}$ & $\begin{array}{l}\text { Approximate } \\
\text { depth of } \\
\text { measurement, } \\
\text { in feet }\end{array}$ \\
\hline 5 & 01408155 & $\begin{array}{l}\text { Metedeconk River at } \\
\text { Laurelton }\end{array}$ & $\begin{array}{l}\text { October } 1992 \text { - } \\
\text { January } 1994\end{array}$ & $\begin{array}{l}\text { Left channel } \\
\text { at Route } 70 \text {, } \\
\text { mid-depth }\end{array}$ & 3 \\
\hline 9 & 01408160 & $\begin{array}{l}\text { Metedeconk River near } \\
\text { Laurelton }\end{array}$ & $\begin{array}{l}\text { October } 1992- \\
\text { December } 1993\end{array}$ & $\begin{array}{l}\text { Left bank near } \\
\text { surface, and } \\
\text { left bank near } \\
\text { bottom }\end{array}$ & $\begin{array}{l}1 \\
6\end{array}$ \\
\hline
\end{tabular}




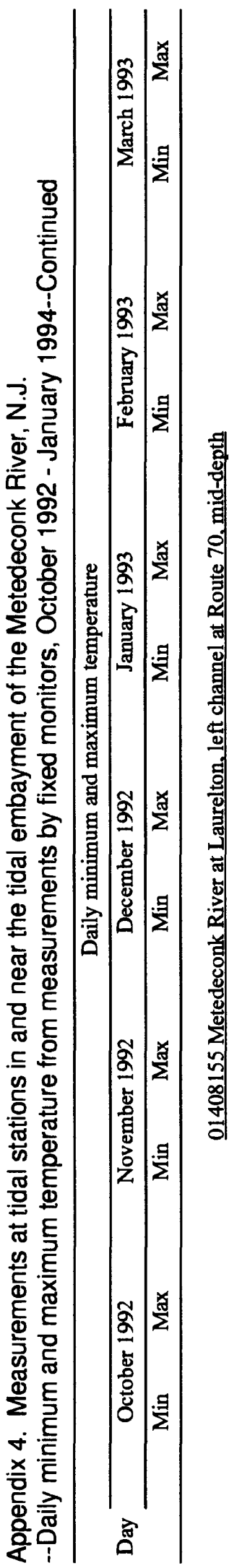

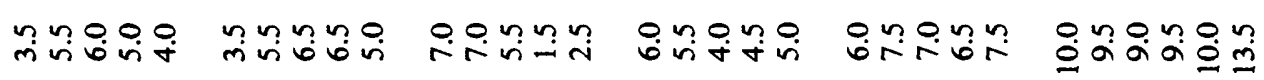

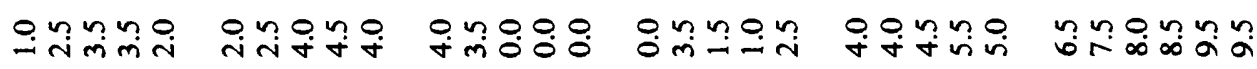

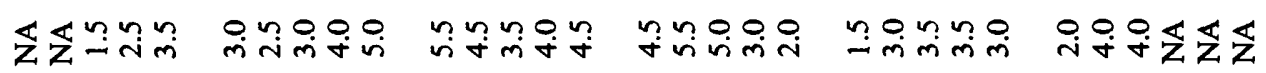

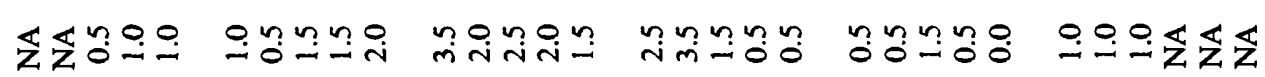

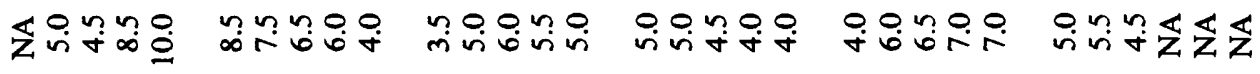

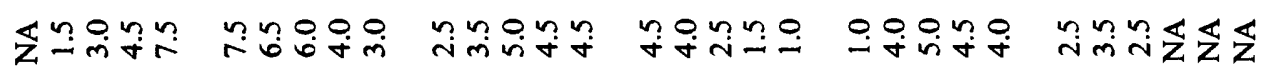

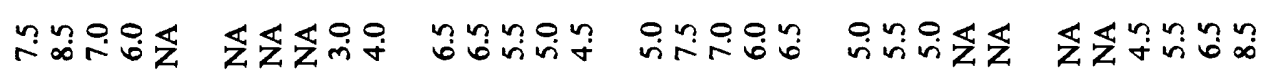

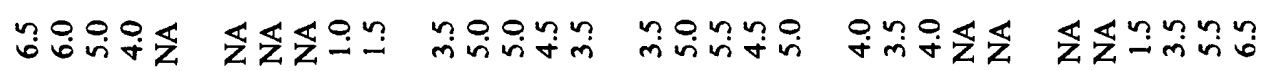

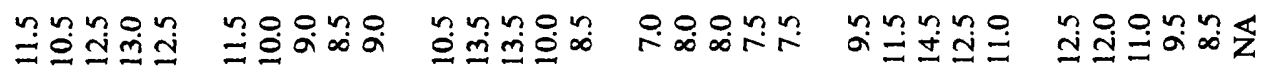

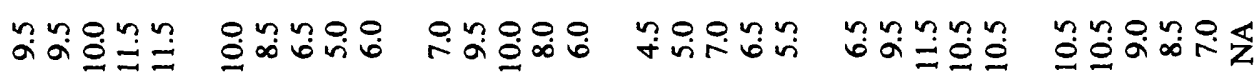

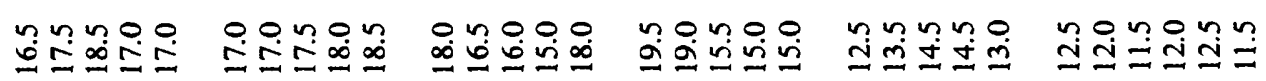

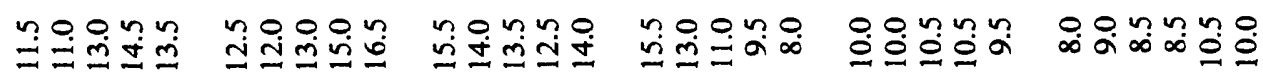

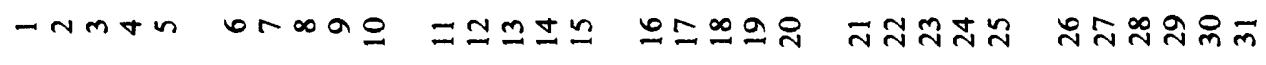




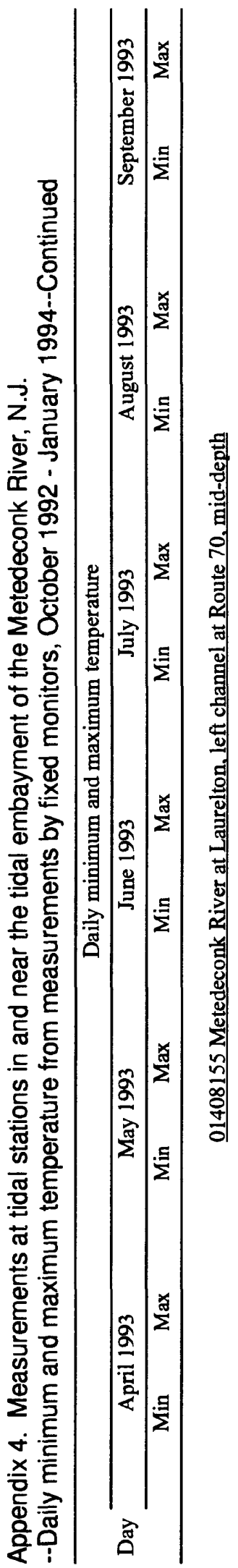

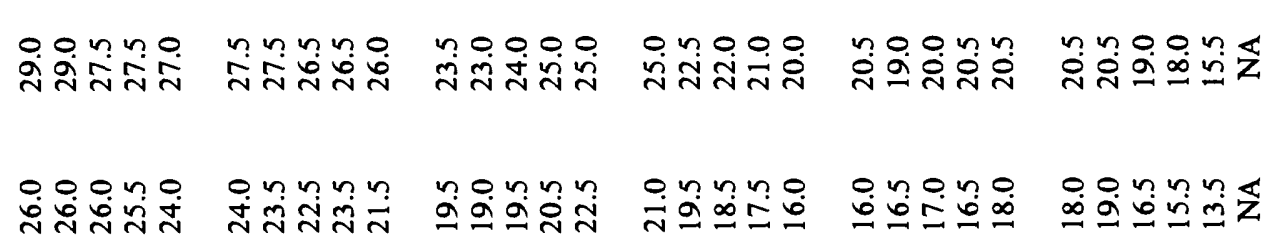

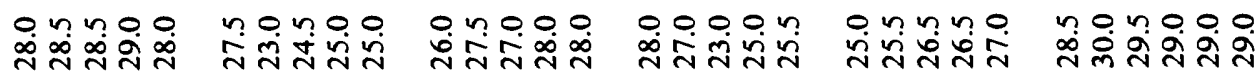

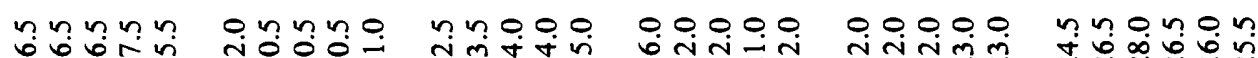

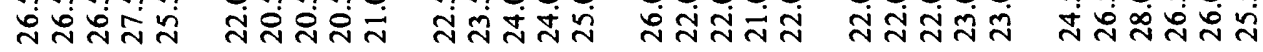

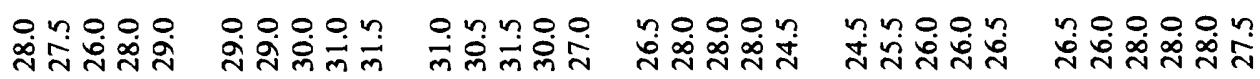

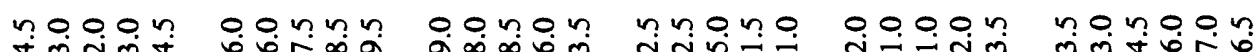

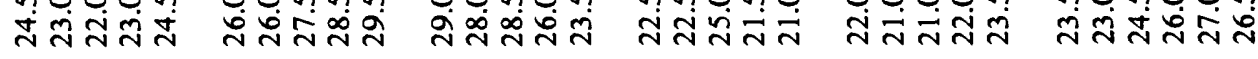

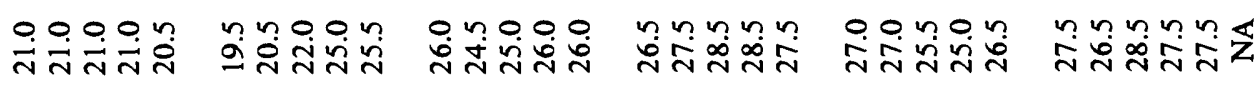

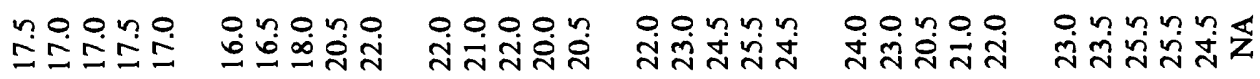

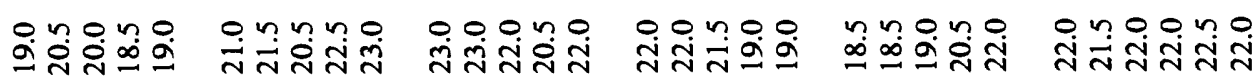

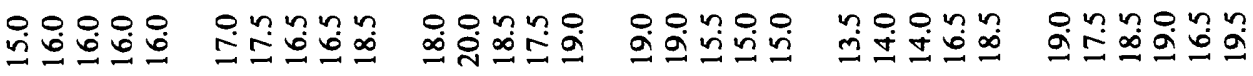

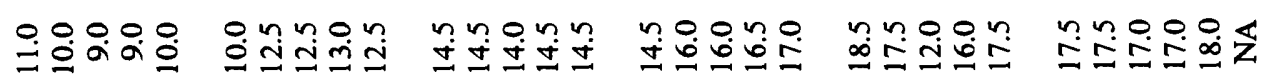

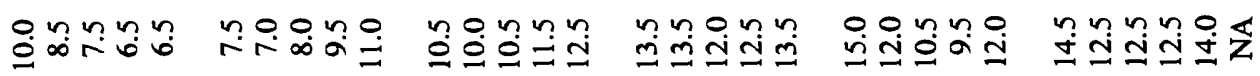

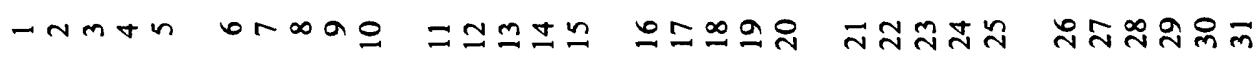




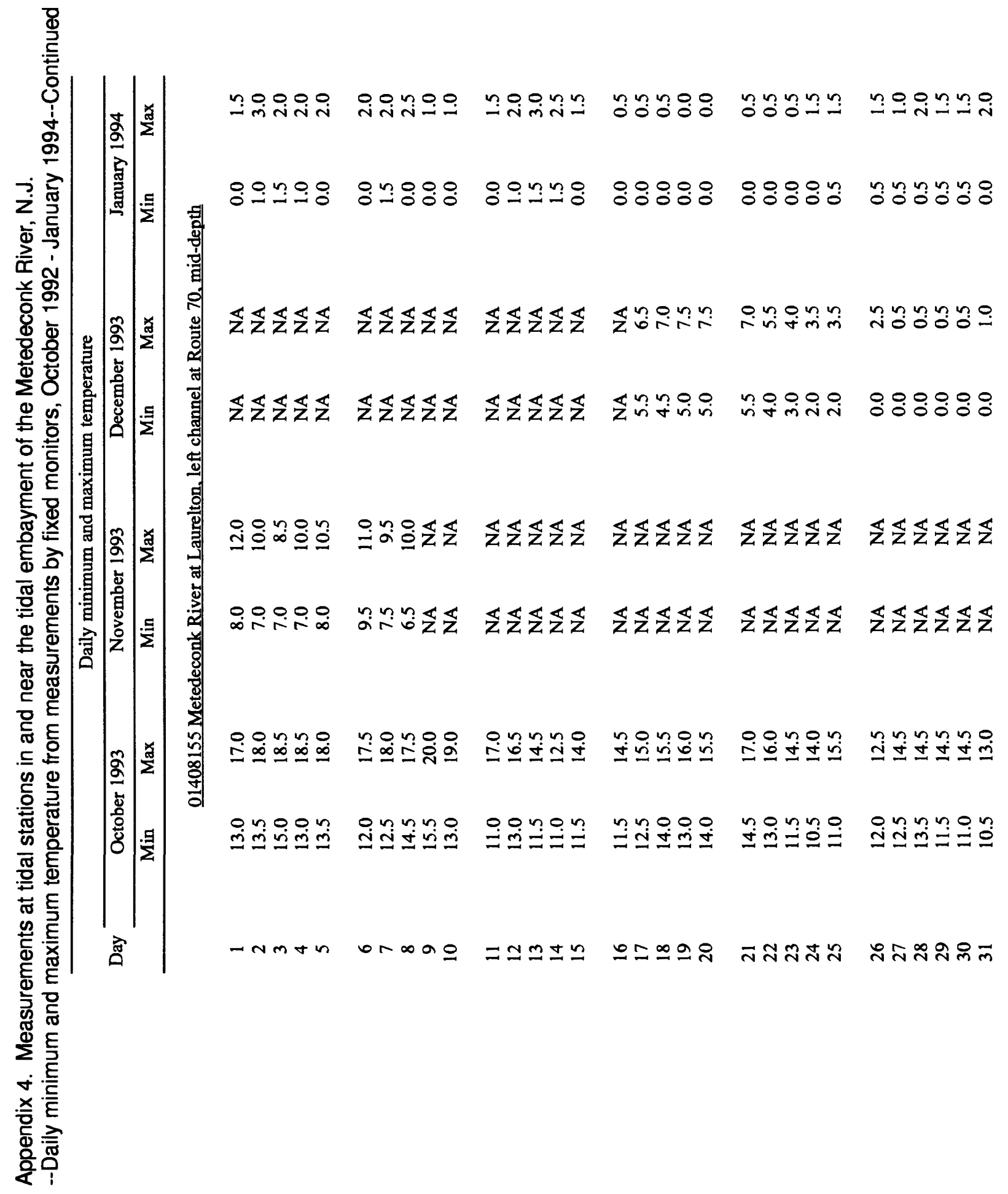




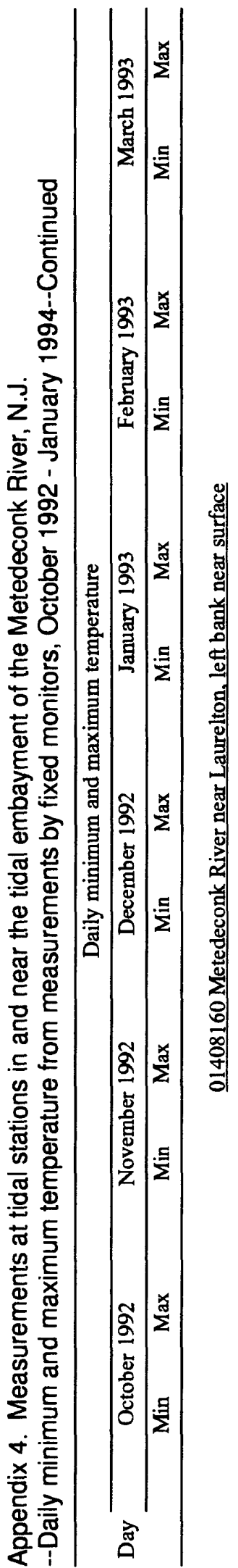

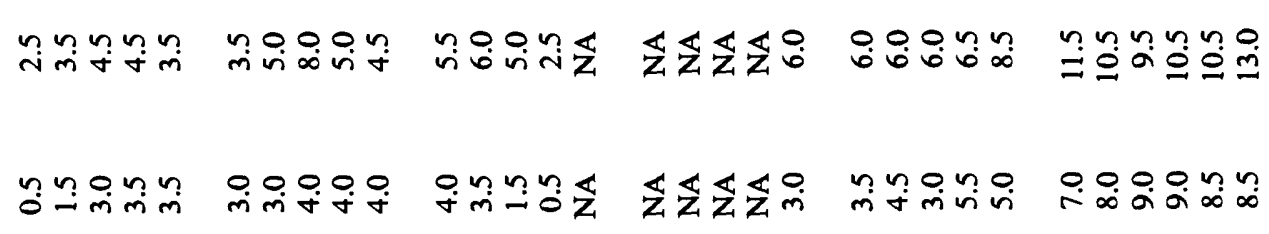

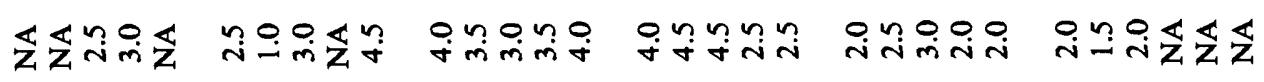

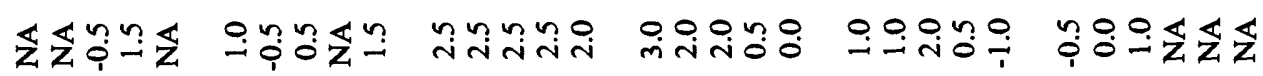

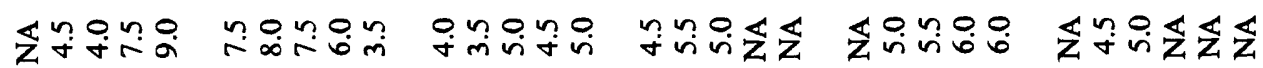

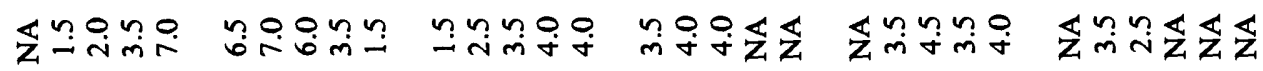

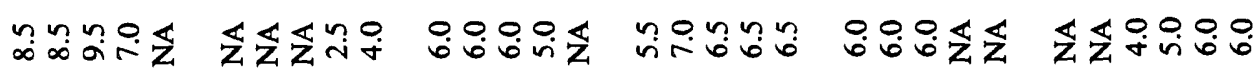

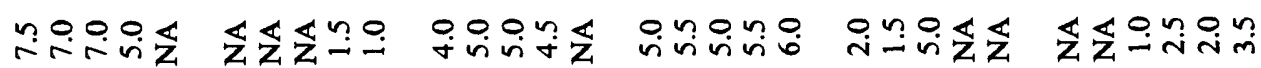

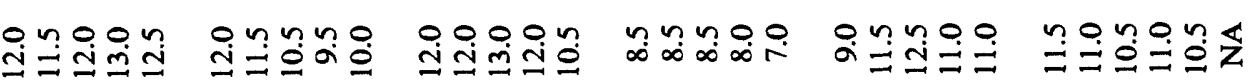

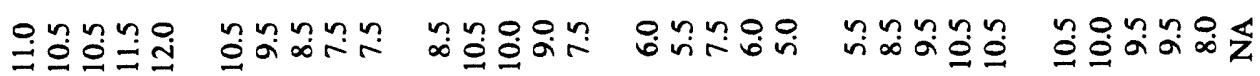

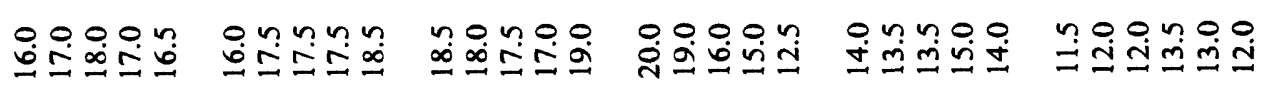

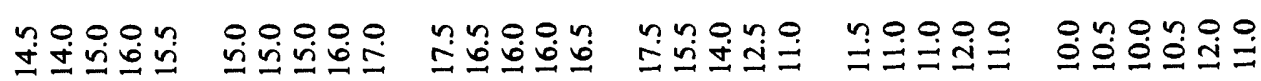

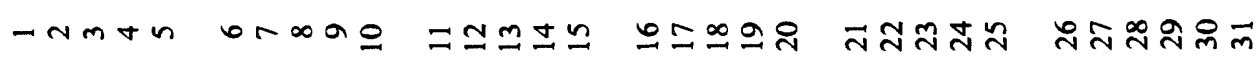




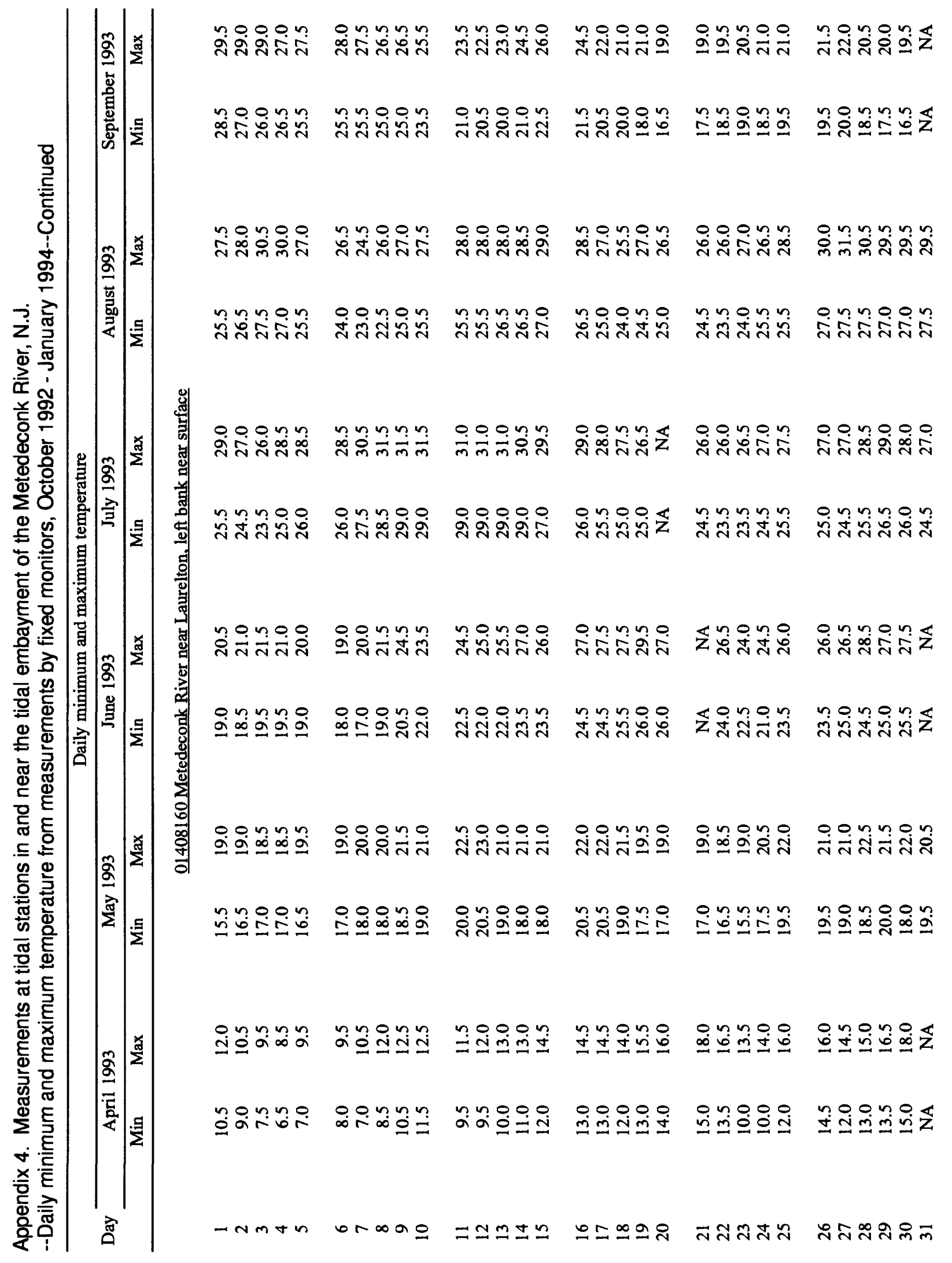




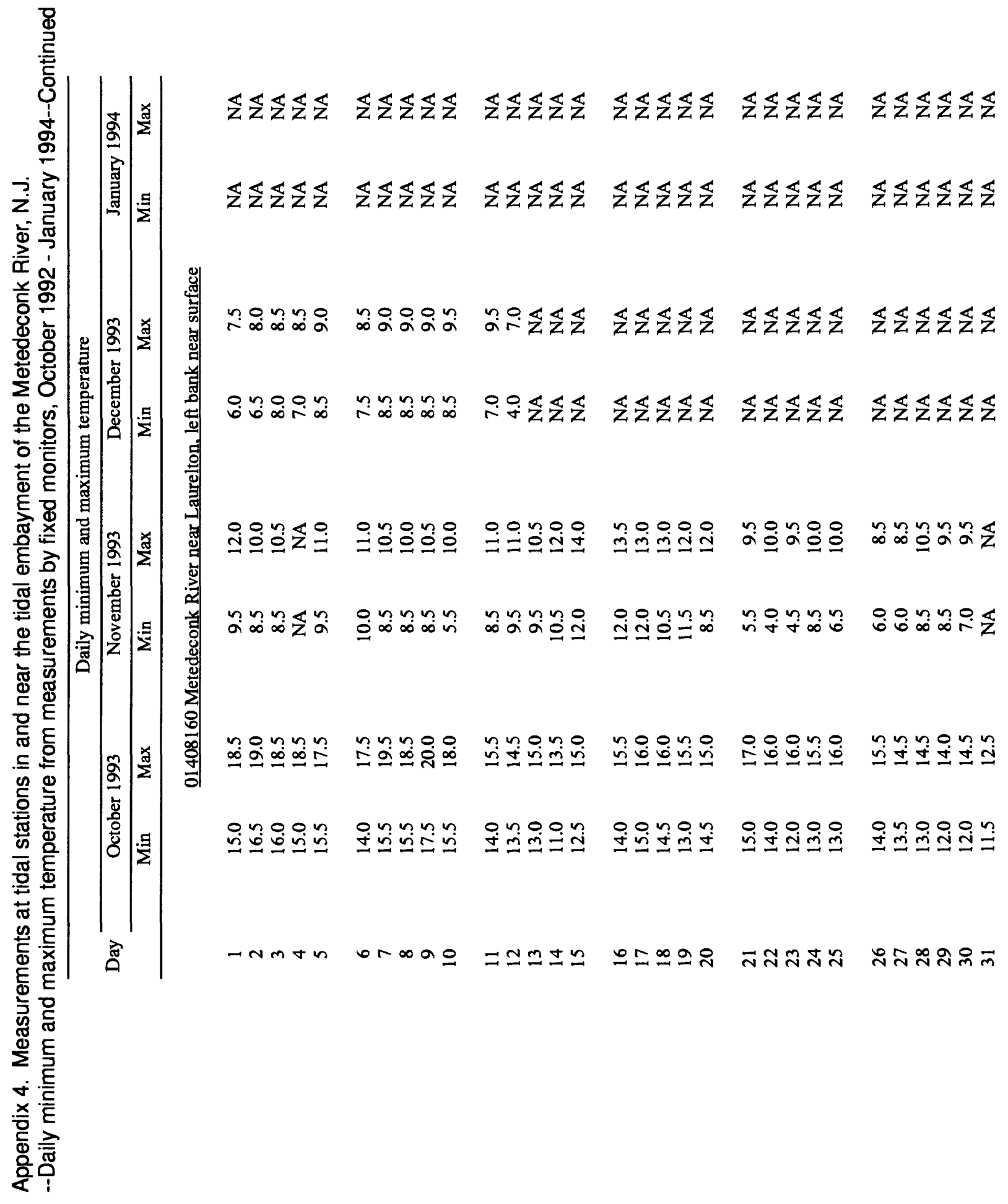




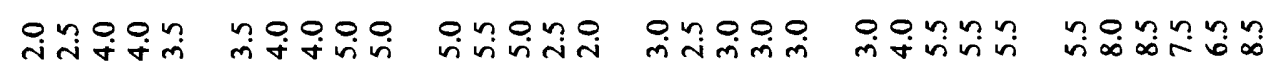

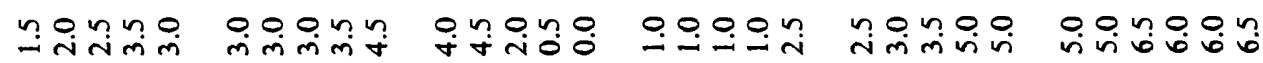

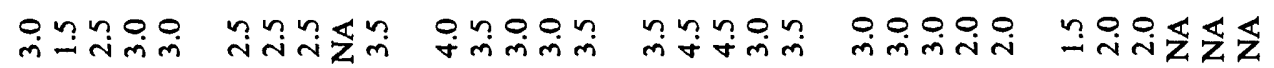

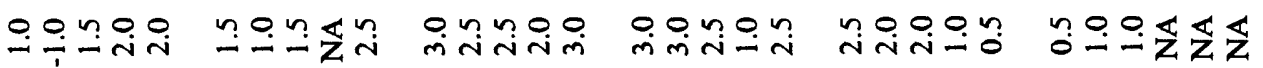

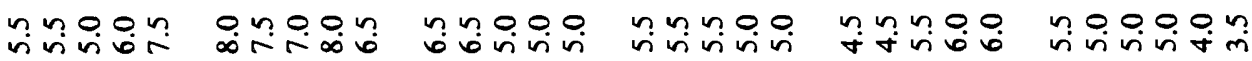

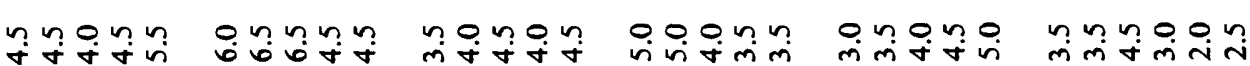

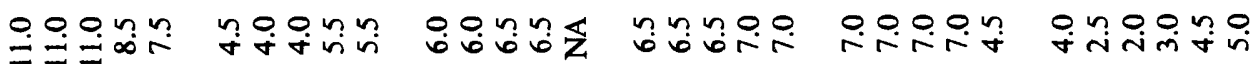

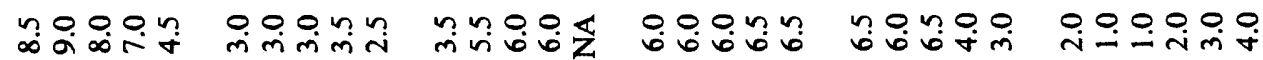

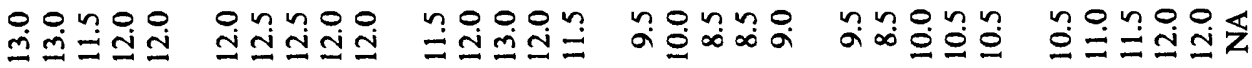

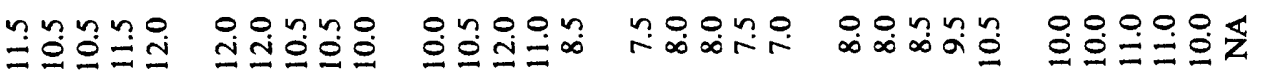

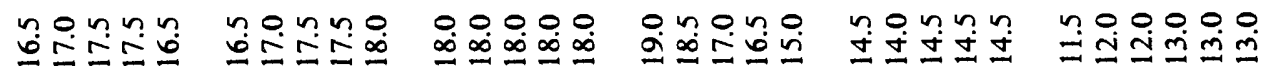

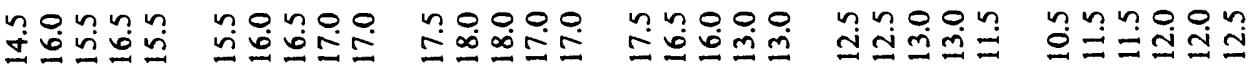

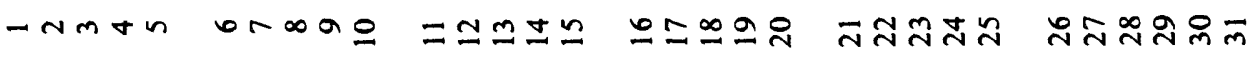




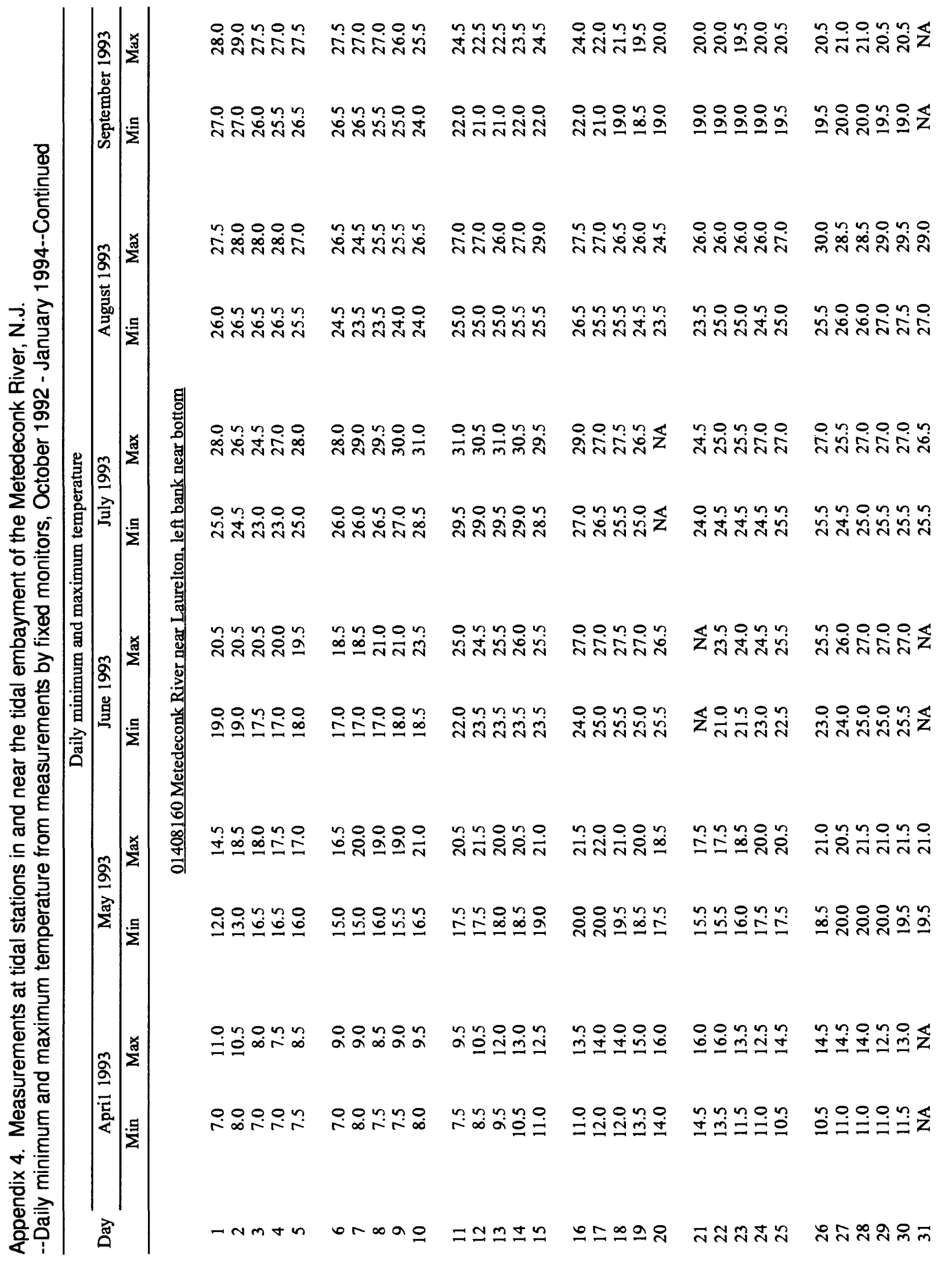




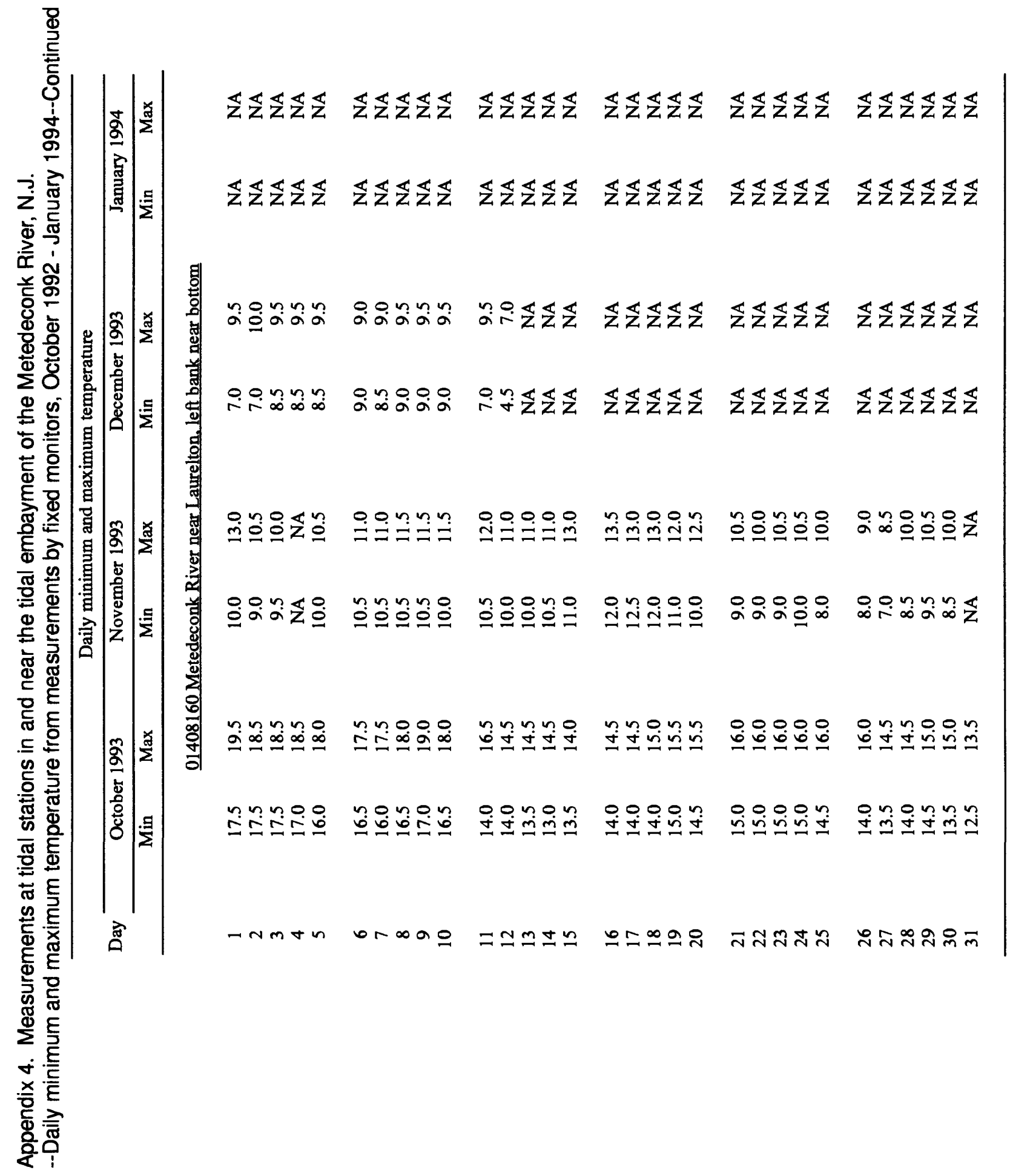


Appendix 5. Measurements at tidal stations in and near the tidal embayment of the Metedeconk River, N.J.--Daily minimum and maximum specific conductance from measurements by fixed monitors, October 1992 - January 1994

[Values of specific conductance are presented for the stations and periods listed below. Values are in microsiemens per centimeter at 25 degrees Celsius and rounded to the nearest hundred. USGS, U.S. Geological Survey; Min, daily minimum; Max, daily maximum; NA, not available or not applicable]

\begin{tabular}{|c|c|c|c|c|c|}
\hline $\begin{array}{l}\text { Index } \\
\text { number } \\
\text { (fig. 2) }\end{array}$ & $\begin{array}{l}\text { USGS station } \\
\text { number }\end{array}$ & USGS station name & Period of operation & $\begin{array}{l}\text { Location in } \\
\text { cross section }\end{array}$ & $\begin{array}{l}\text { Approximate } \\
\text { depth of } \\
\text { measurement, } \\
\text { in feet }\end{array}$ \\
\hline 5 & 01408155 & $\begin{array}{l}\text { Metedeconk River at } \\
\text { Laurelton }\end{array}$ & $\begin{array}{l}\text { October } 1992- \\
\text { January } 1994\end{array}$ & $\begin{array}{l}\text { Left channel } \\
\text { at Route } 70 \text {, } \\
\text { mid-depth }\end{array}$ & 3 \\
\hline 9 & 01408160 & $\begin{array}{l}\text { Metedeconk River near } \\
\text { Laurelton }\end{array}$ & $\begin{array}{l}\text { October } 1992- \\
\text { December } 1993\end{array}$ & $\begin{array}{l}\text { Left bank near } \\
\text { surface, and } \\
\text { left bank near } \\
\text { bottom }\end{array}$ & $\begin{array}{l}1 \\
6\end{array}$ \\
\hline
\end{tabular}




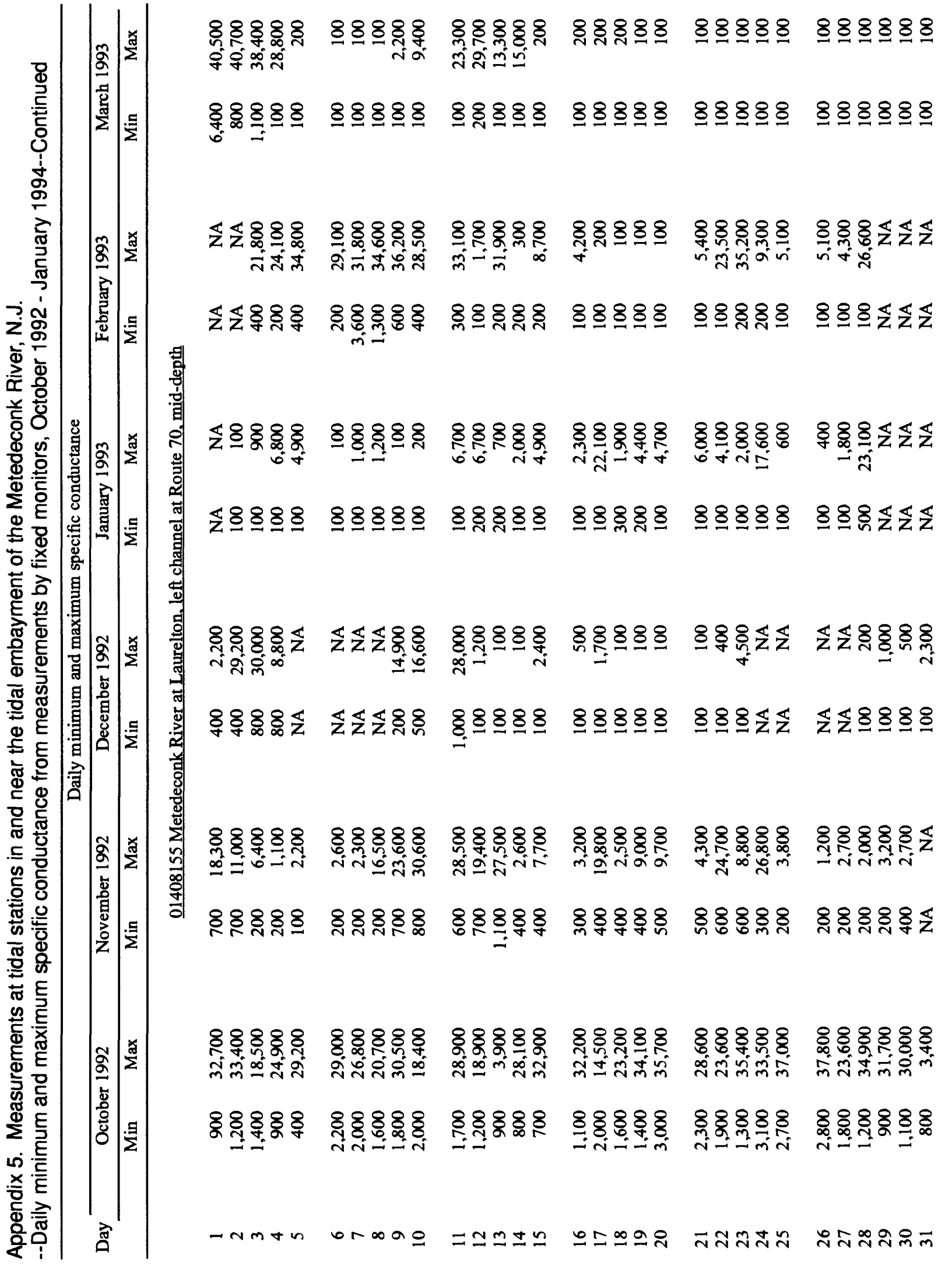




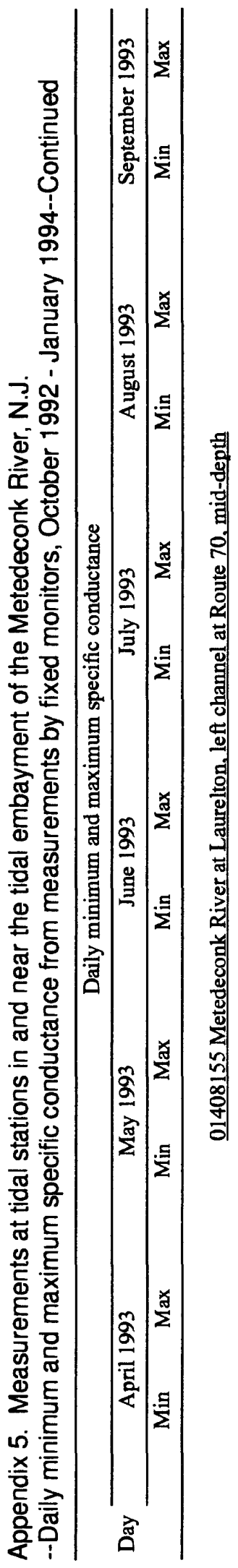

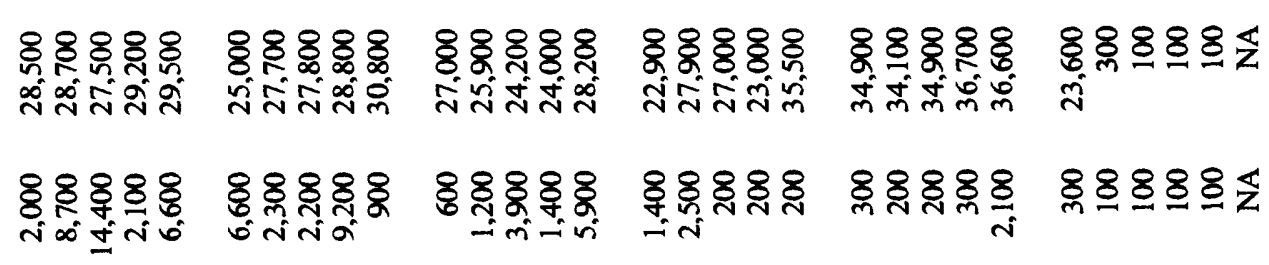

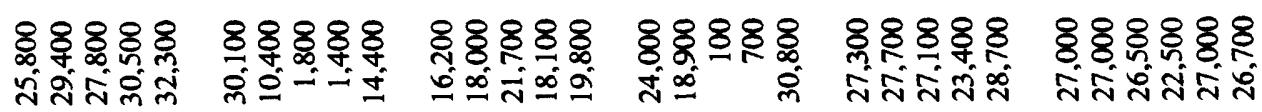

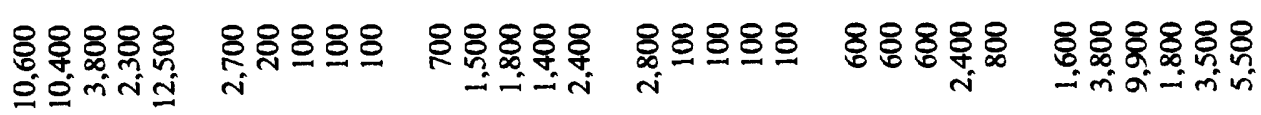

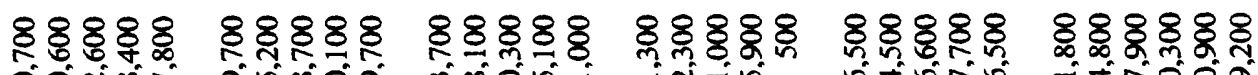

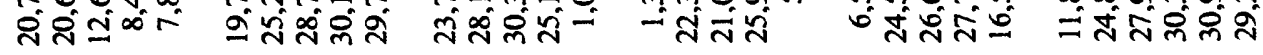

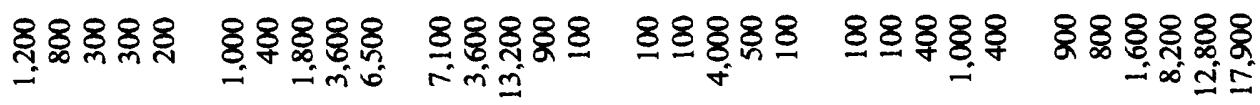

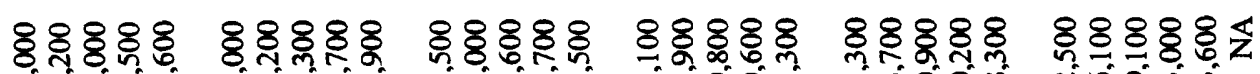

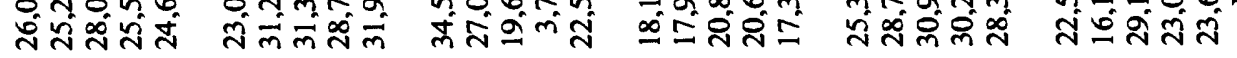

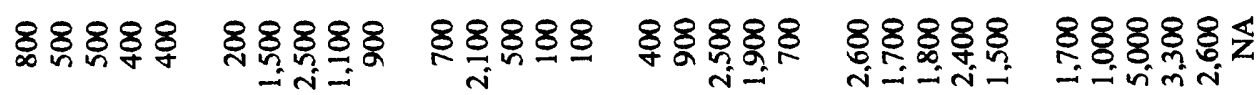

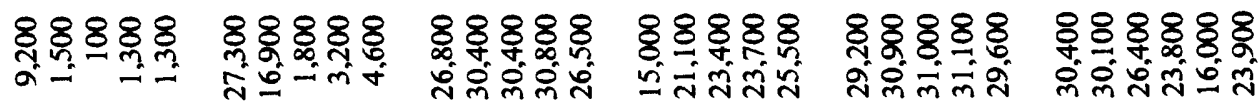

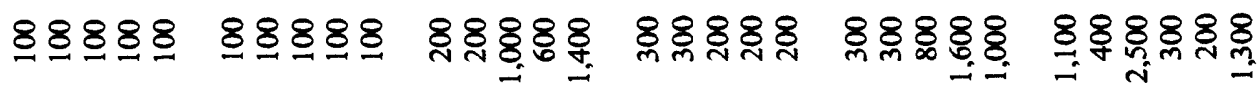

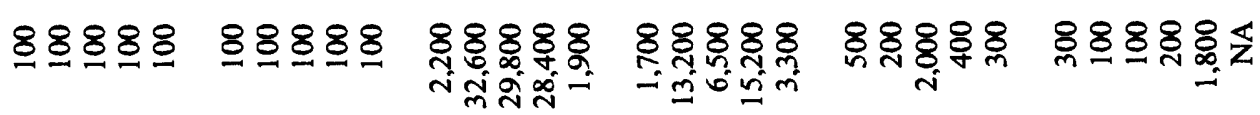

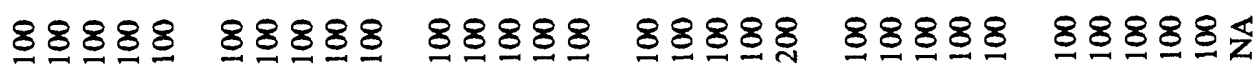

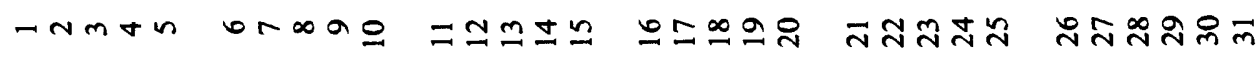




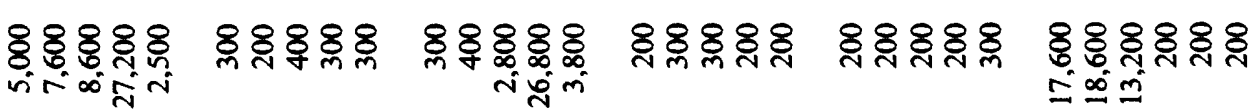

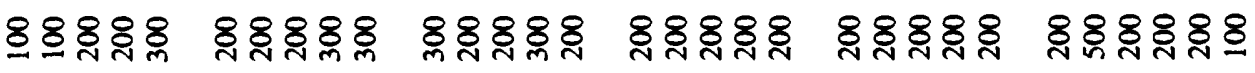

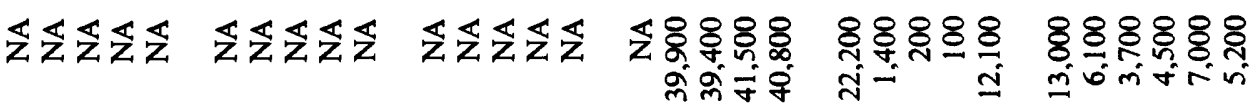

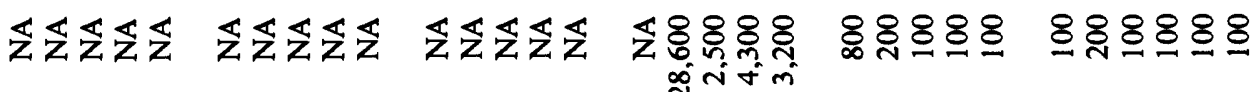

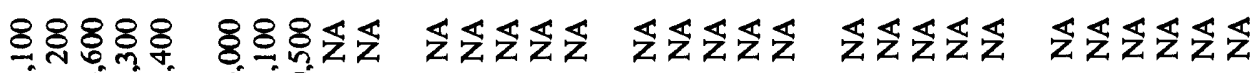

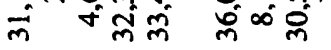

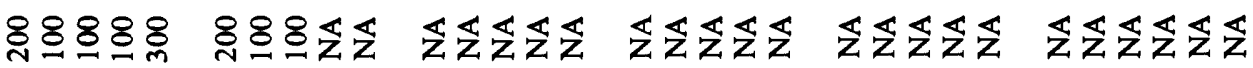

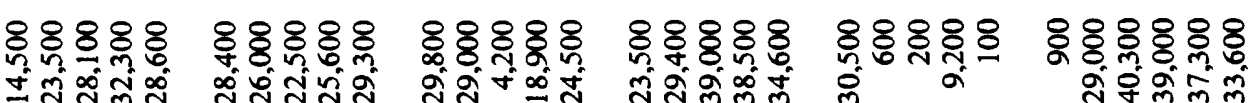

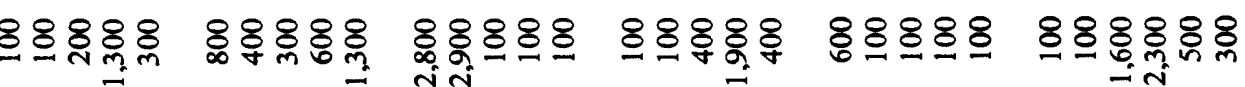

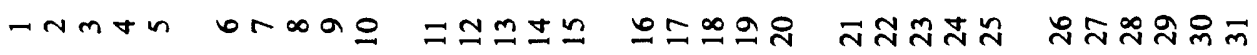




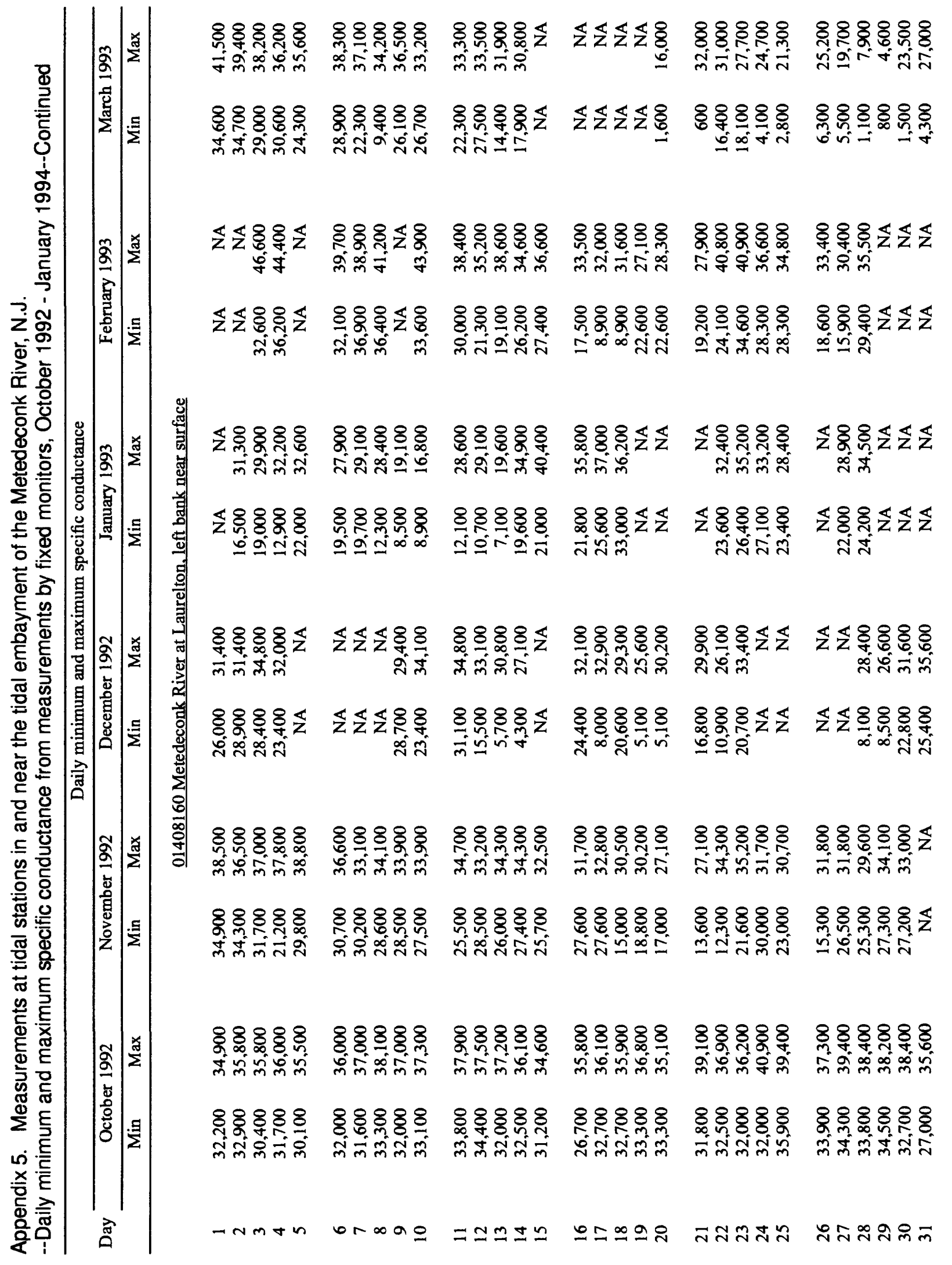




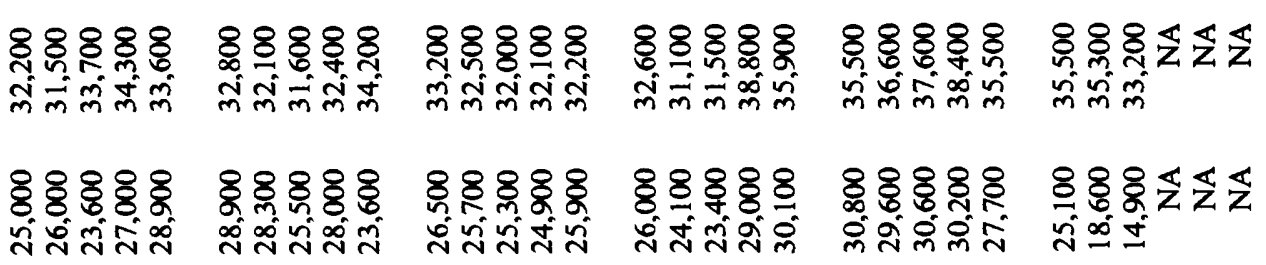

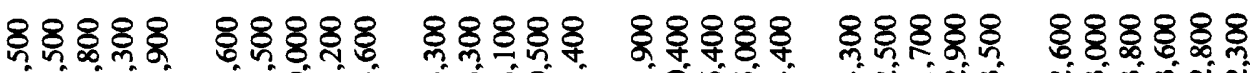

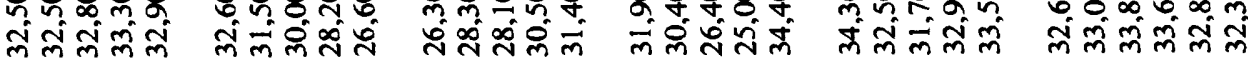

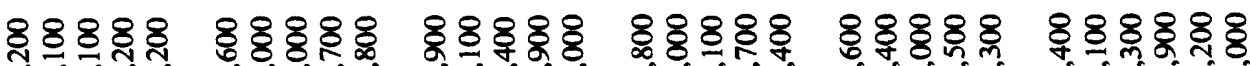

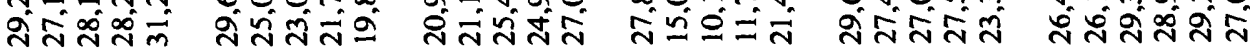

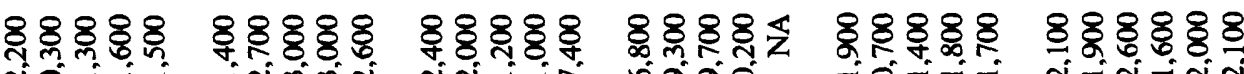

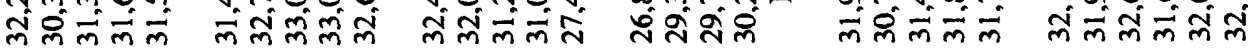

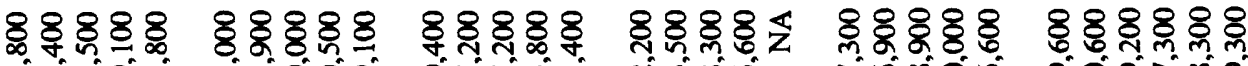

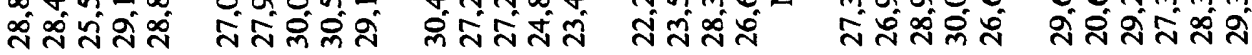

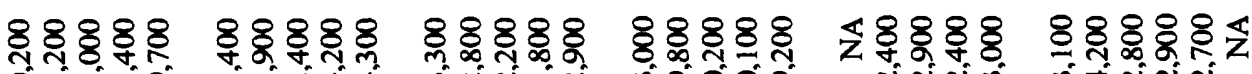

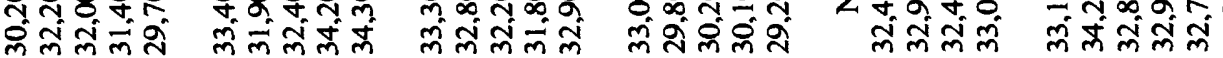

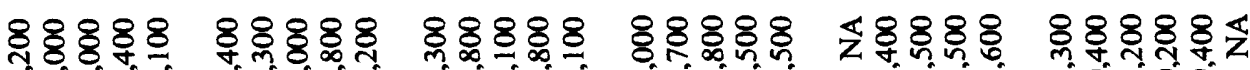

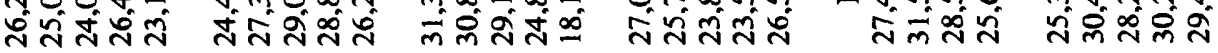

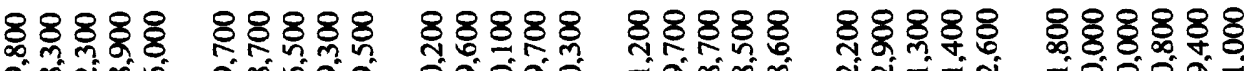

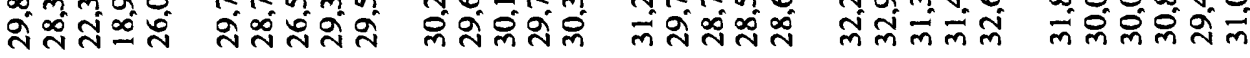

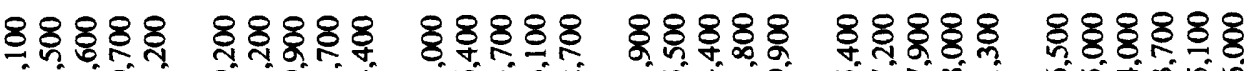

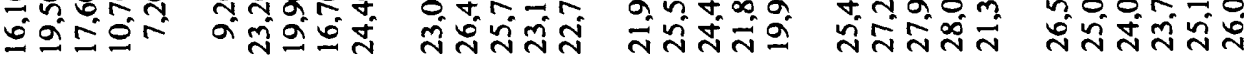

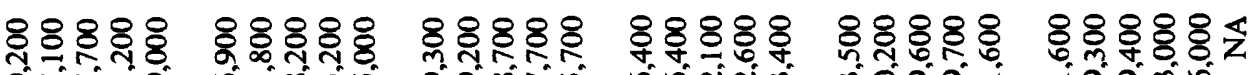

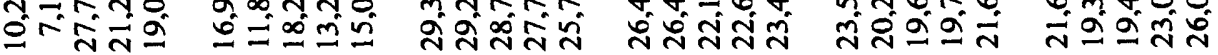

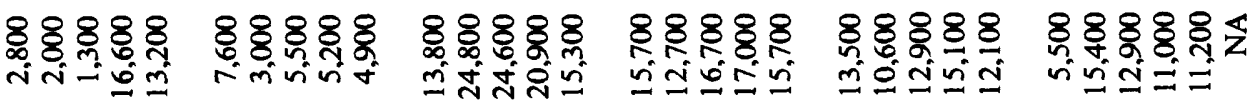

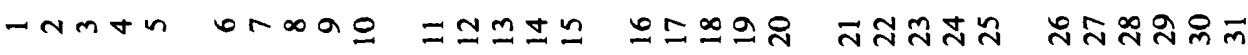




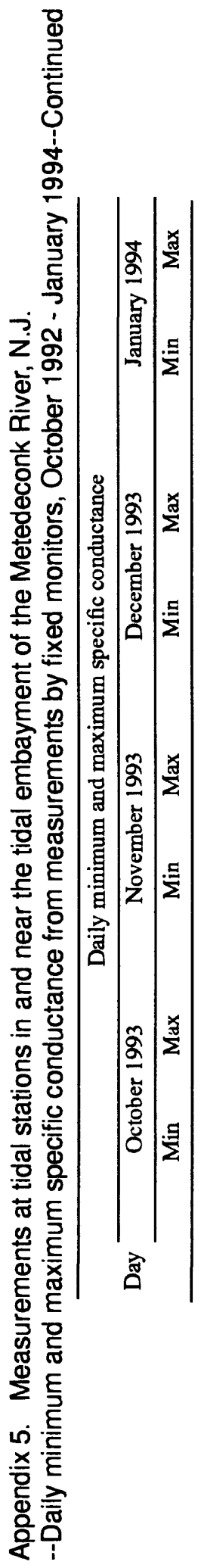

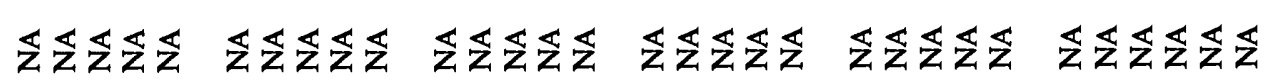

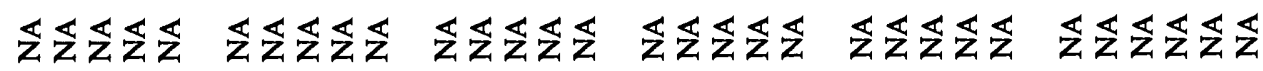

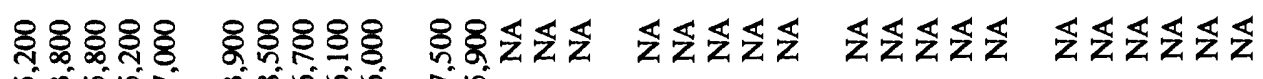
ñmminm mंm

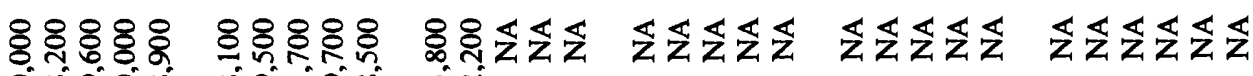

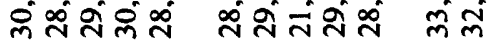

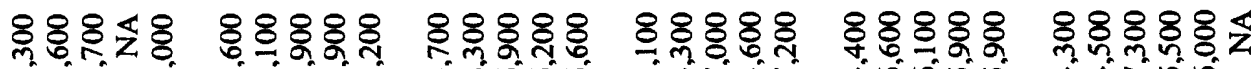

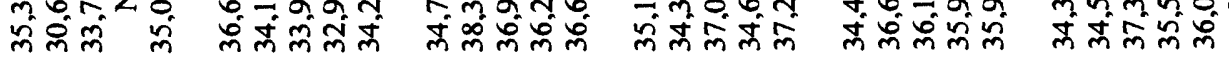

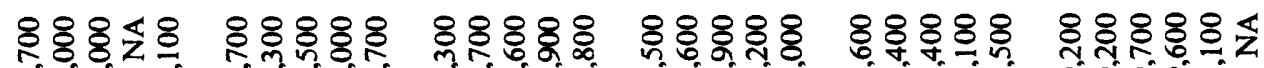

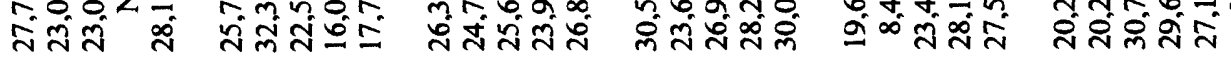

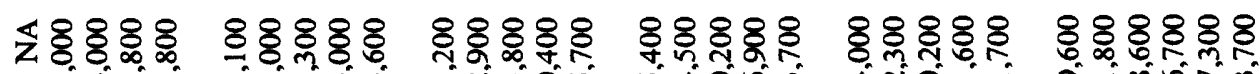

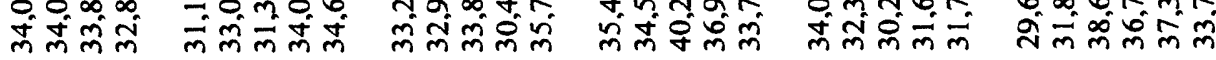

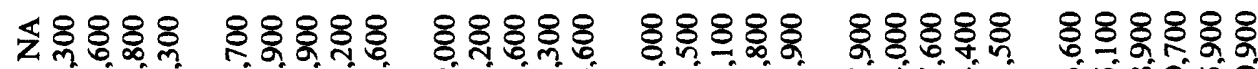

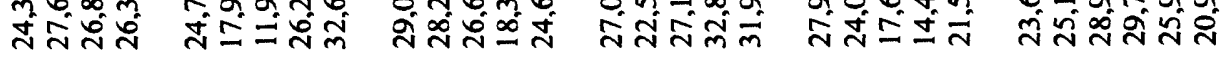

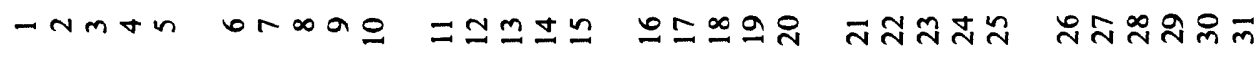




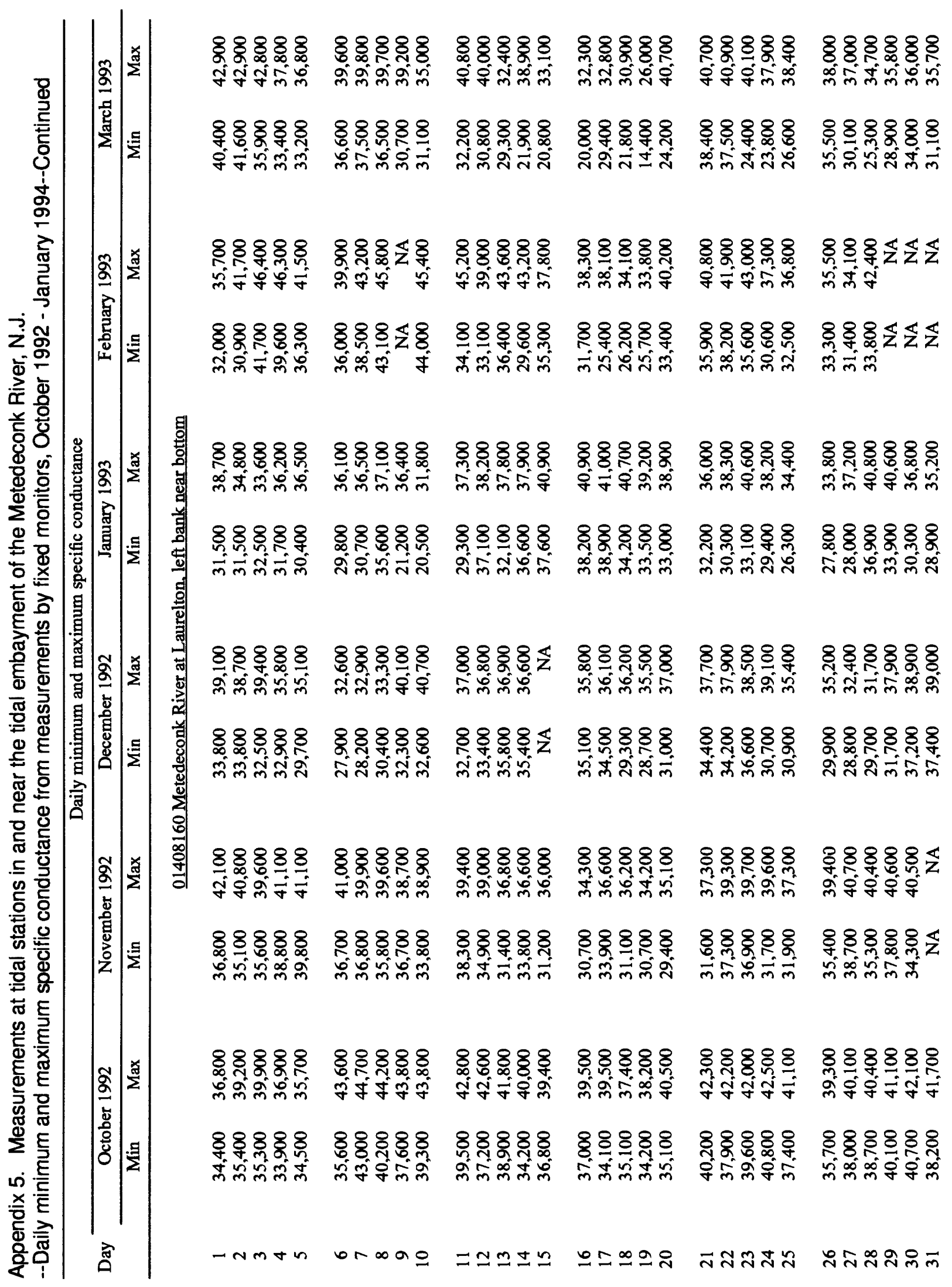




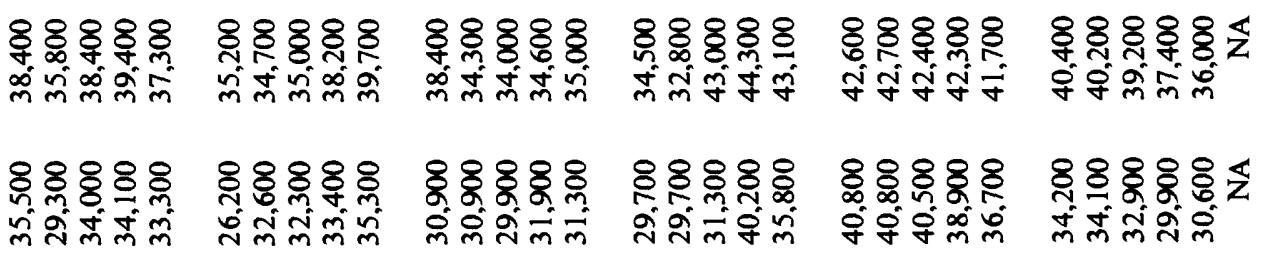

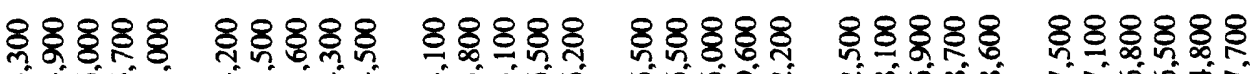

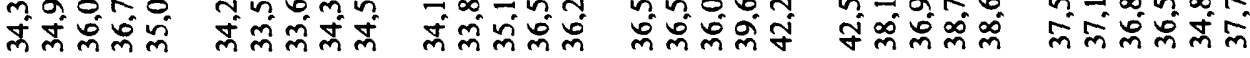

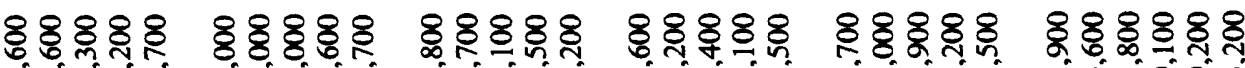

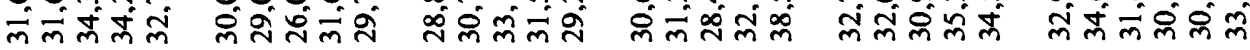

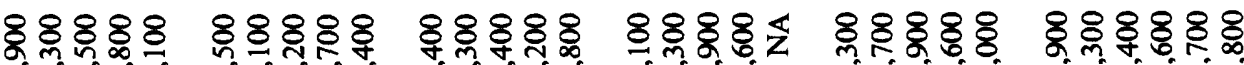

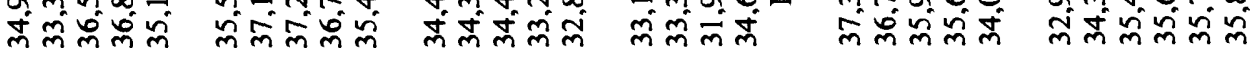

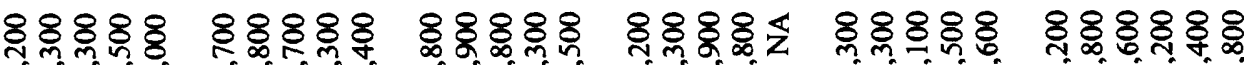

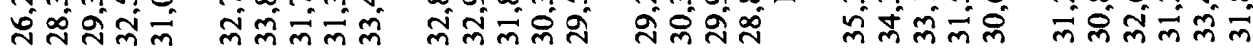

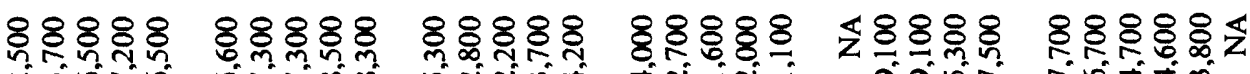

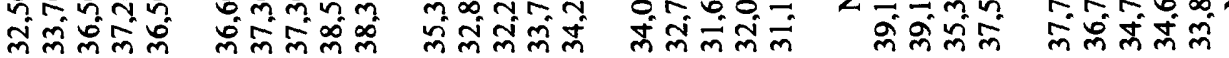

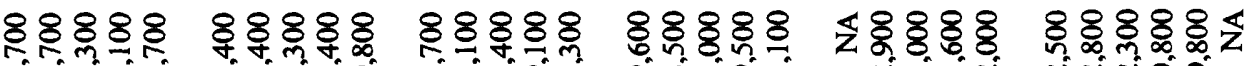

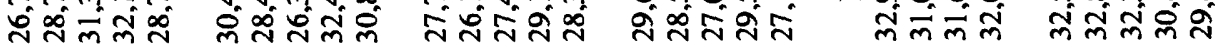

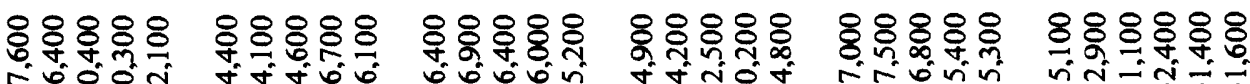
mं

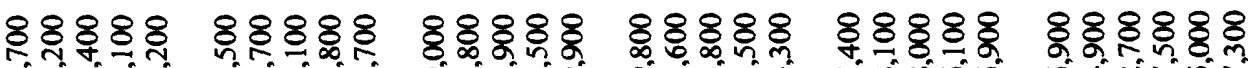

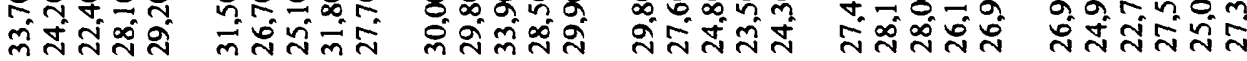

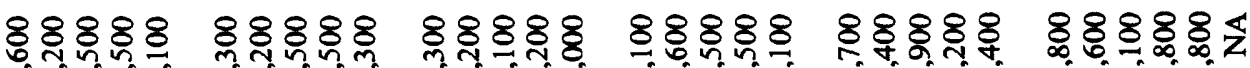

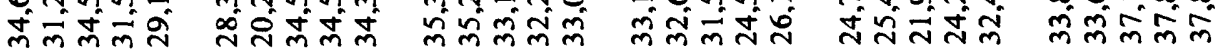

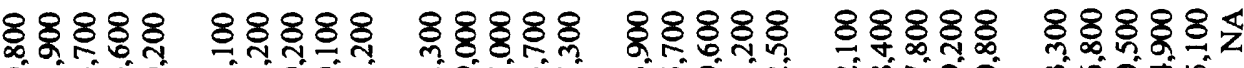

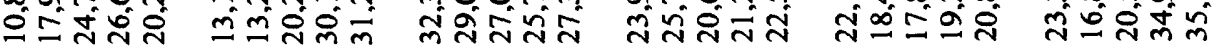

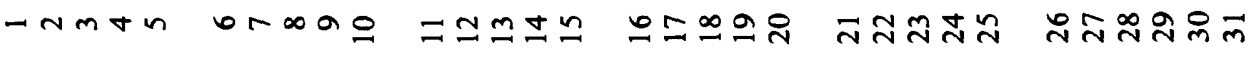




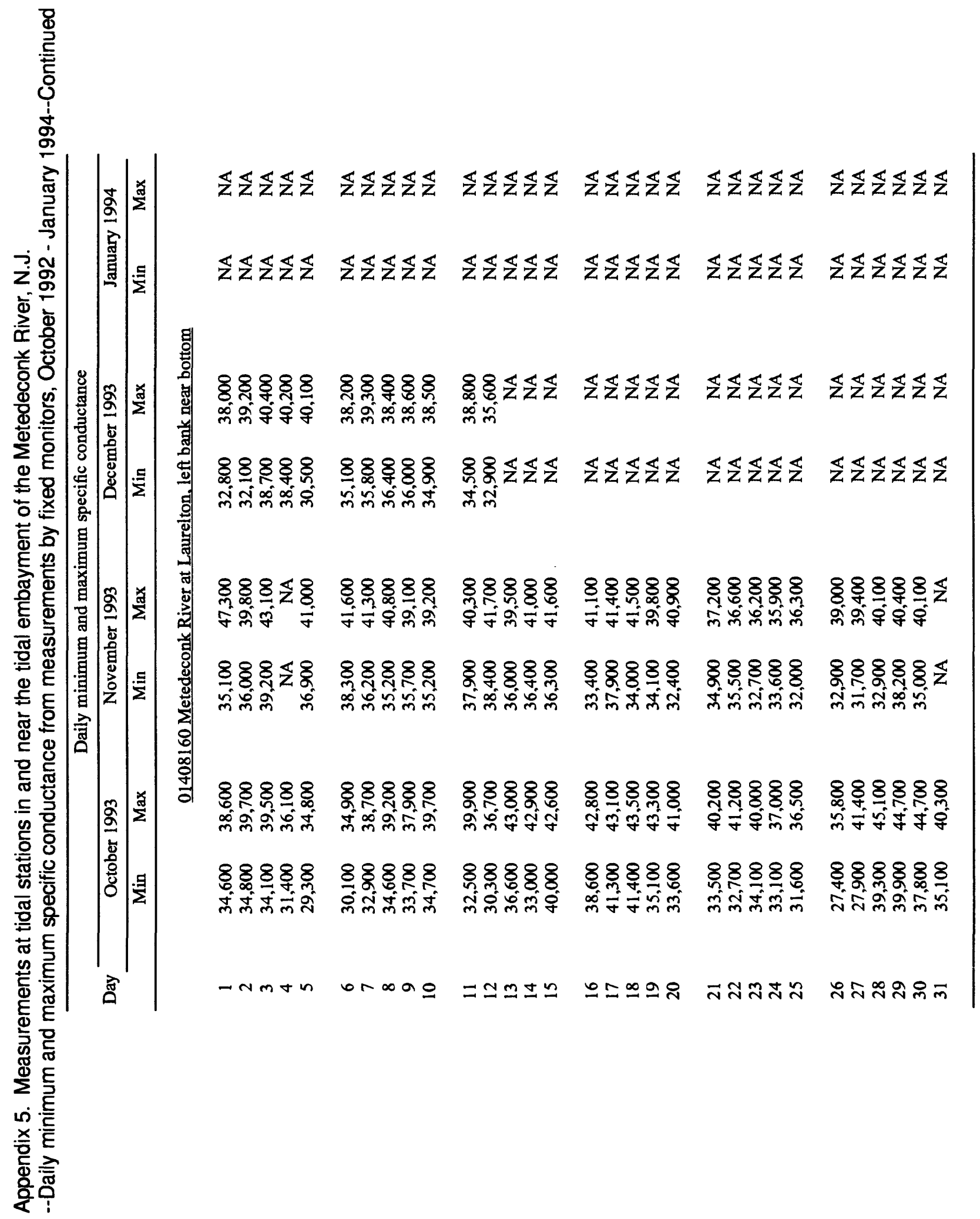


Appendix 6. Measurements at tidal stations in and near the tidal embayment of the Metedeconk River, N.J.--Daily minimum and maximum values of water-quality characteristics from measurements by movable monitors, November 1992 - January 1994

[Monitors were deployed at the stations listed below for the indicated periods. USGS, U.S. Geological Survey; Min, daily minimum; Max, daily maximum; NA, not available or not applicable]

\begin{tabular}{|c|c|c|c|c|c|}
\hline $\begin{array}{l}\text { Index } \\
\text { number } \\
\text { (fig. 2) }\end{array}$ & USGS station number & USGS station name & $\begin{array}{c}\text { Periods of } \\
\text { deployment } \\
\text { (year/month/day) }\end{array}$ & $\begin{array}{l}\text { Location in } \\
\text { cross section }\end{array}$ & $\begin{array}{l}\text { Approximate } \\
\text { depth of } \\
\text { measurement, } \\
\text { in feet }\end{array}$ \\
\hline 3 & 400412074082600 & $\begin{array}{l}\text { Metedeconk River } \\
\text { near Bricktown }\end{array}$ & $\begin{array}{l}93 / 08 / 31-93 / 09 / 10 \\
93 / 09 / 22-93 / 10 / 19 \\
93 / 10 / 21-93 / 11 / 17 \\
93 / 12 / 02-94 / 01 / 29\end{array}$ & $\begin{array}{l}\text { In channel, } \\
\text { near bottom }\end{array}$ & 5 \\
\hline 4 & 400405074080600 & $\begin{array}{l}\text { Metedeconk River at } \\
\text { Laurelton Gardens }\end{array}$ & $\begin{array}{l}92 / 11 / 19-92 / 11 / 29 \\
92 / 12 / 15-92 / 12 / 19 \\
93 / 01 / 21-93 / 01 / 28 \\
93 / 02 / 04-93 / 02 / 08 \\
93 / 03 / 02-93 / 03 / 15 \\
93 / 04 / 08-93 / 04 / 22 \\
93 / 04 / 22-93 / 05 / 20 \\
93 / 05 / 20-93 / 06 / 03 \\
93 / 06 / 08-93 / 06 / 21 \\
93 / 07 / 01-93 / 07 / 20 \\
93 / 07 / 28-93 / 08 / 11 \\
93 / 08 / 11-93 / 08 / 26 \\
93 / 09 / 29-93 / 10 / 19\end{array}$ & $\begin{array}{c}\text { Edge of } \\
\text { channel, } \\
\text { near bottom }\end{array}$ & 1 \\
\hline 9 & 01408160 & $\begin{array}{l}\text { Metedeconk River } \\
\text { near Laurelton }\end{array}$ & $\begin{array}{l}93 / 07 / 01-93 / 07 / 07 \\
93 / 09 / 10-93 / 09 / 15 \\
93 / 11 / 04-93 / 11 / 15\end{array}$ & $\begin{array}{l}\text { Left bank near } \\
\text { surface; and } \\
\text { left bank near } \\
\text { bottom }\end{array}$ & $\begin{array}{l}1 \\
6\end{array}$ \\
\hline
\end{tabular}


Appendix 6. Measurements at tidal stations in and near the tidal embayment of the Metedeconk River, N.J.--Daily minimum and maximum values of water-quality characteristics from measurements by movable monitors, November 1992 - January 1994--Continued

The following characteristics were measured. Intervals between measurements were 15, 30 , or 60 minutes. Days with partial records are included.

\begin{tabular}{llc}
\hline \multicolumn{1}{c}{ Characteristic } & \multicolumn{1}{c}{ Unit } & Rounding \\
\hline & & \\
Temperature & Degrees Celsius & 0.5 \\
Specific conductance & Microsiemens per centimeter at & 100 \\
& 25 degrees Celsius & \\
$\mathrm{pH}$ & Standard units & 0.1 \\
Dissolved-oxygen & Milligrams per liter & 0.1 \\
concentration & & \\
\hline
\end{tabular}


Appendix 6. Measurements at tidal stations in and near the tidal embayment of the Metedeconk River, N.J.--Daily minimum and maximum values of water-quality characteristics from measurements by movable monitors, November 1992 - January 1994--Continued

\begin{tabular}{|c|c|c|c|c|c|c|c|c|}
\hline \multirow{3}{*}{$\begin{array}{c}\text { Date } \\
\text { year/month/day }\end{array}$} & \multicolumn{8}{|c|}{ Daily minimum and maximum } \\
\hline & \multicolumn{2}{|c|}{ Temperature } & \multicolumn{2}{|c|}{ Specific conductance } & \multicolumn{2}{|c|}{ pH } & \multicolumn{2}{|c|}{$\begin{array}{l}\text { Dissolved-oxygen } \\
\text { concentration }\end{array}$} \\
\hline & Min & $\operatorname{Max}$ & Min & $\operatorname{Max}$ & Min & Max & Min & $\operatorname{Max}$ \\
\hline
\end{tabular}

400412074082600 Metedeconk River near Bricktown, in channel, near bottom

\begin{tabular}{|c|c|c|c|c|c|c|c|c|}
\hline $93 / 08 / 31$ & 24.0 & 24.5 & 100 & 100 & 6.3 & 6.5 & 7.3 & 8.4 \\
\hline $93 / 09 / 01$ & 23.0 & 24.5 & 100 & 100 & 6.0 & 6.3 & 4.8 & 7.9 \\
\hline $93 / 09 / 02$ & 23.5 & 24.5 & 100 & 100 & 6.0 & 6.2 & 4.2 & 7.1 \\
\hline $93 / 09 / 03$ & 23.0 & 24.5 & 100 & 5,800 & 5.9 & 6.3 & 3.8 & 6.7 \\
\hline $93 / 09 / 04$ & 23.5 & 25.5 & 100 & 10,300 & 5.8 & 6.4 & 3.2 & 6.3 \\
\hline 93/09/05 & 22.5 & 24.0 & 100 & 100 & 6.0 & 6.2 & 4.8 & 7.6 \\
\hline $93 / 09 / 06$ & 21.5 & 23.5 & 100 & 100 & 6.1 & 6.3 & 5.2 & 7.7 \\
\hline $93 / 09 / 07$ & 21.5 & 23.0 & 100 & 100 & 6.0 & 6.2 & 5.0 & 7.6 \\
\hline $93 / 09 / 08$ & 21.5 & 22.0 & 100 & 100 & 6.1 & 6.3 & 5.6 & 7.5 \\
\hline 93/09/09 & 21.0 & 24.5 & 100 & 16,400 & 6.1 & 6.7 & 4.0 & 7.9 \\
\hline $93 / 09 / 10$ & 21.0 & 24.5 & 100 & 17,100 & 5.9 & 6.7 & 2.8 & 5.3 \\
\hline $93 / 09 / 22$ & 17.0 & 17.0 & 100 & 100 & NA & NA & NA & NA \\
\hline $93 / 09 / 23$ & 16.5 & 17.5 & 100 & 100 & NA & NA & NA & NA \\
\hline $93 / 09 / 24$ & 17.0 & 18.5 & 100 & 100 & NA & NA & NA & NA \\
\hline $93 / 09 / 25$ & 16.0 & 17.5 & 100 & 100 & NA & NA & NA & NA \\
\hline $93 / 09 / 26$ & 16.5 & 19.0 & 100 & 100 & NA & NA & NA & NA \\
\hline $93 / 09 / 27$ & 19.0 & 19.5 & 100 & 100 & NA & NA & NA & NA \\
\hline $93 / 09 / 28$ & 17.0 & 19.0 & 100 & 100 & NA & NA & NA & NA \\
\hline $93 / 09 / 29$ & 16.0 & 17.0 & 100 & 100 & NA & NA & NA & NA \\
\hline $93 / 09 / 30$ & 14.0 & 16.0 & 100 & 100 & NA & NA & NA & NA \\
\hline $93 / 10 / 01$ & 13.5 & 15.0 & 100 & 100 & NA & NA & NA & NA \\
\hline $93 / 10 / 02$ & 13.0 & 15.5 & 100 & 100 & NA & NA & NA & NA \\
\hline $93 / 10 / 03$ & 14.5 & 16.0 & 100 & 100 & NA & NA & NA & NA \\
\hline $93 / 10 / 04$ & 13.5 & 15.5 & 100 & 100 & NA & NA & NA & NA \\
\hline $93 / 10 / 05$ & 13.5 & 15.5 & 100 & 100 & NA & NA & NA & NA \\
\hline 93/10/06 & 12.5 & 14.5 & 100 & 100 & NA & NA & NA & NA \\
\hline $93 / 10 / 07$ & 12.5 & 15.0 & 100 & 100 & NA & NA & NA & NA \\
\hline $93 / 10 / 08$ & 14.5 & 16.0 & 100 & 100 & NA & NA & NA & NA \\
\hline $93 / 10 / 09$ & 15.0 & 18.0 & 100 & 13,200 & NA & NA & NA & NA \\
\hline $93 / 10 / 10$ & 13.0 & 16.5 & 100 & 1,000 & NA & NA & NA & NA \\
\hline $93 / 10 / 11$ & 12.0 & 13.0 & 100 & 100 & NA & NA & NA & NA \\
\hline $93 / 10 / 12$ & 12.0 & 13.0 & 100 & 100 & NA & NA & NA & NA \\
\hline $93 / 10 / 13$ & 12.0 & 13.0 & 100 & 100 & NA & NA & NA & NA \\
\hline $93 / 10 / 14$ & 11.0 & 11.5 & 100 & 100 & NA & NA & NA & NA \\
\hline $93 / 10 / 15$ & 11.5 & 13.0 & 100 & 100 & NA & NA & NA & NA \\
\hline $93 / 10 / 16$ & 11.5 & 13.5 & 100 & 100 & NA & NA & NA & NA \\
\hline $93 / 10 / 17$ & 12.5 & 13.5 & 100 & 100 & NA & NA & NA & NA \\
\hline $93 / 10 / 18$ & 13.5 & 15.5 & 100 & 100 & NA & NA & NA & NA \\
\hline $93 / 10 / 19$ & 13.0 & 14.0 & 100 & 200 & NA & NA & NA & NA \\
\hline
\end{tabular}


Appendix 6. Measurements at tidal stations in and near the tidal embayment of the Metedeconk River, N.J.--Daily minimum and maximum values of water-quality characteristics from measurements by movable monitors, November 1992 - January 1994--Continued

\begin{tabular}{|c|c|c|c|c|c|c|c|c|}
\hline \multirow{3}{*}{$\begin{array}{c}\text { Date } \\
\text { year/month/day }\end{array}$} & \multicolumn{8}{|c|}{ Daily minimum and maximum } \\
\hline & \multicolumn{2}{|c|}{ Temperature } & \multicolumn{2}{|c|}{ Specific conductance } & \multicolumn{2}{|c|}{$\mathrm{pH}$} & \multicolumn{2}{|c|}{$\begin{array}{l}\text { Dissolved-oxygen } \\
\text { concentration }\end{array}$} \\
\hline & Min & $\operatorname{Max}$ & Min & Max & Min & $\operatorname{Max}$ & Min & $\operatorname{Max}$ \\
\hline
\end{tabular}

400412074082600 Metedeconk River near Bricktown, in channel, near bottom (continued)

\begin{tabular}{|c|c|c|c|c|c|c|c|c|}
\hline $93 / 10 / 21$ & 15.5 & 16.0 & 100 & 100 & NA & NA & NA & NA \\
\hline $93 / 10 / 22$ & 13.5 & 15.5 & 100 & 100 & NA & NA & NA & NA \\
\hline $93 / 10 / 23$ & 12.0 & 13.5 & 100 & 100 & NA & NA & NA & NA \\
\hline $93 / 10 / 24$ & 11.0 & 12.5 & 100 & 100 & NA & NA & NA & NA \\
\hline $93 / 10 / 25$ & 11.5 & 13.0 & 100 & 100 & NA & NA & NA & NA \\
\hline $93 / 10 / 26$ & 12.0 & 12.5 & 100 & 100 & NA & NA & NA & NA \\
\hline $93 / 10 / 27$ & 12.0 & 14.0 & 100 & 24,300 & NA & NA & NA & NA \\
\hline $93 / 10 / 28$ & 13.5 & 14.0 & 19,500 & 25,100 & NA & NA & NA & NA \\
\hline $93 / 10 / 29$ & 10.5 & 13.5 & 100 & 19,900 & NA & NA & NA & NA \\
\hline $93 / 10 / 30$ & 11.0 & 11.5 & 100 & 100 & NA & NA & NA & NA \\
\hline $93 / 10 / 31$ & 11.0 & 11.0 & 100 & 100 & NA & NA & NA & NA \\
\hline $93 / 11 / 01$ & 9.0 & 11.0 & 100 & 6,700 & NA & NA & NA & NA \\
\hline $93 / 11 / 02$ & 8.0 & 9.0 & 100 & 100 & NA & NA & NA & NA \\
\hline $93 / 11 / 03$ & 7.5 & 8.0 & 100 & 100 & NA & NA & NA & NA \\
\hline $93 / 11 / 04$ & 7.0 & 8.5 & 100 & 100 & NA & NA & NA & NA \\
\hline $93 / 11 / 05$ & 8.0 & 10.0 & 100 & 100 & NA & NA & NA & NA \\
\hline $93 / 11 / 06$ & 9.5 & 10.5 & 100 & 100 & NA & NA & NA & NA \\
\hline $93 / 11 / 07$ & 8.0 & 9.5 & 100 & 100 & NA & NA & NA & NA \\
\hline $93 / 11 / 08$ & 6.5 & 8.0 & 100 & 100 & NA & NA & NA & NA \\
\hline $93 / 11 / 09$ & 6.0 & 7.5 & 100 & 100 & NA & NA & NA & NA \\
\hline $93 / 11 / 10$ & 6.5 & 7.5 & 100 & 100 & NA & NA & NA & NA \\
\hline $93 / 11 / 11$ & 6.0 & 7.0 & 100 & 100 & NA & NA & NA & NA \\
\hline $93 / 11 / 12$ & 7.0 & 9.0 & 100 & 900 & NA & NA & NA & NA \\
\hline $93 / 11 / 13$ & 7.5 & 9.0 & 100 & 100 & NA & NA & NA & NA \\
\hline $93 / 11 / 14$ & 9.0 & 11.0 & 100 & 200 & NA & NA & NA & NA \\
\hline $93 / 11 / 15$ & 11.0 & 14.0 & 100 & 100 & NA & NA & NA & NA \\
\hline $93 / 11 / 16$ & 11.5 & 13.5 & 100 & 200 & NA & NA & NA & NA \\
\hline $93 / 11 / 17$ & 10.0 & 11.5 & 100 & 100 & NA & NA & NA & NA \\
\hline $93 / 12 / 02$ & 7.5 & 7.5 & 100 & 100 & NA & NA & NA & NA \\
\hline $93 / 12 / 03$ & 7.0 & 8.5 & 100 & 100 & NA & NA & NA & NA \\
\hline $93 / 12 / 04$ & 7.5 & 8.0 & 100 & 200 & NA & NA & NA & NA \\
\hline $93 / 12 / 05$ & 8.0 & 9.5 & 100 & 1,300 & NA & NA & NA & NA \\
\hline $93 / 12 / 06$ & 7.5 & 8.5 & 100 & 100 & NA & NA & NA & NA \\
\hline $93 / 12 / 07$ & 6.5 & 7.5 & 100 & 100 & NA & NA & NA & NA \\
\hline $93 / 12 / 08$ & 7.0 & 7.5 & 100 & 100 & NA & NA & NA & NA \\
\hline $93 / 12 / 09$ & 6.0 & 7.0 & 100 & 100 & NA & NA & NA & NA \\
\hline $93 / 12 / 10$ & 6.0 & 7.0 & 100 & 100 & NA & NA & NA & NA \\
\hline $93 / 12 / 11$ & 6.0 & 8.0 & 100 & 100 & NA & NA & NA & NA \\
\hline $93 / 12 / 12$ & 3.5 & 5.5 & 100 & 100 & NA & NA & NA & NA \\
\hline $93 / 12 / 13$ & 3.0 & 4.0 & 100 & 100 & NA & NA & NA & NA \\
\hline
\end{tabular}


Appendix 6. Measurements at tidal stations in and near the tidal embayment of the Metedeconk River, N.J.--Daily minimum and maximum values of water-quality characteristics from measurements by movable monitors, November 1992 - January 1994--Continued

\begin{tabular}{|c|c|c|c|c|c|c|c|c|}
\hline \multirow{3}{*}{$\begin{array}{c}\text { Date } \\
\text { year/month/day }\end{array}$} & \multicolumn{8}{|c|}{ Daily minimum and maximum } \\
\hline & \multicolumn{2}{|c|}{ Temperature } & \multicolumn{2}{|c|}{ Specific conductance } & \multicolumn{2}{|c|}{$\mathrm{pH}$} & \multicolumn{2}{|c|}{$\begin{array}{l}\text { Dissolved-oxygen } \\
\text { concentration }\end{array}$} \\
\hline & Min & $\operatorname{Max}$ & Min & $\operatorname{Max}$ & Min & Max & Min & $\operatorname{Max}$ \\
\hline
\end{tabular}

400412074082600 Metedeconk River near Bricktown, in channel, near bottom (continued)

\begin{tabular}{|c|c|c|c|c|c|c|c|c|}
\hline $93 / 12 / 14$ & 3.5 & 5.5 & 100 & 32,800 & NA & NA & NA & NA \\
\hline $93 / 12 / 15$ & 5.0 & 5.5 & 100 & 32,000 & NA & NA & NA & NA \\
\hline $93 / 12 / 16$ & 5.5 & 6.0 & 100 & 24,100 & NA & NA & NA & NA \\
\hline $93 / 12 / 17$ & 5.5 & 6.5 & 24,100 & 35,500 & NA & NA & NA & NA \\
\hline $93 / 12 / 18$ & 6.0 & 6.5 & 30,500 & 33,900 & NA & NA & NA & NA \\
\hline $93 / 12 / 19$ & 5.0 & 6.5 & 200 & 31,900 & NA & NA & NA & NA \\
\hline $93 / 12 / 20$ & 4.5 & 5.5 & 100 & 200 & NA & NA & NA & NA \\
\hline $93 / 12 / 21$ & 5.5 & 7.0 & 100 & 12,100 & NA & NA & NA & NA \\
\hline $93 / 12 / 22$ & 4.5 & 6.0 & 100 & 100 & NA & NA & NA & NA \\
\hline $93 / 12 / 23$ & 3.5 & 4.5 & 100 & 100 & NA & NA & NA & NA \\
\hline $93 / 12 / 24$ & 2.5 & 3.5 & 100 & 100 & NA & NA & NA & NA \\
\hline $93 / 12 / 25$ & 2.5 & 3.5 & 100 & 100 & NA & NA & NA & NA \\
\hline $93 / 12 / 26$ & 1.0 & 3.0 & 100 & 200 & NA & NA & NA & NA \\
\hline $93 / 12 / 27$ & 0.5 & 1.0 & 200 & 200 & NA & NA & NA & NA \\
\hline $93 / 12 / 28$ & 0.5 & 1.5 & 100 & 200 & NA & NA & NA & NA \\
\hline $93 / 12 / 29$ & 0.5 & 1.0 & 100 & 200 & NA & NA & NA & NA \\
\hline $93 / 12 / 30$ & 0.5 & 1.0 & 200 & 200 & NA & NA & NA & NA \\
\hline $93 / 12 / 31$ & 0.0 & 1.5 & 100 & 200 & NA & NA & NA & NA \\
\hline $94 / 01 / 01$ & 1.0 & 2.5 & 200 & 200 & NA & NA & NA & NA \\
\hline $94 / 01 / 02$ & 2.5 & 3.5 & 200 & 300 & NA & NA & NA & NA \\
\hline $94 / 01 / 03$ & 2.5 & 3.0 & 200 & 300 & NA & NA & NA & NA \\
\hline $94 / 01 / 04$ & 2.0 & 3.5 & 200 & 14,200 & NA & NA & NA & NA \\
\hline $94 / 01 / 05$ & 1.5 & 2.0 & 200 & 12,600 & NA & NA & NA & NA \\
\hline $94 / 01 / 06$ & 1.0 & 2.0 & 200 & 300 & NA & NA & NA & NA \\
\hline $94 / 01 / 07$ & 2.0 & 2.5 & 200 & 300 & NA & NA & NA & NA \\
\hline $94 / 01 / 08$ & 1.0 & 2.5 & 300 & 500 & NA & NA & NA & NA \\
\hline $94 / 01 / 09$ & 0.5 & 1.5 & 300 & 400 & NA & NA & NA & NA \\
\hline $94 / 01 / 10$ & 0.5 & 1.5 & 300 & 400 & NA & NA & NA & NA \\
\hline $94 / 01 / 11$ & 0.5 & 2.0 & 300 & 300 & NA & NA & NA & NA \\
\hline $94 / 01 / 12$ & 2.0 & 2.5 & 300 & 300 & NA & NA & NA & NA \\
\hline $94 / 01 / 13$ & 2.0 & 2.5 & 300 & 400 & NA & NA & NA & NA \\
\hline $94 / 01 / 14$ & 2.0 & 3.0 & 300 & 400 & NA & NA & NA & NA \\
\hline $94 / 01 / 15$ & 0.0 & 2.5 & 300 & 300 & NA & NA & NA & NA \\
\hline $94 / 01 / 16$ & 0.0 & 1.0 & 300 & 300 & NA & NA & NA & NA \\
\hline $94 / 01 / 17$ & 0.0 & 1.5 & 200 & 400 & NA & NA & NA & NA \\
\hline $94 / 01 / 18$ & 0.0 & 1.5 & 200 & 400 & NA & NA & NA & NA \\
\hline $94 / 01 / 19$ & 0.0 & 0.5 & 200 & 300 & NA & NA & NA & NA \\
\hline $94 / 01 / 20$ & 0.0 & 0.5 & 200 & 300 & NA & NA & NA & NA \\
\hline $94 / 01 / 21$ & 0.0 & 0.5 & 200 & 200 & NA & NA & NA & NA \\
\hline $94 / 01 / 22$ & 0.0 & 1.0 & 200 & 200 & NA & NA & NA & NA \\
\hline $94 / 01 / 23$ & 0.5 & 1.0 & 200 & 300 & NA & NA & NA & NA \\
\hline $94 / 01 / 24$ & 1.0 & 2.5 & 200 & 300 & NA & NA & NA & NA \\
\hline
\end{tabular}


Appendix 6. Measurements at tidal stations in and near the tidal embayment of the Metedeconk River, N.J.--Daily minimum and maximum values of water-quality characteristics from measurements by movable monitors, November 1992 - January 1994-Continued

\begin{tabular}{|c|c|c|c|c|c|c|c|c|}
\hline \multirow{3}{*}{$\begin{array}{c}\text { Date } \\
\text { year/month/day }\end{array}$} & \multicolumn{8}{|c|}{ Daily minimum and maximum } \\
\hline & \multicolumn{2}{|c|}{ Temperature } & \multicolumn{2}{|c|}{ Specific conductance } & \multicolumn{2}{|c|}{$\mathrm{pH}$} & \multicolumn{2}{|c|}{$\begin{array}{l}\text { Dissolved-oxygen } \\
\text { concentration }\end{array}$} \\
\hline & Min & $\operatorname{Max}$ & Min & $\operatorname{Max}$ & Min & $\operatorname{Max}$ & Min & $\operatorname{Max}$ \\
\hline
\end{tabular}

400412074082600 Metedeconk River near Bricktown, in channel, near bottom (continued)

$\begin{array}{lllllllll}94 / 01 / 25 & 1.5 & 2.5 & 200 & 300 & \text { NA } & \text { NA } & \text { NA } & \text { NA } \\ 94 / 01 / 26 & 1.0 & 2.0 & 300 & 300 & \text { NA } & \text { NA } & \text { NA } & \text { NA } \\ 94 / 01 / 27 & 0.5 & 1.5 & 200 & 300 & \text { NA } & \text { NA } & \text { NA } & \text { NA } \\ 94 / 01 / 28 & 1.5 & 2.5 & 200 & 400 & \text { NA } & \text { NA } & \text { NA } & \text { NA } \\ 94 / 01 / 29 & 1.0 & 1.5 & 200 & 300 & \text { NA } & \text { NA } & \text { NA } & \text { NA }\end{array}$

400405074080600 Metedeconk River at Laurelton Gardens, edge of channel, near bottom

$\begin{array}{lrrrrrrrr}92 / 11 / 19 & 6.5 & 7.5 & 500 & 1,800 & 6.4 & 6.7 & 8.5 & 10.7 \\ 92 / 11 / 20 & 6.0 & 8.0 & 500 & 9,400 & 6.5 & 7.0 & 8.7 & 11.0 \\ 92 / 11 / 21 & 6.5 & 9.5 & 200 & 2,300 & 6.5 & 6.6 & 9.6 & 11.3 \\ 92 / 11 / 22 & 9.0 & 11.0 & 200 & 2,100 & 6.4 & 6.7 & 6.8 & 10.9 \\ 92 / 11 / 23 & 11.0 & 15.0 & 200 & 12,100 & 6.4 & 6.9 & 8.1 & 10.0 \\ 92 / 11 / 24 & 11.0 & 12.5 & 100 & 11,200 & 6.3 & 6.8 & 7.2 & 9.6 \\ 92 / 11 / 25 & 10.5 & 11.0 & 100 & 600 & 6.1 & 6.4 & 8.8 & 9.8 \\ 92 / 11 / 26 & 10.5 & 12.0 & 100 & 200 & 6.1 & 6.3 & 8.9 & 9.4 \\ 92 / 11 / 27 & 10.5 & 12.0 & 100 & 300 & 6.1 & 6.3 & 8.2 & 9.1 \\ 92 / 11 / 28 & 9.0 & 11.0 & 100 & 300 & 6.1 & 6.2 & 8.9 & 9.9 \\ 92 / 11 / 29 & 8.5 & 9.5 & 100 & 600 & 6.2 & 6.3 & 9.4 & 10.1 \\ & & & & & & & & \\ 92 / 12 / 15 & 4.5 & 5.0 & 100 & 200 & \text { NA } & \text { NA } & \text { NA } & \text { NA } \\ 92 / 12 / 16 & 3.5 & 5.5 & 100 & 200 & \text { NA } & \text { NA } & \text { NA } & \text { NA } \\ 92 / 12 / 17 & 5.5 & 8.0 & 100 & 200 & \text { NA } & \text { NA } & \text { NA } & \text { NA } \\ 92 / 12 / 18 & 6.0 & 7.5 & 200 & 200 & \text { NA } & \text { NA } & \text { NA } & \text { NA } \\ 92 / 12 / 19 & 5.0 & 6.0 & 200 & 200 & \text { NA } & \text { NA } & \text { NA } & \text { NA } \\ & & & & & & & & \\ 93 / 01 / 21 & 4.0 & 5.0 & 200 & 200 & \text { NA } & \text { NA } & \text { NA } & \text { NA } \\ 93 / 01 / 22 & 5.0 & 6.5 & 100 & 200 & \text { NA } & \text { NA } & \text { NA } & \text { NA } \\ 93 / 01 / 23 & 5.0 & 6.5 & 100 & 200 & \text { NA } & \text { NA } & \text { NA } & \text { NA } \\ 93 / 01 / 24 & 4.0 & 7.5 & 100 & 200 & \text { NA } & \text { NA } & \text { NA } & \text { NA } \\ 93 / 01 / 25 & 4.0 & 7.0 & 200 & 400 & \text { NA } & \text { NA } & \text { NA } & \text { NA } \\ 93 / 01 / 26 & 1.0 & 6.5 & 200 & 200 & \text { NA } & \text { NA } & \text { NA } & \text { NA } \\ 93 / 01 / 27 & 3.0 & 5.5 & 100 & 200 & \text { NA } & \text { NA } & \text { NA } & \text { NA } \\ 93 / 01 / 28 & 2.0 & 4.5 & 200 & 200 & \text { NA } & \text { NA } & \text { NA } & \text { NA } \\ 93 / 02 / 04 & 0.0 & 3.0 & 100 & 500 & \text { NA } & \text { NA } & \text { NA } & \text { NA } \\ 93 / 02 / 05 & 0.5 & 4.5 & 200 & 600 & \text { NA } & \text { NA } & \text { NA } & \text { NA } \\ 93 / 02 / 06 & 0.5 & 3.0 & 200 & 800 & \text { NA } & \text { NA } & \text { NA } & \text { NA } \\ 93 / 02 / 07 & 0.5 & 3.0 & 300 & 4,400 & \text { NA } & \text { NA } & \text { NA } & \text { NA } \\ 93 / 02 / 08 & 2.0 & 3.5 & 700 & 1,600 & \text { NA } & \text { NA } & \text { NA } & \text { NA }\end{array}$


Appendix 6. Measurements at tidal stations in and near the tidal embayment of the Metedeconk River, N.J.--Daily minimum and maximum values of water-quality characteristics from measurements by movable monitors, November 1992 - January 1994--Continued

\begin{tabular}{|c|c|c|c|c|c|c|c|c|}
\hline \multirow{3}{*}{$\begin{array}{c}\text { Date } \\
\text { year/month/day }\end{array}$} & \multicolumn{8}{|c|}{ Daily minimum and maximum } \\
\hline & \multicolumn{2}{|c|}{ Temperature } & \multicolumn{2}{|c|}{ Specific conductance } & \multicolumn{2}{|c|}{$\mathrm{pH}$} & \multicolumn{2}{|c|}{$\begin{array}{l}\text { Dissolved-oxygen } \\
\text { concentration }\end{array}$} \\
\hline & Min & $\operatorname{Max}$ & Min & Max & Min & $\operatorname{Max}$ & Min & $\operatorname{Max}$ \\
\hline
\end{tabular}

400405074080600 Metedeconk River at Laurelton Gardens, edge of channel, near bottom (continued)

\begin{tabular}{|c|c|c|c|c|c|c|c|c|}
\hline $93 / 03 / 02$ & 3.0 & 5.5 & 300 & 700 & NA & NA & NA & NA \\
\hline $93 / 03 / 03$ & 4.0 & 6.5 & 200 & 300 & NA & NA & NA & NA \\
\hline $93 / 03 / 04$ & 4.0 & 6.5 & 200 & 300 & NA & NA & NA & NA \\
\hline $93 / 03 / 05$ & 3.0 & 4.0 & 100 & 200 & NA & NA & NA & NA \\
\hline 93/03/06 & 2.0 & 4.0 & 200 & 200 & NA & NA & NA & NA \\
\hline $93 / 03 / 07$ & 2.5 & 7.0 & 100 & 200 & NA & NA & NA & NA \\
\hline $93 / 03 / 08$ & 4.5 & 7.5 & 100 & 200 & NA & NA & NA & NA \\
\hline $93 / 03 / 09$ & 4.5 & 6.5 & 100 & 200 & NA & NA & NA & NA \\
\hline $93 / 03 / 10$ & 3.5 & 6.0 & 100 & 200 & NA & NA & NA & NA \\
\hline $93 / 03 / 11$ & 4.0 & 7.0 & 100 & 200 & NA & NA & NA & NA \\
\hline $93 / 03 / 12$ & 2.0 & 8.0 & 200 & 300 & NA & NA & NA & NA \\
\hline $93 / 03 / 13$ & 0.5 & 5.5 & 100 & 5,600 & NA & NA & NA & NA \\
\hline $93 / 03 / 14$ & 0.0 & 2.0 & 500 & 12,200 & NA & NA & NA & NA \\
\hline $93 / 03 / 15$ & 0.0 & 4.5 & 300 & 600 & NA & NA & NA & NA \\
\hline $93 / 04 / 08$ & 11.5 & 12.5 & 100 & 100 & 6.1 & 6.5 & 10.1 & 10.7 \\
\hline $93 / 04 / 09$ & 9.5 & 12.5 & 100 & 100 & 6.0 & 6.1 & 9.7 & 10.5 \\
\hline $93 / 04 / 10$ & 11.0 & 12.0 & 100 & 100 & 6.1 & 6.2 & 9.5 & 9.9 \\
\hline $93 / 04 / 11$ & 11.0 & 14.0 & 100 & 100 & 6.0 & 6.3 & 9.1 & 10.1 \\
\hline $93 / 04 / 12$ & 10.5 & 14.5 & 100 & 1,400 & 6.1 & 6.4 & 9.4 & 10.0 \\
\hline $93 / 04 / 13$ & 10.5 & 15.0 & 100 & 700 & 6.2 & 6.5 & 9.7 & 10.3 \\
\hline $93 / 04 / 14$ & 12.0 & 14.5 & 100 & 300 & 6.1 & 6.3 & 8.9 & 10.0 \\
\hline $93 / 04 / 15$ & 12.0 & 14.5 & 100 & 100 & 5.9 & 6.2 & 8.6 & 9.9 \\
\hline $93 / 04 / 16$ & 13.0 & 14.5 & 100 & 200 & 5.8 & 6.4 & 7.7 & 9.7 \\
\hline $93 / 04 / 17$ & 13.5 & 16.0 & 100 & 200 & 5.8 & 6.4 & 8.2 & 9.5 \\
\hline $93 / 04 / 18$ & 11.5 & 16.5 & 100 & 100 & 5.8 & 6.4 & 8.7 & 10.3 \\
\hline $93 / 04 / 19$ & 12.0 & 17.0 & 100 & 6,100 & 6.0 & 6.8 & 8.4 & 9.9 \\
\hline $93 / 04 / 20$ & 13.5 & 17.5 & 100 & 300 & 5.9 & 6.4 & 7.4 & 9.4 \\
\hline $93 / 04 / 21$ & 14.5 & 18.5 & 100 & 100 & 5.6 & 6.4 & 6.9 & 9.7 \\
\hline $93 / 04 / 22$ & 15.0 & 16.5 & 100 & 100 & 6.0 & 6.3 & 7.5 & 9.1 \\
\hline $93 / 04 / 22$ & 12.0 & 15.0 & 100 & 100 & NA & NA & NA & NA \\
\hline $93 / 04 / 23$ & 10.0 & 11.5 & 100 & 100 & NA & NA & NA & NA \\
\hline $93 / 04 / 24$ & 9.5 & 15.0 & 100 & 100 & NA & NA & NA & NA \\
\hline $93 / 04 / 25$ & 11.5 & 17.5 & 100 & 100 & NA & NA & NA & NA \\
\hline $93 / 04 / 26$ & 15.0 & 17.5 & 100 & 100 & NA & NA & NA & NA \\
\hline $93 / 04 / 27$ & 12.0 & 18.5 & 100 & 100 & NA & NA & NA & NA \\
\hline $93 / 04 / 28$ & 12.0 & 17.0 & 100 & 100 & NA & NA & NA & NA \\
\hline $93 / 04 / 29$ & 12.5 & 17.0 & 100 & 100 & NA & NA & NA & NA \\
\hline $93 / 04 / 30$ & 13.5 & 18.0 & 100 & 100 & NA & NA & $\mathrm{NA}$ & NA \\
\hline $93 / 05 / 01$ & 14.5 & 19.5 & 100 & 100 & NA & NA & NA & NA \\
\hline $93 / 05 / 02$ & 15.5 & 19.5 & 100 & 100 & NA & NA & NA & NA \\
\hline
\end{tabular}


Appendix 6. Measurements at tidal stations in and near the tidal embayment of the Metedeconk River, N.J.--Daily minimum and maximum values of water-quality characteristics from measurements by movable monitors, November 1992 - January 1994--Continued

\begin{tabular}{|c|c|c|c|c|c|c|c|c|}
\hline \multirow{3}{*}{$\begin{array}{c}\text { Date } \\
\text { year/month/day }\end{array}$} & \multicolumn{8}{|c|}{ Daily minimum and maximum } \\
\hline & \multicolumn{2}{|c|}{ Temperature } & \multicolumn{2}{|c|}{ Specific conductance } & \multicolumn{2}{|c|}{$\mathrm{pH}$} & \multicolumn{2}{|c|}{$\begin{array}{l}\text { Dissolved-oxygen } \\
\text { concentration }\end{array}$} \\
\hline & Min & $\operatorname{Max}$ & Min & $\operatorname{Max}$ & Min & $\operatorname{Max}$ & Min & $\operatorname{Max}$ \\
\hline
\end{tabular}

400405074080600 Metedeconk River at Laurelton Gardens, edge of channel, near bottom (continued)

\begin{tabular}{|c|c|c|c|c|c|c|c|c|}
\hline $93 / 05 / 03$ & 15.5 & 19.5 & 100 & 100 & NA & NA & NA & NA \\
\hline $93 / 05 / 04$ & 15.5 & 18.5 & 100 & 100 & NA & NA & NA & NA \\
\hline 93/05/05 & 15.5 & 18.5 & 100 & 100 & NA & NA & NA & NA \\
\hline $93 / 05 / 06$ & 16.5 & 22.0 & 100 & 100 & NA & NA & NA & NA \\
\hline $93 / 05 / 07$ & 17.5 & 21.0 & 100 & 200 & NA & NA & NA & NA \\
\hline $93 / 05 / 08$ & 16.5 & 20.5 & 100 & 100 & NA & NA & NA & NA \\
\hline $93 / 05 / 09$ & 16.0 & 22.5 & 100 & 100 & NA & NA & NA & NA \\
\hline $93 / 05 / 10$ & 18.0 & 22.5 & 100 & 100 & NA & NA & NA & NA \\
\hline $93 / 05 / 11$ & 17.5 & 23.5 & 100 & 100 & NA & NA & NA & NA \\
\hline $93 / 05 / 12$ & 19.5 & 24.0 & 100 & 2,100 & NA & NA & NA & NA \\
\hline $93 / 05 / 13$ & 18.5 & 21.5 & 200 & 800 & NA & NA & NA & NA \\
\hline $93 / 05 / 14$ & 16.5 & 21.0 & 100 & 600 & NA & NA & NA & NA \\
\hline $93 / 05 / 15$ & 17.0 & 21.5 & 100 & 12,200 & NA & NA & NA & NA \\
\hline $93 / 05 / 16$ & 17.5 & 21.5 & 200 & 300 & NA & NA & NA & NA \\
\hline $93 / 05 / 17$ & 18.5 & 22.5 & 100 & 3,200 & NA & NA & NA & NA \\
\hline $93 / 05 / 18$ & 15.0 & 20.0 & 100 & 300 & NA & NA & NA & NA \\
\hline $93 / 05 / 19$ & 14.5 & 16.0 & 100 & 100 & NA & NA & NA & NA \\
\hline $93 / 05 / 20$ & 14.5 & 15.0 & 100 & 100 & NA & NA & NA & NA \\
\hline $93 / 05 / 20$ & 15.0 & 16.0 & 100 & 700 & 6.2 & 6.4 & 7.3 & 9.1 \\
\hline $93 / 05 / 21$ & 13.5 & 17.5 & 100 & 15,100 & 6.1 & 6.7 & 4.3 & 9.3 \\
\hline $93 / 05 / 22$ & 14.0 & 18.5 & 200 & 900 & 6.1 & 6.7 & 7.9 & 9.3 \\
\hline $93 / 05 / 23$ & 14.0 & 20.5 & 200 & 5,900 & 6.3 & 6.7 & 7.5 & 9.9 \\
\hline $93 / 05 / 24$ & 16.0 & 21.5 & 200 & 10,400 & 6.2 & 6.9 & 6.9 & 9.5 \\
\hline $93 / 05 / 25$ & 18.0 & 22.5 & 100 & 1,600 & 6.2 & 6.5 & 5.6 & 9.4 \\
\hline $93 / 05 / 26$ & 18.0 & 22.5 & 100 & 900 & 6.2 & 6.9 & 7.0 & 10.0 \\
\hline $93 / 05 / 27$ & 17.0 & 21.0 & 200 & 2,500 & 6.2 & 6.7 & 6.4 & 9.6 \\
\hline $93 / 05 / 28$ & 17.0 & 22.0 & 200 & 500 & 6.2 & 6.7 & 6.4 & 9.8 \\
\hline $93 / 05 / 29$ & 18.5 & 22.5 & 200 & 900 & 6.3 & 6.8 & 6.8 & 9.1 \\
\hline $93 / 05 / 30$ & 15.5 & 22.0 & 100 & 8,800 & 6.4 & 6.8 & 7.2 & 10.0 \\
\hline $93 / 05 / 31$ & 17.5 & 20.5 & 400 & 18,900 & 6.2 & 7.1 & 5.8 & 8.4 \\
\hline $93 / 06 / 01$ & 17.0 & 21.5 & 600 & 15,700 & 6.3 & 6.9 & 5.5 & 9.2 \\
\hline $93 / 06 / 02$ & 16.5 & 20.5 & 300 & 9,200 & 6.2 & 6.7 & 6.4 & 9.4 \\
\hline $93 / 06 / 03$ & 16.5 & 21.0 & 200 & 600 & 6.2 & 6.4 & 7.0 & 8.9 \\
\hline $93 / 06 / 08$ & 18.0 & 22.5 & 200 & 600 & NA & NA & NA & NA \\
\hline $93 / 06 / 09$ & 19.0 & 25.5 & 100 & 4,600 & NA & NA & NA & NA \\
\hline $93 / 06 / 10$ & 20.5 & 26.5 & 100 & 2,000 & NA & NA & NA & NA \\
\hline $93 / 06 / 11$ & 21.5 & 26.0 & 200 & 1,100 & NA & NA & NA & NA \\
\hline $93 / 06 / 12$ & 20.0 & 25.5 & 100 & 700 & NA & NA & NA & NA \\
\hline $93 / 06 / 13$ & 19.5 & 25.5 & 100 & 200 & NA & NA & NA & NA \\
\hline $93 / 06 / 14$ & 19.0 & 26.0 & 100 & 100 & NA & NA & NA & NA \\
\hline
\end{tabular}


Appendix 6. Measurements at tidal stations in and near the tidal embayment of the Metedeconk River, N.J.--Daily minimum and maximum values of water-quality characteristics from measurements by movable monitors, November 1992 - January 1994--Continued

\begin{tabular}{|c|c|c|c|c|c|c|c|c|}
\hline \multirow{3}{*}{$\begin{array}{c}\text { Date } \\
\text { year/month/day }\end{array}$} & \multicolumn{8}{|c|}{ Daily minimum and maximum } \\
\hline & \multicolumn{2}{|c|}{ Temperature } & \multicolumn{2}{|c|}{ Specific conductance } & \multicolumn{2}{|c|}{$\mathrm{pH}$} & \multicolumn{2}{|c|}{$\begin{array}{l}\text { Dissolved-oxygen } \\
\text { concentration }\end{array}$} \\
\hline & Min & Max & Min & $\operatorname{Max}$ & Min & $\operatorname{Max}$ & Min & Max \\
\hline
\end{tabular}

400405074080600 Metedeconk River at Laurelton Gardens, edge of channel, near bottom (continued)

\begin{tabular}{|c|c|c|c|c|c|c|c|c|}
\hline $93 / 06 / 15$ & 20.5 & 25.0 & 100 & 11,000 & NA & NA & NA & NA \\
\hline $93 / 06 / 16$ & 21.0 & 27.0 & 200 & 4,400 & NA & NA & NA & NA \\
\hline $93 / 06 / 17$ & 21.5 & 27.0 & 200 & 7,600 & NA & NA & NA & NA \\
\hline $93 / 06 / 18$ & 22.5 & 28.0 & 200 & 12,900 & NA & NA & NA & NA \\
\hline $93 / 06 / 19$ & 23.0 & 29.0 & 300 & 3,200 & NA & NA & NA & NA \\
\hline $93 / 06 / 20$ & 22.0 & 26.5 & 200 & 5,300 & NA & NA & NA & NA \\
\hline $93 / 06 / 21$ & 22.5 & 25.5 & 200 & 2,100 & NA & NA & NA & NA \\
\hline $93 / 07 / 01$ & 25.0 & 28.0 & 300 & 5,800 & NA & NA & NA & NA \\
\hline $93 / 07 / 02$ & 22.0 & 25.5 & 100 & 6,300 & NA & NA & NA & NA \\
\hline $93 / 07 / 03$ & 21.0 & 26.0 & 200 & 1,400 & NA & NA & NA & NA \\
\hline $93 / 07 / 04$ & 22.0 & 28.5 & 100 & 1,000 & NA & NA & NA & NA \\
\hline $93 / 07 / 05$ & 23.5 & 29.5 & 100 & 3,100 & NA & NA & NA & NA \\
\hline $93 / 07 / 06$ & 24.5 & 29.0 & 100 & 2,500 & NA & NA & NA & NA \\
\hline $93 / 07 / 07$ & 25.0 & 30.0 & 100 & 500 & NA & NA & NA & NA \\
\hline $93 / 07 / 08$ & 26.0 & 30.5 & 200 & 900 & NA & NA & NA & NA \\
\hline 93/07/09 & 27.0 & 31.5 & 300 & 4,300 & NA & NA & NA & NA \\
\hline $93 / 07 / 10$ & 27.0 & 31.5 & 500 & 10,400 & NA & NA & NA & NA \\
\hline $93 / 07 / 11$ & 27.0 & 30.5 & 900 & 15,300 & NA & NA & NA & NA \\
\hline $93 / 07 / 12$ & 26.0 & 29.5 & 400 & 3,000 & NA & NA & NA & NA \\
\hline $93 / 07 / 13$ & 26.5 & 31.0 & 600 & 12,100 & NA & NA & NA & NA \\
\hline $93 / 07 / 14$ & 24.5 & 29.0 & 200 & 2,600 & NA & NA & NA & NA \\
\hline $93 / 07 / 15$ & 23.5 & 28.0 & 100 & 300 & NA & NA & NA & NA \\
\hline $93 / 07 / 16$ & 22.0 & 27.5 & 100 & 100 & NA & NA & NA & NA \\
\hline $93 / 07 / 17$ & 21.5 & 26.5 & 100 & 11,000 & NA & NA & NA & NA \\
\hline $93 / 07 / 18$ & 22.5 & 27.5 & 1,200 & 13,100 & NA & NA & NA & NA \\
\hline $93 / 07 / 19$ & 21.0 & 26.5 & 300 & 12,500 & NA & NA & NA & NA \\
\hline $93 / 07 / 20$ & 21.0 & 21.0 & 100 & 400 & NA & NA & NA & NA \\
\hline $93 / 07 / 28$ & 24.5 & 28.0 & 200 & 16,000 & NA & NA & NA & NA \\
\hline $93 / 07 / 29$ & 24.5 & 28.5 & 400 & 14,400 & NA & NA & NA & NA \\
\hline $93 / 07 / 30$ & 25.5 & 28.0 & 600 & 16,600 & NA & NA & NA & NA \\
\hline $93 / 07 / 31$ & 23.0 & 27.5 & 700 & 16,900 & NA & NA & NA & NA \\
\hline $93 / 08 / 01$ & 23.5 & 27.5 & 600 & 11,500 & NA & NA & NA & NA \\
\hline $93 / 08 / 02$ & 24.0 . & 29.0 & 400 & 17,600 & NA & NA & NA & NA \\
\hline $93 / 08 / 03$ & 24.5 & 28.5 & 300 & 4,700 & NA & NA & NA & NA \\
\hline $93 / 08 / 04$ & 24.5 & 28.5 & 300 & 3,300 & NA & NA & NA & NA \\
\hline 93/08/05 & 23.5 & 26.5 & 700 & 3,400 & NA & NA & NA & NA \\
\hline 93/08/06 & 20.5 & 25.5 & 200 & 3,800 & NA & NA & NA & NA \\
\hline $93 / 08 / 07$ & 20.0 & 22.5 & 100 & 300 & NA & NA & NA & NA \\
\hline $93 / 08 / 08$ & 20.0 & 24.5 & 100 & 200 & NA & NA & NA & NA \\
\hline 93/08/09 & 20.0 & 25.0 & 100 & 100 & NA & NA & NA & NA \\
\hline
\end{tabular}


Appendix 6. Measurements at tidal stations in and near the tidal embayment of the Metedeconk River, N.J.--Daily minimum and maximum values of water-quality characteristics from measurements by movable monitors, November 1992 - January 1994--Continued

\begin{tabular}{|c|c|c|c|c|c|c|c|c|}
\hline \multirow{3}{*}{$\begin{array}{c}\text { Date } \\
\text { year/month/day }\end{array}$} & \multicolumn{8}{|c|}{ Daily minimum and maximum } \\
\hline & \multicolumn{2}{|c|}{ Temperature } & \multicolumn{2}{|c|}{ Specific conductance } & \multicolumn{2}{|c|}{$\mathrm{pH}$} & \multicolumn{2}{|c|}{$\begin{array}{l}\text { Dissolved-oxygen } \\
\text { concentration }\end{array}$} \\
\hline & Min & $\operatorname{Max}$ & Min & $\operatorname{Max}$ & Min & $\operatorname{Max}$ & Min & $\operatorname{Max}$ \\
\hline
\end{tabular}

400405074080600 Metedeconk River at Laurelton Gardens, edge of channel, near bottom (continued)

\begin{tabular}{|c|c|c|c|c|c|c|c|c|}
\hline $93 / 08 / 10$ & 20.5 & 25.5 & 100 & 200 & NA & NA & NA & NA \\
\hline $93 / 08 / 11$ & 21.0 & 24.5 & 200 & 200 & NA & NA & NA & NA \\
\hline $93 / 08 / 11$ & 24.0 & 26.5 & 100 & 700 & NA & NA & NA & NA \\
\hline $93 / 08 / 12$ & 21.5 & 26.0 & 200 & 5,000 & NA & NA & NA & NA \\
\hline $93 / 08 / 13$ & 22.0 & 25.5 & 200 & 10,600 & NA & NA & NA & NA \\
\hline $93 / 08 / 14$ & 22.5 & 26.5 & 300 & 10,200 & NA & NA & NA & NA \\
\hline $93 / 08 / 15$ & 23.5 & 28.0 & 400 & 9,800 & NA & NA & NA & NA \\
\hline $93 / 08 / 16$ & 24.5 & 27.5 & 300 & 9,800 & NA & NA & NA & NA \\
\hline $93 / 08 / 17$ & 21.5 & 25.0 & 100 & 3,200 & NA & NA & NA & NA \\
\hline $93 / 08 / 18$ & 21.5 & 23.0 & 100 & 100 & NA & NA & NA & NA \\
\hline $93 / 08 / 19$ & 21.0 & 24.5 & 100 & 100 & NA & NA & NA & NA \\
\hline $93 / 08 / 20$ & 21.0 & 25.5 & 100 & 25,800 & NA & NA & NA & NA \\
\hline $93 / 08 / 21$ & 21.5 & 24.5 & 300 & 3,300 & NA & NA & NA & NA \\
\hline $93 / 08 / 22$ & 21.0 & 25.0 & 200 & 8,900 & NA & NA & NA & NA \\
\hline $93 / 08 / 23$ & 21.0 & 26.0 & 200 & 19,900 & NA & NA & NA & NA \\
\hline $93 / 08 / 24$ & 21.5 & 26.0 & 200 & 18,100 & NA & NA & NA & NA \\
\hline $93 / 08 / 25$ & 22.5 & 26.5 & 200 & 16,900 & NA & NA & NA & NA \\
\hline $93 / 08 / 26$ & 23.0 & 25.5 & 300 & 1,300 & NA & NA & NA & NA \\
\hline $93 / 09 / 29$ & 15.5 & 18.5 & 100 & 100 & NA & NA & NA & NA \\
\hline $93 / 09 / 30$ & 13.5 & 15.5 & 100 & 100 & NA & NA & NA & NA \\
\hline $93 / 10 / 01$ & 12.5 & 17.0 & 100 & 100 & NA & NA & NA & NA \\
\hline $93 / 10 / 02$ & 13.0 & 17.0 & 100 & 5,200 & NA & NA & NA & NA \\
\hline $93 / 10 / 03$ & 14.5 & 17.5 & 100 & 400 & NA & NA & NA & NA \\
\hline $93 / 10 / 04$ & 13.0 & 17.5 & 100 & 21,800 & NA & NA & NA & NA \\
\hline $93 / 10 / 05$ & 13.0 & 16.5 & 200 & 700 & NA & NA & NA & NA \\
\hline $93 / 10 / 06$ & 11.5 & 16.0 & 100 & 300 & NA & NA & NA & NA \\
\hline $93 / 10 / 07$ & 12.0 & 18.0 & 100 & 300 & NA & NA & NA & NA \\
\hline $93 / 10 / 08$ & 14.0 & 17.5 & 100 & 200 & NA & NA & NA & NA \\
\hline $93 / 10 / 09$ & 14.5 & 19.5 & 100 & 22,600 & NA & NA & NA & NA \\
\hline $93 / 10 / 10$ & 13.5 & 19.0 & 1,200 & 16,800 & NA & NA & NA & NA \\
\hline $93 / 10 / 11$ & 10.5 & 15.0 & 1,000 & 14,800 & NA & NA & NA & NA \\
\hline $93 / 10 / 12$ & 12.5 & 13.5 & 300 & 4,800 & NA & NA & NA & NA \\
\hline $93 / 10 / 13$ & 11.5 & 14.5 & 100 & 300 & NA & NA & NA & NA \\
\hline $93 / 10 / 14$ & 11.0 & 12.5 & 100 & 200 & NA & NA & NA & NA \\
\hline $93 / 10 / 15$ & 11.5 & 14.0 & 100 & 200 & NA & NA & NA & NA \\
\hline $93 / 10 / 16$ & 11.5 & 14.5 & 100 & 100 & NA & NA & NA & NA \\
\hline $93 / 10 / 17$ & 12.5 & 14.5 & 100 & 2,200 & NA & NA & NA & NA \\
\hline $93 / 10 / 18$ & 13.5 & 16.5 & 100 & 35,800 & NA & NA & NA & NA \\
\hline $93 / 10 / 19$ & 13.0 & 16.0 & 300 & 34,000 & NA & NA & NA & NA \\
\hline
\end{tabular}


Appendix 6. Measurements at tidal stations in and near the tidal embayment of the Metedeconk River, N.J.--Daily minimum and maximum values of water-quality characteristics from measurements by movable monitors, November 1992 - January 1994--Continued

\begin{tabular}{|c|c|c|c|c|c|c|c|c|}
\hline \multirow{3}{*}{$\begin{array}{c}\text { Date } \\
\text { year/month/day }\end{array}$} & \multicolumn{8}{|c|}{ Daily minimum and maximum } \\
\hline & \multicolumn{2}{|c|}{ Temperature } & \multicolumn{2}{|c|}{ Specific conductance } & \multicolumn{2}{|c|}{$\mathrm{pH}$} & \multicolumn{2}{|c|}{$\begin{array}{l}\text { Dissolved-oxygen } \\
\text { concentration }\end{array}$} \\
\hline & Min & $\operatorname{Max}$ & Min & $\operatorname{Max}$ & Min & $\operatorname{Max}$ & Min & $\operatorname{Max}$ \\
\hline \multicolumn{9}{|c|}{ 01408160 Metedeconk River near Laurelton, left bank near surface } \\
\hline $93 / 07 / 01$ & 26.7 & 28.0 & 29,300 & 30,300 & 8.0 & 8.2 & 7.5 & 9.6 \\
\hline $93 / 07 / 02$ & 24.5 & 26.5 & 28,800 & 30,200 & 7.8 & 8.0 & 6.3 & 7.5 \\
\hline $93 / 07 / 03$ & 24.0 & 25.5 & 26,100 & 31,700 & 7.5 & 8.0 & 5.5 & 8.8 \\
\hline $93 / 07 / 04$ & 25.0 & 27.5 & 30,000 & 31,500 & 7.7 & 8.1 & 6.8 & 10.0 \\
\hline 93/07/05 & 26.0 & 28.0 & 29,500 & 31,900 & 7.4 & 7.9 & 4.6 & 8.4 \\
\hline $93 / 07 / 06$ & 26.0 & 28.0 & 27,600 & 31,800 & 7.2 & 7.7 & 4.9 & 7.4 \\
\hline $93 / 07 / 07$ & 27.5 & 29.5 & 27,200 & 32,500 & 7.3 & 7.7 & 3.3 & 7.2 \\
\hline $93 / 09 / 10$ & 23.5 & 25.5 & 29,200 & 33,100 & 7.6 & 8.2 & 6.5 & 8.8 \\
\hline $93 / 09 / 11$ & 21.5 & 23.5 & 31,000 & 34,000 & 7.5 & 8.2 & 5.1 & 9.9 \\
\hline $93 / 09 / 12$ & 21.0 & 22.5 & 30,400 & 33,400 & 7.8 & 8.4 & 6.5 & 10.9 \\
\hline $93 / 09 / 13$ & 20.5 & 23.0 & 25,300 & 33,000 & 7.5 & 8.4 & 5.2 & 11.2 \\
\hline $93 / 09 / 14$ & 21.0 & 24.5 & 23,800 & 32,900 & 7.6 & 8.3 & 4.7 & 10.5 \\
\hline $93 / 09 / 15$ & 22.5 & 24.0 & 25,200 & 33,100 & 7.7 & 8.1 & 4.8 & 7.6 \\
\hline $93 / 11 / 04$ & 10.0 & 10.5 & 30,900 & 35,300 & 8.0 & 8.2 & 9.2 & 10.1 \\
\hline $93 / 11 / 05$ & 9.0 & 11.0 & 25,600 & 35,100 & 7.8 & 8.2 & 9.1 & 9.8 \\
\hline $93 / 11 / 06$ & 10.0 & 11.0 & 29,400 & 36,500 & 8.0 & 8.1 & 8.6 & 9.3 \\
\hline $93 / 11 / 07$ & 8.5 & 10.0 & 31,200 & 34,800 & 8.0 & 8.1 & 8.6 & 9.1 \\
\hline $93 / 11 / 08$ & 8.5 & 10.0 & 25,700 & 34,600 & 7.8 & 8.1 & 8.7 & 9.7 \\
\hline $93 / 11 / 09$ & 8.5 & 10.0 & 11,600 & 33,500 & 7.3 & 8.2 & 9.2 & 10.3 \\
\hline $93 / 11 / 10$ & 8.0 & 10.0 & 25,800 & 34,800 & 8.0 & 8.2 & 9.6 & 10.8 \\
\hline $93 / 11 / 11$ & 8.5 & 10.5 & 28,200 & 34,000 & 8.1 & 8.2 & 10.1 & 10.9 \\
\hline $93 / 11 / 12$ & 9.0 & 11.0 & 27,200 & 38,900 & 8.0 & 8.2 & 8.6 & 10.5 \\
\hline $93 / 11 / 13$ & 9.5 & 10.5 & 35,800 & 37,400 & 8.1 & 8.2 & 9.0 & 10.2 \\
\hline $93 / 11 / 14$ & 10.5 & 12.0 & 31,800 & 37,600 & 8.1 & 8.2 & 9.0 & 10.4 \\
\hline $93 / 11 / 15$ & 12.0 & 13.5 & 27,200 & 37,400 & 8.0 & 8.2 & 8.7 & 9.8 \\
\hline
\end{tabular}

01408160 Metedeconk River near Laurelton, left bank near bottom

$\begin{array}{lllllllll}93 / 07 / 01 & 25.0 & 27.0 & 30,700 & 32,400 & 7.4 & 7.8 & 5.9 & 6.2 \\ 93 / 07 / 02 & 24.5 & 26.0 & 30,100 & 32,700 & 7.3 & 7.9 & 2.4 & 6.4 \\ 93 / 07 / 03 & 22.5 & 24.5 & 31,900 & 36,100 & 7.2 & 7.6 & 0.5 & 4.8 \\ 93 / 07 / 04 & 22.5 & 24.5 & 34,000 & 36,800 & 7.2 & 7.5 & 0.5 & 4.5 \\ 93 / 07 / 05 & 24.0 & 27.5 & 30,600 & 35,300 & 7.2 & 7.8 & 0.8 & 7.3 \\ 93 / 07 / 06 & 26.0 & 27.5 & 31,900 & 35,100 & 7.2 & 7.8 & 1.7 & 7.1 \\ 93 / 07 / 07 & 25.5 & 26.5 & 34,500 & 35,900 & 7.1 & 7.3 & 0.8 & 2.7 \\ 93 / 09 / 10 & 24.5 & 24.5 & 37,100 & 39,100 & 7.2 & 7.4 & \text { NA } & \text { NA } \\ 93 / 09 / 11 & 22.5 & 24.0 & 33,900 & 38,500 & 7.3 & 8.0 & \text { NA } & \text { NA } \\ 93 / 09 / 12 & 21.0 & 22.5 & 32,600 & 34,100 & 7.8 & 8.2 & \text { NA } & \text { NA } \\ 93 / 09 / 13 & 21.5 & 22.5 & 32,200 & 34,000 & 7.7 & 8.3 & \text { NA } & \text { NA }\end{array}$


Appendix 6. Measurements at tidal stations in and near the tidal embayment of the Metedeconk River, N.J.--Daily minimum and maximum values of water-quality characteristics from measurements by movable monitors, November 1992 - January 1994--Continued

\begin{tabular}{|c|c|c|c|c|c|c|c|c|}
\hline \multirow{3}{*}{$\begin{array}{c}\text { Date } \\
\text { year/month/day }\end{array}$} & \multicolumn{8}{|c|}{ Daily minimum and maximum } \\
\hline & \multicolumn{2}{|c|}{ Temperature } & \multicolumn{2}{|c|}{ Specific conductance } & \multicolumn{2}{|c|}{$\mathrm{pH}$} & \multicolumn{2}{|c|}{$\begin{array}{c}\text { Dissolved-oxygen } \\
\text { concentration }\end{array}$} \\
\hline & Min & Max & Min & Max & Min & Max & Min & Max \\
\hline \multicolumn{9}{|c|}{ 01408160 Metedeconk River near Laurelton, left bank near bottom (continued) } \\
\hline $93 / 09 / 14$ & 21.5 & 23.5 & 33,000 & 34,500 & 7.3 & 8.1 & NA & NA \\
\hline $93 / 09 / 15$ & 22.0 & 22.5 & 33,900 & 34,900 & 7.3 & 7.7 & NA & NA \\
\hline $93 / 11 / 04$ & 10.0 & 10.5 & 37,100 & 40,300 & NA & NA & 7.4 & 10.2 \\
\hline $93 / 11 / 05$ & 10.5 & 11.0 & 40,000 & 41,800 & NA & NA & 5.8 & 8.0 \\
\hline $93 / 11 / 06$ & 10.5 & 11.0 & 41,000 & 42,300 & NA & NA & 5.4 & 7.1 \\
\hline $93 / 11 / 07$ & 11.0 & 11.0 & 39,600 & 42,200 & NA & NA & 6.0 & 7.5 \\
\hline $93 / 11 / 08$ & 10.5 & 11.5 & 37,100 & 41,400 & NA & NA & 5.3 & 7.8 \\
\hline $93 / 11 / 09$ & 11.0 & 12.0 & 37,400 & 40,800 & NA & NA & 5.3 & 8.5 \\
\hline $93 / 11 / 10$ & 10.5 & 12.0 & 36,900 & 40,100 & NA & NA & 5.6 & 9.8 \\
\hline $93 / 11 / 11$ & 10.5 & 12.0 & 39,900 & 41,100 & NA & NA & 5.4 & 8.1 \\
\hline $93 / 11 / 12$ & 10.0 & 10.5 & 40,000 & 42,900 & NA & NA & 6.3 & 10.1 \\
\hline $93 / 11 / 13$ & 10.0 & 11.0 & 37,300 & 42,700 & NA & NA & 8.3 & 10.2 \\
\hline $93 / 11 / 14$ & 10.5 & 11.0 & 38,100 & 41,700 & NA & NA & 6.7 & 9.2 \\
\hline $93 / 11 / 15$ & 11.0 & 12.0 & 41,000 & 42,700 & NA & NA & 6.9 & 8.7 \\
\hline
\end{tabular}


Appendix 7. Measurements at tidal stations in and near the tidal embayment of the Metedeconk River, N.J.--Water-quality data from manual measurements, October 1992 - October 1993

[Water-quality measurements were made at the stations listed below. USGS, U.S. Geological Survey; NA, not applicable or not available; >, greater than]

\begin{tabular}{|c|c|c|c|}
\hline $\begin{array}{l}\text { Index } \\
\text { number } \\
\text { (fig. 2) }\end{array}$ & USGS station number & USGS station name & $\begin{array}{l}\text { Location of the } \\
\text { channel in the } \\
\text { cross section, in } \\
\text { percent distance } \\
\text { from left to right } \\
\text { bank }\end{array}$ \\
\hline 3 & 400412074082600 & Metedeconk River near Bricktown & 95 \\
\hline 4 & 400405074080600 & Metedeconk River at Laurelton Gardens & 5 \\
\hline 5 & 01408155 & Metedeconk River at Laurelton & $\begin{array}{c}25,75 \\
\text { (two channels) }\end{array}$ \\
\hline 6 & 400353074074900 & $\begin{array}{l}\text { Metedeconk River } 1,200 \text { feet downstream } \\
\text { from Route } 70 \text { at Laurelton }\end{array}$ & 50 \\
\hline 7 & 400343074073400 & $\begin{array}{l}\text { Metedeconk River } 0.6 \text { miles downstream } \\
\text { from Route } 70 \text { at Laurelton }\end{array}$ & 50 \\
\hline 8 & 400337074071600 & $\begin{array}{l}\text { Metedeconk River } 0.9 \text { miles downstream } \\
\text { from Route } 70 \text { near Laurelton }\end{array}$ & 50 \\
\hline 9 & 01408160 & Metedeconk River near Laurelton & 50 \\
\hline 10 & 400313074055200 & $\begin{array}{l}\text { Metedeconk River at Eagle Point at } \\
\text { Adamston }\end{array}$ & 50 \\
\hline 11 & 400314074044500 & Metedeconk River at Metedeconk & 50 \\
\hline 12 & 400311074035200 & Metedeconk River at West Mantoloking & 50 \\
\hline 13 & 01408168 & Barnegat Bay at Mantoloking & NA \\
\hline 14 & 400337074033500 & Barnegat Bay near Point Pleasant & NA \\
\hline
\end{tabular}


Appendix 7. Measurements at tidal stations in and near the tidal embayment of the Metedeconk River, N.J.--Water-quality data from manual measurements, October 1992 - October 1993-Continued

The following information is presented:

\begin{tabular}{|c|c|c|}
\hline Characteristic & Unit & Rounding \\
\hline $\begin{array}{l}\text { Date and time of } \\
\text { measurement }\end{array}$ & NA & NA \\
\hline Distance from left bank & $\begin{array}{l}\text { Percent of distance from left to } \\
\text { right bank }\end{array}$ & 1 \\
\hline Sample depth & Feet & 0.5 \\
\hline Total depth & Feet & 0.5 \\
\hline Temperature & Degrees Celsius & 0.5 \\
\hline $\mathrm{pH}$ & Standard units & 0.1 \\
\hline $\begin{array}{l}\text { Dissolved-oxygen } \\
\text { concentration }\end{array}$ & Milligrams per liter & 0.1 \\
\hline $\begin{array}{l}\text { Dissolved-oxygen } \\
\text { concentration as a percent } \\
\text { of saturation }\end{array}$ & Percent & 1 \\
\hline Barometric pressure & Millimeters of mercury & 5 \\
\hline Specific conductance & $\begin{array}{l}\text { Microsiemens per centimeter at } \\
25 \text { degrees Celsius }\end{array}$ & NA \\
\hline Secchi-disk depth & Feet & 0.5 \\
\hline Tidal-water level & $\begin{array}{l}\text { Feet above the National Geo- } \\
\text { detic Vertical Datum of } 1929\end{array}$ & 0.01 \\
\hline
\end{tabular}




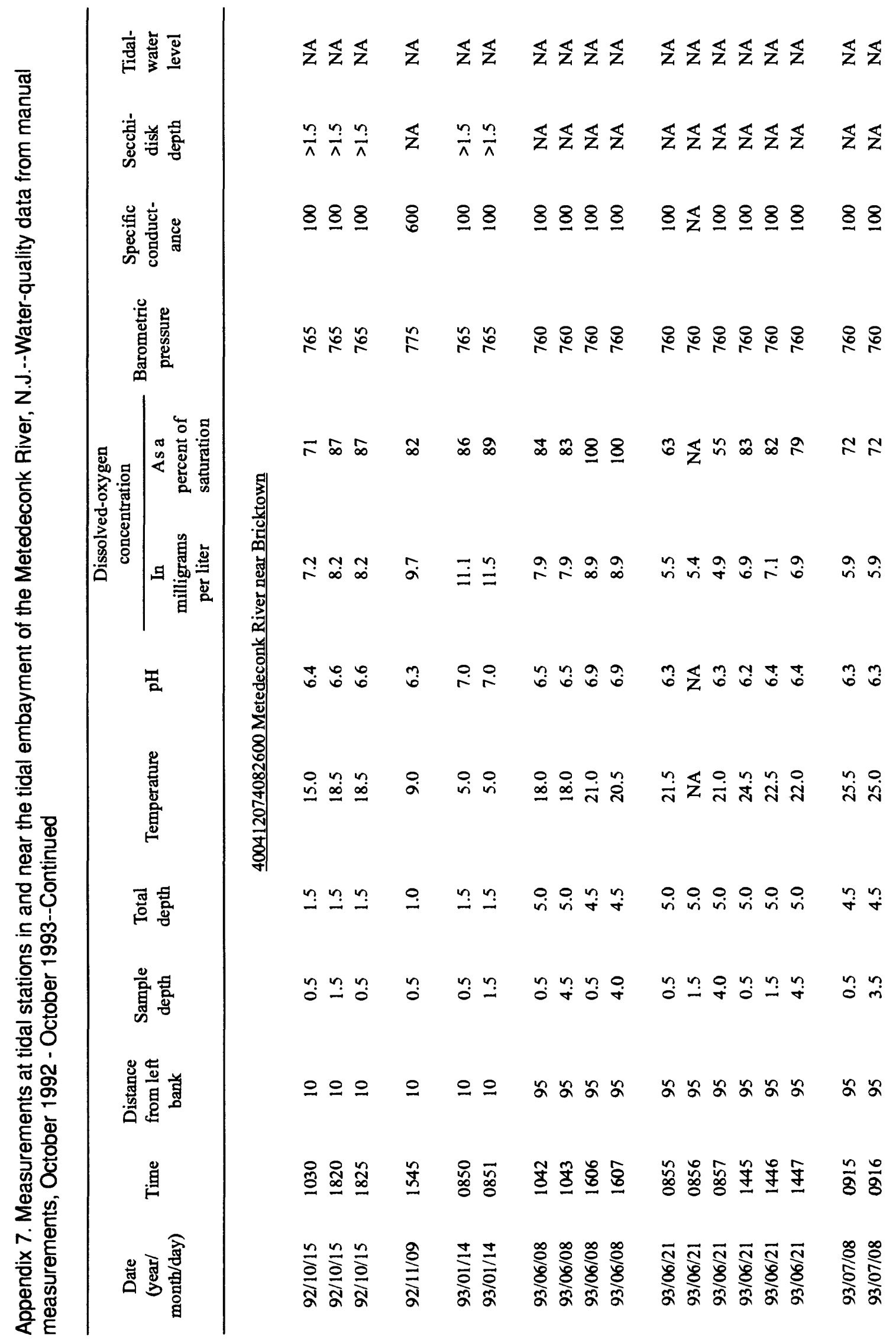




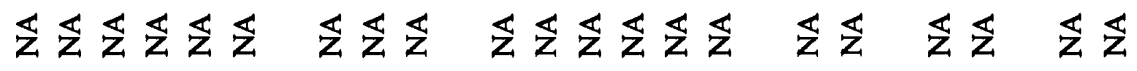

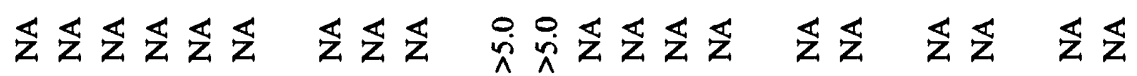

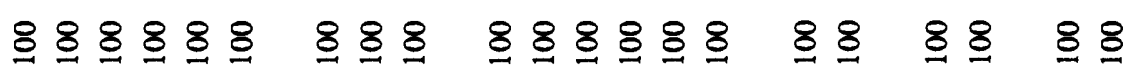

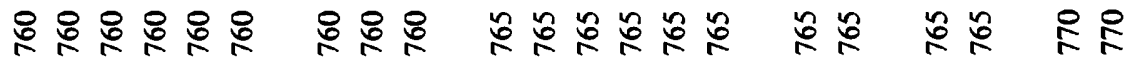

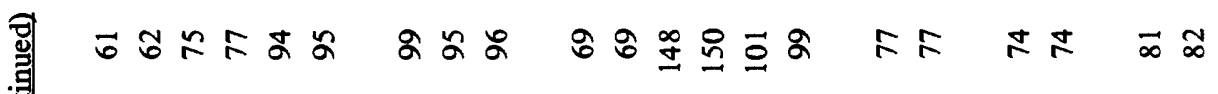

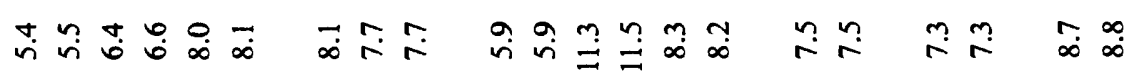

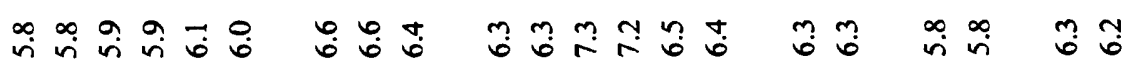

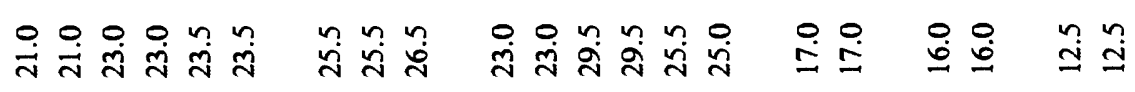

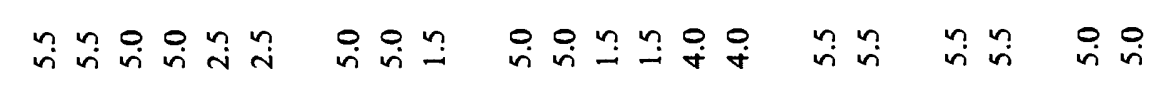

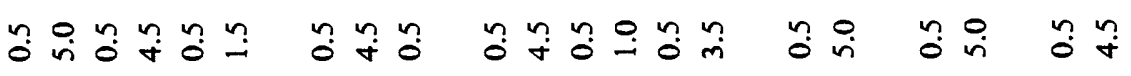

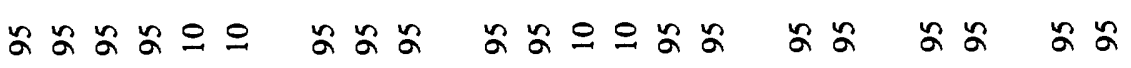

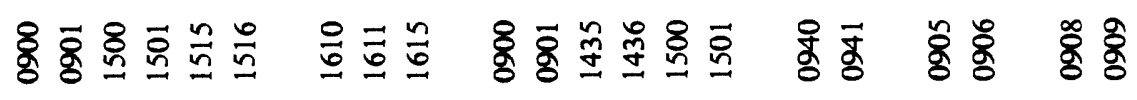

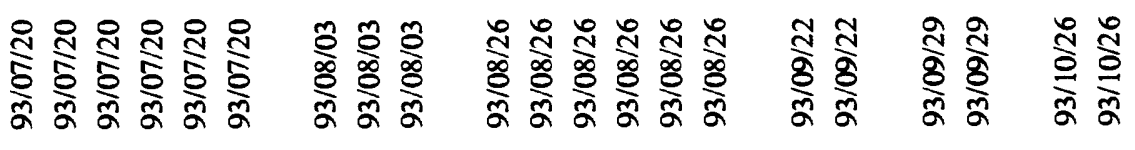




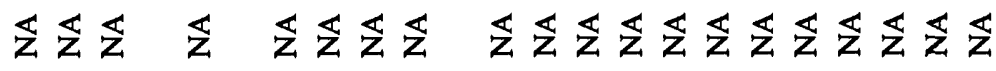

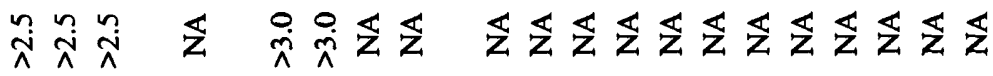

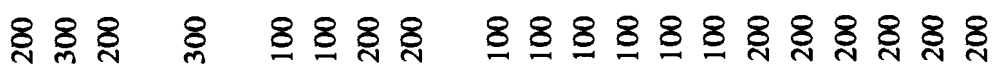

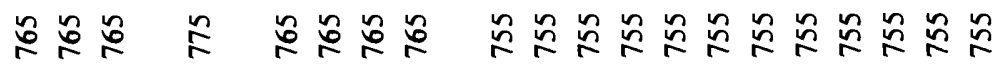

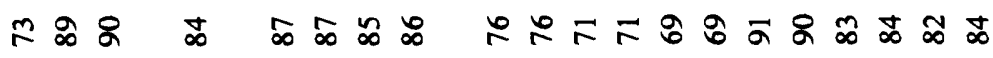

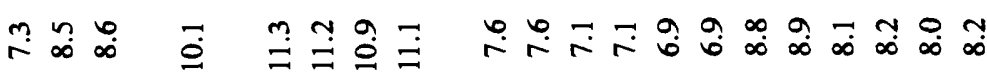

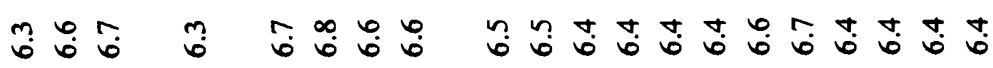

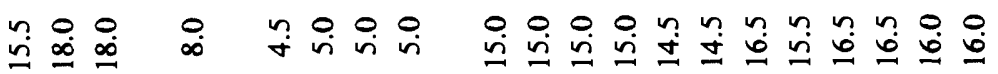

กับ

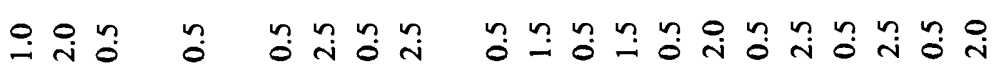

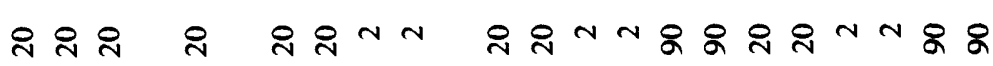

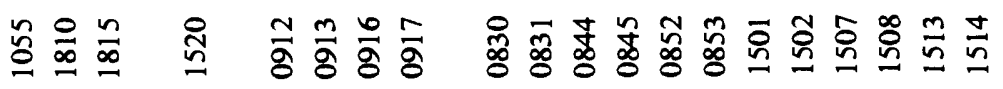

능 닝 สু 


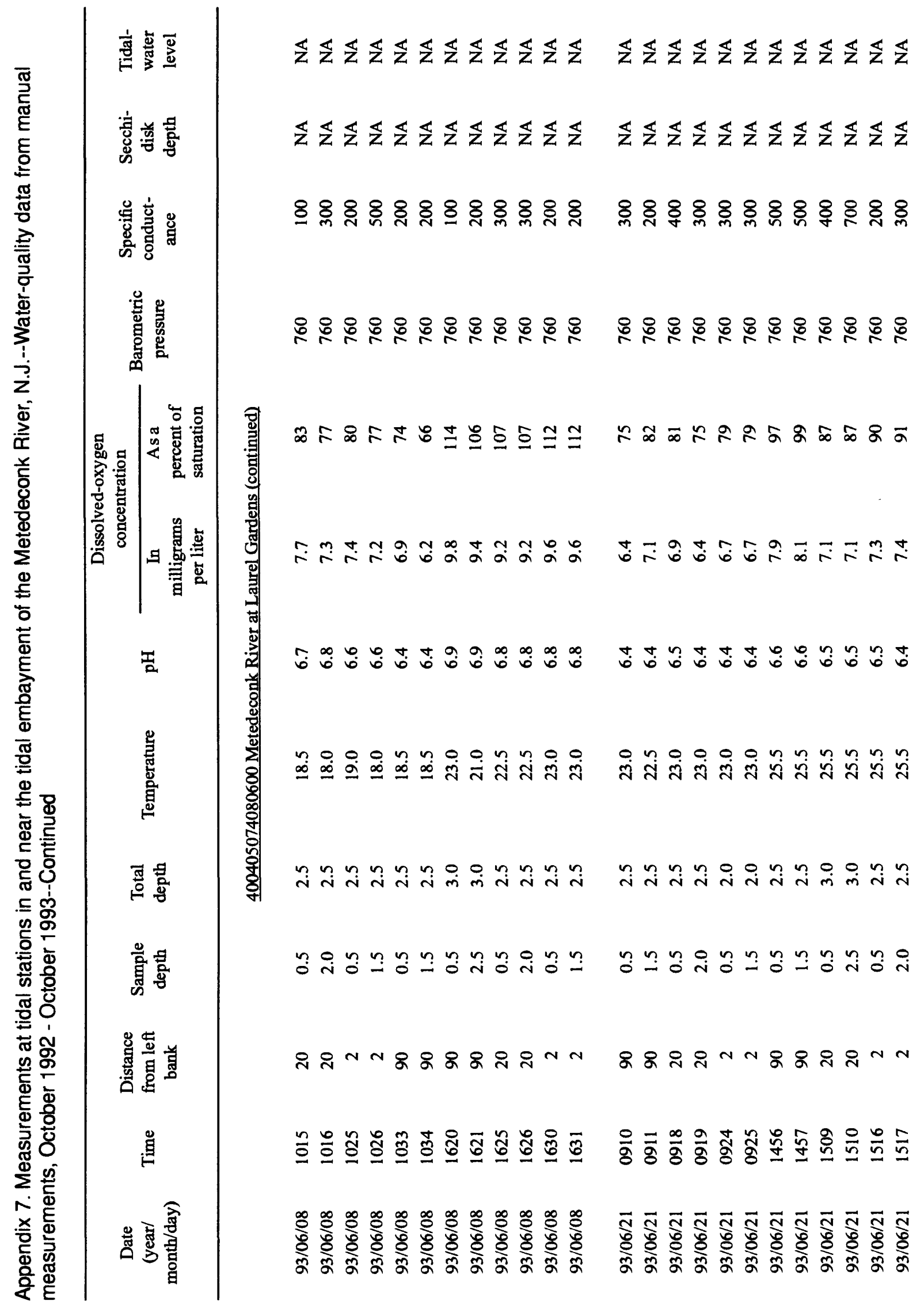




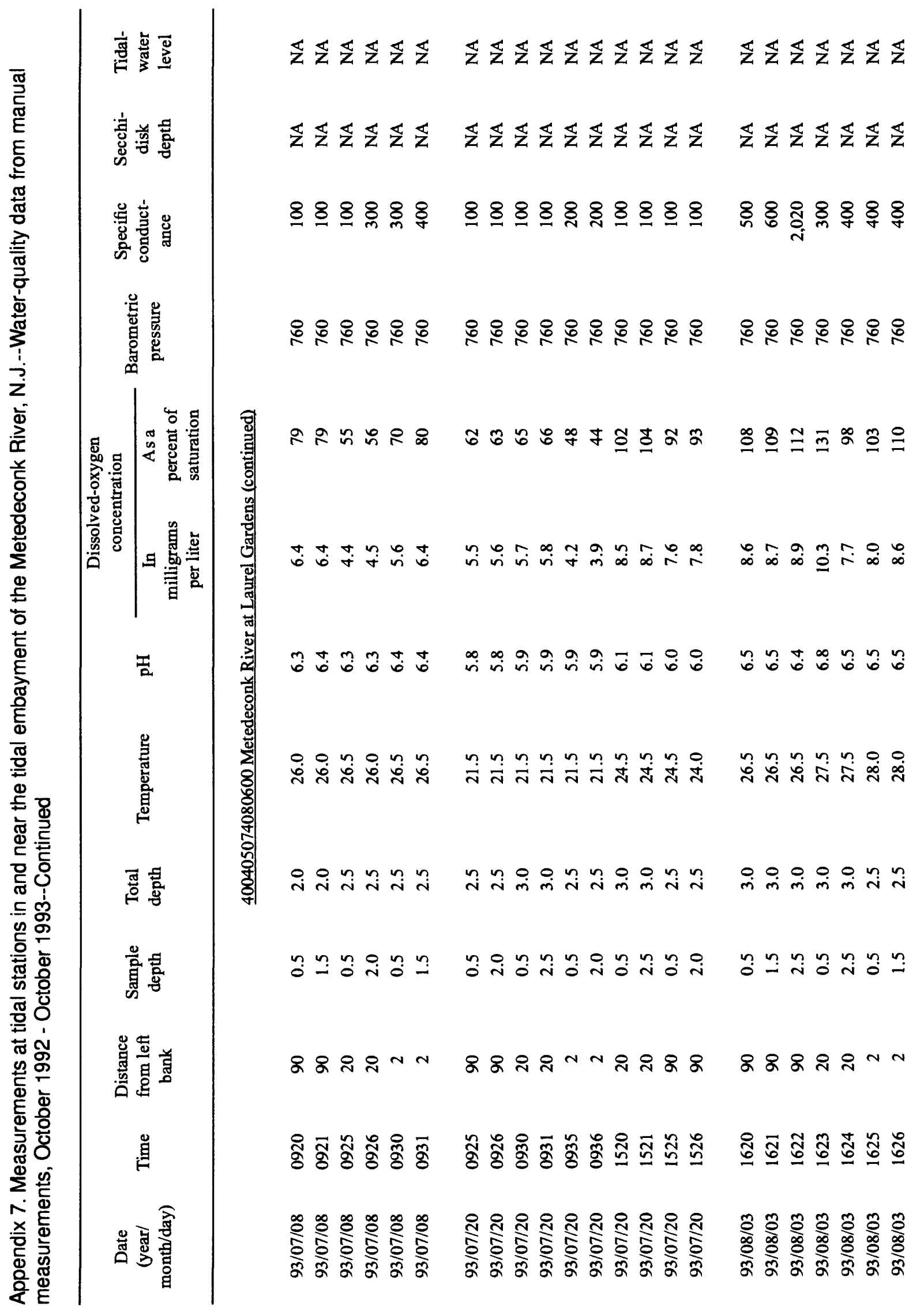




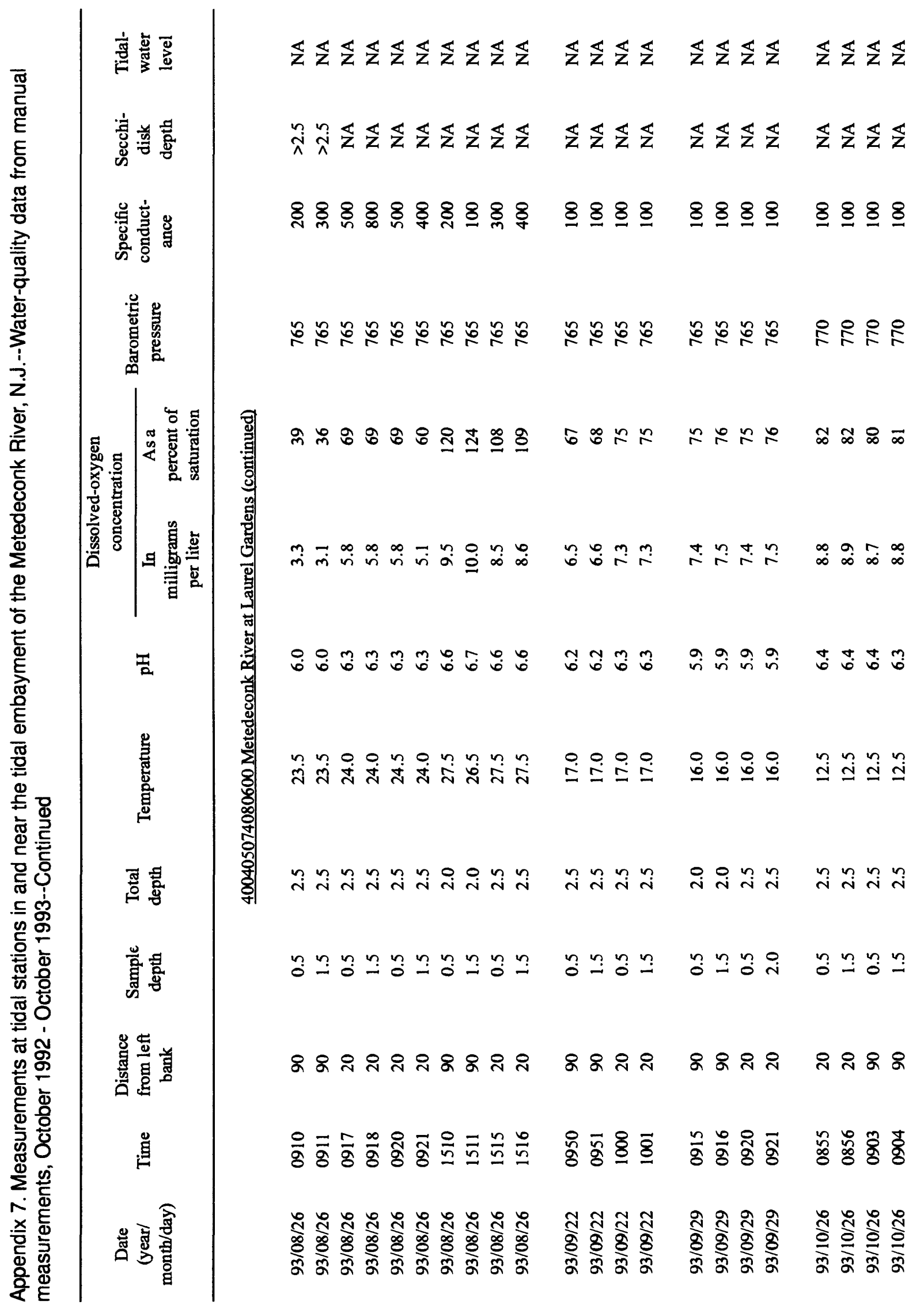




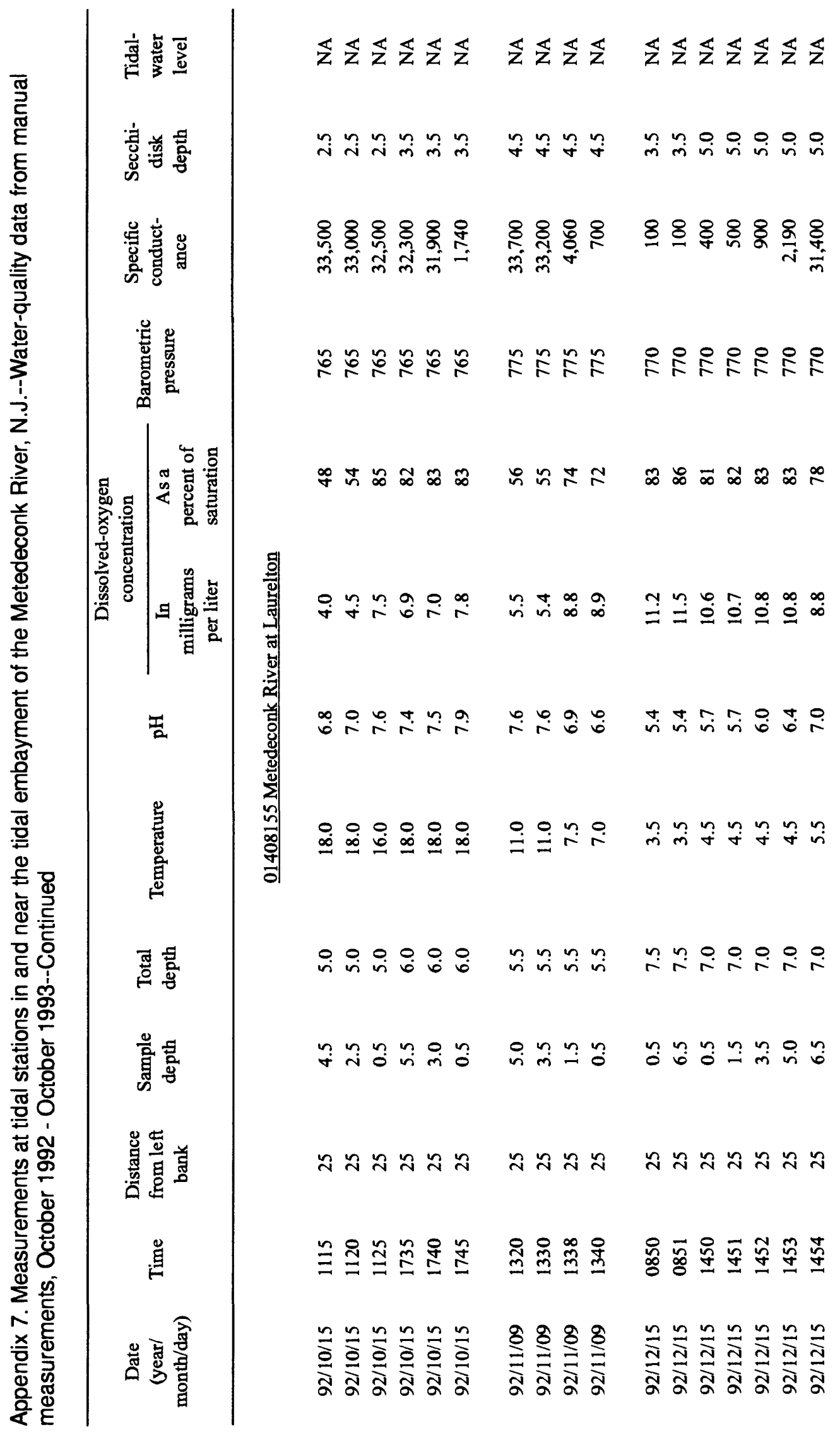




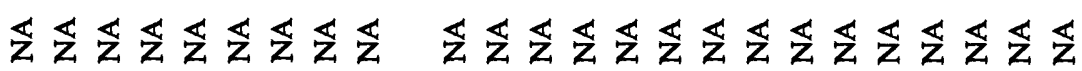

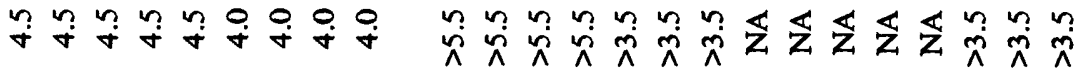

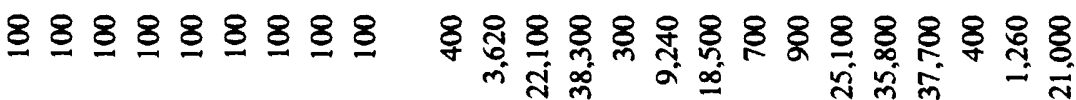

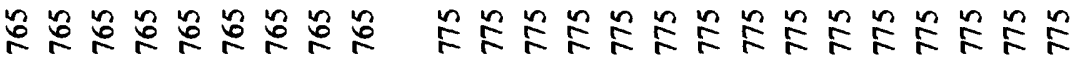

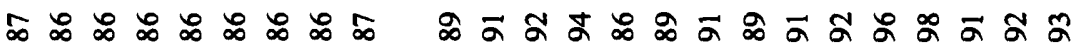

व्⿹

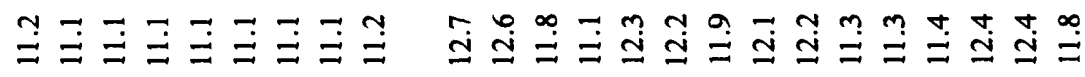

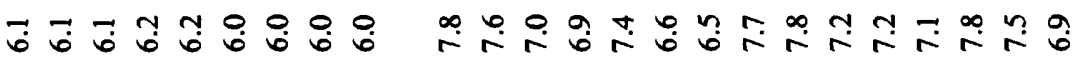

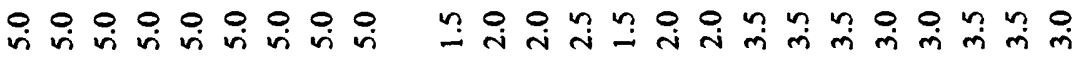

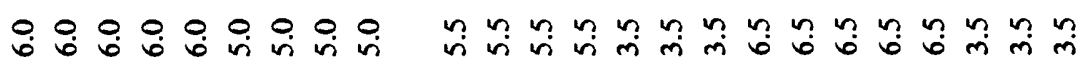

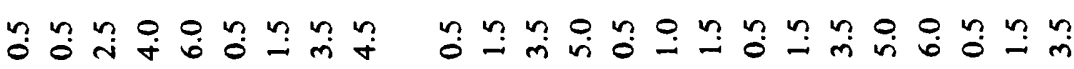

งูง

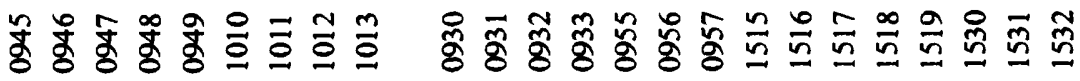

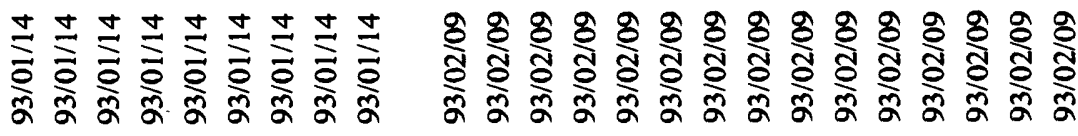




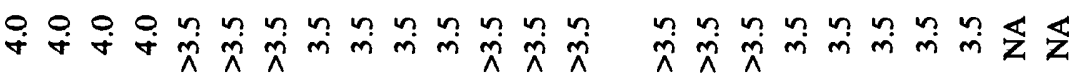

용ㅇㅇㅇㅇㅇㅇㅇㅇㅇㅇㅇㅇㅇㅇㅇㅇㅇㅇㅇㅇㅇㅁ

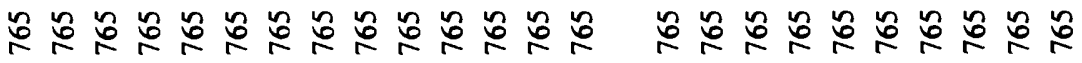

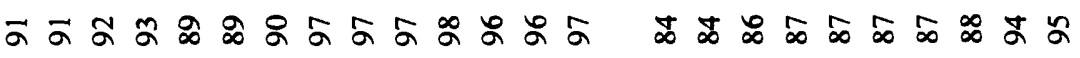

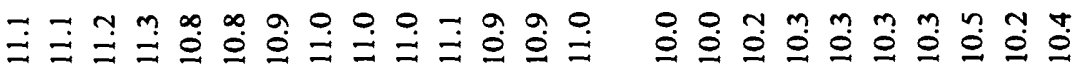

我

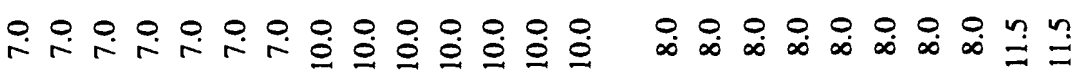

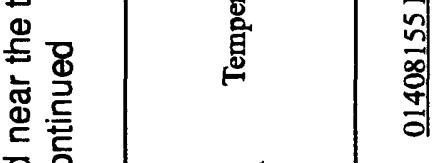

현 형

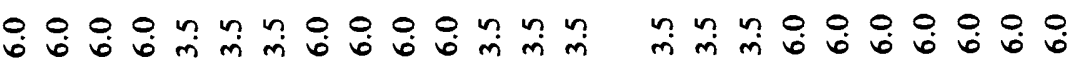

总㖥

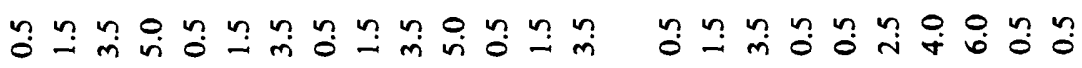

$$
\operatorname{rin} \frac{1}{2}
$$




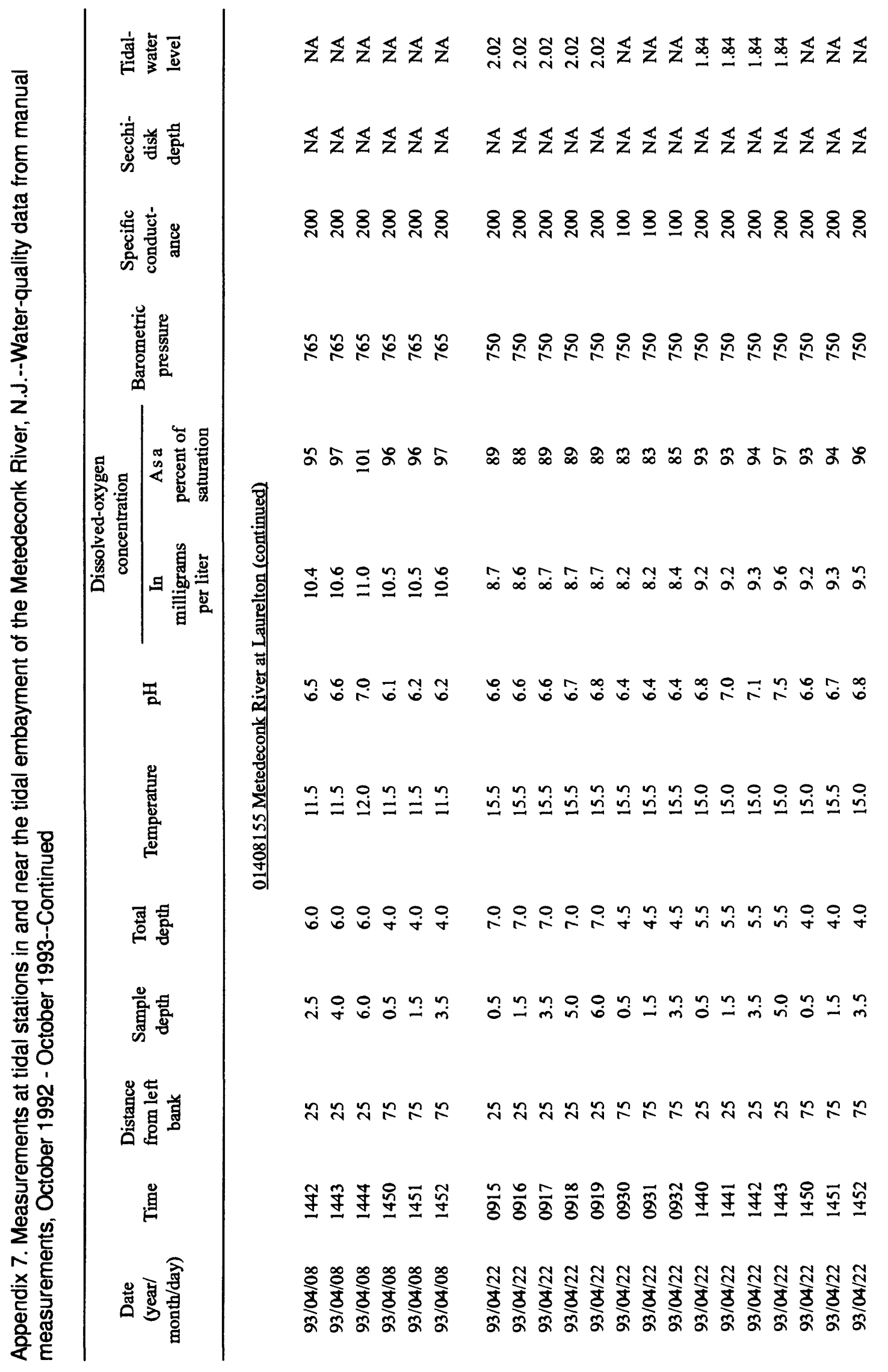




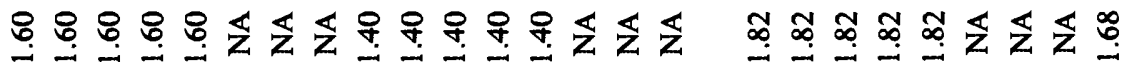

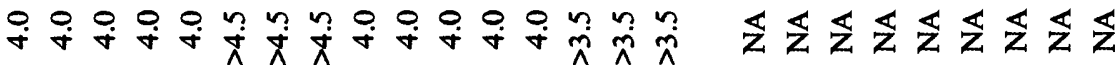

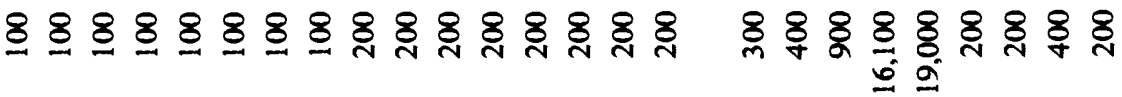

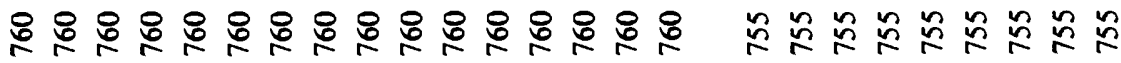

क人

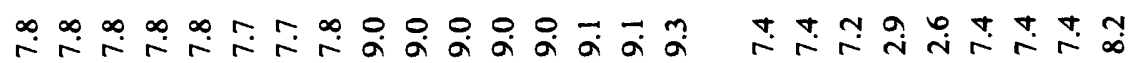

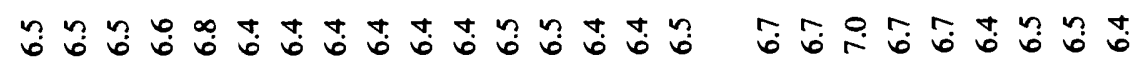

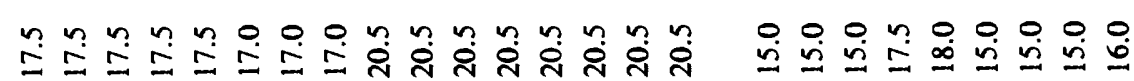

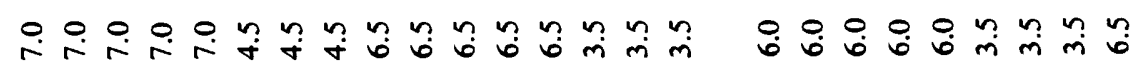

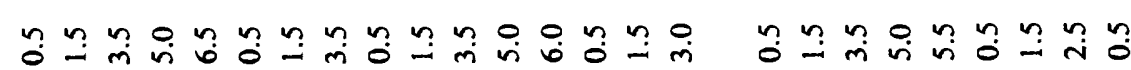

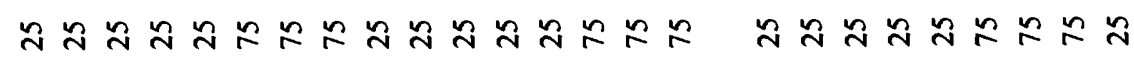

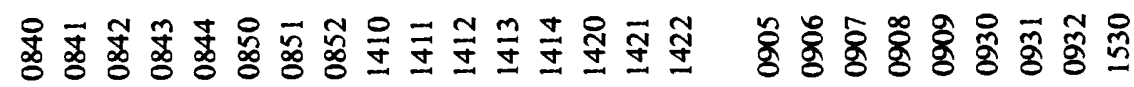

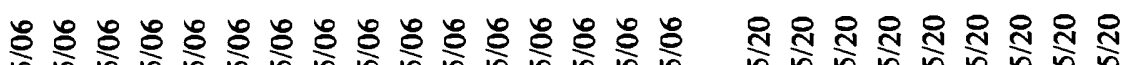

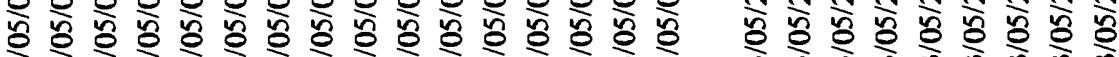

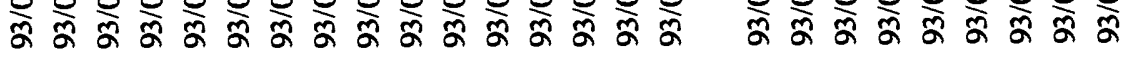




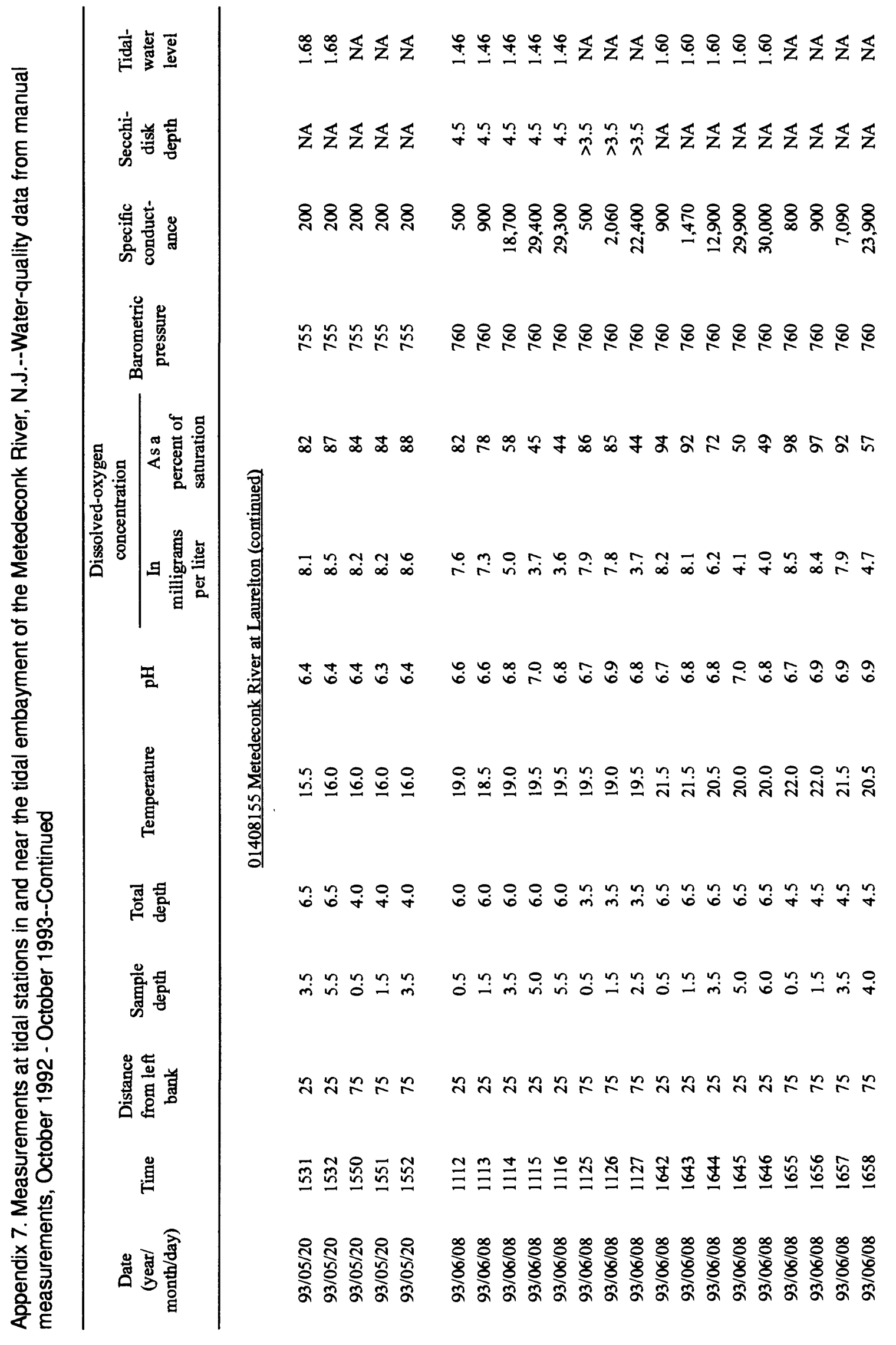




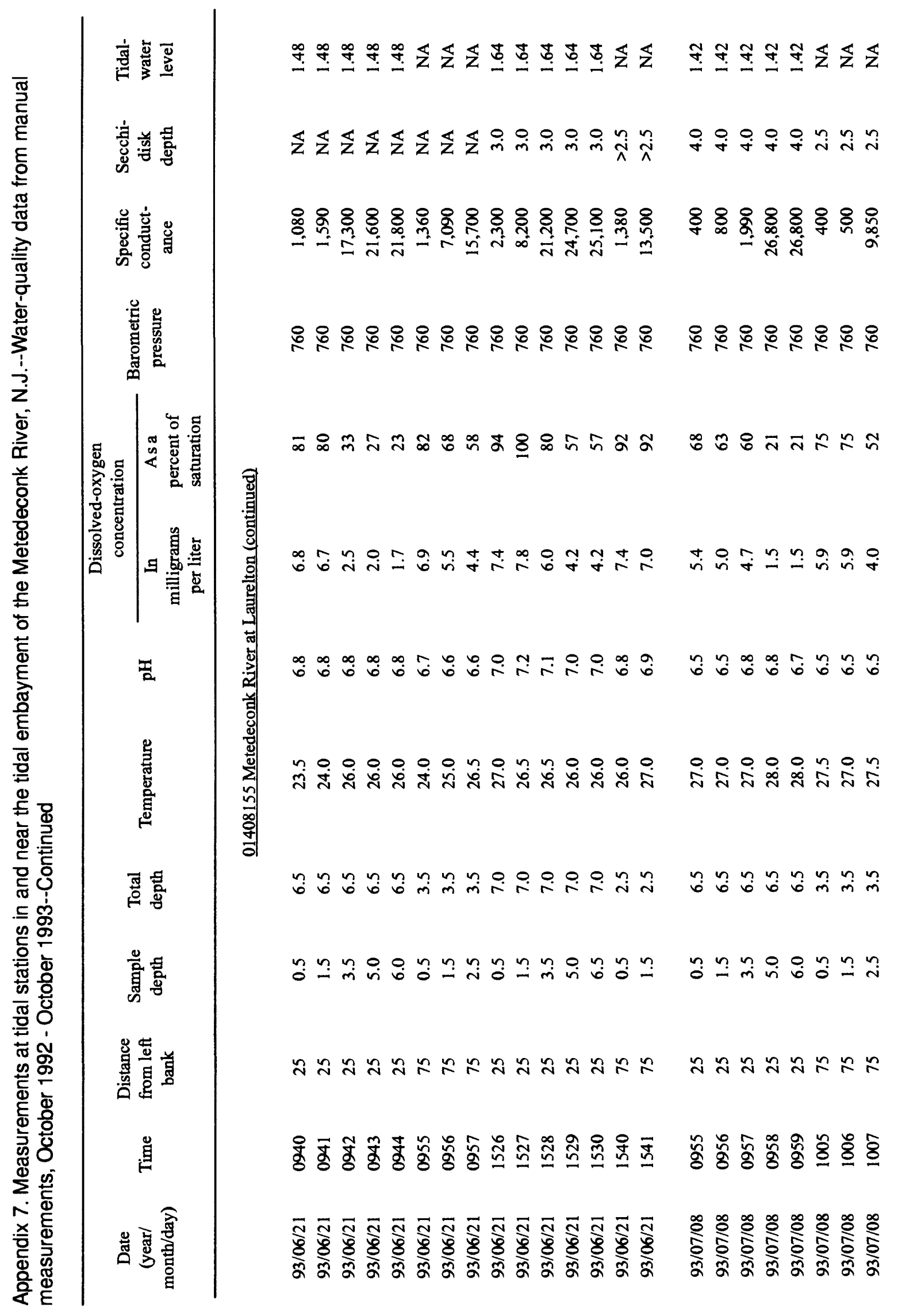




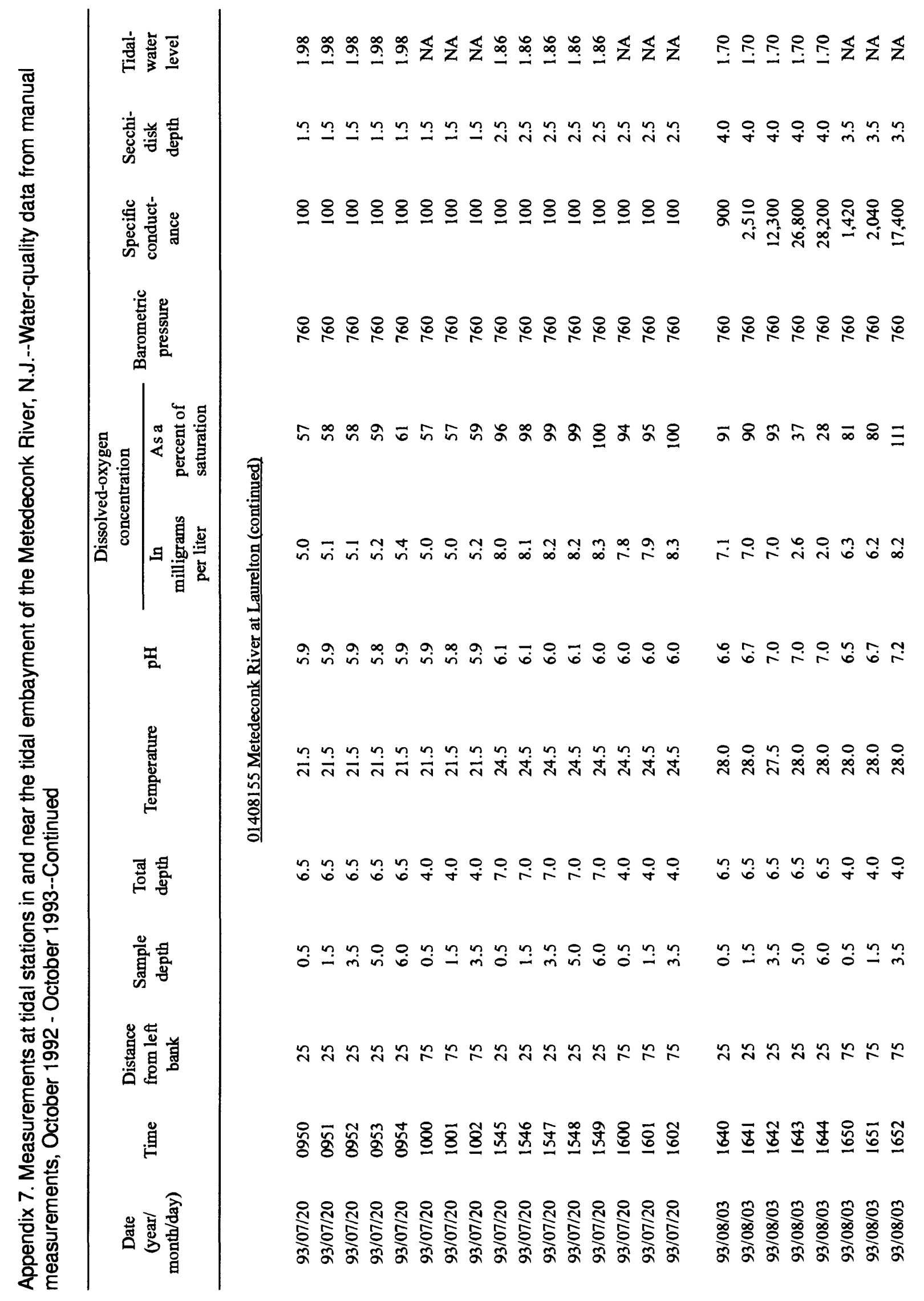




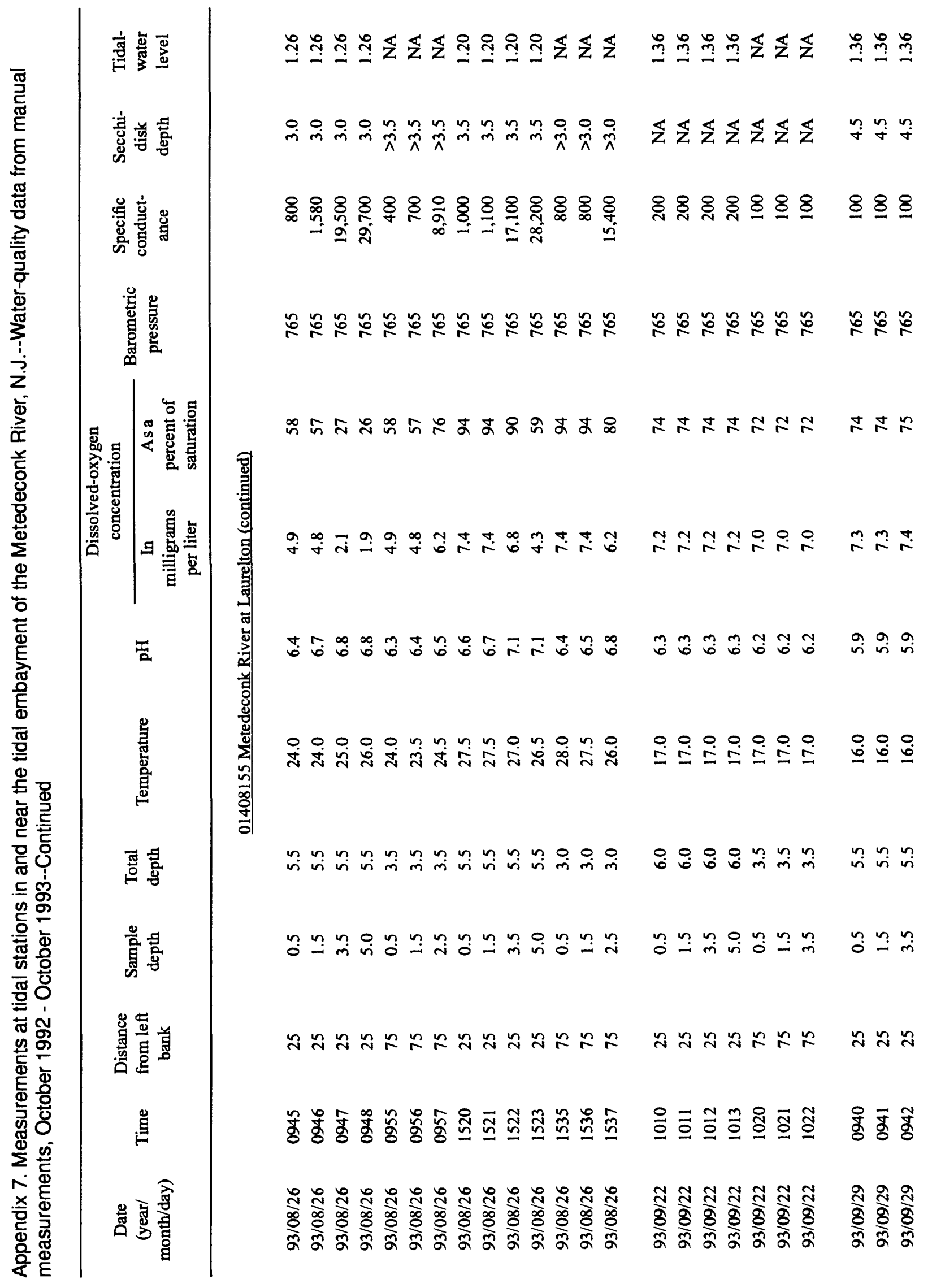




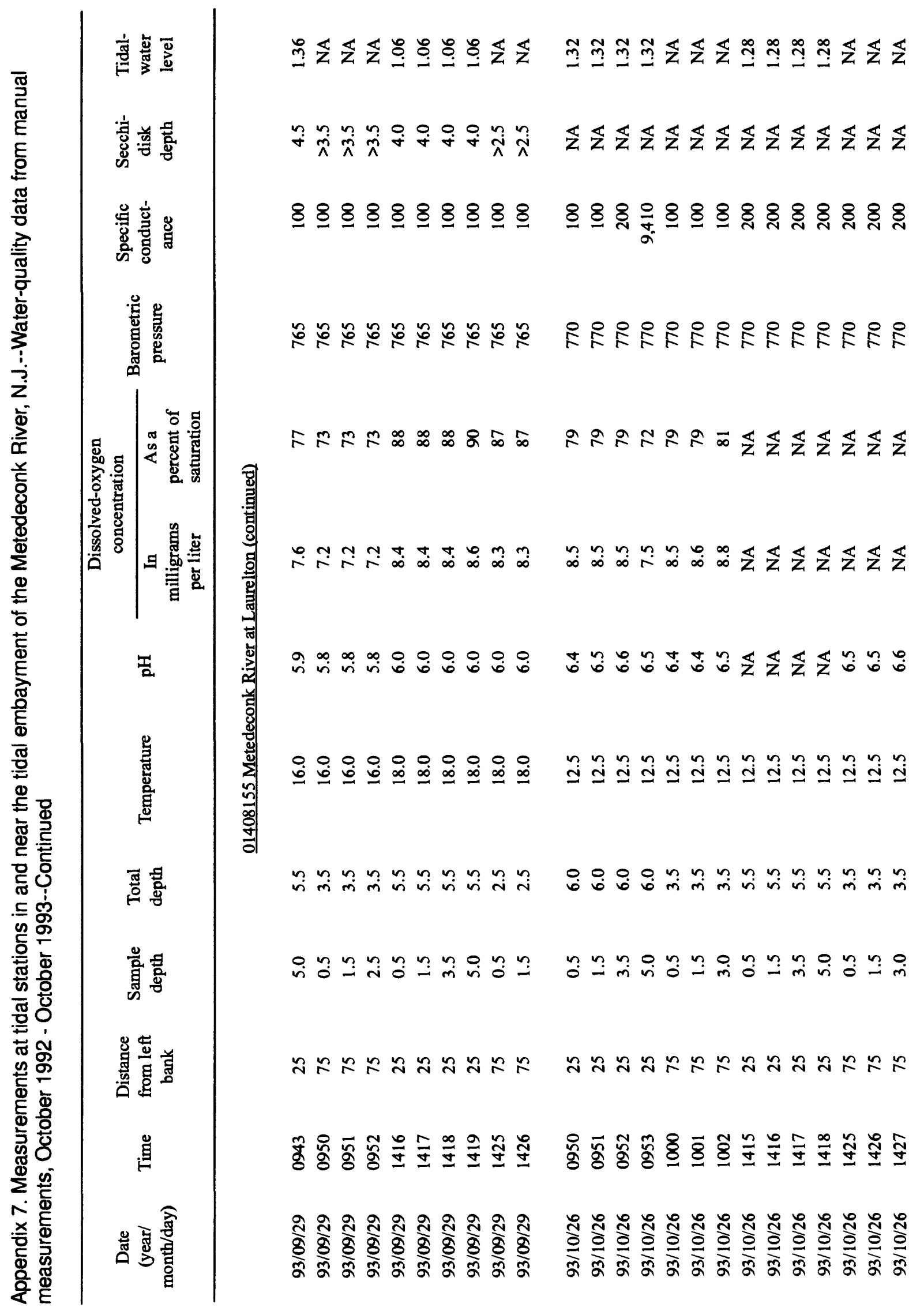




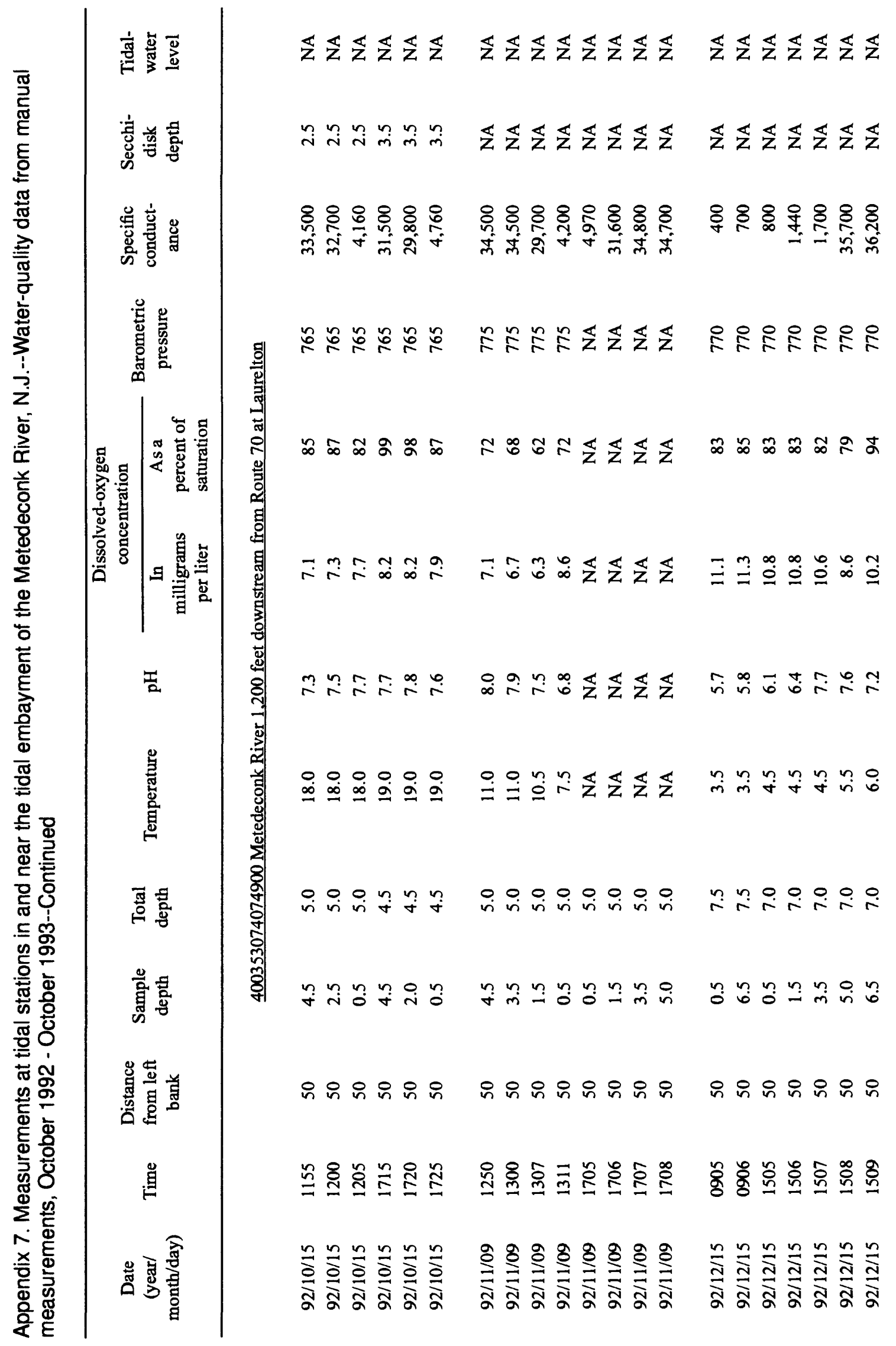




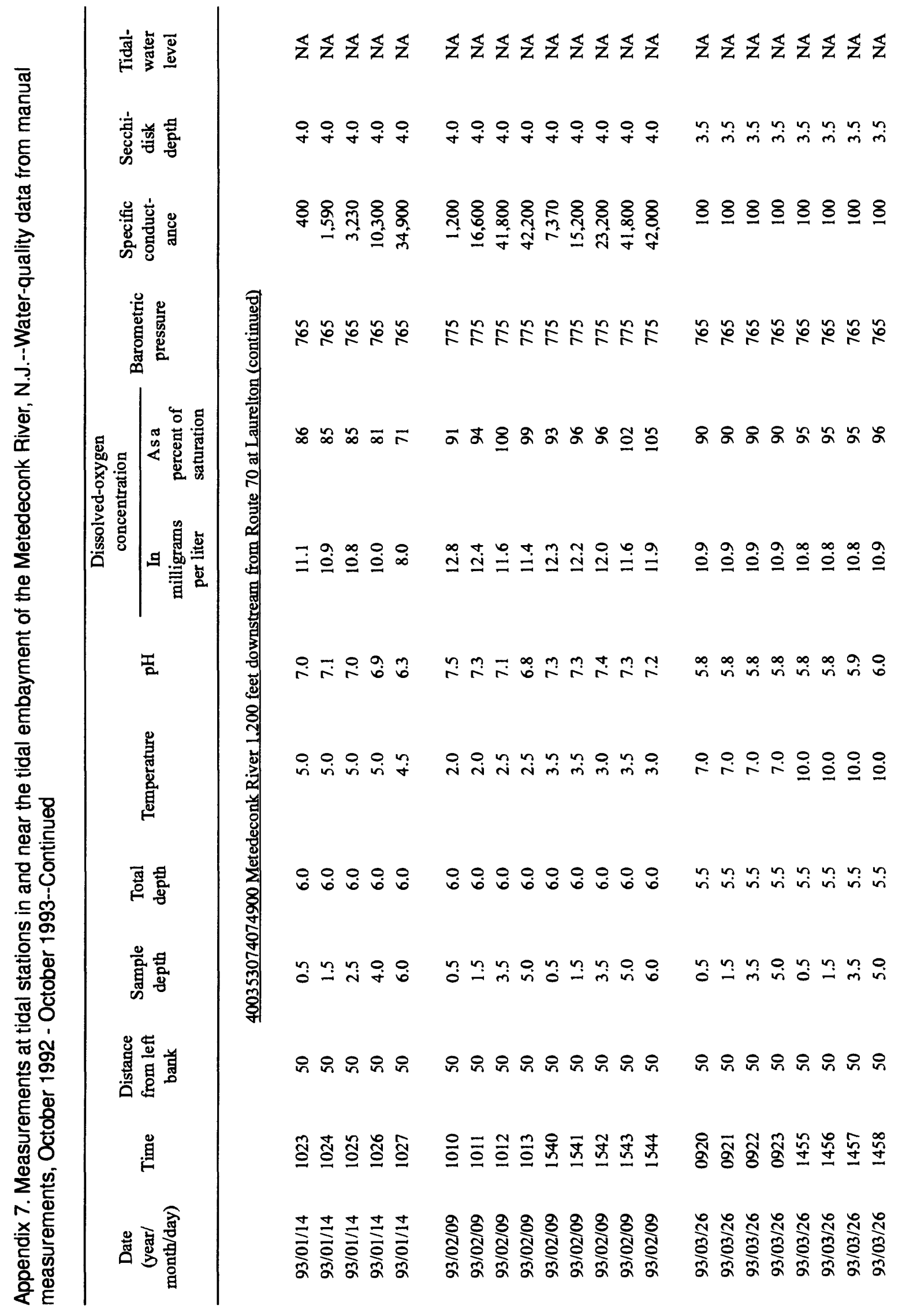




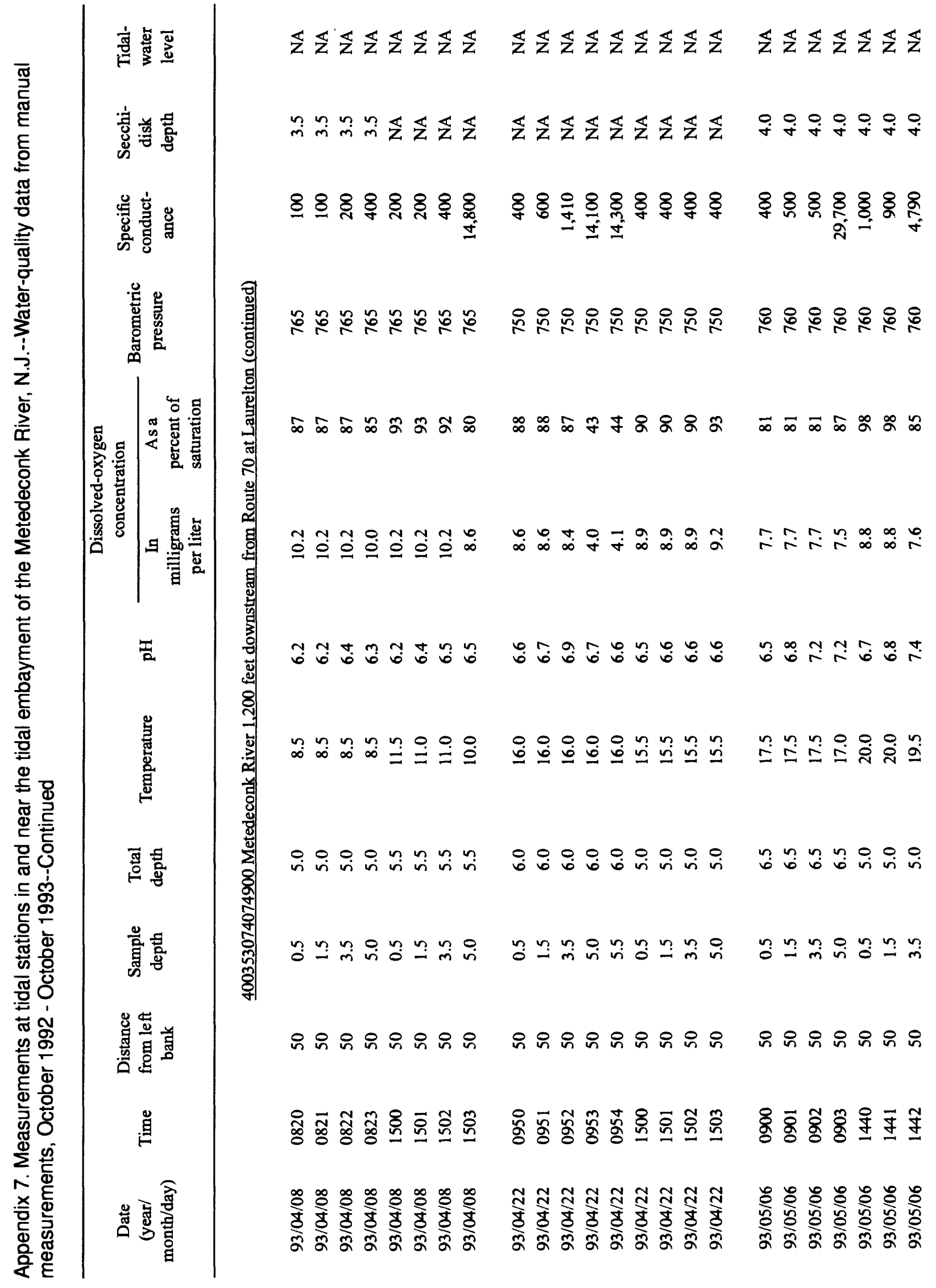




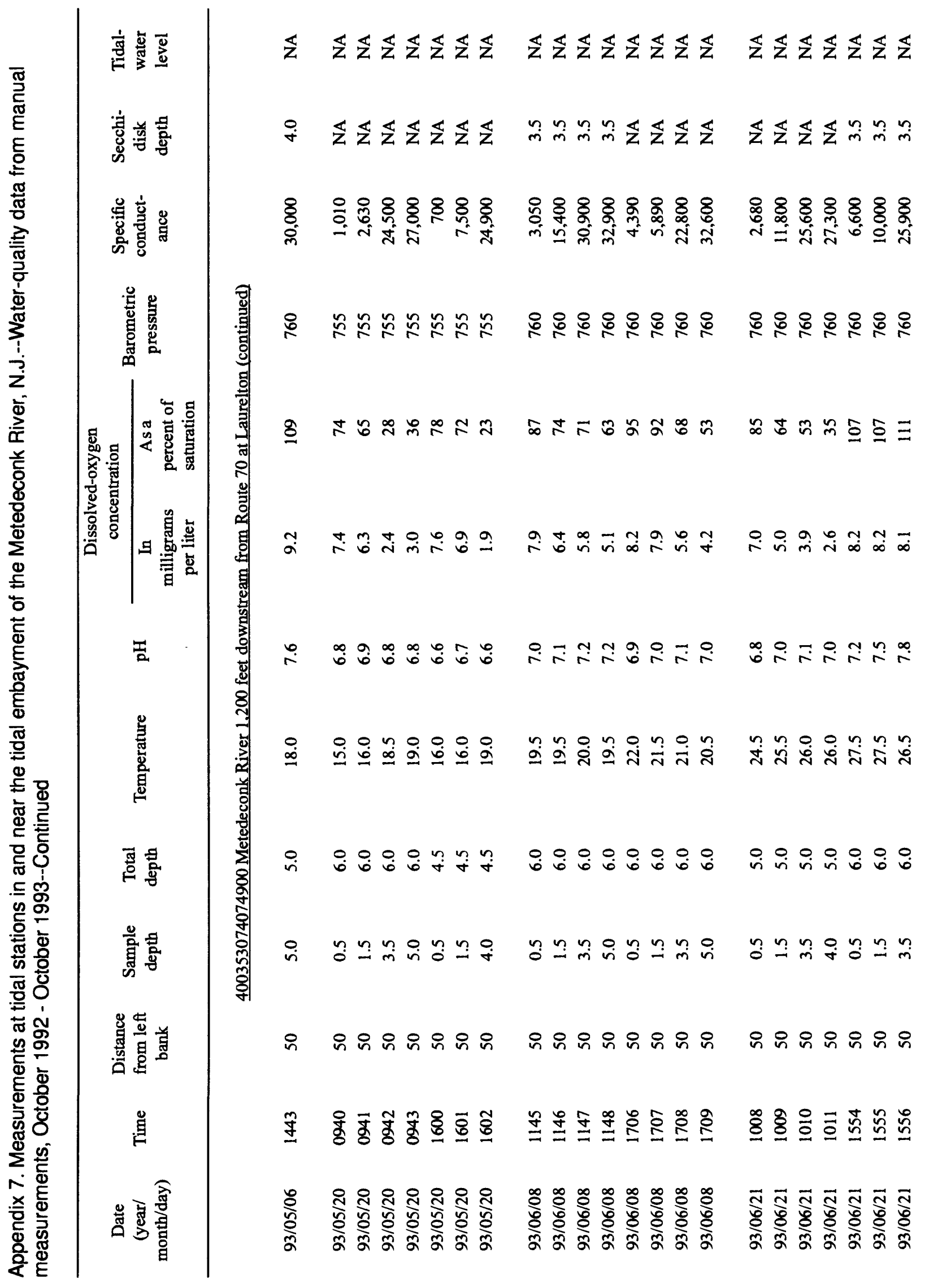




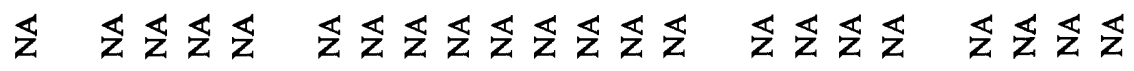

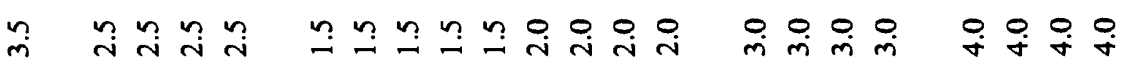

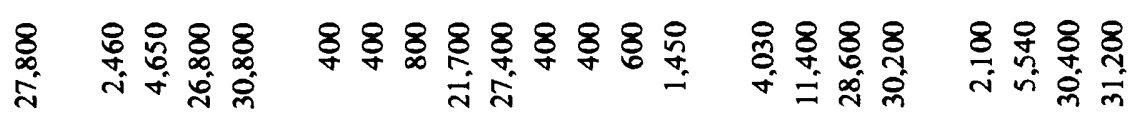

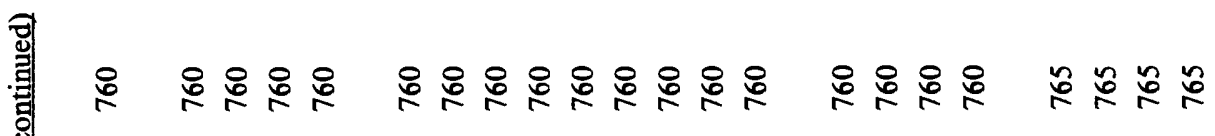

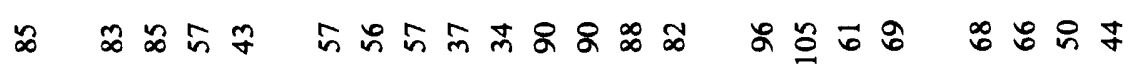

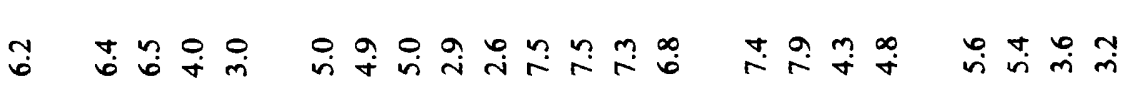

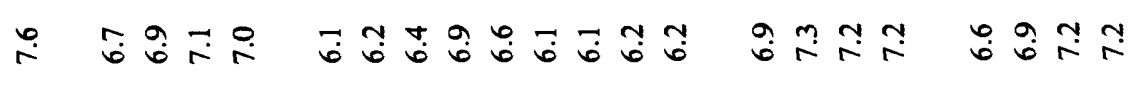

每

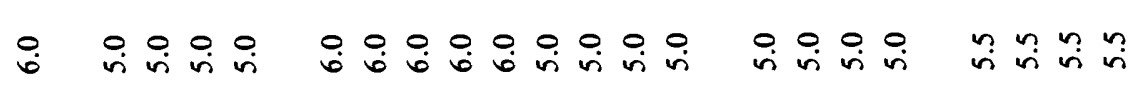

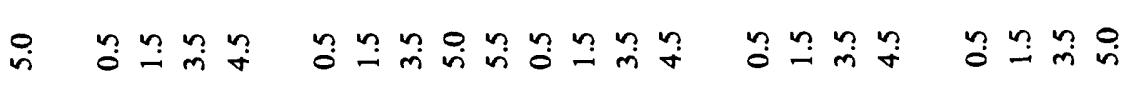

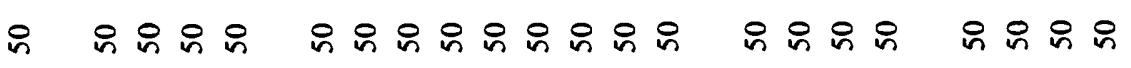

点

సี

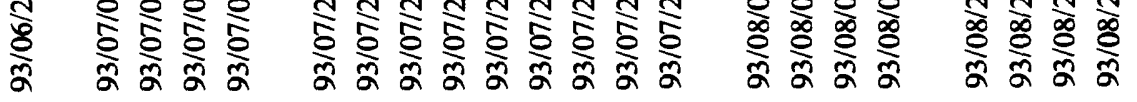




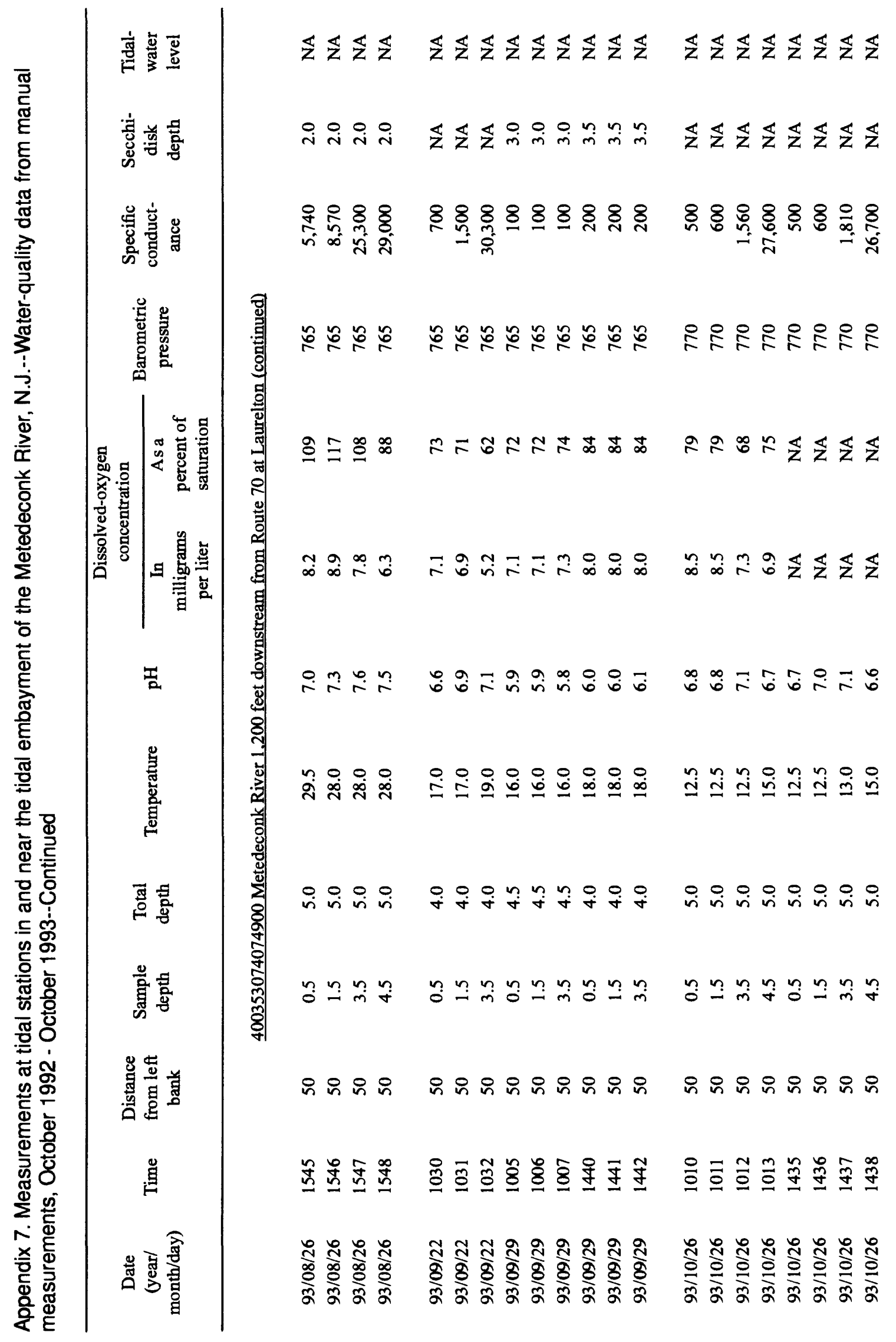




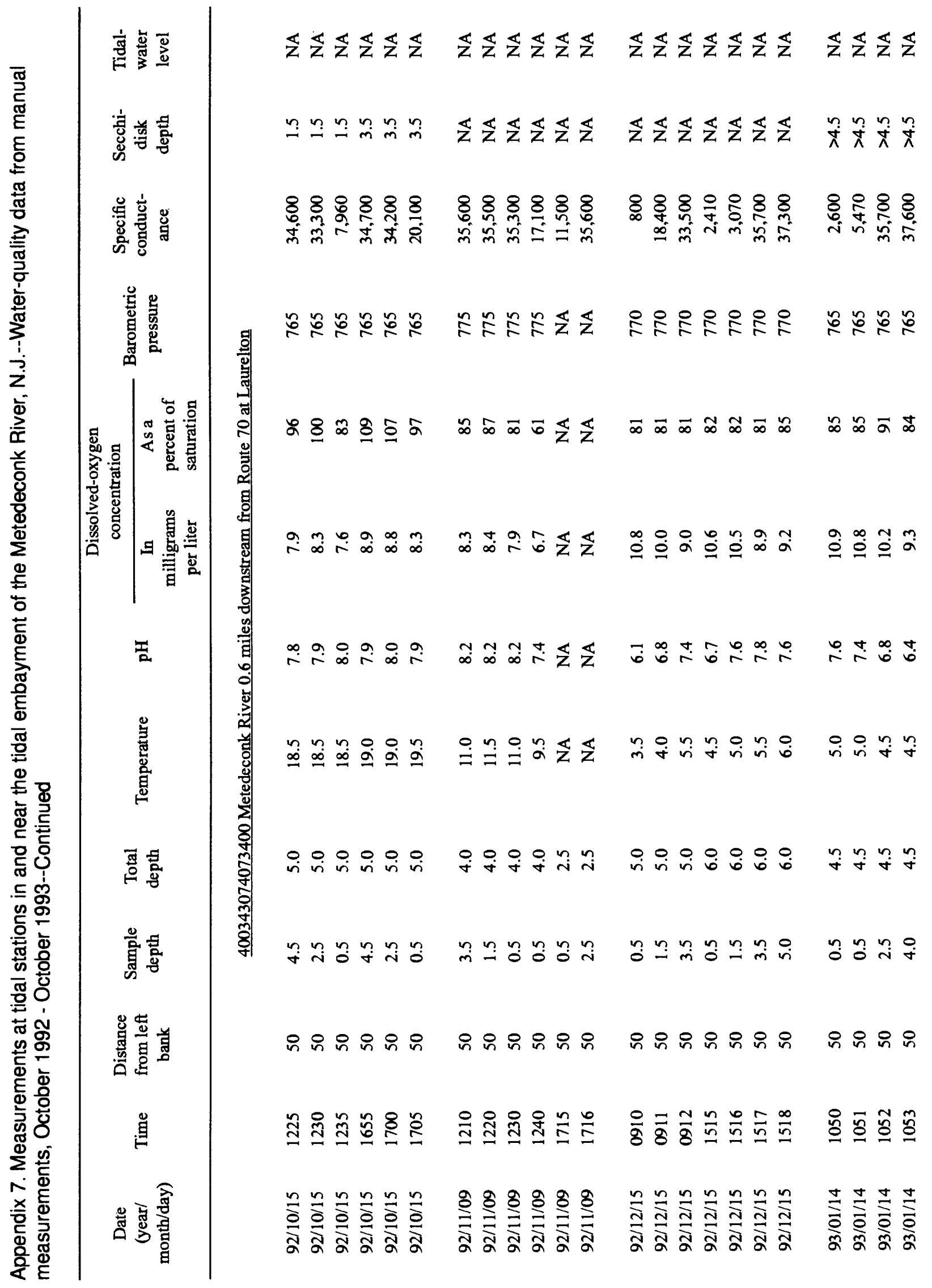




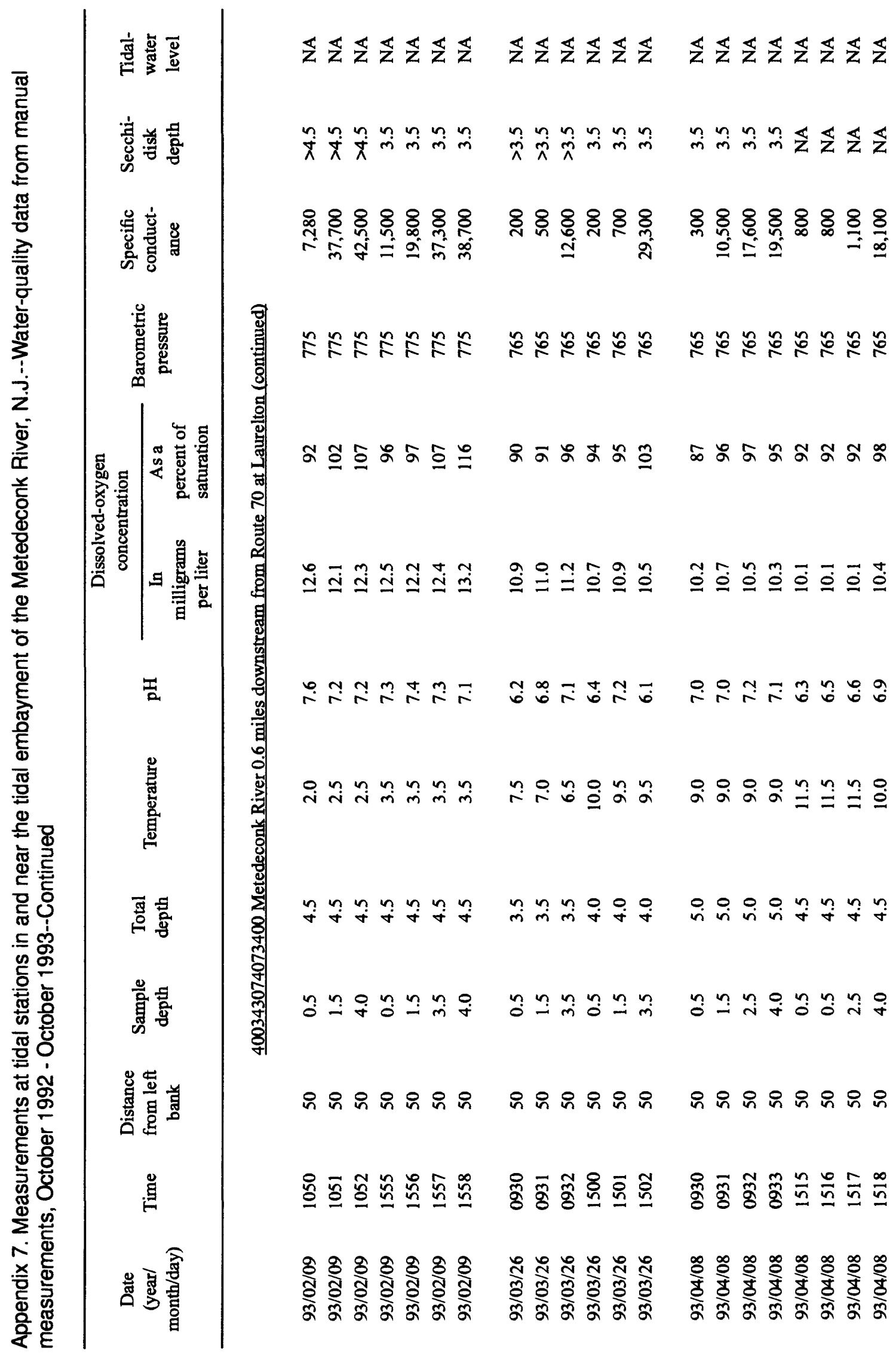




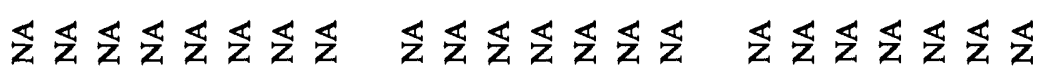

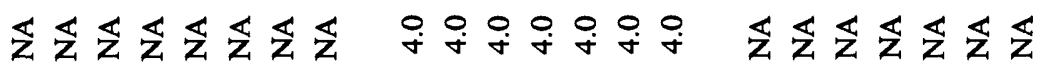

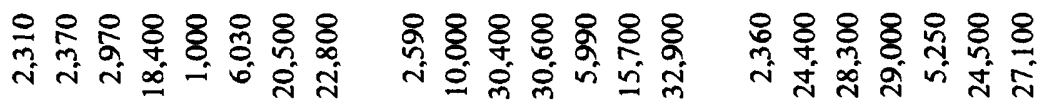

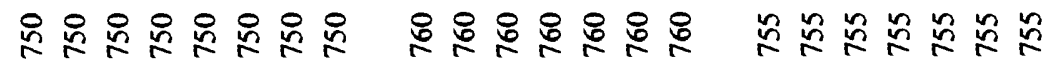

闹

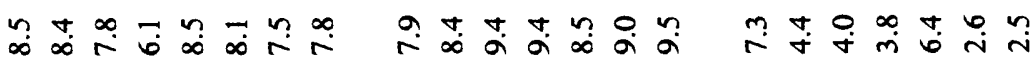

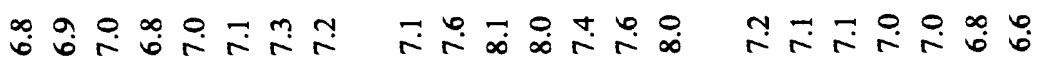

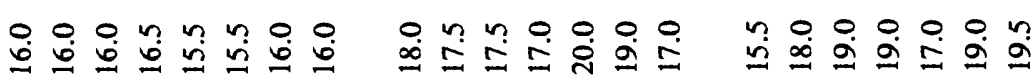

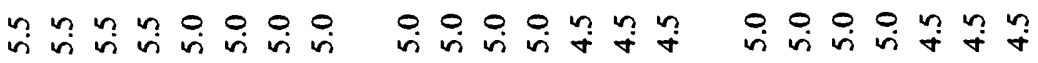

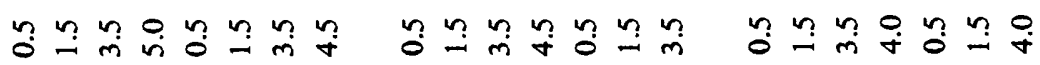

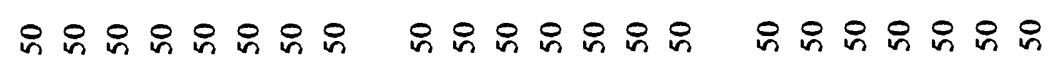

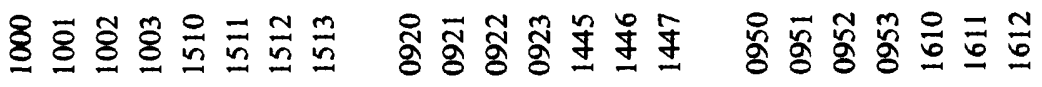

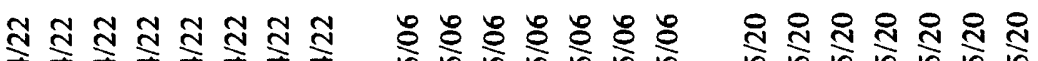

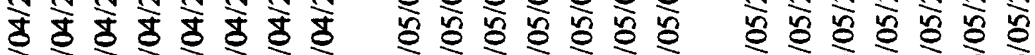

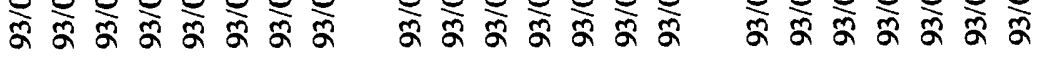




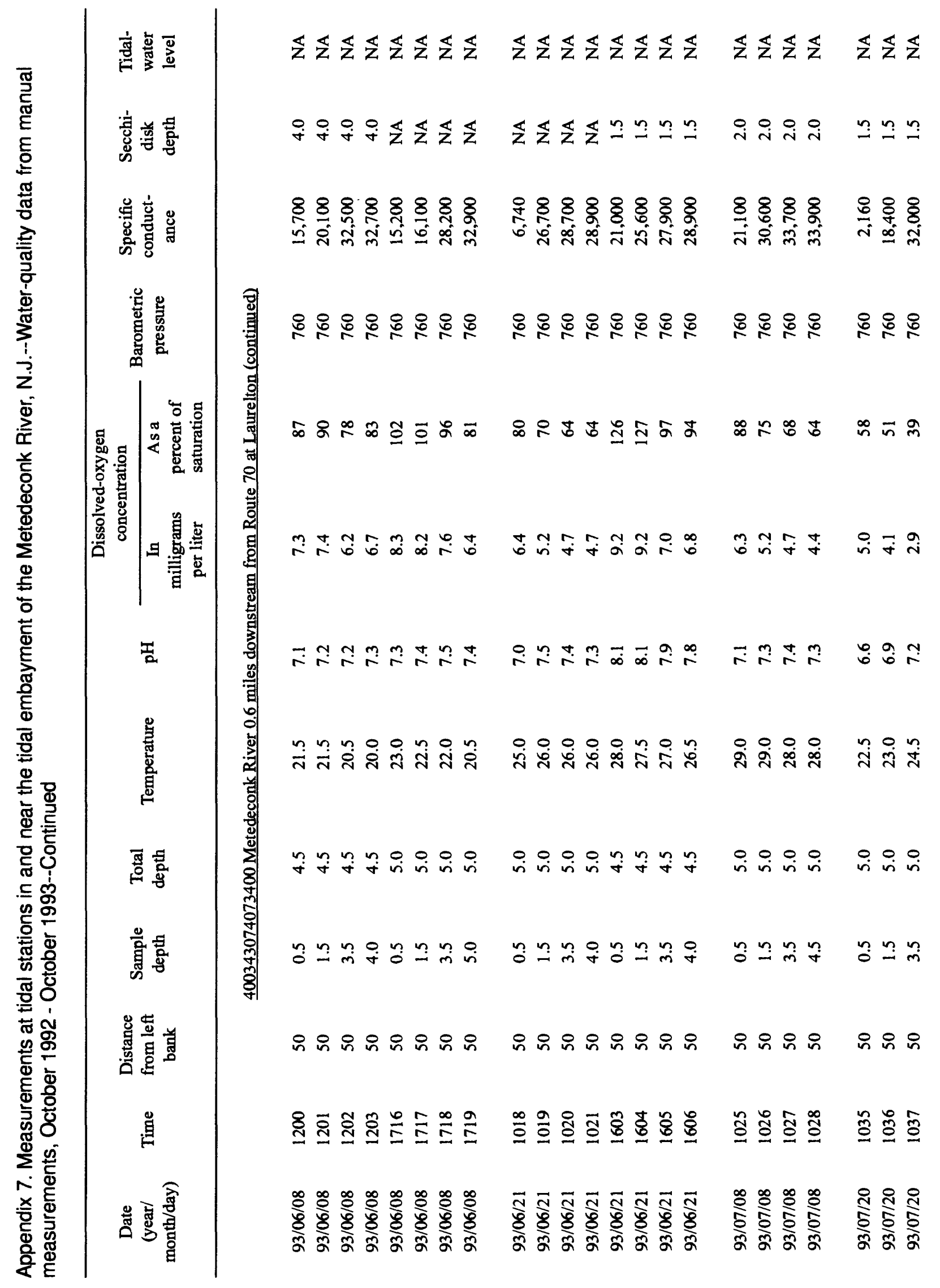




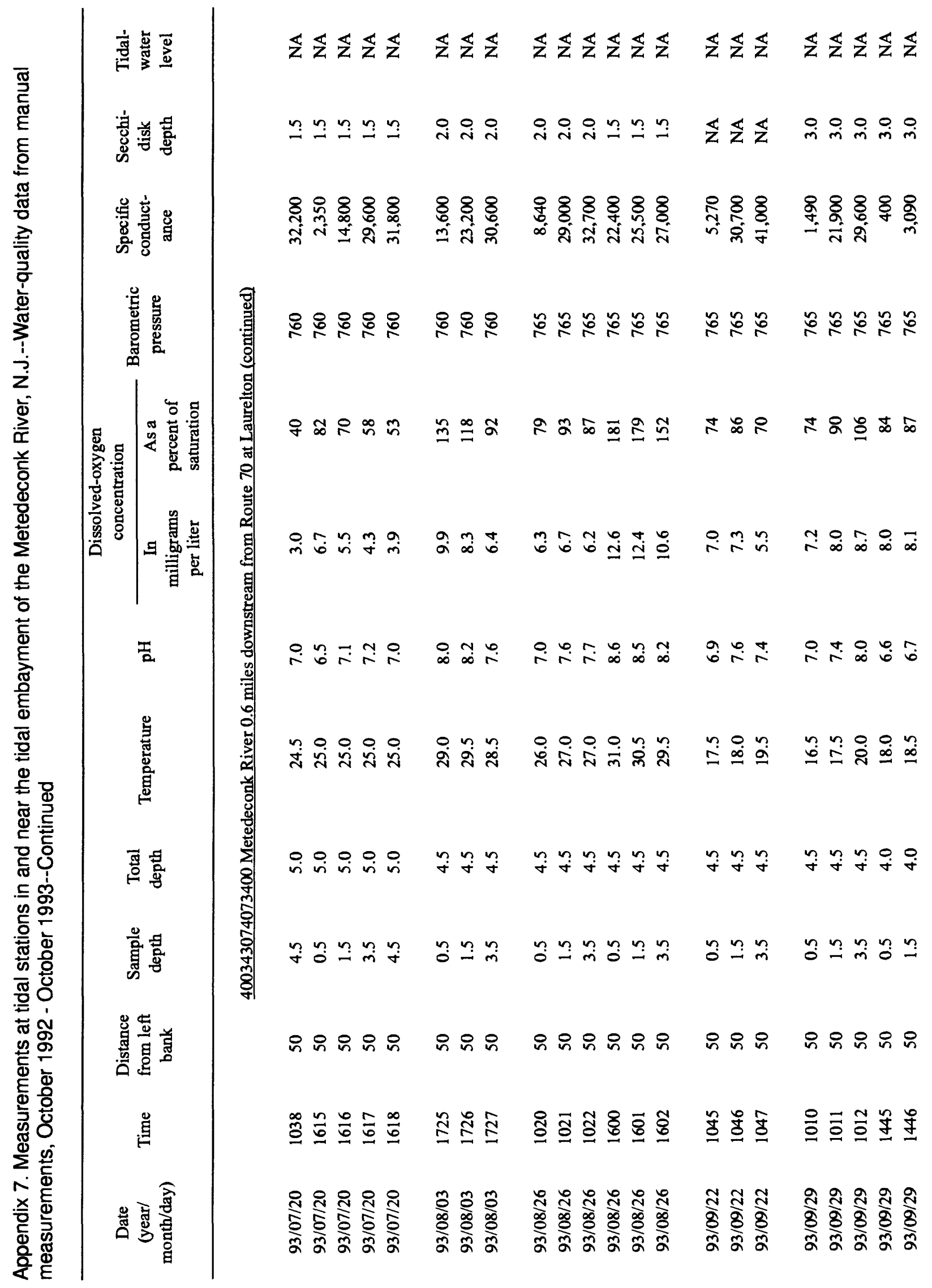




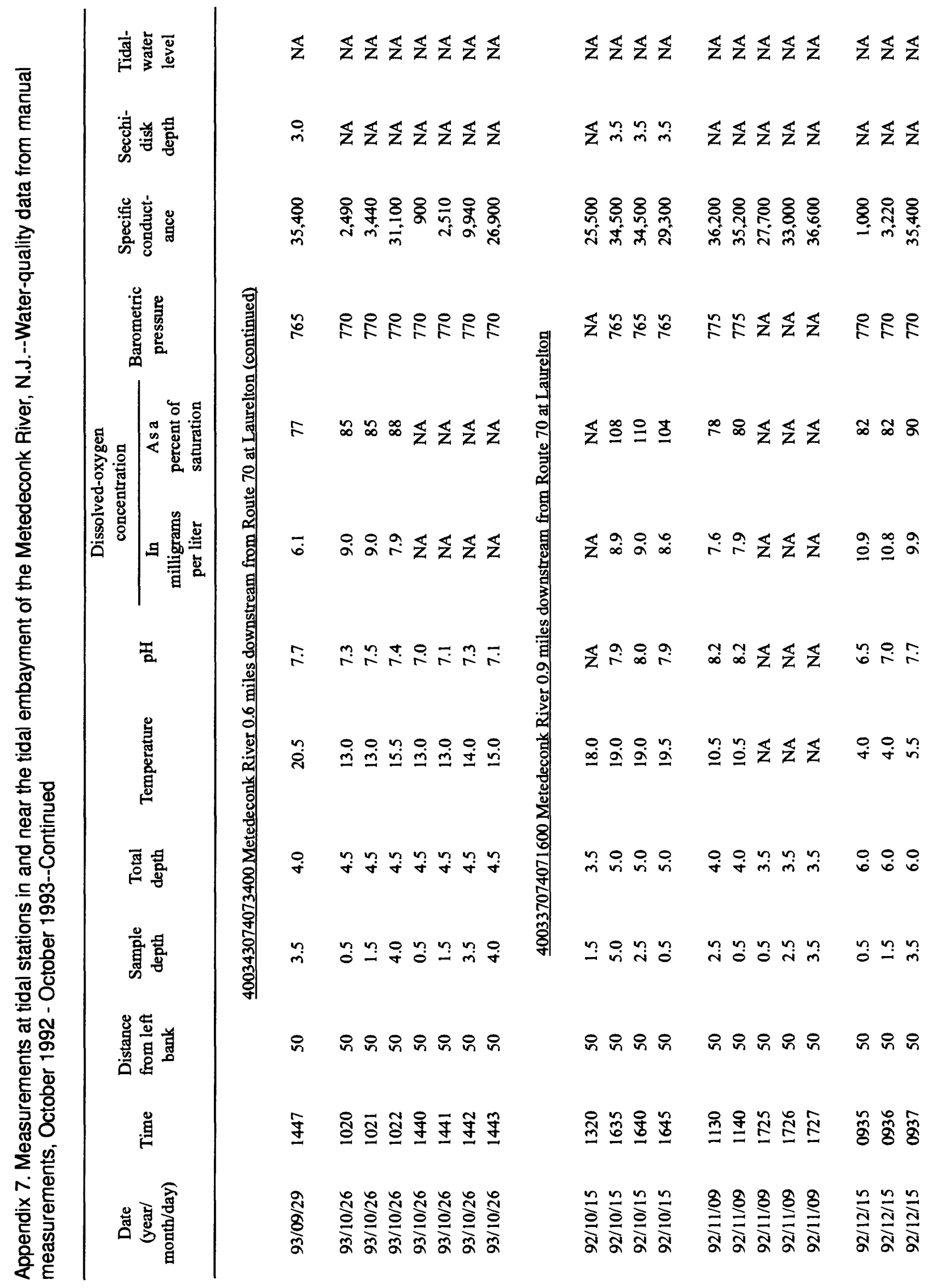




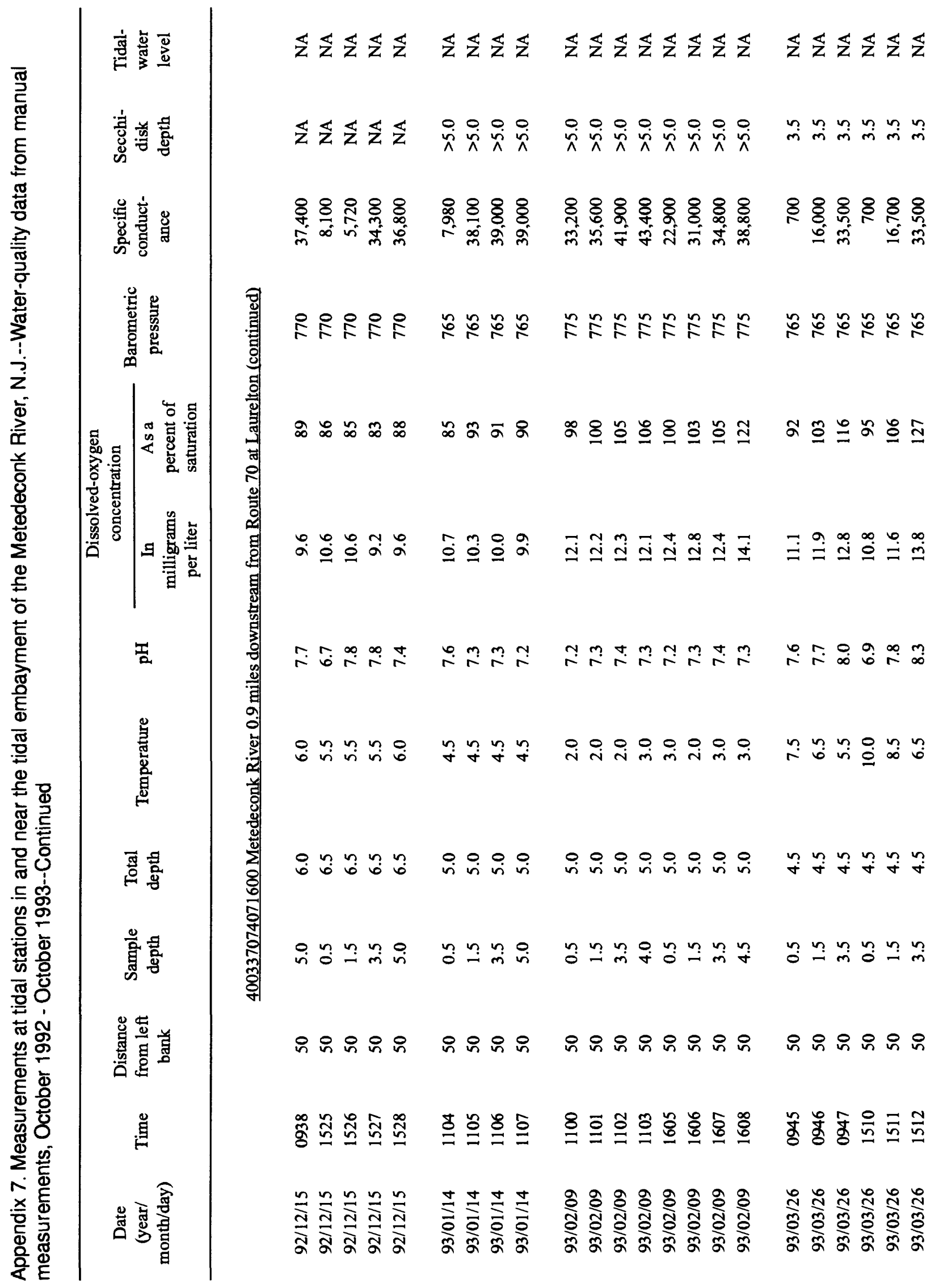




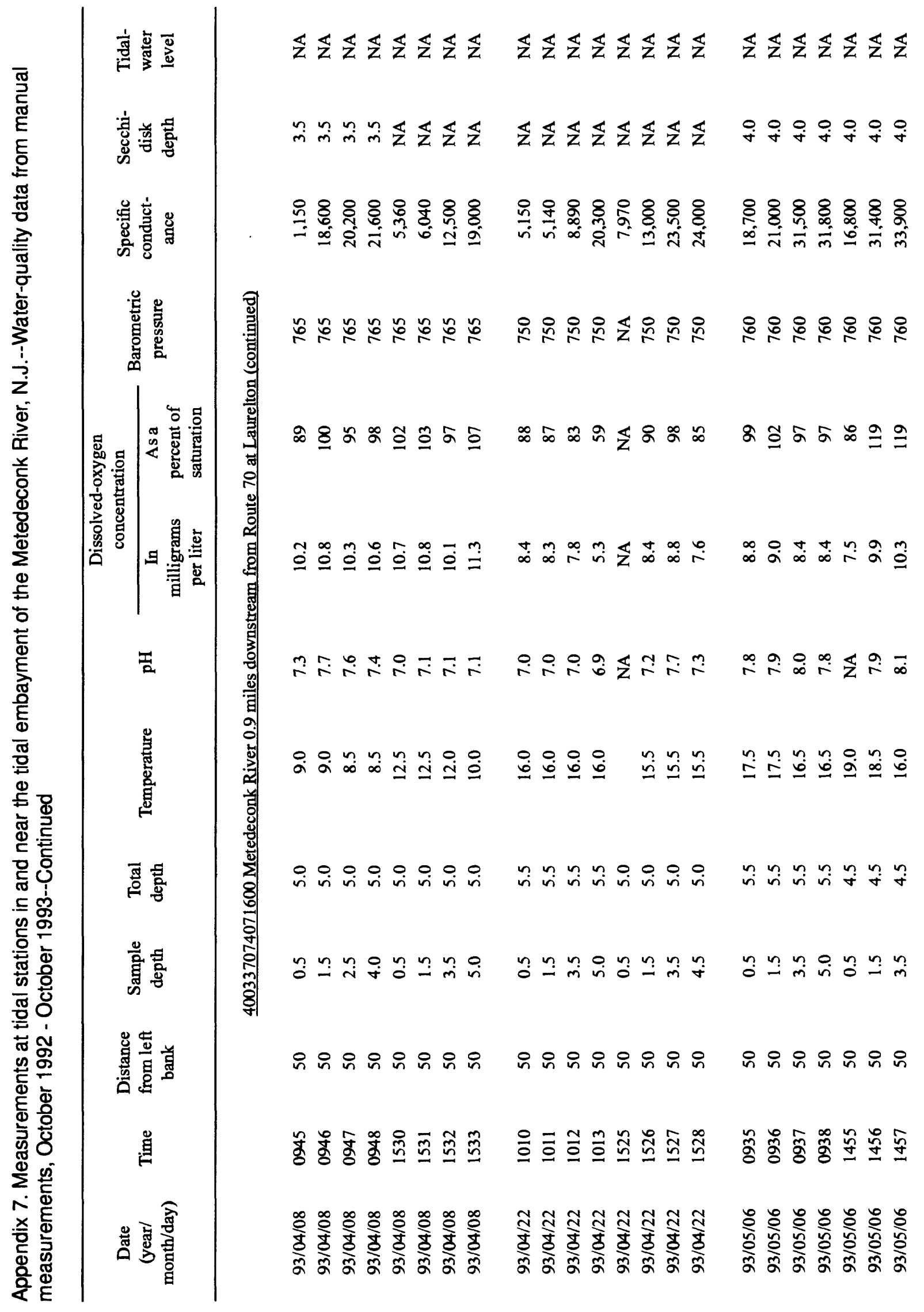




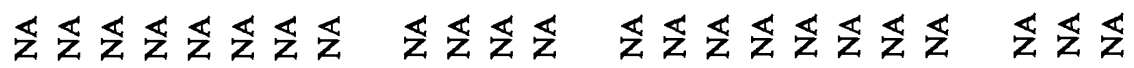

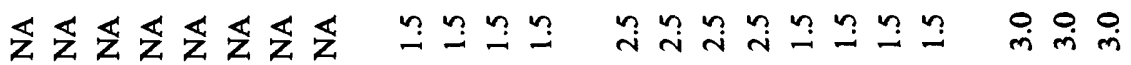

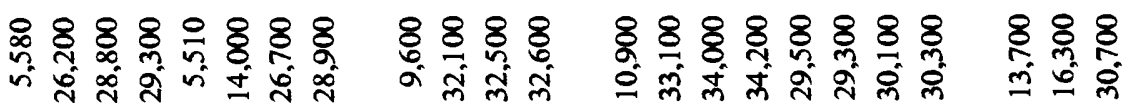

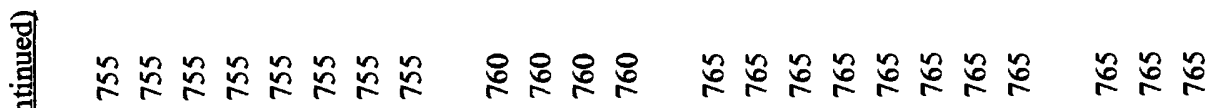

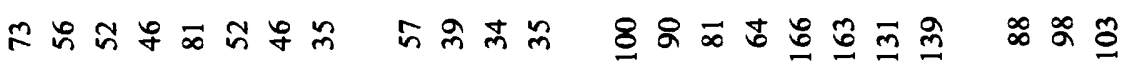

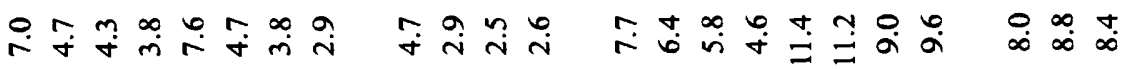

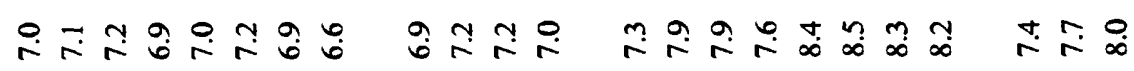

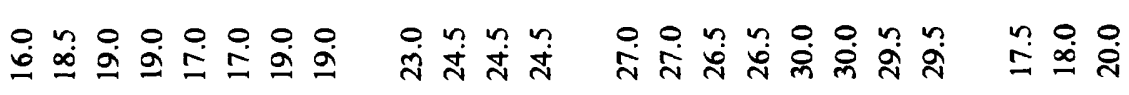

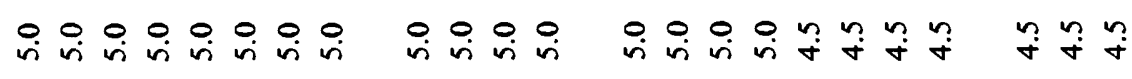

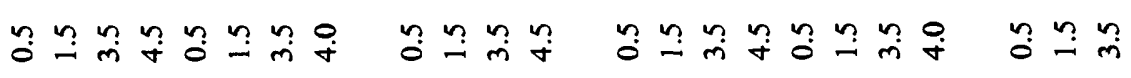

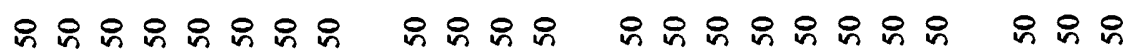

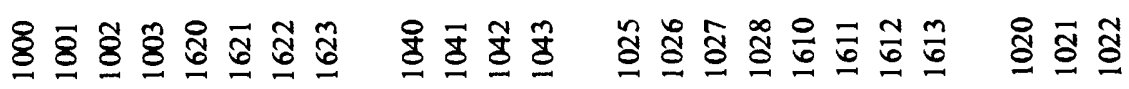

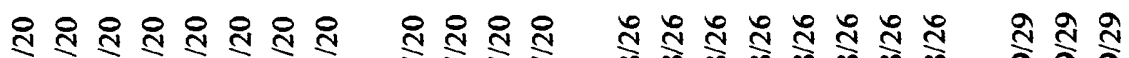

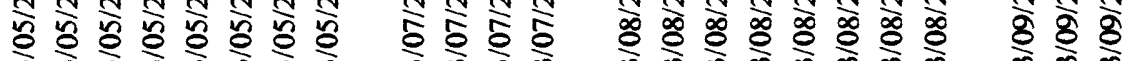

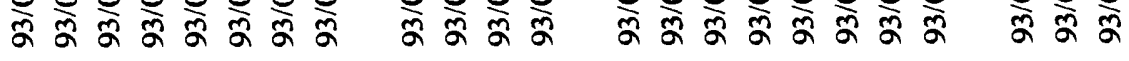




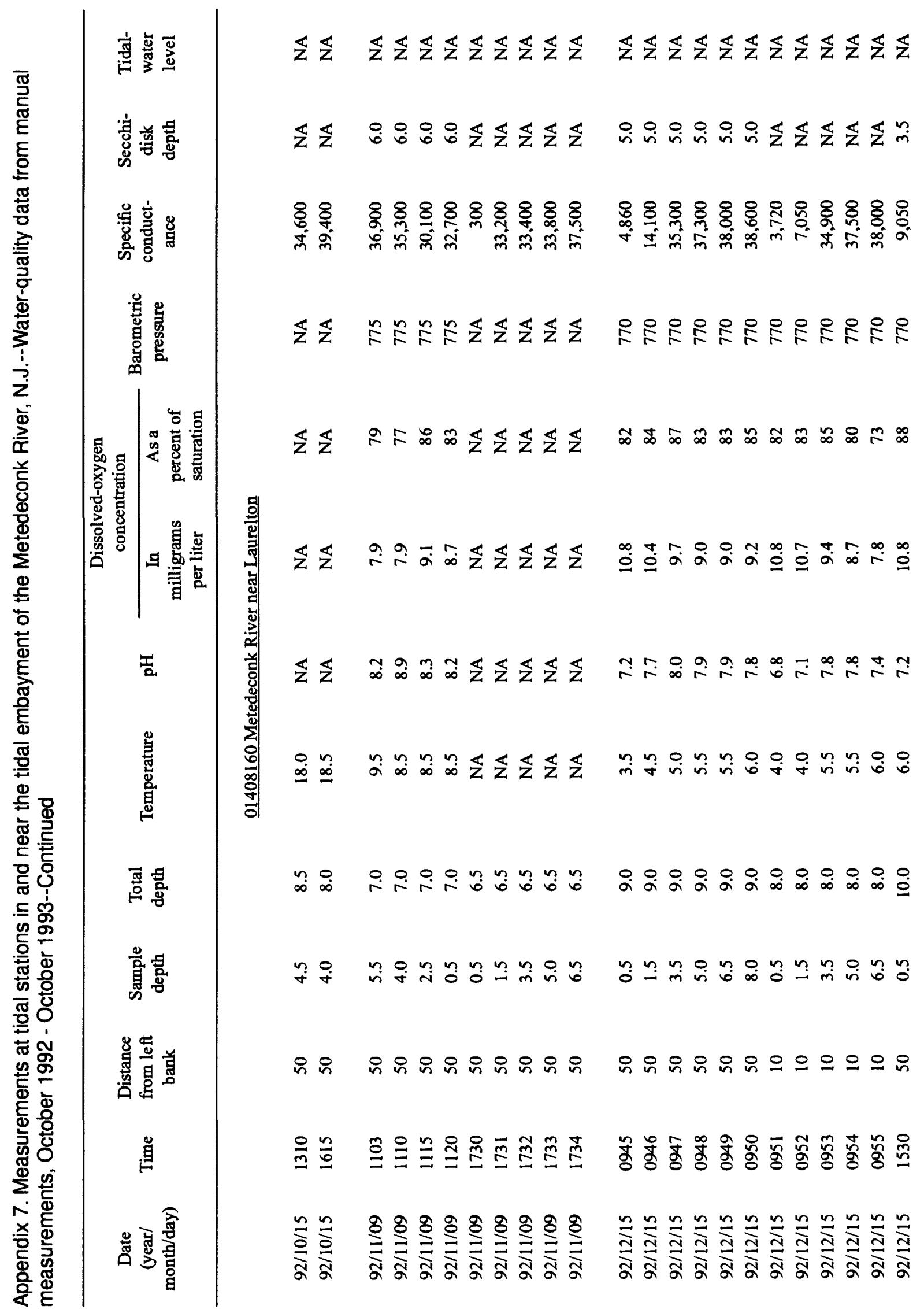




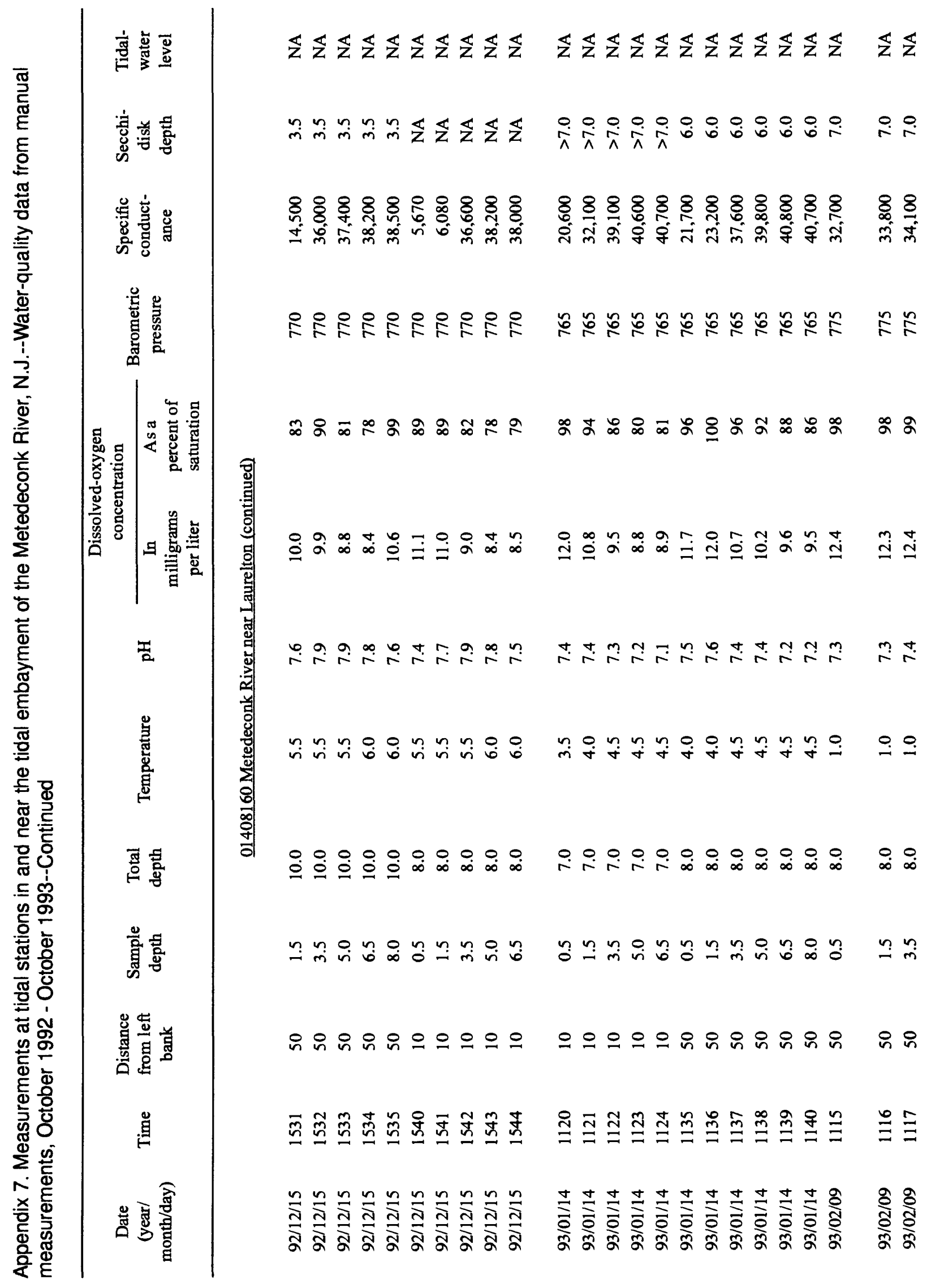




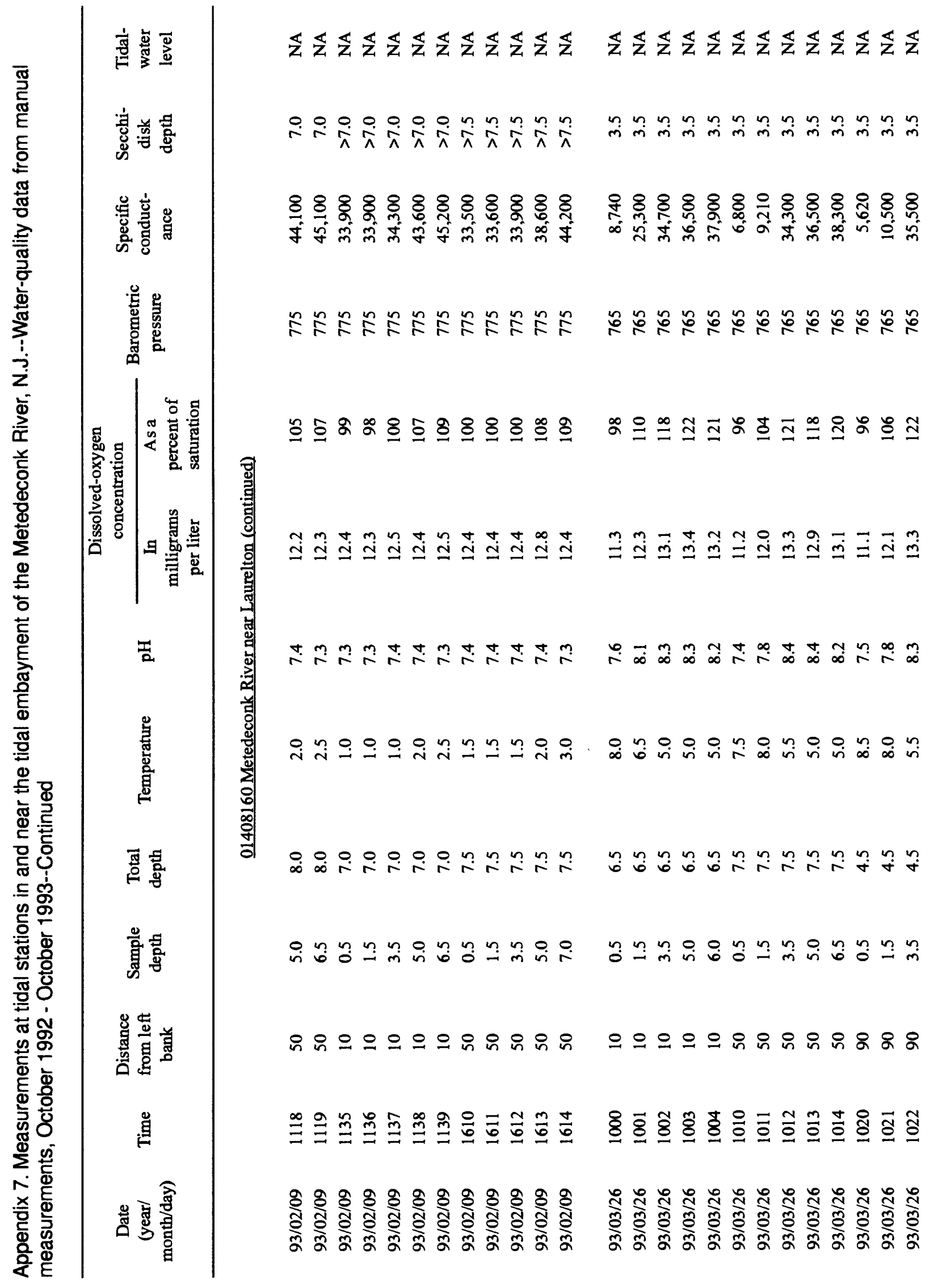




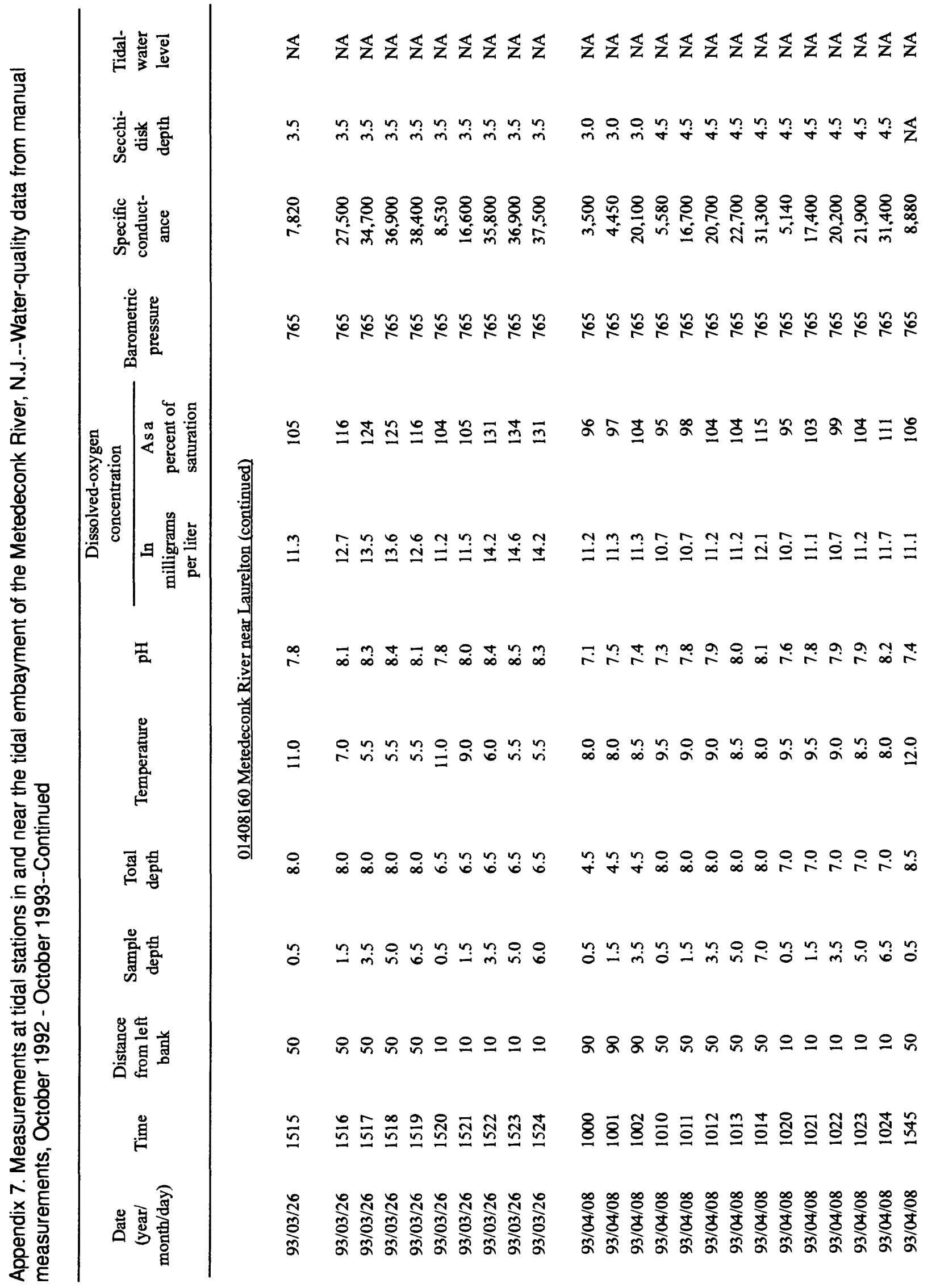




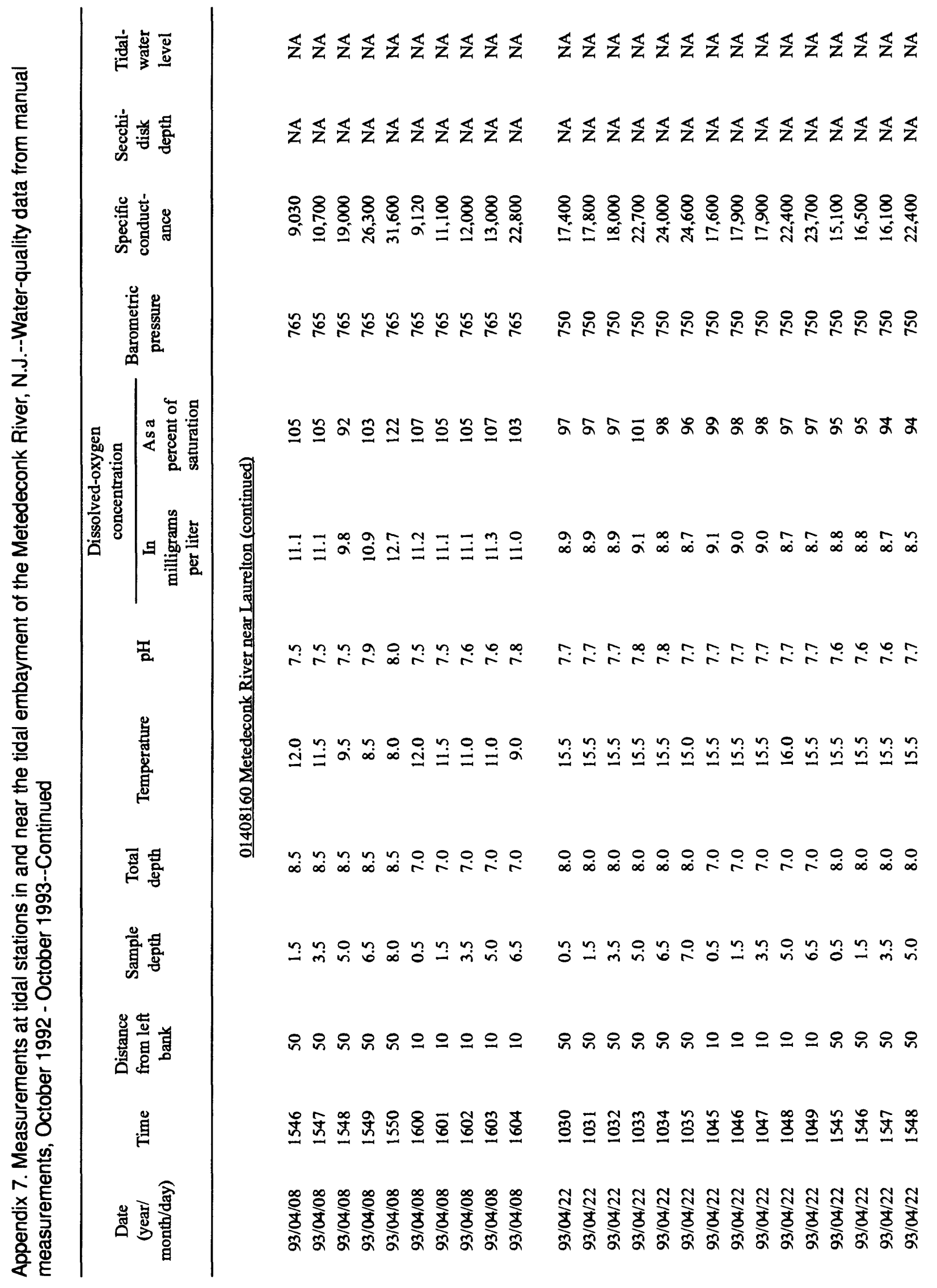




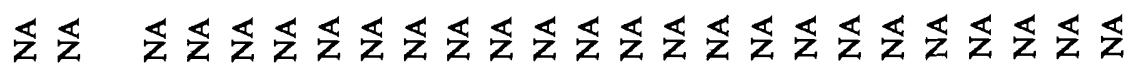

不

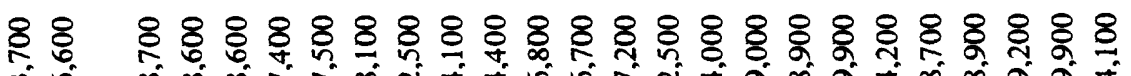

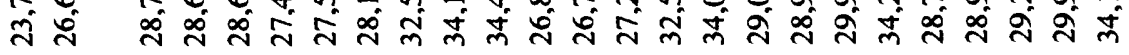

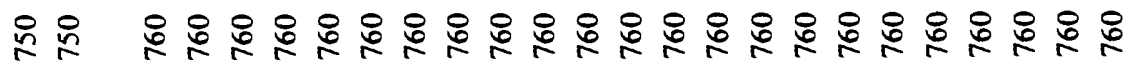

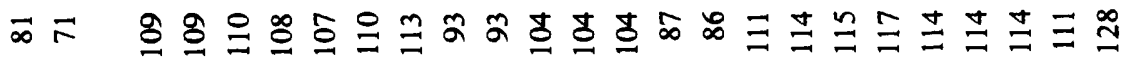

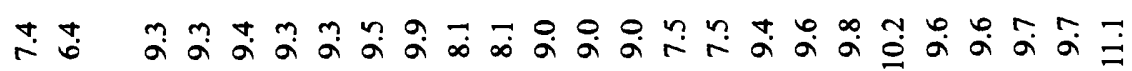

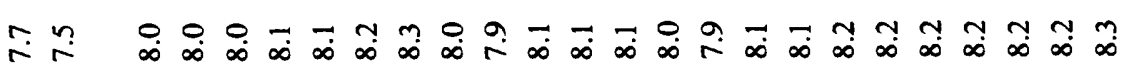

일

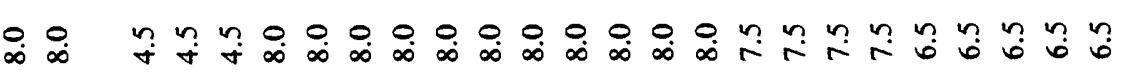

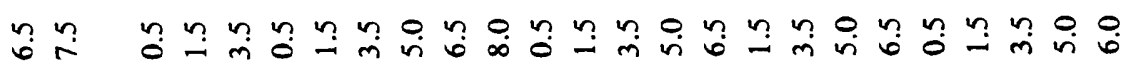

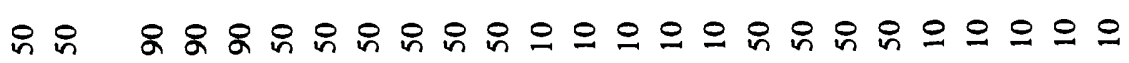

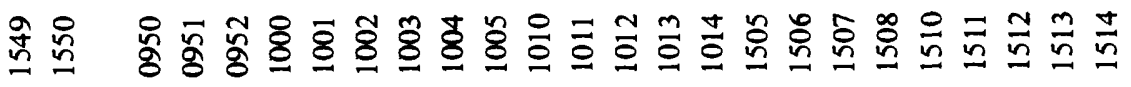

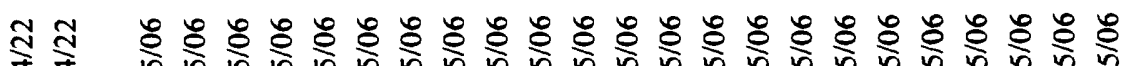

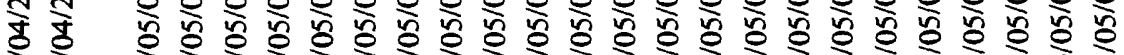

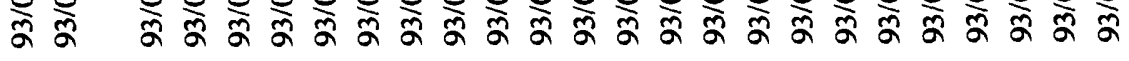




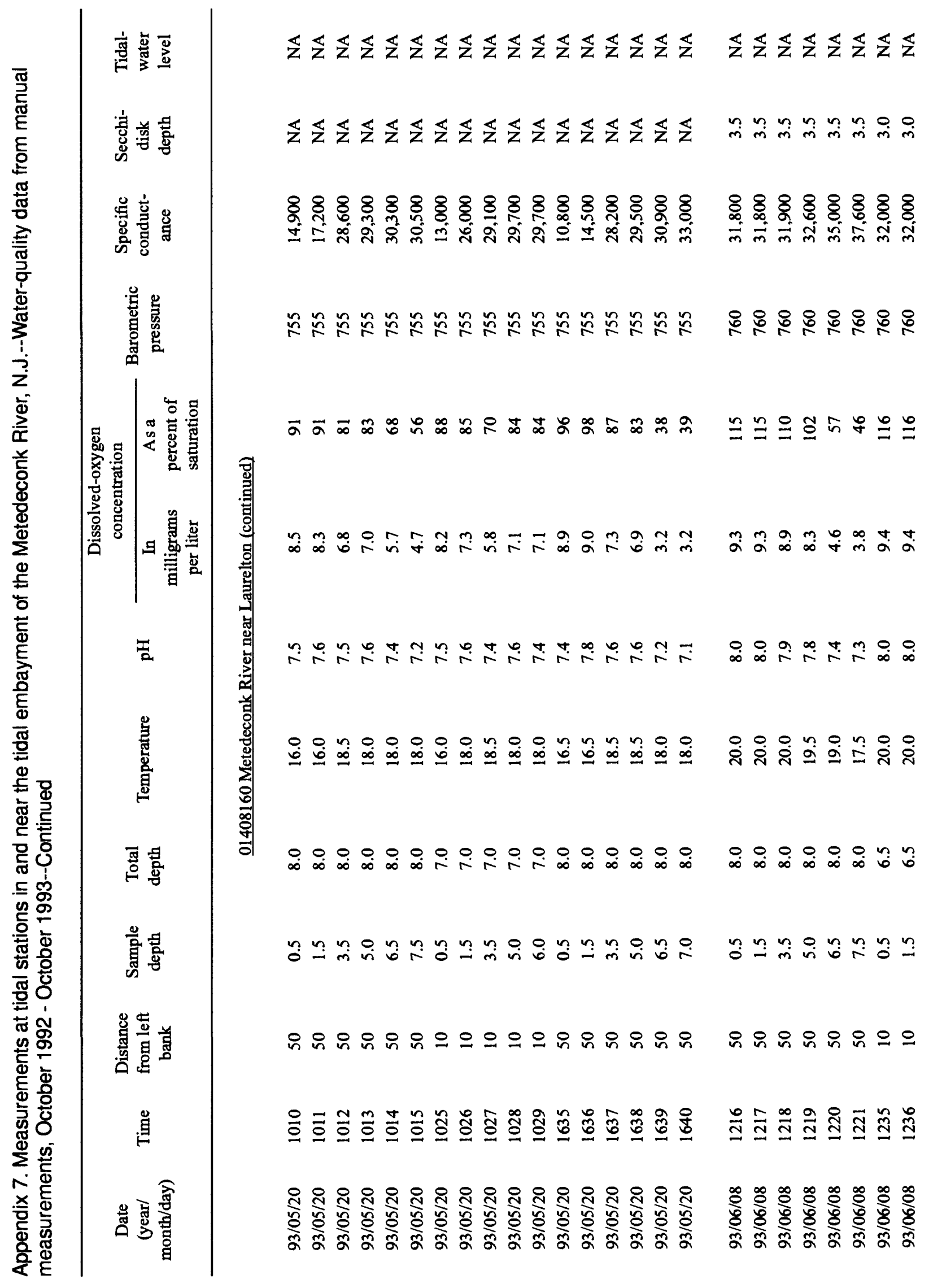




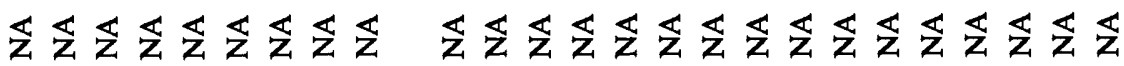

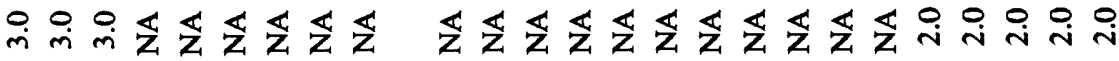

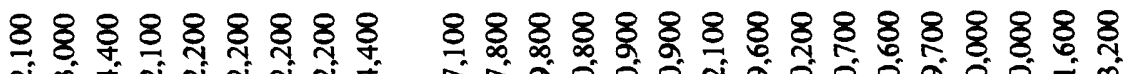

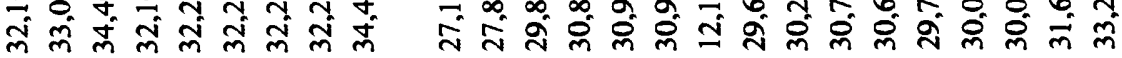

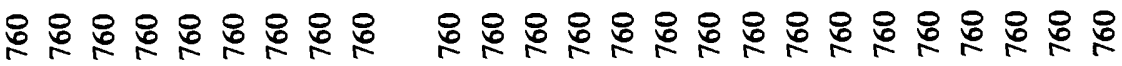

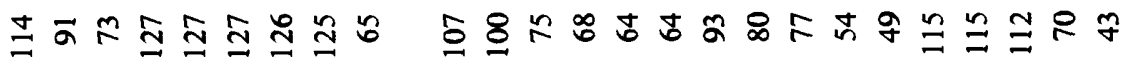

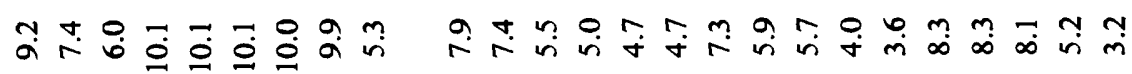

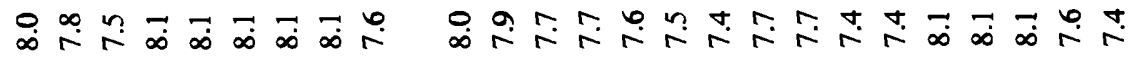

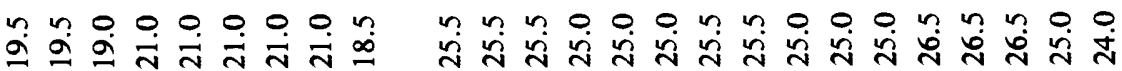

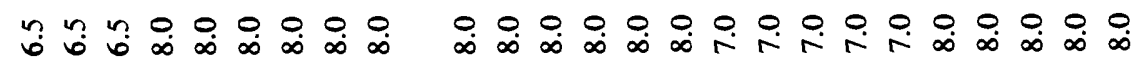

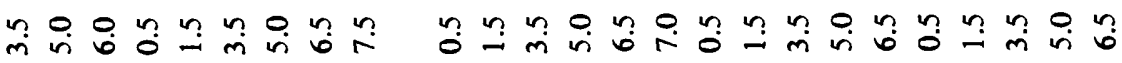

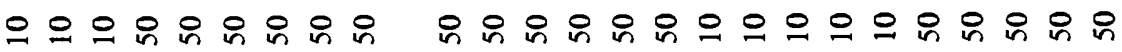

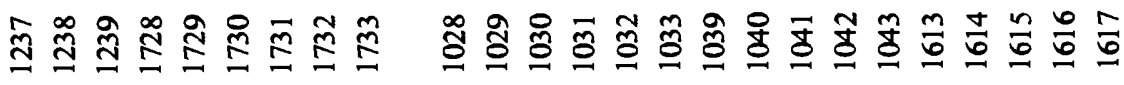

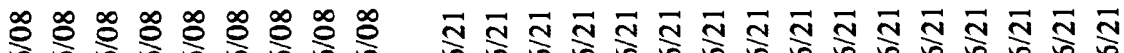

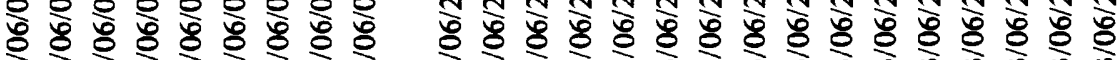
ă ă 


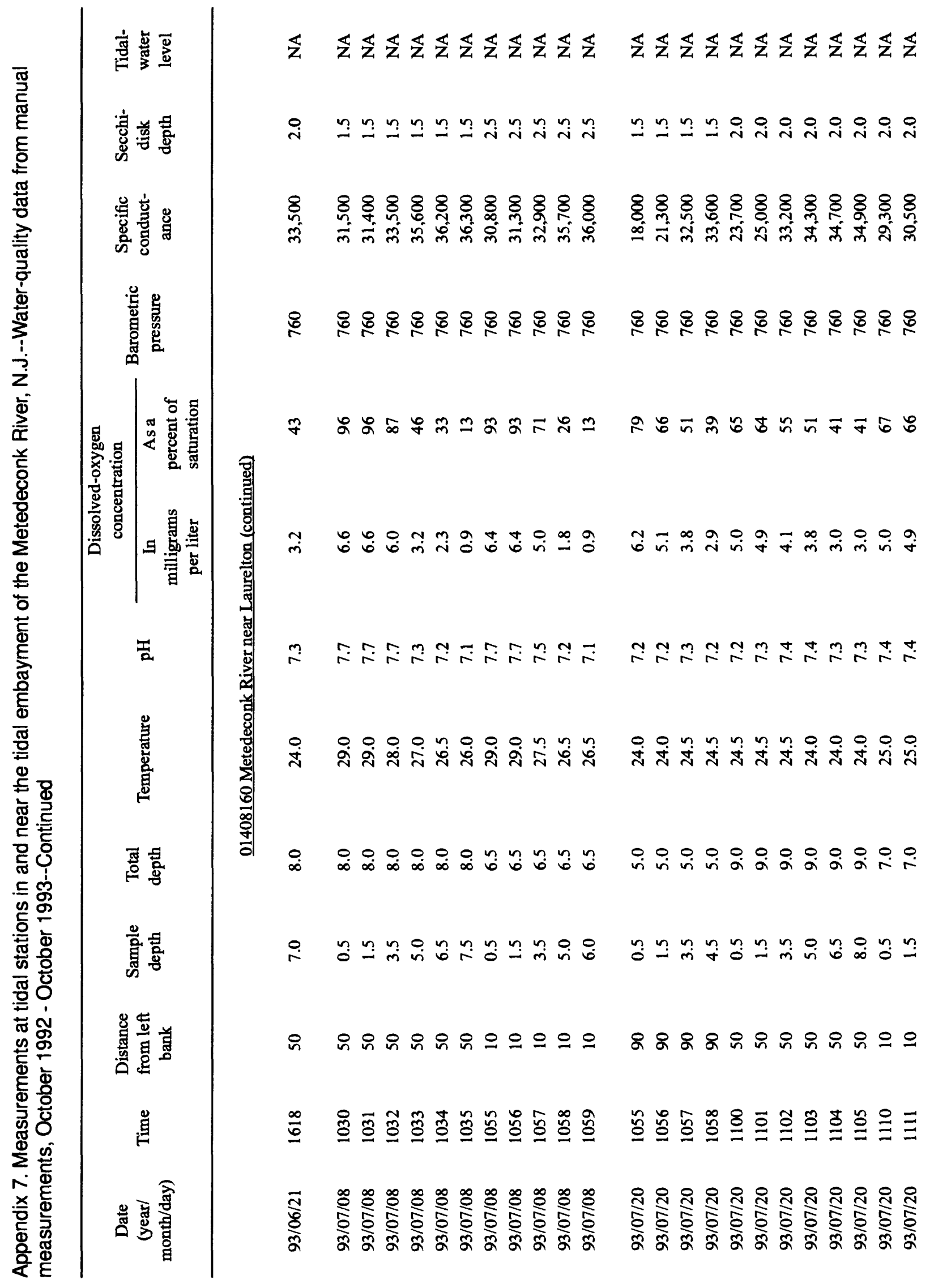




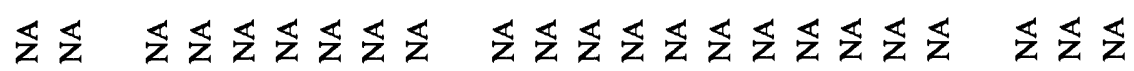

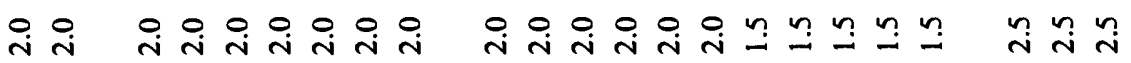

8 \& 8888

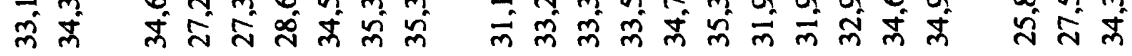

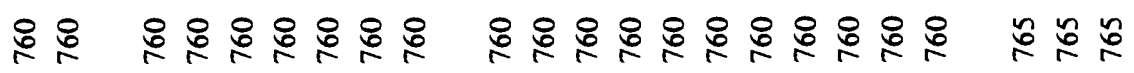

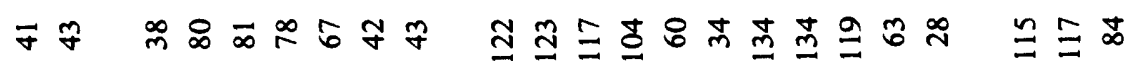

近

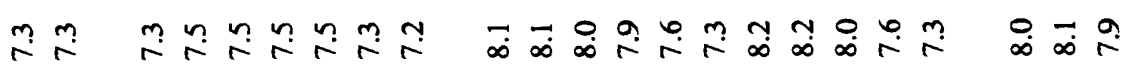

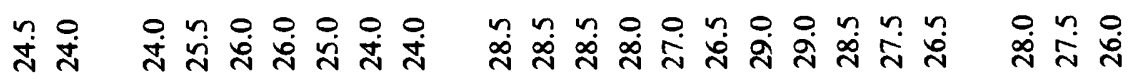

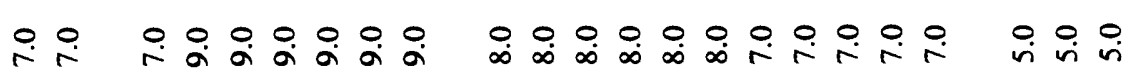

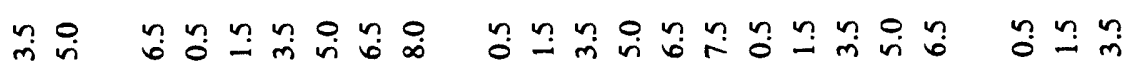

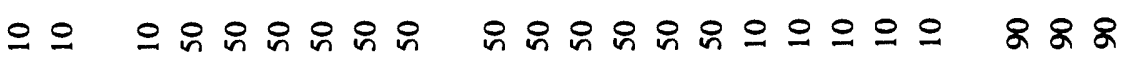

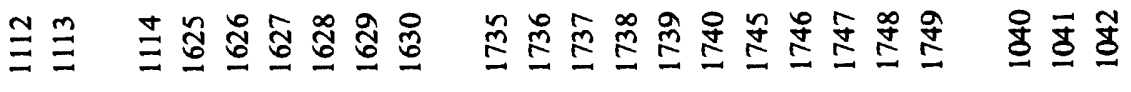

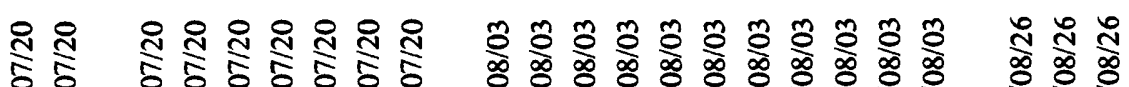

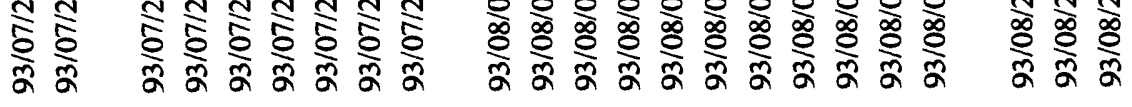




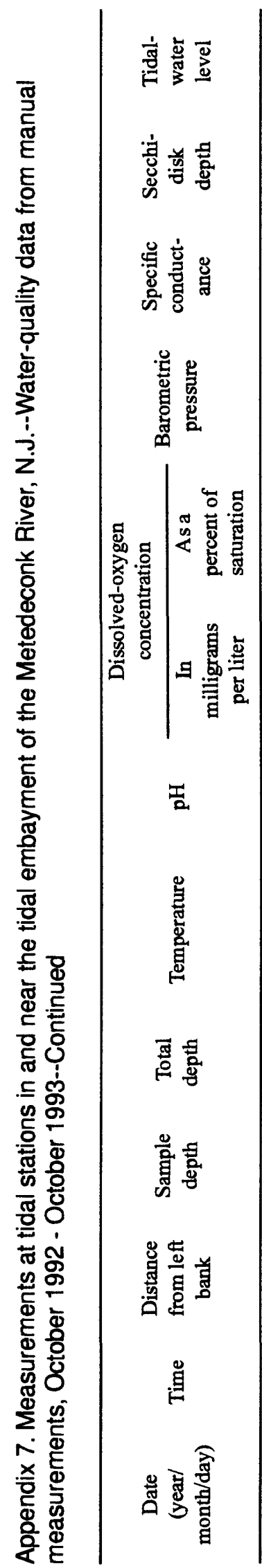

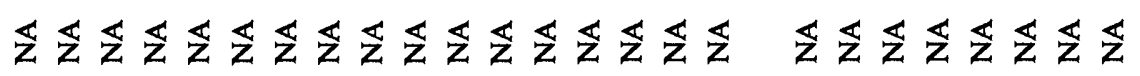

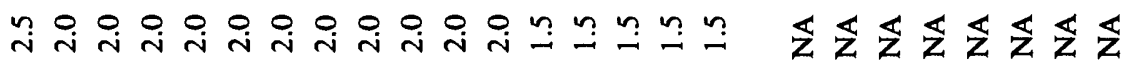

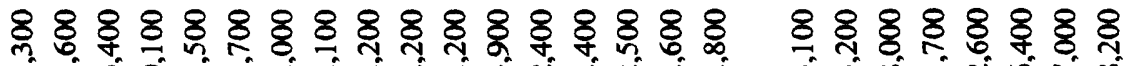

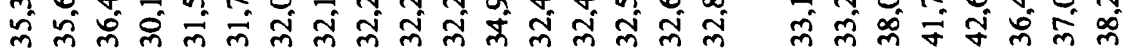

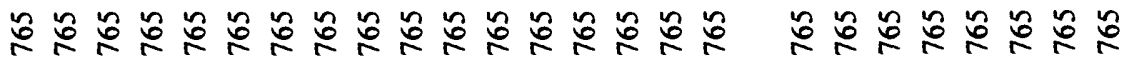

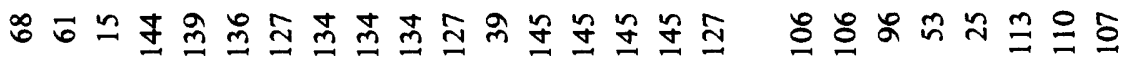

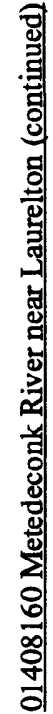

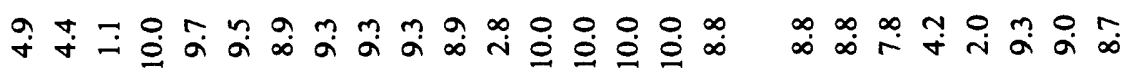

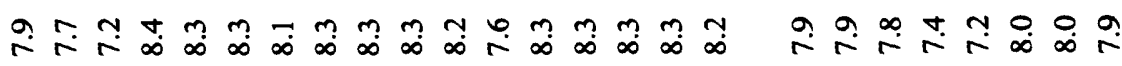

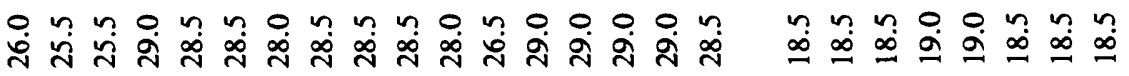

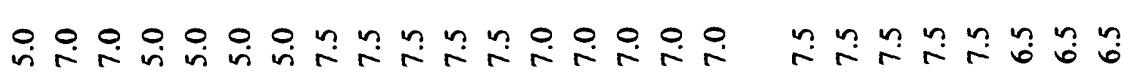
そํํำกำ

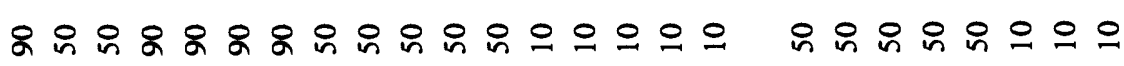

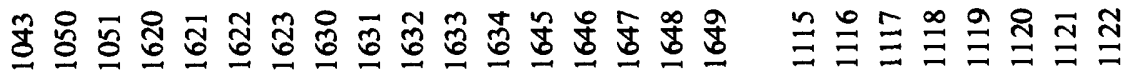

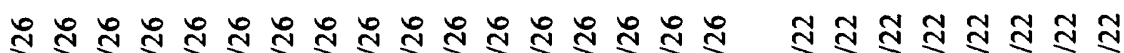

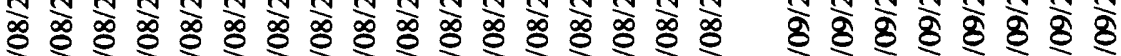

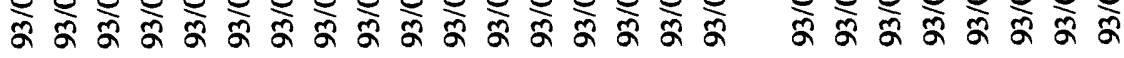




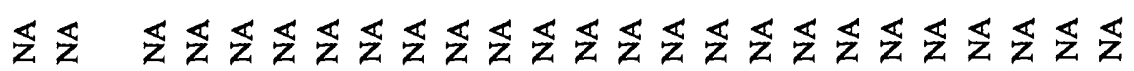

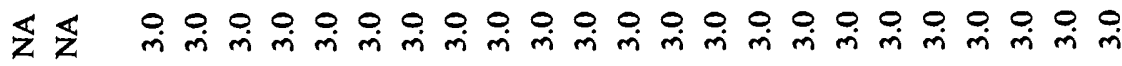

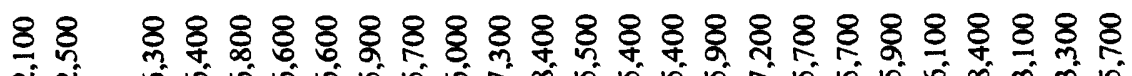

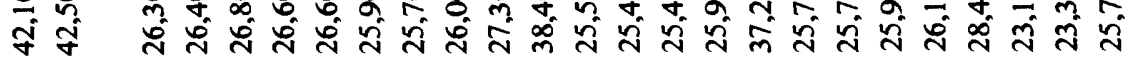

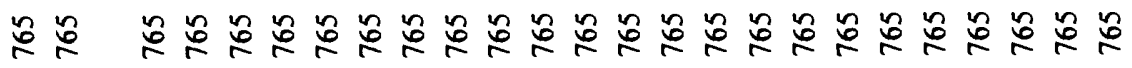

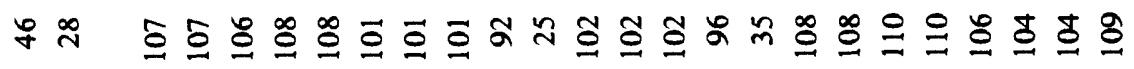

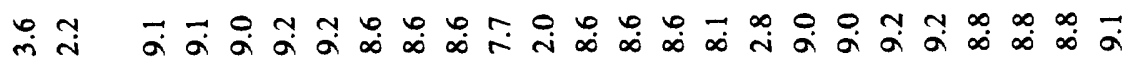

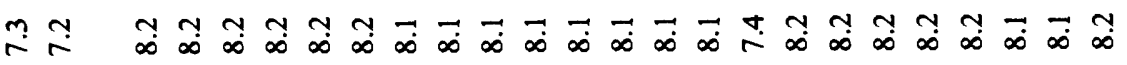

ఏ

ที่

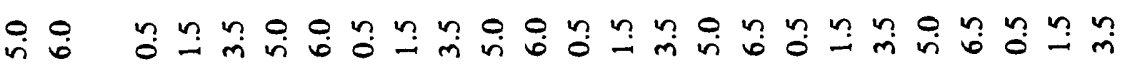

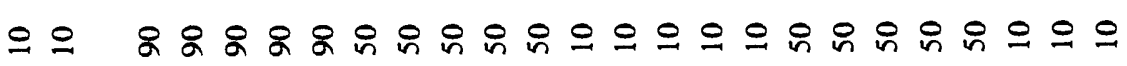

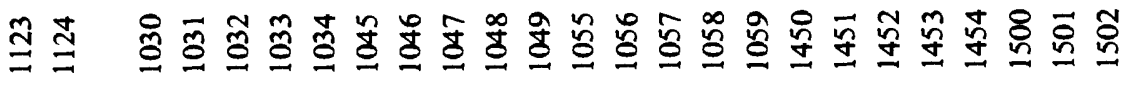

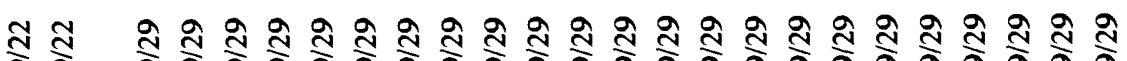

के के के के के के के के के के के के के के के के के

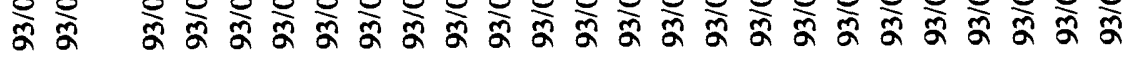




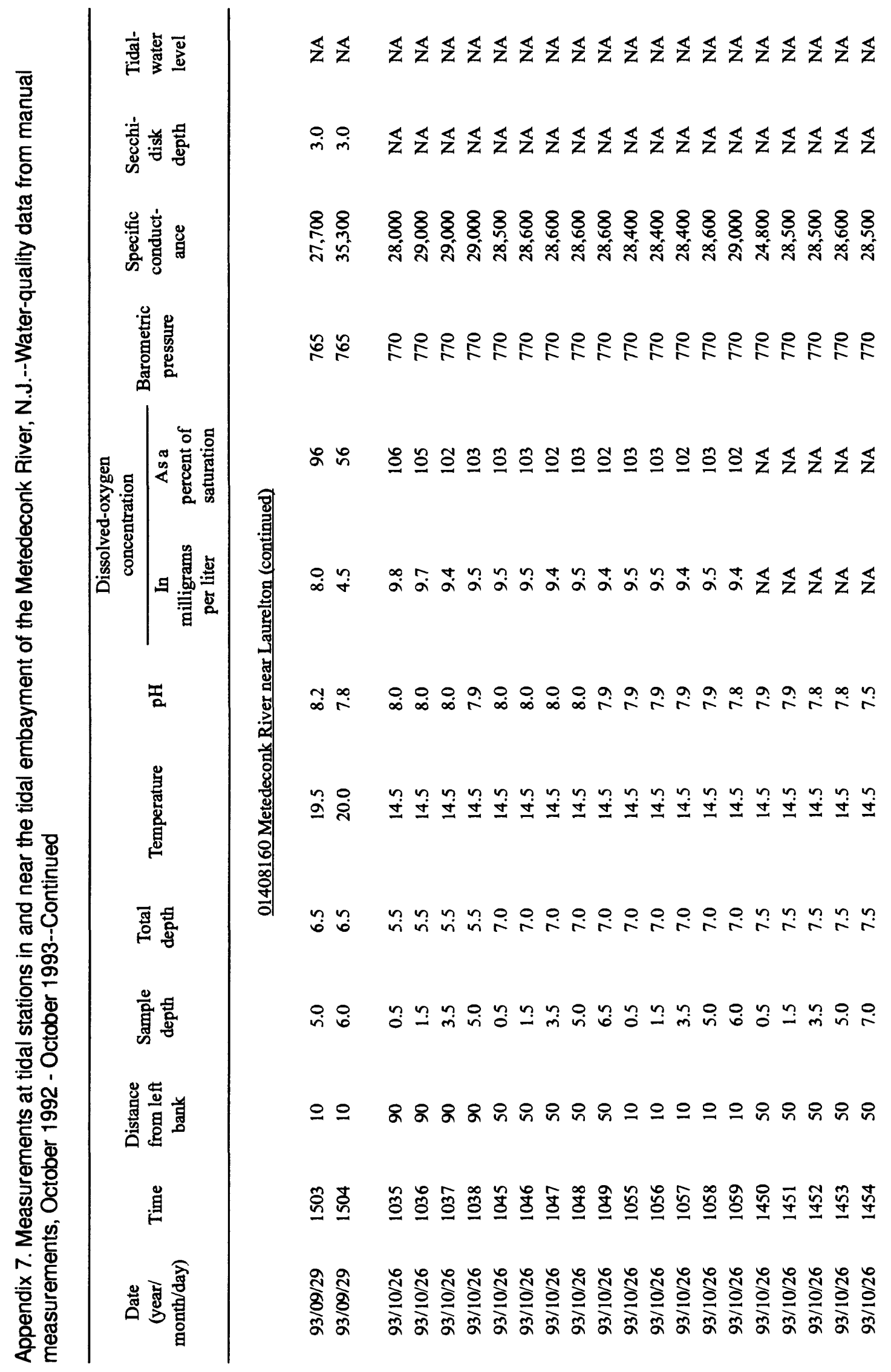




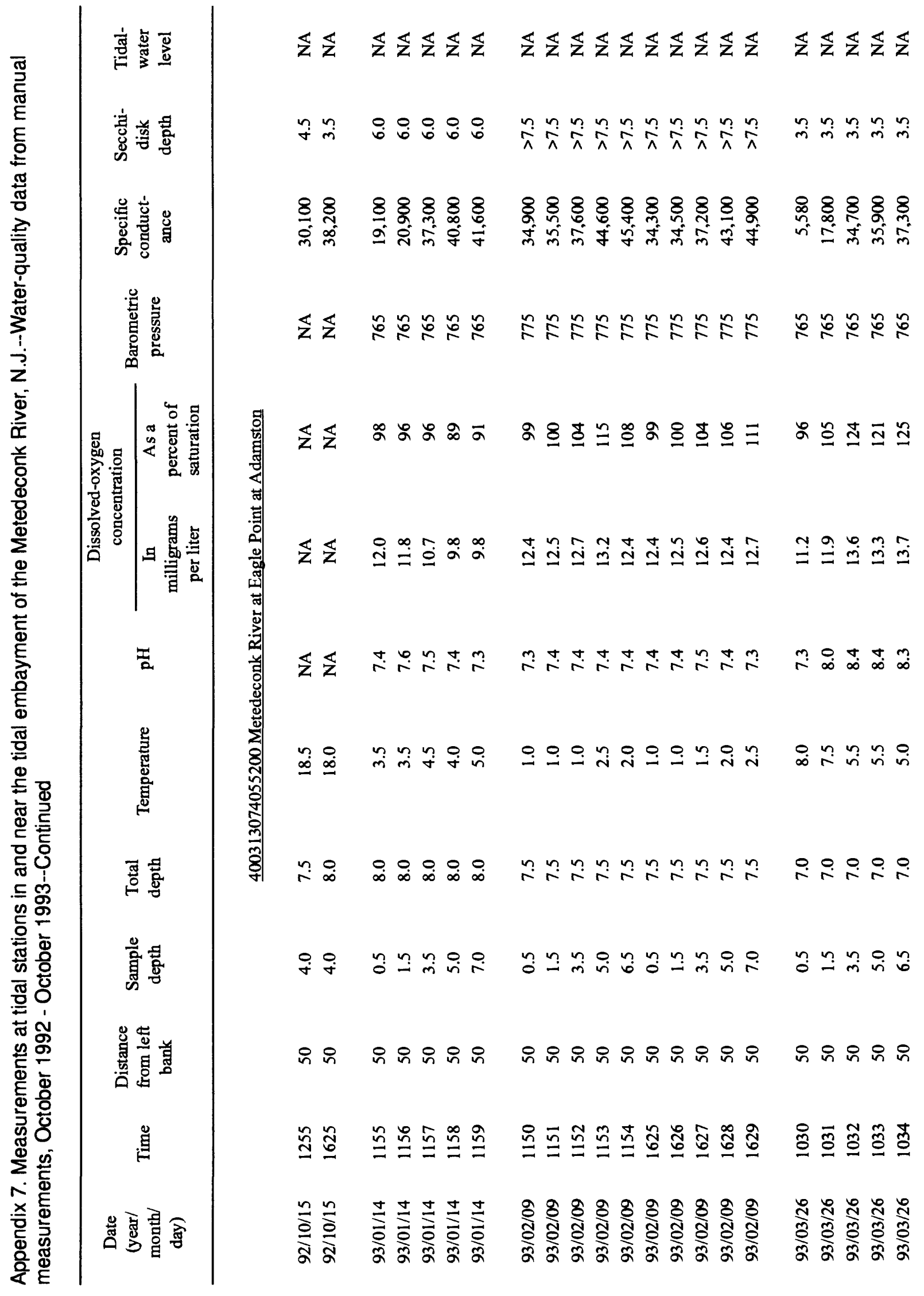




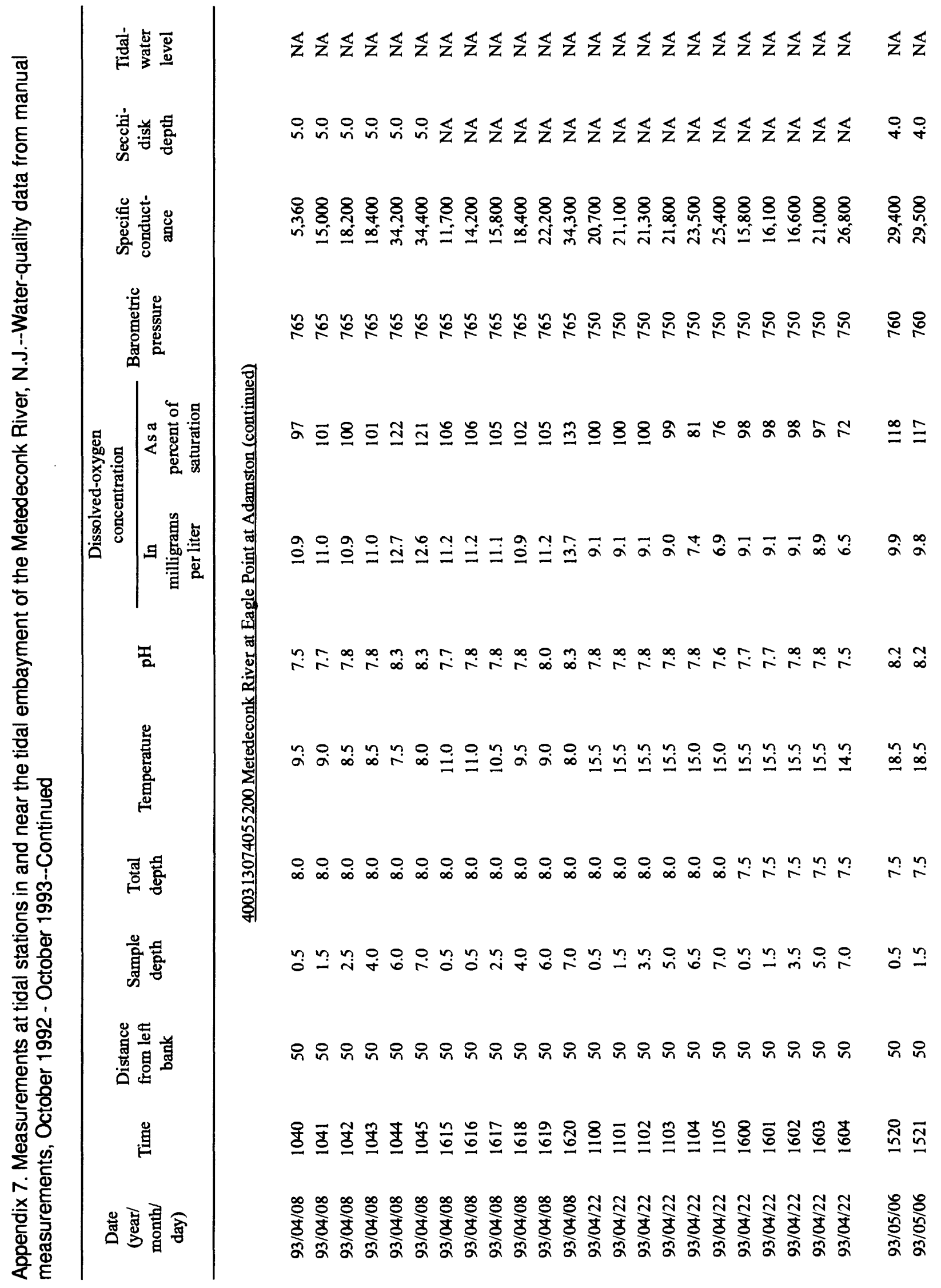




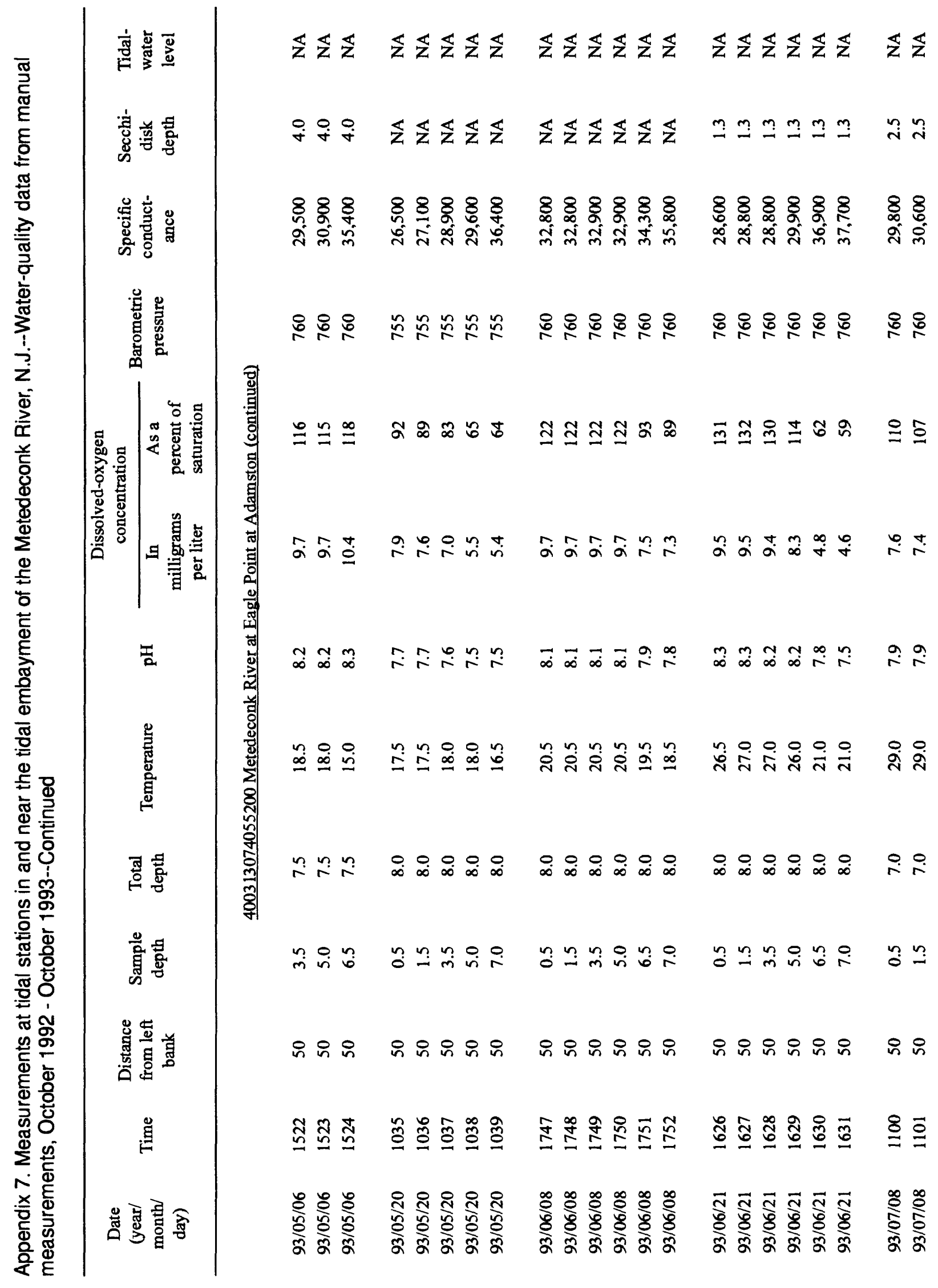




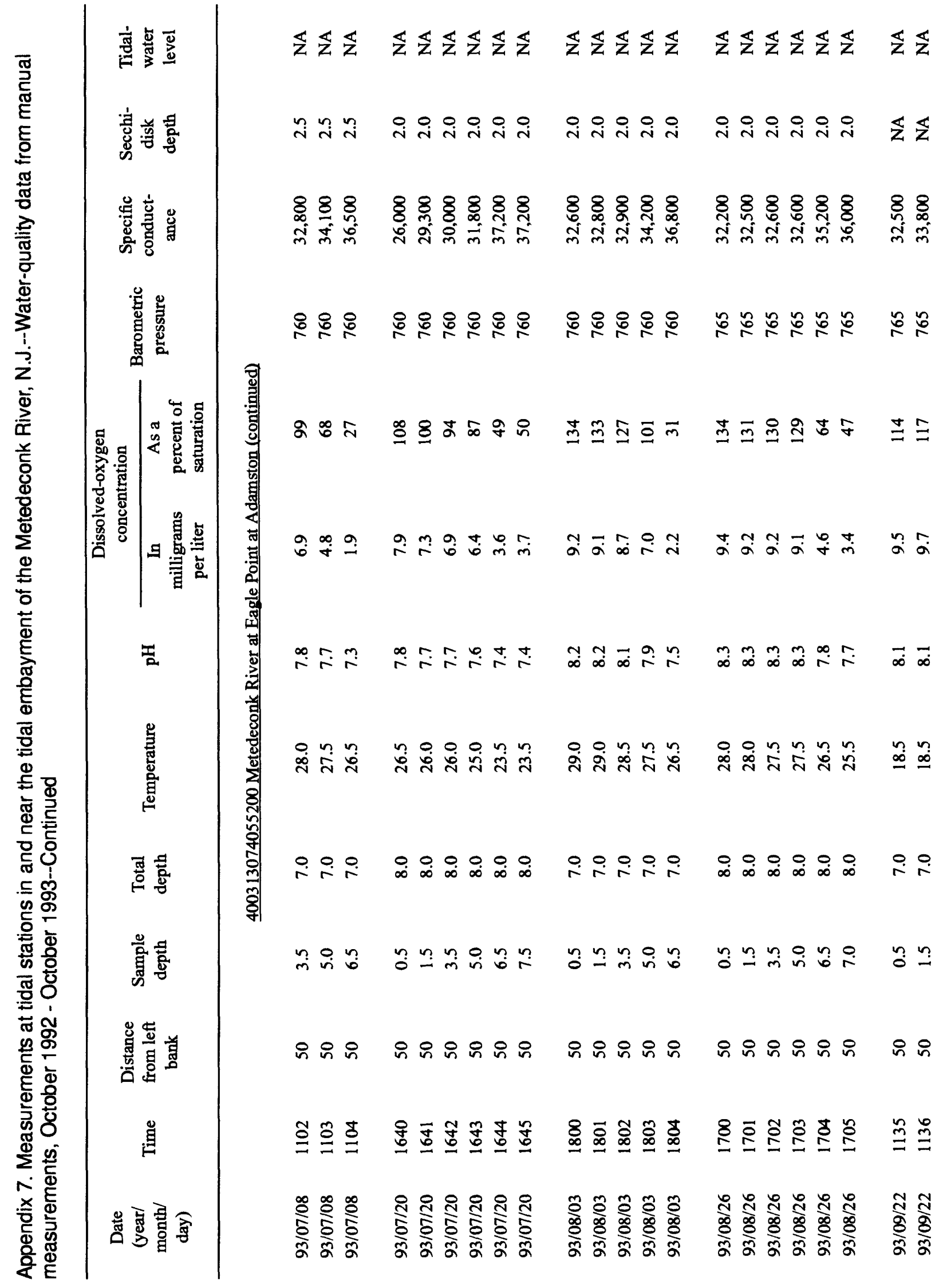




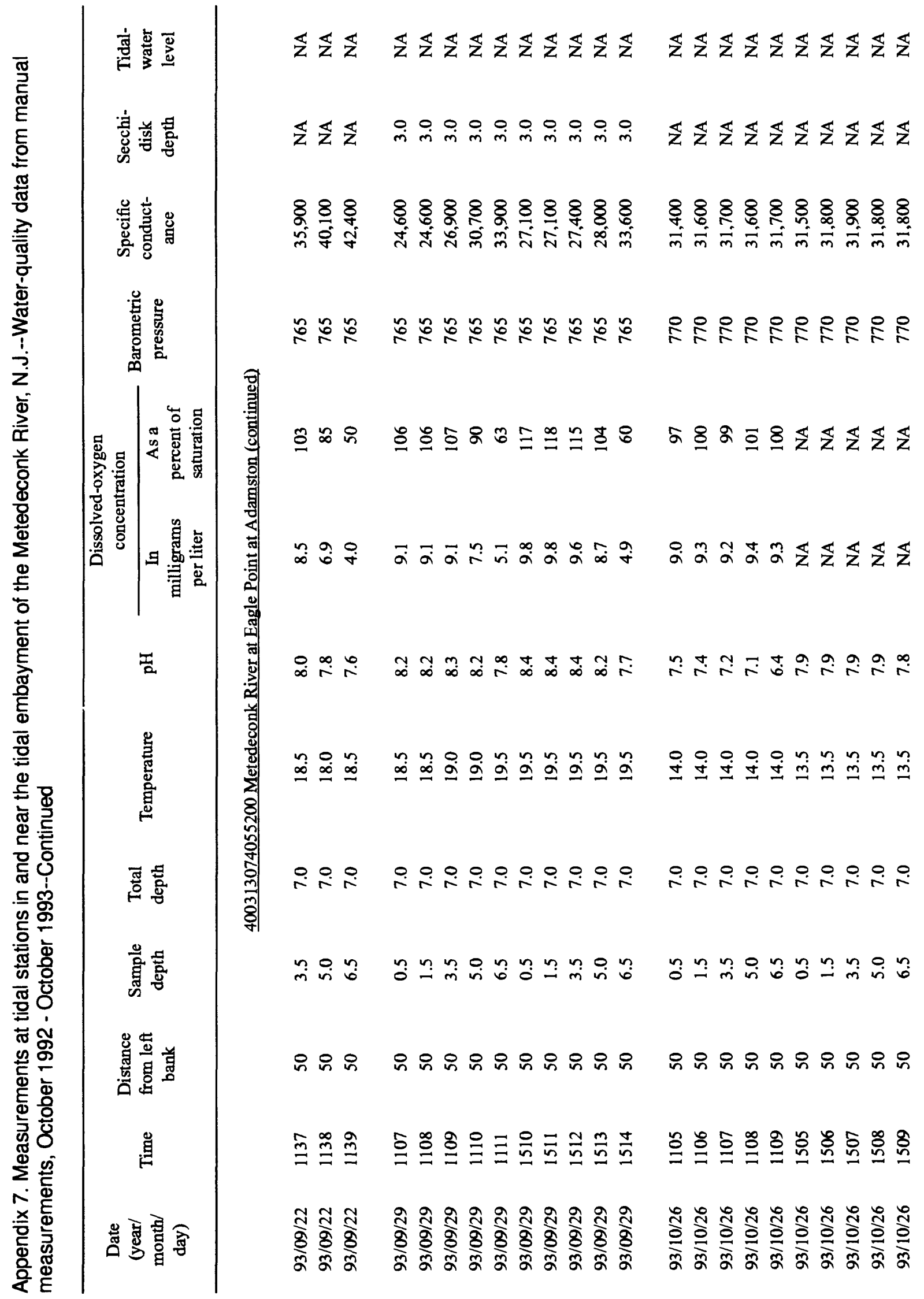




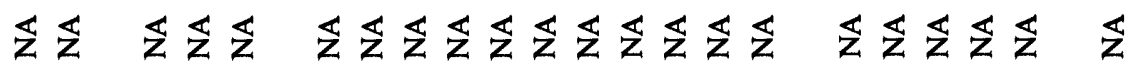

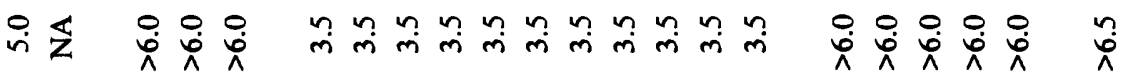

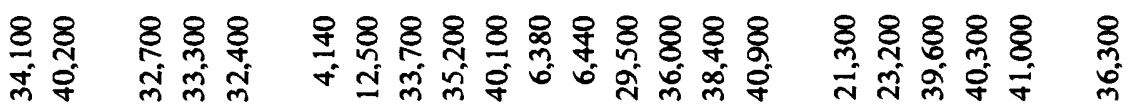

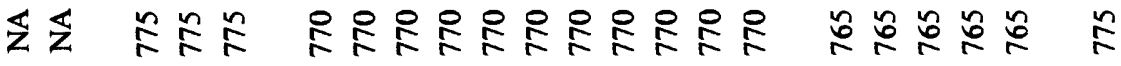

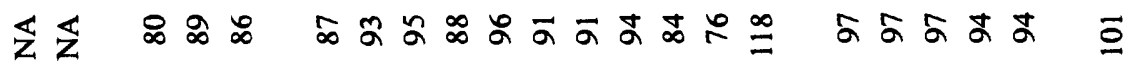

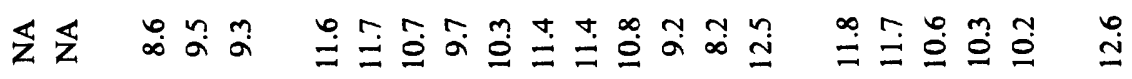

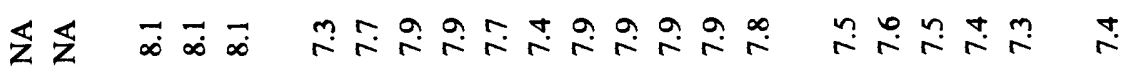

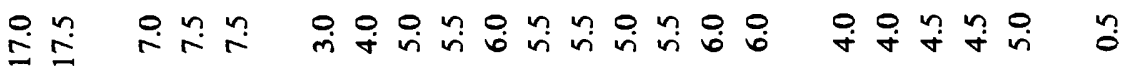

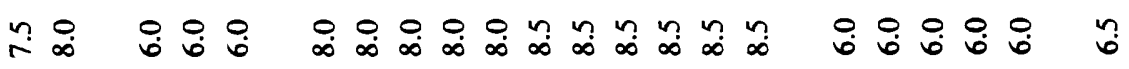

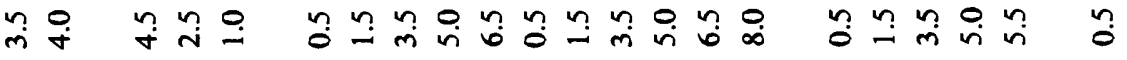

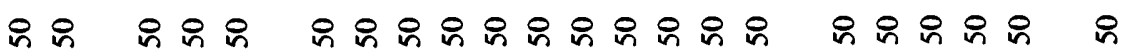

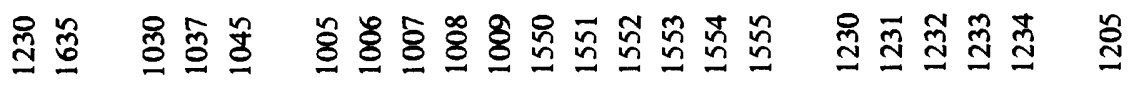

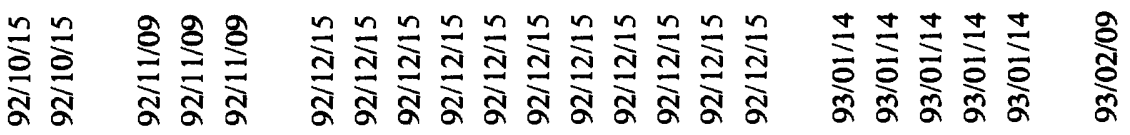




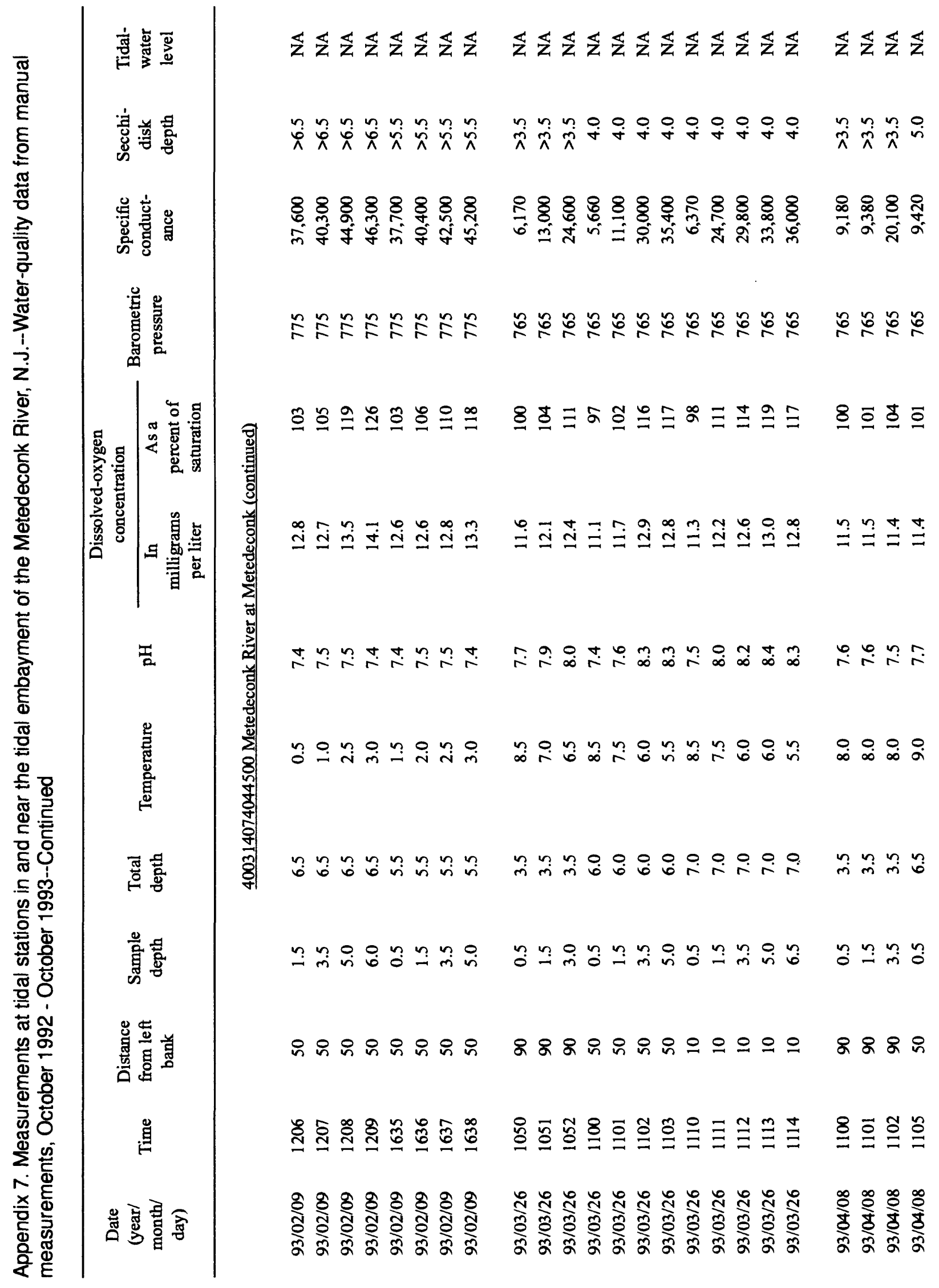




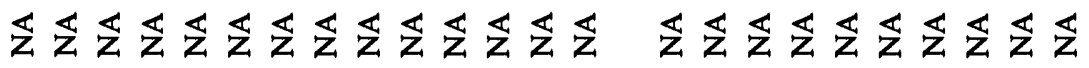

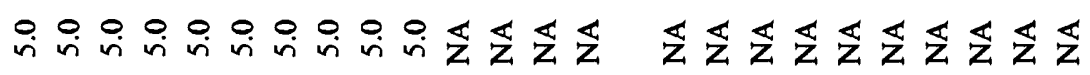

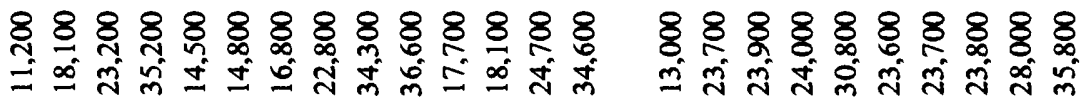

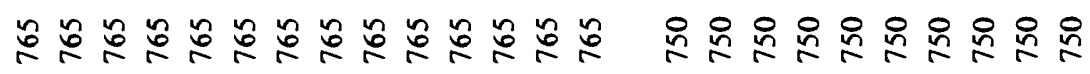

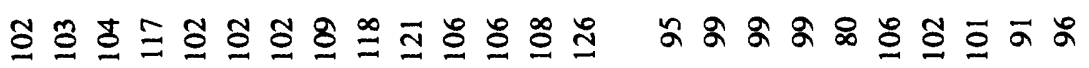

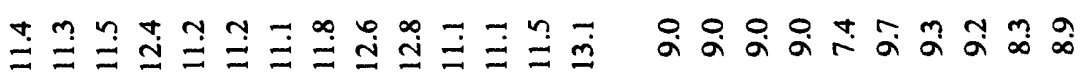

₹웅요

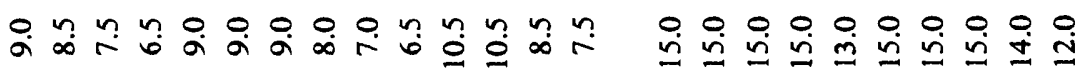

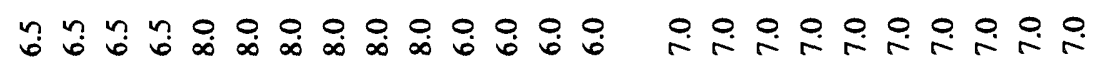

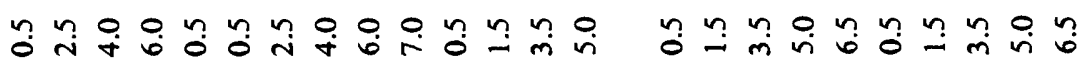

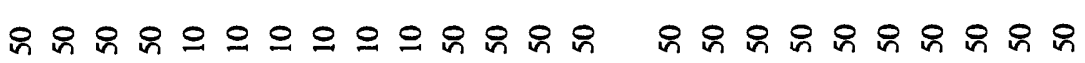

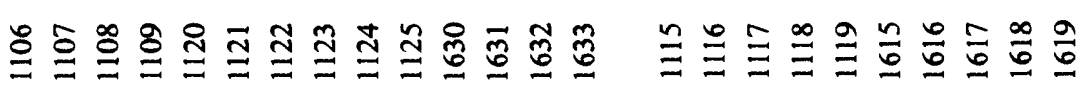

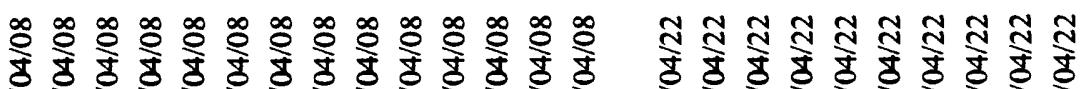

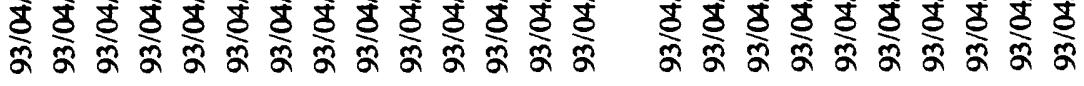




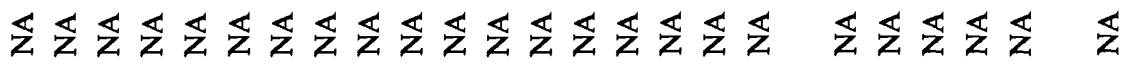

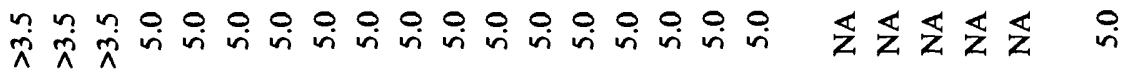

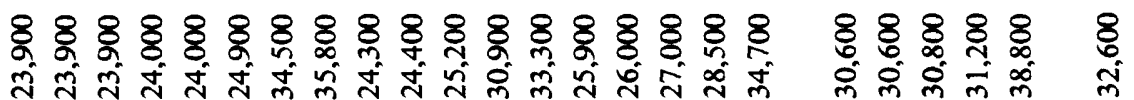

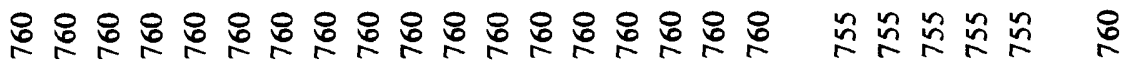

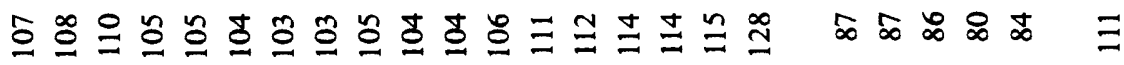

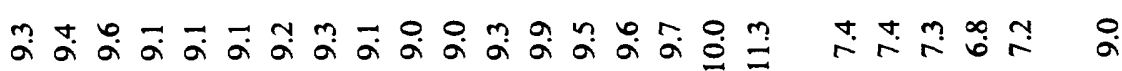

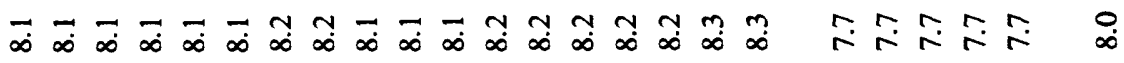

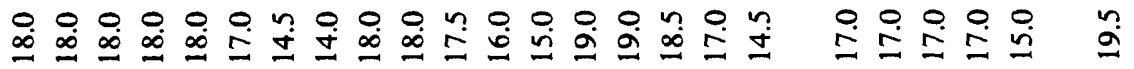

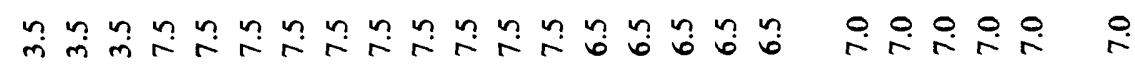

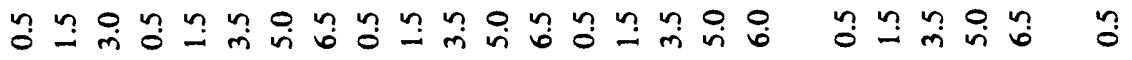

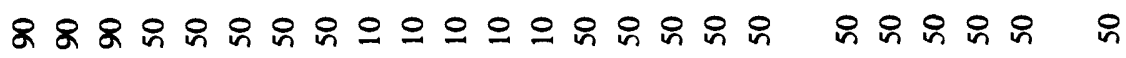

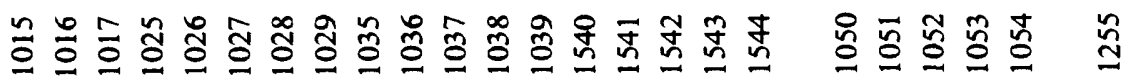

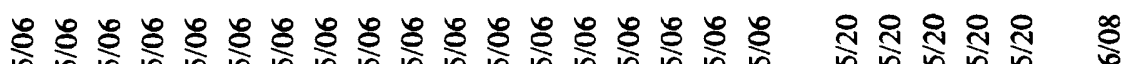

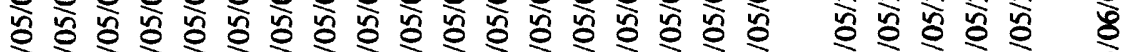

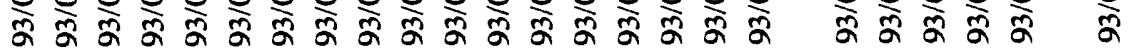




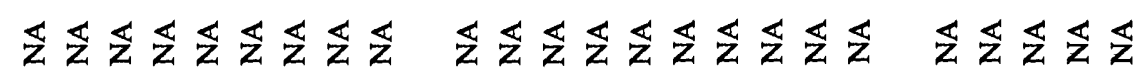

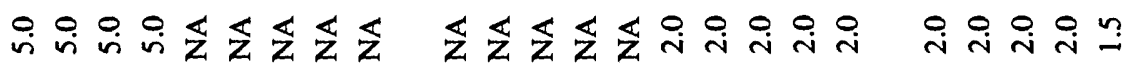

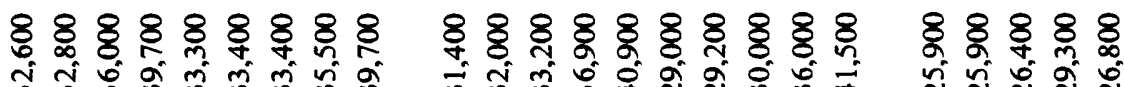

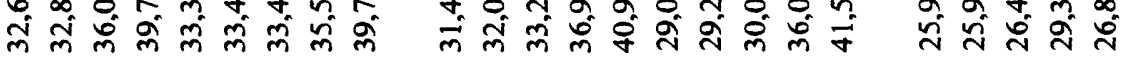

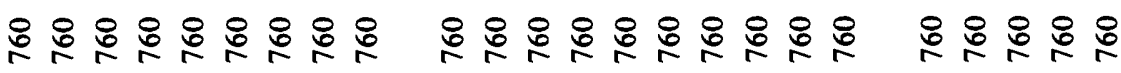

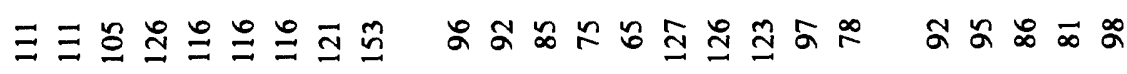

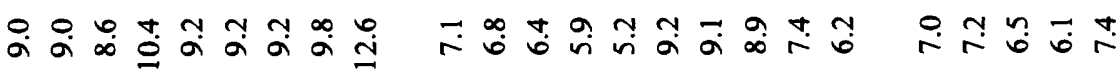

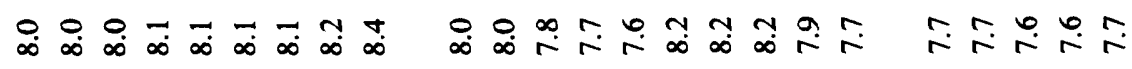

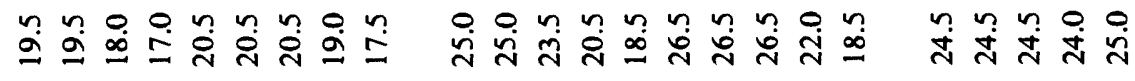

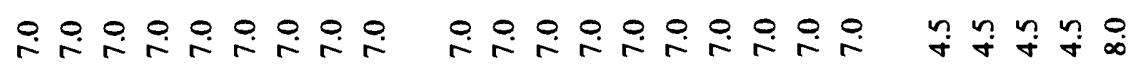

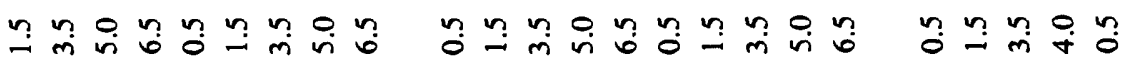

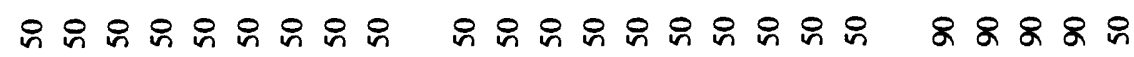

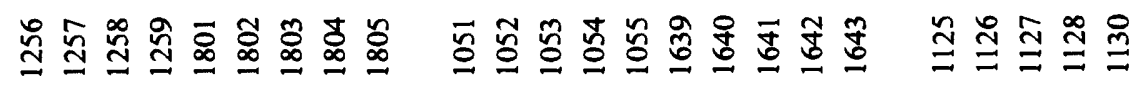

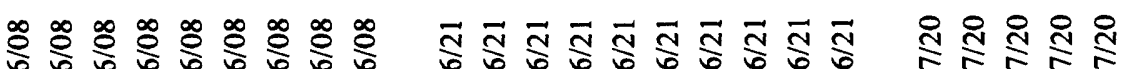

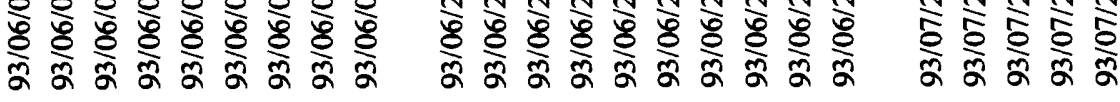




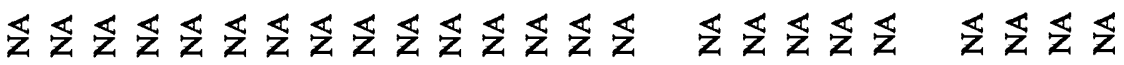

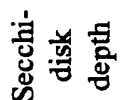

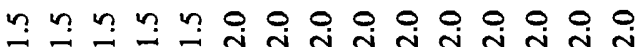

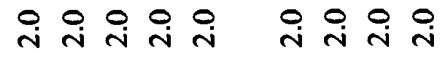

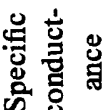

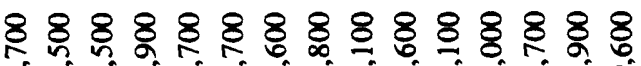

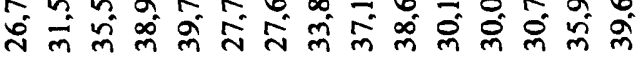

88 \& \& \& 8 \& 8

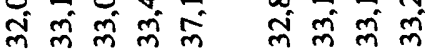

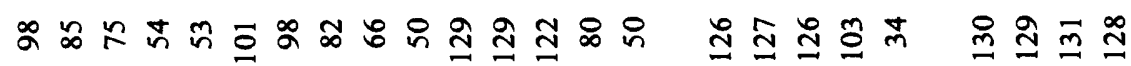

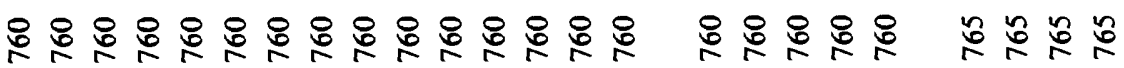

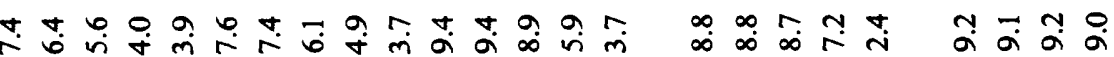

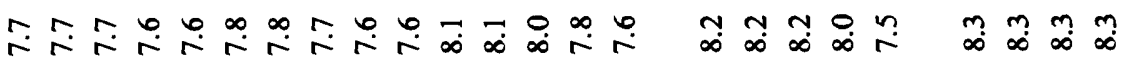

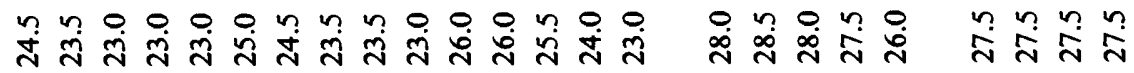

엉 엉 0 엉

育章

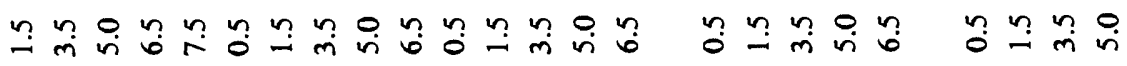

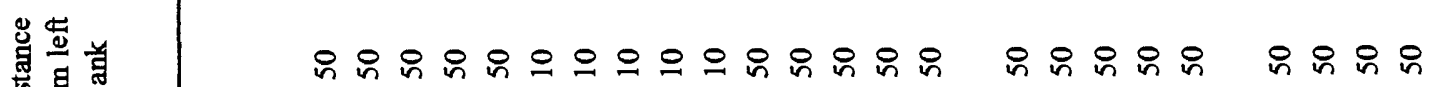

要莡

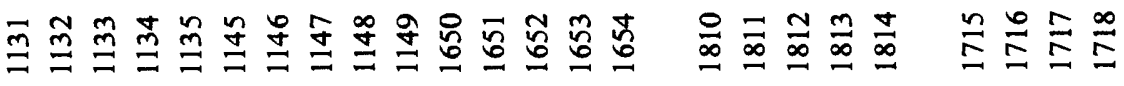

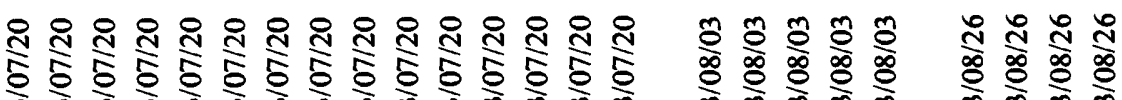

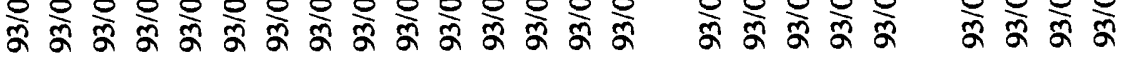




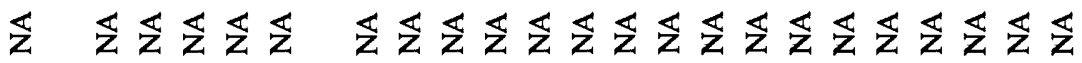

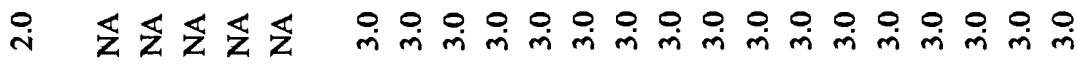

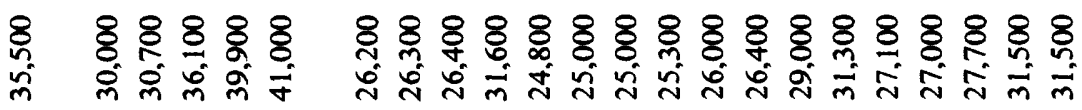

そ

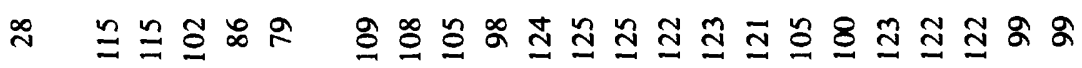

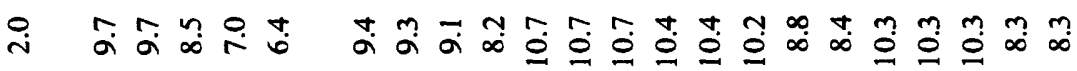

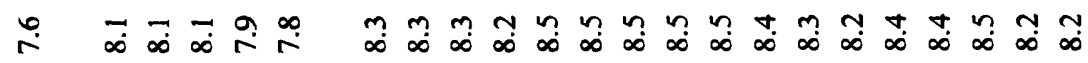

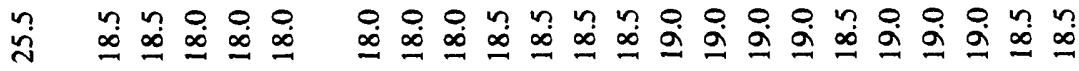

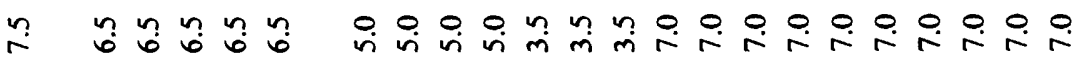

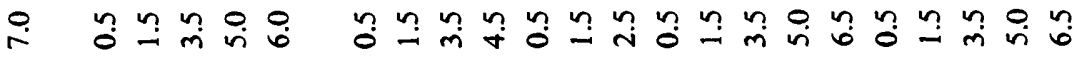

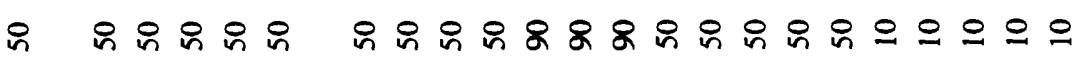

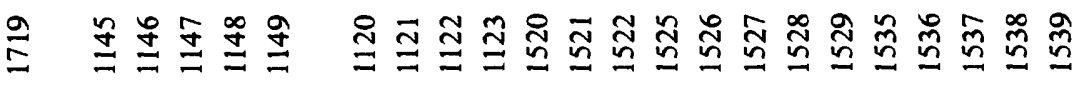

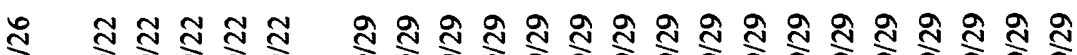

क्षे के के के के के के के के के के के के के के के

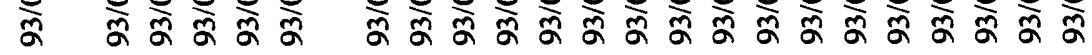




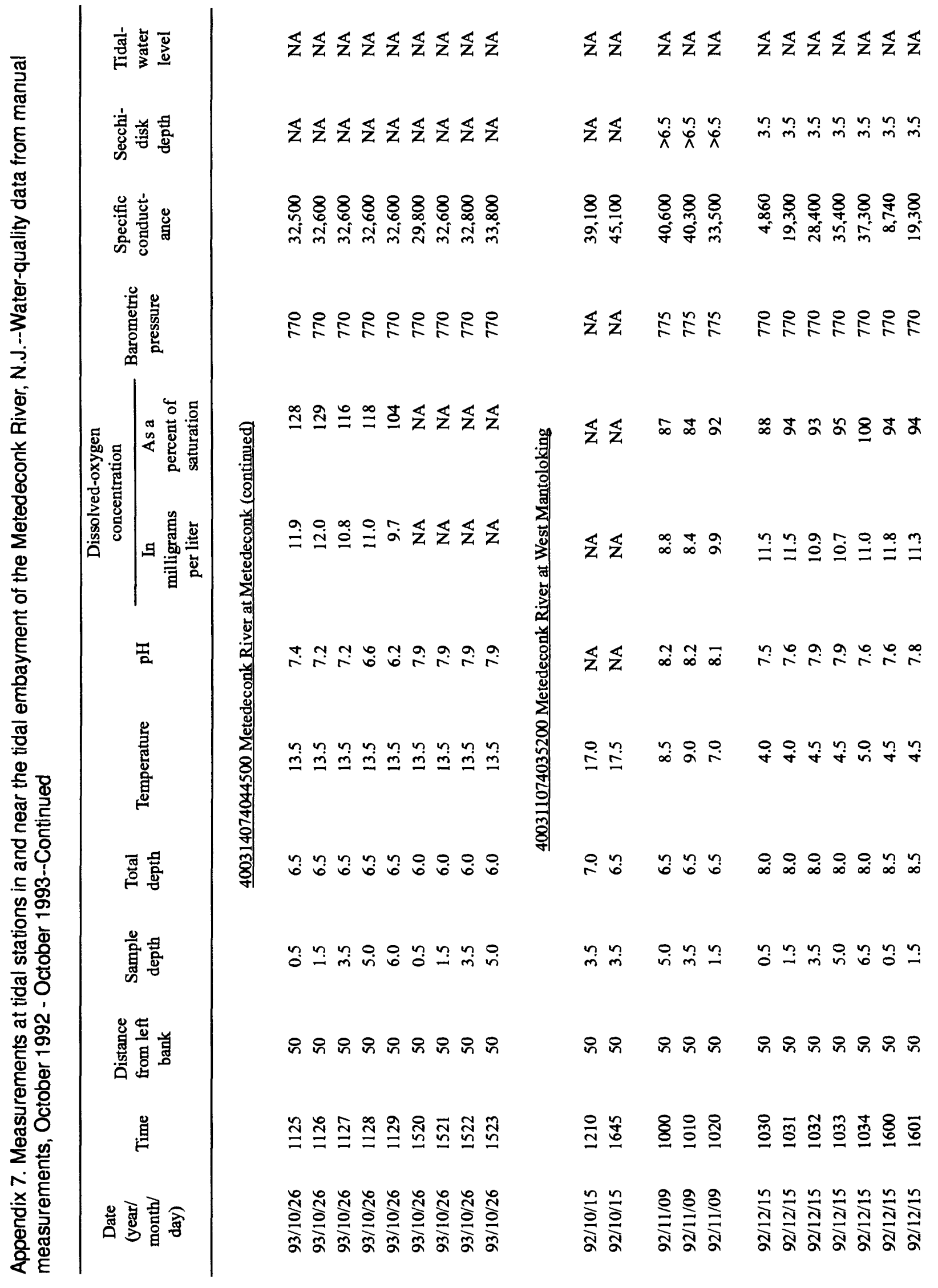




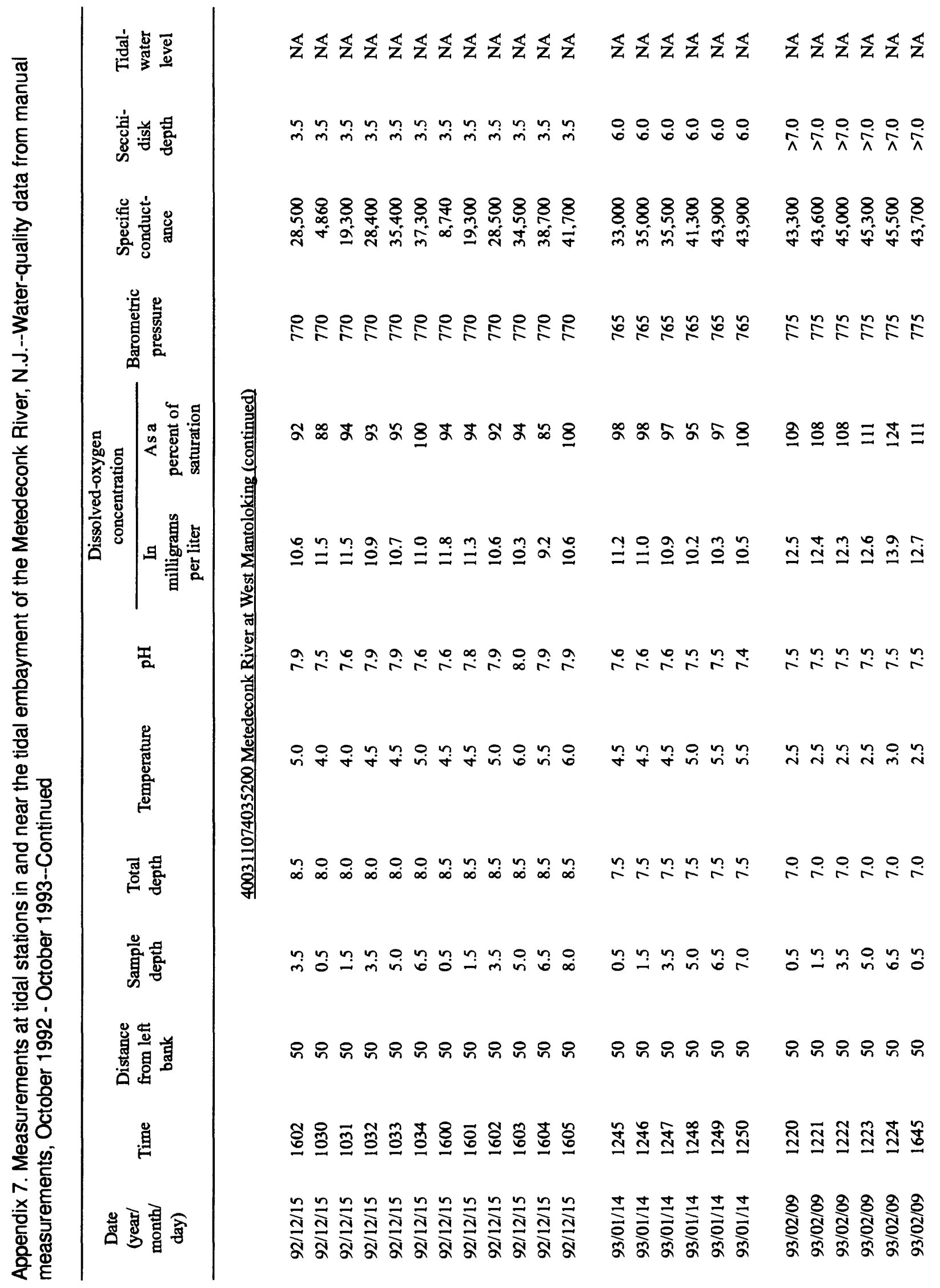




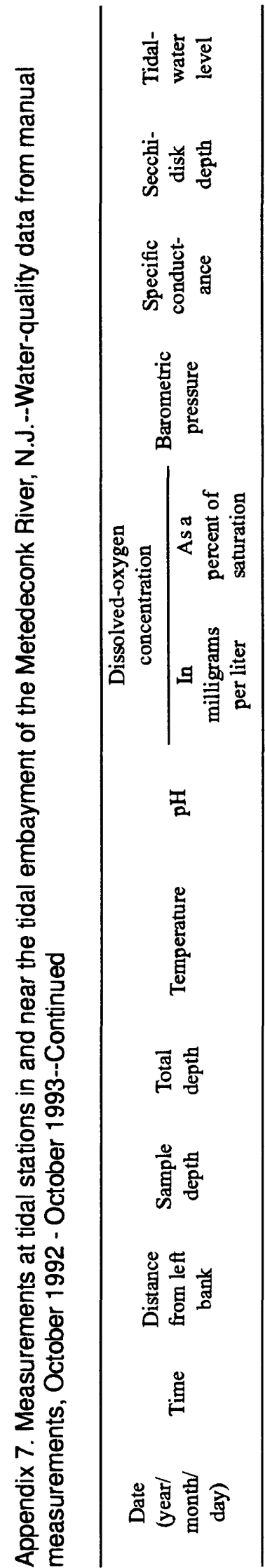

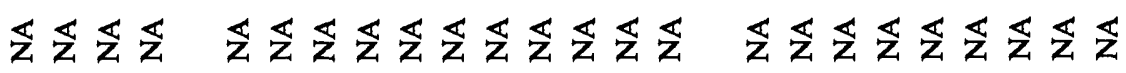

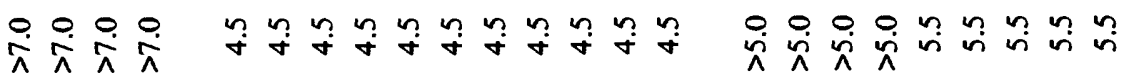

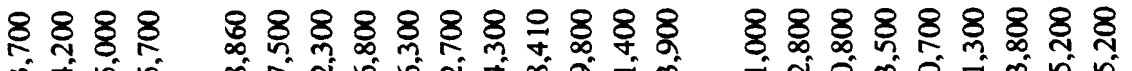

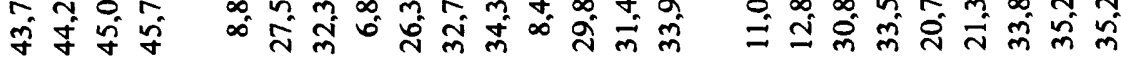

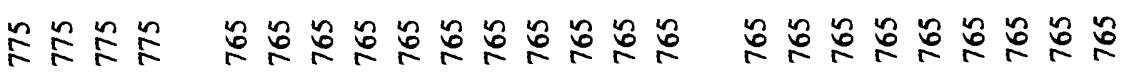

氙

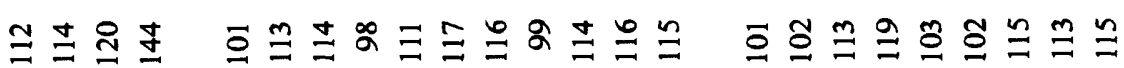

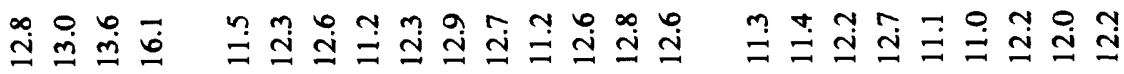

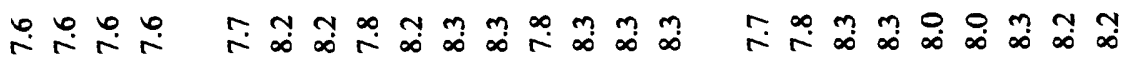

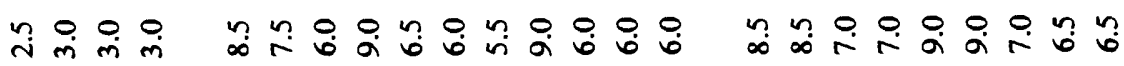

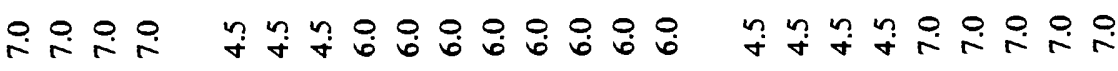

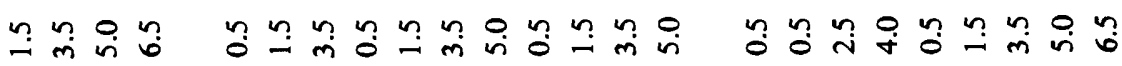

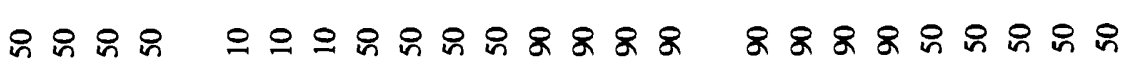

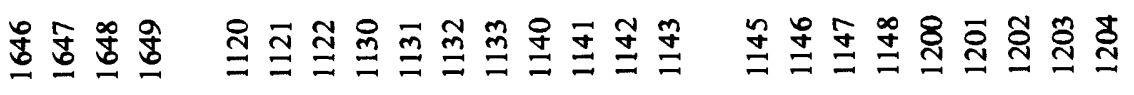

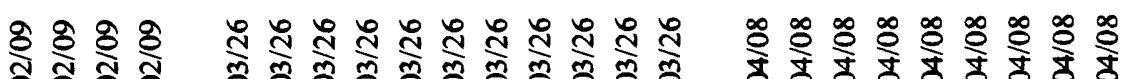

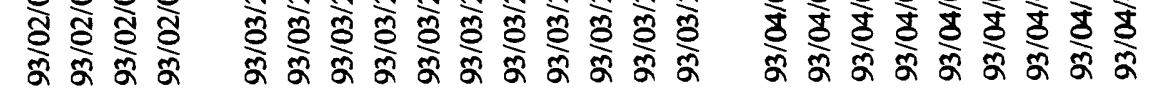




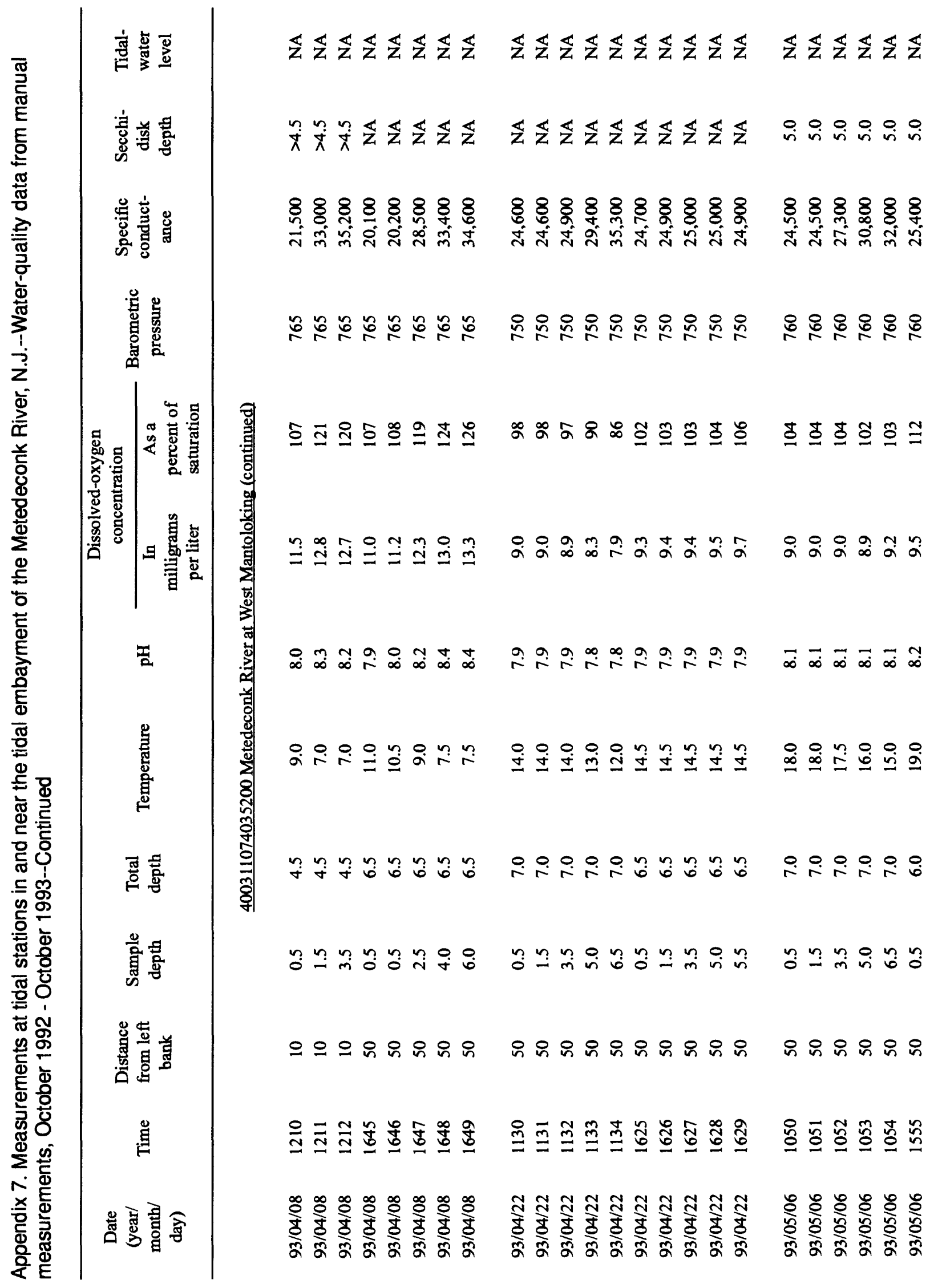




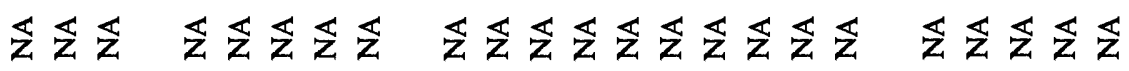

密罒产

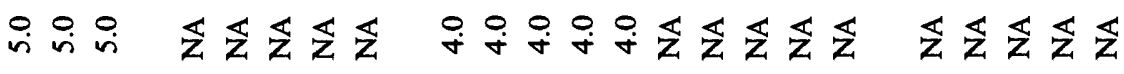

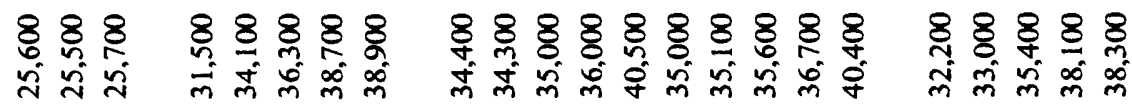

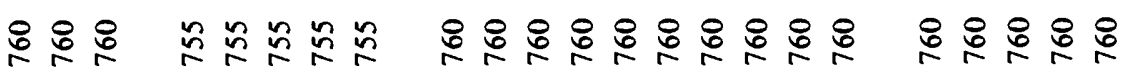

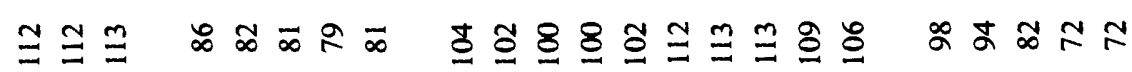

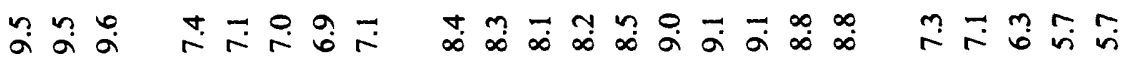

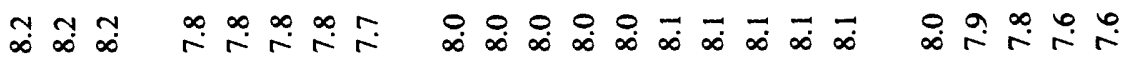

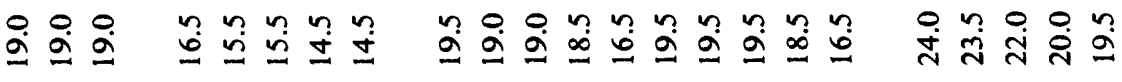

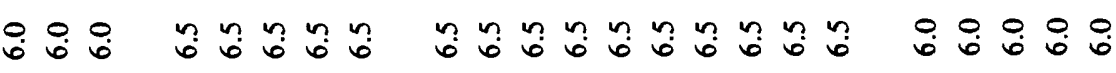

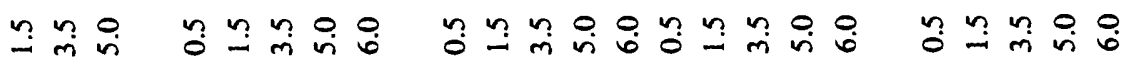

क⿻ 丷⿹

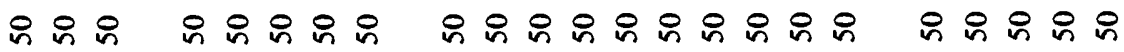

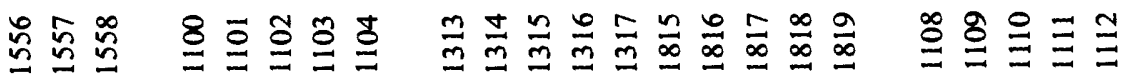

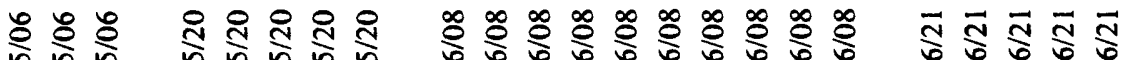
ऊัे

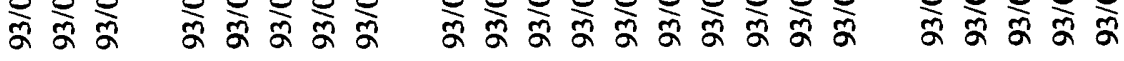




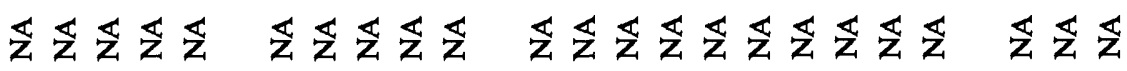

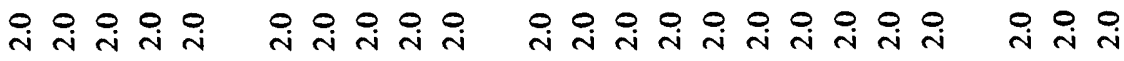

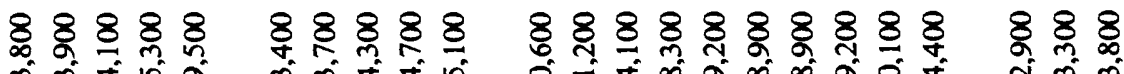

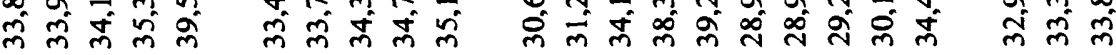

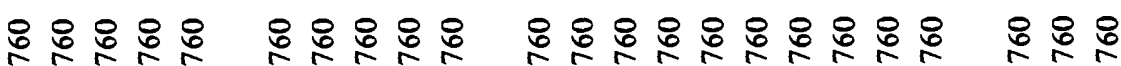

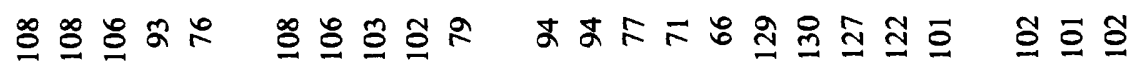

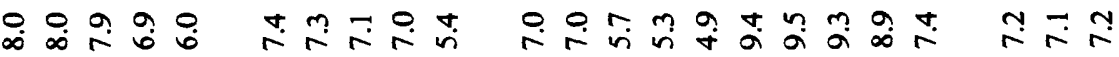

웅 어

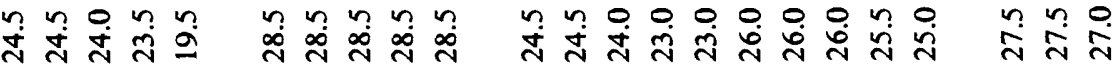

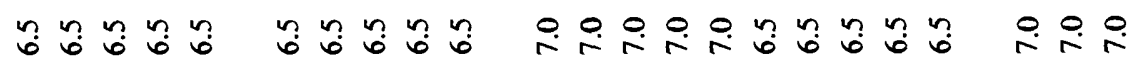

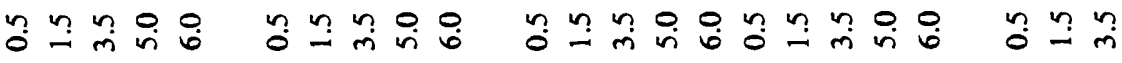

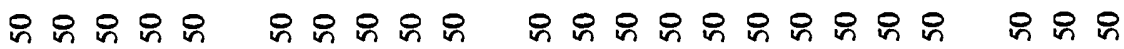

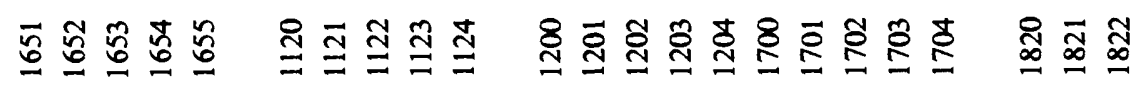

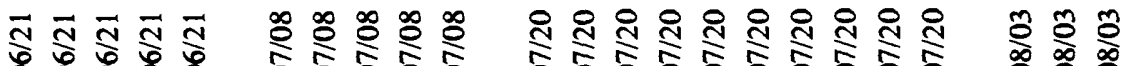

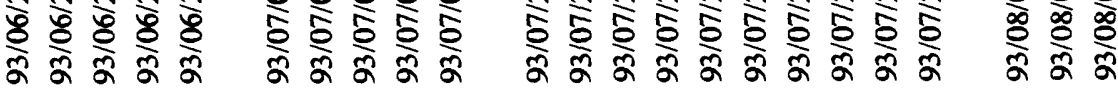




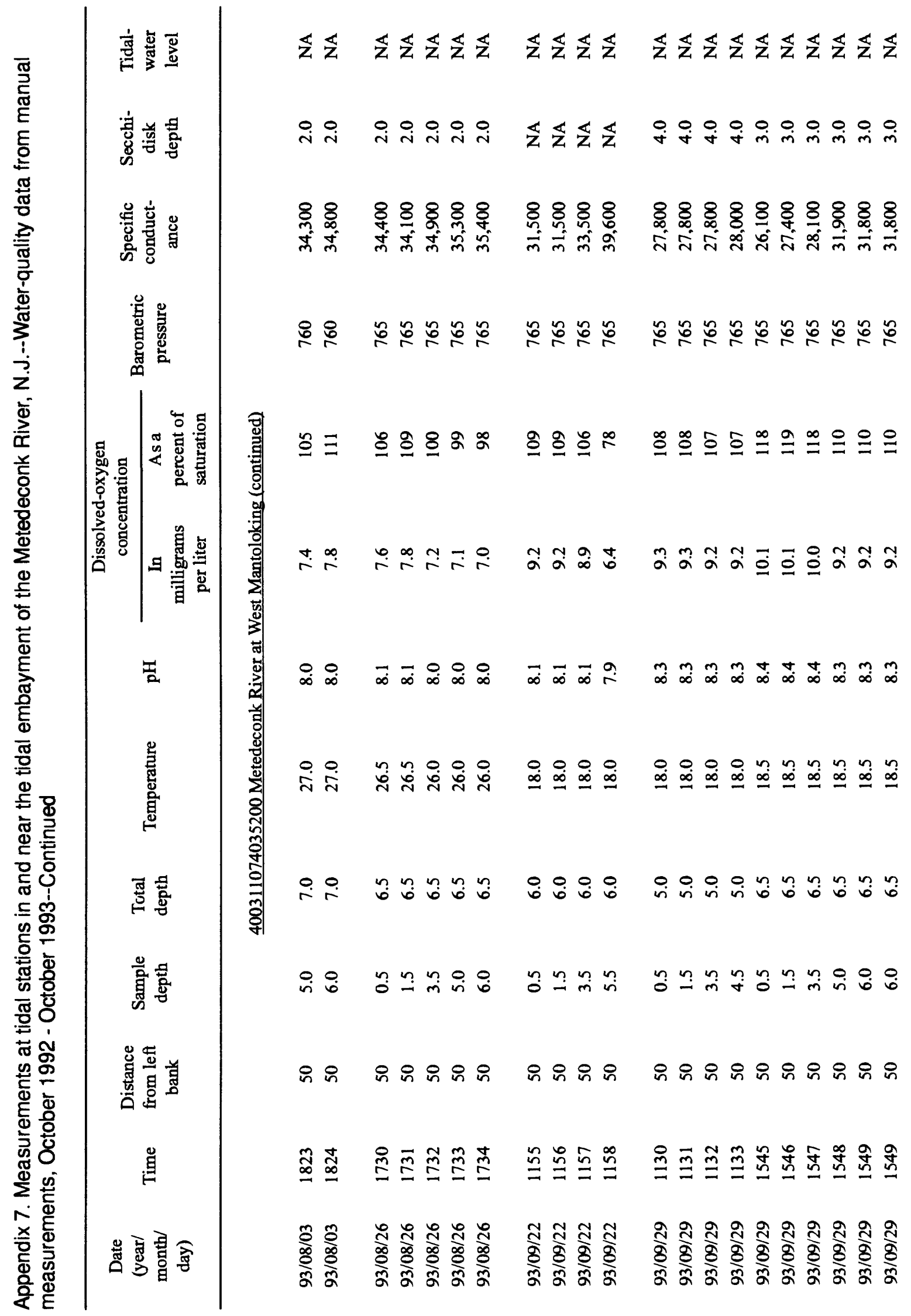




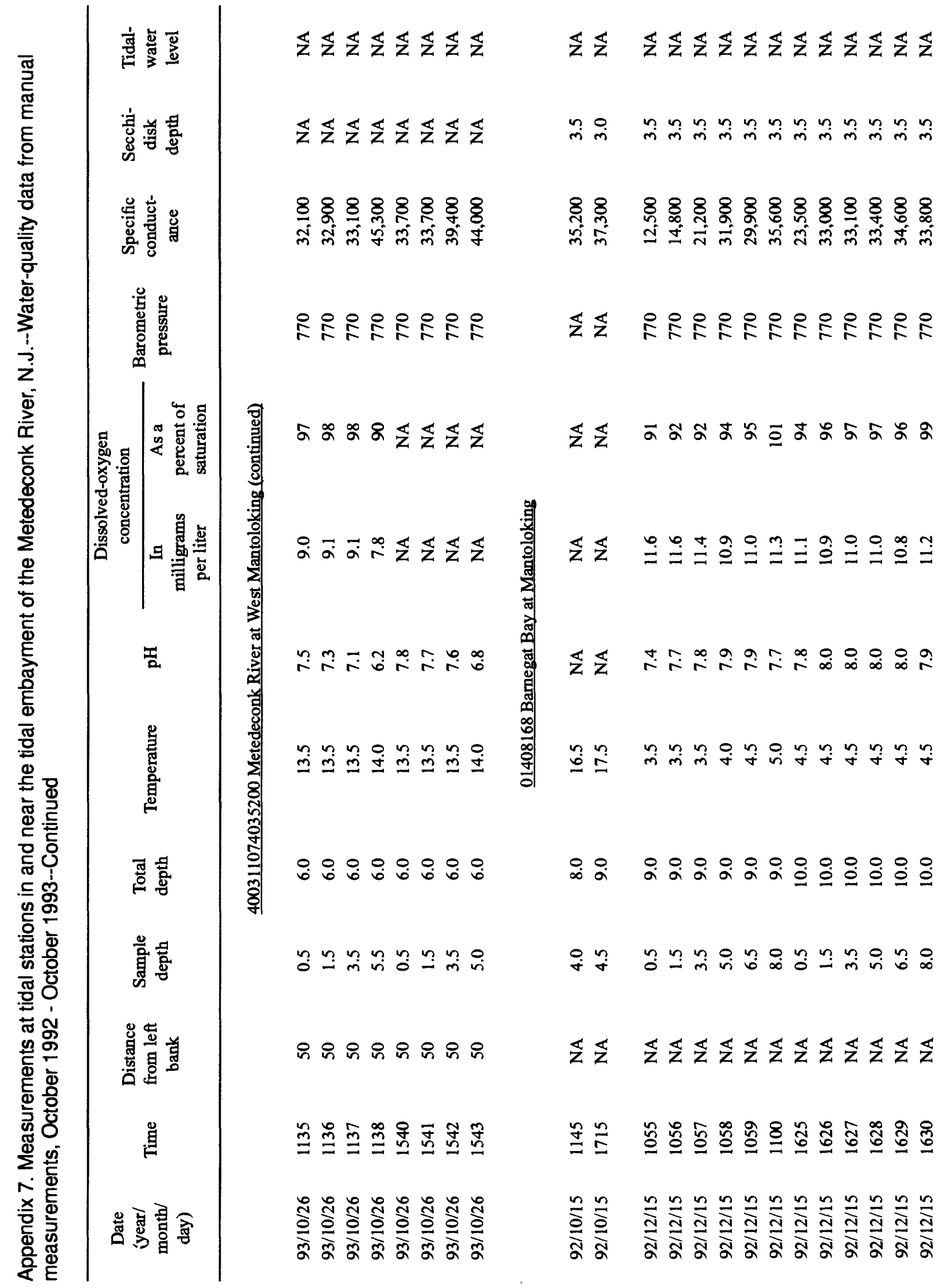




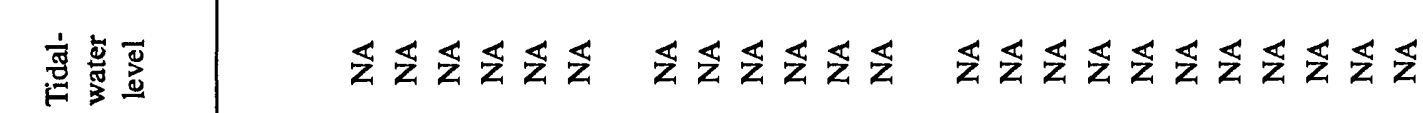

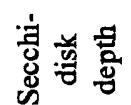

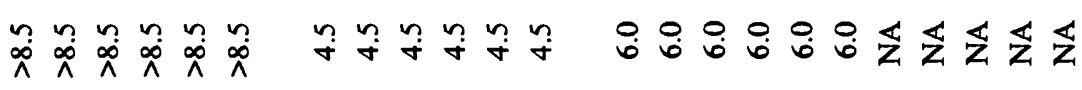

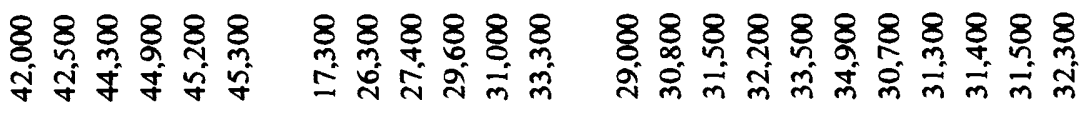

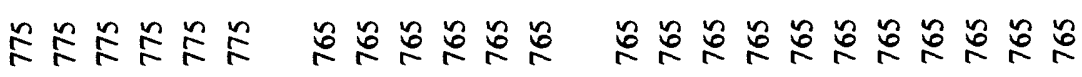

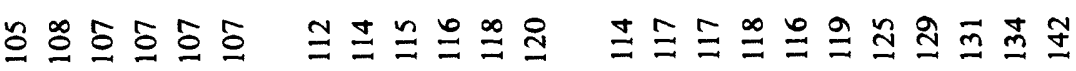

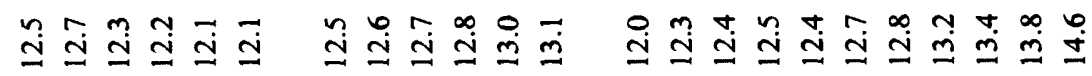

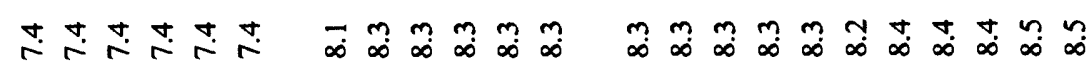

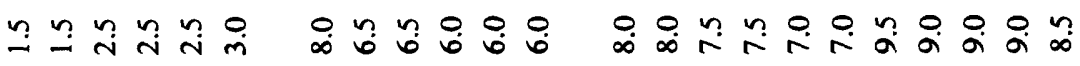

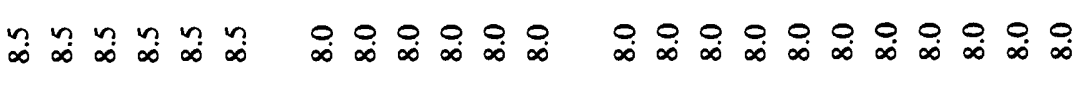

要

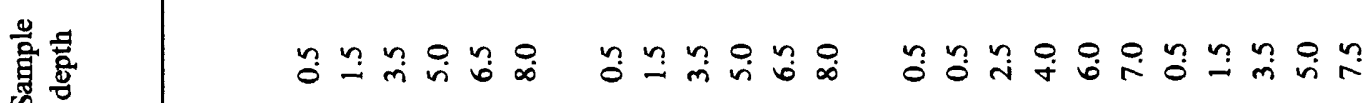

증

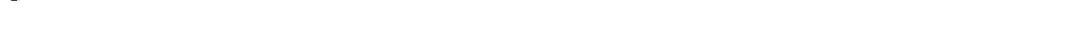

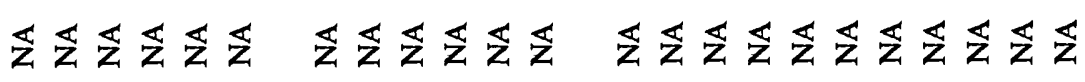

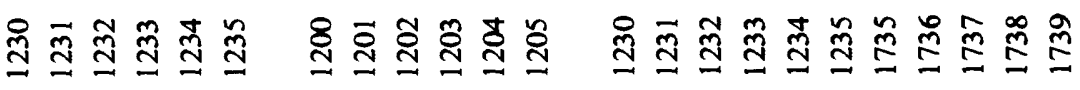

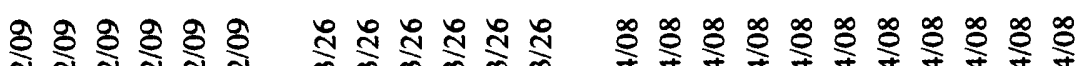

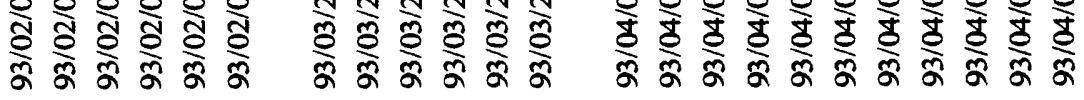




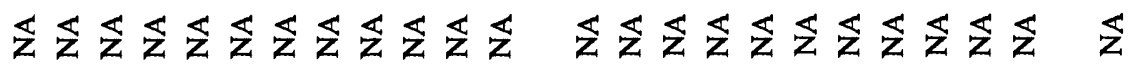

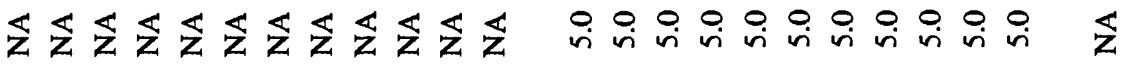

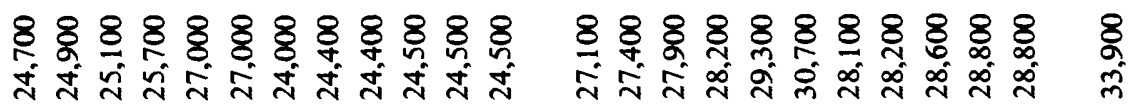

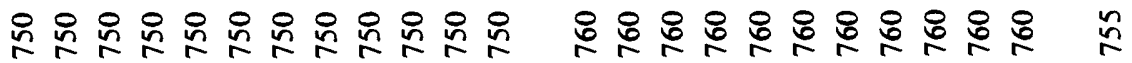

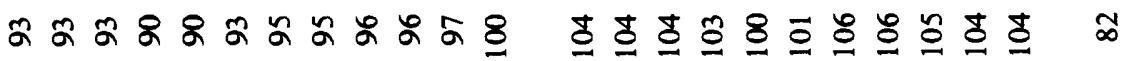

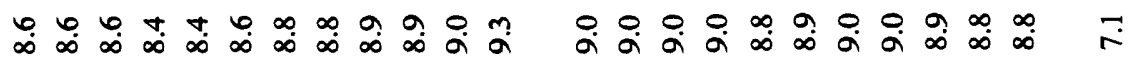

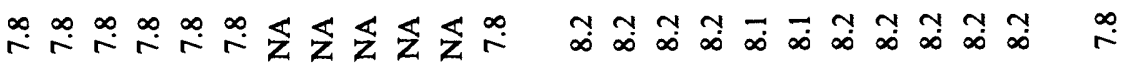

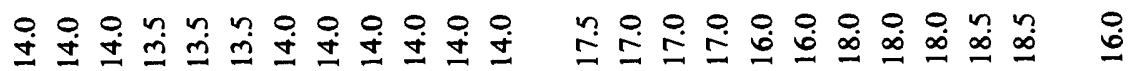

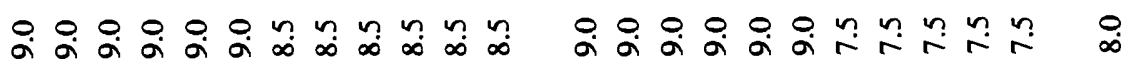

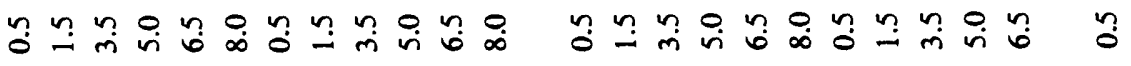

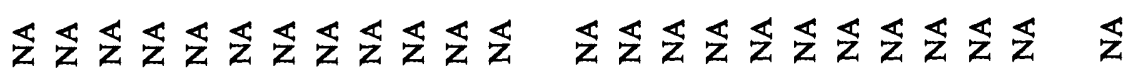

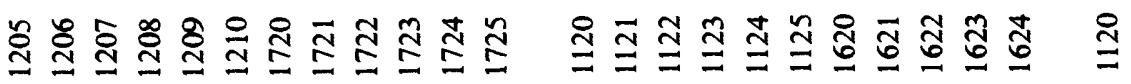

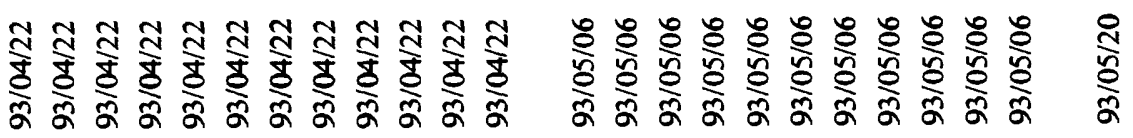




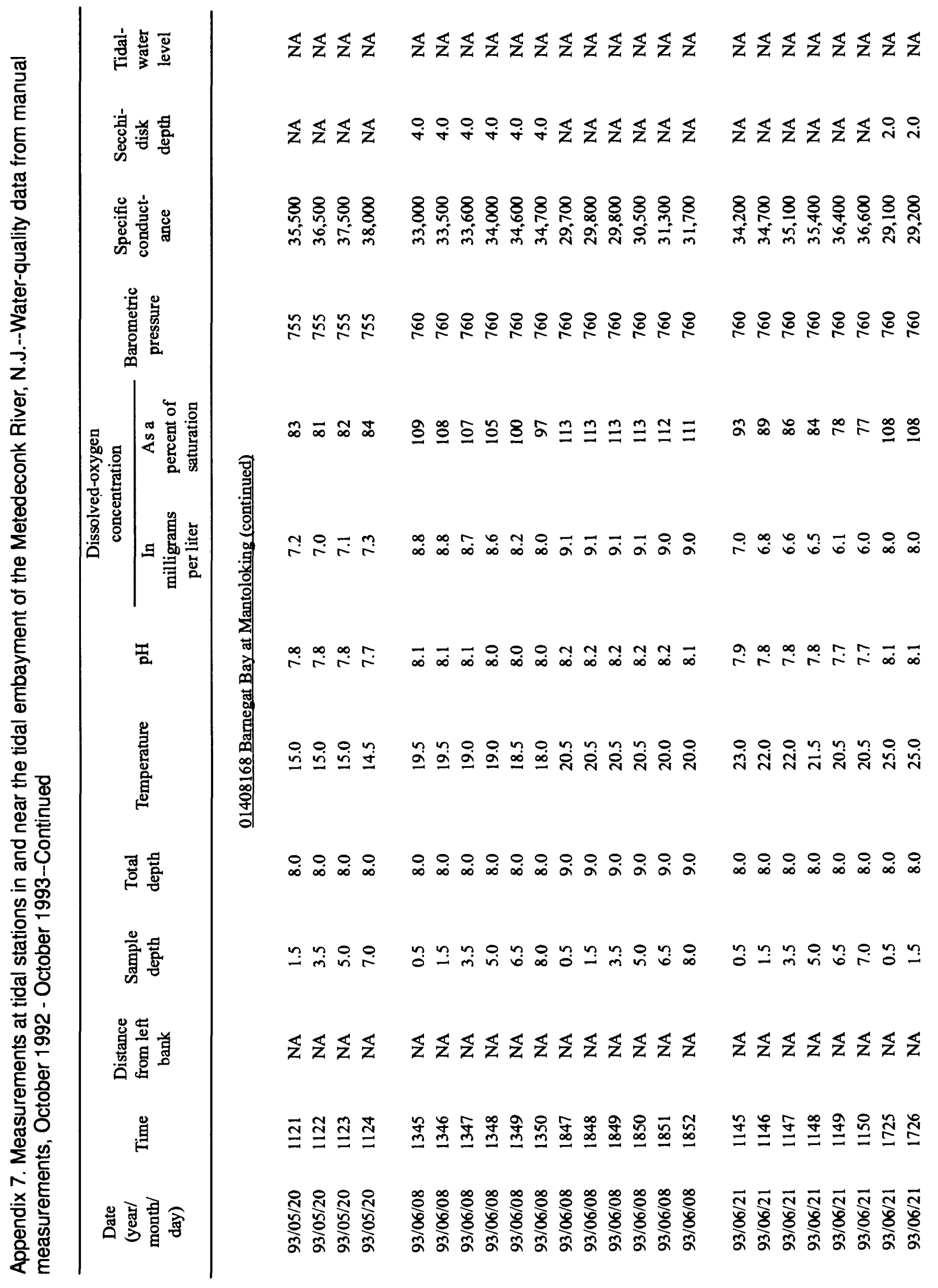




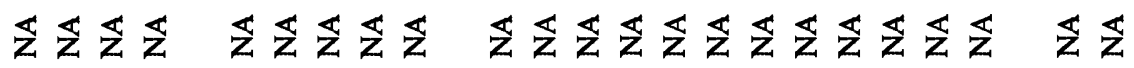

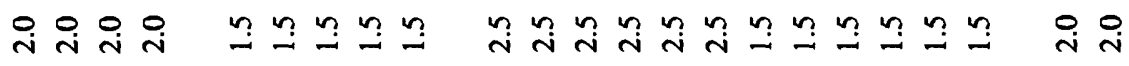

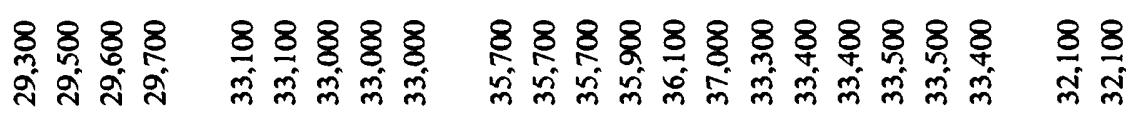

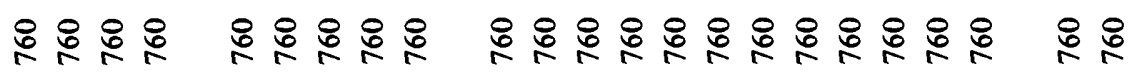

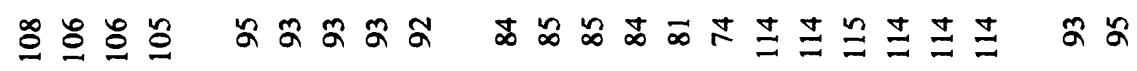

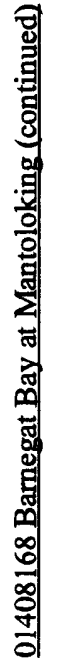

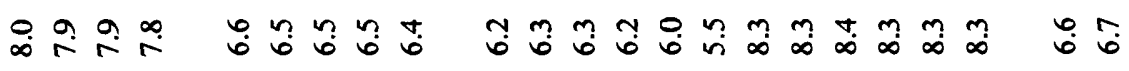

展

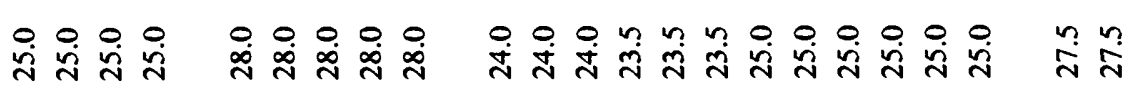

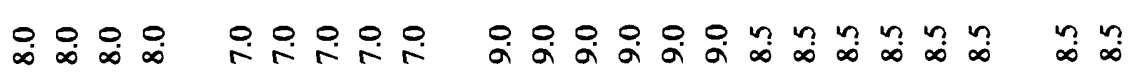

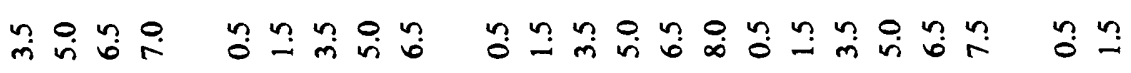

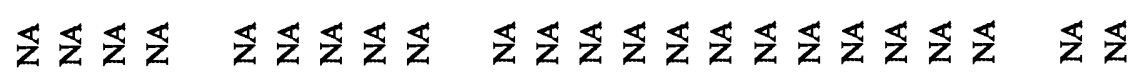

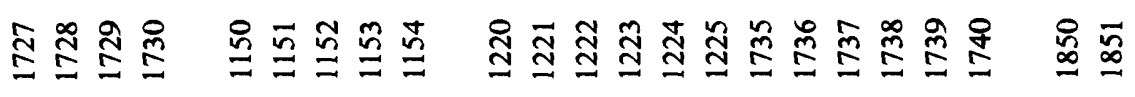

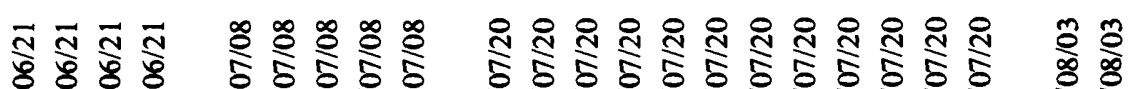

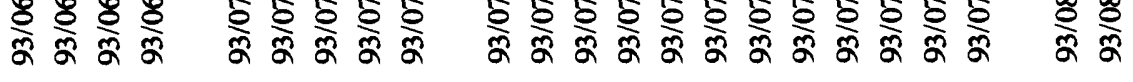




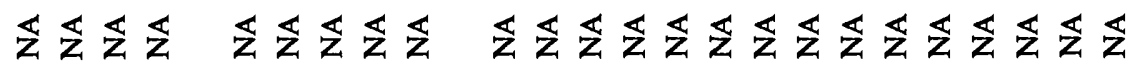

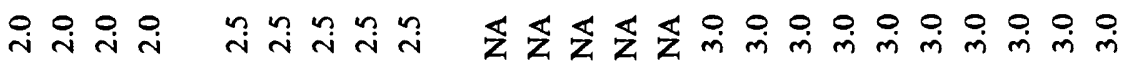

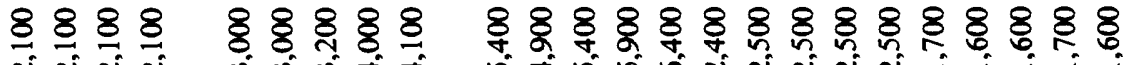

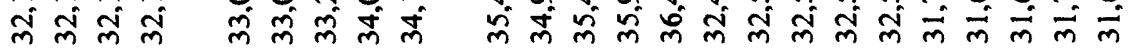

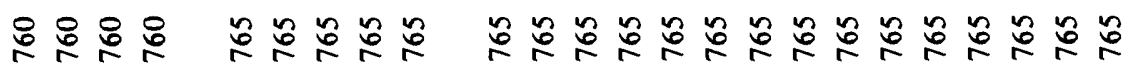

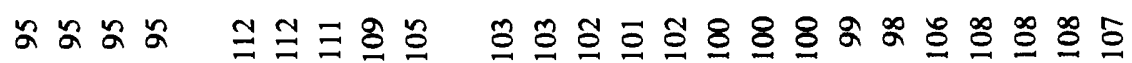

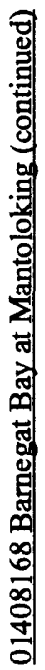

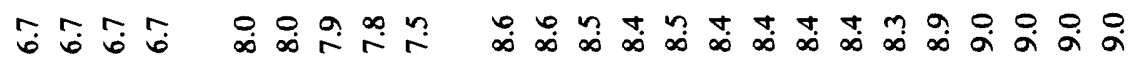

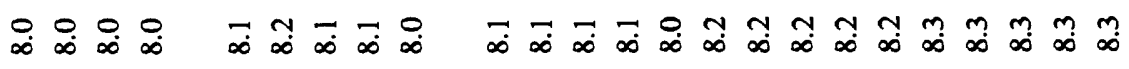

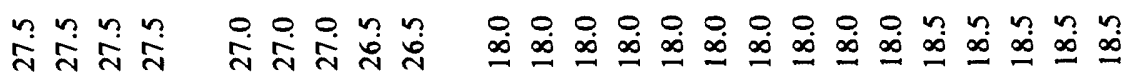
4⿻一𠃋十

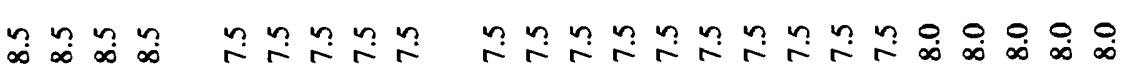

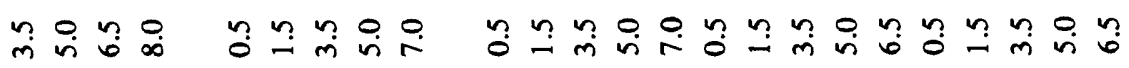

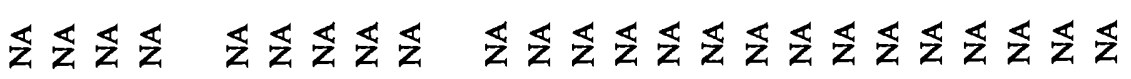

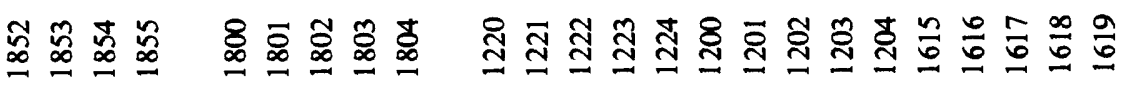

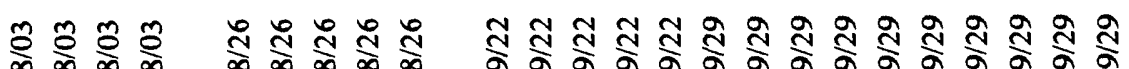

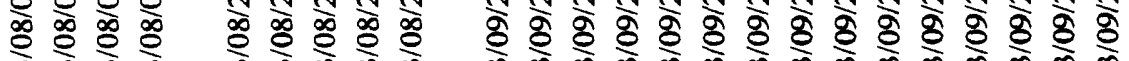

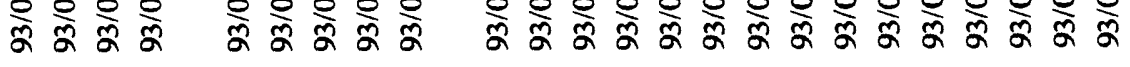




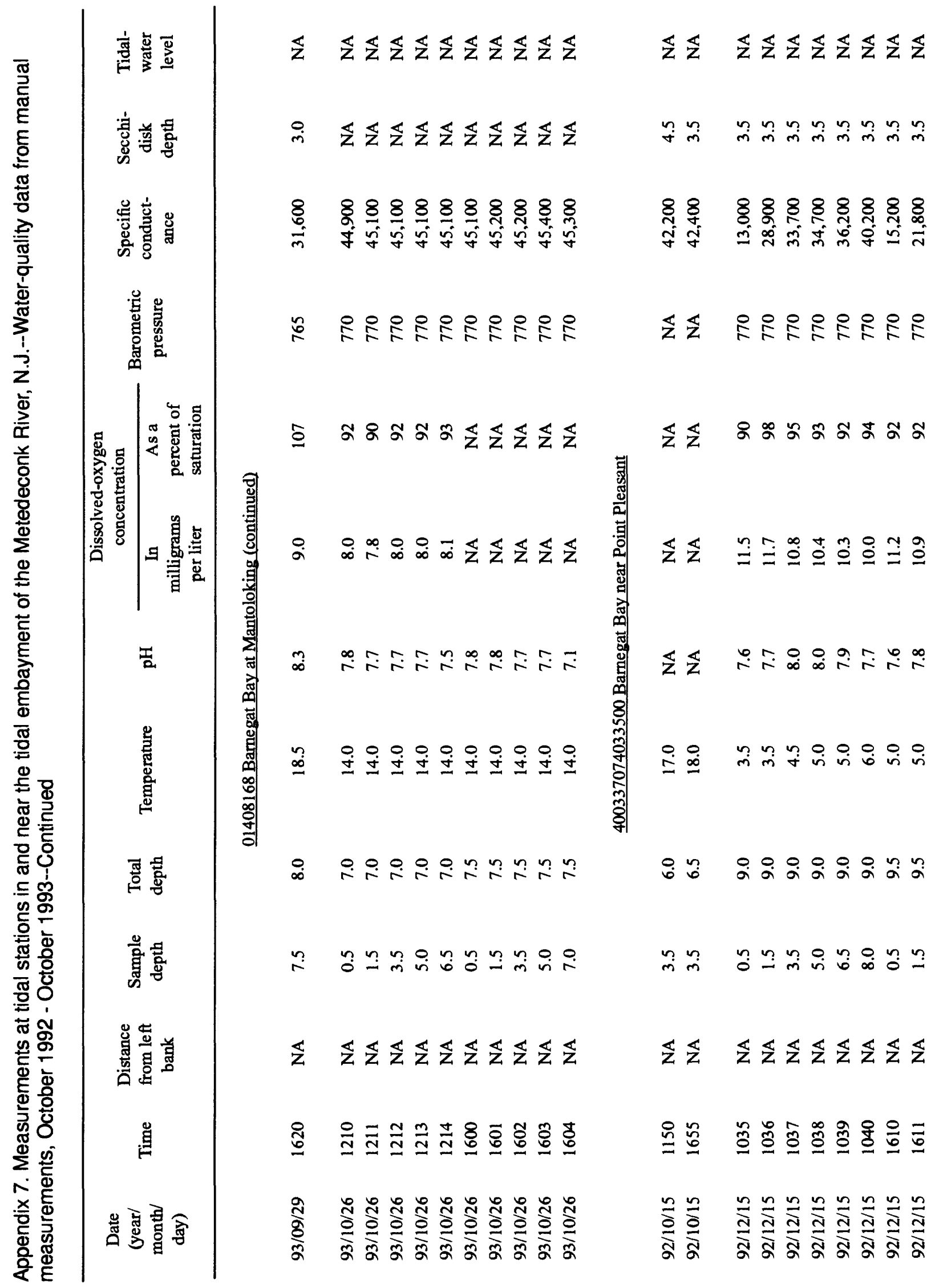




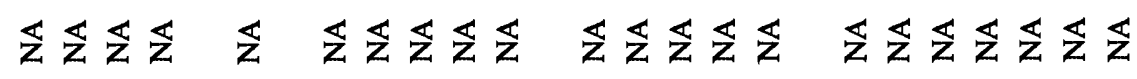

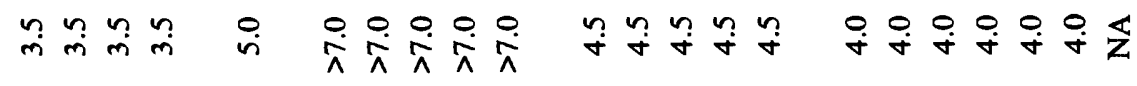

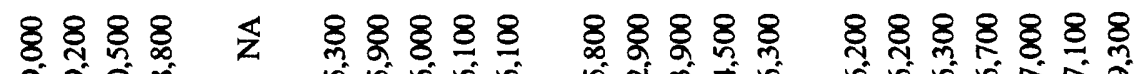

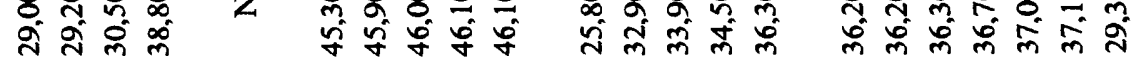

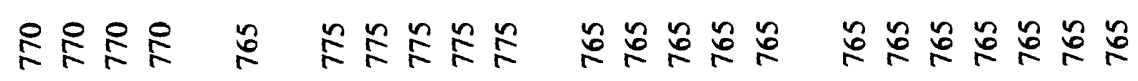

氧

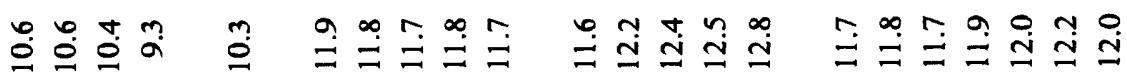

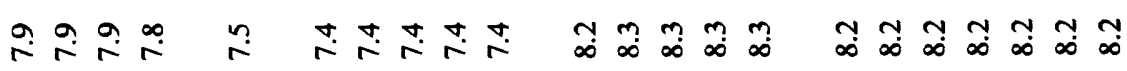

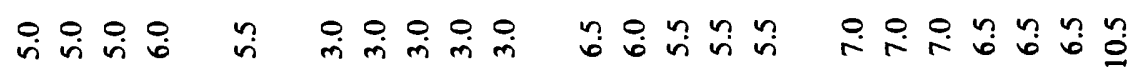

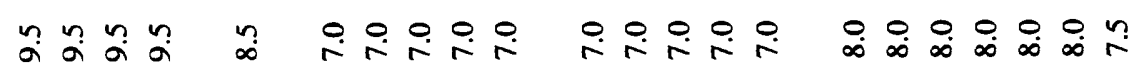

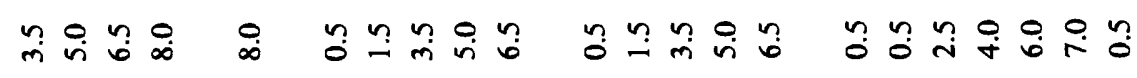

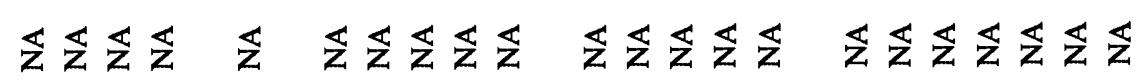

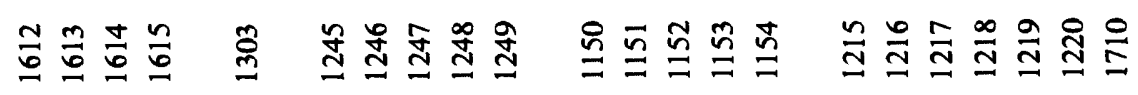

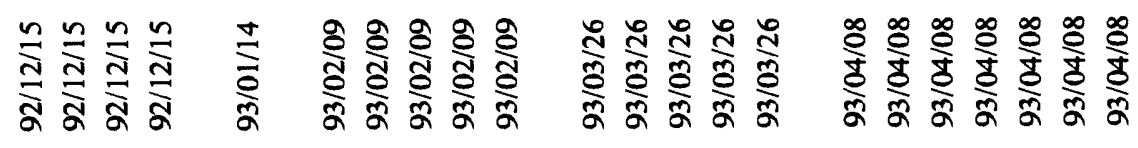




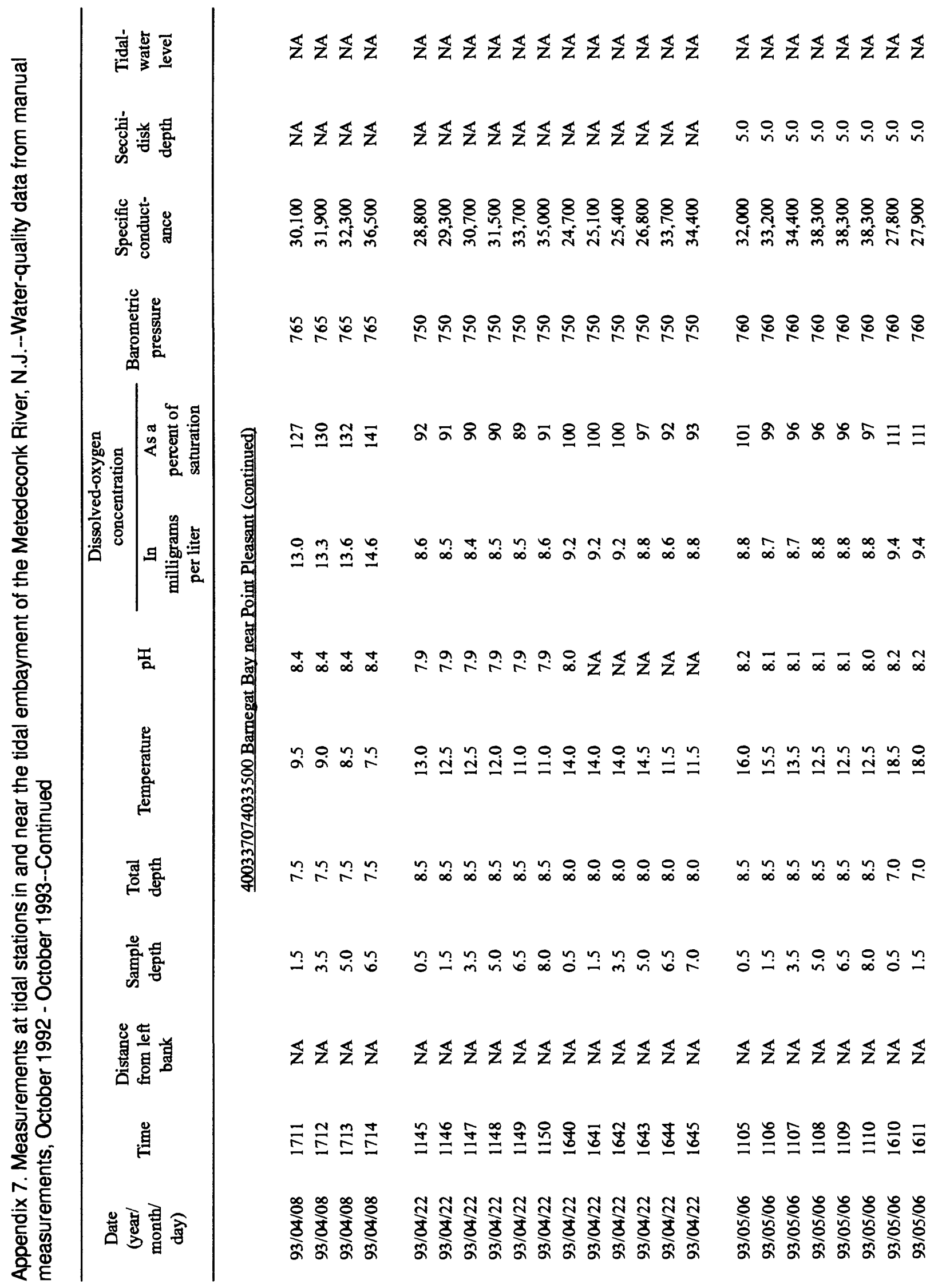




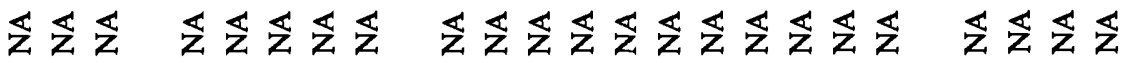

育尊意

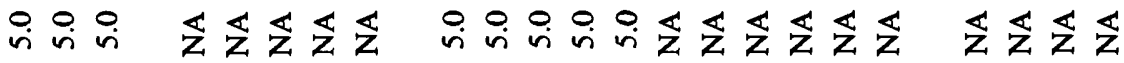

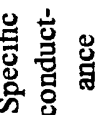

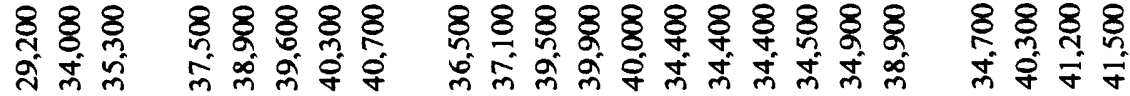

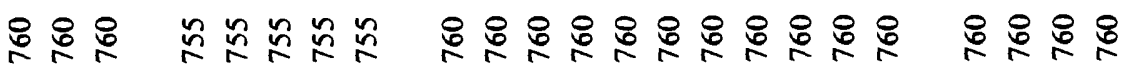

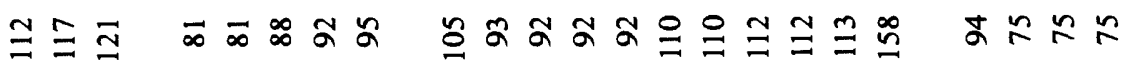

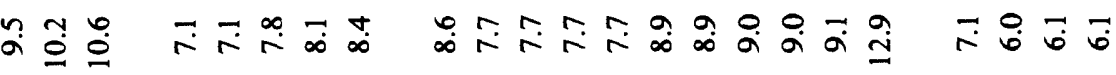

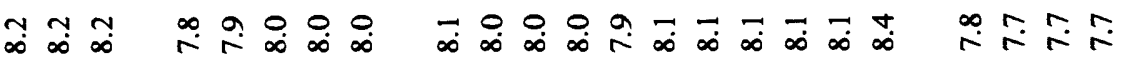

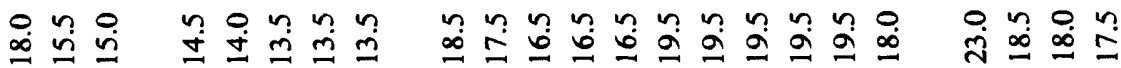

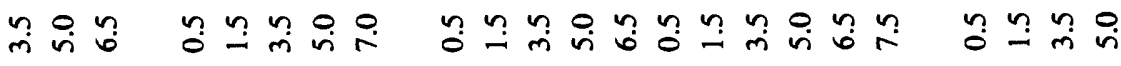

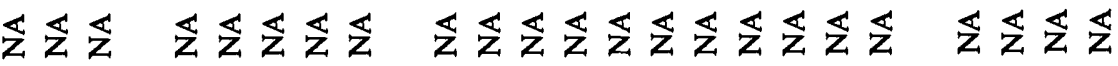

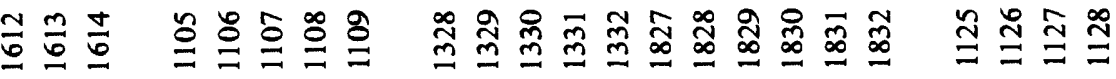

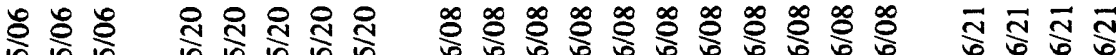

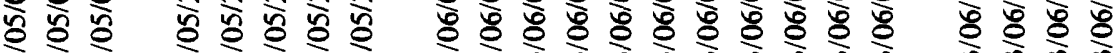

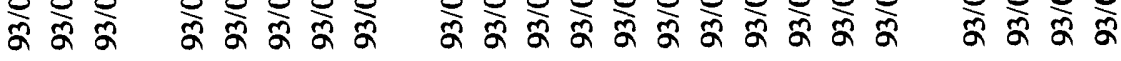




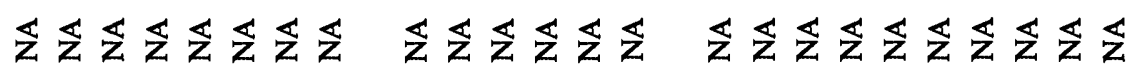

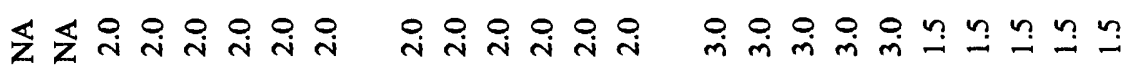

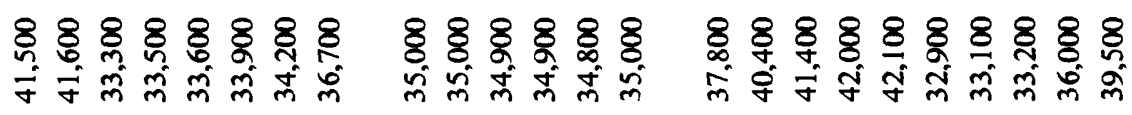

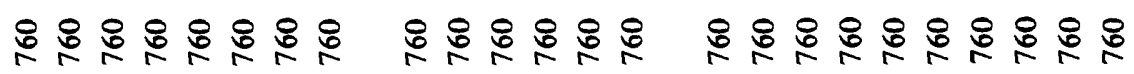

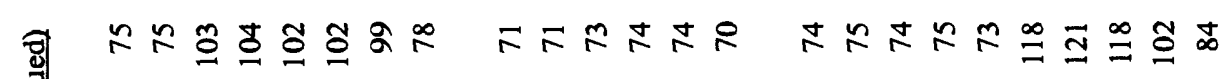

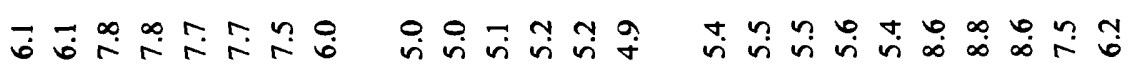

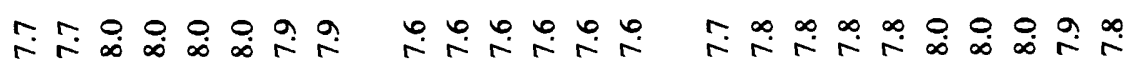

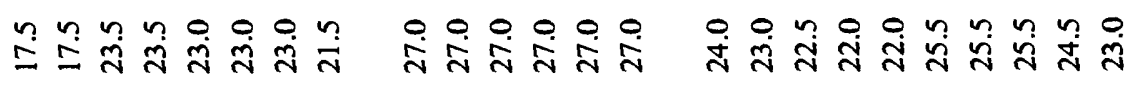

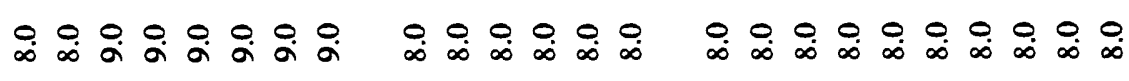

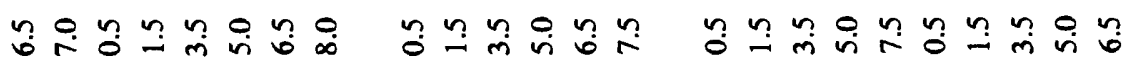

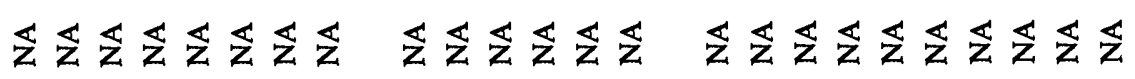

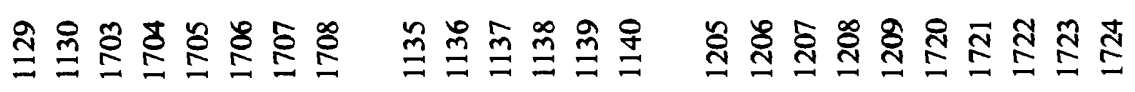

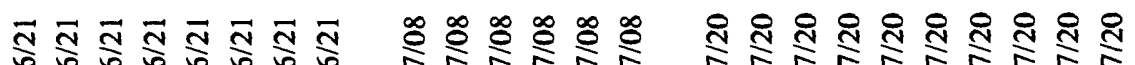

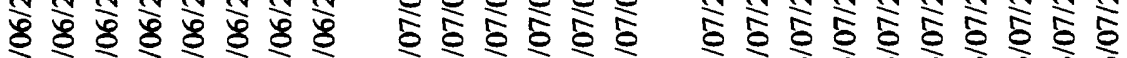

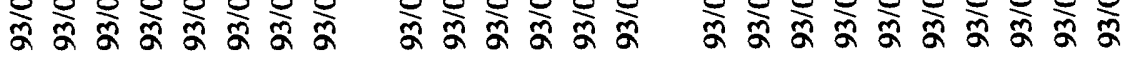




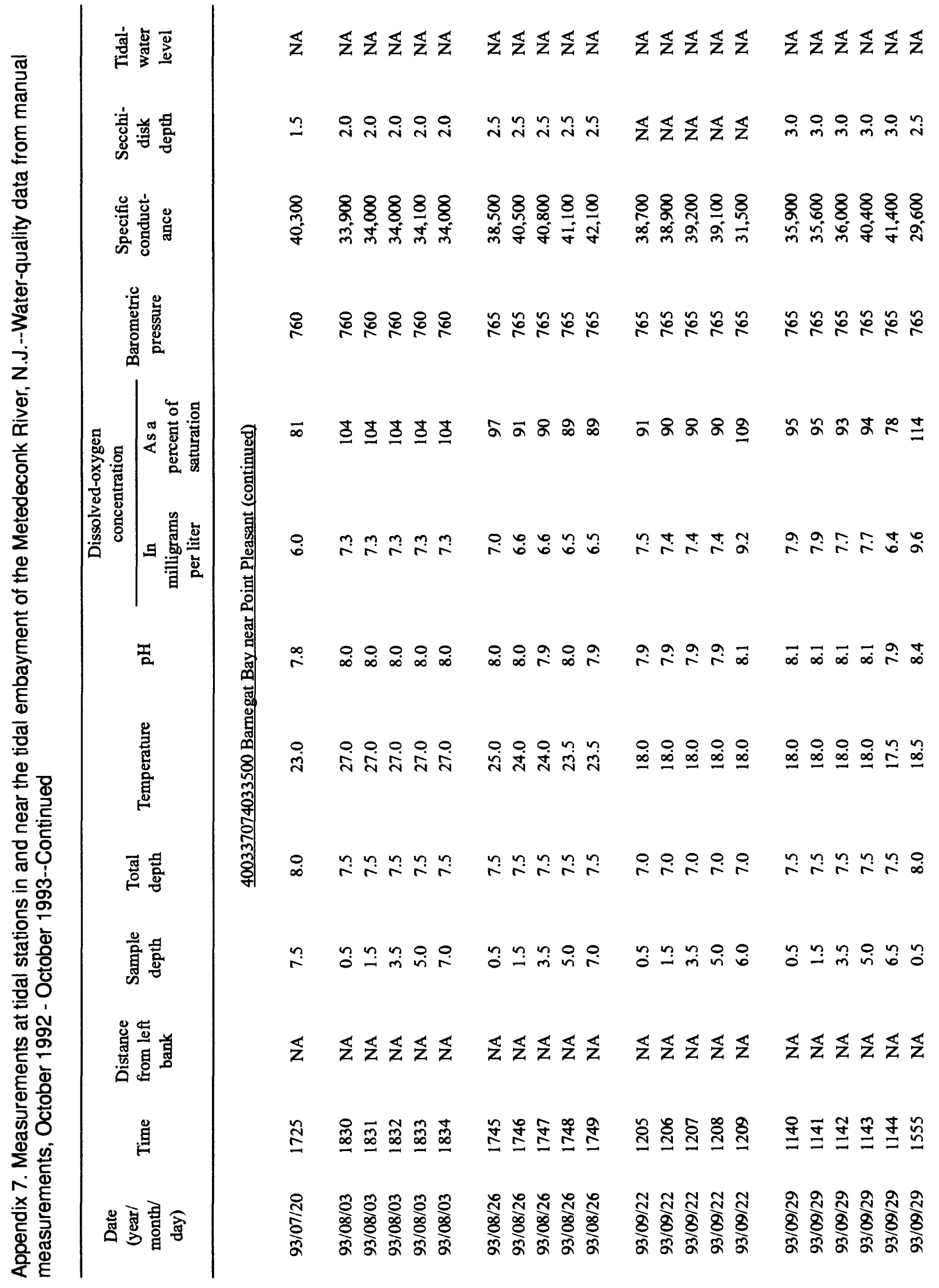




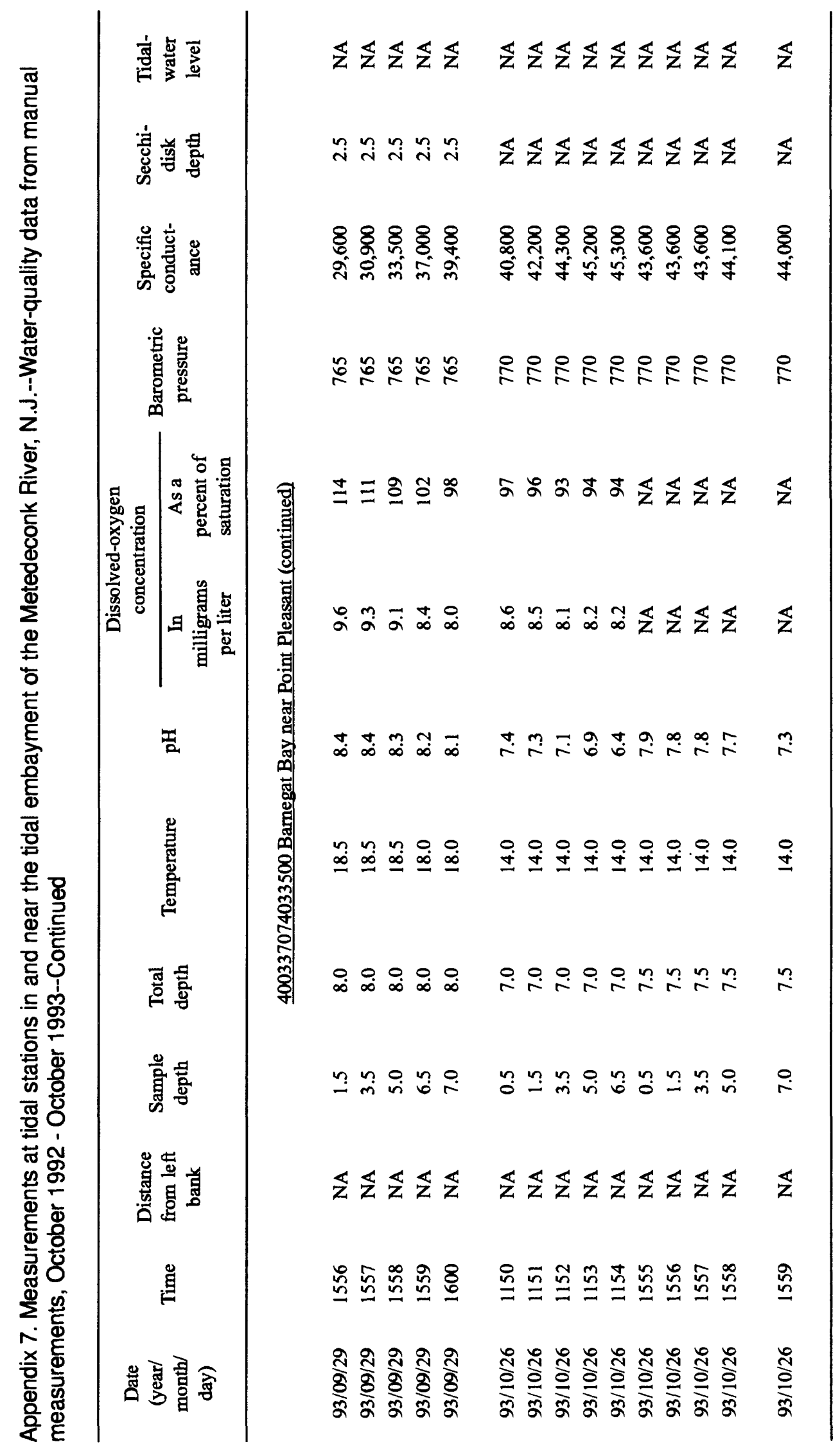


Appendix 8. Measurements at tidal stations in and near the tidal embayment of the Metedeconk River, N.J.--Results of laboratory measurements of dissolved constituents, October 1992 October 1993

[Laboratory analyses were conducted on water samples at the stations listed below. USGS, U.S. Geological Survey; NA, not applicable or not available]

\begin{tabular}{|c|c|c|c|}
\hline $\begin{array}{l}\text { Index } \\
\text { number } \\
\text { (fig. 2) }\end{array}$ & USGS station number & USGS station name & $\begin{array}{l}\text { Location of the } \\
\text { channel in the } \\
\text { cross section, in } \\
\text { percent distance } \\
\text { from left to right } \\
\text { bank }\end{array}$ \\
\hline 3 & 400412074082600 & Metedeconk River near Bricktown & 95 \\
\hline 4 & 400405074080600 & Metedeconk River at Laurelton Gardens & 5 \\
\hline 5 & 01408155 & Metedeconk River at Laurelton & $\begin{array}{c}25,75 \\
\text { (two channels) }\end{array}$ \\
\hline 6 & 400353074074900 & $\begin{array}{l}\text { Metedeconk River } 1,200 \text { feet downstream } \\
\text { from Route } 70 \text { at Laurelton }\end{array}$ & 50 \\
\hline 7 & 400343074073400 & $\begin{array}{l}\text { Metedeconk River } 0.6 \text { miles downstream } \\
\text { from Route } 70 \text { at Laurelton }\end{array}$ & 50 \\
\hline 8 & 400337074071600 & $\begin{array}{l}\text { Metedeconk River } 0.9 \text { miles downstream } \\
\text { from Route } 70 \text { near Laurelton }\end{array}$ & 50 \\
\hline 9 & 01408160 & Metedeconk River near Laurelton & 50 \\
\hline 10 & 400313074055200 & $\begin{array}{l}\text { Metedeconk River at Eagle Point at } \\
\text { Adamston }\end{array}$ & 50 \\
\hline 11 & 400314074044500 & Metedeconk River at Metedeconk & 50 \\
\hline 12 & 400311074035200 & Metedeconk River at West Mantoloking & 50 \\
\hline 13 & 01408168 & Barnegat Bay at Mantoloking & NA \\
\hline 14 & 400337074033500 & Barnegat Bay near Point Pleasant & NA \\
\hline
\end{tabular}


Appendix 8. Measurements at tidal stations in and near the tidal embayment of the Metedeconk River, N.J.--Results of laboratory measurements of dissolved constituents, October 1992 October 1993--Continued

The following information is presented:

\begin{tabular}{lll}
\hline \multicolumn{1}{c}{ Characteristic } & \multicolumn{1}{c}{ Unit } & Rounding \\
\hline $\begin{array}{l}\text { Date and time of water } \\
\text { sample }\end{array}$ & NA & NA \\
$\begin{array}{l}\text { Distance from left bank } \\
\text { Sample depth }\end{array}$ & $\begin{array}{c}\text { Percent of distance from left to } \\
\text { right bank }\end{array}$ & 1 \\
$\begin{array}{l}\text { Specific conductance } \\
\text { Feet }\end{array}$ & $\begin{array}{c}\text { Microsiemens per centimeter at } \\
25 \text { degrees Celsius }\end{array}$ & 1 \\
$\begin{array}{l}\text { Salinity } \\
\text { Dissolved-chloride } \\
\text { concentration }\end{array}$ & Parts per thousand & 0.5 \\
$\begin{array}{c}\text { Dissolved-solids } \\
\text { concentration }\end{array}$ & Milligrams per liter as chloride & 1 \\
& Milligrams per liter & 1 \\
\hline
\end{tabular}


Appendix 8. Measurements at tidal stations in and near the tidal embayment of the Metedeconk River, N.J.--Results of laboratory measurements of dissolved constituents, October 1992 October 1993--Continued

\begin{tabular}{|c|c|c|c|c|c|c|c|}
\hline $\begin{array}{c}\text { Date } \\
\text { (year/month/ } \\
\text { day) }\end{array}$ & Time & $\begin{array}{c}\text { Distance } \\
\text { from left } \\
\text { bank }\end{array}$ & $\begin{array}{c}\text { Sample } \\
\text { depth }\end{array}$ & $\begin{array}{c}\text { Specific } \\
\text { conductance }\end{array}$ & Salinity & $\begin{array}{l}\text { Dissolved- } \\
\text { chloride } \\
\text { concentration }\end{array}$ & $\begin{array}{l}\text { Dissolved- } \\
\text { solids } \\
\text { concentration }\end{array}$ \\
\hline
\end{tabular}

400412074082600 Metedeconk River near Bricktown

$\begin{array}{llllllll}92 / 10 / 15 & 1030 & 10 & 0.5 & 290 & 0.08 & 28 & 82 \\ 92 / 10 / 15 & 1035 & 10 & 1.5 & 134 & 0.09 & 16 & 84\end{array}$

$\underline{400405074080600}$ Metedeconk River at Laurelton Gardens

$\begin{array}{rrrrrrrr}92 / 10 / 15 & 1055 & 20 & 0.5 & 139 & 0.11 & 23 & 90 \\ 92 / 10 / 15 & 1100 & 20 & 2.0 & 239 & 0.12 & 46 & 144\end{array}$

01408155 Metedeconk River at Laurelton

$\begin{array}{rrrrrrrr}92 / 10 / 15 & 1125 & 25 & 1.5 & 1,100 & 0.76 & 292 & 635 \\ 92 / 10 / 15 & 1115 & 25 & 3.5 & 30,400 & 19.80 & 11,100 & 19,600 \\ 93 / 02 / 09 & 0930 & 25 & 0.5 & 405 & 0.22 & 95 & 195 \\ 93 / 03 / 26 & 0900 & 25 & 0.5 & 92 & 0.05 & 15 & 40 \\ 93 / 04 / 08 & 0855 & 25 & 0.5 & 99 & 0.05 & 17 & 70 \\ 93 / 04 / 22 & 0915 & 25 & 0.5 & 134 & 0.07 & 23 & 3 . \\ 93 / 05 / 06 & 0900 & 25 & 0.5 & 118 & 0.07 & 18 & 30 \\ 93 / 05 / 20 & 0905 & 25 & 0.5 & 204 & 0.11 & 42 & 80 \\ 93 / 06 / 08 & 1112 & 25 & 0.5 & 536 & 0.30 & 138 & 280 \\ 93 / 06 / 21 & 0940 & 25 & 0.5 & 1,250 & 0.73 & 297 & 640 \\ 93 / 07 / 08 & 0955 & 25 & 0.5 & 406 & 0.23 & 101 & 220 \\ 93 / 08 / 03 & 1640 & 25 & 0.5 & 1,460 & 0.85 & 434 & 835 \\ 93 / 08 / 26 & 0945 & 25 & 0.5 & 819 & 0.45 & 316 & 424 \\ 93 / 10 / 26 & 0950 & 25 & 0.5 & 165 & 0.08 & 392 & 60\end{array}$

400353074074900 Metedeconk River 1,200 feet downstream from Route 70 at Laurelton

$\begin{array}{rrrrrrrr}92 / 10 / 15 & 1135 & 50 & 1.5 & 2,860 & 1.70 & 768 & 1,330 \\ 92 / 10 / 15 & 1205 & 50 & 3.5 & 31,000 & 20.20 & 11,400 & 20,300 \\ 93 / 01 / 14 & 1040 & 50 & 1.5 & 416 & 0.23 & 172 & 330 \\ 93 / 09 / 22 & 1030 & 50 & 0.5 & 778 & 0.36 & 202 & 380\end{array}$


Appendix 8. Measurements at tidal stations in and near the tidal embayment of the Metedeconk River, N.J.--Results of laboratory measurements of dissolved constituents, October 1992 October 1993--Continued

\begin{tabular}{|c|c|c|c|c|c|c|c|}
\hline $\begin{array}{c}\text { Date } \\
\text { (year/month/ } \\
\text { day) }\end{array}$ & Time & $\begin{array}{c}\text { Distance } \\
\text { from left } \\
\text { bank }\end{array}$ & $\begin{array}{c}\text { Sample } \\
\text { depth }\end{array}$ & $\begin{array}{c}\text { Specific } \\
\text { conductance }\end{array}$ & Salinity & $\begin{array}{l}\text { Dissolved- } \\
\text { chloride } \\
\text { concentration }\end{array}$ & $\begin{array}{l}\text { Dissolved- } \\
\text { solids } \\
\text { concentration }\end{array}$ \\
\hline
\end{tabular}

400343074073400 Metedeconk River 0.6 miles downstream from Route 70 at Laurelton

$\begin{array}{rrrrrrrr}92 / 10 / 15 & 1235 & 50 & 1.5 & 14,600 & 15.90 & 4,600 & 8,870 \\ 92 / 10 / 15 & 1225 & 50 & 3.5 & 32,400 & 23.20 & 11,700 & 24,100 \\ 92 / 12 / 15 & 0910 & 50 & 0.5 & 2,130 & 1.16 & 568 & 1,130 \\ 93 / 07 / 20 & 1035 & 50 & 0.5 & 1,300 & 0.77 & 365 & 688 \\ 93 / 09 / 29 & 1010 & 50 & 0.5 & 1,540 & 0.77 & 28 & 712\end{array}$

400337074071600 Metedeconk River 0.9 miles downstream from Route 70 near Laurelton

$\begin{array}{rrrrrrrr}92 / 10 / 15 & 1330 & 50 & 1.0 & 29,400 & 21.70 & 10,200 & 21,200 \\ 92 / 10 / 15 & 1320 & 50 & 2.5 & 25,200 & 22.70 & 9,400 & 17,100\end{array}$

$\underline{01408160 \text { Metedeconk River near Laurelton }}$

$\begin{array}{llllllll}92 / 10 / 15 & 1315 & 50 & 1.5 & 33,800 & 21.50 & 11,600 & 22,200 \\ 92 / 10 / 15 & 1310 & 50 & 6.5 & 35,300 & 21.90 & 11,400 & 23,000\end{array}$

400313074055200 Metedeconk River at Eagle Point at Adamston

$\begin{array}{rrrrrrrr}92 / 10 / 15 & 1300 & 50 & 2.5 & 21,600 & 11.10 & 6,900 & 14,400 \\ 92 / 10 / 15 & 1255 & 50 & 6.0 & 33,000 & 22.60 & 11,200 & 21,700\end{array}$

400314074044500 Metedeconk River at Metedeconk

$\begin{array}{llllllll}92 / 10 / 15 & 1235 & 50 & 2.5 & 31,500 & 19.90 & 10,400 & 20,300 \\ 92 / 10 / 15 & 1230 & 50 & 5.5 & 38,900 & 25.10 & 12,500 & 24,900\end{array}$

400311074035200 Metedeconk River at West Mantoloking

$\begin{array}{llllllll}92 / 10 / 15 & 1215 & 50 & 2.5 & 32,600 & 22.00 & 11,700 & 21,600 \\ 92 / 10 / 15 & 1210 & 50 & 5.0 & 42,200 & 26.80 & 14,900 & 25,800\end{array}$

01408168 Barnegat Bay at Mantoloking

$\begin{array}{llllllll}92 / 10 / 15 & 1150 & \text { NA } & 1.5 & 36,800 & 24.30 & 12,200 & 24,400 \\ 92 / 10 / 15 & 1145 & \text { NA } & 6.5 & 36,700 & 24.60 & 14,800 & 25,200\end{array}$


Appendix 8. Measurements at tidal stations in and near the tidal embayment of the Metedeconk River, N.J.--Results of laboratory measurements of dissolved constituents, October 1992 October 1993--Continued

\begin{tabular}{cccccccc}
\hline $\begin{array}{c}\text { Date } \\
\text { (year/month/ } \\
\text { day) }\end{array}$ & Time & $\begin{array}{c}\text { Distance } \\
\text { from left } \\
\text { bank }\end{array}$ & $\begin{array}{c}\text { Sample } \\
\text { depth }\end{array}$ & $\begin{array}{c}\text { Specific } \\
\text { conductance }\end{array}$ & Salinity & $\begin{array}{c}\text { Dissolved- } \\
\text { chloride } \\
\text { concentration }\end{array}$ & $\begin{array}{c}\text { Dissolved- } \\
\text { solids } \\
\text { concentration }\end{array}$ \\
\hline \multicolumn{7}{c}{ 00337074033500 Bamegat Bay near Point Pleasant } \\
& & & & & & \\
$92 / 10 / 15$ & 1200 & NA & 1.5 & 42,000 & 29.60 & 15,400 & 30,700 \\
$92 / 10 / 15$ & 1155 & NA & 6.5 & 42,500 & 30.10 & 16,300 & 31,400 \\
\hline
\end{tabular}


Appendix 9. Measurements at nontidal stations on tributaries to the tidal embayment of the Toms River, N.J.--Daily mean streamflow, October 1992 - September 1994

[Values of streamflow in cubic feet per second are presented for the continuous-record station listed below. USGS, U.S. Geological Survey; NA, not applicable]

\begin{tabular}{ccc}
$\begin{array}{c}\text { Index } \\
\text { number } \\
\text { (fig. 3) }\end{array}$ & USGS station number & USGS station name \\
\hline 17 & 01408500 & Toms River near Toms River \\
\hline
\end{tabular}




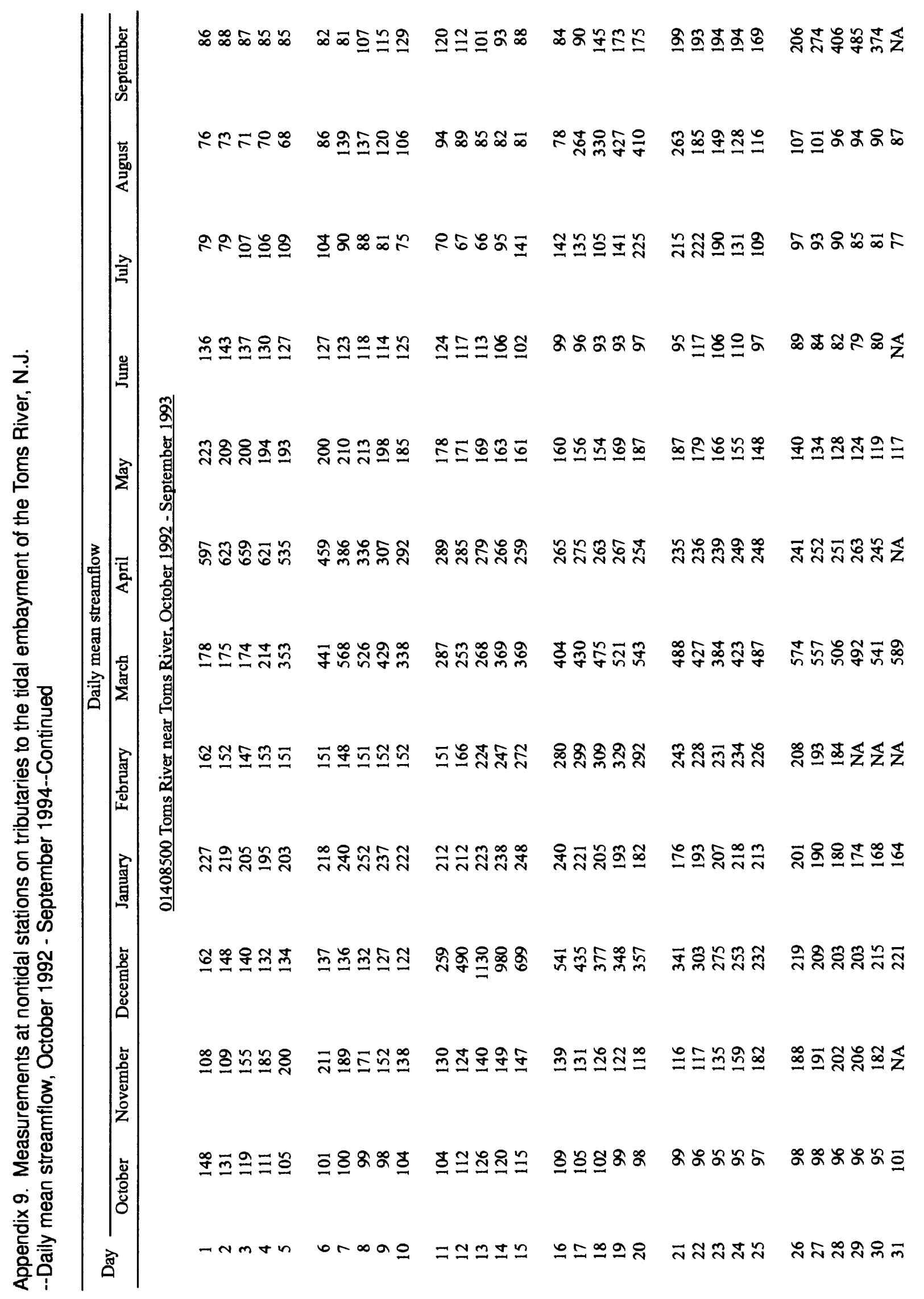




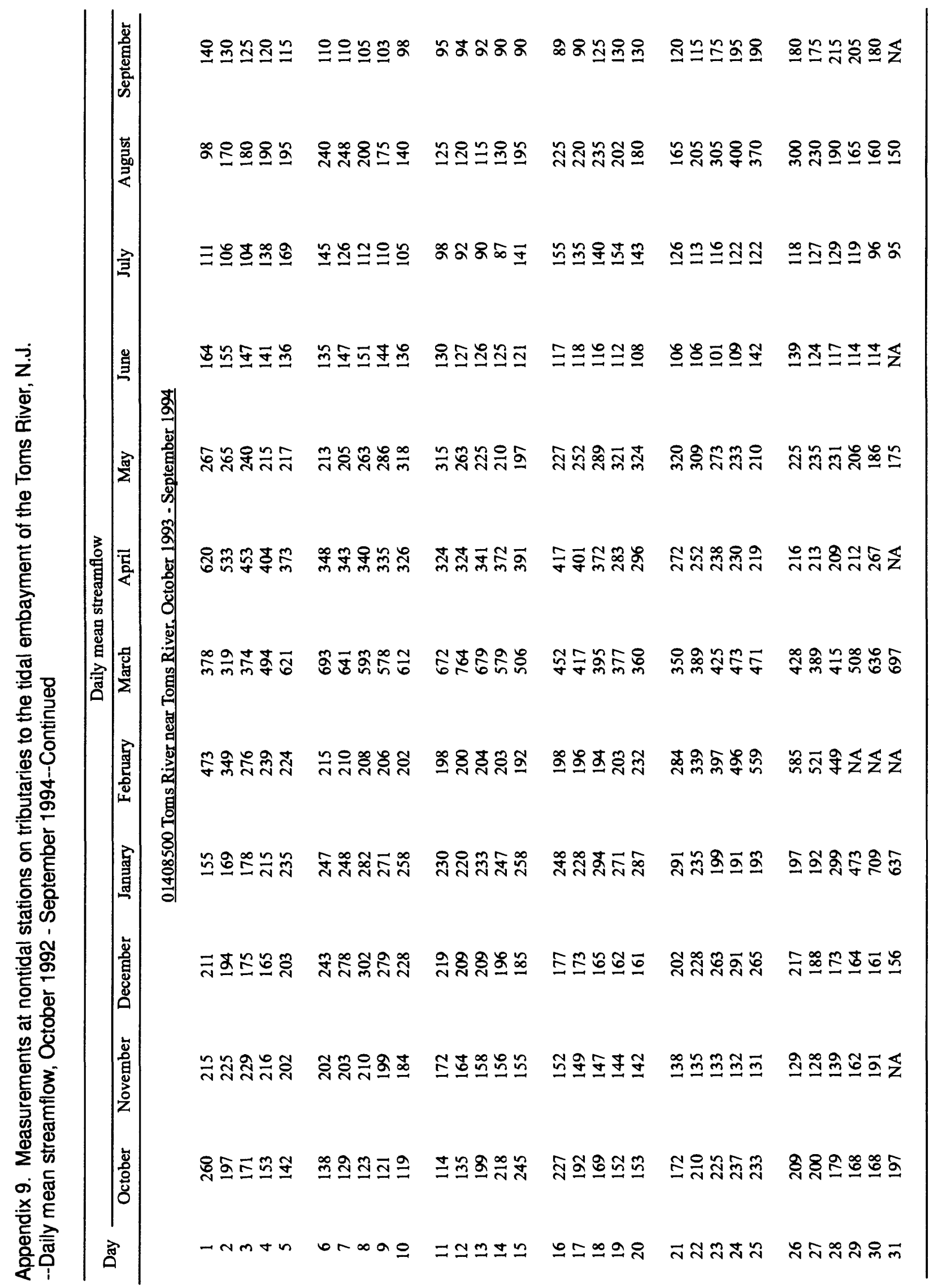


Appendix 10. Measurements at nontidal stations on tributaries to the tidal embayment of the Toms River, N.J.--Measured values of discrete streamflow, October 1992 - August 1993

[Streamflow measurements were made at the stations listed below. USGS, U.S. Geological Survey]

\begin{tabular}{ccc}
\hline $\begin{array}{c}\text { Index } \\
\text { number } \\
\text { (fig. 3) }\end{array}$ & USGS station number & USGS station name \\
\hline & & \\
15 & 01408630 & Davenport Branch near Toms River \\
16 & 01408600 & Wrangle Brook near Toms River \\
\hline
\end{tabular}

The following information is presented:

\begin{tabular}{lll}
\multicolumn{1}{c}{ Characteristic } & Unit & Rounding \\
\hline $\begin{array}{l}\text { Date and time of streamflow } \\
\text { measurement }\end{array}$ & NA & NA \\
Stage & Feet above local datum \\
Streamflow & Cubic feet per second & 0.01 \\
& & 1 \\
\hline
\end{tabular}


Appendix 10. Measurements at nontidal stations on tributaries to the tidal embayment of the Toms River, N.J.--Measured values of discrete streamflow, October 1992 August 1993--Continued

\begin{tabular}{cccc}
\hline $\begin{array}{c}\text { Date } \\
\text { (year/month/day) }\end{array}$ & Time & Stage & Streamflow \\
\hline
\end{tabular}

01408630 Davenport Branch near Toms River

$\begin{array}{llll}92 / 10 / 30 & 1347 & 0.56 & 12 \\ 92 / 12 / 02 & 1104 & 0.57 & 12 \\ 92 / 12 / 21 & 0912 & 0.68 & 24 \\ 93 / 01 / 20 & 1520 & 0.60 & 17 \\ 93 / 02 / 19 & 1058 & 0.63 & 19 \\ 93 / 03 / 23 & 0931 & 0.72 & 29 \\ 93 / 04 / 29 & 1442 & 0.68 & 25 \\ 93 / 06 / 18 & 1340 & 0.53 & 13 \\ 93 / 08 / 30 & 1357 & 0.50 & 11\end{array}$

01408600 Wrangle Brook near Toms River

$\begin{array}{llll}92 / 10 / 29 & 1100 & 2.85 & 26 \\ 92 / 12 / 02 & 1253 & 2.95 & 30 \\ 92 / 12 / 21 & 1542 & 3.44 & 40 \\ 93 / 01 / 21 & 1425 & 3.10 & 37 \\ 93 / 02 / 11 & 1528 & 3.02 & 32 \\ 93 / 03 / 23 & 1101 & 3.63 & 62 \\ 93 / 04 / 29 & 1144 & 3.33 & 48 \\ 93 / 06 / 18 & 1442 & 2.51 & 21 \\ 93 / 08 / 30 & 1154 & 2.72 & 25\end{array}$


Appendix 11. Measurements at nontidal stations on tributaries to the tidal embayment of the Toms River, N.J.--Daily minimum and maximum values of water-quality characteristics from measurements by movable monitors, July 1993 - August 1993

[Measurements were made at the station listed below. USGS, U.S. Geological Survey; Min, daily minimum; Max, daily maximum]

\begin{tabular}{|c|c|c|c|c|c|}
\hline $\begin{array}{l}\text { Index } \\
\text { number } \\
\text { (fig. 3) }\end{array}$ & USGS station number & USGS station name & $\begin{array}{c}\text { Period of } \\
\text { deployment } \\
\text { (year/month/day) }\end{array}$ & $\begin{array}{l}\text { Location in } \\
\text { cross section }\end{array}$ & $\begin{array}{l}\text { Approximate } \\
\text { depth of } \\
\text { measurement, } \\
\text { in feet }\end{array}$ \\
\hline 17 & 01408500 & $\begin{array}{l}\text { Toms River near Toms } \\
\text { River }\end{array}$ & $93 / 07 / 28-93 / 08 / 03$ & Near bottom & 5 \\
\hline
\end{tabular}

The following characteristics were measured. The interval between measurements was 60 minutes. Days with partial records are included.

\begin{tabular}{llc}
\hline \multicolumn{1}{c}{ Characteristic } & \multicolumn{1}{c}{ Unit } & Rounding \\
\hline & & \\
Temperature & Degrees Celsius & 0.5 \\
Specific conductance & Microsiemens per centimeter at & 100 \\
& 25 degrees Celsius & \\
pH & Standard units & 0.1 \\
Dissolved-oxygen & Milligrams per liter & 0.1 \\
concentration & & \\
& & \\
\hline
\end{tabular}


Appendix 11. Measurements at nontidal stations on tributaries to the tidal embayment of the Toms River, N.J.--Daily minimum and maximum values of water-quality characteristics from measurements by movable monitors, July 1993 - August 1993--Continued

\begin{tabular}{|c|c|c|c|c|c|c|c|c|}
\hline \multirow{3}{*}{$\begin{array}{c}\text { Date } \\
\text { year/month/day }\end{array}$} & \multicolumn{8}{|c|}{ Daily minimum and maximum } \\
\hline & \multicolumn{2}{|c|}{ Temperature } & \multicolumn{2}{|c|}{ Specific conductance } & \multicolumn{2}{|c|}{ pH } & \multicolumn{2}{|c|}{$\begin{array}{l}\text { Dissolved-oxygen } \\
\text { concentration }\end{array}$} \\
\hline & Min & Max & Min & $\operatorname{Max}$ & Min & Max & Min & $\operatorname{Max}$ \\
\hline \multicolumn{9}{|c|}{ 01408500 Toms River near Toms River, near bottom } \\
\hline $93 / 07 / 28$ & 23.0 & 23.5 & 100 & 100 & 5.3 & 5.6 & 7.4 & 8.1 \\
\hline $93 / 07 / 29$ & 21.5 & 24.0 & 100 & 100 & 5.3 & 5.4 & 7.3 & 8.0 \\
\hline $93 / 07 / 30$ & 21.5 & 23.5 & 100 & 100 & 5.4 & 5.4 & 7.3 & 7.9 \\
\hline $93 / 07 / 31$ & 20.5 & 22.0 & 100 & 100 & 5.4 & 5.5 & 7.4 & 8.1 \\
\hline $93 / 08 / 01$ & 20.5 & 22.0 & 100 & 100 & 5.5 & 5.5 & 7.6 & 8.0 \\
\hline $93 / 08 / 02$ & 20.5 & 23.0 & 100 & 100 & 5.5 & 5.5 & 7.5 & 8.1 \\
\hline $93 / 08 / 03$ & 21.5 & 21.5 & 100 & 100 & 5.4 & 5.5 & 7.4 & 7.5 \\
\hline
\end{tabular}


Appendix 12. Measurements at nontidal stations on tributaries to the tidal embayment of the Toms River, N.J.--Water-quality data from manual measurements, October 1992 - October 1993

[Water-quality data are presented for the stations listed below. USGS, U.S. Geological Survey; NA, not applicable or not available; <, less than; E, estimated from relation between streamflow at 01408600, Wrangle Brook near Toms River, and at 01408500, Toms River near Toms River]

\begin{tabular}{ccc}
\hline $\begin{array}{c}\text { Index number } \\
\text { (fig. 3) }\end{array}$ & USGS station number & USGS station name \\
\hline 15 & 01408630 & $\begin{array}{c}\text { Davenport Branch near Toms } \\
\text { River }\end{array}$ \\
16 & 01408600 & Wrangle Brook near Toms River \\
17 & 01408500 & Toms River near Toms River
\end{tabular}

The following information is presented:

\begin{tabular}{lll}
\hline \multicolumn{1}{c}{ Characteristic } & \multicolumn{1}{c}{ Unit } & Rounding \\
\hline $\begin{array}{l}\text { Date and time of } \\
\text { measurement }\end{array}$ & NA & NA \\
Stage & Feet above a local datum & 0.01 \\
Streamflow & Cubic feet per second & 1 \\
$\begin{array}{l}\text { Temperature } \\
\text { pH }\end{array}$ & Degrees Celsius & 0.5 \\
$\begin{array}{l}\text { Dissolved-oxygen } \\
\text { concentration }\end{array}$ & Standard units & 0.1 \\
$\begin{array}{l}\text { Dissolved-oxygen } \\
\text { concentration as a percent } \\
\text { of saturation }\end{array}$ & Milligrams per liter & 0.1 \\
$\begin{array}{l}\text { Barometric pressure } \\
\text { Specific conductance }\end{array}$ & Mercent & 1 \\
& Millimeters of mercury & 5 \\
\hline
\end{tabular}




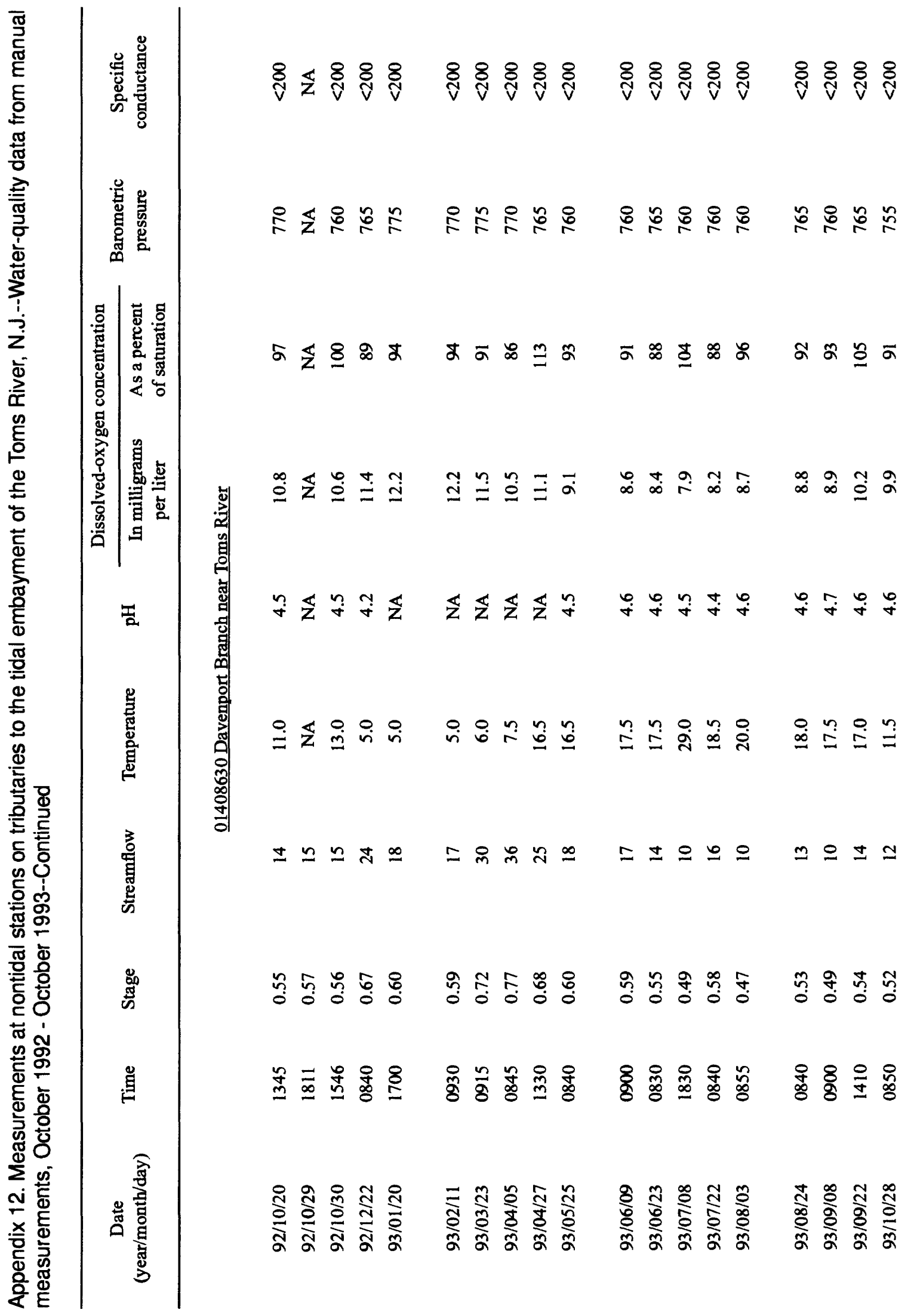




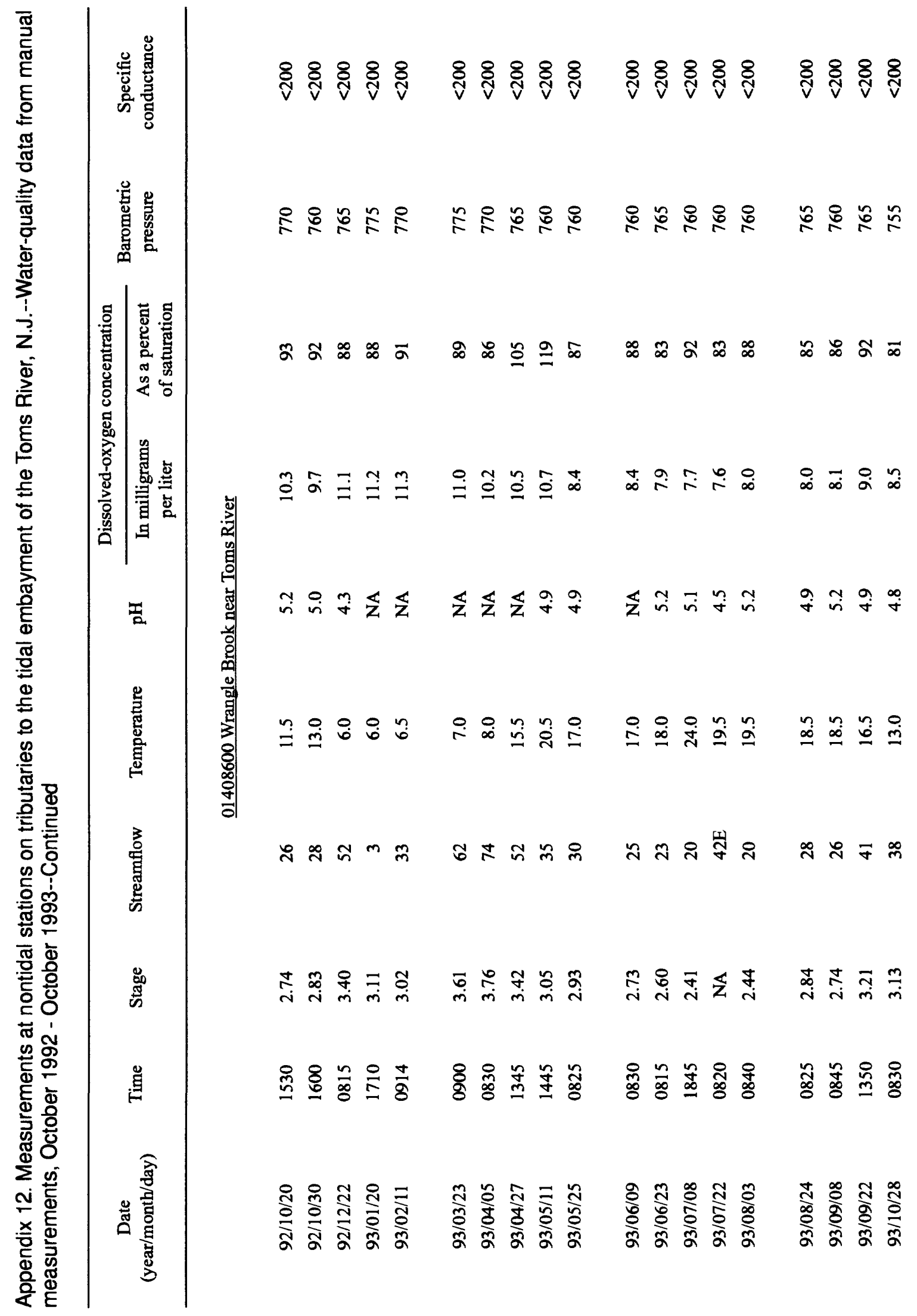




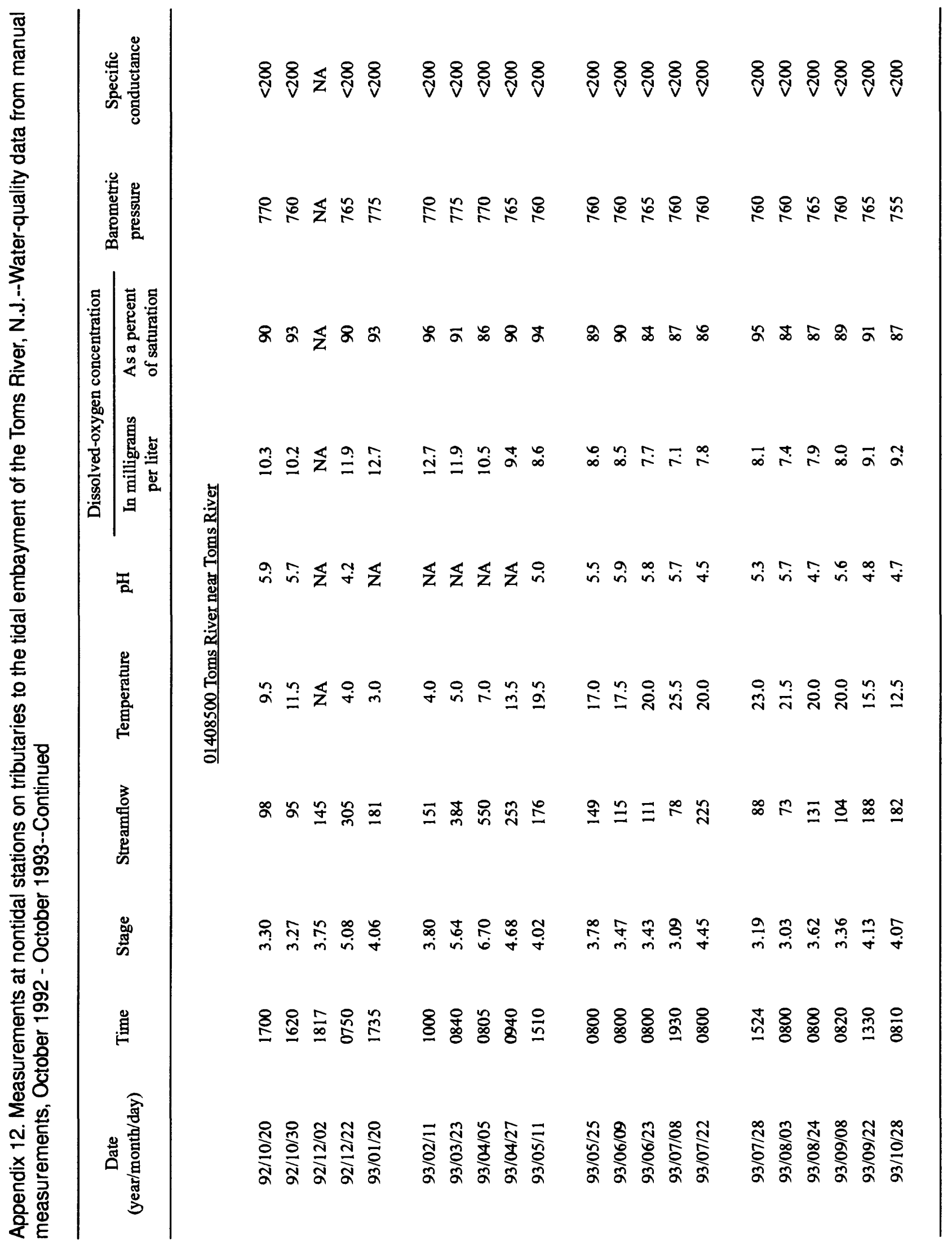


Appendix 13. Measurements at tidal stations in and near the tidal embayment of the Toms River, N.J.--Daily minimum and maximum tidal-water level, October 1992 - November 1993

[Values of tidal-water level are given in feet above the National Geodetic Vertical Datum of 1929 for the station listed below. Min, daily minimum; Max, daily maximum; USGS, U.S. Geological Survey; NA, not available or not applicable]

\begin{tabular}{ccc}
\hline $\begin{array}{c}\text { Index } \\
\text { number } \\
\text { (fig. 3) }\end{array}$ & USGS station number & USGS station name \\
\hline 22 & 01408719 & $\begin{array}{c}\text { Toms River at Cedar Point } \\
\text { at South Toms River }\end{array}$ \\
\hline
\end{tabular}




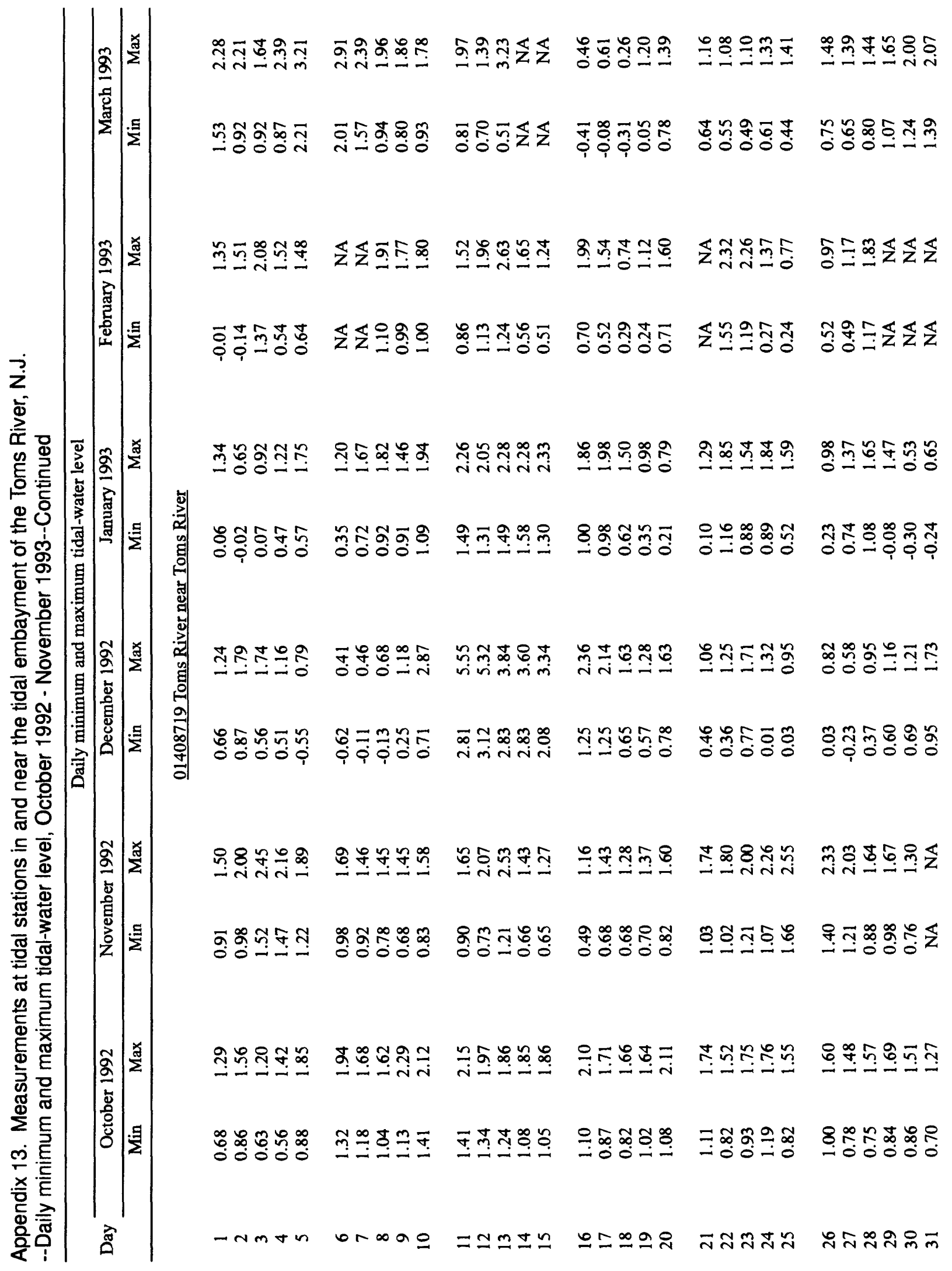




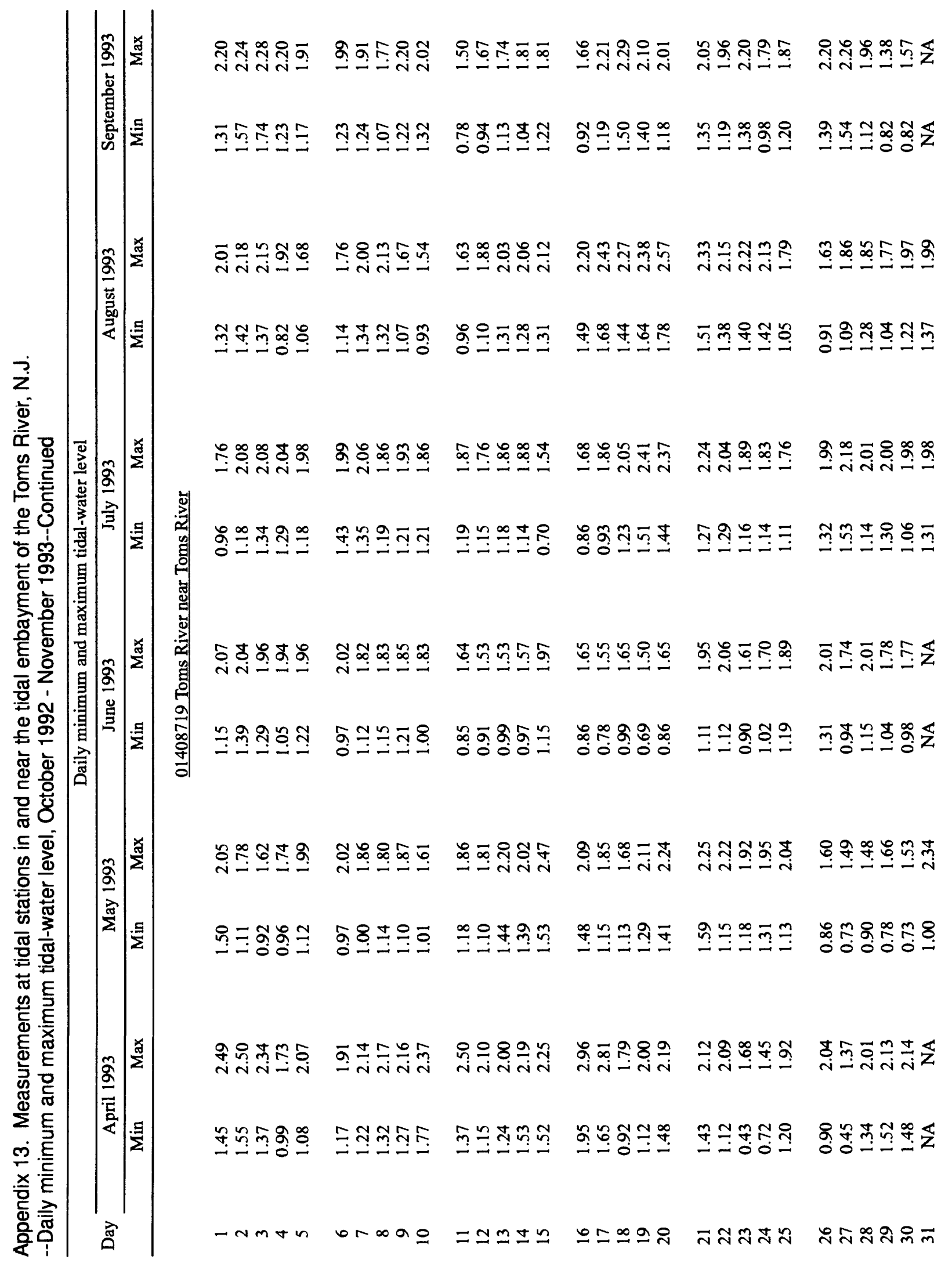




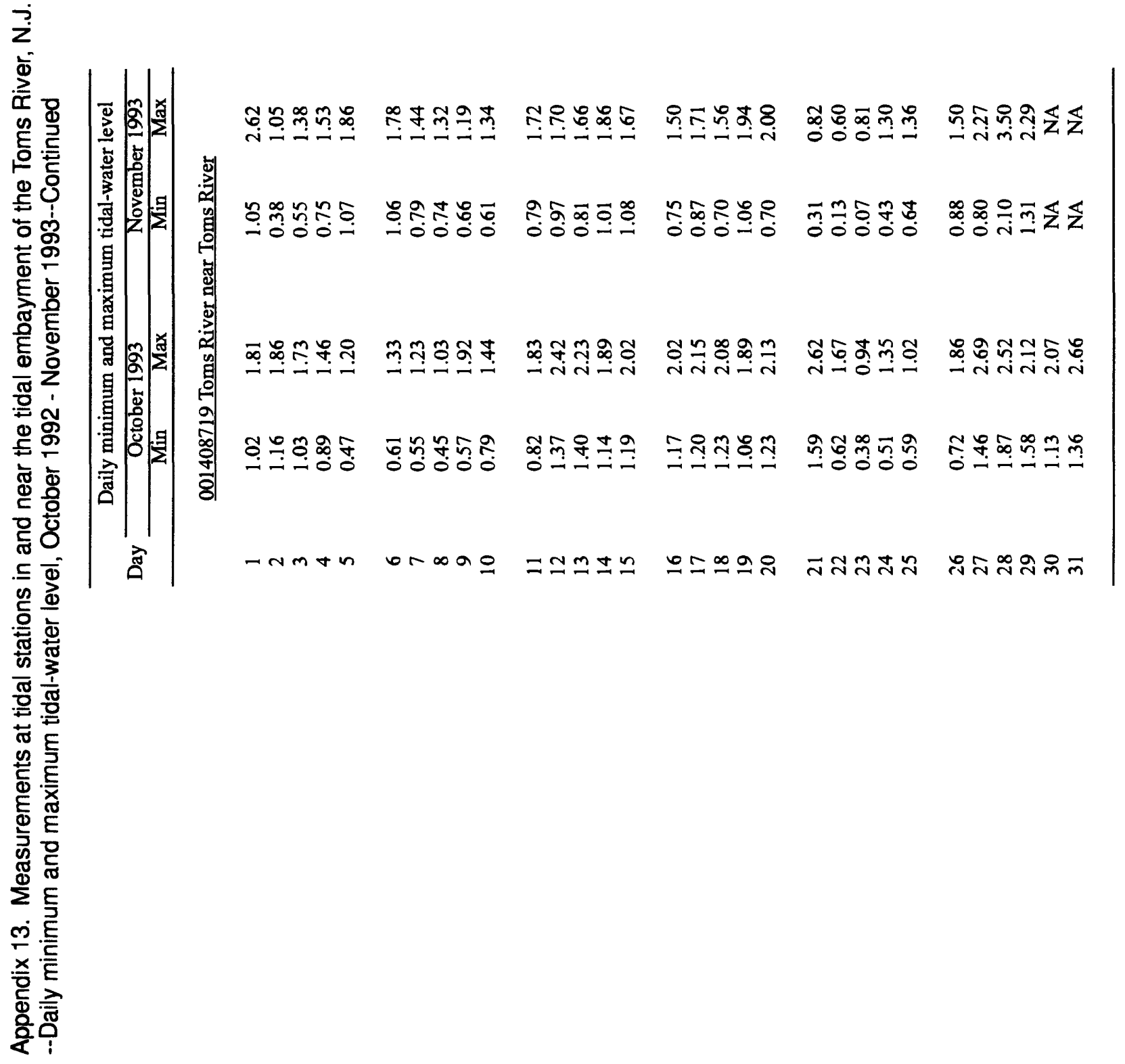


Appendix 14. Measurements at tidal stations in and near the tidal embayment of the Toms River, N.J.--Daily minimum and maximum temperature from measurements by fixed monitors,

October 1992 - January 1994

[Values of temperature are presented for the stations and periods listed below. Values are in degrees Celsius and rounded to the nearest 0.5 degree. USGS, U.S. Geological Survey; Min, daily minimum; Max, daily maximum; NA, not available or not applicable]

\begin{tabular}{|c|c|c|c|c|c|}
\hline $\begin{array}{l}\text { Index } \\
\text { number } \\
\text { (fig. 3) }\end{array}$ & $\begin{array}{l}\text { USGS station } \\
\text { number }\end{array}$ & USGS station name & Period of operation & $\begin{array}{l}\text { Location in } \\
\text { cross section }\end{array}$ & $\begin{array}{l}\text { Approximate } \\
\text { depth of } \\
\text { measurement, } \\
\text { in feet }\end{array}$ \\
\hline 21 & 01408700 & $\begin{array}{c}\text { Toms River at Toms } \\
\text { River }\end{array}$ & $\begin{array}{l}\text { October } 1992 \text { - } \\
\text { January } 1994\end{array}$ & Mid-depth & 3 \\
\hline 22 & 01408719 & $\begin{array}{c}\text { Toms River at Cedar } \\
\text { Point at South } \\
\text { Toms River }\end{array}$ & $\begin{array}{l}\text { December } 1992- \\
\text { October } 1993\end{array}$ & $\begin{array}{l}\text { In channel near } \\
\text { surface, and } \\
\text { in channel near } \\
\text { bottom }\end{array}$ & $\begin{array}{l}1 \\
6\end{array}$ \\
\hline
\end{tabular}




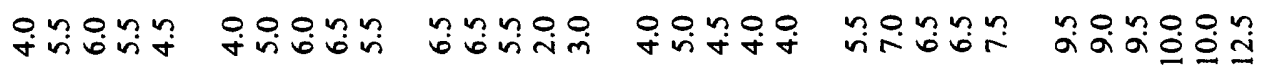

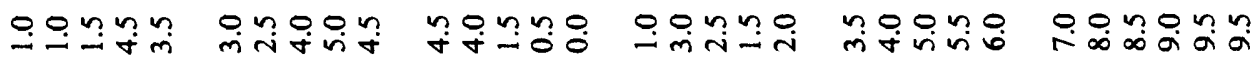

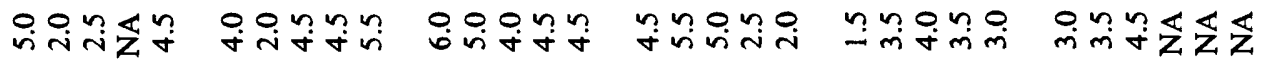

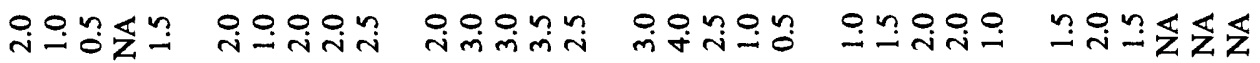
물

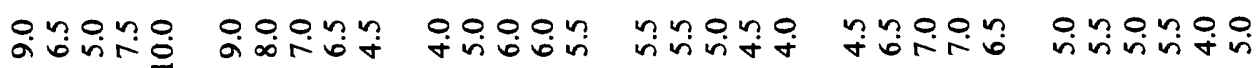
nูy

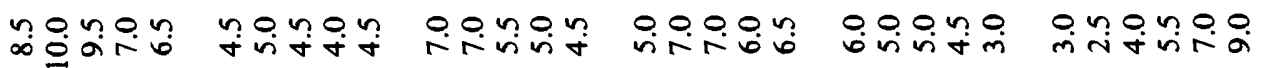

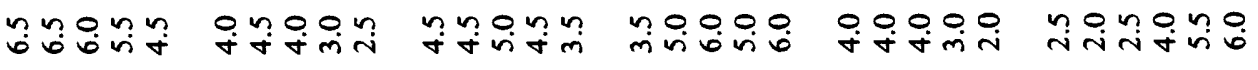

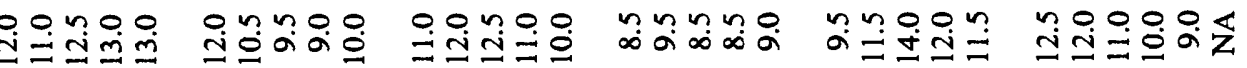

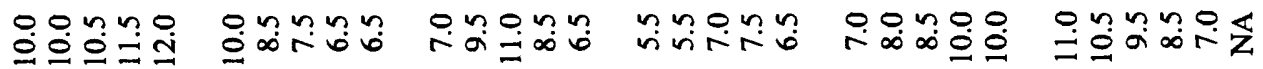

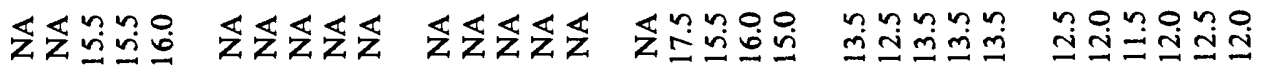

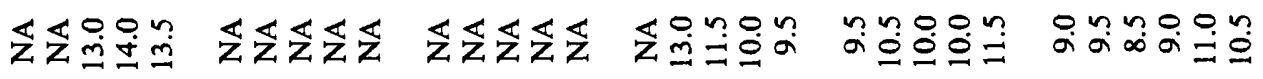

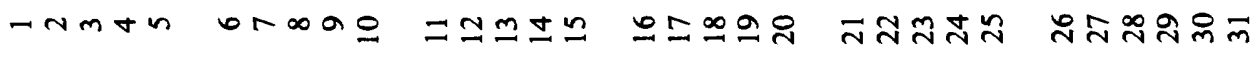




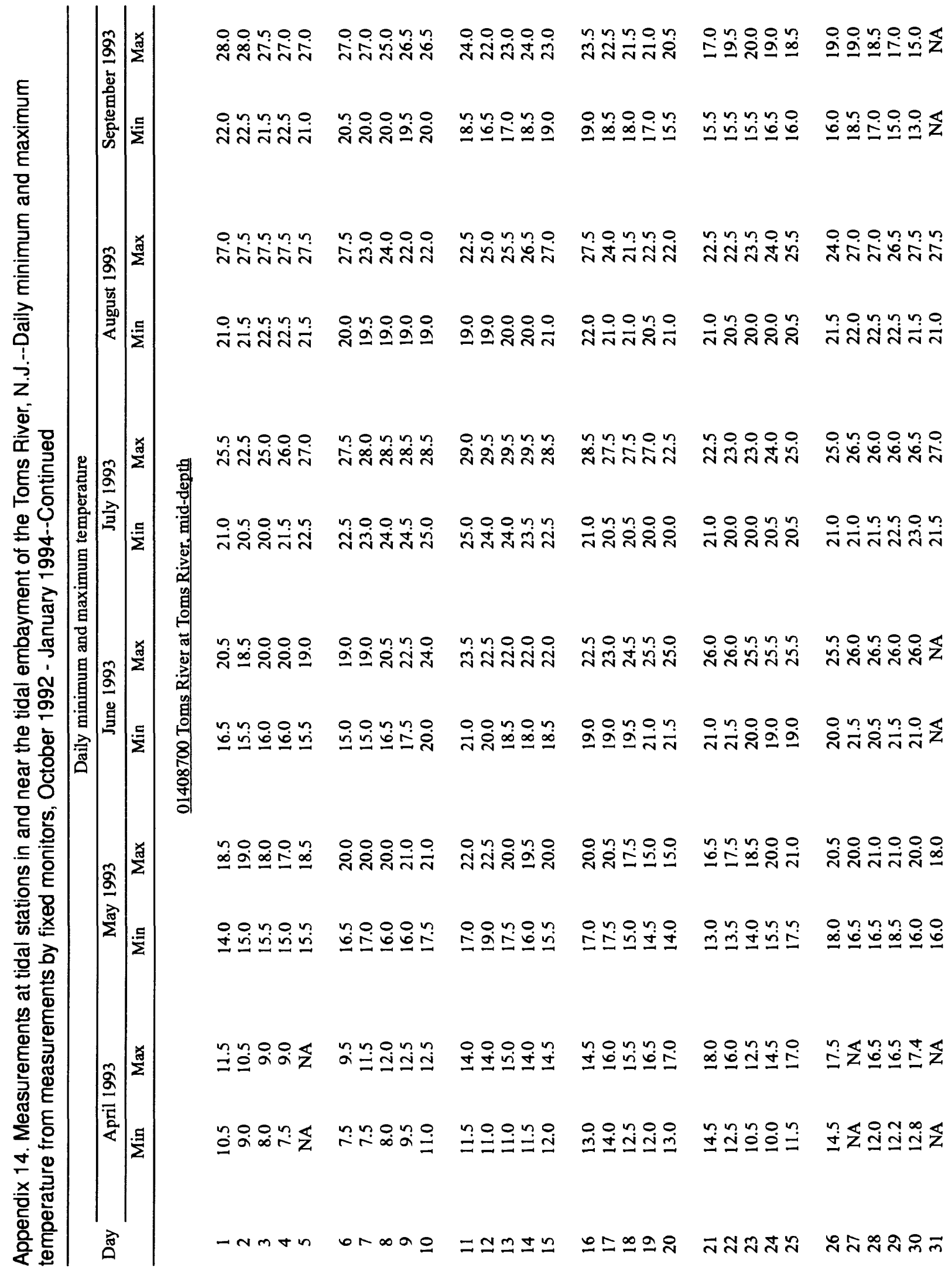




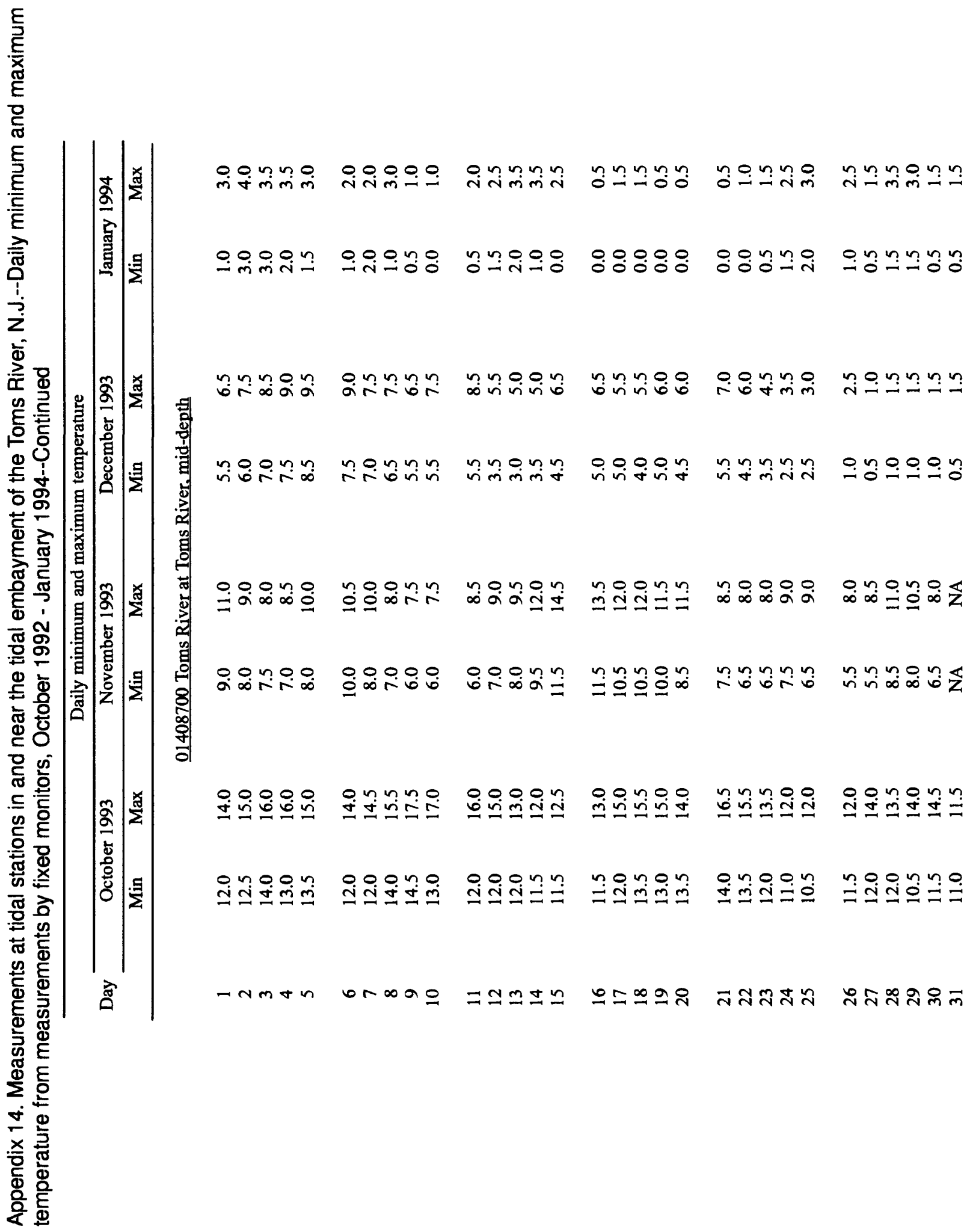




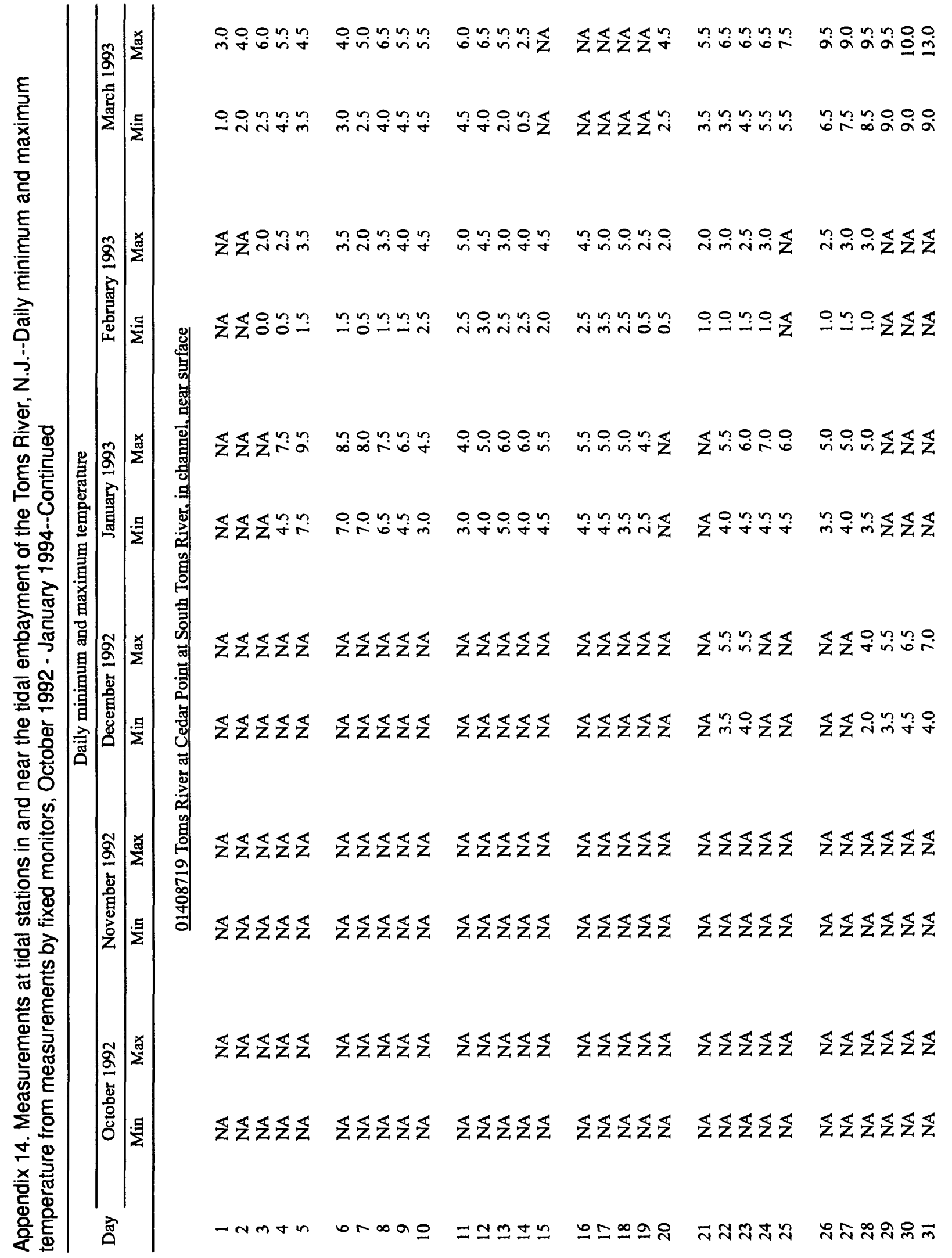




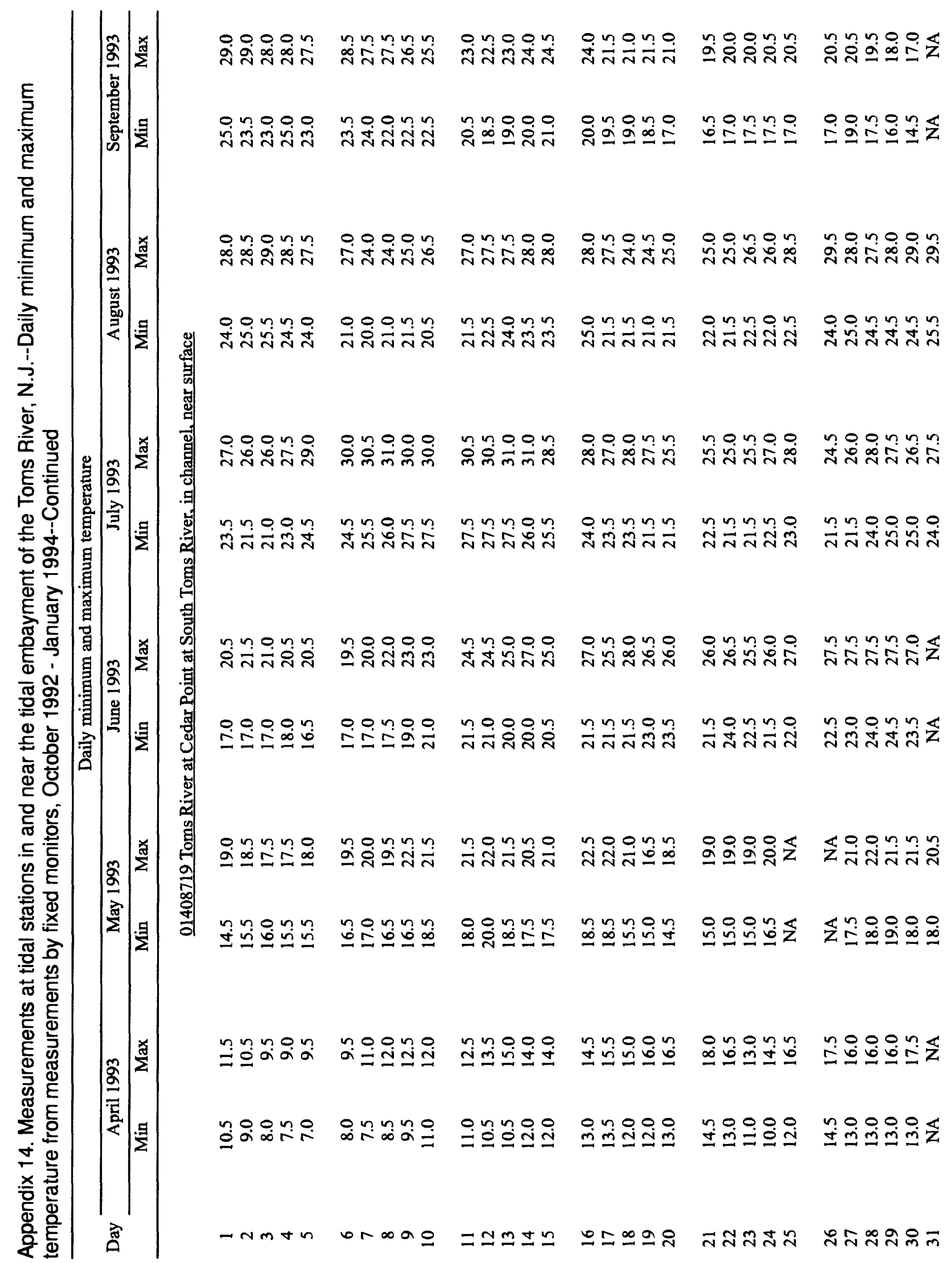




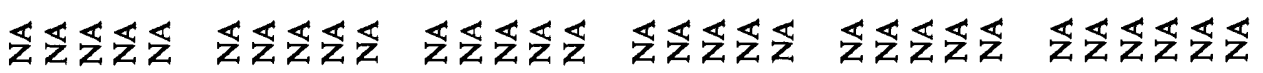

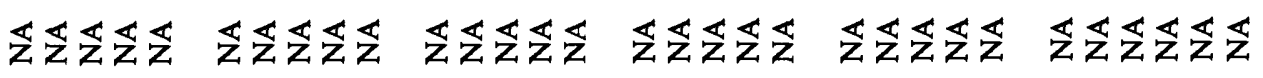

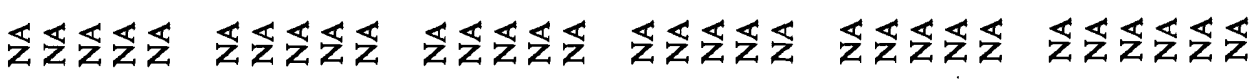

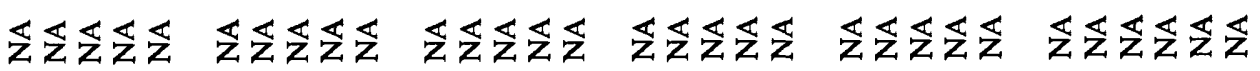

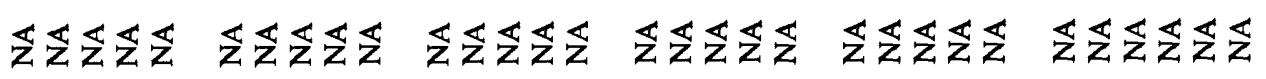

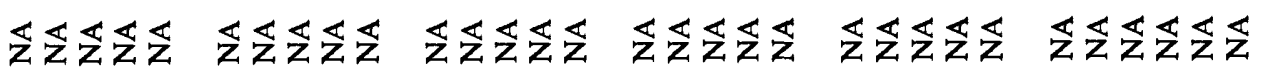

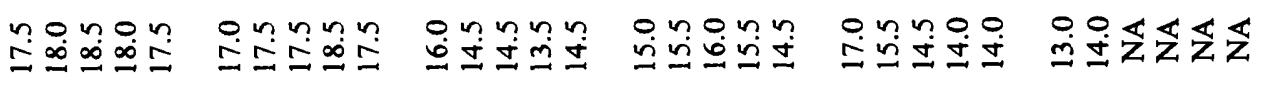

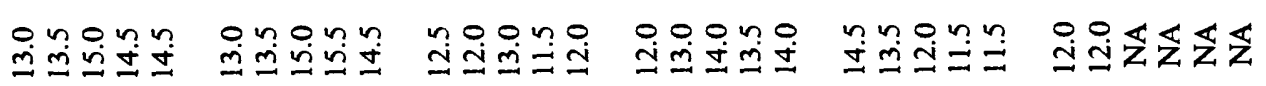
옹 要

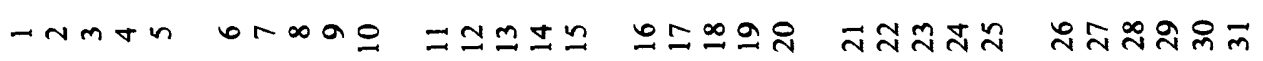




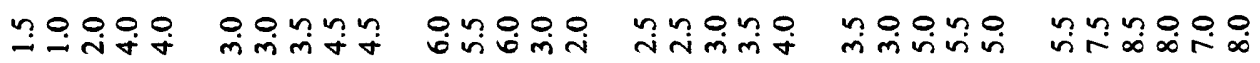

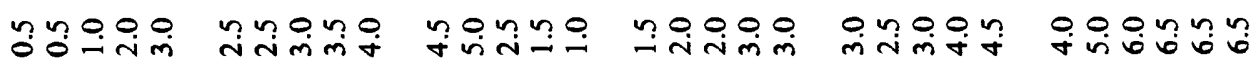

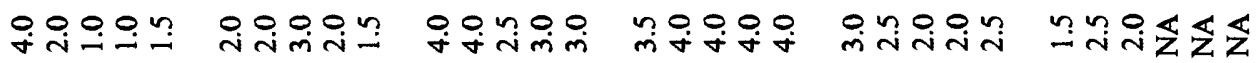

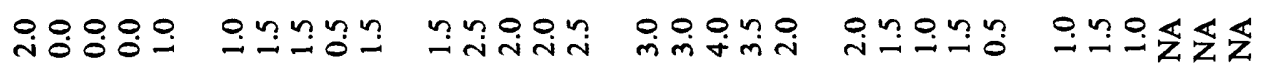

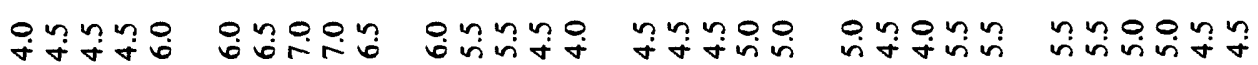

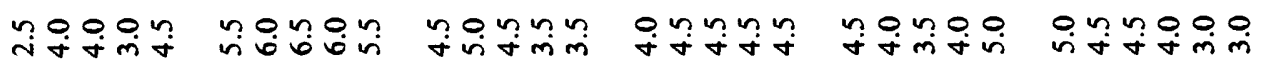

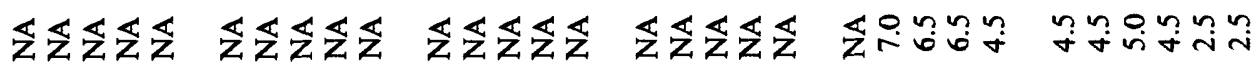

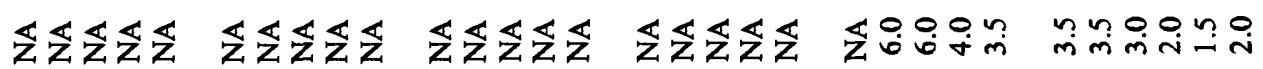

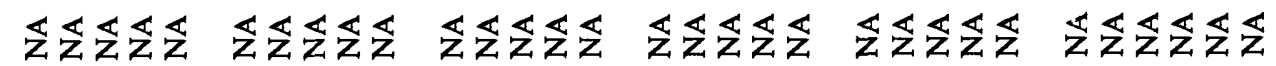

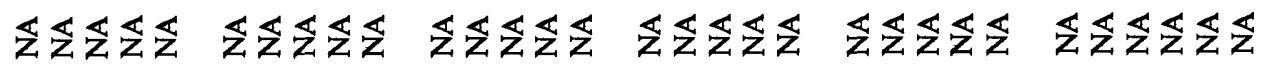

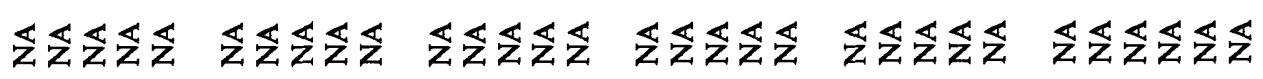

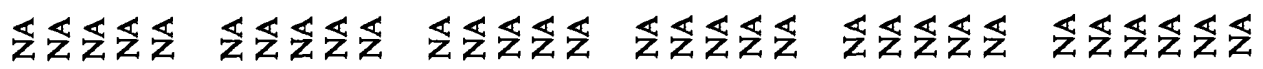

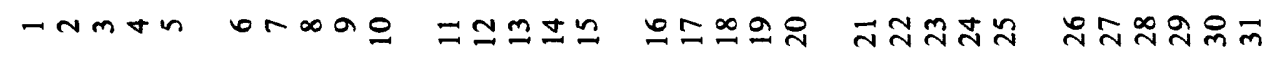




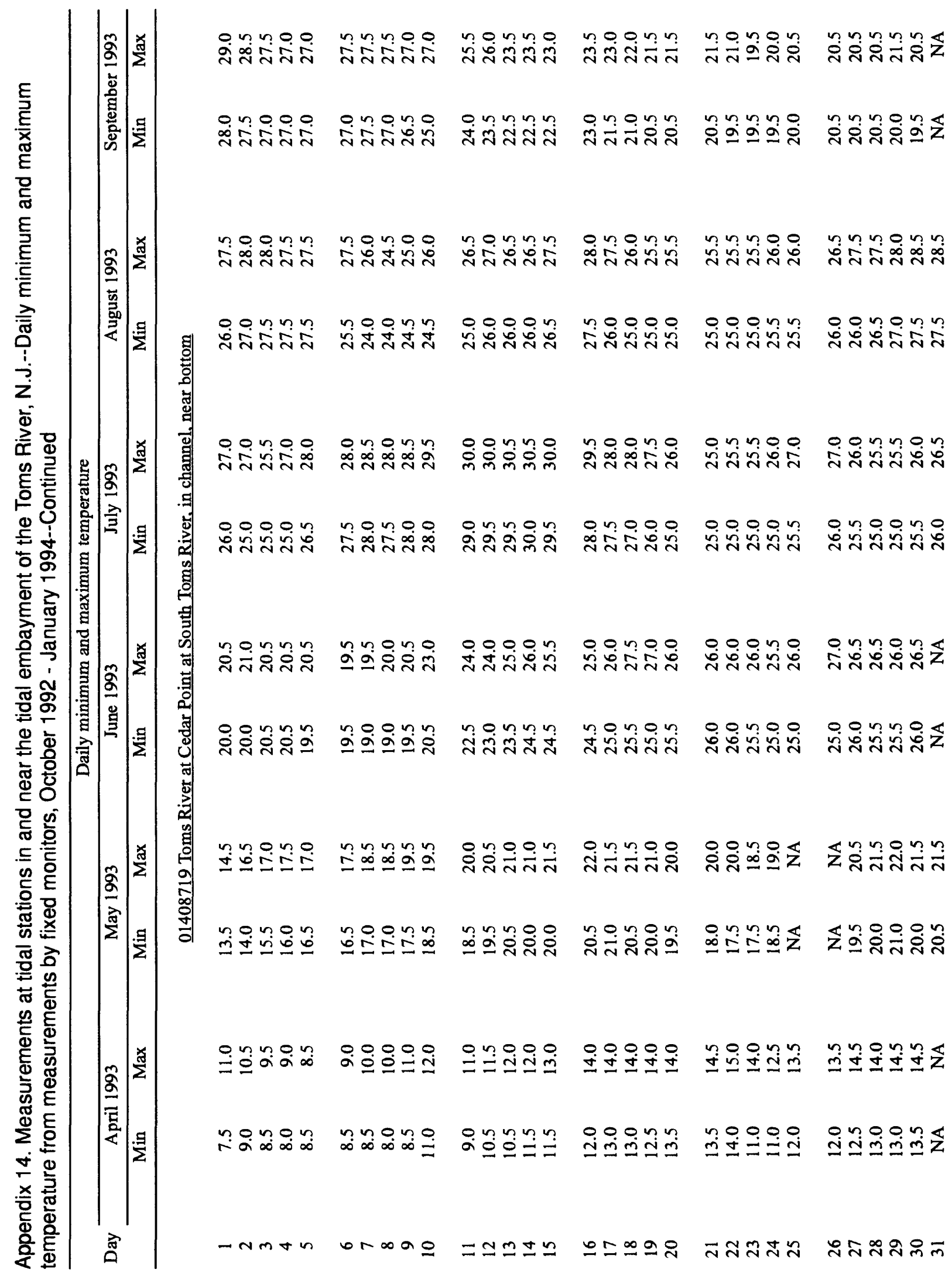




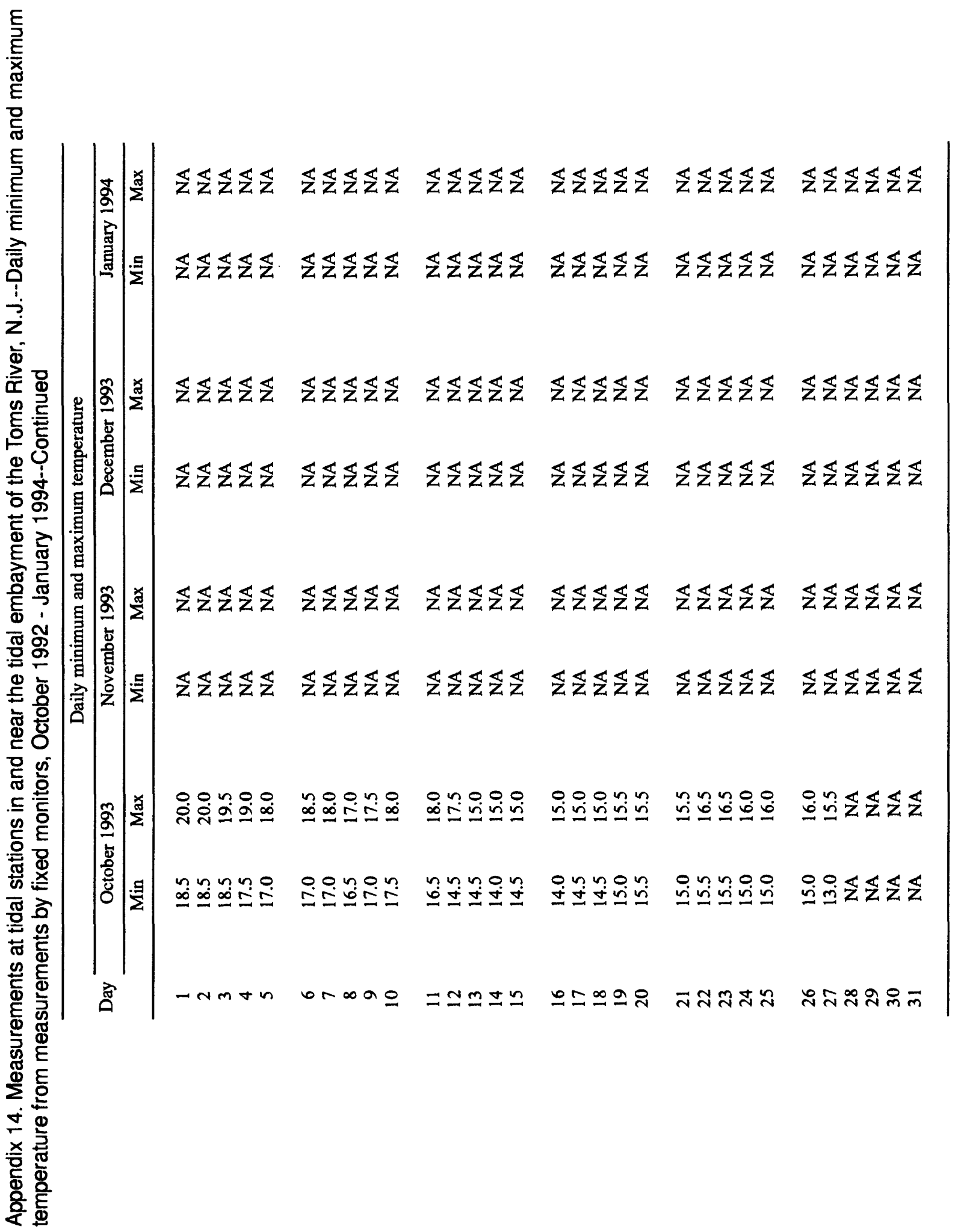


Appendix 15. Measurements at tidal stations in and near the tidal embayment of the Toms River, N.J.--Daily minimum and maximum specific conductance from measurements by fixed monitors, October 1992 - January 1994

[Values of specific conductance are presented for the stations and periods listed below. Values are in microsiemens per centimeter at 25 degrees Celsius and rounded to the nearest hundred. Min, daily minimum; Max, daily maximum; NA, not available or not applicable]

\begin{tabular}{|c|c|c|c|c|c|}
\hline $\begin{array}{l}\text { Index } \\
\text { number } \\
\text { (fig. 3) }\end{array}$ & $\begin{array}{l}\text { USGS station } \\
\text { number }\end{array}$ & USGS station name & Period of operation & $\begin{array}{l}\text { Location in } \\
\text { cross section }\end{array}$ & $\begin{array}{l}\text { Approximate } \\
\text { depth of } \\
\text { measurement, } \\
\text { in feet }\end{array}$ \\
\hline 21 & 01408700 & $\begin{array}{l}\text { Toms River at Toms } \\
\text { River }\end{array}$ & $\begin{array}{l}\text { October } 1992 \text { - } \\
\text { January } 1994\end{array}$ & Mid-depth & 3 \\
\hline 22 & 01408719 & $\begin{array}{l}\text { Toms River at Cedar } \\
\text { Point at South } \\
\text { Toms River }\end{array}$ & $\begin{array}{l}\text { December } 1992- \\
\text { October } 1993\end{array}$ & $\begin{array}{l}\text { In channel near } \\
\text { surface, and } \\
\text { in channel near } \\
\text { bottom }\end{array}$ & $\begin{array}{l}1 \\
6\end{array}$ \\
\hline
\end{tabular}




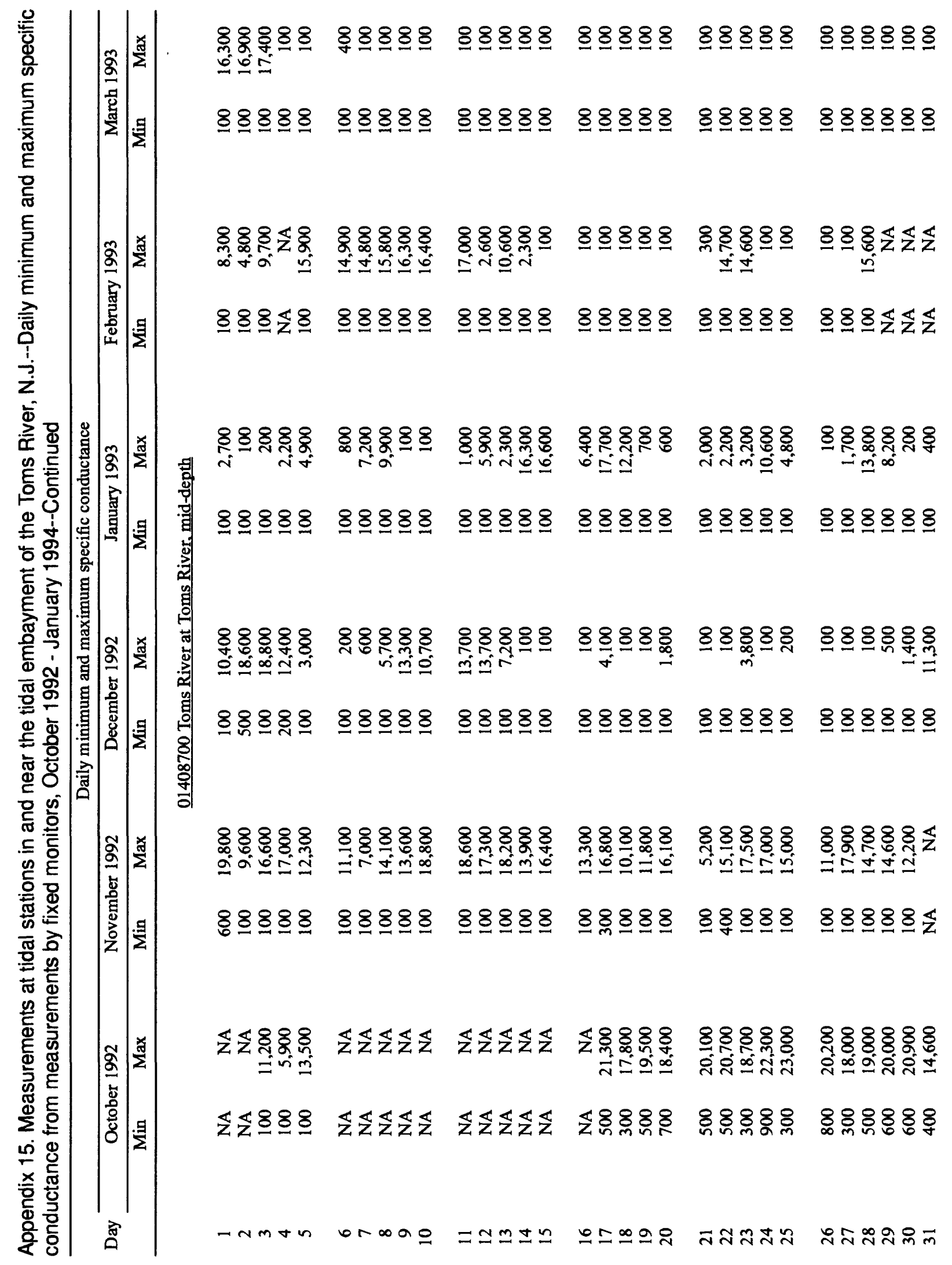




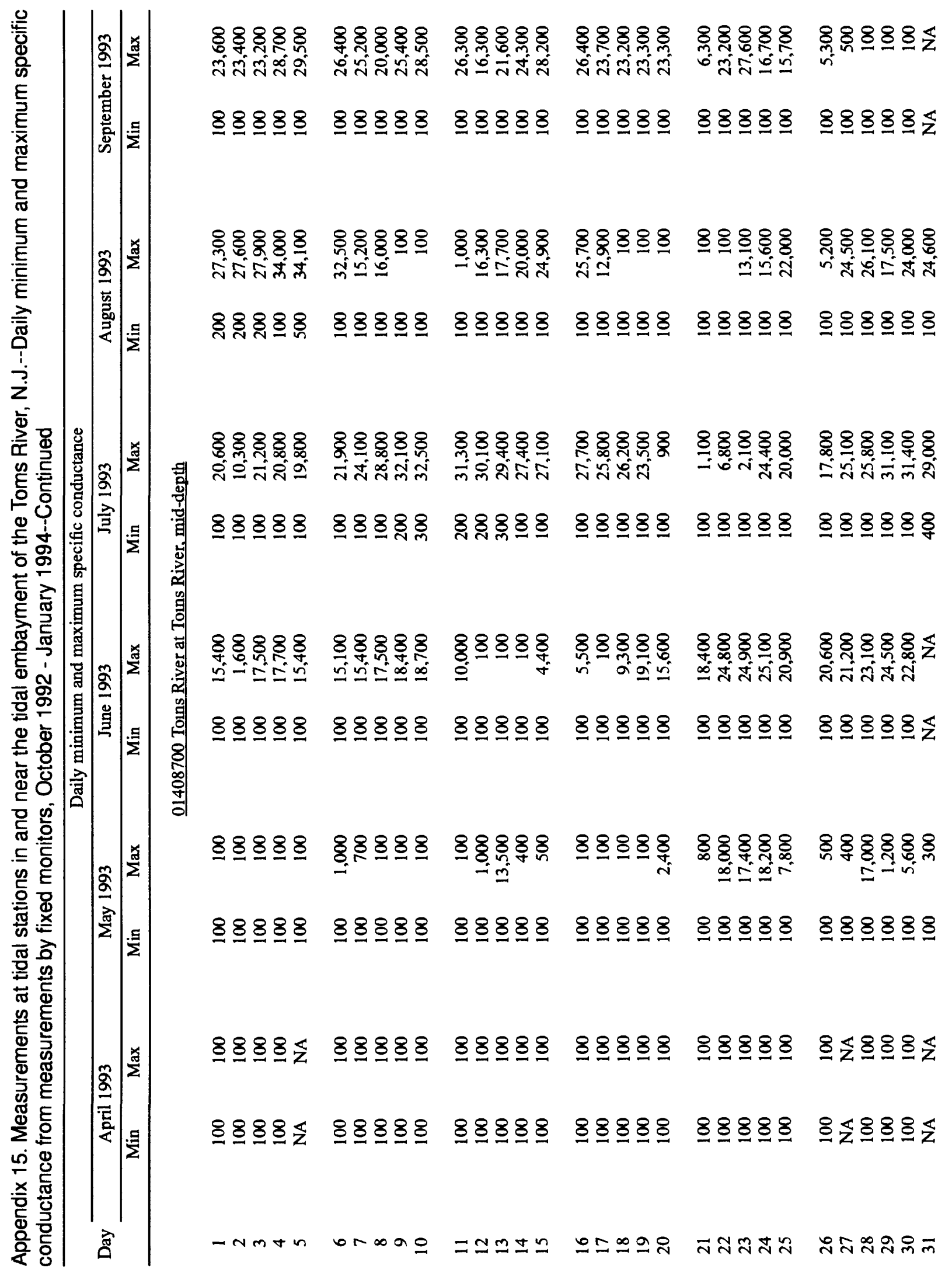




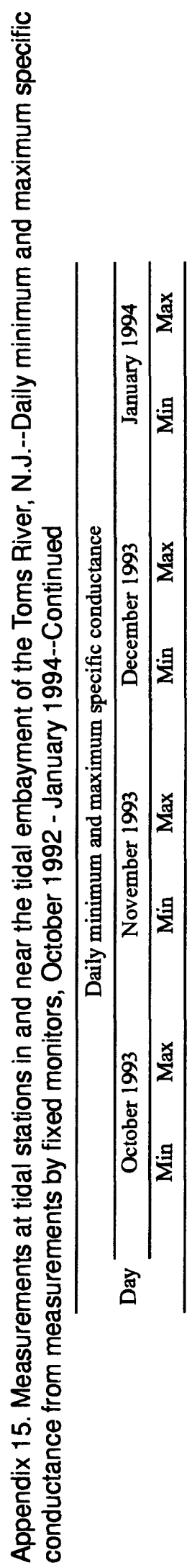

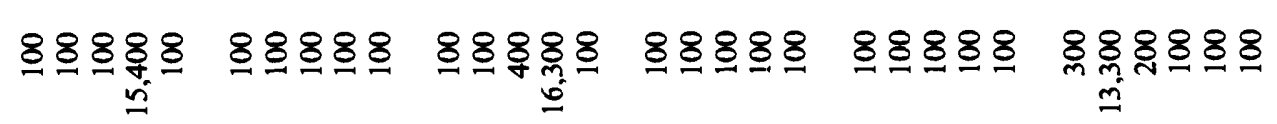

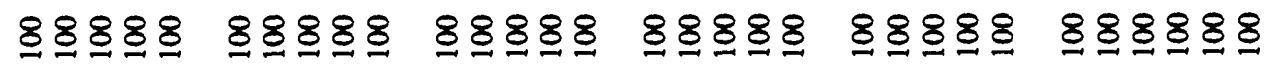

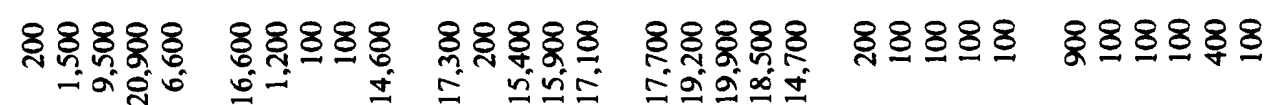

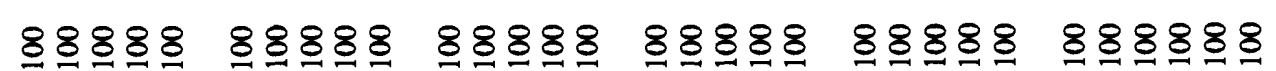

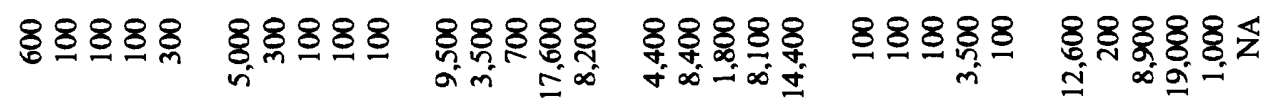

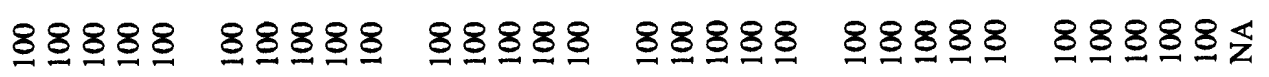

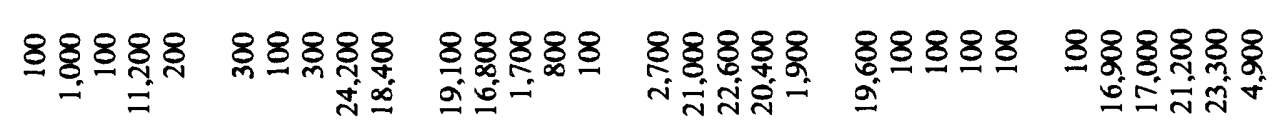

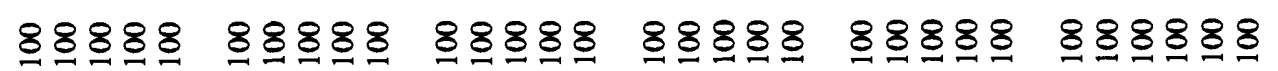
ーnm+n คrmaอ 


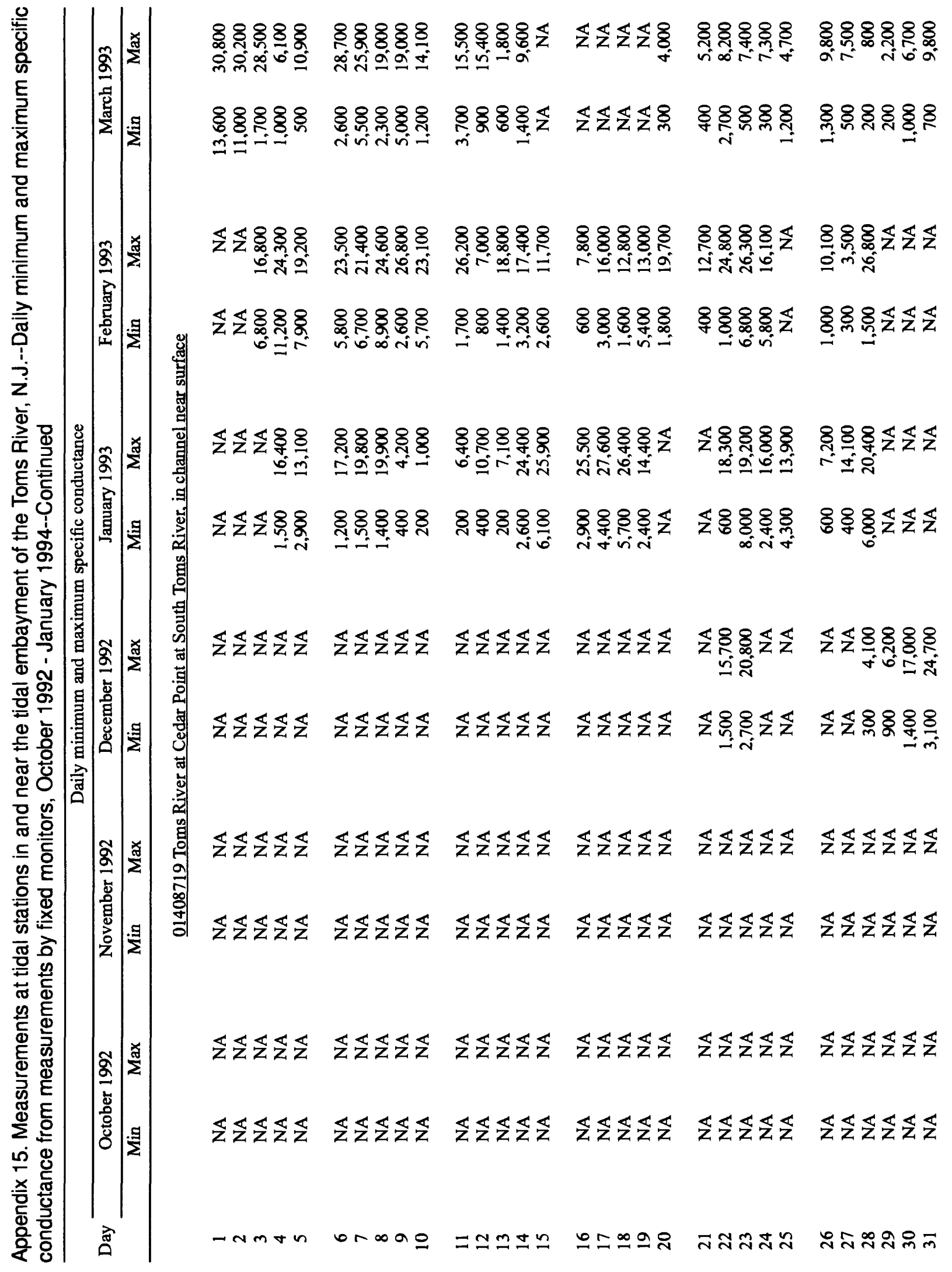




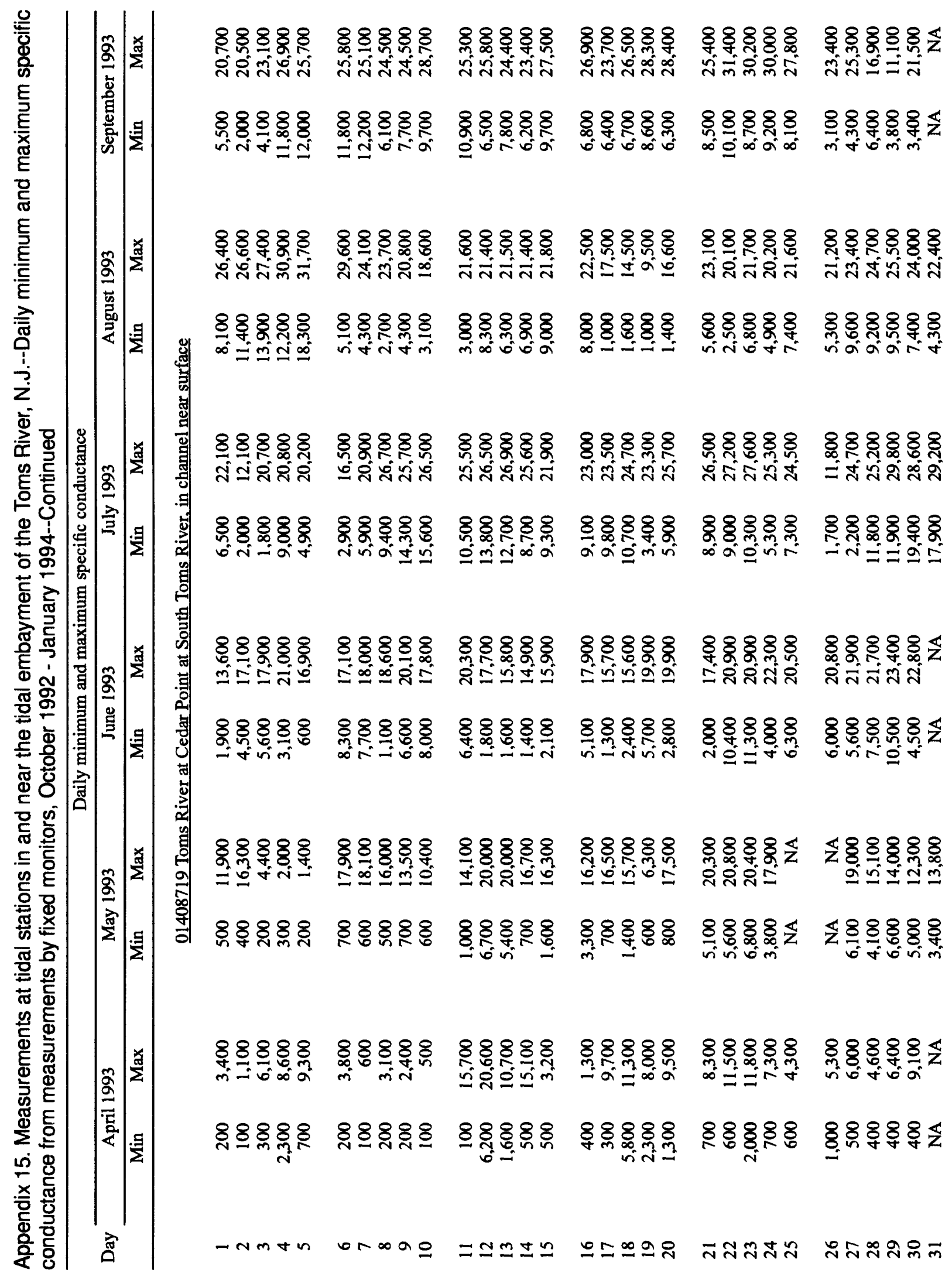




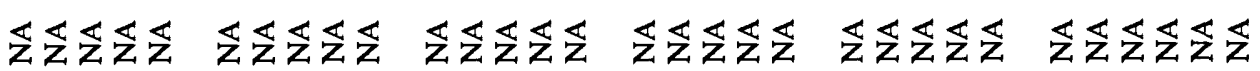

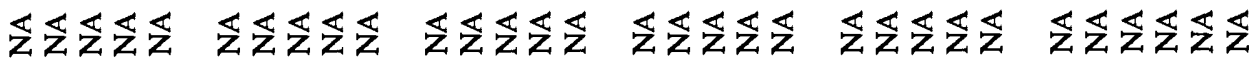

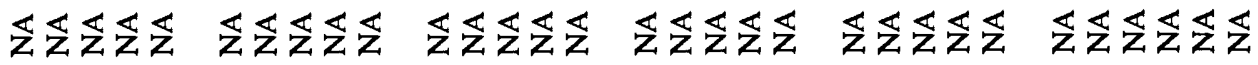

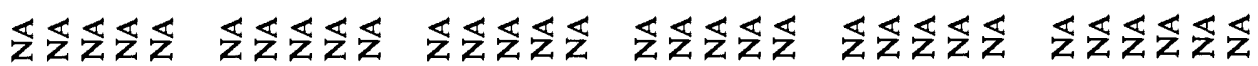

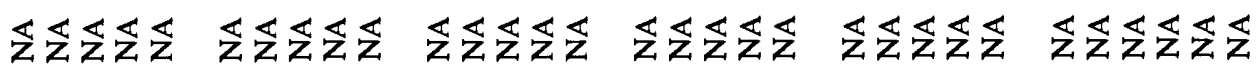

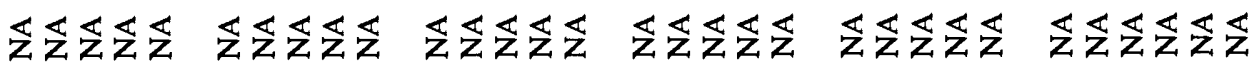

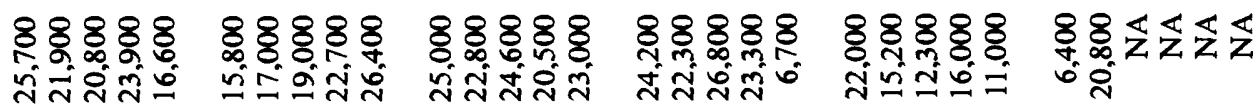

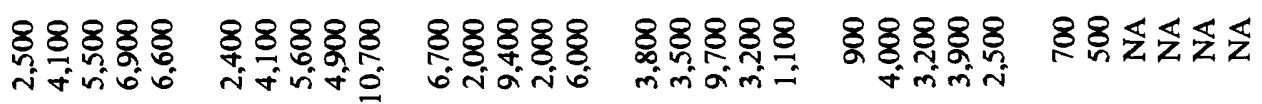

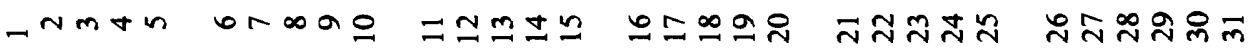
ब苛 ค 용 जह $\stackrel{\mathbb{\Phi}}{\Sigma}$ 은 ம 증 㺃 힝 웅 엉 


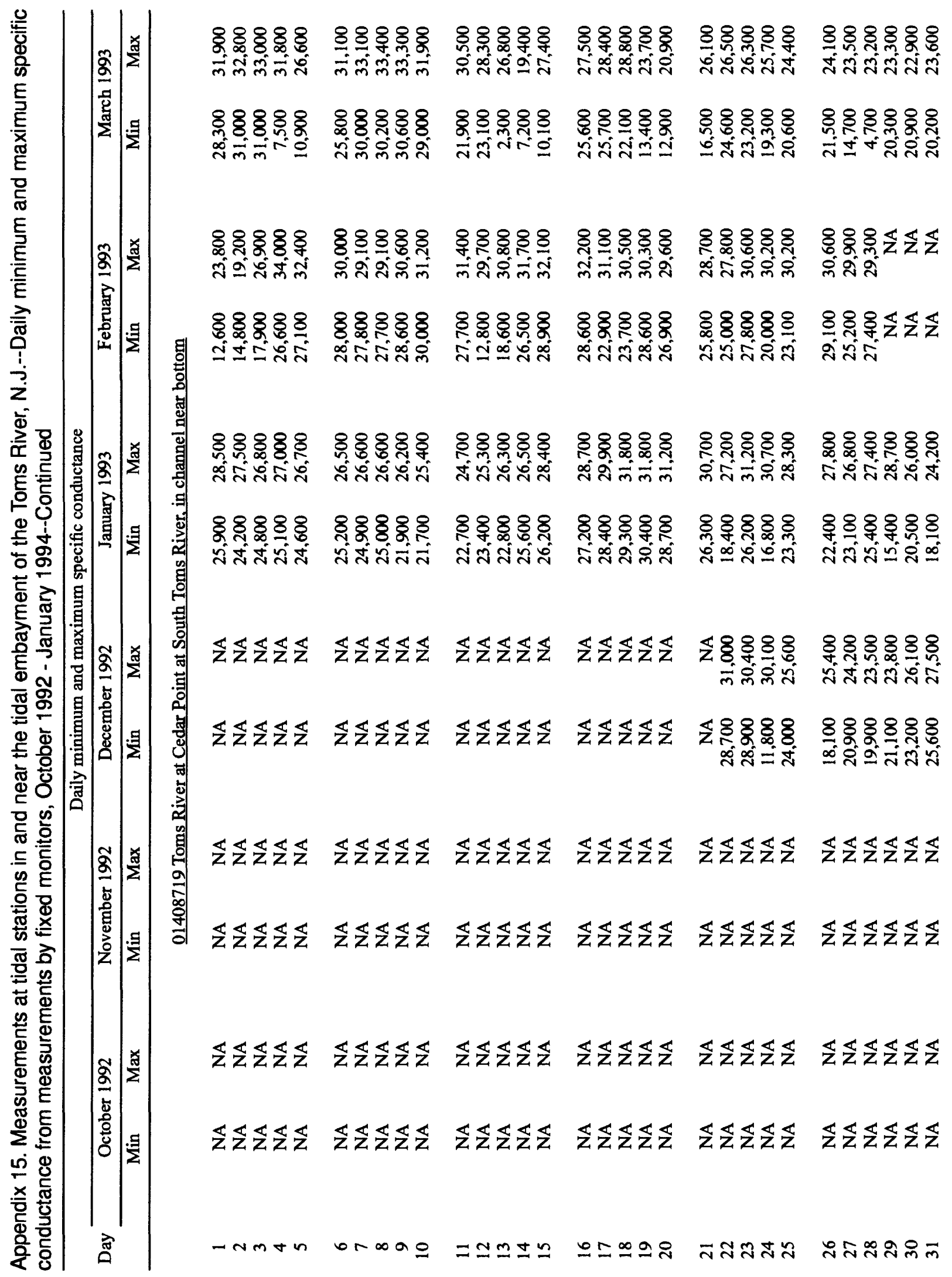




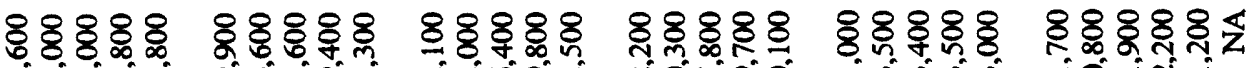

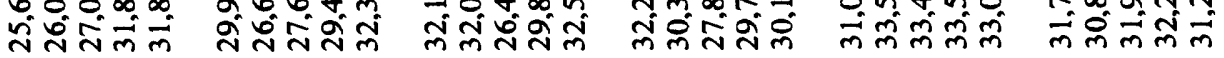

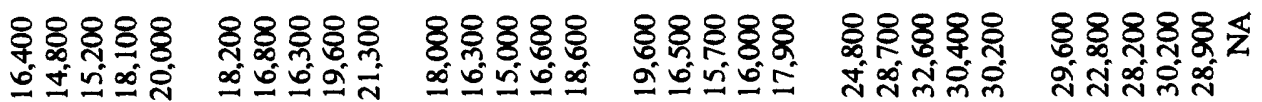

\begin{tabular}{|c|c|c|c|c|c|}
\hline & 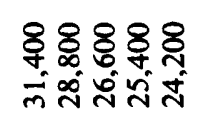 & 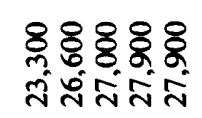 & 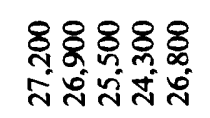 & 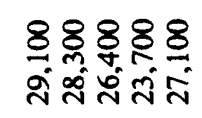 & 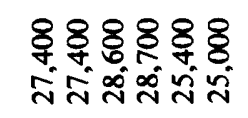 \\
\hline & & & & & \\
\hline
\end{tabular}

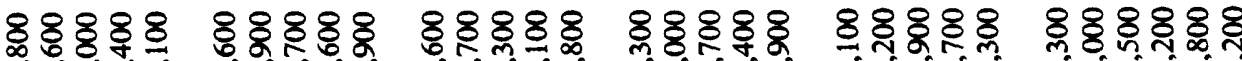

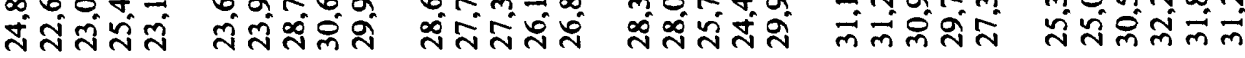

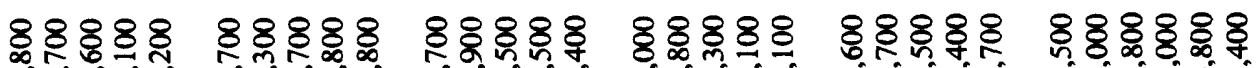

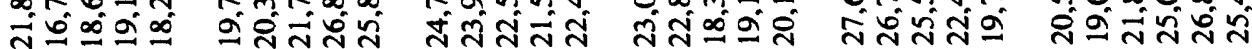

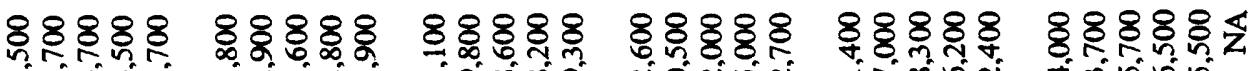

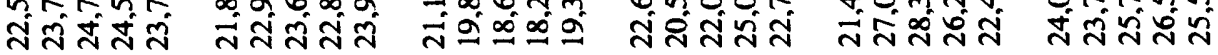

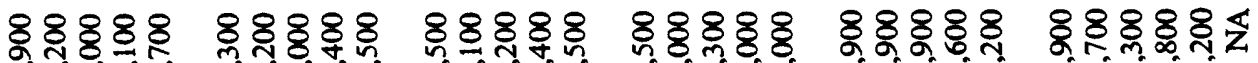

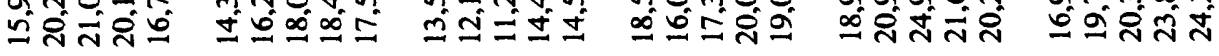

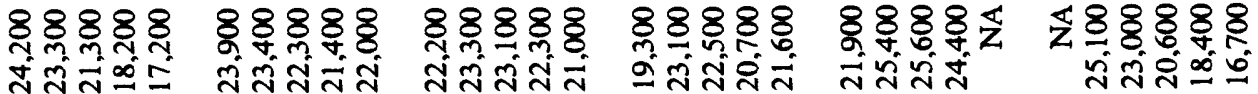

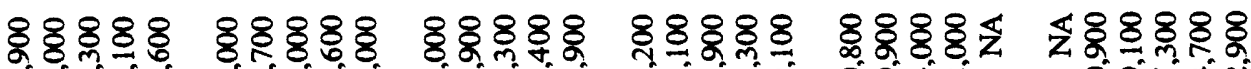

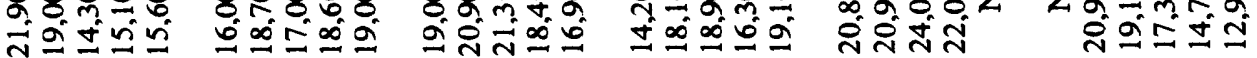

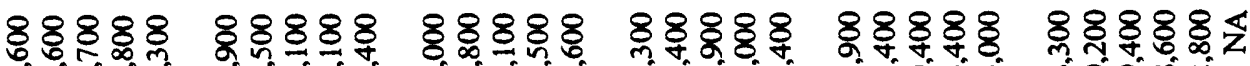

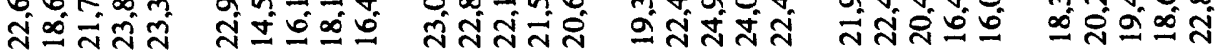

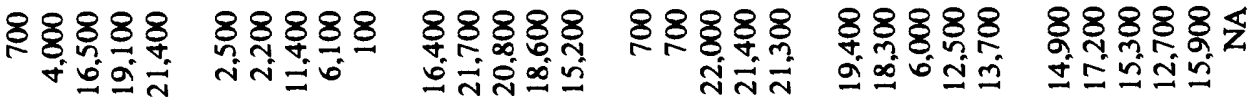

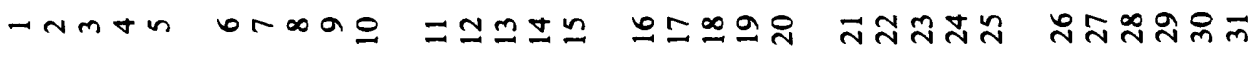




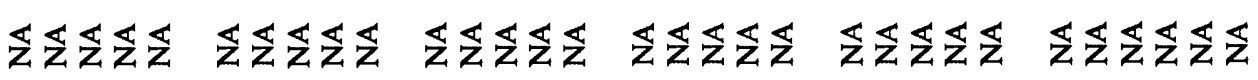

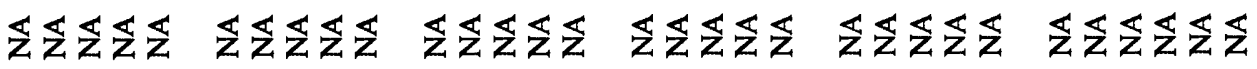

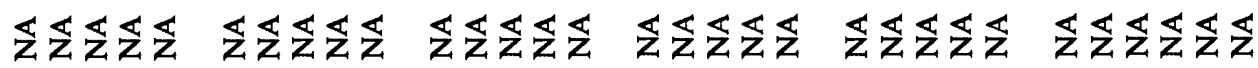

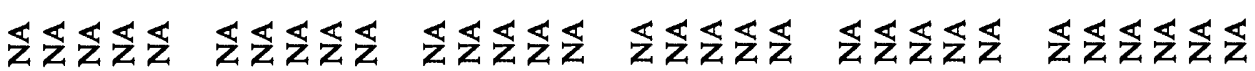

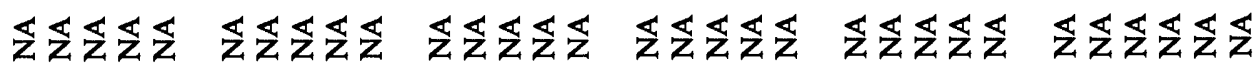

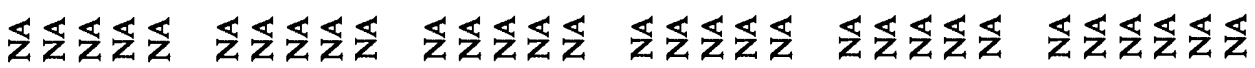

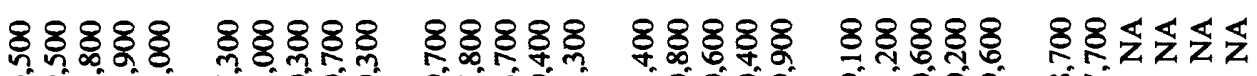

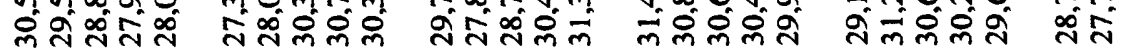

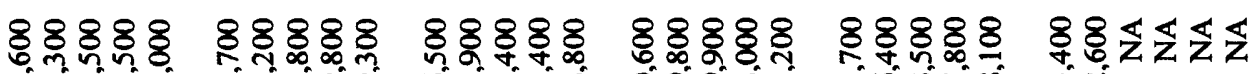

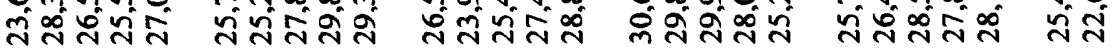

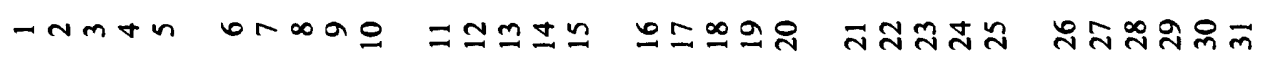


Appendix 16. Measurements at tidal stations in and near the tidal embayment of the Toms River, N.J.--Daily minimum and maximum values of water-quality characteristics from measurements by movable monitors, October 1992 - January 1994

[Monitors were deployed at the stations listed below for the indicated periods. USGS, U.S. Geological Survey; Min, daily minimum; Max, daily maximum; NA, not available or not applicable]

\begin{tabular}{|c|c|c|c|c|c|}
\hline $\begin{array}{l}\text { Index } \\
\text { number } \\
\text { (fig. 3) }\end{array}$ & USGS station number & USGS station name & $\begin{array}{c}\text { Periods of } \\
\text { operation } \\
\text { (year/month/day) }\end{array}$ & $\begin{array}{l}\text { Location in } \\
\text { cross section }\end{array}$ & $\begin{array}{l}\text { Approximate } \\
\text { depth of } \\
\text { measurement, } \\
\text { in feet }\end{array}$ \\
\hline 18 & 01408685 & $\begin{array}{l}\text { Toms River at Garden } \\
\text { State Parkway }\end{array}$ & $93 / 08 / 31-93 / 09 / 08$ & Near bottom & 5 \\
\hline 19 & 01408690 & $\begin{array}{l}\text { Toms River } 0.2 \text { miles } \\
\text { downstream from } \\
\text { Garden State } \\
\text { Parkway }\end{array}$ & $\begin{array}{l}93 / 07 / 28-93 / 08 / 03 \\
93 / 08 / 11-93 / 08 / 24 \\
93 / 09 / 22-93 / 10 / 19 \\
93 / 10 / 19-93 / 11 / 18 \\
93 / 12 / 02-94 / 01 / 28\end{array}$ & Near bottom & 5 \\
\hline 20 & 01408695 & $\begin{array}{l}\text { Toms River near } \\
\text { South Toms River }\end{array}$ & $\begin{array}{l}92 / 10 / 30-92 / 11 / 24 \\
92 / 12 / 02-92 / 12 / 22 \\
92 / 12 / 22-92 / 12 / 28 \\
93 / 01 / 20-93 / 02 / 11 \\
93 / 04 / 05-93 / 04 / 19 \\
93 / 04 / 27-93 / 05 / 25 \\
93 / 05 / 25-93 / 06 / 23 \\
93 / 06 / 23-93 / 08 / 11\end{array}$ & Mid-depth & 3 \\
\hline 23 & 01408722 & $\begin{array}{l}\text { Toms River near Toms } \\
\text { River }\end{array}$ & $\begin{array}{l}93 / 06 / 15-93 / 06 / 23 \\
93 / 10 / 21-93 / 10 / 28\end{array}$ & $\begin{array}{l}\text { Near surface, } \\
\text { and } \\
\text { near bottom }\end{array}$ & $\begin{array}{l}1 \\
6\end{array}$ \\
\hline
\end{tabular}


Appendix 16. Measurements at tidal stations in and near the tidal embayment of the Toms River, N.J.--Daily minimum and maximum values of water-quality characteristics from measurements by movable monitors, October 1992 - January 1994--Continued

The following characteristics were measured. Intervals between measurements were 15, 30 , or 60 minutes. Days with partial records are included.

\begin{tabular}{llc}
\hline \multicolumn{1}{c}{ Characteristic } & Unit & Rounding \\
\hline & & \\
Temperature & Degrees Celsius & 0.5 \\
Specific conductance & Microsiemens per centimeter at & 100 \\
25 degrees Celsius & \\
pH & Standard units & 0.1 \\
$\begin{array}{c}\text { Dissolved-oxygen } \\
\text { concentration }\end{array}$ & Milligrams per liter & 0.1 \\
& & \\
\hline
\end{tabular}


Appendix 16. Measurements at tidal stations in and near the tidal embayment of the Toms River, N.J.--Daily minimum and maximum values of water-quality characteristics from measurements by movable monitors, October 1992 - January 1994--Continued

\begin{tabular}{|c|c|c|c|c|c|c|c|c|}
\hline \multirow{3}{*}{$\begin{array}{c}\text { Date } \\
\text { year/month/day }\end{array}$} & \multicolumn{8}{|c|}{ Daily minimum and maximum } \\
\hline & \multicolumn{2}{|c|}{ Temperature } & \multicolumn{2}{|c|}{ Specific conductance } & \multicolumn{2}{|c|}{ pH } & \multicolumn{2}{|c|}{$\begin{array}{l}\text { Dissolved-oxygen } \\
\text { concentration }\end{array}$} \\
\hline & Min & $\operatorname{Max}$ & Min & $\operatorname{Max}$ & Min & $\operatorname{Max}$ & Min & $\operatorname{Max}$ \\
\hline \multicolumn{9}{|c|}{01408685 Toms River at Garden State Parkway, near bottom } \\
\hline $93 / 08 / 31$ & 22.0 & 22.5 & 100 & 100 & 5.2 & 5.3 & 7.2 & 7.9 \\
\hline $93 / 09 / 01$ & 20.5 & 23.0 & 100 & 100 & 5.1 & 5.2 & 6.8 & 7.9 \\
\hline $93 / 09 / 02$ & 21.0 & 22.0 & 100 & 100 & 5.1 & 5.3 & 6.5 & 7.3 \\
\hline $93 / 09 / 03$ & 21.0 & 23.5 & 100 & 100 & 5.2 & 5.3 & 6.6 & 7.6 \\
\hline $93 / 09 / 04$ & 21.5 & 22.5 & 100 & 100 & 5.2 & 5.3 & 6.2 & 7.3 \\
\hline $93 / 09 / 05$ & 20.5 & 22.0 & 100 & 100 & 5.2 & 5.3 & 6.7 & 7.8 \\
\hline 93/09/06 & 19.5 & 22.0 & 100 & 100 & 5.3 & 5.3 & 7.0 & 8.1 \\
\hline $93 / 09 / 07$ & 19.5 & 20.5 & 100 & 100 & 5.3 & 5.3 & 7.0 & 8.3 \\
\hline $93 / 09 / 08$ & 19.5 & 20.0 & 100 & 100 & 5.3 & 5.3 & 7.2 & 7.5 \\
\hline
\end{tabular}

01408690 Toms River 0.2 miles downstream from Garden State Parkway, near bottom

$\begin{array}{lrllrllll}93 / 07 / 28 & 23.0 & 24.0 & 100 & 15,300 & 5.1 & 7.0 & 5.6 & 8.0 \\ 93 / 07 / 29 & 22.0 & 25.0 & 100 & 26,400 & 5.1 & 7.0 & 3.8 & 7.7 \\ 93 / 07 / 30 & 21.5 & 25.0 & 100 & 27,500 & 5.1 & 5.8 & 2.4 & 7.5 \\ 93 / 07 / 31 & 20.5 & 22.5 & 100 & 500 & 5.1 & 6.6 & 6.3 & 7.7 \\ 93 / 08 / 01 & 20.0 & 24.0 & 100 & 15,300 & 5.1 & 6.8 & 5.1 & 7.8 \\ 93 / 08 / 02 & 20.5 & 24.0 & 100 & 19,500 & 5.1 & 5.5 & 4.5 & 7.9 \\ 93 / 08 / 03 & 21.5 & 23.0 & 100 & 15,300 & 5.1 & 7.0 & 6.0 & 6.9 \\ & & & & & & & & \\ 93 / 08 / 11 & 20.5 & 21.0 & 100 & 100 & \text { NA } & \text { NA } & \text { NA } & \text { NA } \\ 93 / 08 / 12 & 18.5 & 21.0 & 100 & 100 & \text { NA } & \text { NA } & \text { NA } & \text { NA } \\ 93 / 08 / 13 & 19.0 & 21.0 & 100 & 100 & \text { NA } & \text { NA } & \text { NA } & \text { NA } \\ 93 / 08 / 14 & 19.5 & 21.5 & 100 & 100 & \text { NA } & \text { NA } & \text { NA } & \text { NA } \\ 93 / 08 / 15 & 20.0 & 22.5 & 100 & 100 & \text { NA } & \text { NA } & \text { NA } & \text { NA } \\ 93 / 08 / 16 & 20.5 & 22.5 & 100 & 6,600 & \text { NA } & \text { NA } & \text { NA } & \text { NA } \\ 93 / 08 / 17 & 20.5 & 21.5 & 100 & 100 & \text { NA } & \text { NA } & \text { NA } & \text { NA } \\ 93 / 08 / 18 & 20.5 & 21.0 & 100 & 100 & \text { NA } & \text { NA } & \text { NA } & \text { NA } \\ 93 / 08 / 19 & 20.0 & 21.5 & 100 & 100 & \text { NA } & \text { NA } & \text { NA } & \text { NA } \\ 93 / 08 / 20 & 20.5 & 21.5 & 100 & 100 & \text { NA } & \text { NA } & \text { NA } & \text { NA } \\ 93 / 08 / 21 & 21.0 & 22.0 & 100 & 100 & \text { NA } & \text { NA } & \text { NA } & \text { NA } \\ 93 / 08 / 22 & 20.0 & 22.0 & 100 & 100 & \text { NA } & \text { NA } & \text { NA } & \text { NA } \\ 93 / 08 / 23 & 19.5 & 22.0 & 100 & 100 & \text { NA } & \text { NA } & \text { NA } & \text { NA } \\ 93 / 08 / 24 & 19.5 & 20.5 & 100 & 100 & \text { NA } & \text { NA } & \text { NA } & \text { NA } \\ 93 / 09 / 22 & 16.0 & 16.0 & 100 & 100 & \text { NA } & \text { NA } & \text { NA } & \text { NA } \\ 93 / 09 / 23 & 15.5 & 17.0 & 100 & 100 & \text { NA } & \text { NA } & \text { NA } & \text { NA } \\ 93 / 09 / 24 & 16.5 & 17.5 & 100 & 100 & \text { NA } & \text { NA } & \text { NA } & \text { NA } \\ 93 / 09 / 25 & 15.5 & 16.5 & 100 & 100 & \text { NA } & \text { NA } & \text { NA } & \text { NA } \\ 93 / 09 / 26 & 16.0 & 18.5 & 100 & 100 & \text { NA } & \text { NA } & \text { NA } & \text { NA } \\ 93 / 09 / 27 & 18.0 & 19.0 & 100 & 100 & \text { NA } & \text { NA } & \text { NA } & \text { NA } \\ 93 / 09 / 28 & 16.5 & 18.5 & 100 & 100 & \text { NA } & \text { NA } & \text { NA } & \text { NA }\end{array}$


Appendix 16. Measurements at tidal stations in and near the tidal embayment of the Toms River, N.J.--Daily minimum and maximum values of water-quality characteristics from measurements by movable monitors, October 1992 - January 1994--Continued

\begin{tabular}{|c|c|c|c|c|c|c|c|c|}
\hline \multirow{3}{*}{$\begin{array}{c}\text { Date } \\
\text { year/month/day }\end{array}$} & \multicolumn{8}{|c|}{ Daily minimum and maximum } \\
\hline & \multicolumn{2}{|c|}{ Temperature } & \multicolumn{2}{|c|}{ Specific conductance } & \multicolumn{2}{|c|}{$\mathrm{pH}$} & \multicolumn{2}{|c|}{$\begin{array}{l}\text { Dissolved-oxygen } \\
\text { concentration }\end{array}$} \\
\hline & Min & Max & Min & Max & Min & Max & Min & $\operatorname{Max}$ \\
\hline
\end{tabular}

01408690 Toms River 0.2 miles downstream from Garden State Parkway, near bottom (continued)

\begin{tabular}{|c|c|c|c|c|c|c|c|c|}
\hline $93 / 09 / 29$ & 15.0 & 16.5 & 100 & 100 & NA & NA & NA & NA \\
\hline $93 / 09 / 30$ & 13.0 & 15.0 & 100 & 100 & NA & NA & NA & NA \\
\hline $93 / 10 / 01$ & 12.0 & 13.5 & 100 & 100 & NA & NA & NA & NA \\
\hline $93 / 10 / 02$ & 12.5 & 14.0 & 100 & 100 & NA & NA & NA & NA \\
\hline $93 / 10 / 03$ & 14.0 & 15.0 & 100 & 100 & NA & NA & NA & NA \\
\hline $93 / 10 / 04$ & 13.0 & 15.0 & 100 & 100 & NA & NA & NA & NA \\
\hline $93 / 10 / 05$ & 13.0 & 14.5 & 100 & 100 & NA & NA & NA & NA \\
\hline $93 / 10 / 06$ & 12.0 & 13.5 & 100 & 100 & NA & NA & NA & NA \\
\hline $93 / 10 / 07$ & 12.0 & 14.5 & 100 & 100 & NA & NA & NA & NA \\
\hline $93 / 10 / 08$ & 14.0 & 15.0 & 100 & 100 & NA & NA & NA & NA \\
\hline $93 / 10 / 09$ & 14.0 & 16.0 & 100 & 1,000 & NA & NA & NA & NA \\
\hline $93 / 10 / 10$ & 13.0 & 15.5 & 100 & 100 & NA & NA & $\mathrm{NA}$ & NA \\
\hline $93 / 10 / 11$ & 11.5 & 12.5 & 100 & 100 & NA & NA & $\mathrm{NA}$ & NA \\
\hline $93 / 10 / 12$ & 11.5 & 13.0 & 100 & 100 & NA & NA & $\mathrm{NA}$ & NA \\
\hline $93 / 10 / 13$ & 11.5 & 12.5 & 100 & 100 & NA & NA & NA & NA \\
\hline $93 / 10 / 14$ & 11.0 & 12.0 & 100 & 100 & NA & NA & NA & NA \\
\hline $93 / 10 / 15$ & 11.0 & 12.5 & 100 & 100 & NA & NA & NA & NA \\
\hline $93 / 10 / 16$ & 11.5 & 12.5 & 100 & 100 & NA & NA & NA & NA \\
\hline $93 / 10 / 17$ & 12.0 & 13.5 & 100 & 100 & NA & NA & NA & NA \\
\hline $93 / 10 / 18$ & 13.0 & 15.0 & 100 & 100 & NA & NA & NA & NA \\
\hline $93 / 10 / 19$ & 13.0 & 14.0 & 100 & 100 & NA & NA & NA & NA \\
\hline $93 / 10 / 19$ & 13.5 & 13.5 & 100 & 100 & NA & NA & NA & NA \\
\hline $93 / 10 / 20$ & 13.5 & 14.0 & 100 & 100 & NA & NA & NA & NA \\
\hline $93 / 10 / 21$ & 14.0 & 16.0 & 100 & 100 & NA & NA & NA & NA \\
\hline $93 / 10 / 22$ & 13.5 & 15.5 & 100 & 100 & NA & NA & NA & NA \\
\hline $93 / 10 / 23$ & 11.5 & 13.5 & 100 & 100 & NA & NA & NA & NA \\
\hline $93 / 10 / 24$ & 10.5 & 11.5 & 100 & 100 & NA & NA & NA & NA \\
\hline $93 / 10 / 25$ & 10.5 & 11.5 & 100 & 100 & NA & NA & NA & NA \\
\hline $93 / 10 / 26$ & 11.5 & 12.0 & 100 & 100 & NA & NA & NA & NA \\
\hline $93 / 10 / 27$ & 12.0 & 13.0 & 100 & 100 & NA & NA & NA & NA \\
\hline $93 / 10 / 28$ & 11.5 & 13.0 & 100 & 100 & NA & NA & NA & NA \\
\hline $93 / 10 / 29$ & 10.5 & 12.0 & 100 & 100 & NA & NA & NA & NA \\
\hline $93 / 10 / 30$ & 11.0 & 11.5 & 100 & 100 & NA & NA & NA & NA \\
\hline $93 / 10 / 31$ & 10.5 & 11.0 & 100 & 100 & NA & NA & NA & NA \\
\hline $93 / 11 / 01$ & 9.0 & 10.5 & 100 & 100 & NA & NA & NA & NA \\
\hline $93 / 11 / 02$ & 8.0 & 9.0 & 100 & 100 & NA & NA & NA & NA \\
\hline $93 / 11 / 03$ & 7.5 & 8.0 & 100 & 100 & NA & NA & NA & NA \\
\hline $93 / 11 / 04$ & 7.0 & 8.0 & 100 & 100 & NA & NA & NA & NA \\
\hline 93/11/05 & 8.0 & 10.0 & 100 & 100 & NA & NA & NA & NA \\
\hline $93 / 11 / 06$ & 9.5 & 10.0 & 100 & 100 & NA & NA & NA & NA \\
\hline $93 / 11 / 07$ & 8.0 & 9.5 & 100 & 100 & NA & NA & NA & NA \\
\hline
\end{tabular}


Appendix 16. Measurements at tidal stations in and near the tidal embayment of the Toms River, N.J.--Daily minimum and maximum values of water-quality characteristics from measurements by movable monitors, October 1992 - January 1994--Continued

\begin{tabular}{|c|c|c|c|c|c|c|c|c|}
\hline \multirow{3}{*}{$\begin{array}{c}\text { Date } \\
\text { year/month/day }\end{array}$} & \multicolumn{8}{|c|}{ Daily minimum and maximum } \\
\hline & \multicolumn{2}{|c|}{ Temperature } & \multicolumn{2}{|c|}{ Specific conductance } & \multicolumn{2}{|c|}{$\mathrm{pH}$} & \multicolumn{2}{|c|}{$\begin{array}{l}\text { Dissolved-oxygen } \\
\text { concentration }\end{array}$} \\
\hline & Min & $\operatorname{Max}$ & Min & $\operatorname{Max}$ & Min & $\operatorname{Max}$ & Min & $\operatorname{Max}$ \\
\hline
\end{tabular}

01408690 Toms River 0.2 miles downstream from Garden State Parkway, near bottom (continued)

\begin{tabular}{|c|c|c|c|c|c|c|c|c|}
\hline $93 / 11 / 08$ & 7.0 & 8.0 & 100 & 100 & NA & NA & NA & NA \\
\hline $93 / 11 / 09$ & 6.0 & 7.0 & 100 & 100 & NA & NA & NA & NA \\
\hline $93 / 11 / 10$ & 6.0 & 7.0 & 100 & 100 & NA & NA & NA & NA \\
\hline $93 / 11 / 11$ & 6.0 & 7.0 & 100 & 100 & NA & NA & NA & NA \\
\hline $93 / 11 / 12$ & 7.0 & 8.5 & 100 & 100 & NA & NA & NA & NA \\
\hline $93 / 11 / 13$ & 7.5 & 9.0 & 100 & 100 & NA & NA & NA & NA \\
\hline $93 / 11 / 14$ & 9.5 & 11.5 & 100 & 100 & NA & NA & NA & NA \\
\hline $93 / 11 / 15$ & 11.5 & 14.0 & 100 & 100 & NA & NA & NA & NA \\
\hline $93 / 11 / 16$ & 11.5 & 13.5 & 100 & 100 & NA & NA & NA & NA \\
\hline $93 / 11 / 17$ & 10.5 & 11.5 & 100 & 100 & NA & NA & NA & NA \\
\hline $93 / 11 / 18$ & 11.0 & 11.5 & 100 & 100 & NA & NA & NA & NA \\
\hline $93 / 12 / 02$ & 7.0 & 7.0 & 100 & 100 & NA & NA & NA & NA \\
\hline $93 / 12 / 03$ & 7.0 & 8.5 & 100 & 100 & NA & NA & NA & NA \\
\hline $93 / 12 / 04$ & 7.5 & 8.5 & 100 & 100 & NA & NA & NA & NA \\
\hline $93 / 12 / 05$ & 8.5 & 9.5 & 100 & 100 & NA & NA & NA & NA \\
\hline $93 / 12 / 06$ & 8.0 & 9.0 & 100 & 100 & NA & NA & NA & NA \\
\hline $93 / 12 / 07$ & 7.0 & 7.5 & 100 & 100 & NA & NA & NA & NA \\
\hline $93 / 12 / 08$ & 6.5 & 7.5 & 100 & 100 & NA & NA & NA & NA \\
\hline $93 / 12 / 09$ & 5.5 & 6.5 & 100 & 100 & NA & NA & NA & NA \\
\hline $93 / 12 / 10$ & 5.5 & 7.0 & 100 & 100 & NA & NA & NA & NA \\
\hline $93 / 12 / 11$ & 6.0 & 7.5 & 100 & 100 & NA & NA & NA & NA \\
\hline $93 / 12 / 12$ & 4.0 & 6.0 & 100 & 100 & NA & NA & NA & NA \\
\hline $93 / 12 / 13$ & 3.0 & 4.0 & 100 & 100 & NA & NA & NA & NA \\
\hline $93 / 12 / 14$ & 3.5 & 5.0 & 100 & 20,500 & NA & NA & NA & NA \\
\hline $93 / 12 / 15$ & 5.0 & 6.5 & 100 & 100 & NA & NA & NA & NA \\
\hline $93 / 12 / 16$ & 6.0 & 7.0 & 100 & 17,900 & NA & NA & NA & NA \\
\hline $93 / 12 / 17$ & 5.0 & 6.0 & 100 & 29,700 & NA & NA & NA & NA \\
\hline $93 / 12 / 18$ & 4.0 & 5.5 & 100 & 100 & NA & NA & NA & NA \\
\hline $93 / 12 / 19$ & 5.5 & 6.0 & 100 & 100 & NA & NA & NA & NA \\
\hline $93 / 12 / 20$ & 4.5 & 6.0 & 100 & 100 & NA & NA & NA & NA \\
\hline $93 / 12 / 21$ & 6.0 & 7.0 & 100 & 100 & NA & NA & NA & NA \\
\hline $93 / 12 / 22$ & 5.0 & 6.0 & 100 & 100 & NA & NA & NA & NA \\
\hline $93 / 12 / 23$ & 4.0 & 5.0 & 100 & 100 & NA & NA & NA & NA \\
\hline $93 / 12 / 24$ & 3.0 & 3.5 & 100 & 100 & NA & NA & NA & NA \\
\hline $93 / 12 / 25$ & 2.5 & 3.0 & 100 & 100 & NA & NA & NA & NA \\
\hline $93 / 12 / 26$ & 1.5 & 3.0 & 100 & 100 & NA & NA & NA & NA \\
\hline $93 / 12 / 27$ & 1.0 & 1.0 & 100 & 100 & NA & NA & NA & NA \\
\hline $93 / 12 / 28$ & 1.0 & 2.0 & 100 & 100 & NA & NA & NA & NA \\
\hline $93 / 12 / 29$ & 1.5 & 2.0 & 100 & 100 & NA & NA & NA & NA \\
\hline $93 / 12 / 30$ & 1.0 & 1.5 & 100 & 100 & NA & NA & NA & NA \\
\hline $93 / 12 / 31$ & 1.0 & 2.0 & 100 & 100 & NA & NA & NA & NA \\
\hline
\end{tabular}


Appendix 16. Measurements at tidal stations in and near the tidal embayment of the Toms River, N.J.--Daily minimum and maximum values of water-quality characteristics from measurements by movable monitors, October 1992 - January 1994--Continued

\begin{tabular}{|c|c|c|c|c|c|c|c|c|}
\hline \multirow{3}{*}{$\begin{array}{c}\text { Date } \\
\text { year/month/day }\end{array}$} & \multicolumn{8}{|c|}{ Daily minimum and maximum } \\
\hline & \multicolumn{2}{|c|}{ Temperature } & \multicolumn{2}{|c|}{ Specific conductance } & \multicolumn{2}{|c|}{$\mathrm{pH}$} & \multicolumn{2}{|c|}{$\begin{array}{l}\text { Dissolved-oxygen } \\
\text { concentration }\end{array}$} \\
\hline & Min & Max & Min & $\operatorname{Max}$ & Min & Max & Min & Max \\
\hline
\end{tabular}

01408690 Toms River 0.2 miles downstream from Garden State Parkway, near bottom (continued)

$\begin{array}{lllllllll}94 / 01 / 01 & 1.5 & 3.0 & 100 & 100 & \text { NA } & \text { NA } & \text { NA } & \text { NA } \\ 94 / 01 / 02 & 3.0 & 4.0 & 100 & 200 & \text { NA } & \text { NA } & \text { NA } & \text { NA } \\ 94 / 01 / 03 & 3.0 & 3.5 & 100 & 100 & \text { NA } & \text { NA } & \text { NA } & \text { NA } \\ 94 / 01 / 04 & 3.0 & 3.5 & 100 & 100 & \text { NA } & \text { NA } & \text { NA } & \text { NA } \\ 94 / 01 / 05 & 1.5 & 3.0 & 100 & 100 & \text { NA } & \text { NA } & \text { NA } & \text { NA } \\ 94 / 01 / 06 & 1.0 & 2.0 & 100 & 100 & \text { NA } & \text { NA } & \text { NA } & \text { NA } \\ 94 / 01 / 07 & 2.0 & 2.5 & 100 & 100 & \text { NA } & \text { NA } & \text { NA } & \text { NA } \\ 94 / 01 / 08 & 1.5 & 2.5 & 100 & 100 & \text { NA } & \text { NA } & \text { NA } & \text { NA } \\ 94 / 01 / 09 & 0.5 & 1.5 & 100 & 100 & \text { NA } & \text { NA } & \text { NA } & \text { NA } \\ 94 / 01 / 10 & 0.5 & 1.0 & 100 & 100 & \text { NA } & \text { NA } & \text { NA } & \text { NA } \\ 94 / 01 / 11 & 0.5 & 2.0 & 100 & 100 & \text { NA } & \text { NA } & \text { NA } & \text { NA } \\ 94 / 01 / 12 & 2.0 & 2.5 & 100 & 100 & \text { NA } & \text { NA } & \text { NA } & \text { NA } \\ 94 / 01 / 13 & 2.5 & 3.5 & 100 & 100 & \text { NA } & \text { NA } & \text { NA } & \text { NA } \\ 94 / 01 / 14 & 2.5 & 3.5 & 100 & 100 & \text { NA } & \text { NA } & \text { NA } & \text { NA } \\ 94 / 01 / 15 & 0.0 & 2.5 & 100 & 100 & \text { NA } & \text { NA } & \text { NA } & \text { NA } \\ 94 / 01 / 16 & 0.0 & 0.5 & 100 & 100 & \text { NA } & \text { NA } & \text { NA } & \text { NA } \\ 94 / 01 / 17 & 0.5 & 1.5 & 100 & 200 & \text { NA } & \text { NA } & \text { NA } & \text { NA } \\ 94 / 01 / 18 & 0.0 & 1.5 & 100 & 100 & \text { NA } & \text { NA } & \text { NA } & \text { NA } \\ 94 / 01 / 19 & 0.0 & 0.5 & 100 & 100 & \text { NA } & \text { NA } & \text { NA } & \text { NA } \\ 94 / 01 / 20 & 0.0 & 0.5 & 100 & 100 & \text { NA } & \text { NA } & \text { NA } & \text { NA } \\ 94 / 01 / 21 & 0.0 & 0.5 & 100 & 100 & \text { NA } & \text { NA } & \text { NA } & \text { NA } \\ 94 / 01 / 22 & 0.5 & 1.0 & 100 & 100 & \text { NA } & \text { NA } & \text { NA } & \text { NA } \\ 94 / 01 / 23 & 0.5 & 1.5 & 100 & 100 & \text { NA } & \text { NA } & \text { NA } & \text { NA } \\ 94 / 01 / 24 & 1.5 & 3.0 & 100 & 200 & \text { NA } & \text { NA } & \text { NA } & \text { NA } \\ 94 / 01 / 25 & 2.0 & 3.0 & 100 & 100 & \text { NA } & \text { NA } & \text { NA } & \text { NA } \\ 94 / 91 / 26 & 1.5 & 3.0 & 100 & 100 & \text { NA } & \text { NA } & \text { NA } & \text { NA } \\ 94 / 01 / 27 & 1.0 & 1.5 & 100 & 100 & \text { NA } & \text { NA } & \text { NA } & \text { NA } \\ 94 / 01 / 28 & 1.5 & 3.5 & 100 & 200 & \text { NA } & \text { NA } & \text { NA } & \text { NA }\end{array}$

01408695 Toms River near South Toms River, mid-depth

$\begin{array}{rrrrrrrrr}92 / 10 / 30 & 11.0 & 11.5 & 100 & 23,400 & \text { NA } & \text { NA } & \text { NA } & \text { NA } \\ 92 / 10 / 31 & 10.0 & 11.5 & 100 & 200 & \text { NA } & \text { NA } & \text { NA } & \text { NA } \\ 92 / 11 / 01 & 10.0 & 11.0 & 100 & 1,900 & \text { NA } & \text { NA } & \text { NA } & \text { NA } \\ 92 / 11 / 02 & 9.5 & 10.0 & 100 & 100 & \text { NA } & \text { NA } & \text { NA } & \text { NA } \\ 92 / 11 / 03 & 10.0 & 12.5 & 100 & 100 & \text { NA } & \text { NA } & \text { NA } & \text { NA } \\ 92 / 11 / 04 & 11.5 & 13.0 & 100 & 100 & \text { NA } & \text { NA } & \text { NA } & \text { NA } \\ 92 / 11 / 05 & 12.0 & 13.0 & 100 & 100 & \text { NA } & \text { NA } & \text { NA } & \text { NA } \\ 92 / 11 / 06 & 10.0 & 12.0 & 100 & 100 & \text { NA } & \text { NA } & \text { NA } & \text { NA } \\ 92 / 11 / 07 & 8.5 & 10.0 & 100 & 100 & \text { NA } & \text { NA } & \text { NA } & \text { NA } \\ 92 / 11 / 08 & 7.0 & 8.5 & 100 & 100 & \text { NA } & \text { NA } & \text { NA } & \text { NA } \\ 92 / 11 / 09 & 6.0 & 7.5 & 100 & 100 & \text { NA } & \text { NA } & \text { NA } & \text { NA }\end{array}$


Appendix 16. Measurements at tidal stations in and near the tidal embayment of the Toms River, N.J.--Daily minimum and maximum values of water-quality characteristics from measurements by movable monitors, October 1992 - January 1994--Continued

\begin{tabular}{|c|c|c|c|c|c|c|c|c|}
\hline \multirow{3}{*}{$\begin{array}{c}\text { Date } \\
\text { year/month/day }\end{array}$} & \multicolumn{8}{|c|}{ Daily minimum and maximum } \\
\hline & \multicolumn{2}{|c|}{ Temperature } & \multicolumn{2}{|c|}{ Specific conductance } & \multicolumn{2}{|c|}{$\mathrm{pH}$} & \multicolumn{2}{|c|}{$\begin{array}{l}\text { Dissolved-oxygen } \\
\text { concentration }\end{array}$} \\
\hline & Min & $\operatorname{Max}$ & Min & $\operatorname{Max}$ & $\operatorname{Min}$ & $\operatorname{Max}$ & Min & Max \\
\hline
\end{tabular}

01408695 Toms River near South Toms River, mid-depth (continued)

\begin{tabular}{|c|c|c|c|c|c|c|c|}
\hline $92 / 11 / 10$ & 6.0 & 7.5 & 100 & 1,400 & NA & NA & NA \\
\hline $92 / 11 / 11$ & 7.0 & 9.0 & 100 & 9,700 & NA & NA & NA \\
\hline $92 / 11 / 12$ & 9.0 & 12.0 & 100 & 5,800 & NA & NA & NA \\
\hline $92 / 11 / 13$ & 10.5 & 12.5 & 100 & 100 & NA & NA & NA \\
\hline $92 / 11 / 14$ & 8.0 & 10.5 & 100 & 100 & NA & NA & NA \\
\hline $92 / 11 / 15$ & 6.5 & 8.0 & 100 & 100 & NA & NA & NA \\
\hline $92 / 11 / 16$ & 5.5 & 6.5 & 100 & 100 & NA & NA & NA \\
\hline $92 / 11 / 17$ & 5.0 & 7.0 & 100 & 800 & NA & NA & NA \\
\hline $92 / 11 / 18$ & 7.0 & 7.5 & 100 & 100 & NA & NA & NA \\
\hline $92 / 11 / 19$ & 7.0 & 7.5 & 100 & 100 & NA & NA & NA \\
\hline $92 / 11 / 20$ & 6.5 & 7.5 & 100 & 3,900 & NA & NA & NA \\
\hline $92 / 11 / 21$ & 6.5 & 9.0 & 100 & 600 & NA & NA & NA \\
\hline $92 / 11 / 22$ & 9.0 & 11.5 & 100 & 8,400 & NA & NA & NA \\
\hline $92 / 11 / 23$ & 11.5 & 14.0 & 100 & 9,500 & NA & NA & NA \\
\hline $92 / 11 / 24$ & 10.0 & 12.5 & 100 & 20,100 & NA & NA & NA \\
\hline $92 / 12 / 02$ & 6.5 & 9.5 & 100 & 28,400 & NA & NA & NA \\
\hline $92 / 12 / 03$ & 6.0 & 9.0 & 100 & 28,500 & NA & NA & NA \\
\hline $92 / 12 / 04$ & 5.0 & 6.0 & 100 & 100 & NA & NA & NA \\
\hline $92 / 12 / 05$ & 5.0 & 6.5 & 100 & 100 & NA & NA & NA \\
\hline $92 / 12 / 06$ & 4.0 & 5.0 & 100 & 100 & NA & NA & NA \\
\hline $92 / 12 / 07$ & 4.0 & 5.0 & 100 & 100 & NA & NA & NA \\
\hline $92 / 12 / 08$ & 4.0 & 4.5 & 100 & 100 & NA & NA & NA \\
\hline $92 / 12 / 09$ & 2.5 & 3.5 & 100 & 7,900 & NA & NA & NA \\
\hline $92 / 12 / 10$ & 2.5 & 4.5 & 100 & 4,900 & NA & NA & NA \\
\hline $92 / 12 / 11$ & 4.5 & 6.5 & 100 & 21,600 & NA & NA & NA \\
\hline $92 / 12 / 12$ & 5.0 & 7.0 & 100 & 20,700 & NA & NA & NA \\
\hline $92 / 12 / 13$ & 5.0 & 5.5 & 100 & 100 & NA & NA & NA \\
\hline $92 / 12 / 14$ & 4.0 & 5.0 & 100 & 100 & NA & NA & NA \\
\hline $92 / 12 / 15$ & 3.5 & 4.0 & 100 & 100 & NA & NA & NA \\
\hline $92 / 12 / 16$ & 3.5 & 4.5 & 100 & 100 & NA & NA & NA \\
\hline $92 / 12 / 17$ & 4.5 & 6.5 & 100 & 100 & NA & NA & NA \\
\hline $92 / 12 / 18$ & 6.0 & 7.0 & 100 & 100 & NA & NA & NA \\
\hline $92 / 12 / 19$ & 5.0 & 6.0 & 100 & 100 & NA & NA & NA \\
\hline $92 / 12 / 20$ & 5.5 & 6.5 & 100 & 100 & NA & NA & NA \\
\hline $92 / 12 / 21$ & 4.0 & 5.5 & 100 & 100 & NA & $\mathrm{NA}$ & NA \\
\hline $92 / 12 / 22$ & 4.0 & 4.0 & 100 & 100 & NA & NA & NA \\
\hline $92 / 12 / 22$ & 4.0 & 5.0 & 100 & 100 & NA & NA & NA \\
\hline $92 / 12 / 23$ & 4.0 & 5.0 & 100 & 100 & NA & NA & NA \\
\hline $92 / 12 / 24$ & 3.0 & 4.5 & 100 & 100 & NA & NA & NA \\
\hline $92 / 12 / 25$ & 2.0 & 3.0 & 100 & 100 & NA & NA & NA \\
\hline
\end{tabular}


Appendix 16. Measurements at tidal stations in and near the tidal embayment of the Toms River, N.J.--Daily minimum and maximum values of water-quality characteristics from measurements by movable monitors, October 1992 - January 1994--Continued

\begin{tabular}{|c|c|c|c|c|c|c|c|c|}
\hline \multirow{3}{*}{$\begin{array}{c}\text { Date } \\
\text { year/month/day }\end{array}$} & \multicolumn{8}{|c|}{ Daily minimum and maximum } \\
\hline & \multicolumn{2}{|c|}{ Temperature } & \multicolumn{2}{|c|}{ Specific conductance } & \multicolumn{2}{|c|}{$\mathrm{pH}$} & \multicolumn{2}{|c|}{$\begin{array}{l}\text { Dissolved-oxygen } \\
\text { concentration }\end{array}$} \\
\hline & Min & $\operatorname{Max}$ & Min & $\operatorname{Max}$ & Min & $\operatorname{Max}$ & Min & Max \\
\hline \multicolumn{9}{|c|}{ 01408695 Toms River near South Toms River, mid-depth (continued) } \\
\hline $92 / 12 / 26$ & 2.5 & 3.0 & 100 & 100 & NA & NA & NA & NA \\
\hline $92 / 12 / 27$ & 2.0 & 2.5 & 100 & 100 & NA & NA & NA & NA \\
\hline $92 / 12 / 28$ & 2.5 & 4.0 & 100 & 100 & NA & NA & NA & NA \\
\hline $93 / 01 / 20$ & 3.0 & 3.5 & 100 & 100 & NA & NA & NA & NA \\
\hline $93 / 01 / 21$ & 2.5 & 4.5 & 100 & 100 & NA & NA & NA & NA \\
\hline $93 / 01 / 22$ & 4.5 & 6.0 & 100 & 100 & NA & NA & NA & NA \\
\hline $93 / 01 / 23$ & 5.5 & 6.5 & 100 & 100 & NA & NA & NA & NA \\
\hline $93 / 01 / 24$ & 5.0 & 7.0 & 100 & 100 & NA & NA & NA & NA \\
\hline $93 / 01 / 25$ & 5.0 & 6.5 & 100 & 100 & NA & NA & NA & NA \\
\hline $93 / 01 / 26$ & 4.0 & 5.0 & 100 & 100 & NA & NA & NA & NA \\
\hline $93 / 01 / 27$ & 4.0 & 5.5 & 100 & 100 & NA & NA & NA & NA \\
\hline $93 / 01 / 28$ & 3.5 & 5.0 & 100 & 100 & NA & NA & NA & NA \\
\hline $93 / 01 / 29$ & 3.5 & 5.5 & 100 & 100 & NA & NA & NA & NA \\
\hline $93 / 01 / 30$ & 2.5 & 4.0 & 100 & 100 & NA & NA & NA & NA \\
\hline $93 / 01 / 31$ & 3.5 & 5.0 & 100 & 100 & NA & NA & NA & NA \\
\hline $93 / 02 / 01$ & 2.5 & 5.0 & 100 & 100 & NA & NA & NA & NA \\
\hline $93 / 02 / 02$ & 1.0 & 2.5 & 100 & 100 & NA & NA & NA & NA \\
\hline $93 / 02 / 03$ & 0.5 & 2.5 & 100 & 14,000 & NA & NA & NA & NA \\
\hline $93 / 02 / 04$ & 2.5 & 4.0 & 100 & 100 & NA & NA & NA & NA \\
\hline $93 / 02 / 05$ & 2.5 & 4.5 & 100 & 100 & NA & NA & NA & NA \\
\hline $93 / 02 / 06$ & 2.0 & 4.0 & 100 & 100 & NA & NA & NA & NA \\
\hline $93 / 02 / 07$ & 1.0 & 2.0 & 100 & 19,600 & NA & NA & NA & NA \\
\hline $93 / 02 / 08$ & 2.0 & 4.5 & 100 & 20,200 & NA & NA & NA & NA \\
\hline $93 / 02 / 09$ & 2.5 & 4.5 & 100 & 19,100 & NA & NA & NA & NA \\
\hline $93 / 02 / 10$ & 2.5 & 5.5 & 100 & 8,800 & NA & NA & NA & NA \\
\hline $93 / 02 / 11$ & 3.5 & 5.0 & 100 & 100 & NA & NA & NA & NA \\
\hline $93 / 04 / 05$ & 8.0 & 9.5 & 100 & 100 & 4.2 & 4.3 & 10.5 & 11.1 \\
\hline $93 / 04 / 06$ & 7.5 & 9.5 & 100 & 100 & 4.2 & 4.2 & 10.4 & 11.2 \\
\hline $93 / 04 / 07$ & 7.5 & 11.0 & 100 & 100 & 4.2 & 4.3 & 10.4 & 11.2 \\
\hline $93 / 04 / 08$ & 8.5 & 12.0 & 100 & 100 & 4.2 & 4.3 & 10.2 & 11.1 \\
\hline $93 / 04 / 09$ & 9.5 & 12.5 & 100 & 100 & 4.3 & 4.4 & 10.0 & 11.0 \\
\hline $93 / 04 / 10$ & 11.0 & 12.5 & 100 & 100 & 4.3 & 4.4 & 9.4 & 10.4 \\
\hline $93 / 04 / 11$ & 11.0 & 13.5 & 100 & 100 & 4.3 & 4.4 & 9.3 & 10.6 \\
\hline $93 / 04 / 12$ & 11.0 & 14.0 & 100 & 100 & 4.3 & 4.4 & 9.4 & 10.7 \\
\hline $93 / 04 / 13$ & 11.0 & 14.5 & 100 & 100 & 4.3 & 4.4 & 9.5 & 10.8 \\
\hline $93 / 04 / 14$ & 11.0 & 13.5 & 100 & 100 & 4.4 & 4.5 & 9.4 & 10.8 \\
\hline $93 / 04 / 15$ & 12.0 & 14.0 & 100 & 100 & 4.4 & 4.5 & 9.4 & 10.7 \\
\hline $93 / 04 / 16$ & 12.5 & 14.5 & 100 & 100 & 4.4 & 4.5 & 9.2 & 10.3 \\
\hline $93 / 04 / 17$ & 13.5 & 16.0 & 100 & 100 & 4.5 & 4.5 & 8.5 & 10.0 \\
\hline $93 / 04 / 18$ & 12.5 & 15.5 & 100 & 100 & 4.5 & 4.6 & 8.6 & 10.4 \\
\hline
\end{tabular}


Appendix 16. Measurements at tidal stations in and near the tidal embayment of the Toms River, N.J.--Daily minimum and maximum values of water-quality characteristics from measurements by movable monitors, October 1992 - January 1994--Continued

\begin{tabular}{|c|c|c|c|c|c|c|c|c|}
\hline \multirow{3}{*}{$\begin{array}{c}\text { Date } \\
\text { year/month/day }\end{array}$} & \multicolumn{8}{|c|}{ Daily minimum and maximum } \\
\hline & \multicolumn{2}{|c|}{ Temperature } & \multicolumn{2}{|c|}{ Specific conductance } & \multicolumn{2}{|c|}{$\mathrm{pH}$} & \multicolumn{2}{|c|}{$\begin{array}{l}\text { Dissolved-oxygen } \\
\text { concentration }\end{array}$} \\
\hline & Min & $\operatorname{Max}$ & Min & $\operatorname{Max}$ & Min & $\operatorname{Max}$ & Min & $\operatorname{Max}$ \\
\hline
\end{tabular}

01408695 Toms River near South Toms River, mid-depth (continued)

\begin{tabular}{|c|c|c|c|c|c|c|c|c|}
\hline $93 / 04 / 19$ & 12.0 & 16.0 & 100 & 100 & 4.5 & 4.5 & 9.0 & 10.5 \\
\hline $93 / 04 / 27$ & 13.5 & 15.5 & 100 & 100 & NA & NA & NA & NA \\
\hline $93 / 04 / 28$ & 12.0 & 16.0 & 100 & 100 & NA & NA & NA & NA \\
\hline $93 / 04 / 29$ & 12.0 & 16.0 & 100 & 100 & NA & NA & NA & NA \\
\hline $93 / 04 / 30$ & 12.5 & 17.0 & 100 & 100 & NA & NA & NA & NA \\
\hline 93/05/01 & 13.5 & 18.0 & 100 & 100 & NA & NA & NA & NA \\
\hline $93 / 05 / 02$ & 15.0 & 18.5 & 100 & 100 & NA & NA & NA & NA \\
\hline $93 / 05 / 03$ & 15.5 & 17.5 & 100 & 100 & NA & NA & NA & NA \\
\hline $93 / 05 / 04$ & 15.0 & 16.5 & 100 & 100 & NA & NA & NA & NA \\
\hline $93 / 05 / 05$ & 15.0 & 18.0 & 100 & 100 & NA & NA & NA & NA \\
\hline 93/05/06 & 16.0 & 19.5 & 100 & 100 & NA & NA & NA & NA \\
\hline 93/05/07 & 16.5 & 20.0 & 100 & 100 & NA & NA & NA & NA \\
\hline 93/05/08 & 16.0 & 19.5 & 100 & 100 & NA & NA & NA & NA \\
\hline 93/05/09 & 15.5 & 20.5 & 100 & 100 & NA & NA & NA & NA \\
\hline $93 / 05 / 10$ & 17.0 & 20.5 & 100 & 100 & NA & NA & NA & NA \\
\hline $93 / 05 / 11$ & 16.5 & 21.5 & 100 & 100 & NA & NA & NA & NA \\
\hline $93 / 05 / 12$ & 18.5 & 22.5 & 100 & 100 & NA & NA & NA & NA \\
\hline $93 / 05 / 13$ & 17.0 & 20.0 & 100 & 100 & NA & NA & NA & NA \\
\hline $93 / 05 / 14$ & 16.0 & 19.0 & 100 & 100 & NA & NA & NA & NA \\
\hline $93 / 05 / 15$ & 15.0 & 20.0 & 100 & 100 & NA & NA & NA & NA \\
\hline $93 / 05 / 16$ & 16.5 & 20.0 & 100 & 100 & NA & NA & NA & NA \\
\hline $93 / 05 / 17$ & 17.0 & 20.0 & 100 & 100 & NA & NA & NA & NA \\
\hline $93 / 05 / 18$ & 14.5 & 17.0 & 100 & 100 & NA & NA & NA & NA \\
\hline $93 / 05 / 19$ & 14.0 & 15.0 & 100 & 100 & NA & NA & NA & NA \\
\hline $93 / 05 / 20$ & 13.5 & 14.5 & 100 & 100 & NA & NA & NA & NA \\
\hline $93 / 05 / 21$ & 12.5 & 16.0 & 100 & 100 & NA & NA & NA & NA \\
\hline $93 / 05 / 22$ & 13.0 & 16.5 & 100 & 100 & NA & NA & NA & NA \\
\hline $93 / 05 / 23$ & 13.5 & 18.0 & 100 & 100 & NA & NA & NA & NA \\
\hline $93 / 05 / 24$ & 15.0 & 19.5 & 100 & 100 & NA & NA & NA & NA \\
\hline $93 / 05 / 25$ & 17.0 & 17.5 & 100 & 100 & NA & NA & NA & NA \\
\hline $93 / 05 / 25$ & 18.0 & 20.5 & 100 & 100 & 5.2 & 5.5 & 8.1 & 8.8 \\
\hline $93 / 05 / 26$ & 17.5 & 20.0 & 100 & 100 & 5.2 & 5.3 & 7.8 & 8.8 \\
\hline $93 / 05 / 27$ & 16.0 & 19.5 & 100 & 100 & 5.2 & 5.3 & 8.0 & 8.9 \\
\hline $93 / 05 / 28$ & 16.5 & 21.0 & 100 & 100 & 5.2 & 5.3 & 8.0 & 8.8 \\
\hline $93 / 05 / 29$ & 18.0 & 20.5 & 100 & 100 & 5.3 & 5.4 & 7.5 & 8.5 \\
\hline $93 / 05 / 30$ & 16.0 & 19.5 & 100 & 100 & 5.3 & 5.4 & 8.0 & 8.9 \\
\hline $93 / 05 / 31$ & 16.0 & 17.5 & 100 & 100 & 5.3 & 5.4 & 8.0 & 8.9 \\
\hline $93 / 06 / 01$ & 16.0 & 19.0 & 100 & 100 & 5.4 & 5.4 & 7.6 & 8.7 \\
\hline 93/06/02 & 15.0 & 18.5 & 100 & 100 & 5.2 & 5.4 & 7.9 & 9.2 \\
\hline $93 / 06 / 03$ & 15.5 & 18.0 & 100 & 100 & 5.2 & 5.3 & 8.1 & 9.0 \\
\hline
\end{tabular}


Appendix 16. Measurements at tidal stations in and near the tidal embayment of the Toms River, N.J.--Daily minimum and maximum values of water-quality characteristics from measurements by movable monitors, October 1992 - January 1994--Continued

\begin{tabular}{|c|c|c|c|c|c|c|c|c|}
\hline \multirow{3}{*}{$\begin{array}{c}\text { Date } \\
\text { year/month/day }\end{array}$} & \multicolumn{8}{|c|}{ Daily minimum and maximum } \\
\hline & \multicolumn{2}{|c|}{ Temperature } & \multicolumn{2}{|c|}{ Specific conductance } & \multicolumn{2}{|c|}{$\mathrm{pH}$} & \multicolumn{2}{|c|}{$\begin{array}{l}\text { Dissolved-oxygen } \\
\text { concentration }\end{array}$} \\
\hline & Min & $\operatorname{Max}$ & Min & $\operatorname{Max}$ & Min & $\operatorname{Max}$ & Min & $\operatorname{Max}$ \\
\hline
\end{tabular}

01408695 Toms River near South Toms River, mid-depth (continued)

\begin{tabular}{|c|c|c|c|c|c|c|c|c|}
\hline $93 / 06 / 04$ & 16.0 & 17.5 & 100 & 100 & 5.3 & 5.3 & 8.0 & 8.9 \\
\hline $93 / 06 / 05$ & 15.5 & 16.5 & 100 & 100 & 5.3 & 5.3 & 8.0 & 8.6 \\
\hline 93/06/06 & 15.0 & 17.5 & 100 & 100 & 5.3 & 5.4 & 8.1 & 9.1 \\
\hline $93 / 06 / 07$ & 14.5 & 18.0 & 100 & 100 & 5.3 & 5.4 & 8.2 & 9.2 \\
\hline 93/06/08 & 16.0 & 20.5 & 100 & 100 & 5.3 & 5.3 & 7.8 & 9.1 \\
\hline $93 / 06 / 09$ & 18.0 & 22.5 & 100 & 100 & NA & NA & NA & NA \\
\hline $93 / 06 / 10$ & 19.5 & 23.5 & 100 & 100 & NA & NA & NA & NA \\
\hline $93 / 06 / 11$ & 20.0 & 23.0 & 100 & 100 & NA & NA & NA & NA \\
\hline $93 / 06 / 12$ & 19.5 & 22.5 & 100 & 100 & NA & NA & NA & NA \\
\hline $93 / 06 / 13$ & 18.0 & 22.0 & 100 & 100 & NA & NA & NA & NA \\
\hline $93 / 06 / 14$ & 17.5 & 22.0 & 100 & 100 & NA & NA & NA & NA \\
\hline $93 / 06 / 15$ & 18.0 & 21.5 & 100 & 100 & NA & NA & NA & NA \\
\hline $93 / 06 / 16$ & 18.5 & 22.5 & 100 & 100 & NA & NA & NA & NA \\
\hline $93 / 06 / 17$ & 19.0 & 22.5 & 100 & 100 & NA & NA & NA & NA \\
\hline $93 / 06 / 18$ & 19.0 & 23.0 & 100 & 100 & NA & NA & NA & NA \\
\hline $93 / 06 / 19$ & 20.5 & 24.0 & 100 & 100 & NA & NA & NA & NA \\
\hline $93 / 06 / 20$ & 21.0 & 23.0 & 100 & 100 & NA & NA & NA & NA \\
\hline $93 / 06 / 21$ & 20.5 & 23.5 & 100 & 100 & NA & NA & NA & NA \\
\hline $93 / 06 / 22$ & 21.0 & 24.5 & 100 & 11,600 & NA & NA & NA & NA \\
\hline $93 / 06 / 23$ & 20.0 & 22.5 & 100 & 100 & NA & NA & NA & NA \\
\hline $93 / 06 / 23$ & 20.5 & 23.5 & 100 & 100 & NA & NA & NA & NA \\
\hline $93 / 06 / 24$ & 18.5 & 22.5 & 100 & 100 & NA & NA & NA & NA \\
\hline $93 / 06 / 25$ & 18.5 & 22.5 & 100 & 9,700 & NA & NA & NA & NA \\
\hline $93 / 06 / 26$ & 19.5 & 23.5 & 100 & 12,300 & NA & NA & NA & NA \\
\hline $93 / 06 / 27$ & 21.0 & 22.5 & 100 & 100 & NA & NA & NA & NA \\
\hline $93 / 06 / 28$ & 20.0 & 25.0 & 100 & 19,500 & NA & NA & NA & NA \\
\hline $93 / 06 / 29$ & 20.5 & 23.5 & 100 & 1,500 & NA & NA & NA & NA \\
\hline $93 / 06 / 30$ & 20.5 & 22.5 & 100 & 1,200 & NA & NA & NA & NA \\
\hline $93 / 07 / 01$ & 20.0 & 23.5 & 100 & 100 & NA & NA & NA & NA \\
\hline $93 / 07 / 02$ & 20.0 & 21.5 & 100 & 100 & NA & NA & NA & NA \\
\hline $93 / 07 / 03$ & 19.5 & 24.0 & 100 & 12,400 & NA & NA & NA & NA \\
\hline $93 / 07 / 04$ & 21.0 & 24.5 & 100 & 14,300 & NA & NA & NA & NA \\
\hline 93/07/05 & 22.0 & 25.0 & 100 & 1,200 & NA & NA & NA & NA \\
\hline 93/07/06 & 21.5 & 25.5 & 100 & 1,300 & NA & $\mathrm{NA}$ & NA & NA \\
\hline 93/07/07 & 22.5 & 26.0 & 100 & 13,100 & NA & NA & NA & NA \\
\hline 93/07/08 & 23.5 & 27.0 & 100 & 22,100 & NA & NA & NA & NA \\
\hline $93 / 07 / 28$ & 23.0 & 24.5 & 100 & 17,800 & NA & NA & NA & NA \\
\hline $93 / 07 / 29$ & 22.0 & 25.0 & 100 & 29,300 & NA & NA & NA & NA \\
\hline $93 / 07 / 30$ & 22.0 & 25.0 & 100 & 29,400 & NA & NA & NA & NA \\
\hline $93 / 07 / 31$ & 20.5 & 25.0 & 100 & 20,100 & NA & NA & NA & NA \\
\hline $93 / 08 / 01$ & 20.5 & 25.0 & 100 & 20,100 & NA & NA & NA & NA \\
\hline
\end{tabular}


Appendix 16. Measurements at tidal stations in and near the tidal embayment of the Toms River, N.J.--Daily minimum and maximum values of water-quality characteristics from measurements by movable monitors, October 1992 - January 1994--Continued

\begin{tabular}{|c|c|c|c|c|c|c|c|c|}
\hline \multirow{3}{*}{$\begin{array}{c}\text { Date } \\
\text { year/month/day }\end{array}$} & \multicolumn{8}{|c|}{ Daily minimum and maximum } \\
\hline & \multicolumn{2}{|c|}{ Temperature } & \multicolumn{2}{|c|}{ Specific conductance } & \multicolumn{2}{|c|}{$\mathrm{pH}$} & \multicolumn{2}{|c|}{$\begin{array}{l}\text { Dissolved-oxygen } \\
\text { concentration }\end{array}$} \\
\hline & Min & $\operatorname{Max}$ & Min & $\operatorname{Max}$ & Min & $\operatorname{Max}$ & Min & Max \\
\hline
\end{tabular}

01408695 Toms River near South Toms River, mid-depth (continued)

$\begin{array}{lllllllll}93 / 08 / 02 & 20.5 & 26.0 & 100 & 24,400 & \text { NA } & \text { NA } & \text { NA } & \text { NA } \\ 93 / 08 / 03 & 21.5 & 26.0 & 100 & 22,100 & \text { NA } & \text { NA } & \text { NA } & \text { NA } \\ 93 / 08 / 04 & 21.5 & 25.0 & 100 & 18,100 & \text { NA } & \text { NA } & \text { NA } & \text { NA } \\ 93 / 08 / 05 & 21.0 & 25.5 & 100 & 27,000 & \text { NA } & \text { NA } & \text { NA } & \text { NA } \\ 93 / 08 / 06 & 19.5 & 25.0 & 100 & 25,400 & \text { NA } & \text { NA } & \text { NA } & \text { NA } \\ 93 / 08 / 07 & 19.0 & 20.5 & 100 & 100 & \text { NA } & \text { NA } & \text { NA } & \text { NA } \\ 93 / 08 / 08 & 18.5 & 21.0 & 100 & 100 & \text { NA } & \text { NA } & \text { NA } & \text { NA } \\ 93 / 08 / 09 & 19.0 & 21.5 & 100 & 100 & \text { NA } & \text { NA } & \text { NA } & \text { NA } \\ 93 / 08 / 10 & 18.5 & 21.5 & 100 & 100 & \text { NA } & \text { NA } & \text { NA } & \text { NA } \\ 93 / 08 / 11 & 21.0 & 21.0 & 100 & 100 & \text { NA } & \text { NA } & \text { NA } & \text { NA }\end{array}$

01408722 Toms River near Toms River, near surface

$\begin{array}{rrrrrrrrr}93 / 06 / 15 & 25.0 & 26.0 & 16,400 & 19,800 & 8.4 & 8.8 & 8.3 & 10.3 \\ 93 / 06 / 16 & 24.0 & 26.5 & 16,000 & 19,200 & 8.3 & 8.8 & 7.4 & 10.8 \\ 93 / 06 / 17 & 24.5 & 27.0 & 15,000 & 17,800 & 8.3 & 8.8 & 8.6 & 11.8 \\ 93 / 06 / 18 & 25.5 & 28.0 & 12,500 & 19,100 & 8.0 & 8.7 & 8.5 & 10.4 \\ 93 / 06 / 19 & 25.5 & 28.5 & 12,600 & 20,700 & 7.5 & 8.4 & 6.7 & 9.3 \\ 93 / 06 / 20 & 25.5 & 26.5 & 16,500 & 20,200 & 7.1 & 8.1 & 6.1 & 9.0 \\ 93 / 06 / 21 & 24.0 & 27.0 & 11,000 & 20,500 & 6.9 & 8.2 & 6.7 & 9.2 \\ 93 / 06 / 22 & 25.0 & 26.5 & 16,400 & 20,700 & 7.0 & 7.7 & 5.7 & 7.1 \\ 93 / 06 / 23 & 23.5 & 25.0 & 20,400 & 21,400 & 7.3 & 8.2 & 6.0 & 9.5 \\ & & & & & & & & \\ 93 / 10 / 21 & 15.5 & 17.0 & 20,200 & 24,700 & 7.6 & 8.0 & 8.4 & 9.5 \\ 93 / 10 / 22 & 14.0 & 15.5 & 17,800 & 23,800 & 7.3 & 7.7 & 7.9 & 9.0 \\ 93 / 10 / 23 & 13.0 & 16.0 & 12,500 & 22,500 & 6.9 & 7.7 & 8.6 & 9.7 \\ 93 / 10 / 24 & 12.5 & 15.0 & 15,300 & 25,800 & 7.1 & 7.9 & 8.8 & 9.6 \\ 93 / 10 / 25 & 12.5 & 17.0 & 14,000 & 23,000 & 6.9 & 7.7 & 8.8 & 9.6 \\ 93 / 10 / 26 & 13.5 & 15.0 & 13,000 & 21,100 & 7.1 & 7.6 & 8.6 & 9.4 \\ 93 / 10 / 27 & 12.5 & 14.0 & 7,000 & 25,500 & 6.6 & 7.9 & 8.5 & 9.6 \\ 93 / 10 / 28 & 13.5 & 14.0 & 23,100 & 25,800 & 7.6 & 7.8 & 8.0 & 8.7\end{array}$

01408722 Toms River near Toms River, near bottom

$\begin{array}{rrrrrrrrr}93 / 06 / 15 & 24.0 & 25.5 & 19,200 & 21,700 & 7.6 & 8.5 & 4.5 & 8.6 \\ 93 / 06 / 16 & 24.0 & 26.0 & 18,200 & 24,200 & 7.4 & 8.5 & 2.9 & 8.9 \\ 93 / 06 / 17 & 24.5 & 27.0 & 17,100 & 23,000 & 7.2 & 8.8 & 2.0 & 11.1 \\ 93 / 06 / 18 & 25.0 & 27.5 & 12,500 & 24,300 & 7.8 & 8.7 & 3.6 & 9.7 \\ 93 / 06 / 19 & 25.5 & 27.0 & 20,800 & 25,700 & 7.4 & 8.0 & 2.9 & 6.9 \\ 93 / 06 / 20 & 26.0 & 27.0 & 19,300 & 24,400 & 7.2 & 7.8 & 2.7 & 7.6 \\ 93 / 06 / 21 & 25.5 & 26.5 & 19,300 & 22,100 & 7.2 & 7.6 & 5.0 & 7.1 \\ 93 / 06 / 22 & 25.5 & 26.0 & 21,400 & 26,700 & 7.1 & 7.5 & 3.5 & 6.1\end{array}$


Appendix 16. Measurements at tidal stations in and near the tidal embayment of the Toms River, N.J.--Daily minimum and maximum values of water-quality characteristics from measurements by movable monitors, October 1992 - January 1994--Continued

\begin{tabular}{|c|c|c|c|c|c|c|c|c|}
\hline \multirow{3}{*}{$\begin{array}{c}\text { Date } \\
\text { year/month/day }\end{array}$} & \multicolumn{8}{|c|}{ Daily minimum and maximum } \\
\hline & \multicolumn{2}{|c|}{ Temperature } & \multicolumn{2}{|c|}{ Specific conductance } & \multicolumn{2}{|c|}{$\mathrm{pH}$} & \multicolumn{2}{|c|}{$\begin{array}{l}\text { Dissolved-oxygen } \\
\text { concentration }\end{array}$} \\
\hline & Min & $\operatorname{Max}$ & Min & $\operatorname{Max}$ & Min & $\operatorname{Max}$ & Min & $\operatorname{Max}$ \\
\hline \multicolumn{9}{|c|}{ 01408722 Toms River near Toms River, near bottom (continued) } \\
\hline $93 / 06 / 23$ & 24.0 & 26.0 & 22,000 & 26,700 & 7.2 & 7.9 & 3.3 & 8.9 \\
\hline $93 / 10 / 21$ & 15.5 & 15.5 & 26,400 & 29,500 & 7.8 & 8.0 & 7.5 & 8.9 \\
\hline $93 / 10 / 22$ & 15.5 & 16.0 & 24,900 & 31,200 & 7.9 & 8.0 & 7.4 & 9.2 \\
\hline $93 / 10 / 23$ & 15.5 & 16.0 & 27,100 & 31,600 & 7.8 & 8.1 & 6.7 & 9.1 \\
\hline $93 / 10 / 24$ & 15.0 & 16.0 & 27,300 & 30,100 & 7.8 & 8.1 & 7.2 & 8.8 \\
\hline $93 / 10 / 25$ & 14.5 & 15.5 & 25,300 & 29,800 & 7.7 & 8.1 & 6.4 & 9.2 \\
\hline $93 / 10 / 26$ & 14.0 & 15.5 & 19,700 & 29,600 & 7.5 & 7.9 & 7.3 & 9.2 \\
\hline $93 / 10 / 27$ & 13.5 & 14.0 & 19,000 & 27,300 & 7.5 & 7.9 & 8.2 & 9.0 \\
\hline $93 / 10 / 28$ & 13.5 & 14.0 & 27,300 & 31,500 & 7.9 & 8.0 & 8.3 & 8.9 \\
\hline
\end{tabular}


Appendix 17. Measurements at tidal stations in and near the tidal embayment of the Toms River, N.J.--Water-quality data from manual measurements, October 1992 - October 1993

[Water-quality measurements were made at the stations listed below. USGS, U.S. Geological Survey; NA, not applicable or not available; >, greater than]

\begin{tabular}{|c|c|c|c|}
\hline $\begin{array}{l}\text { Index } \\
\text { number } \\
\text { (fig. 3) }\end{array}$ & USGS station number & USGS station name & $\begin{array}{l}\text { Location of the } \\
\text { channel in the } \\
\text { cross section, in } \\
\text { percent distance } \\
\text { from left to righ } \\
\text { bank }\end{array}$ \\
\hline 18 & 01408685 & Toms River at Garden State Parkway & 50 \\
\hline 19 & 01408690 & $\begin{array}{l}\text { Toms River } 0.2 \text { miles downstream from } \\
\text { Garden State Parkway }\end{array}$ & 10 \\
\hline 20 & 01408695 & Toms River near South Toms River & 50 \\
\hline 21 & 01408700 & Toms River at Toms River & $\begin{array}{c}25,75 \\
\text { (two channels) }\end{array}$ \\
\hline 22 & 01408719 & $\begin{array}{l}\text { Toms River at Cedar Point at South Toms } \\
\text { River }\end{array}$ & 50 \\
\hline 23 & 01408722 & Toms River near Toms River & 50 \\
\hline 24 & 01408730 & Toms River at Pine Beach & 50 \\
\hline 25 & 01408735 & $\begin{array}{l}\text { Toms River at Maple Avenue Pier at Island } \\
\text { Heights }\end{array}$ & 50 \\
\hline 26 & 01408740 & Toms River at Island Heights & 50 \\
\hline 27 & 395540074055400 & Barnegat Bay near Ocean Gate & NA \\
\hline 28 & 395611074061800 & Barnegat Bay near Bay Shore & NA \\
\hline 29 & 395703074060100 & Barnegat Bay near Gilford Park & NA \\
\hline
\end{tabular}


Appendix 17. Measurements at tidal stations in and near the tidal embayment of the Toms River, N.J.--Water-quality data from manual measurements, October 1992 - October 1993--Continued

The following information is presented:

\begin{tabular}{|c|c|c|}
\hline Characteristic & Unit & Rounding \\
\hline $\begin{array}{l}\text { Date and time of } \\
\text { measurement }\end{array}$ & NA & NA \\
\hline Distance from left bank & $\begin{array}{l}\text { Percent of distance from left to } \\
\text { right bank }\end{array}$ & 1 \\
\hline Sample depth & Feet & 0.5 \\
\hline Total depth & Feet & 0.5 \\
\hline Temperature & Degrees Celsius & 0.5 \\
\hline $\mathrm{pH}$ & Standard units & 0.1 \\
\hline $\begin{array}{l}\text { Dissolved-oxygen } \\
\text { concentration }\end{array}$ & Milligrams per liter & 0.1 \\
\hline $\begin{array}{l}\text { Dissolved-oxygen } \\
\text { concentration as a percent } \\
\text { of saturation }\end{array}$ & Percent & 1 \\
\hline Barometric pressure & Millimeters of mercury & 5 \\
\hline Specific conductance & $\begin{array}{l}\text { Microsiemens per centimeter at } \\
25 \text { degrees Celsius }\end{array}$ & NA \\
\hline Secchi-disk depth & Feet & 0.5 \\
\hline Tidal-water level & $\begin{array}{l}\text { Feet above the National Geo- } \\
\text { detic Vertical Datum of } 1929\end{array}$ & 0.01 \\
\hline
\end{tabular}




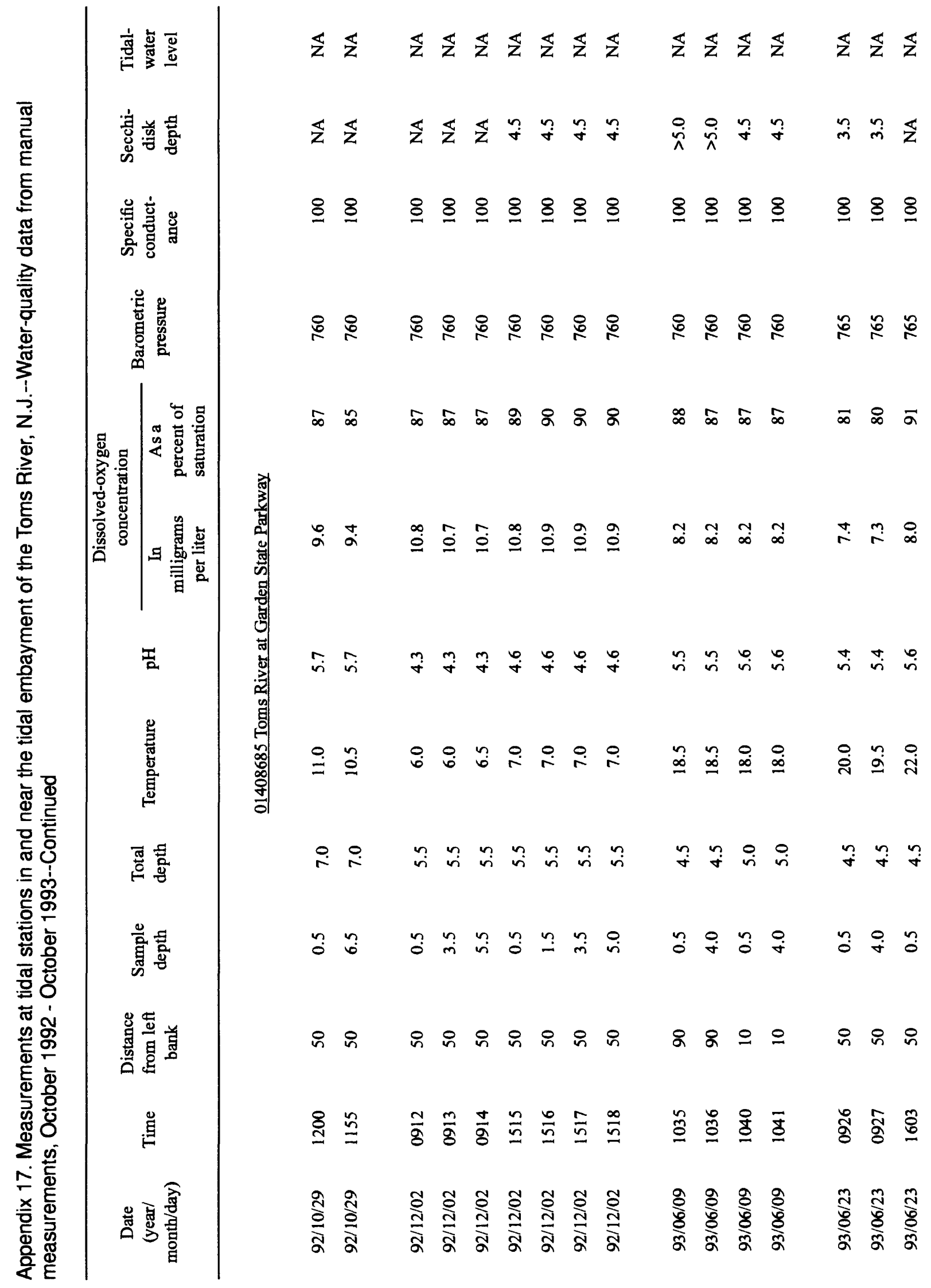




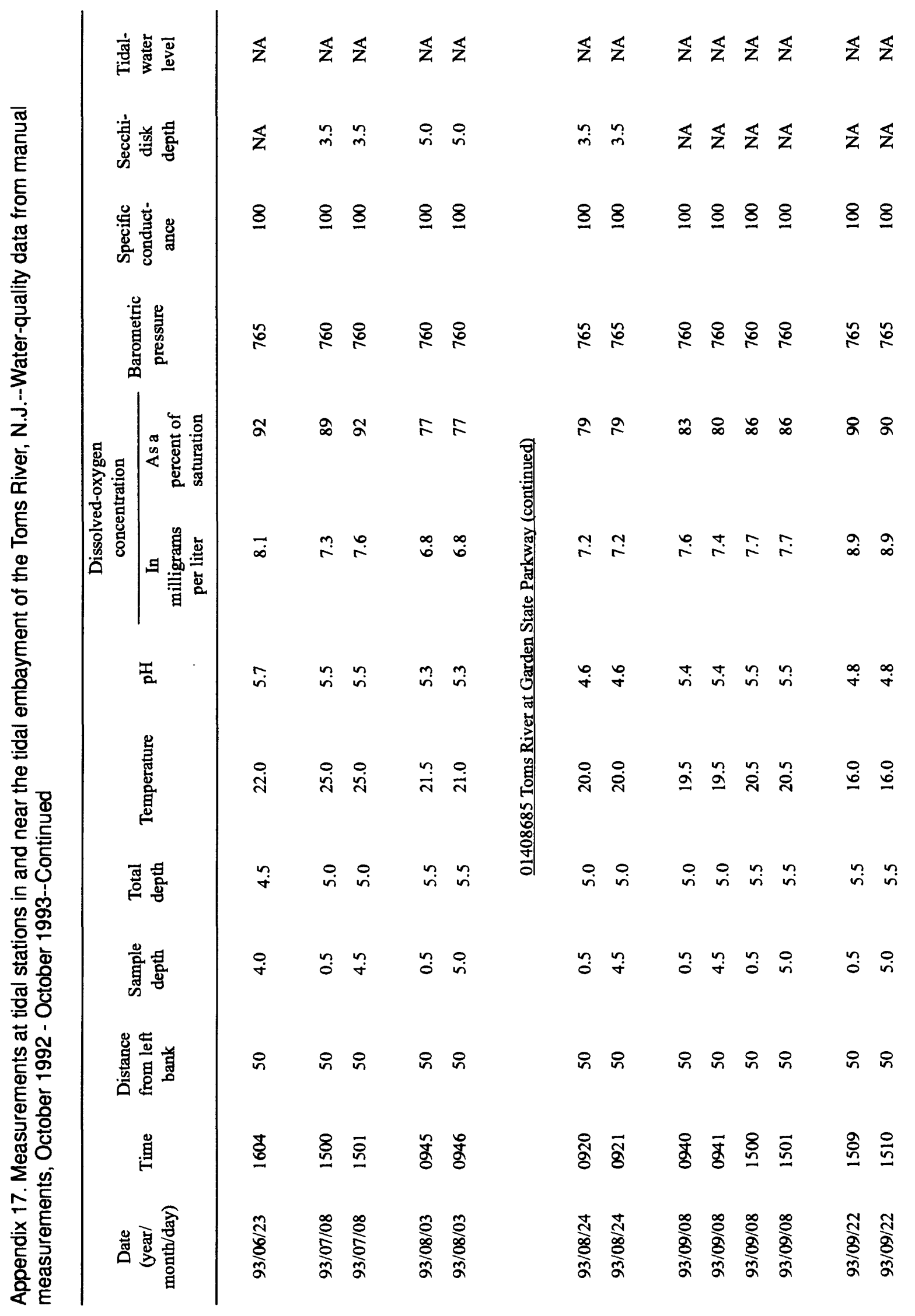




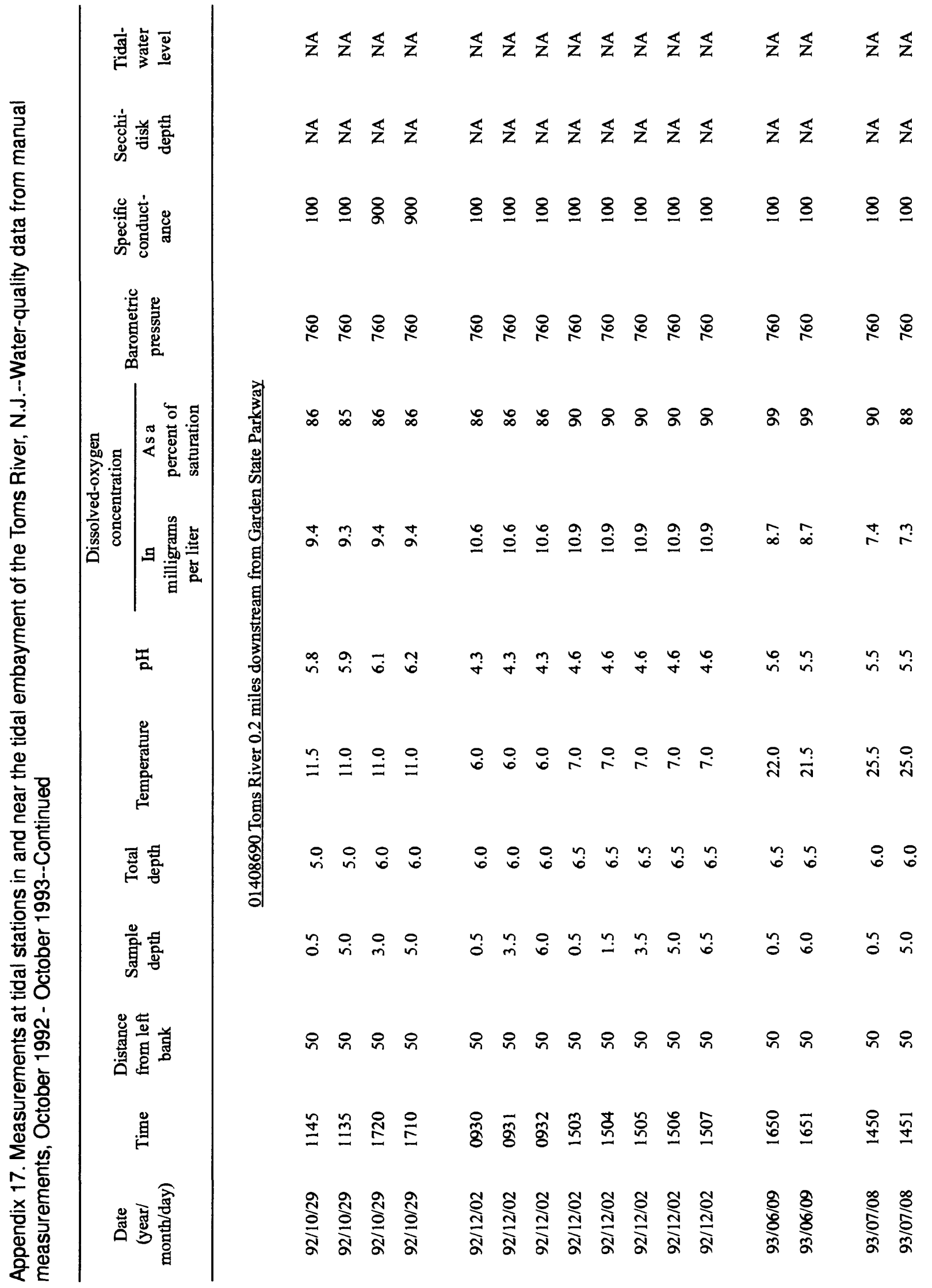




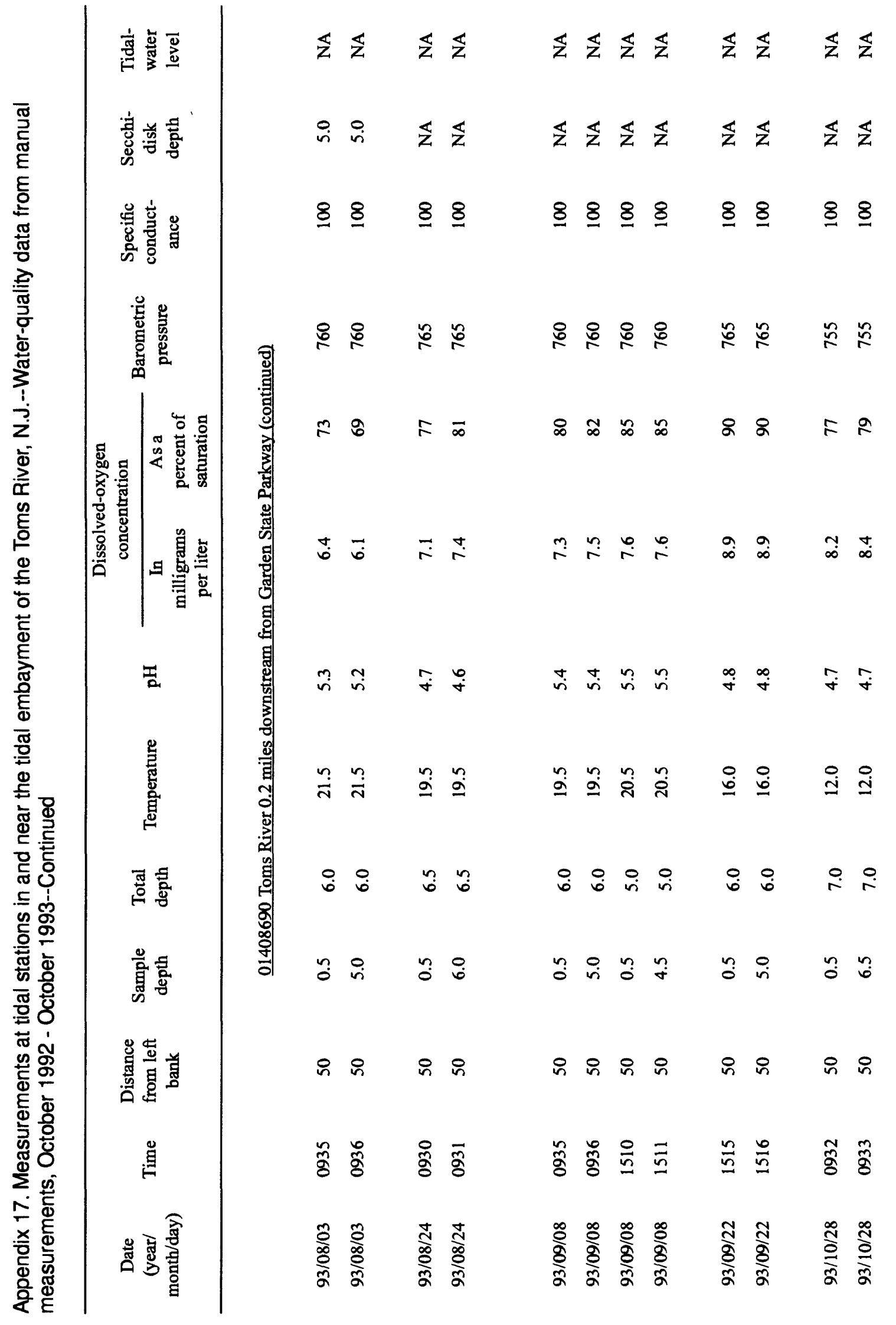




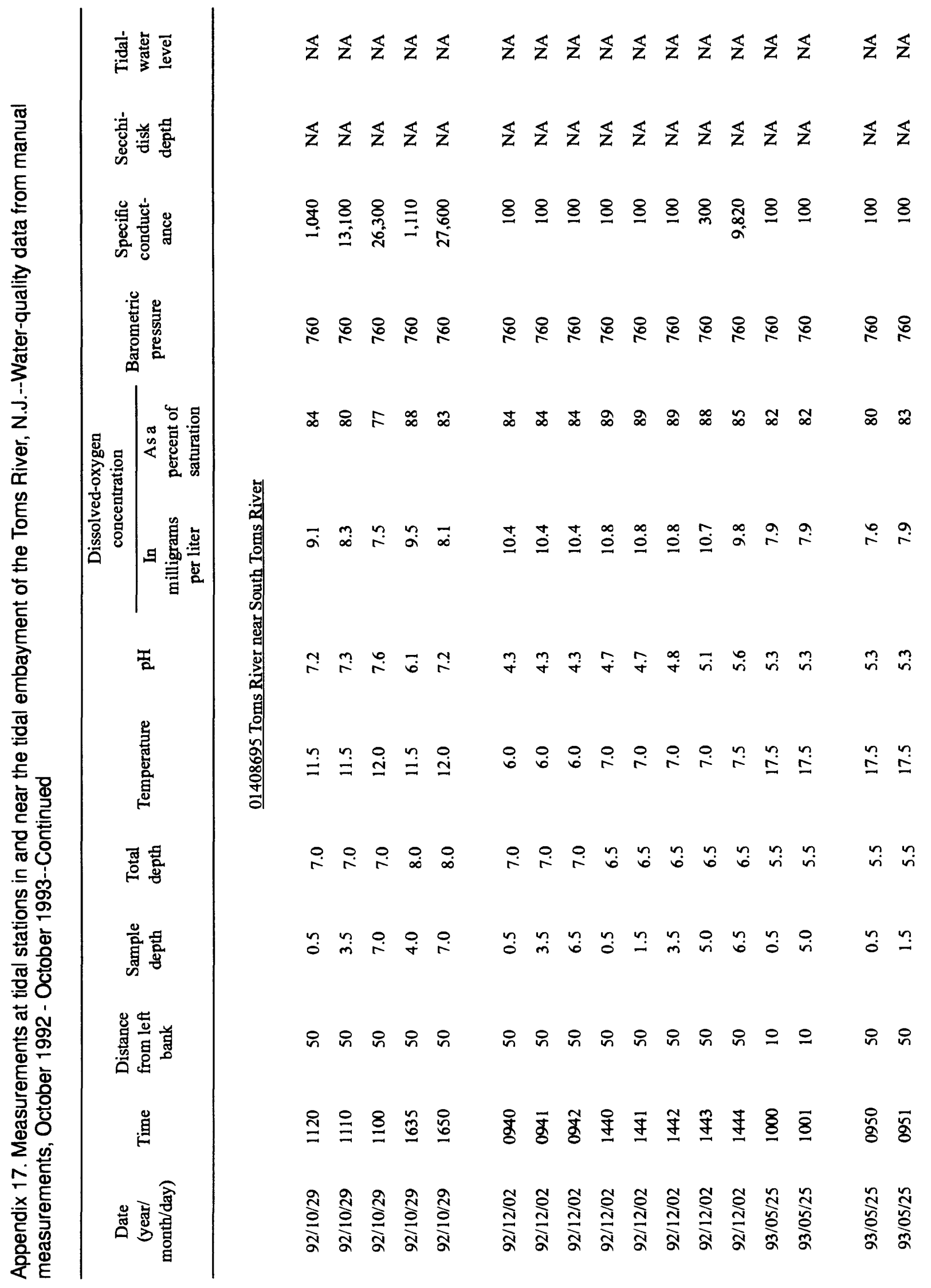




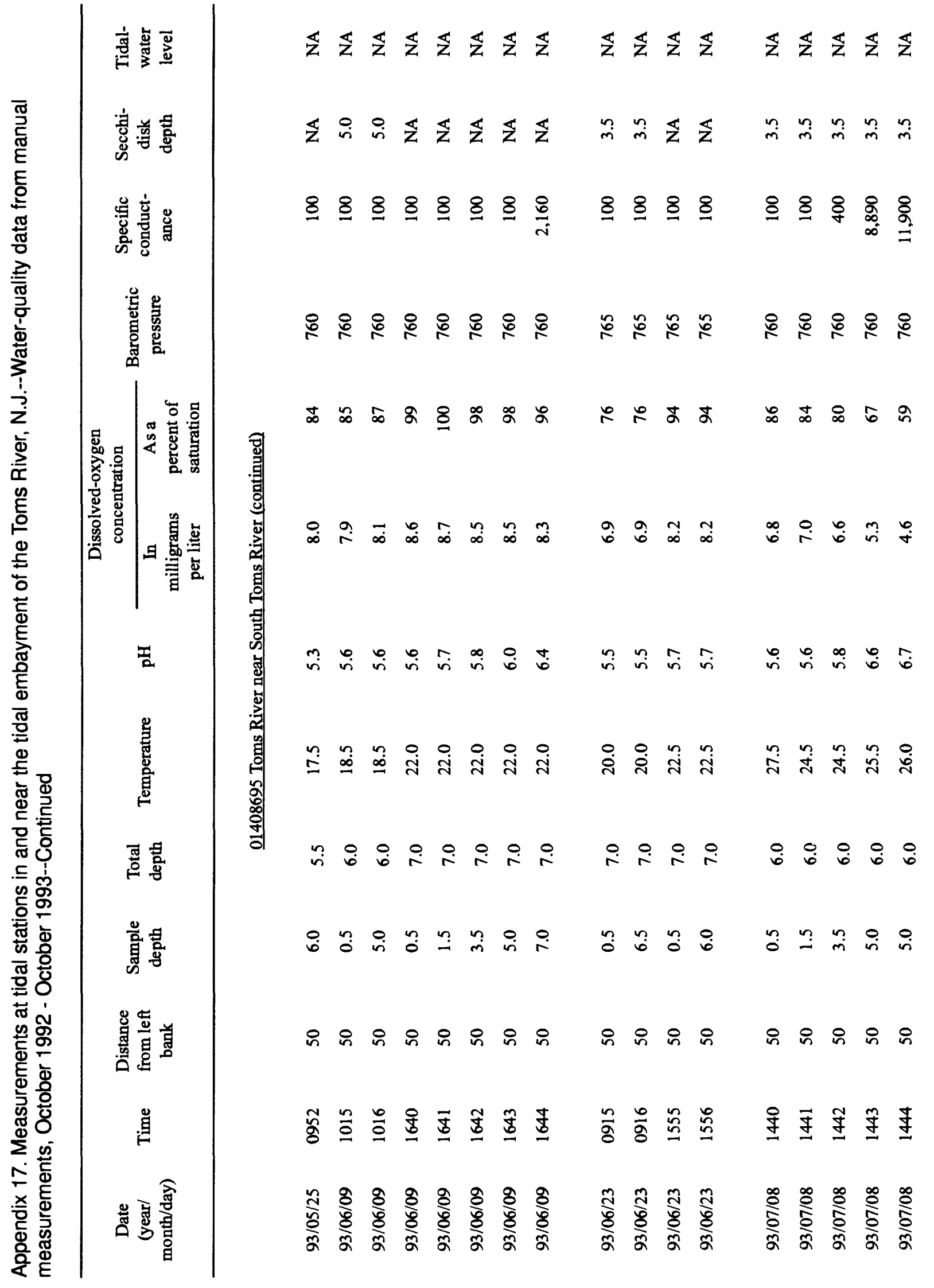




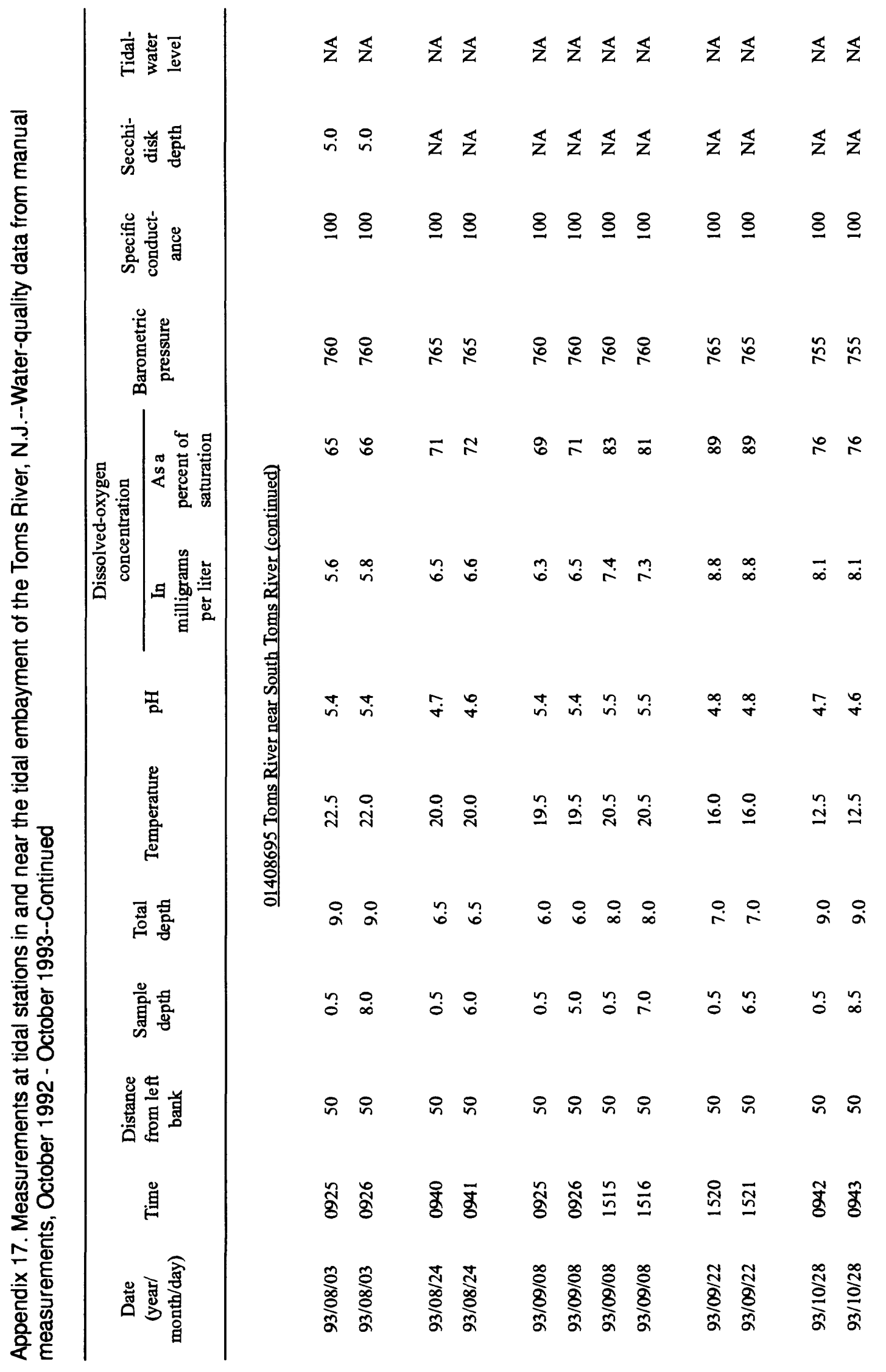




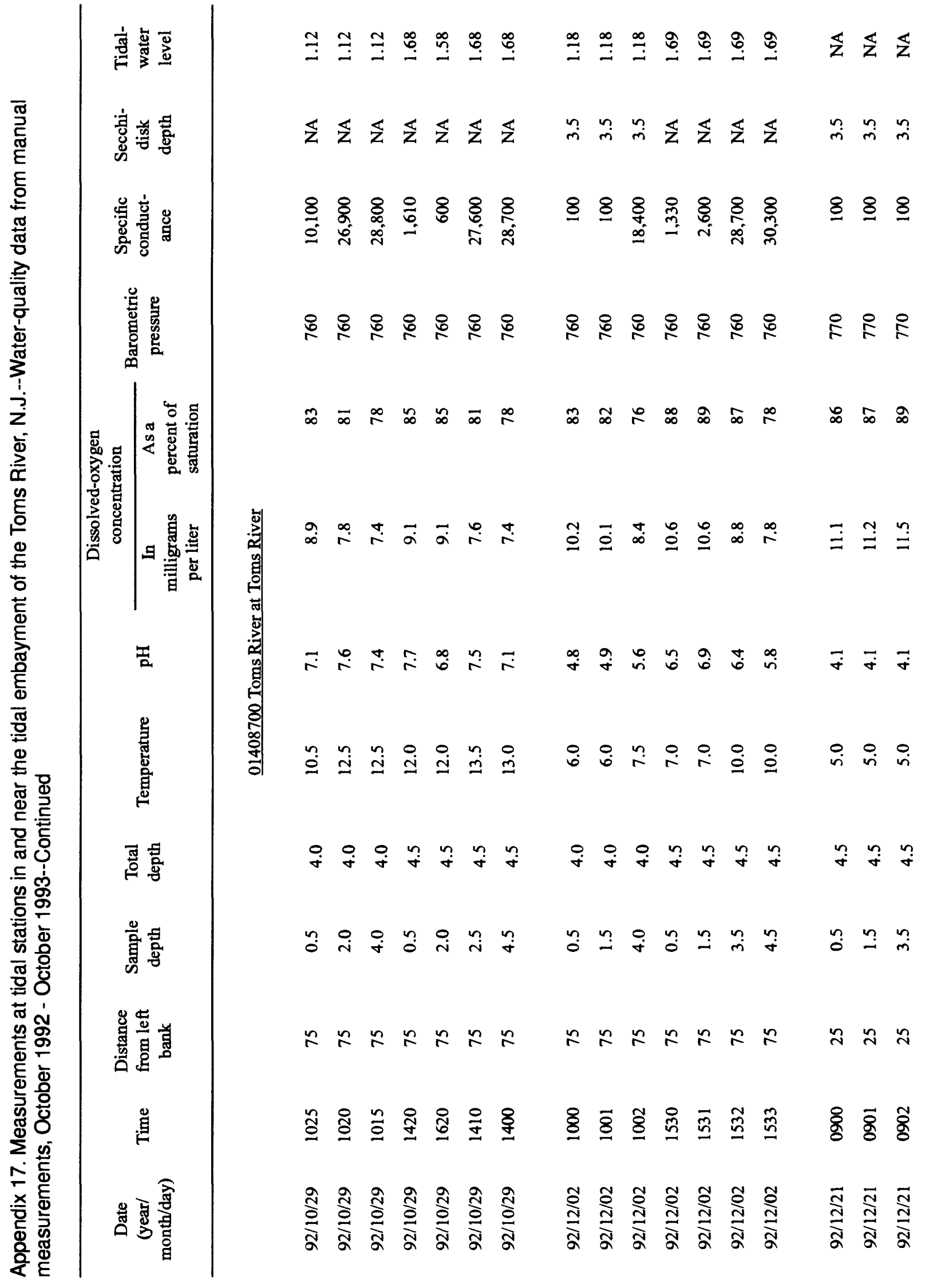




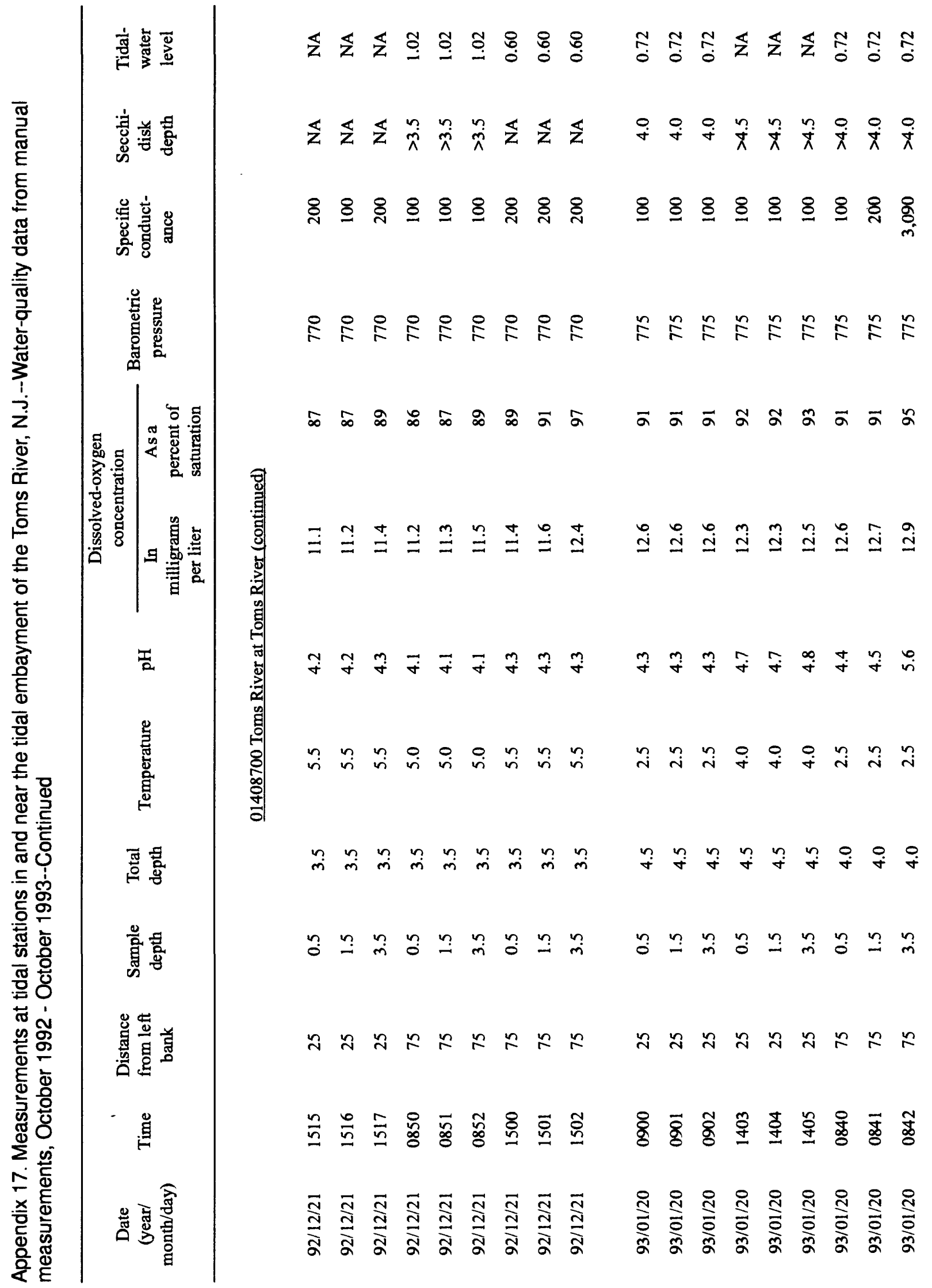




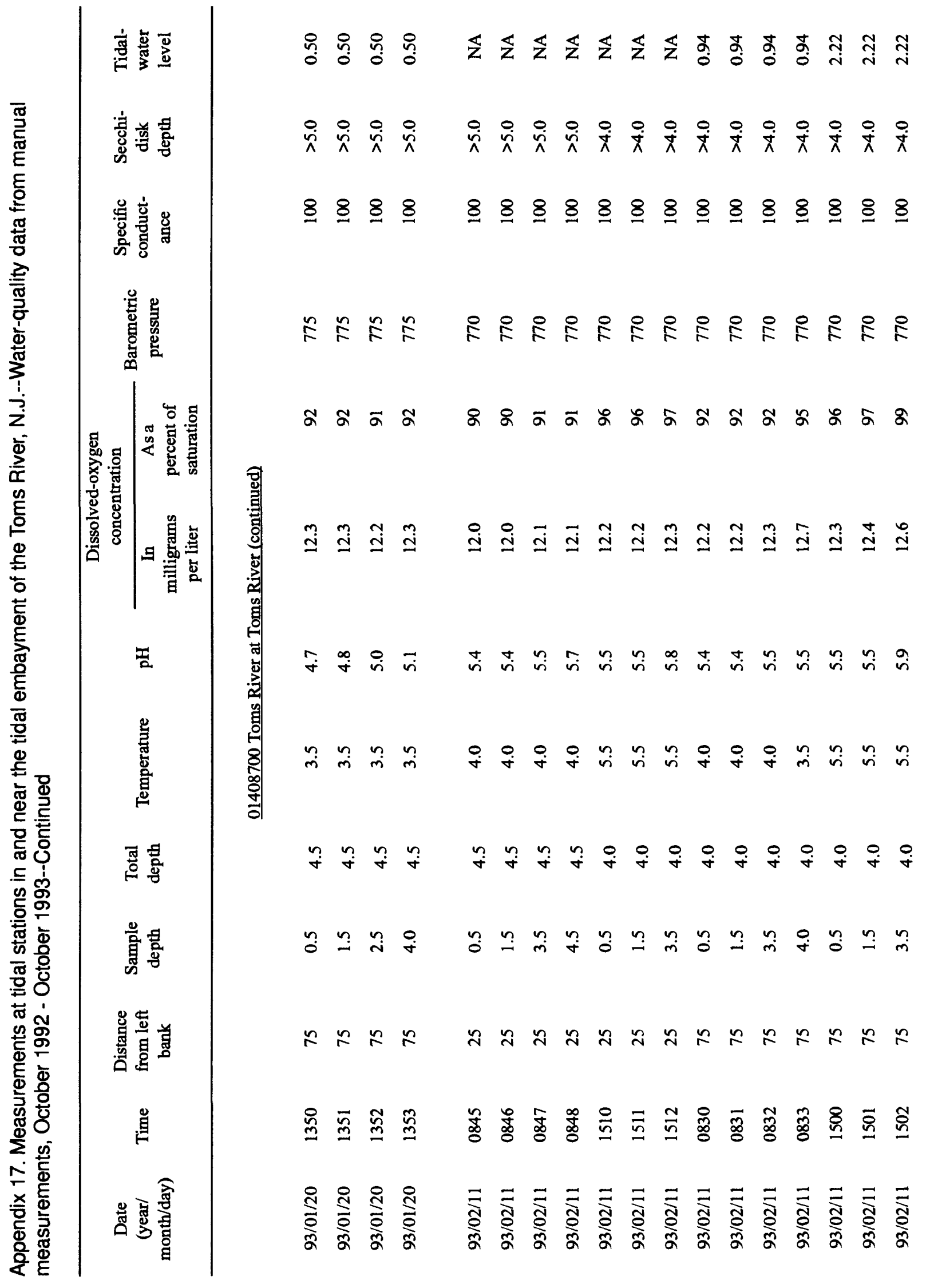




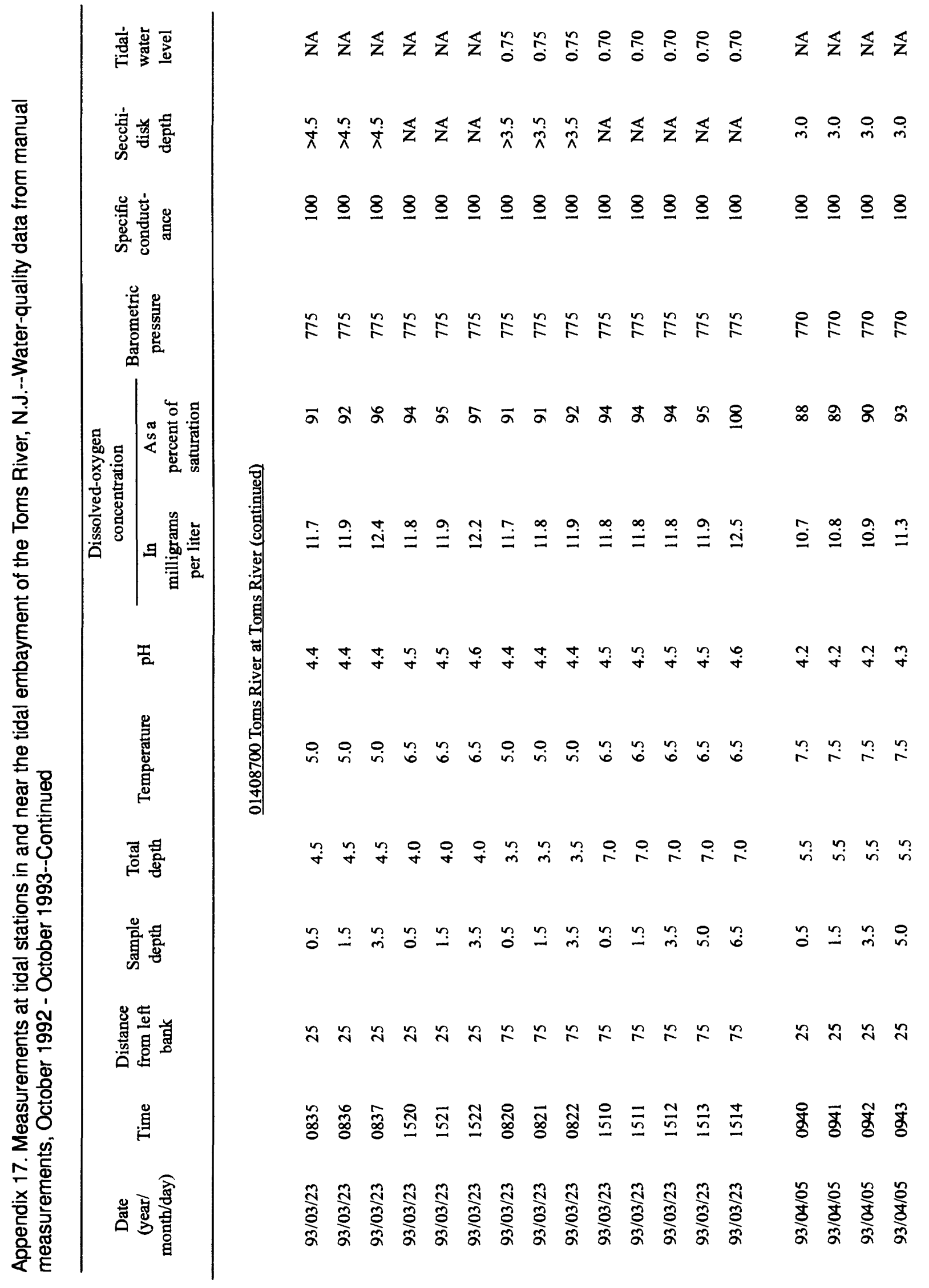




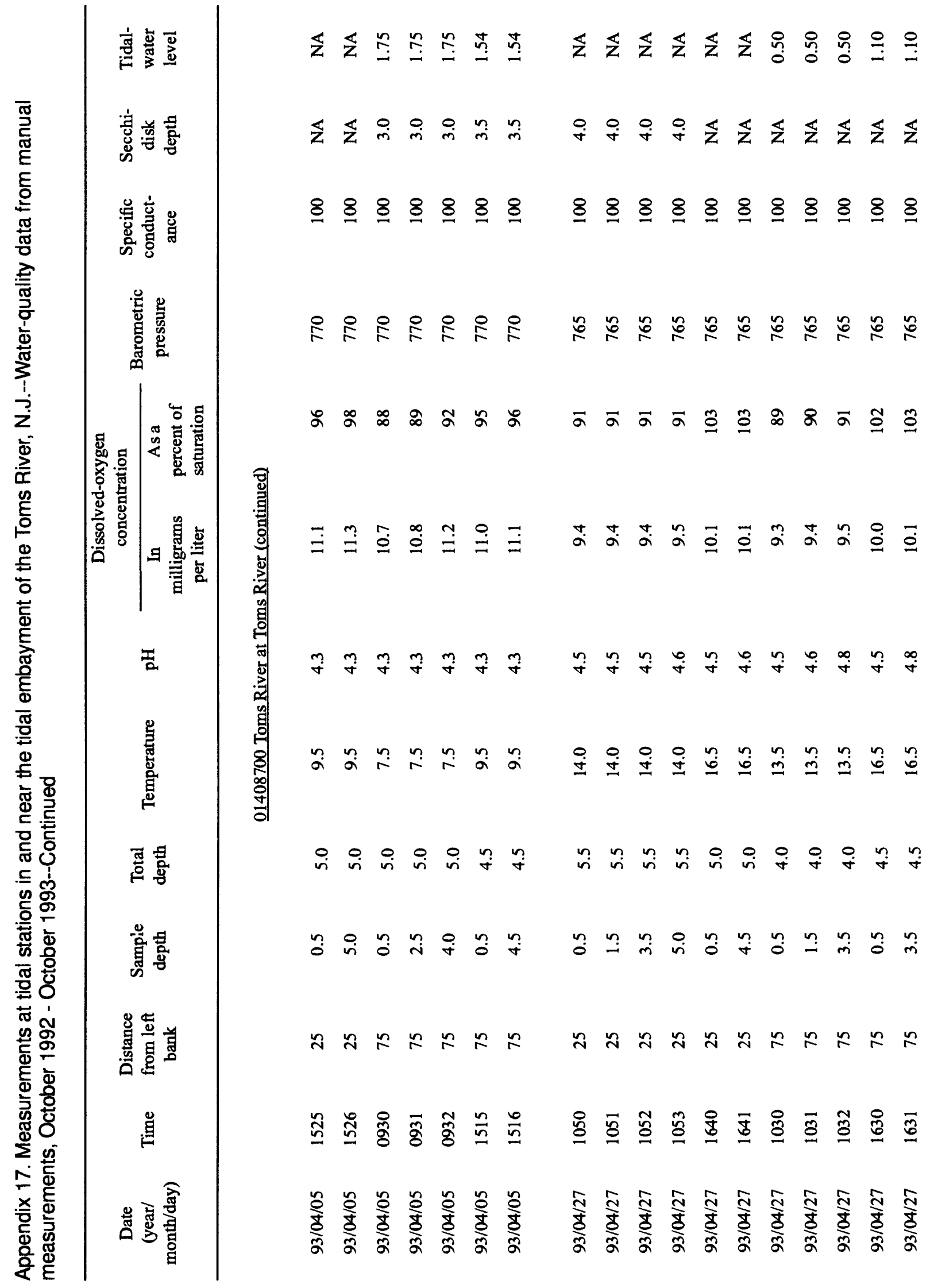




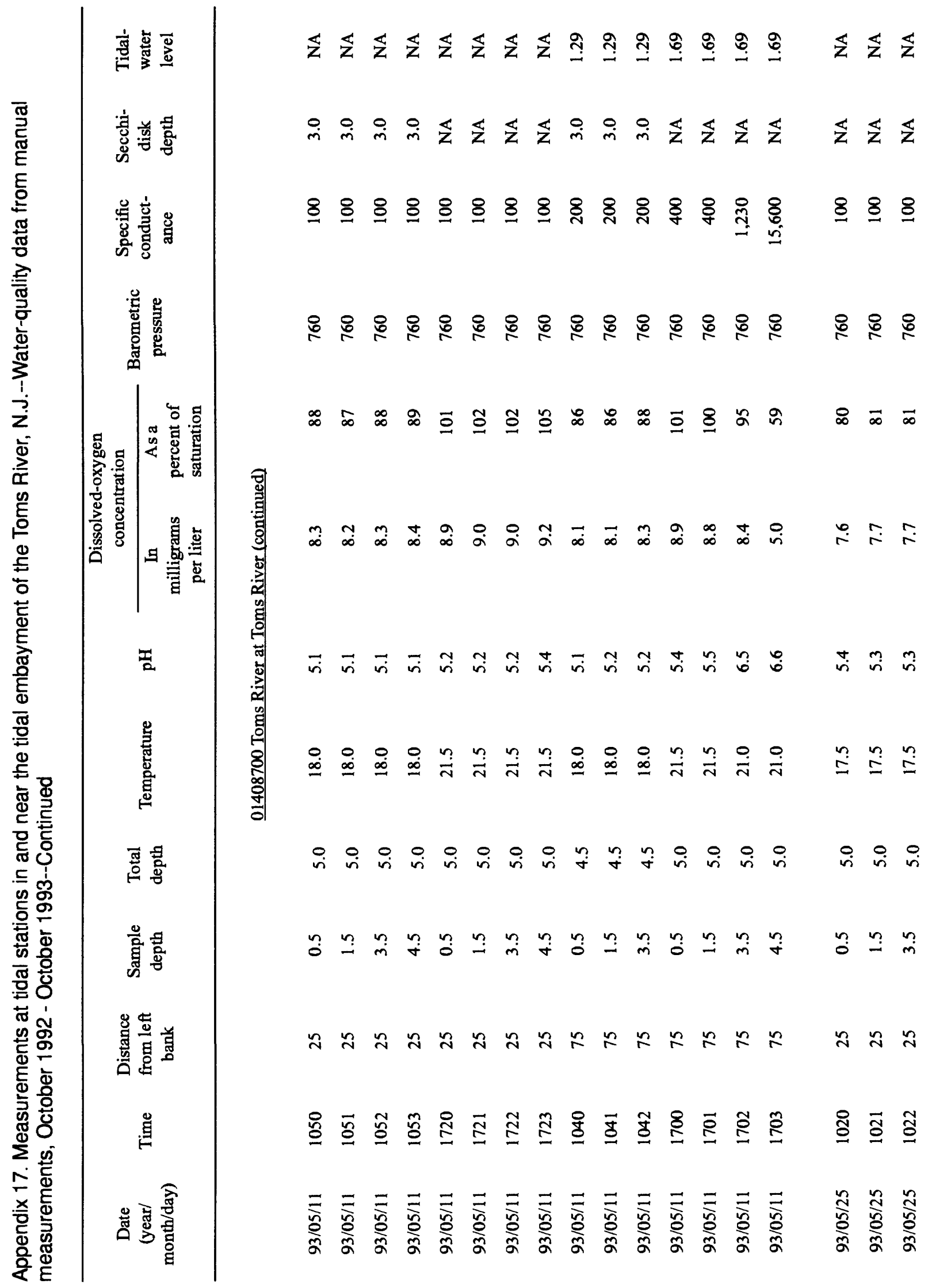




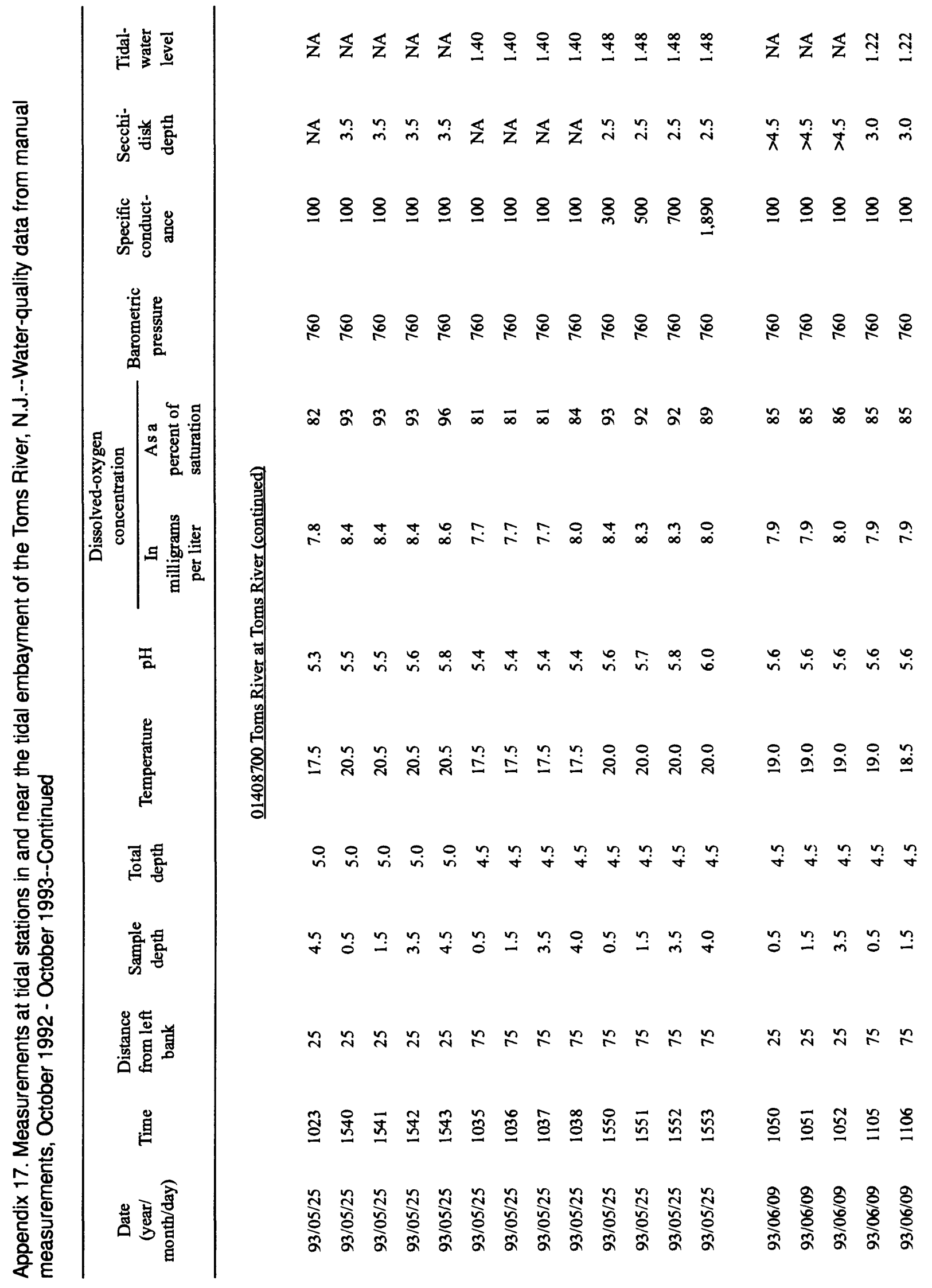




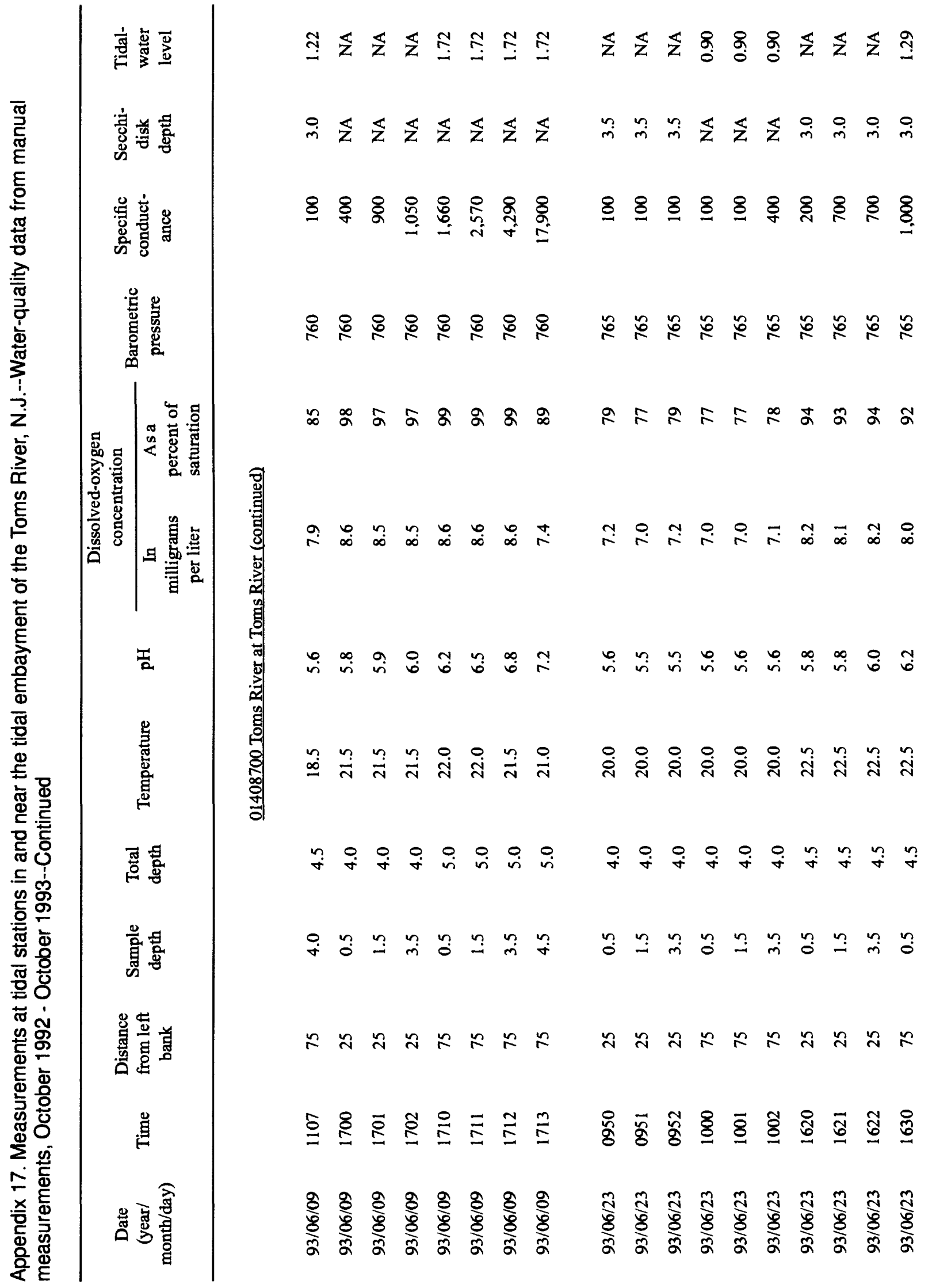




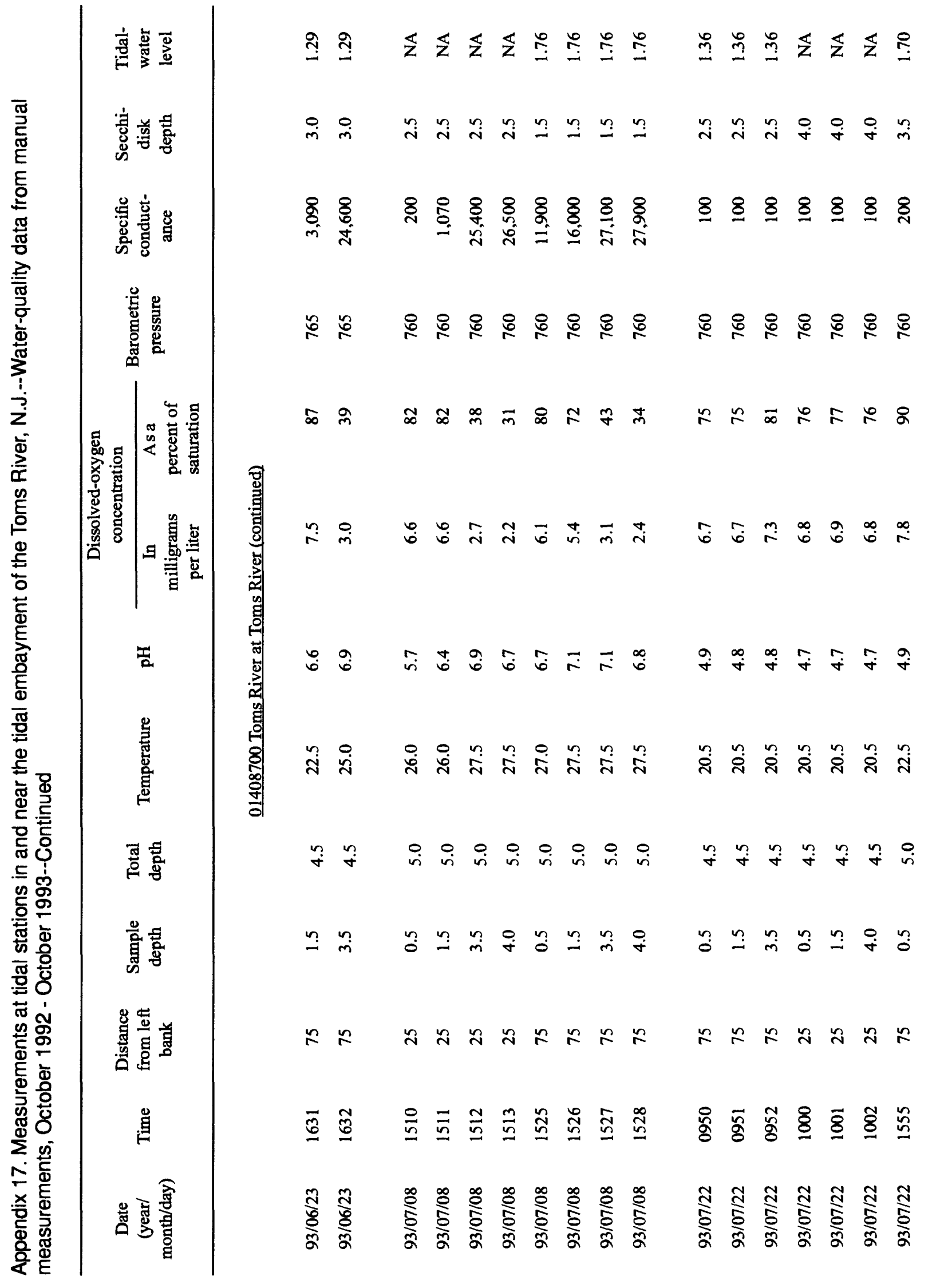




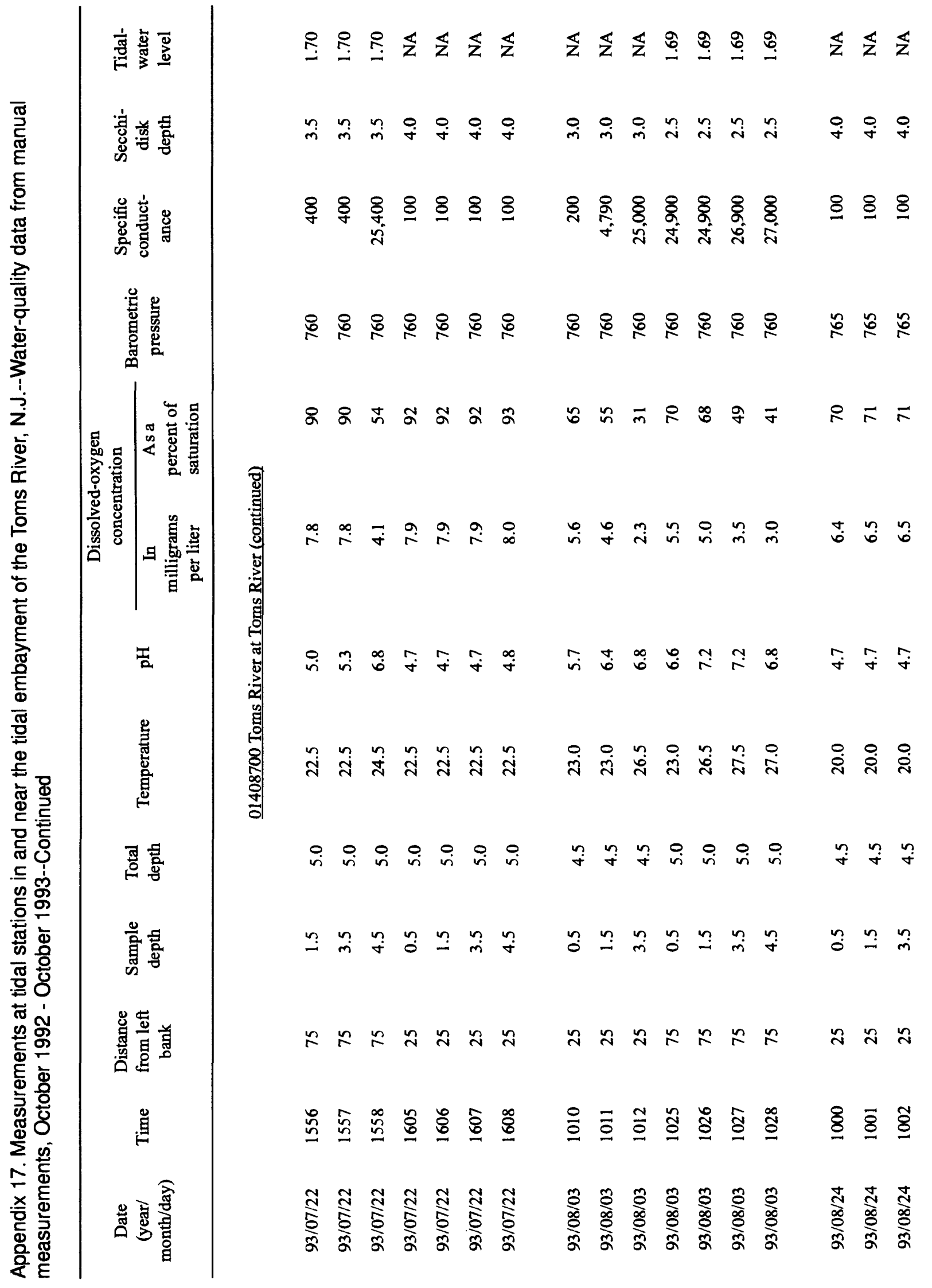




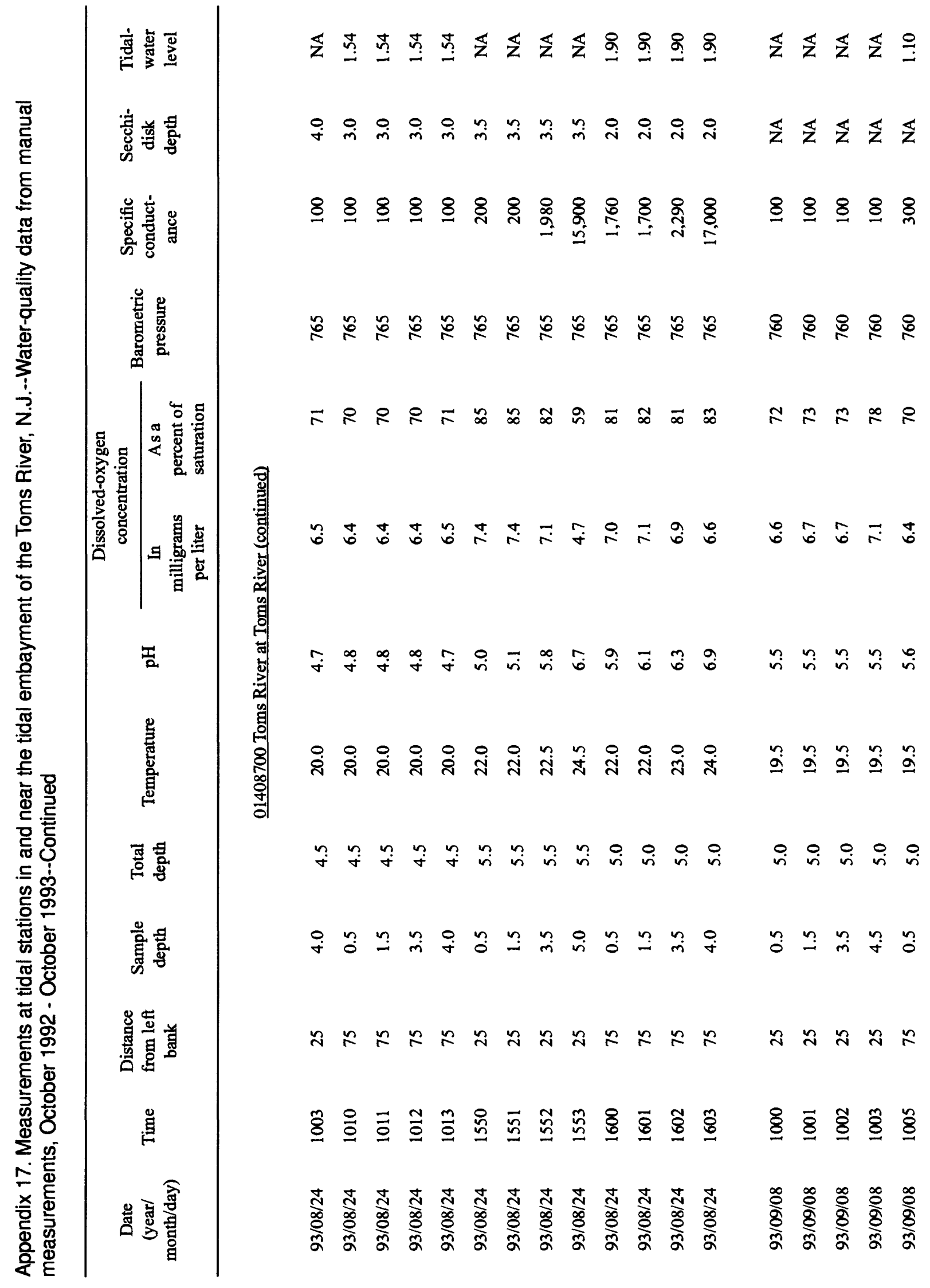




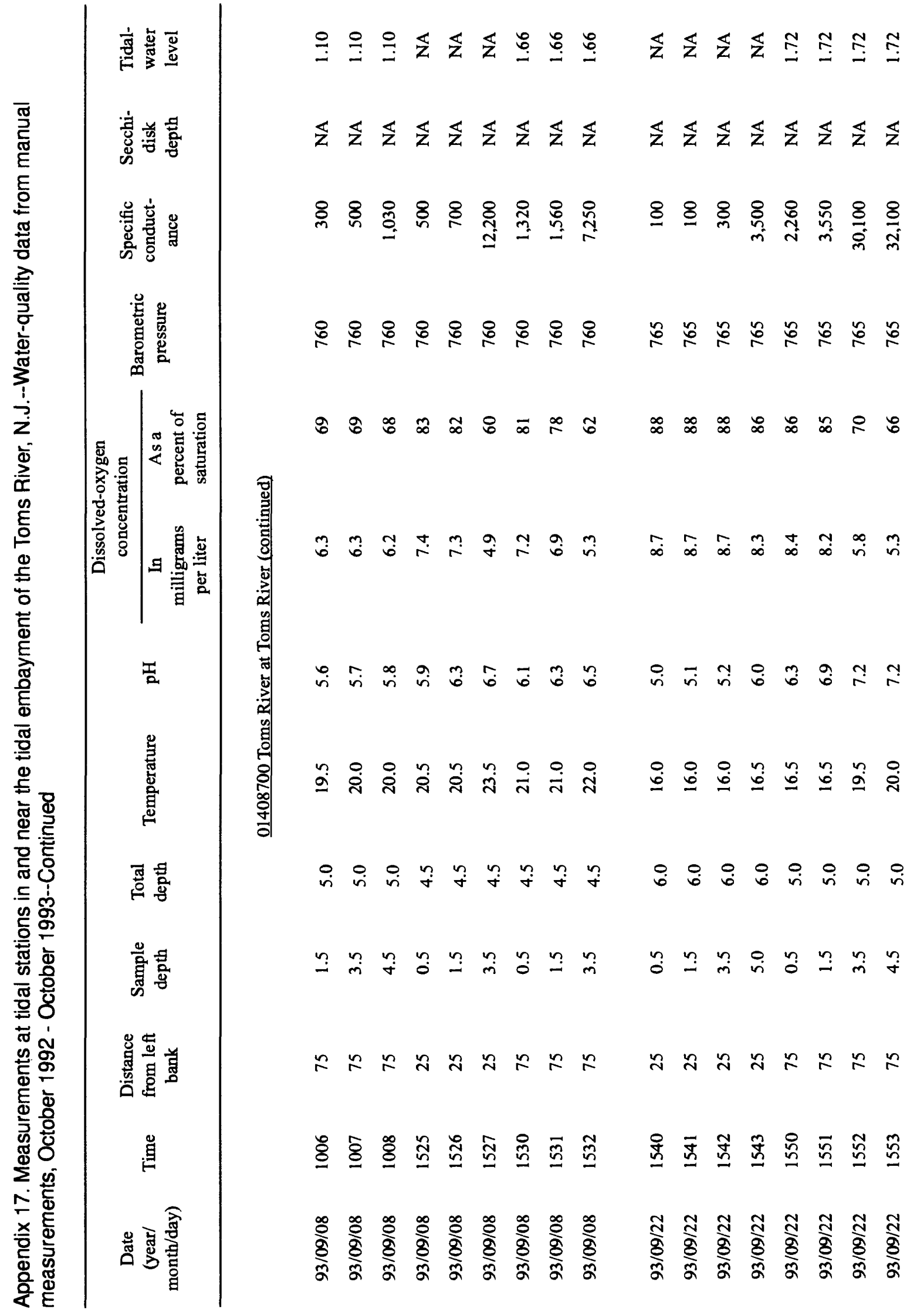




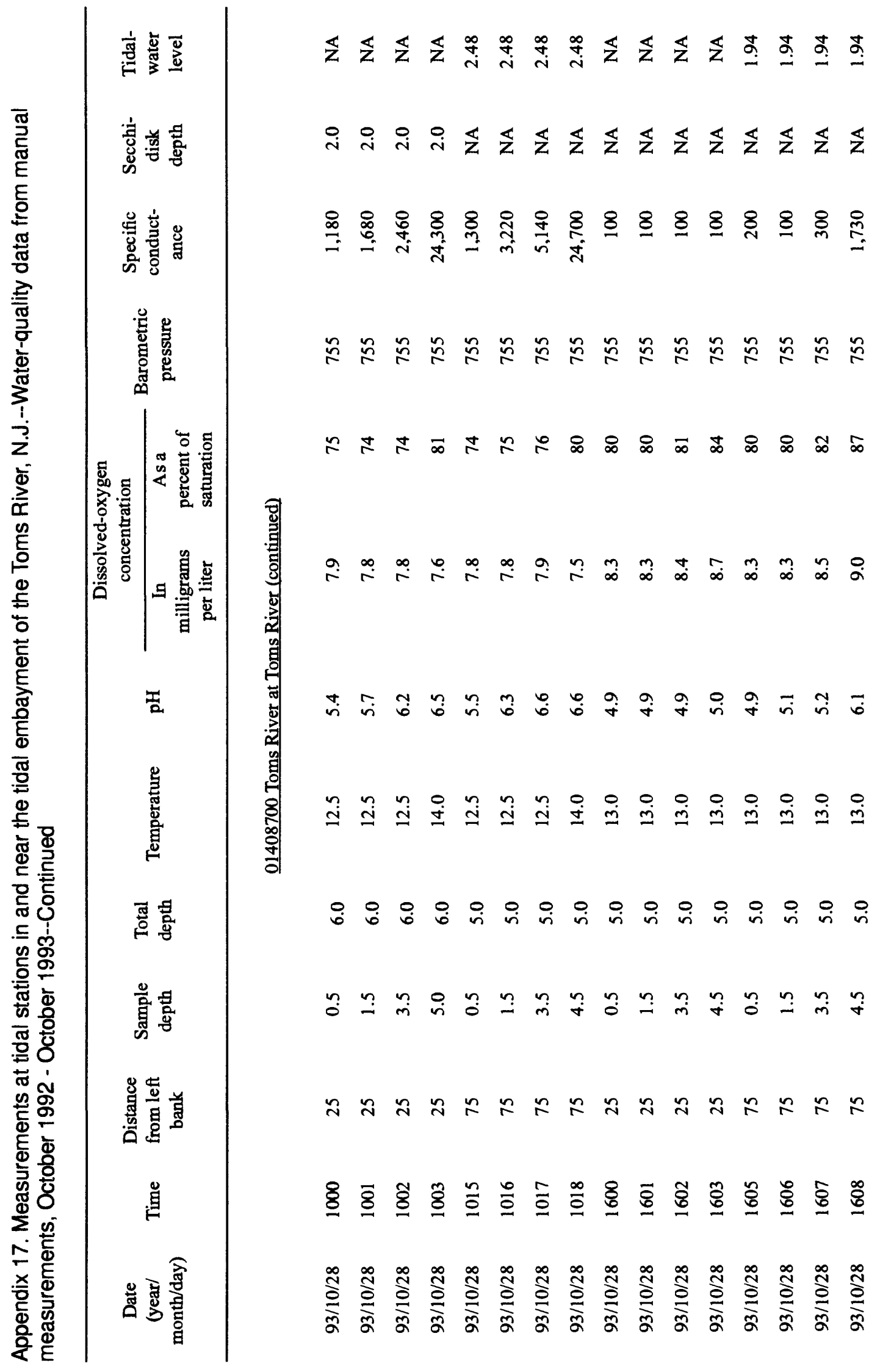




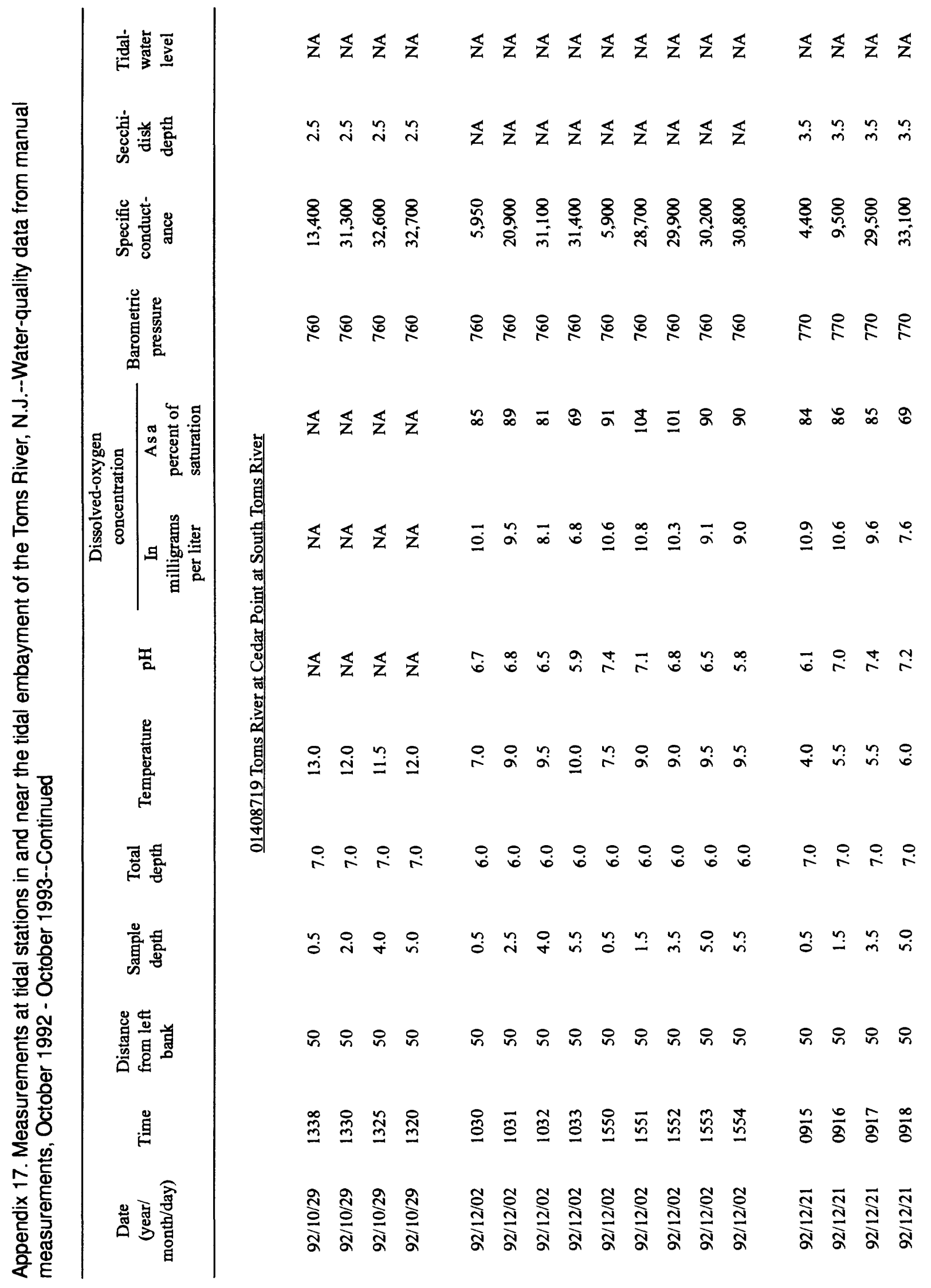




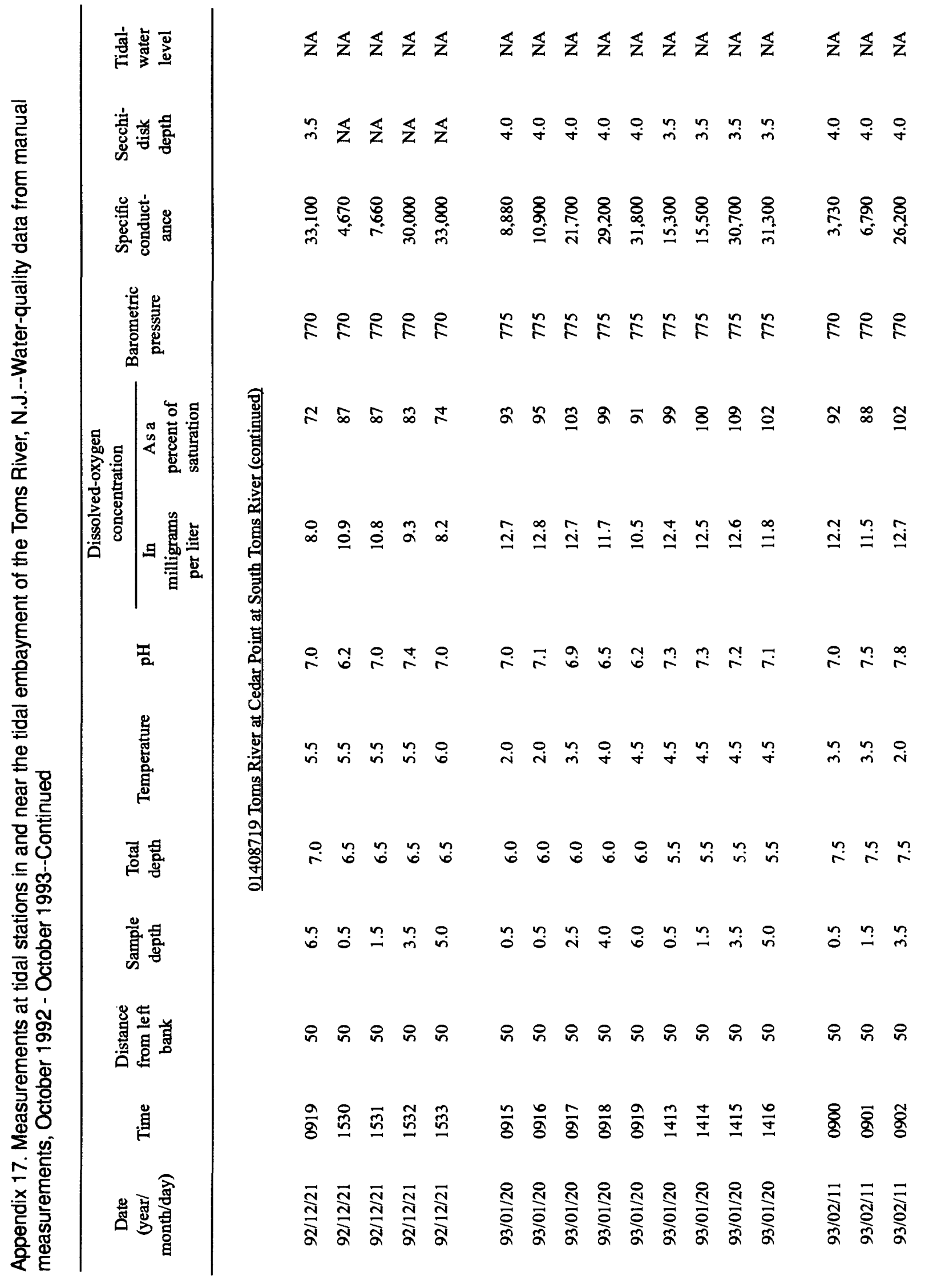




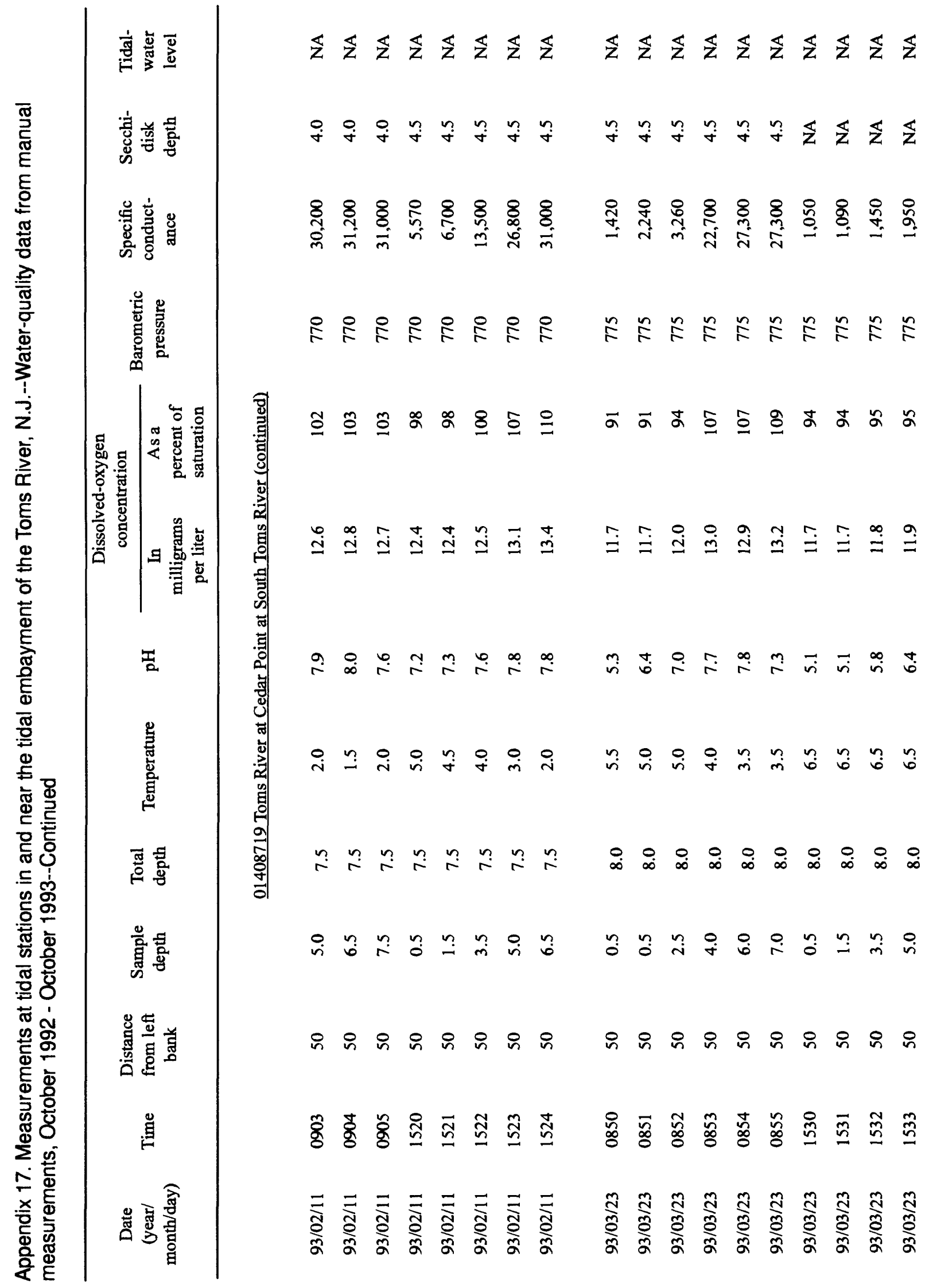




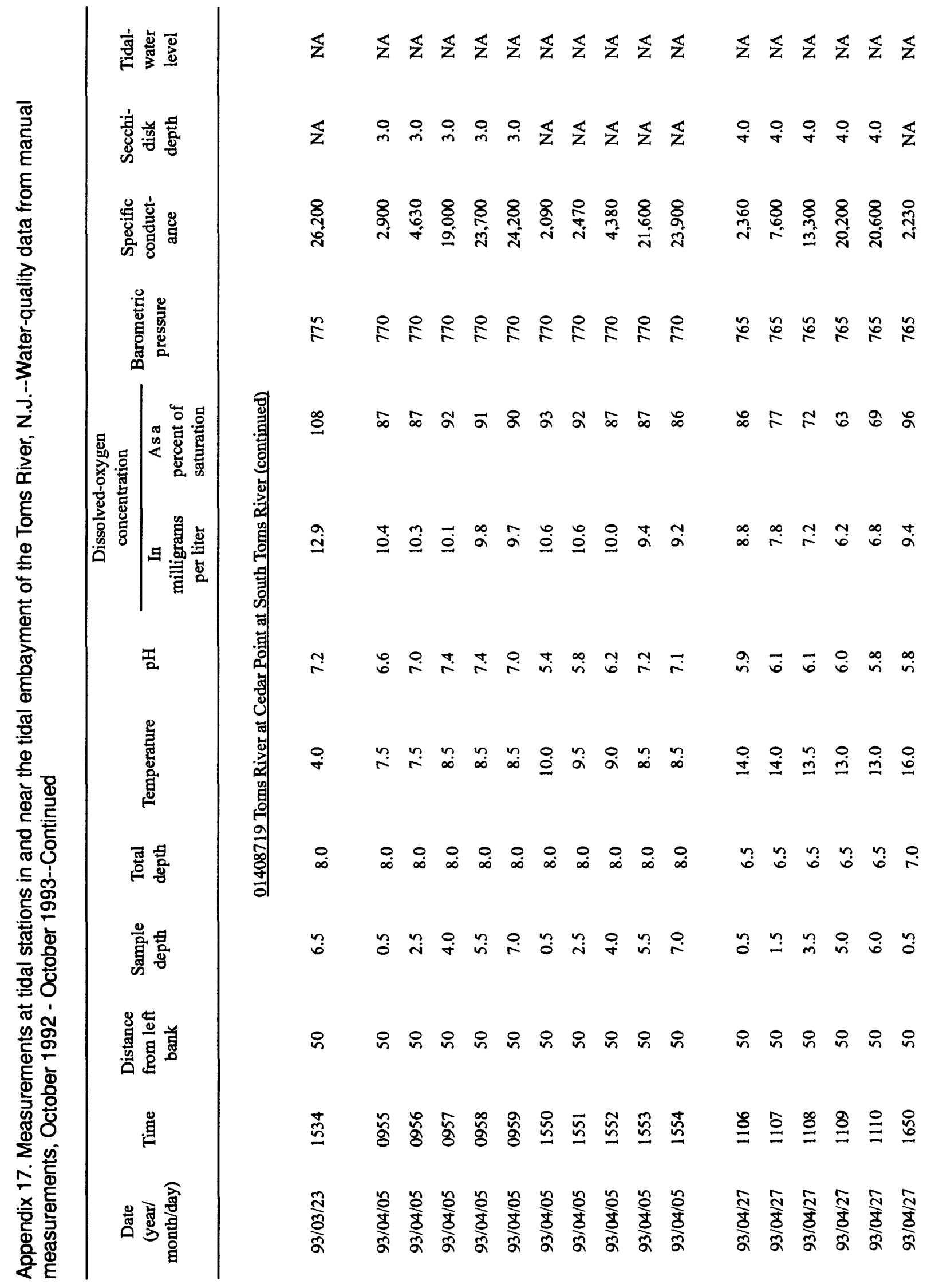




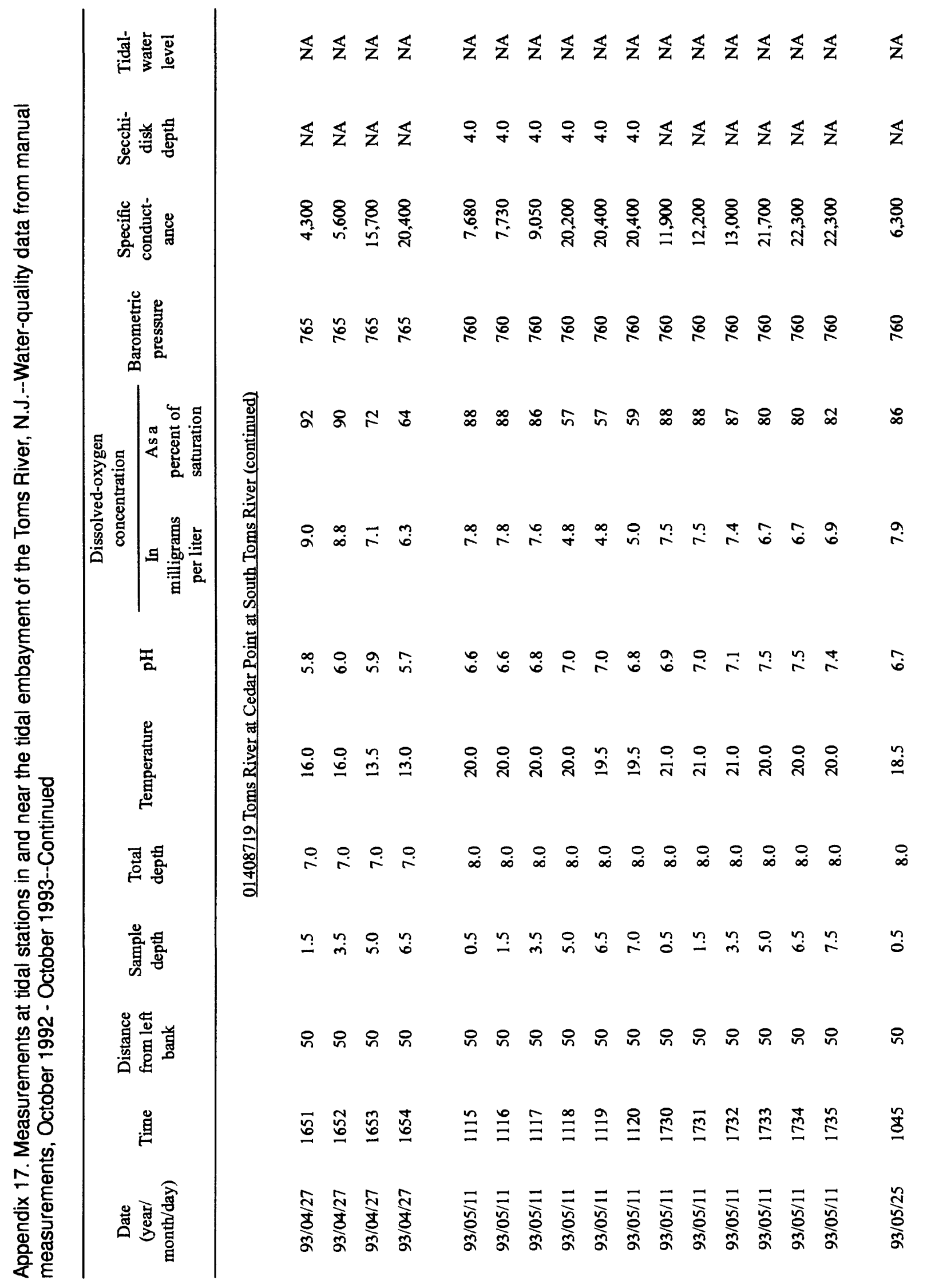




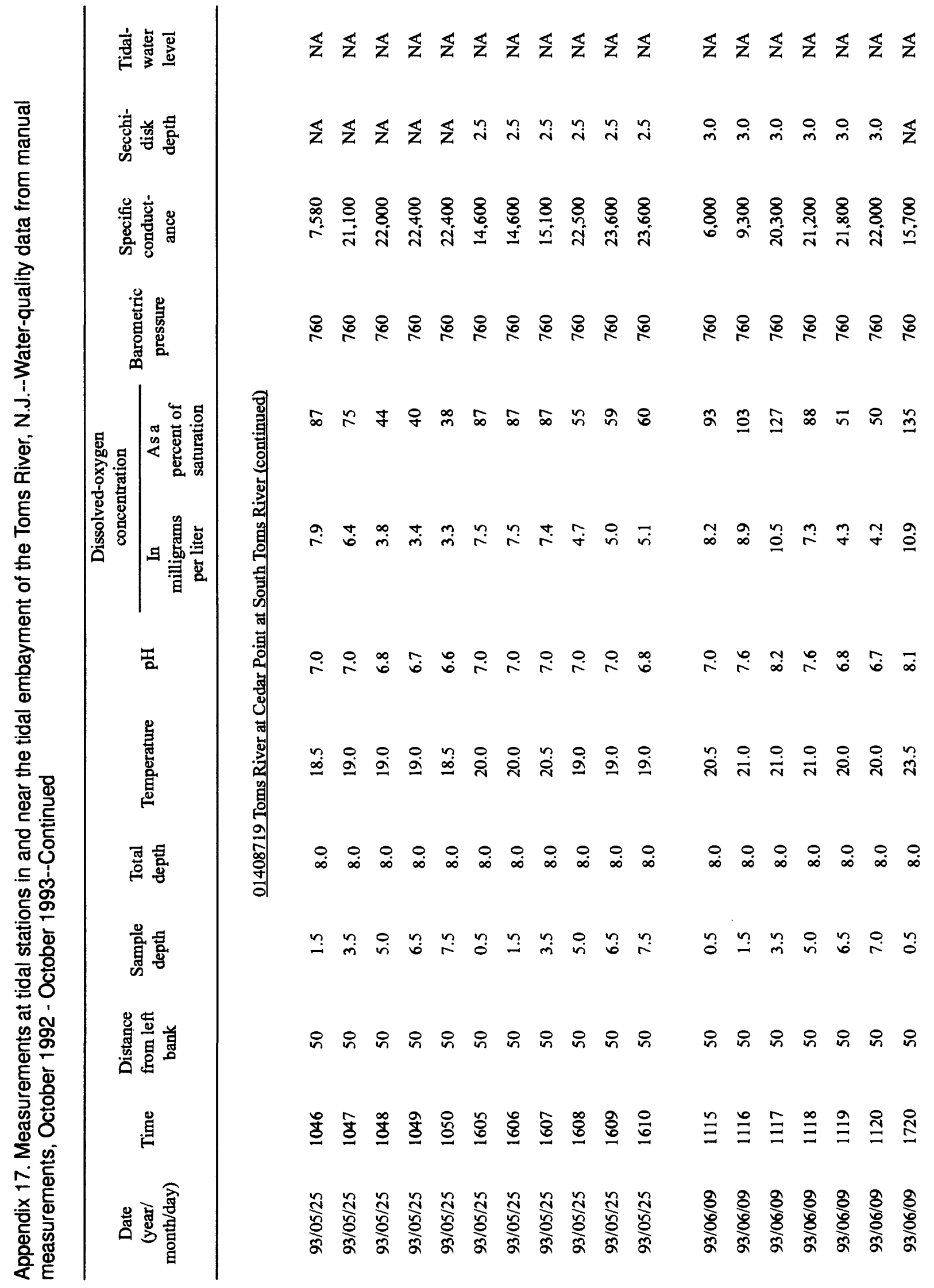




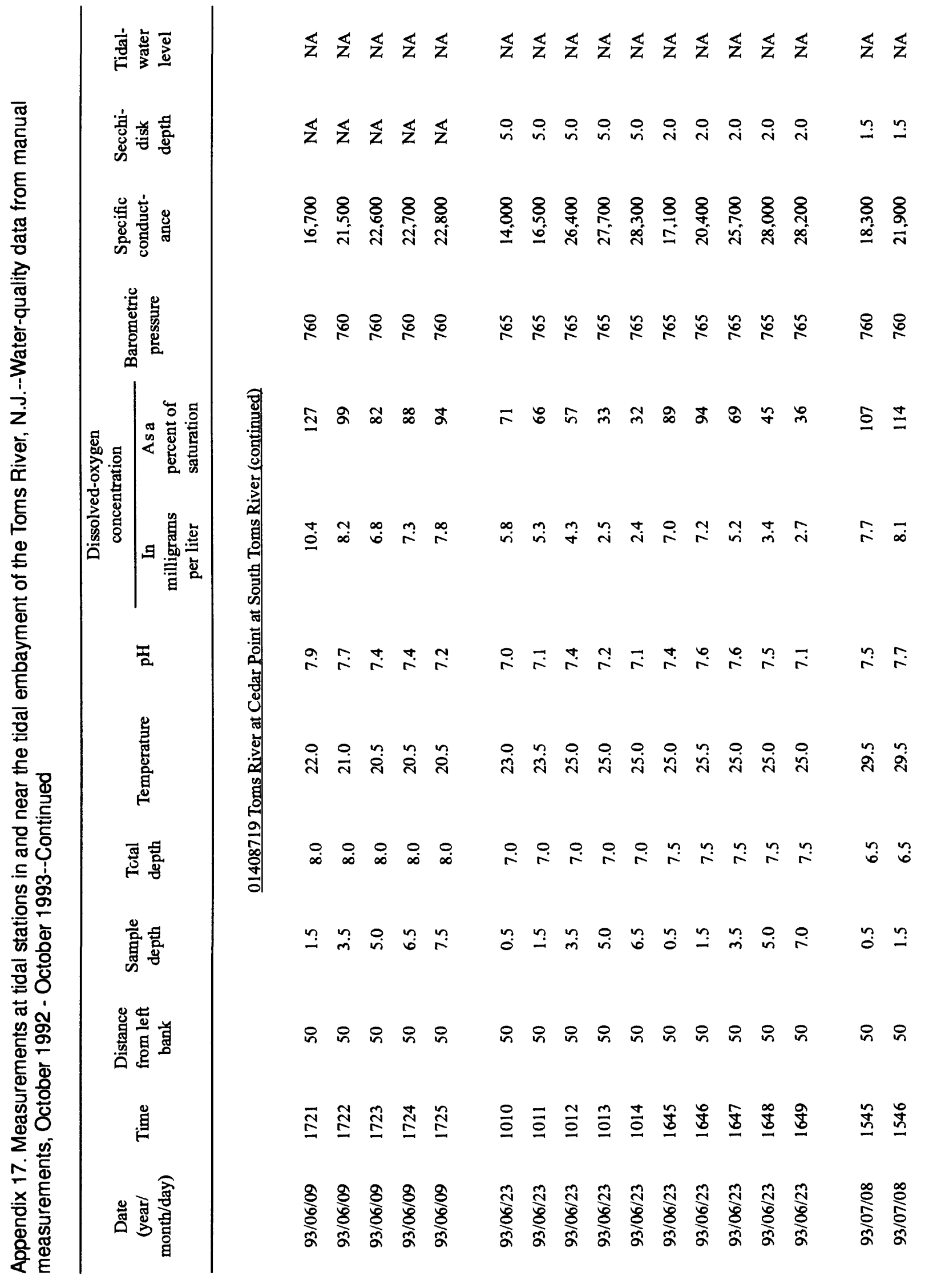




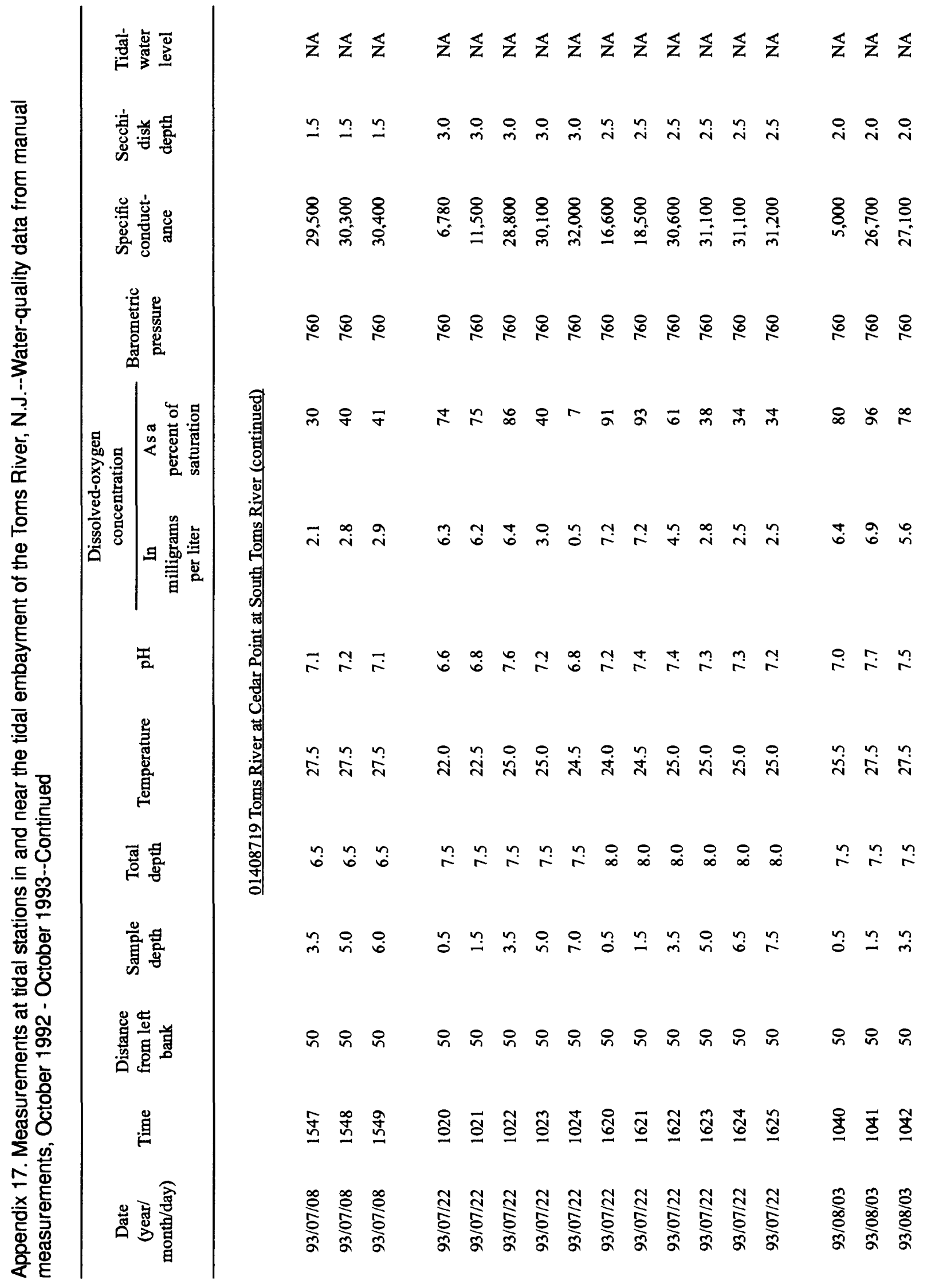




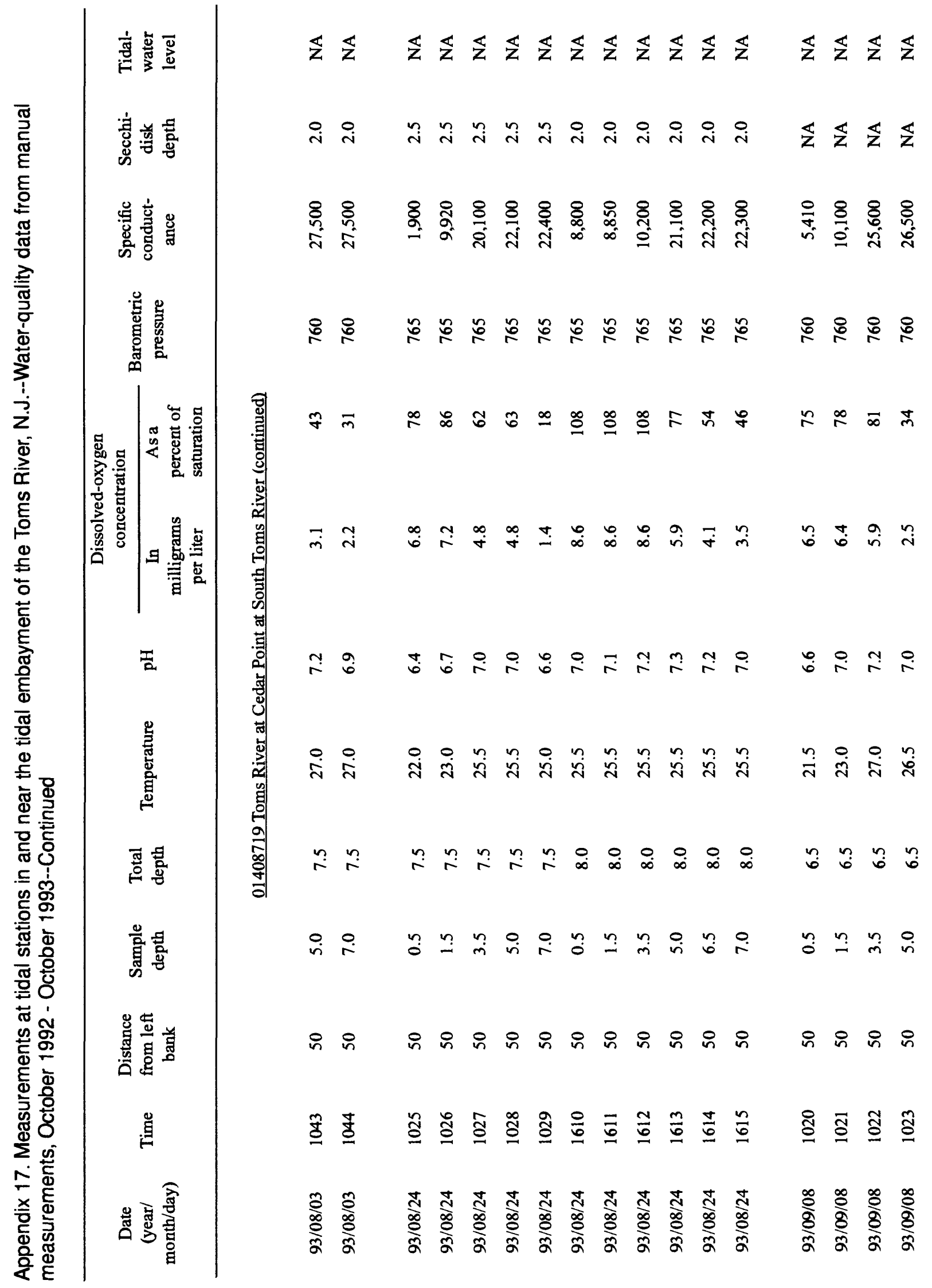




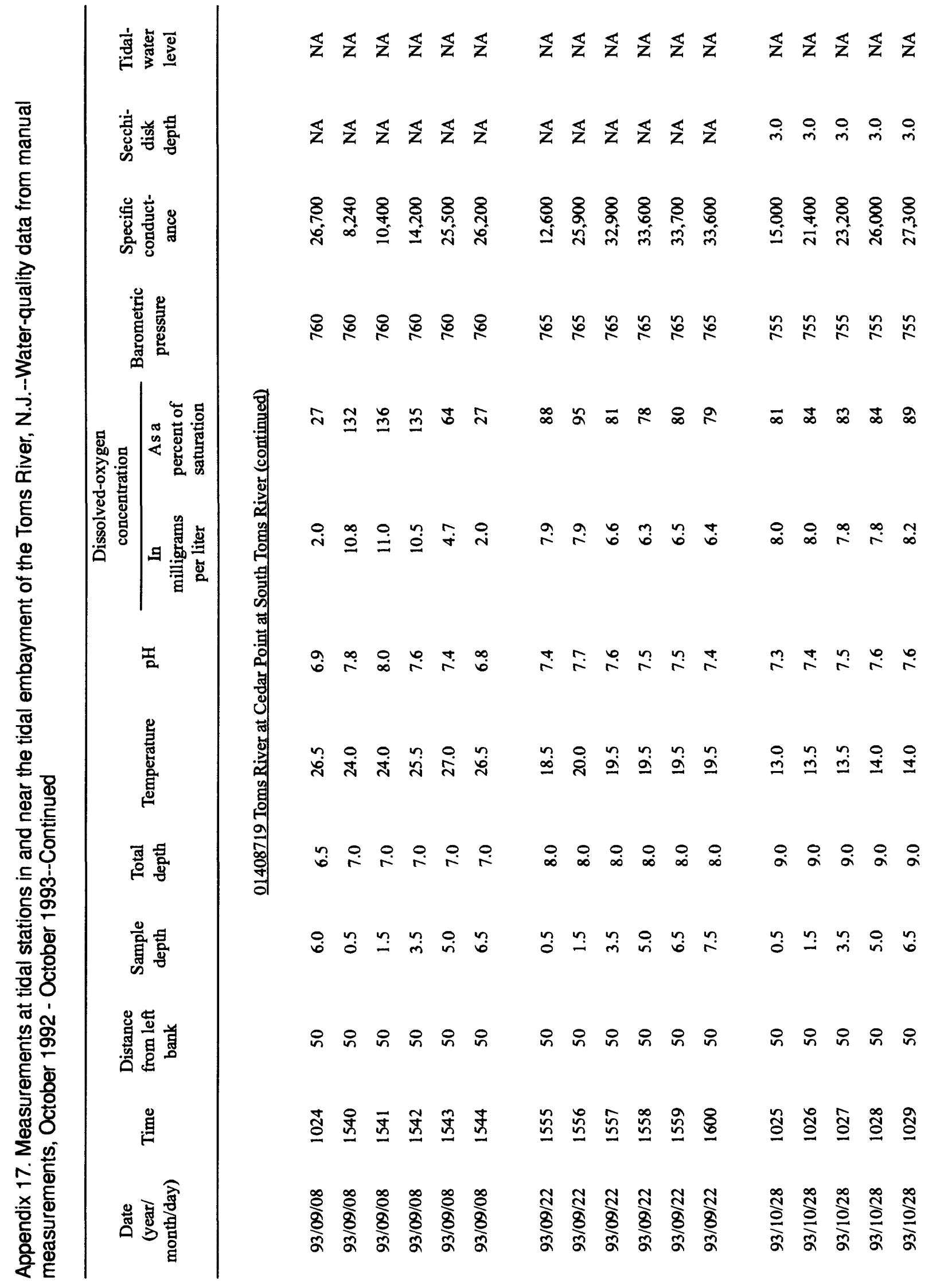




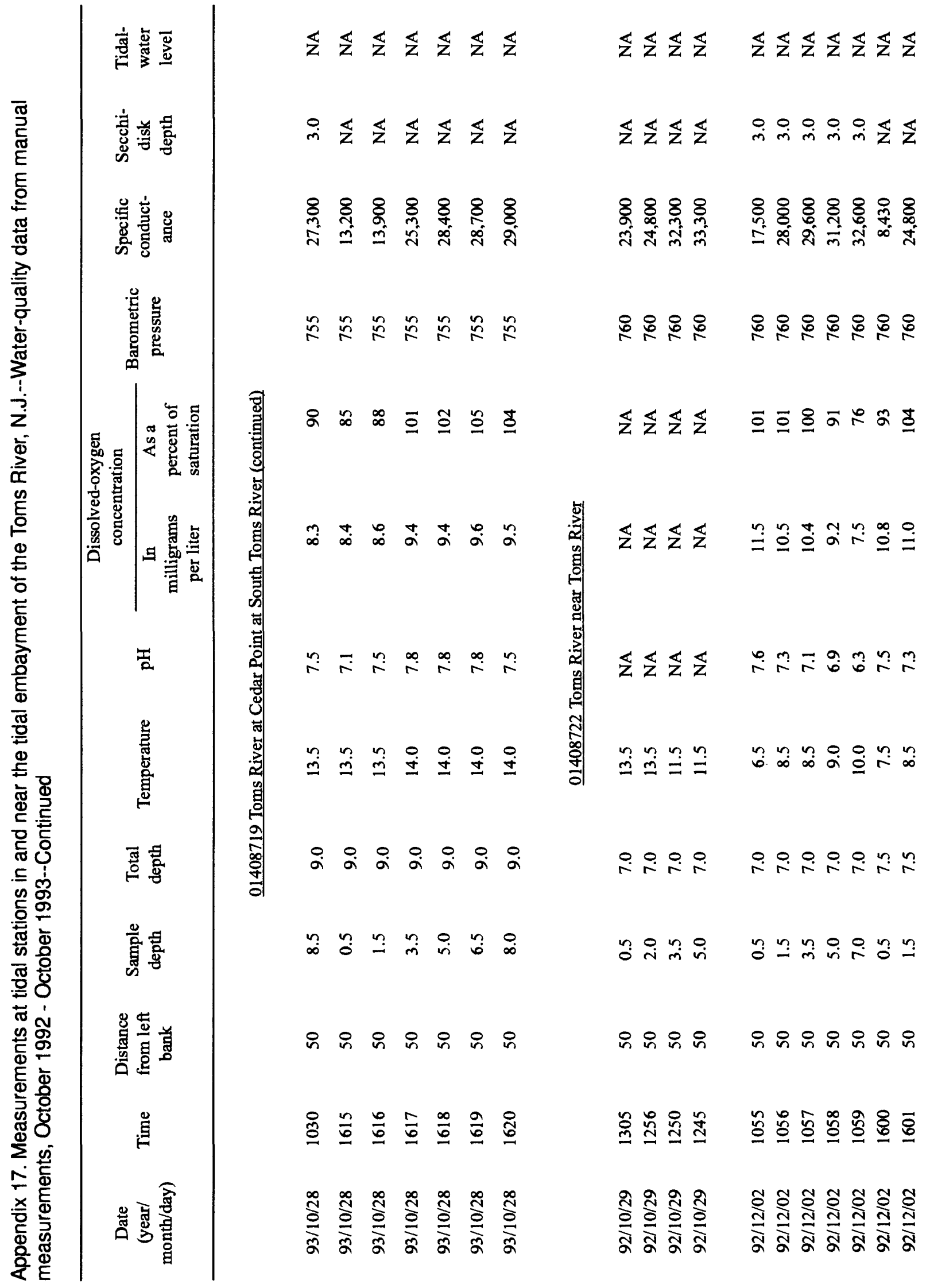




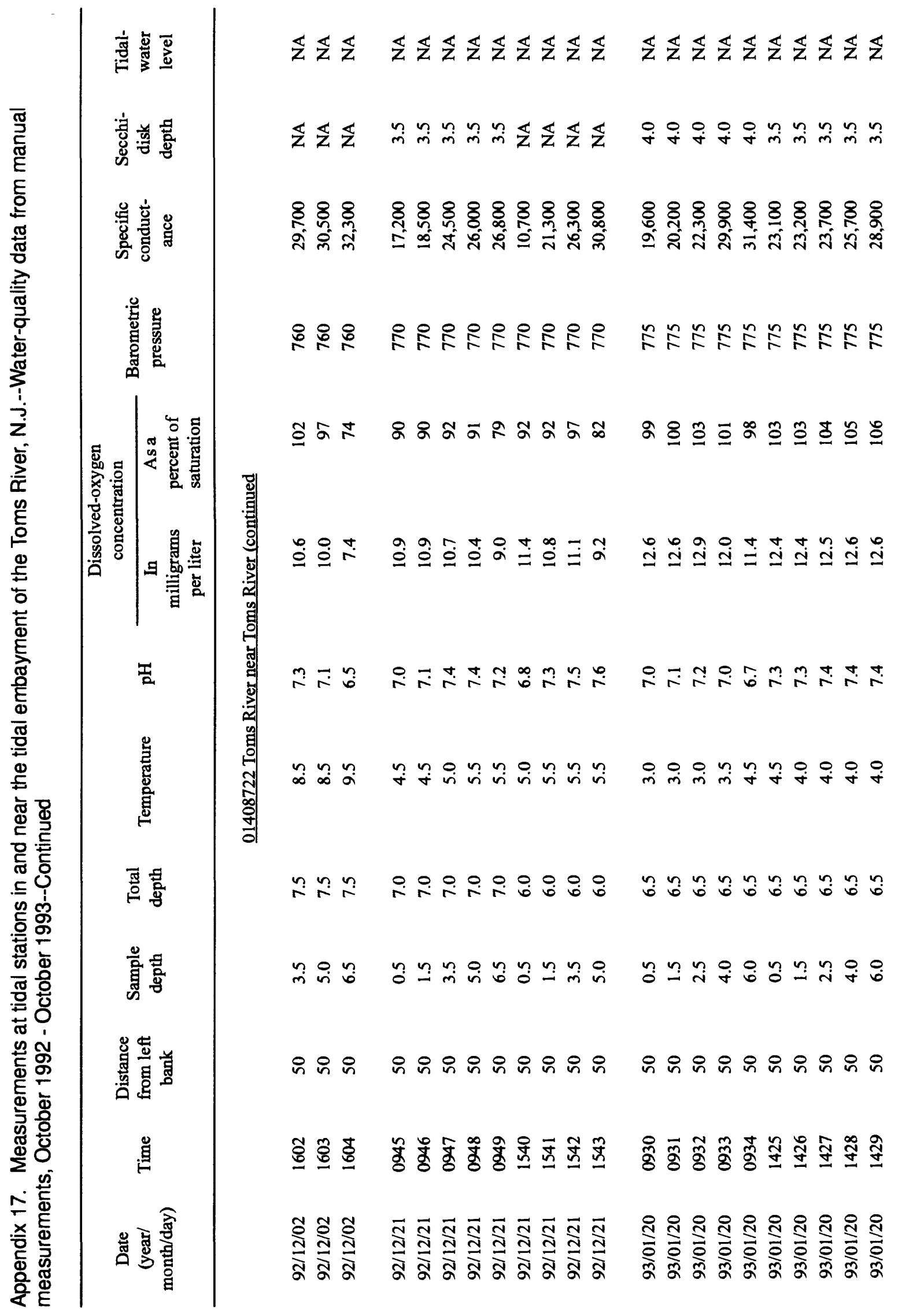




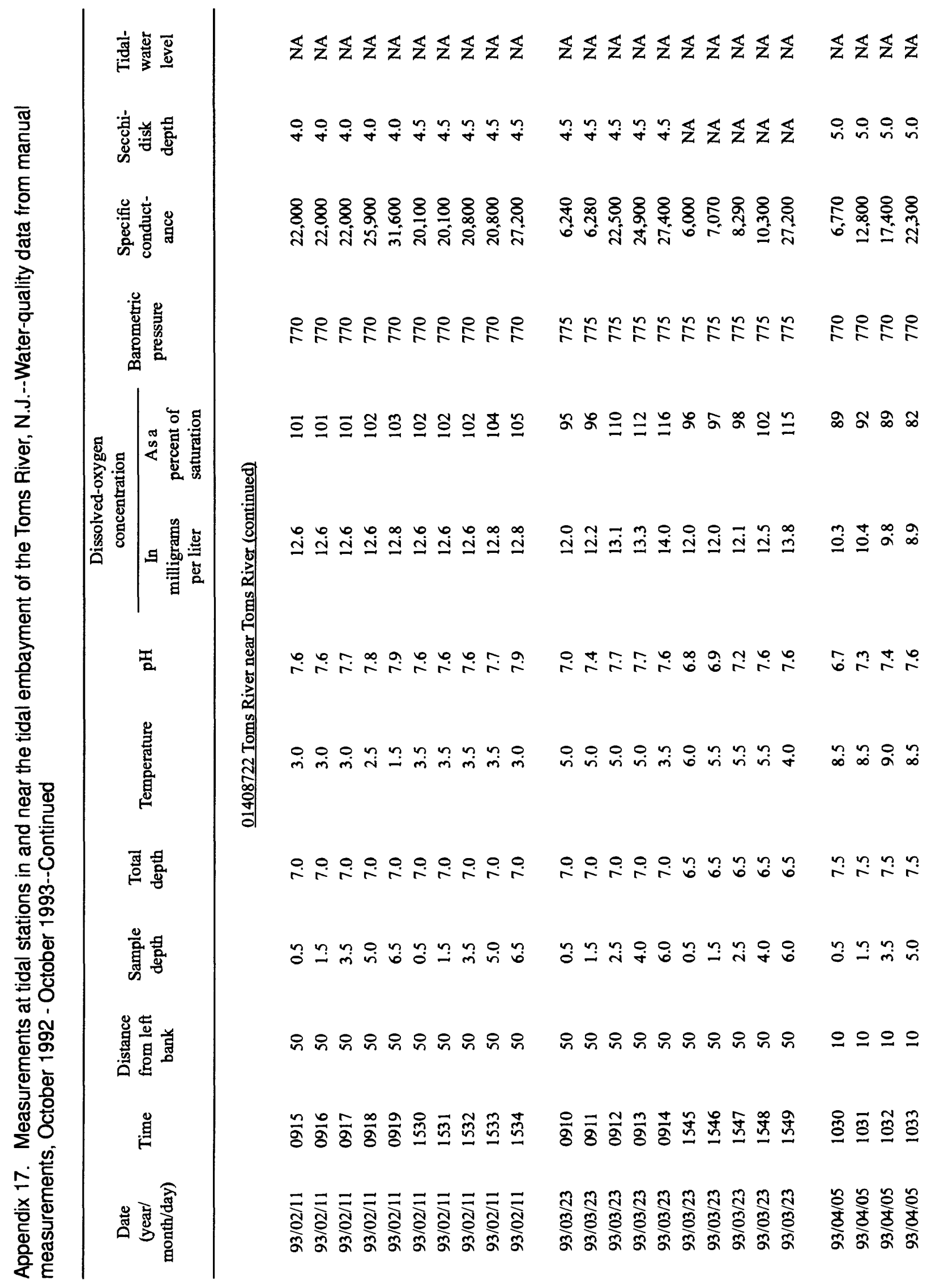




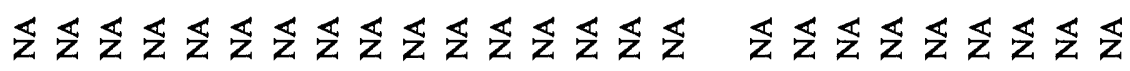

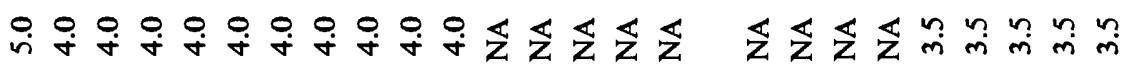

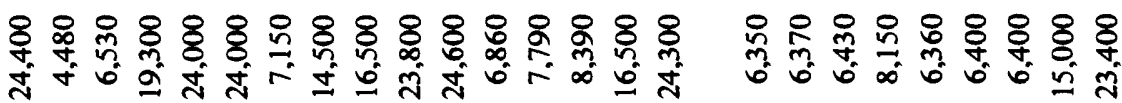

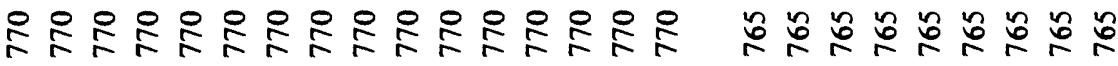

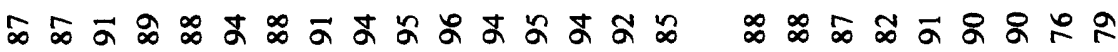

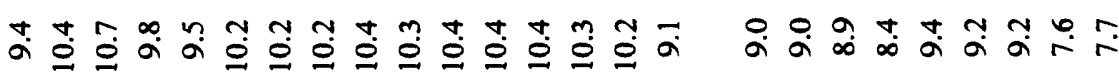

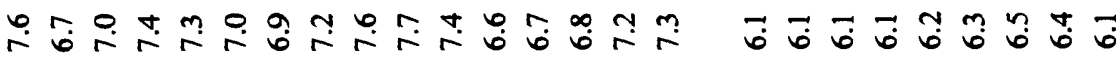

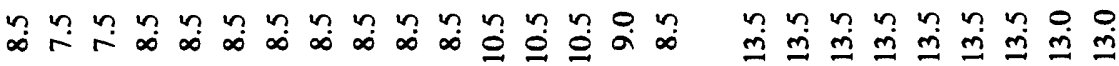

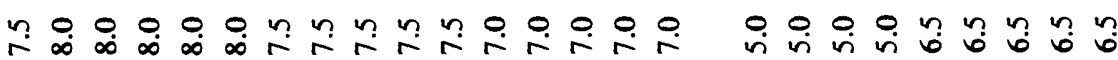

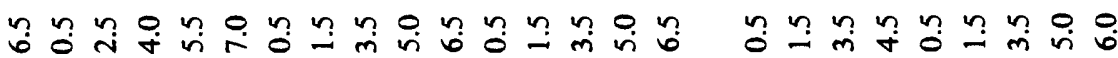

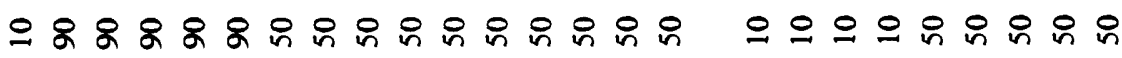

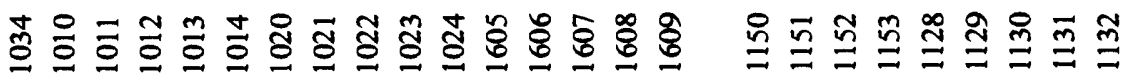

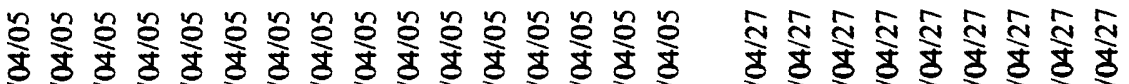
\% 


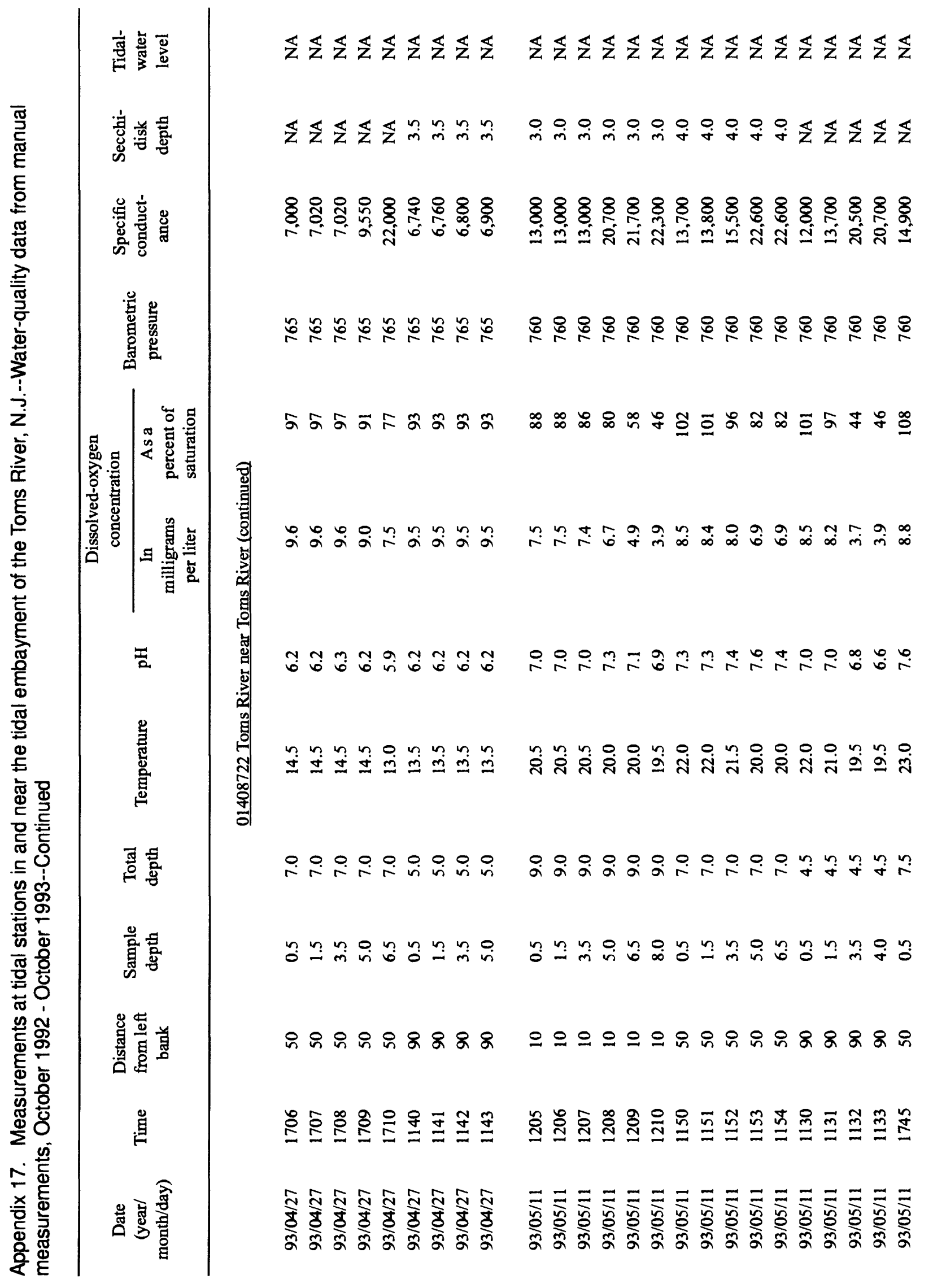




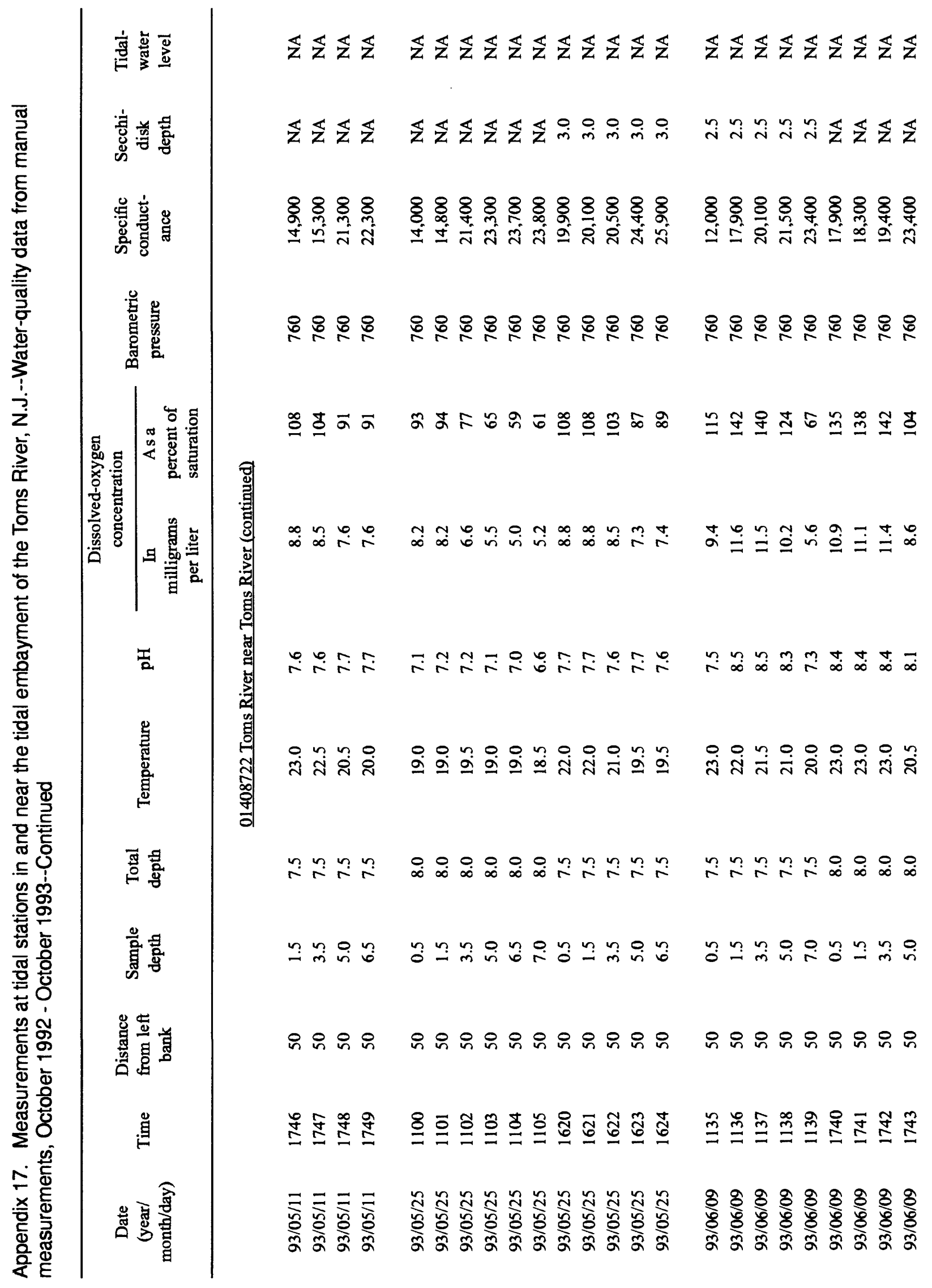




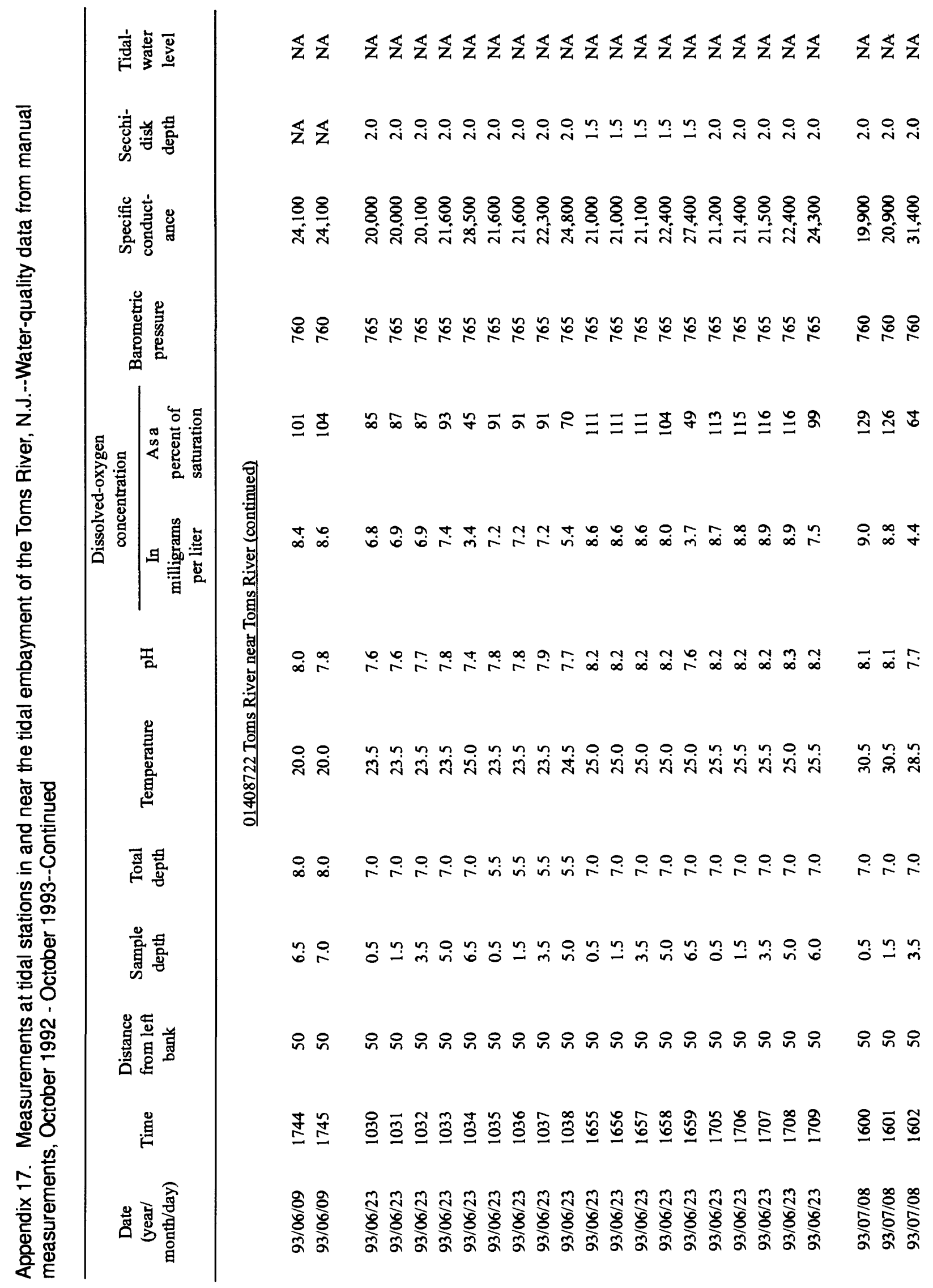




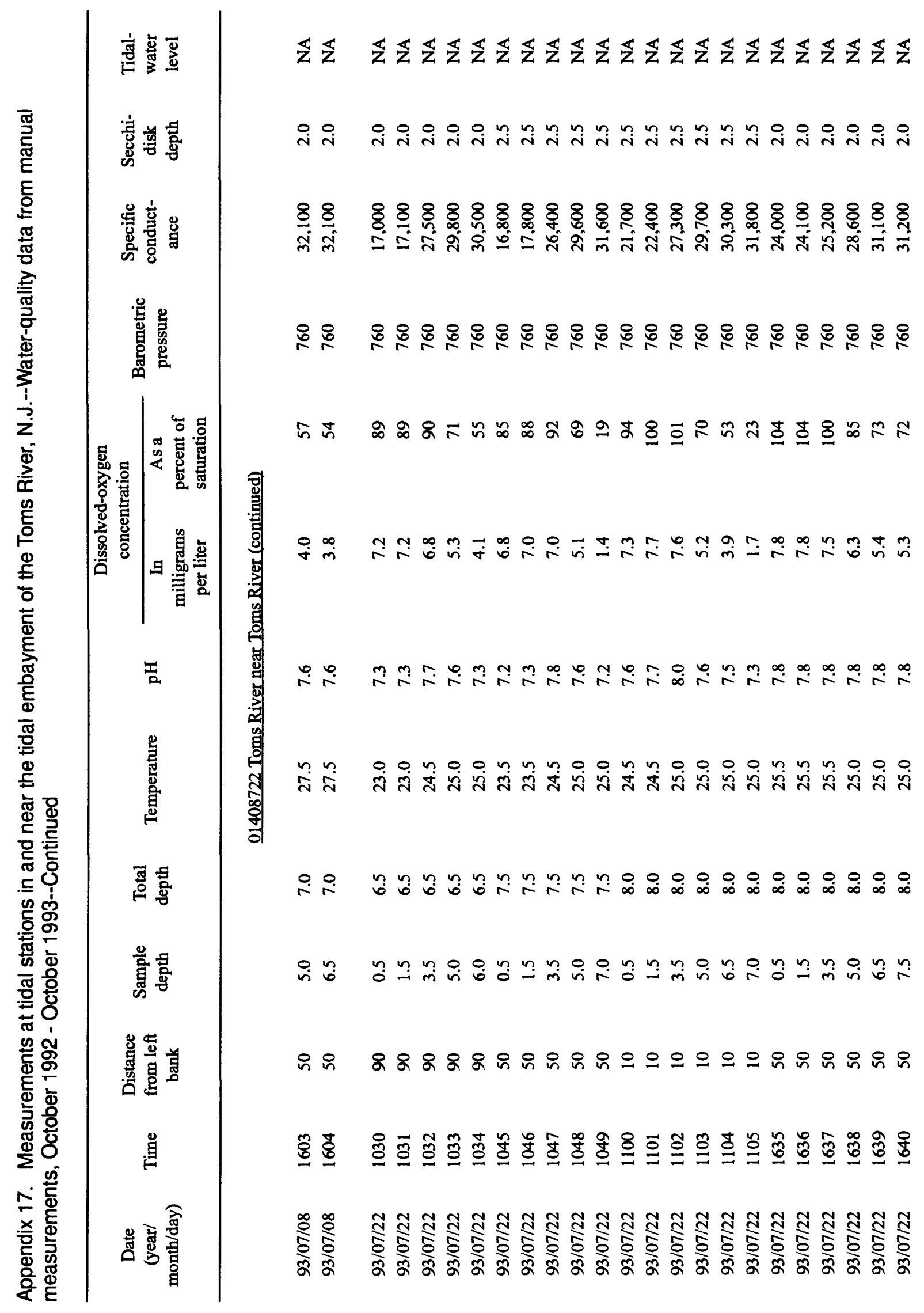




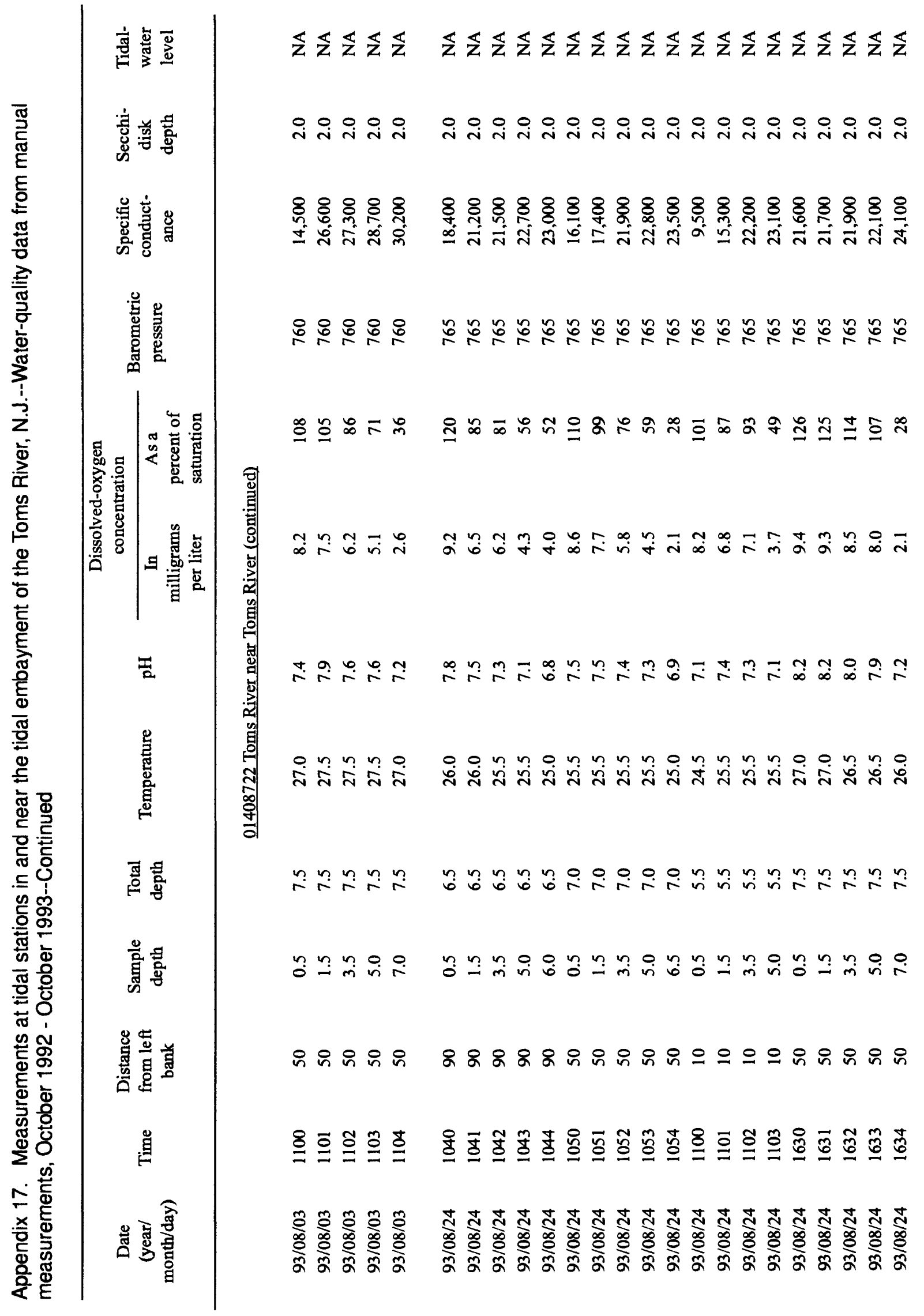




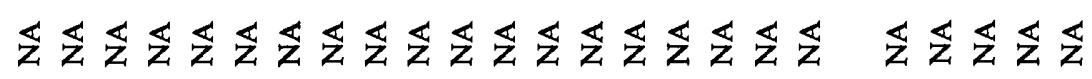

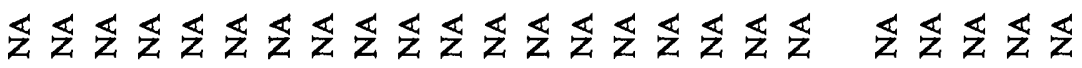

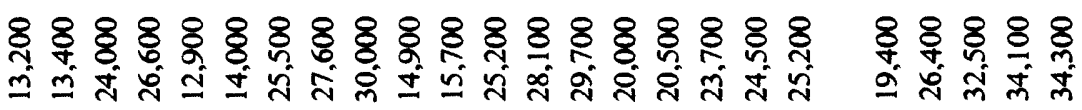

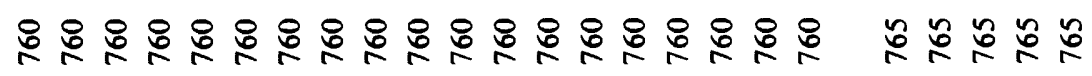

马ి@®

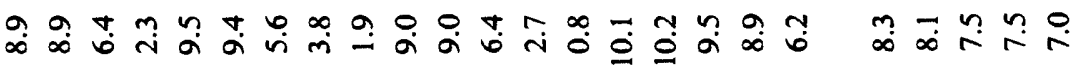

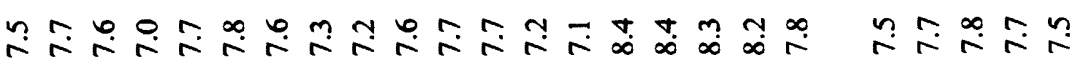

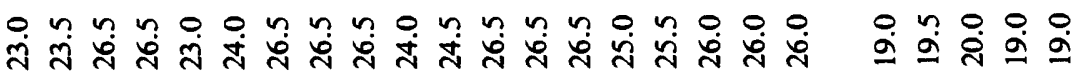

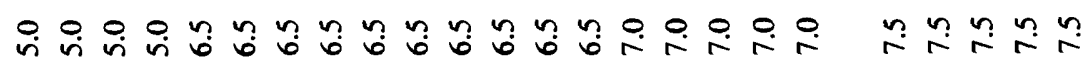

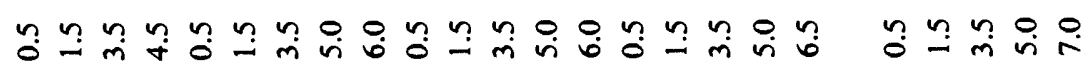

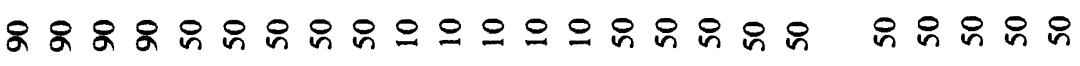

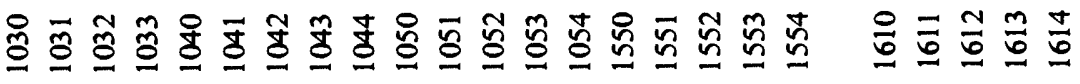

웅 के के के के के 少 


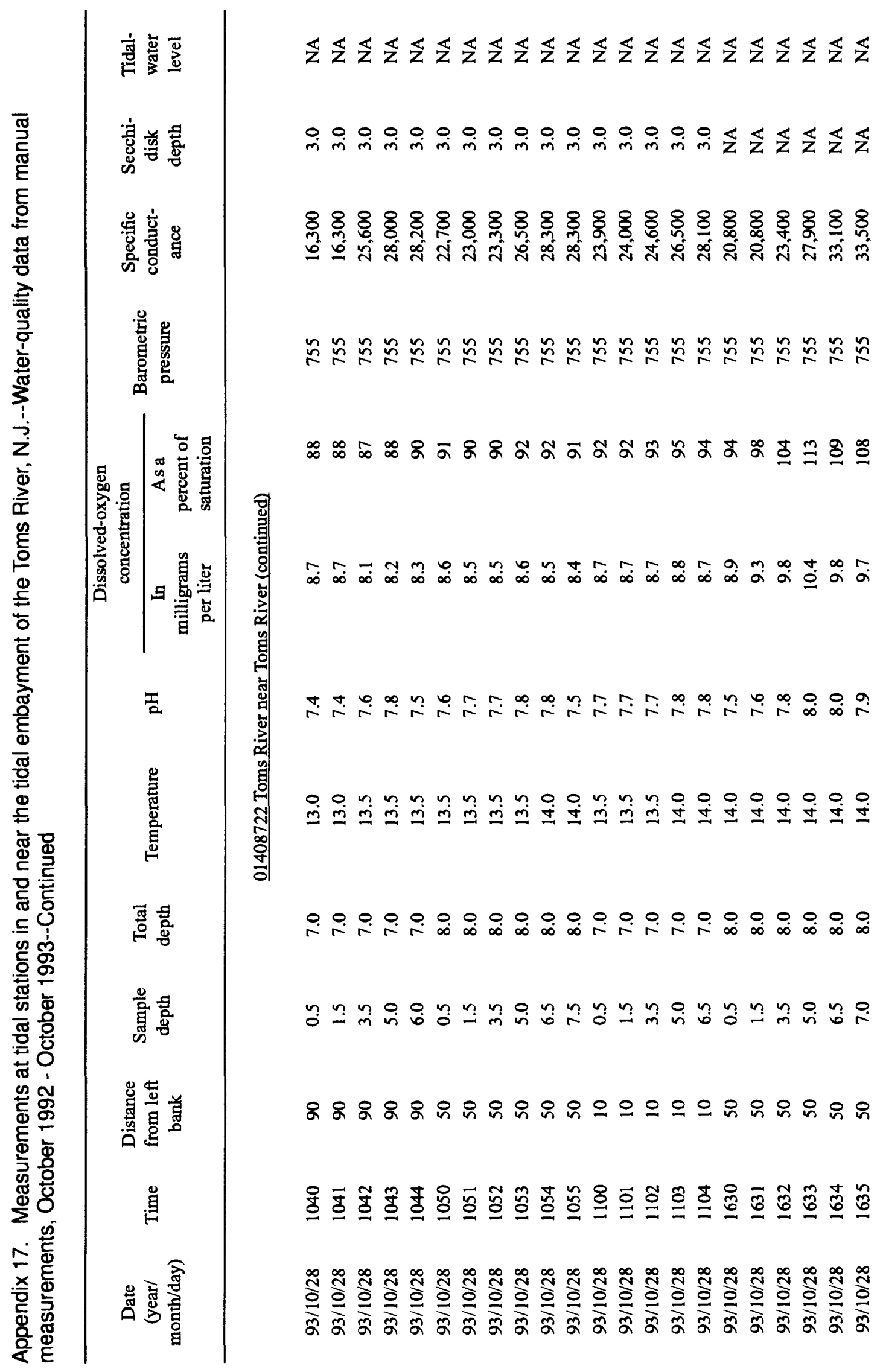




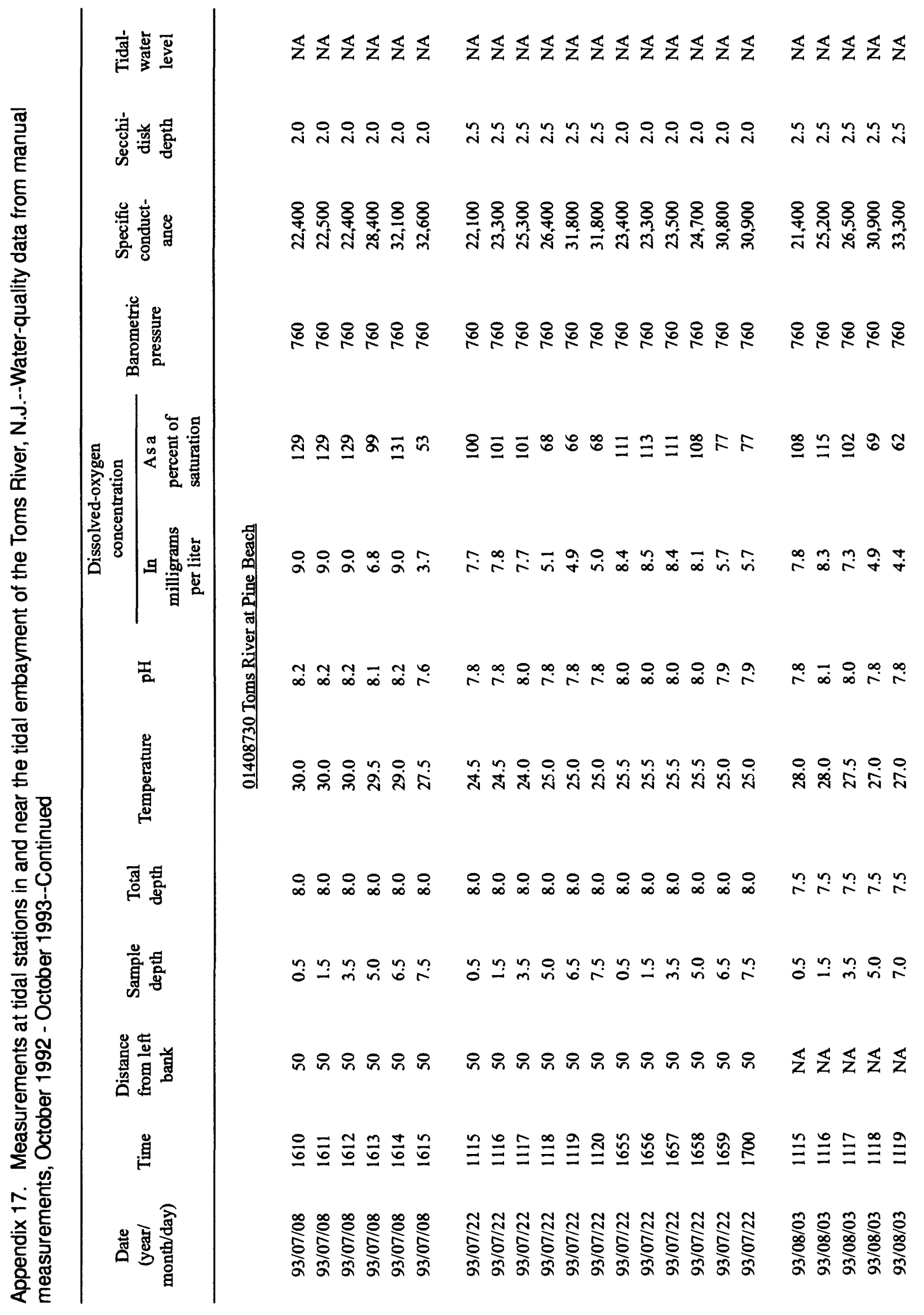




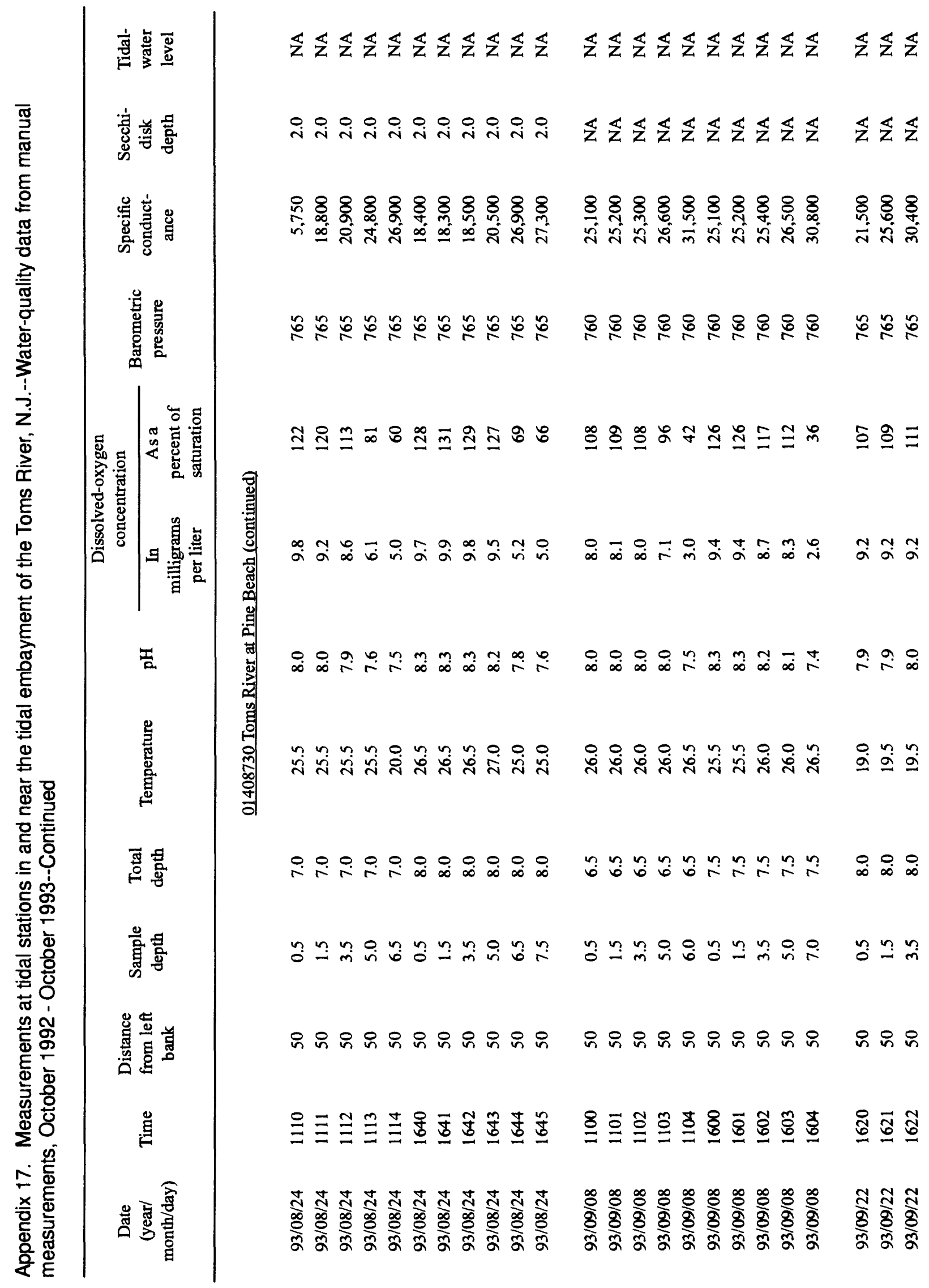




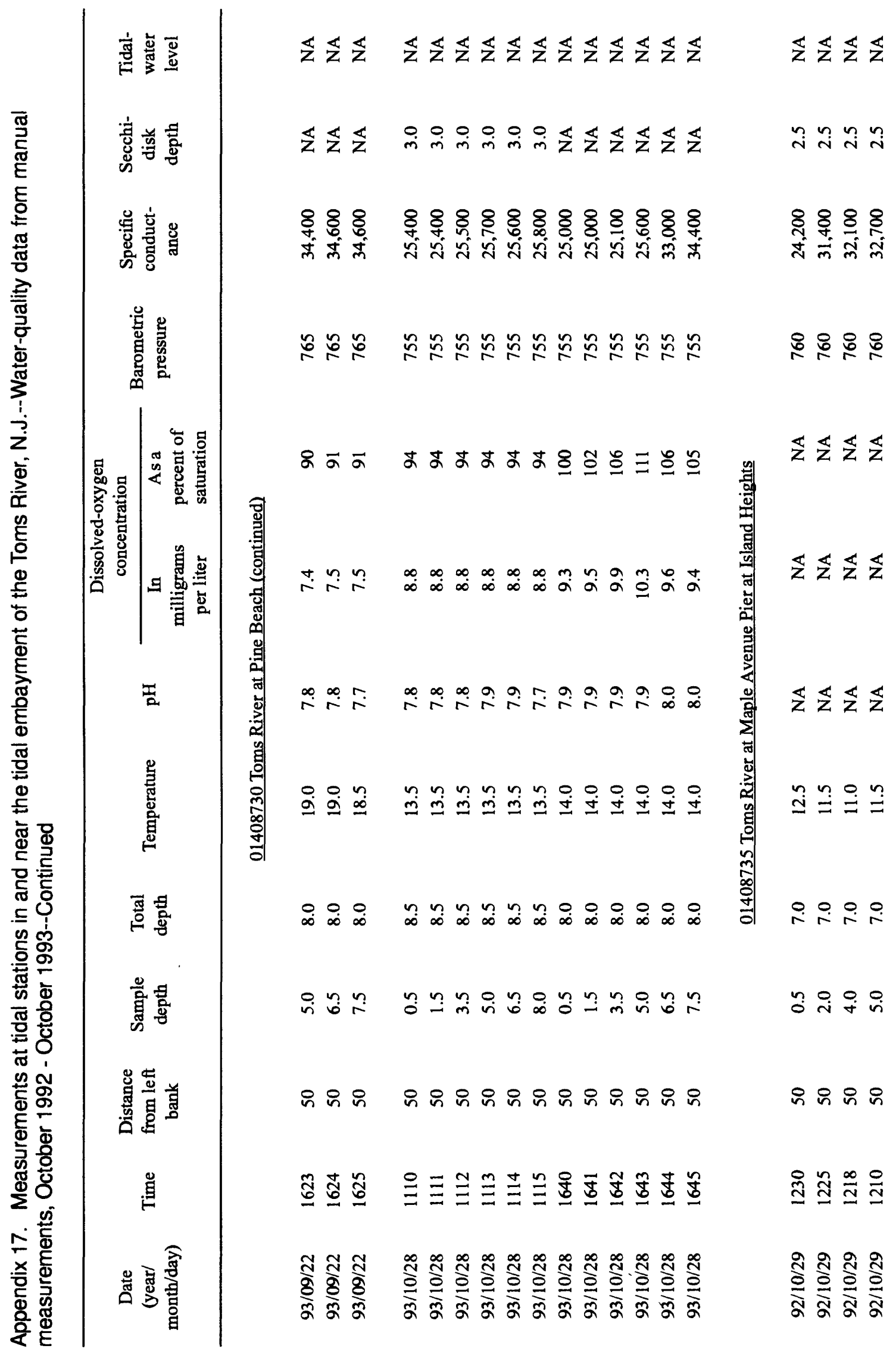




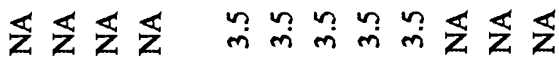

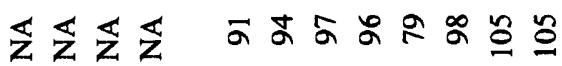

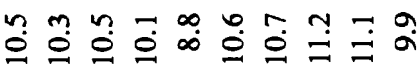

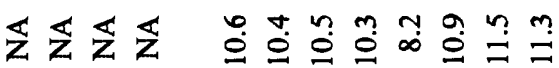

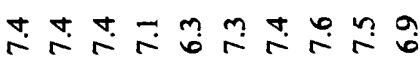

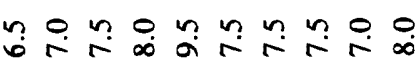

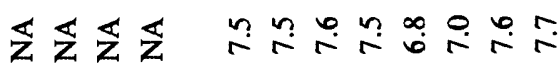

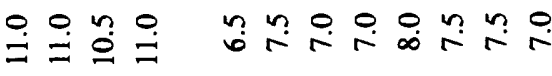

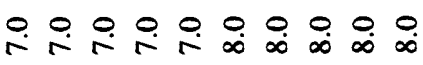

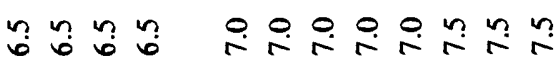

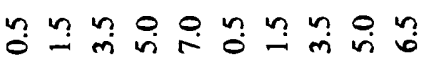

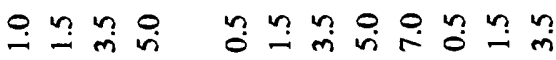

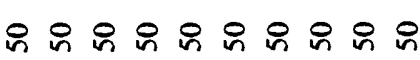

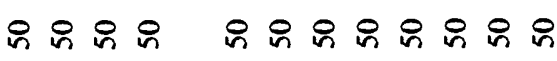

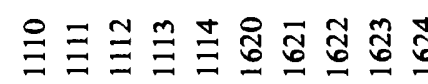

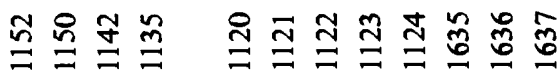

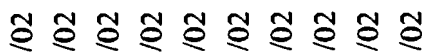

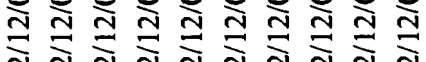

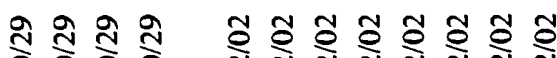

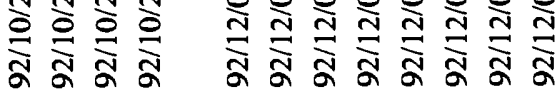




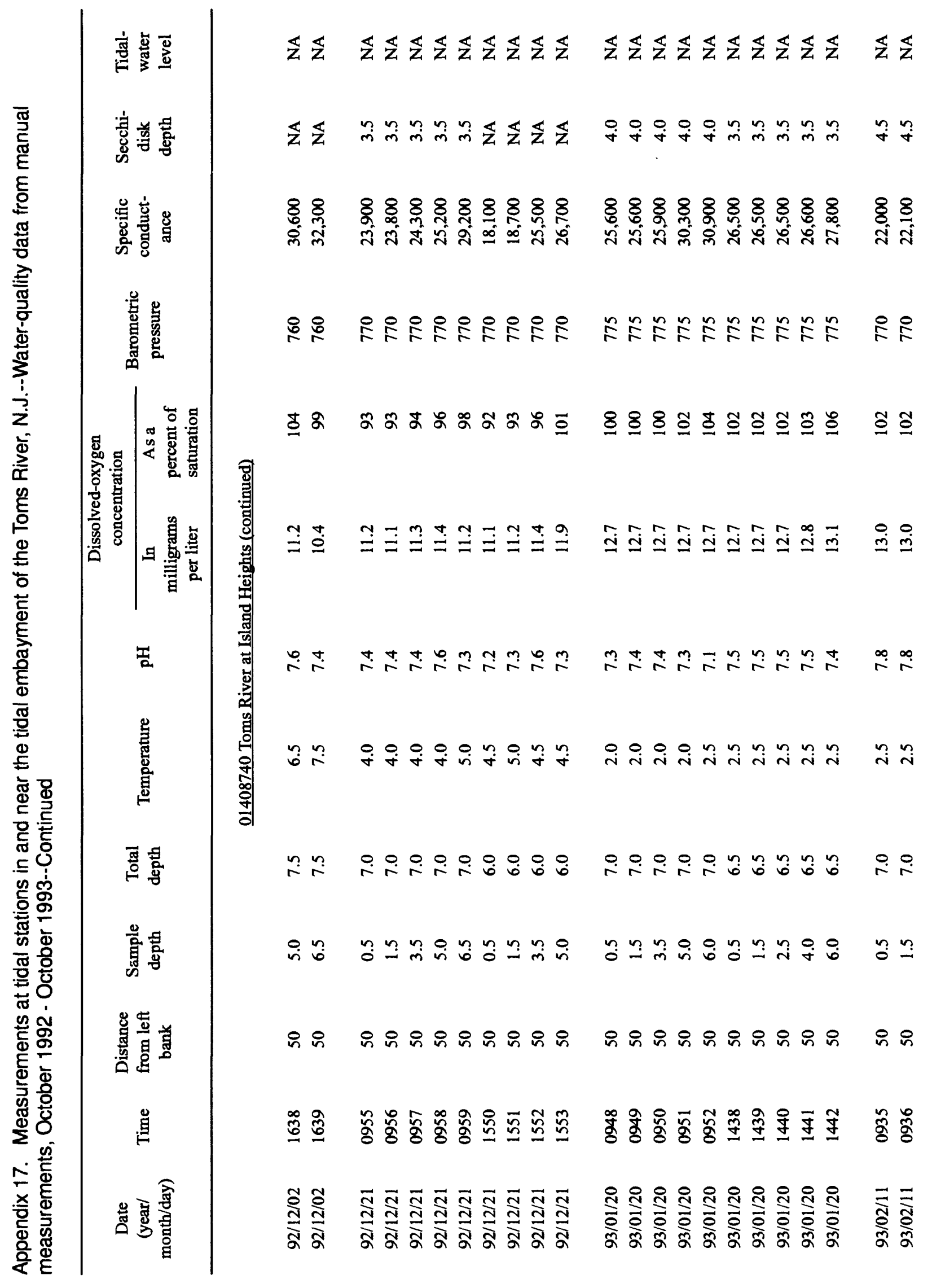




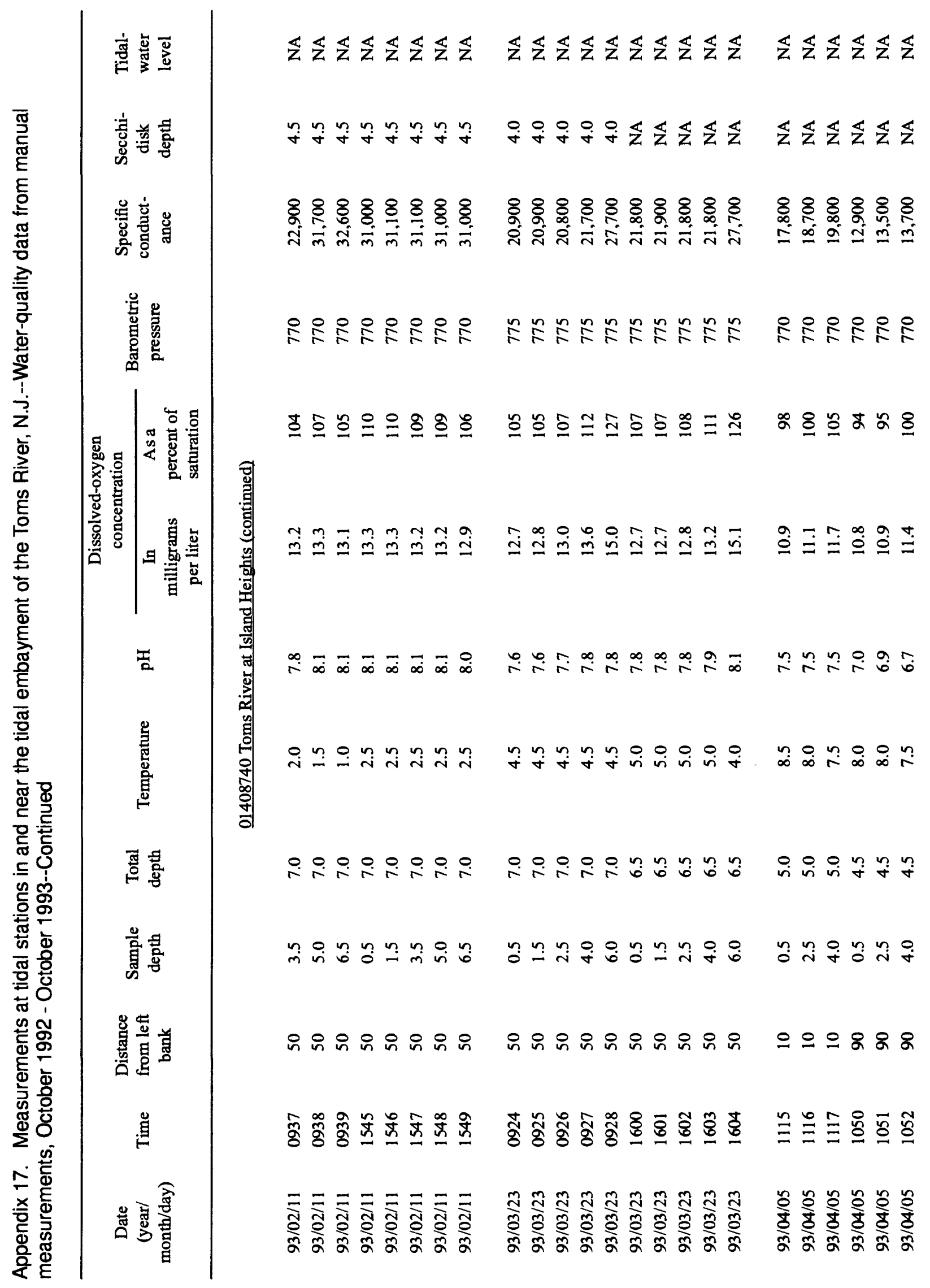




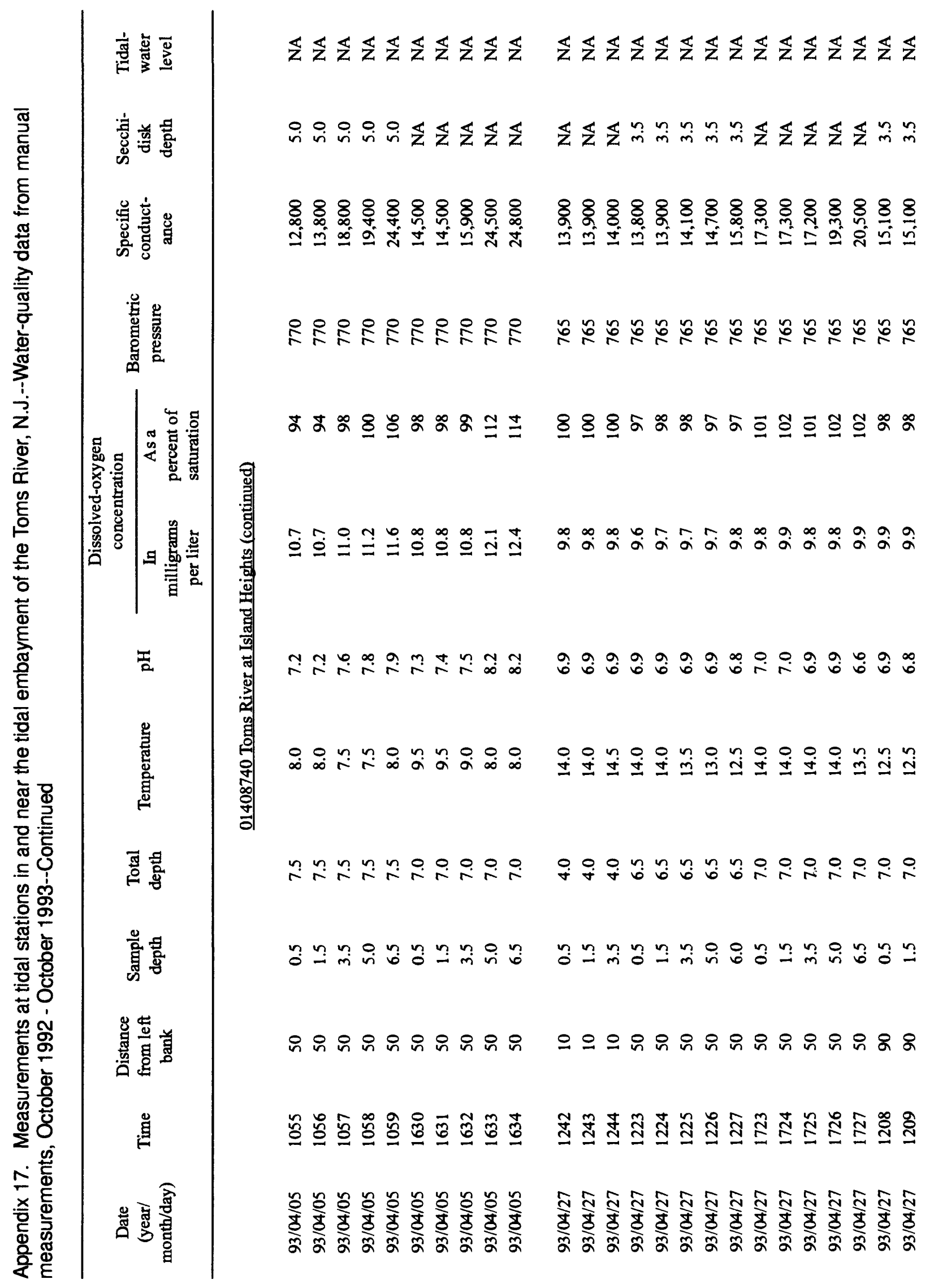




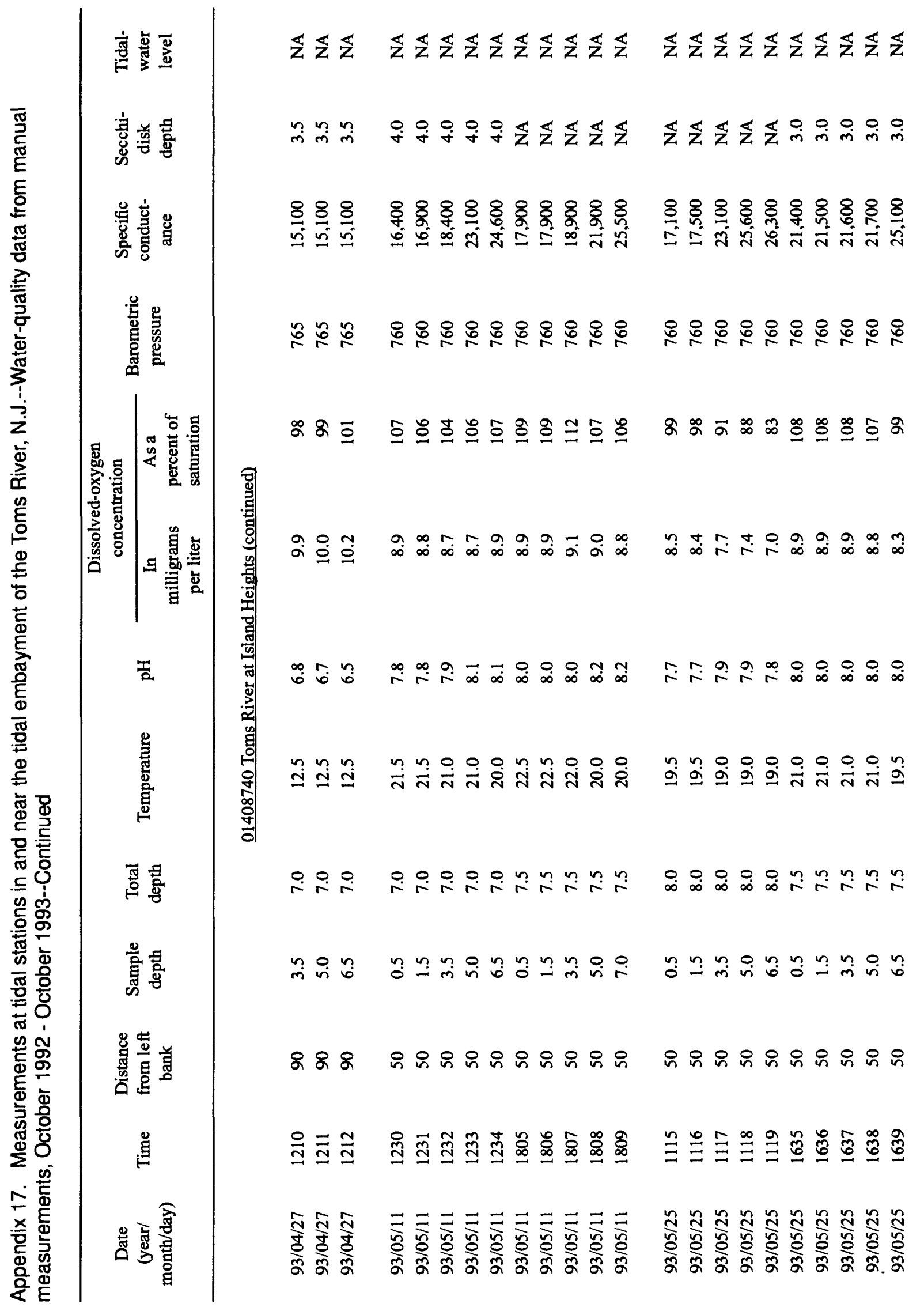




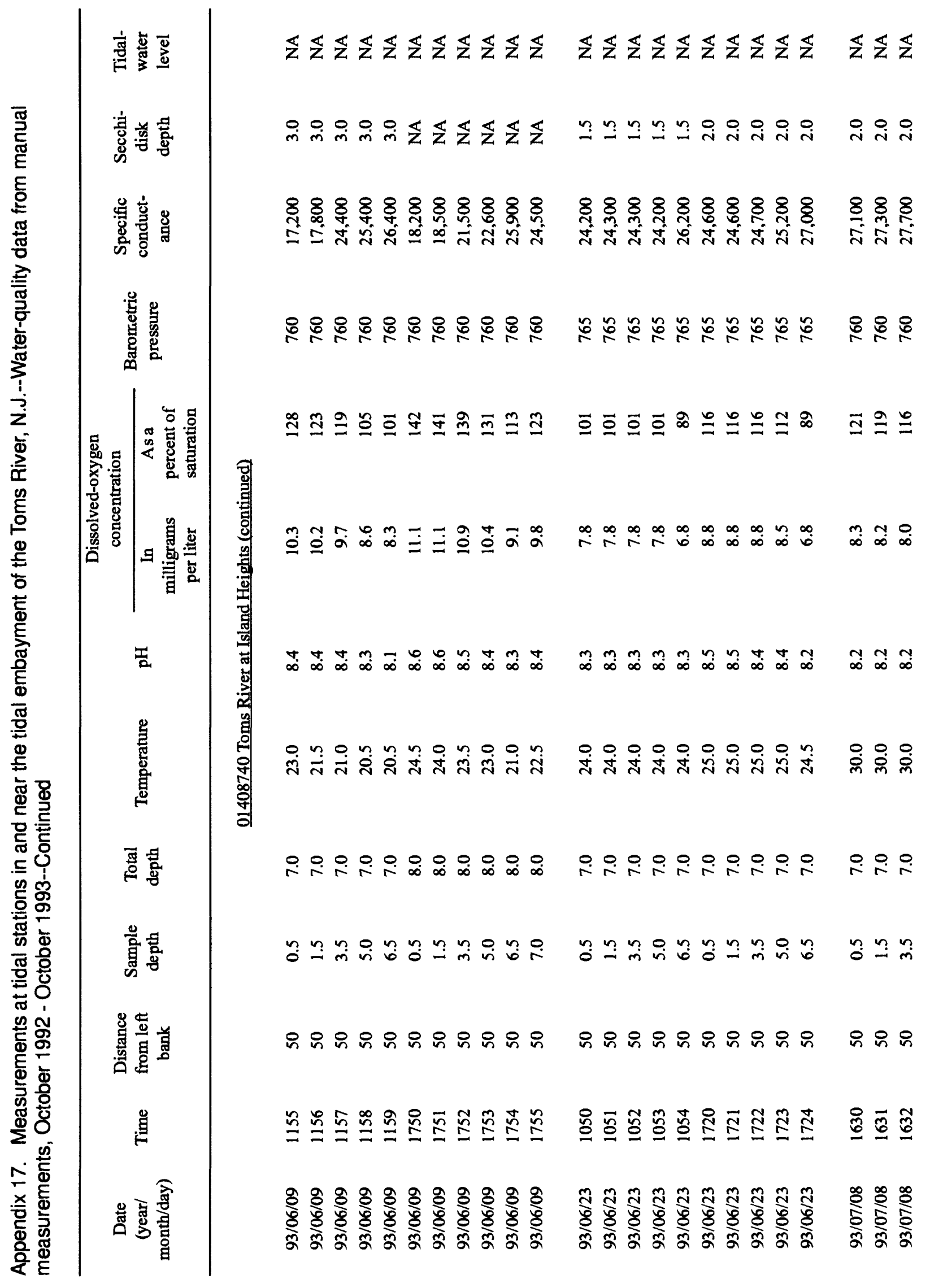




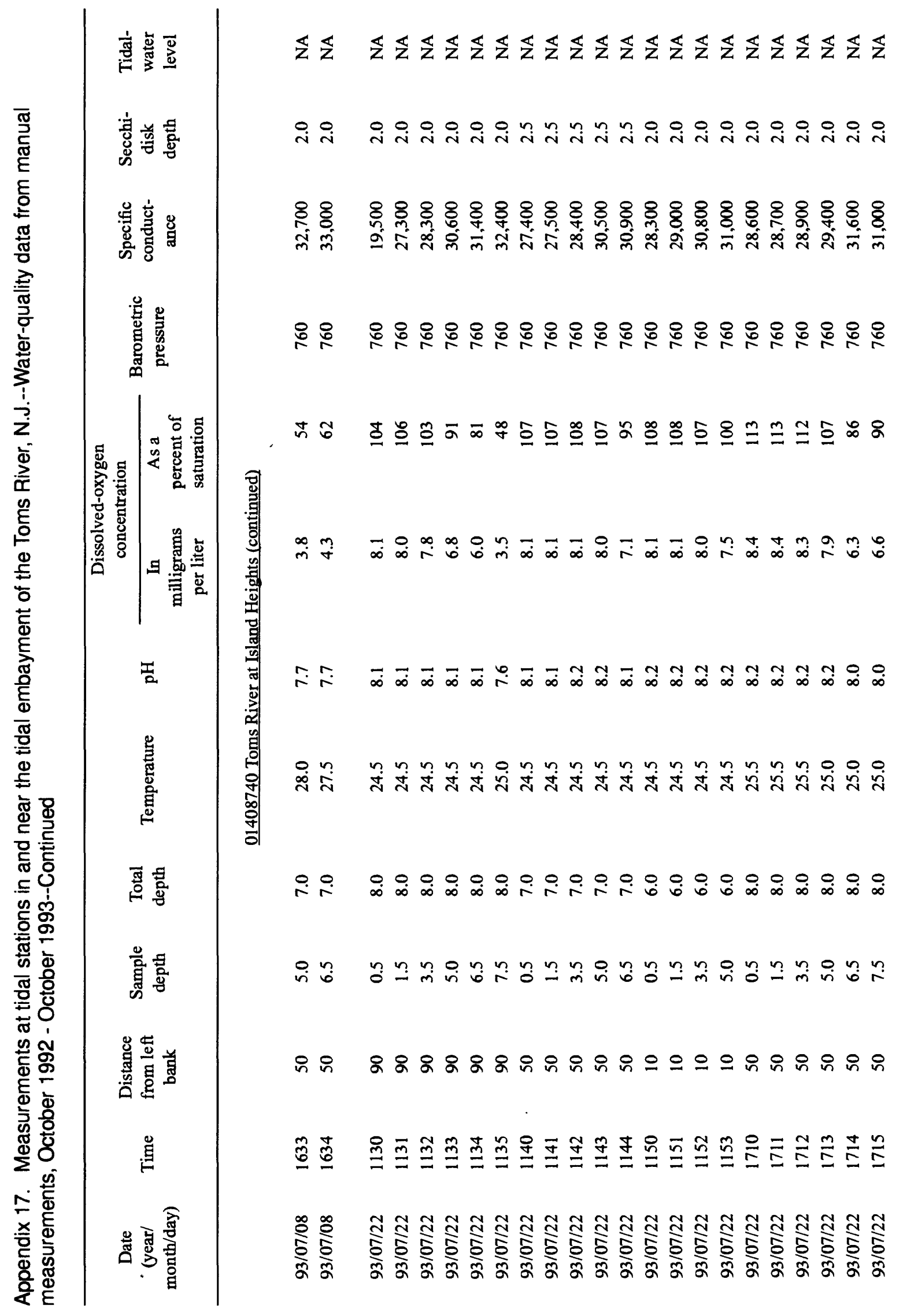




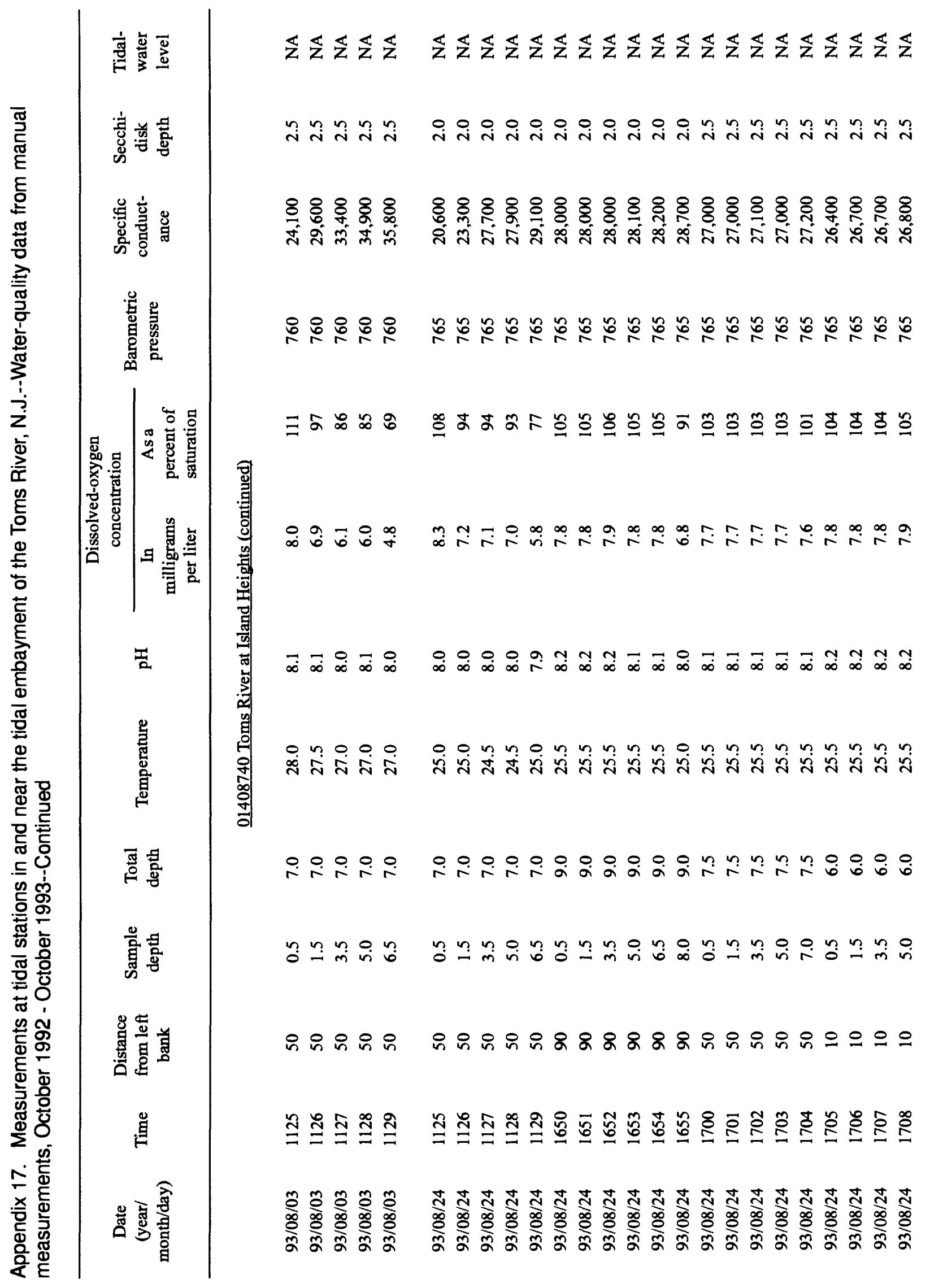




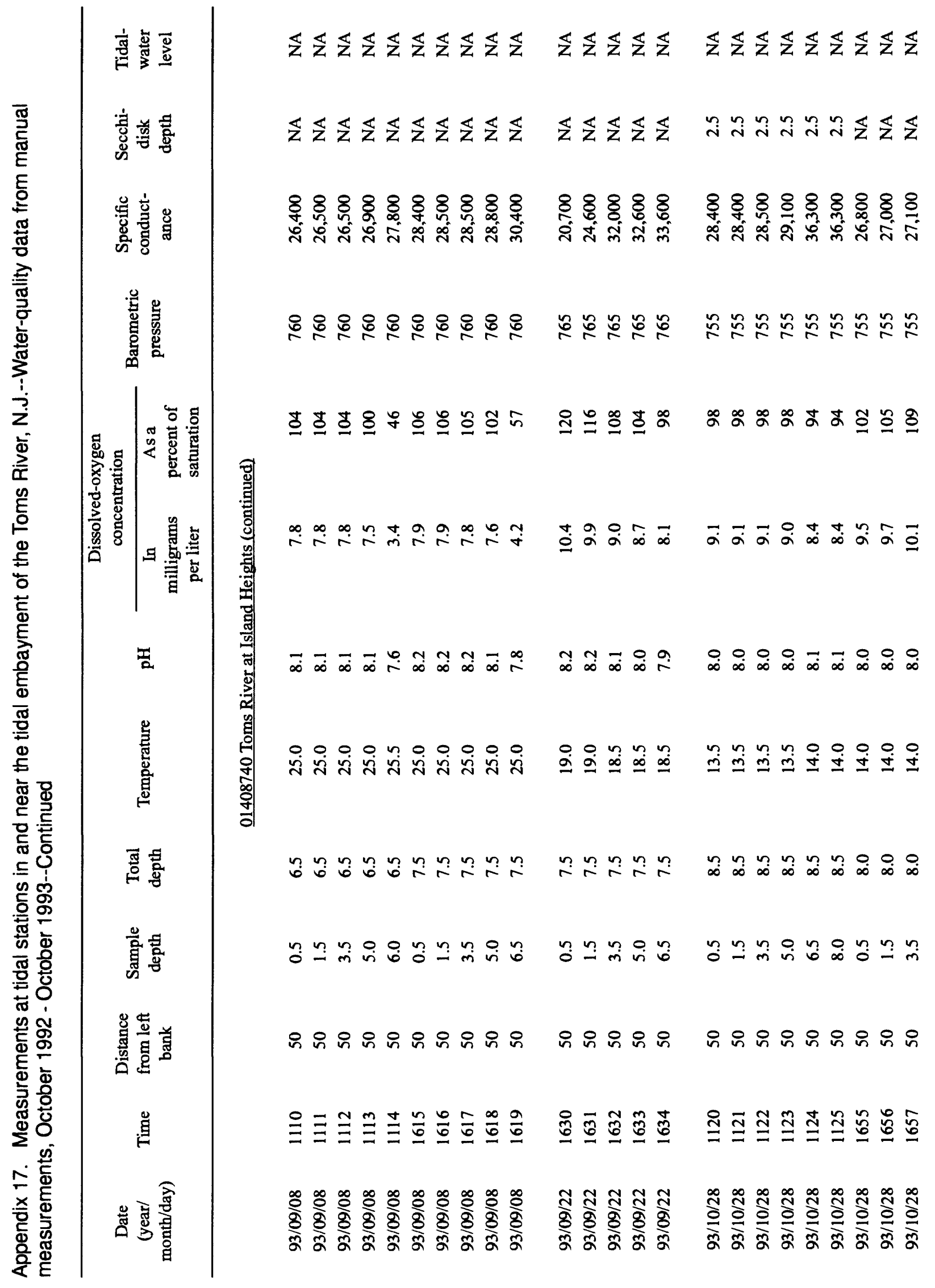




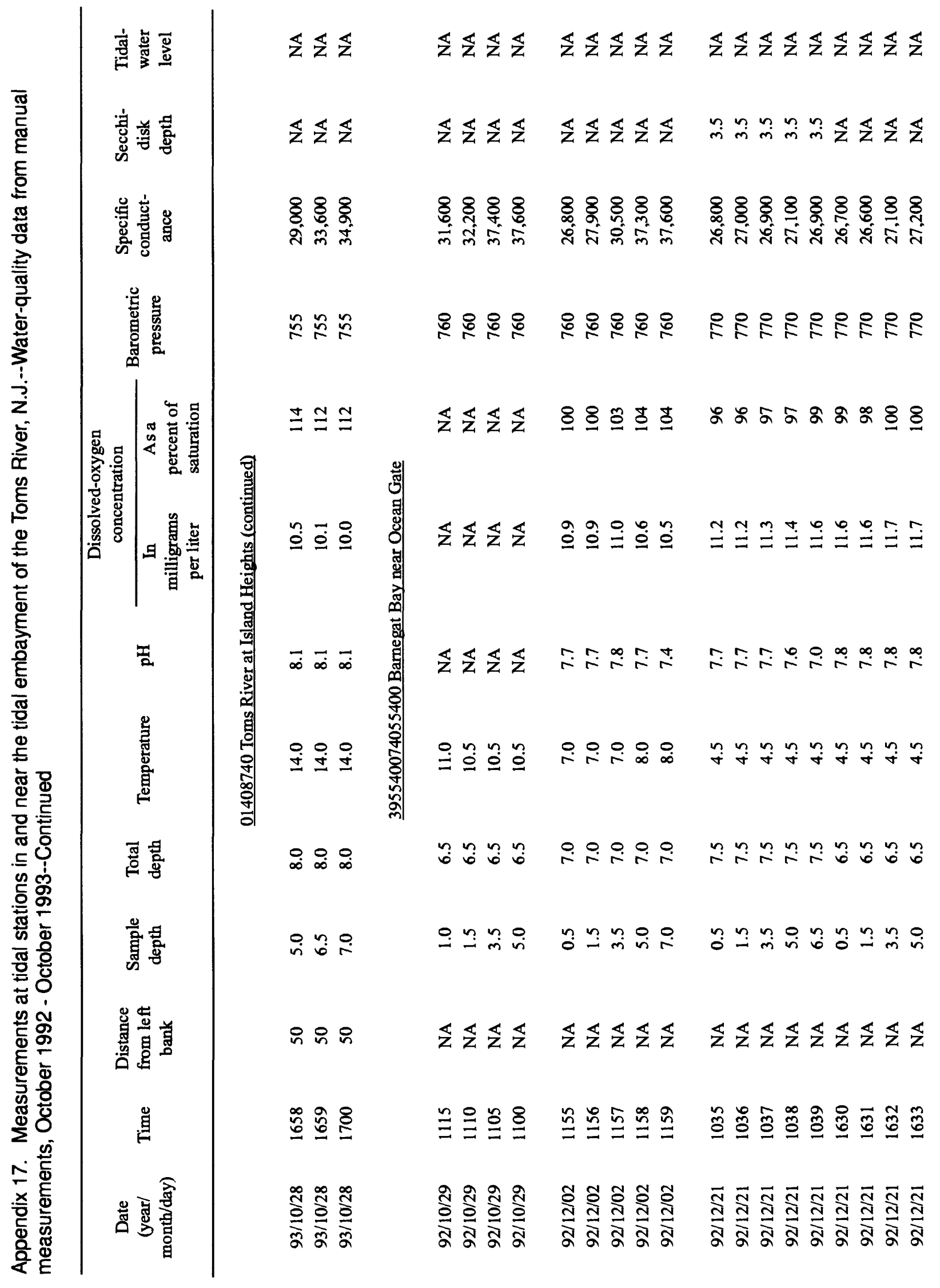




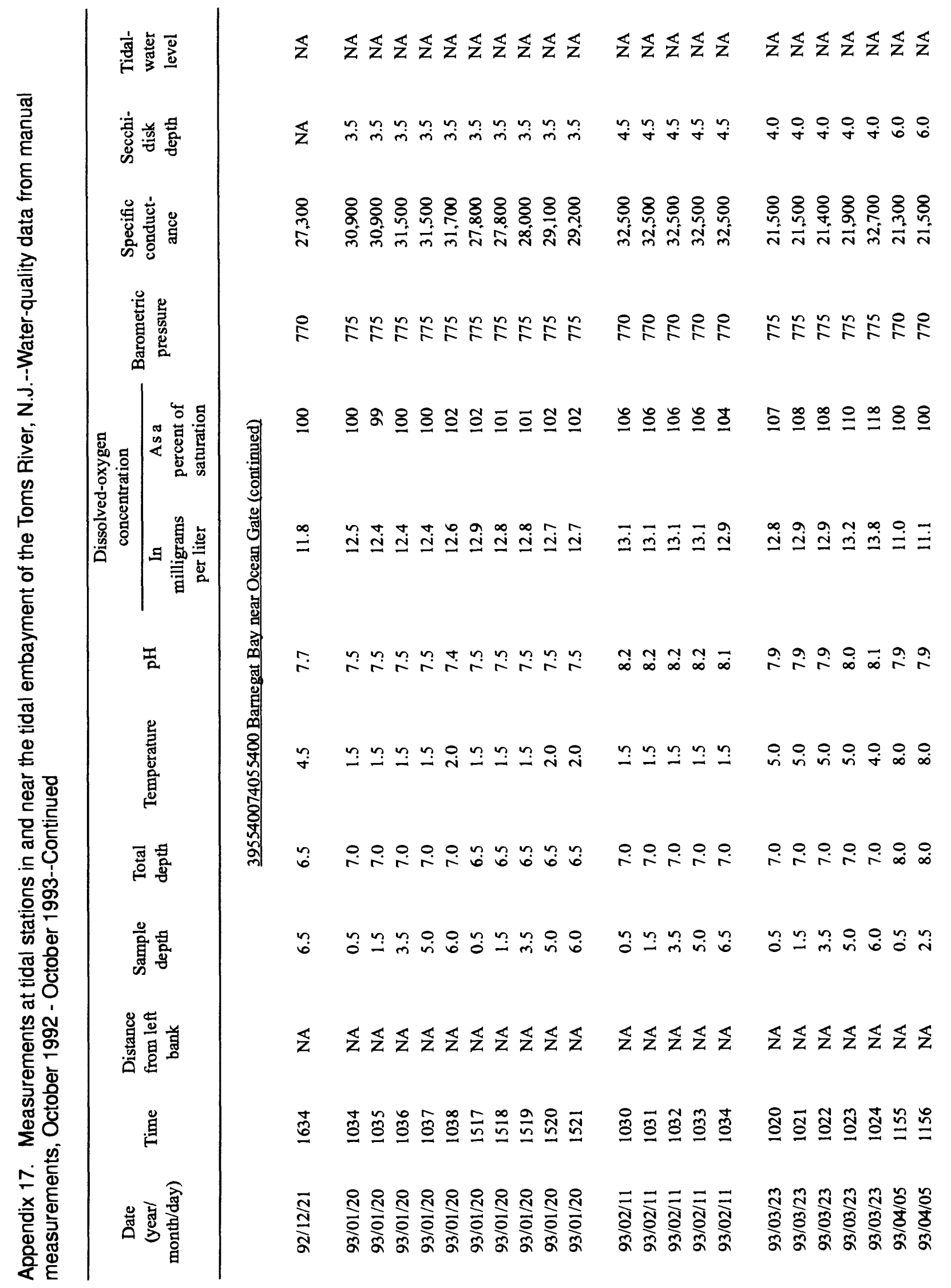




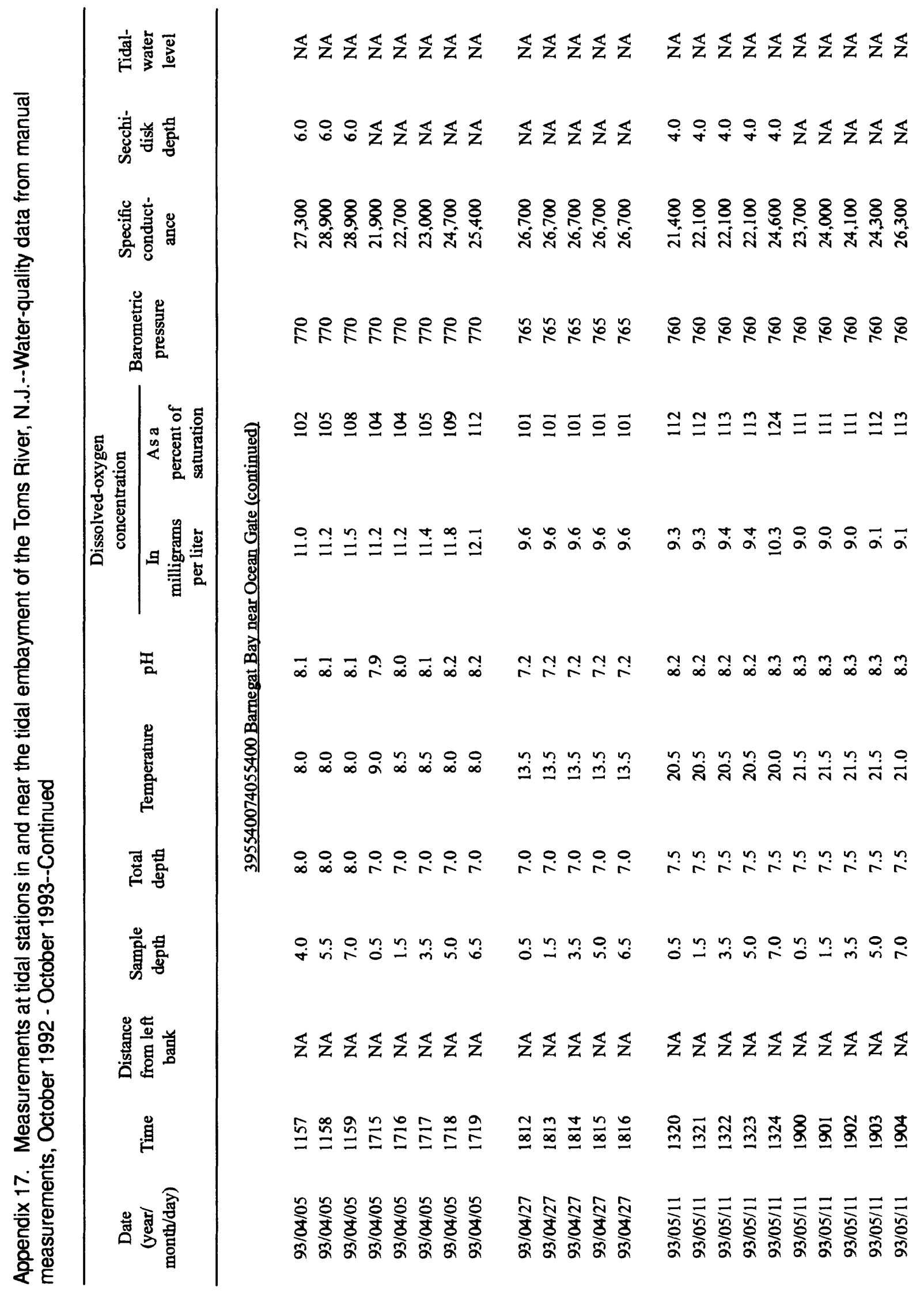




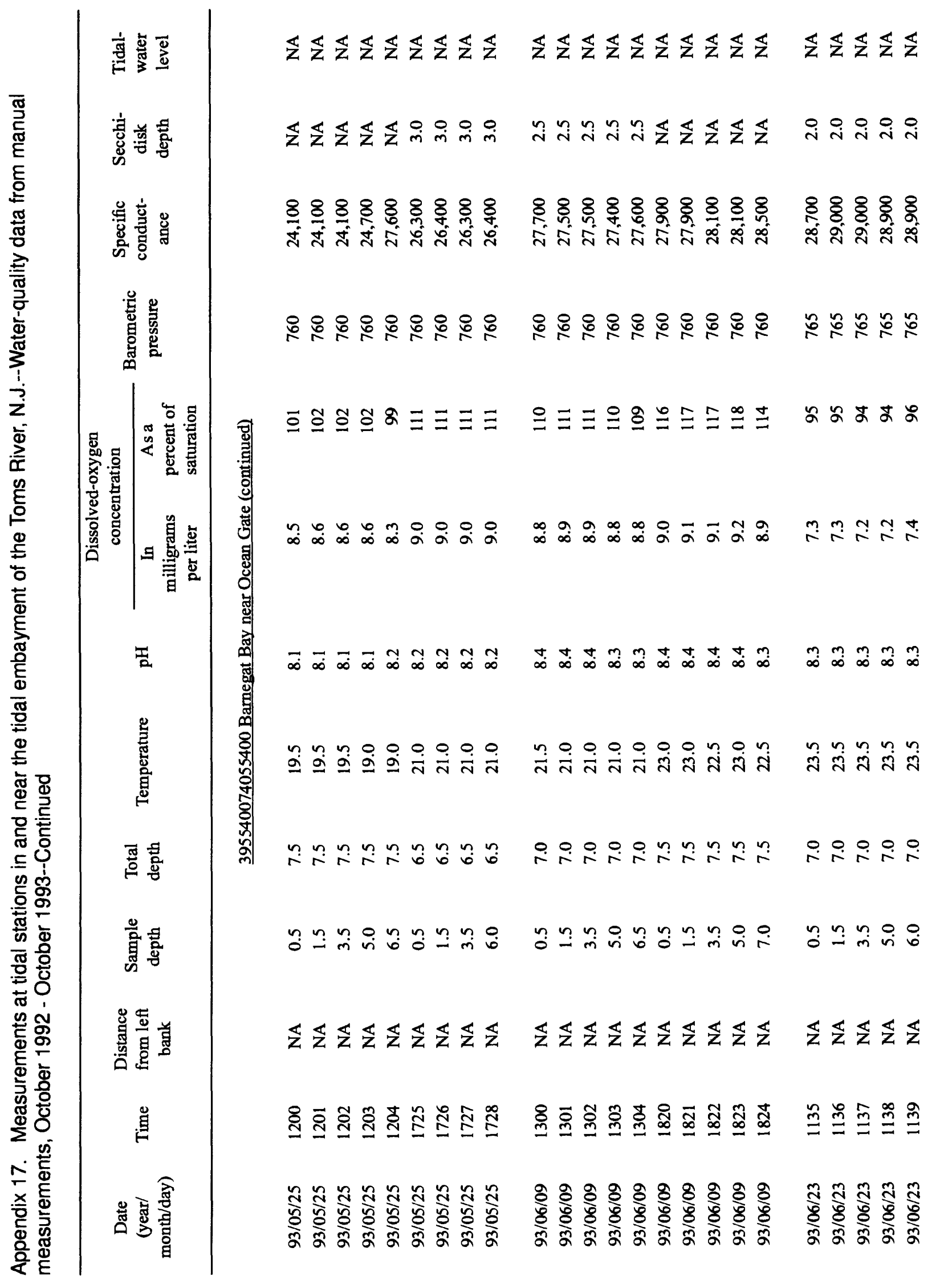




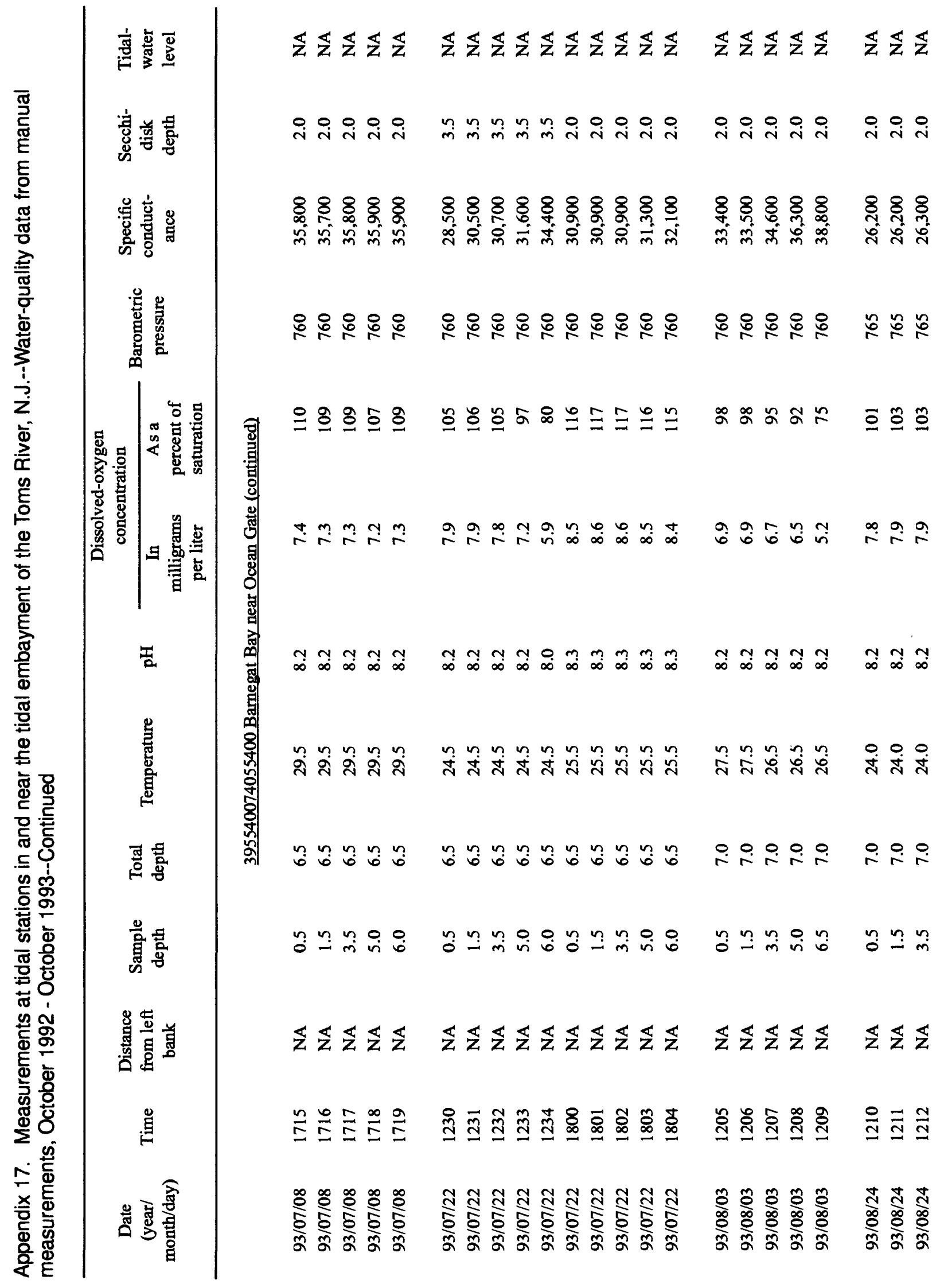




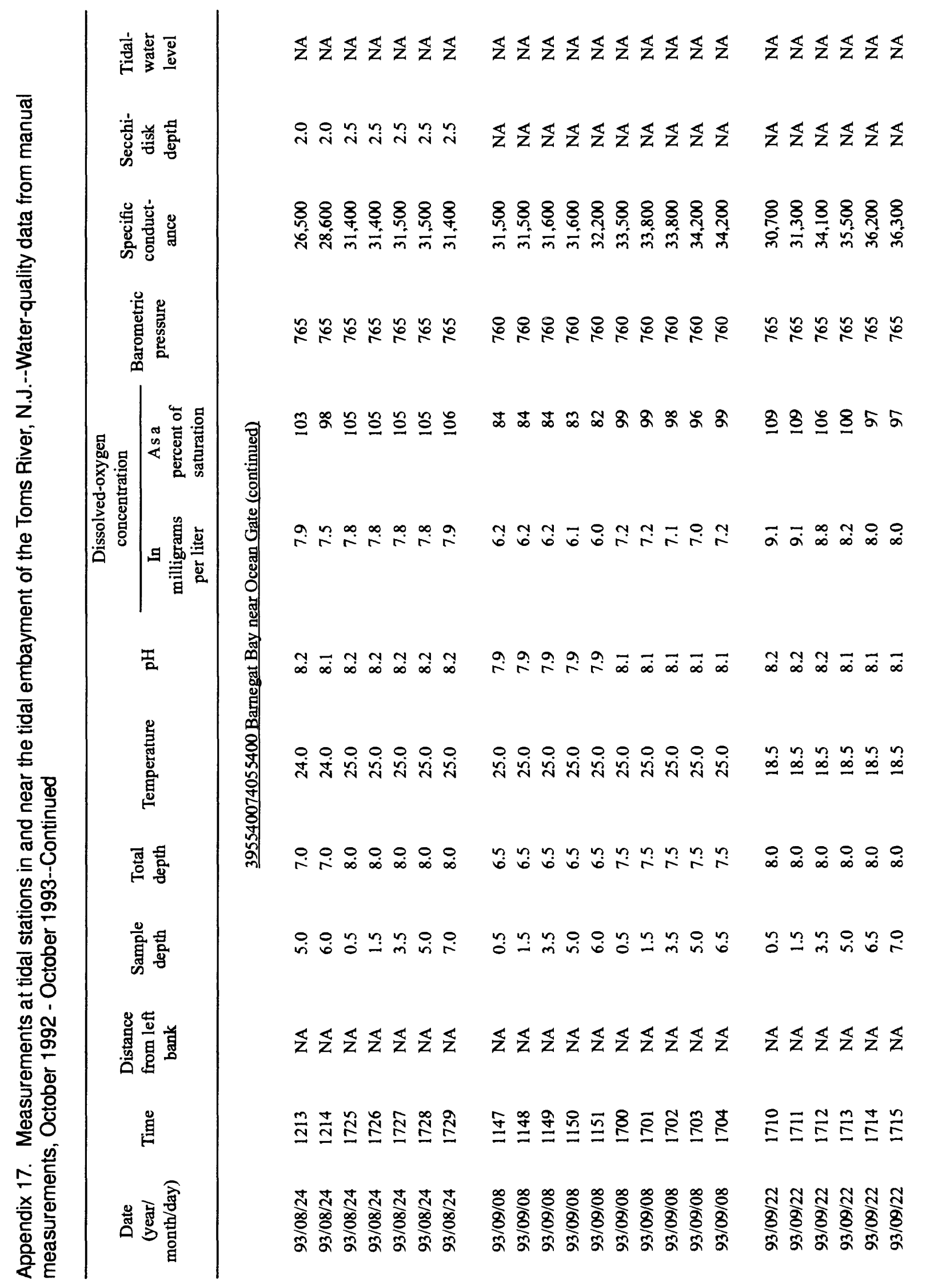




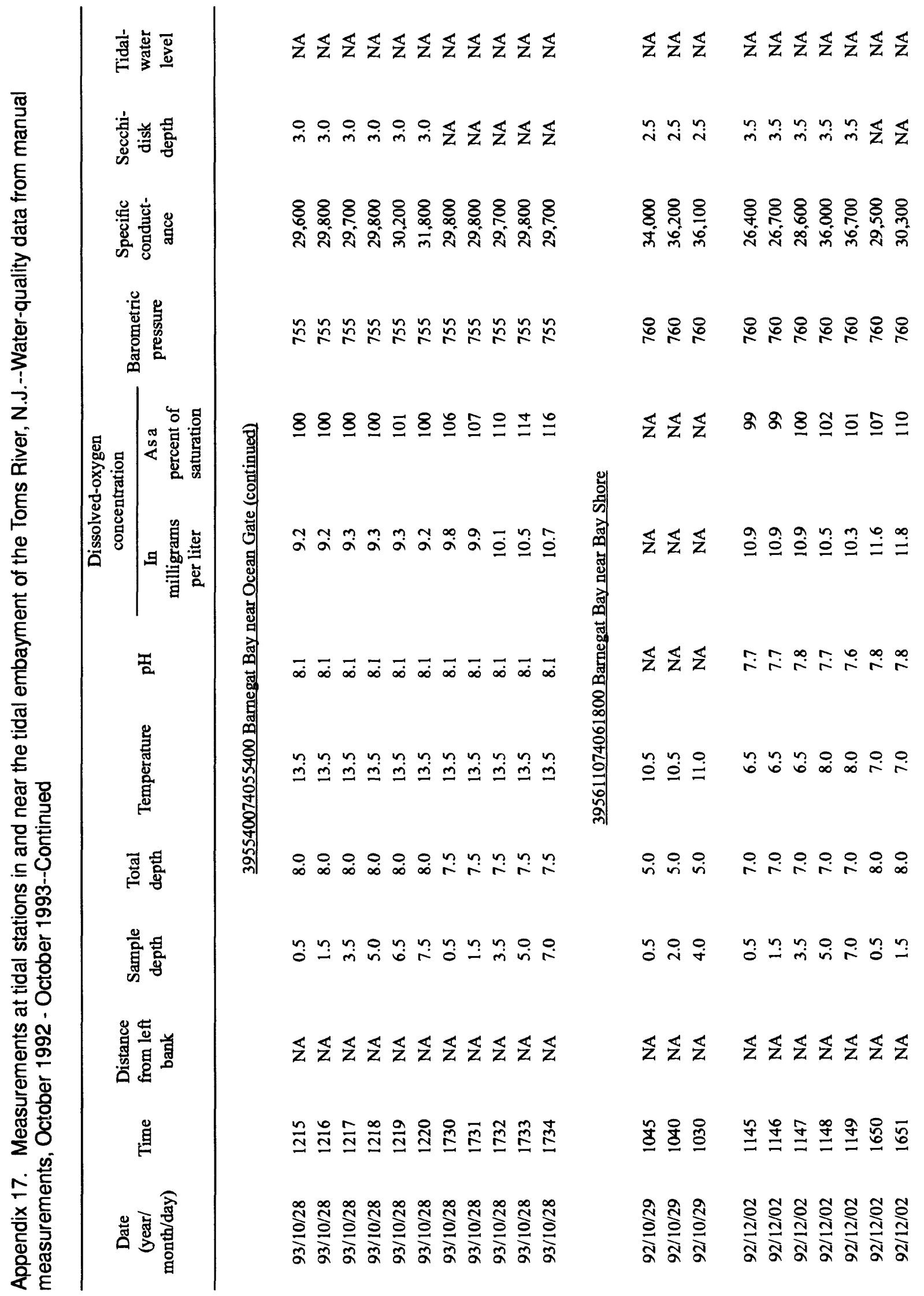




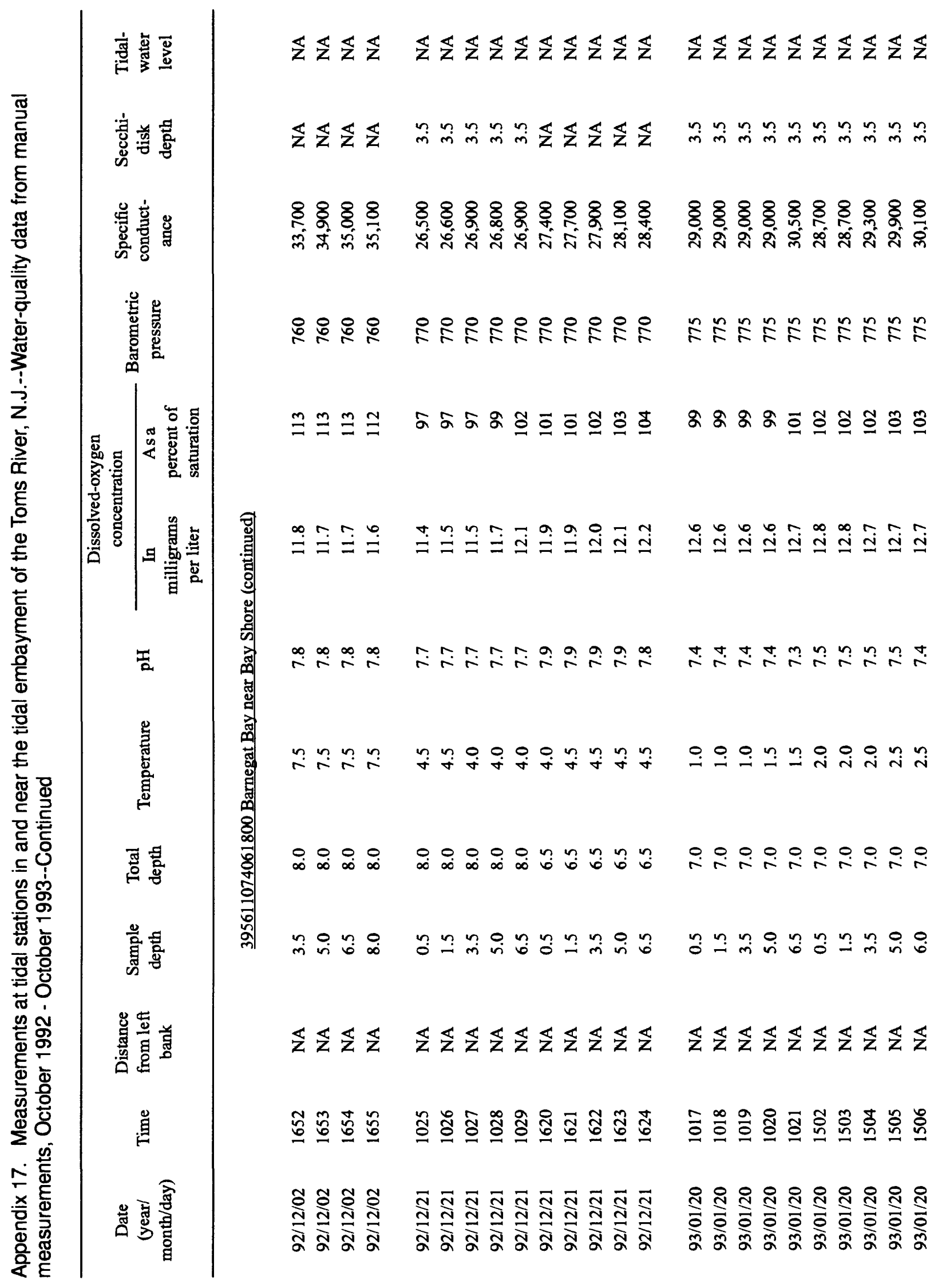




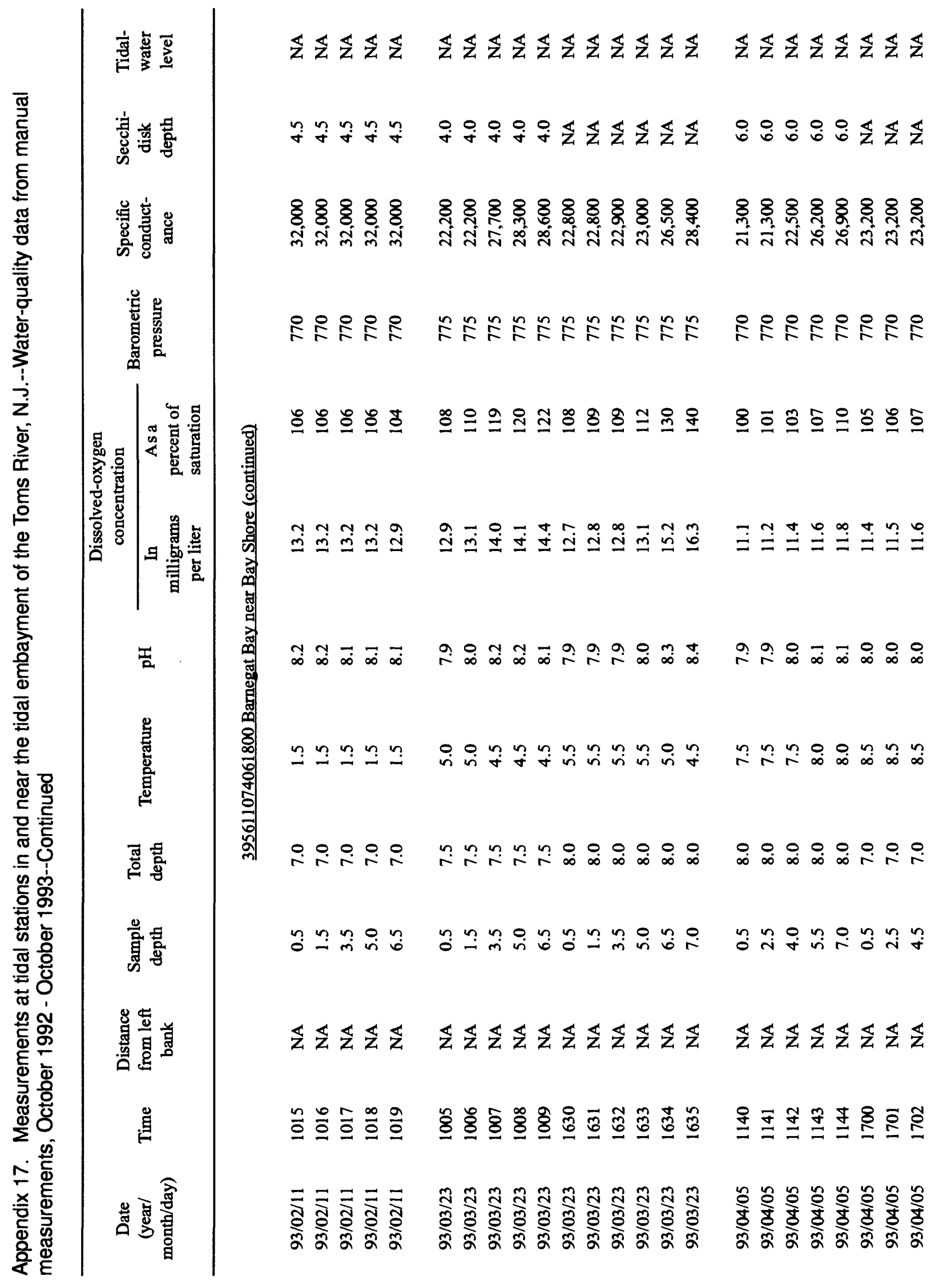




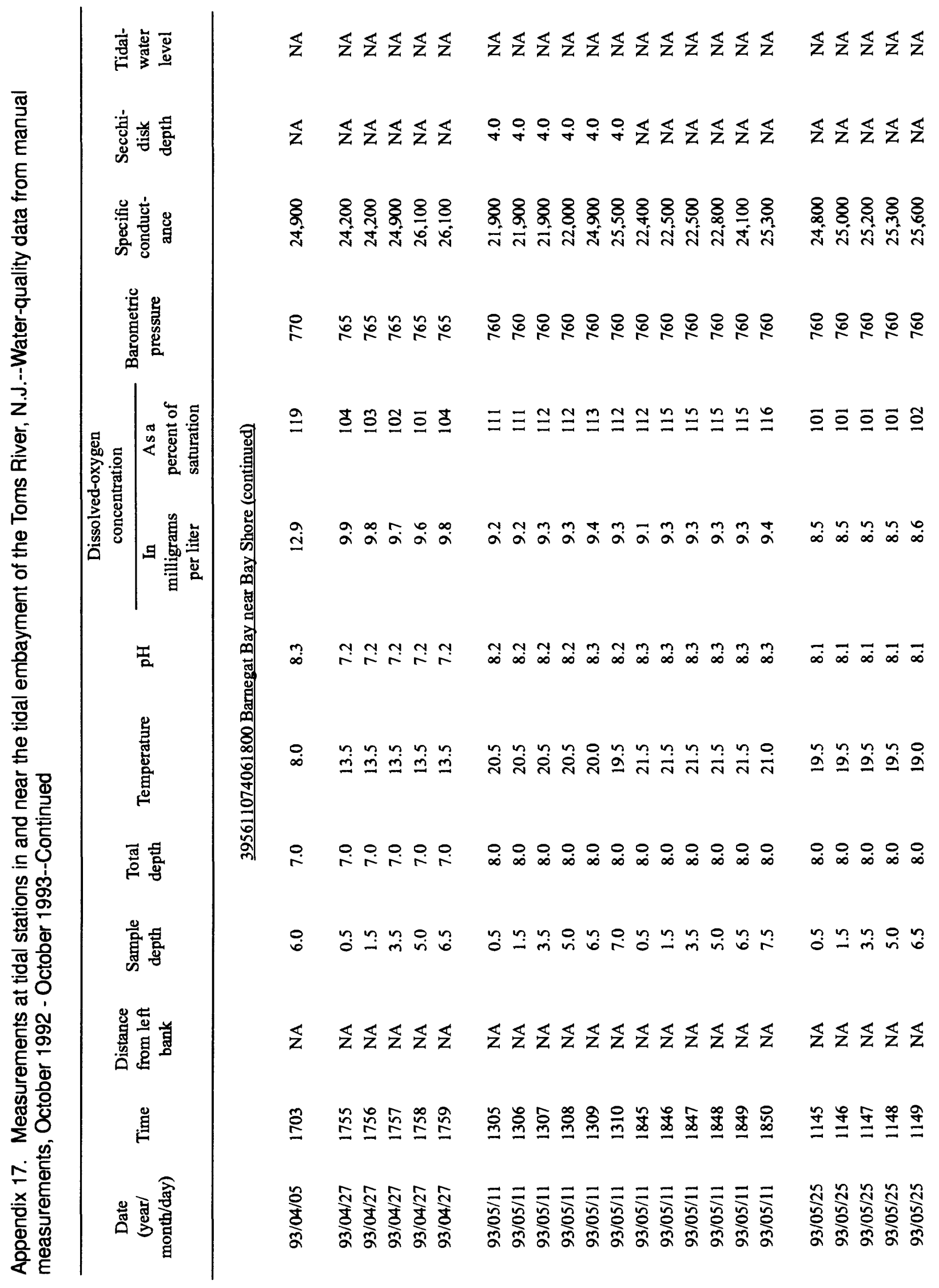




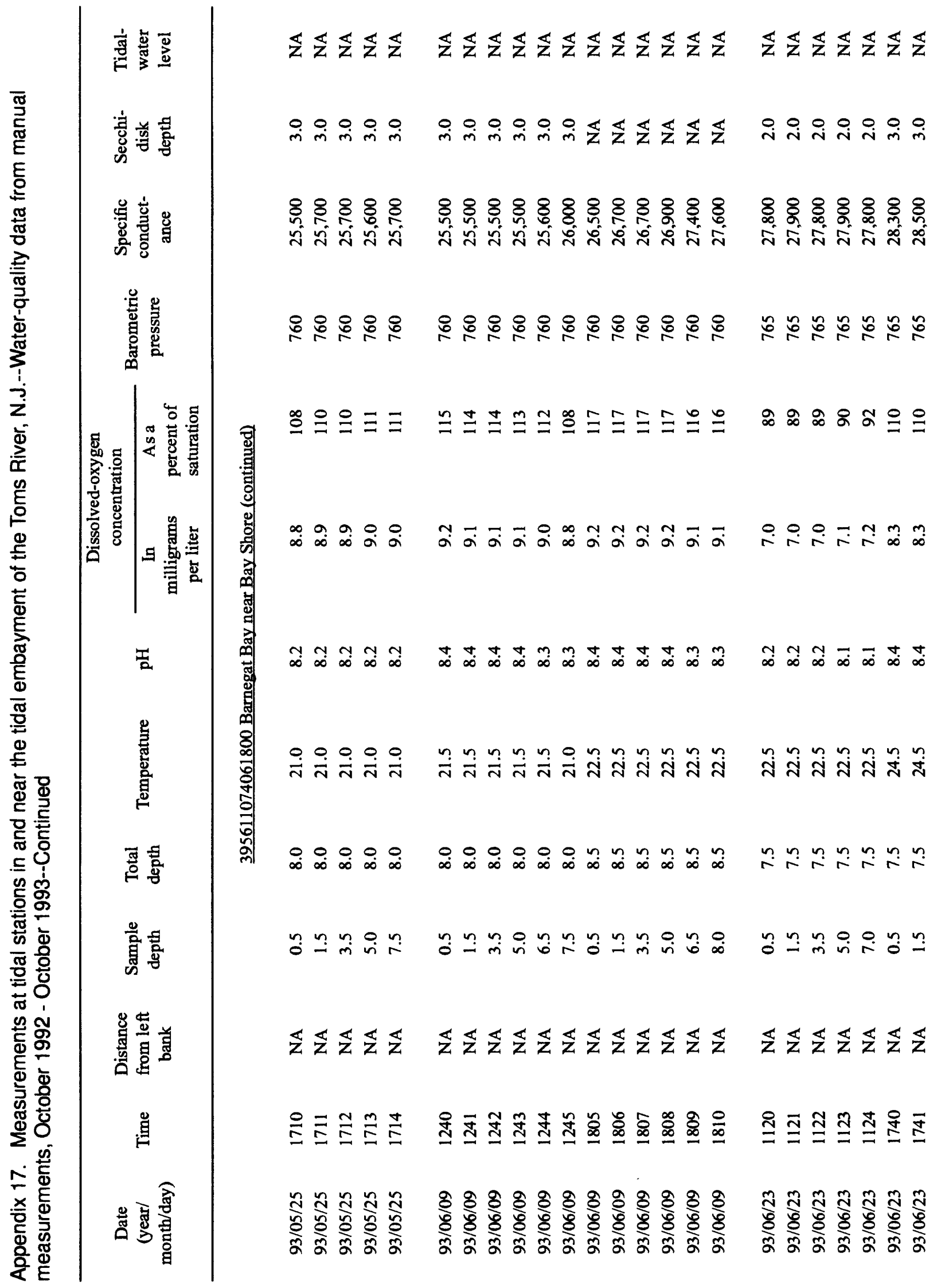




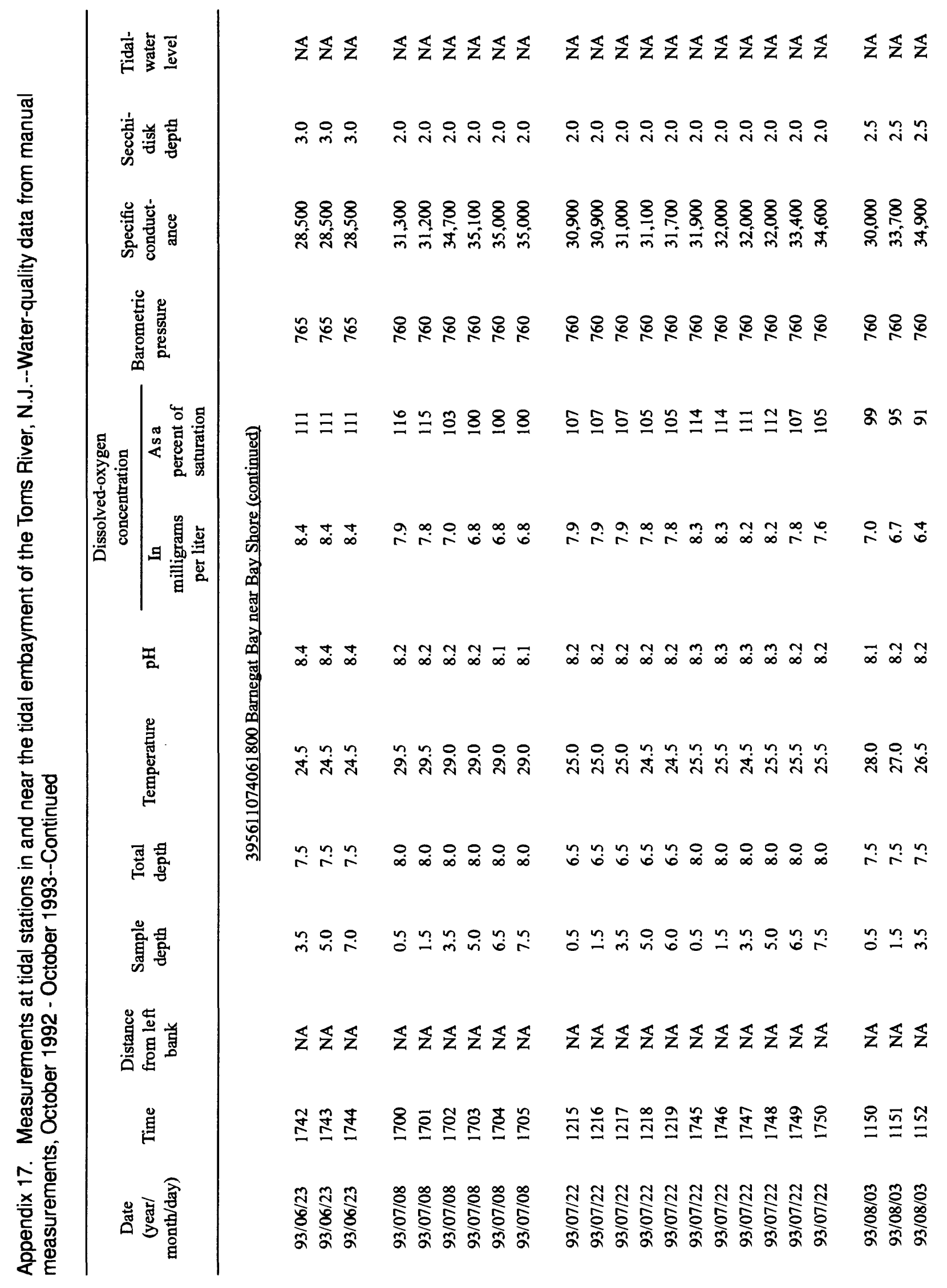




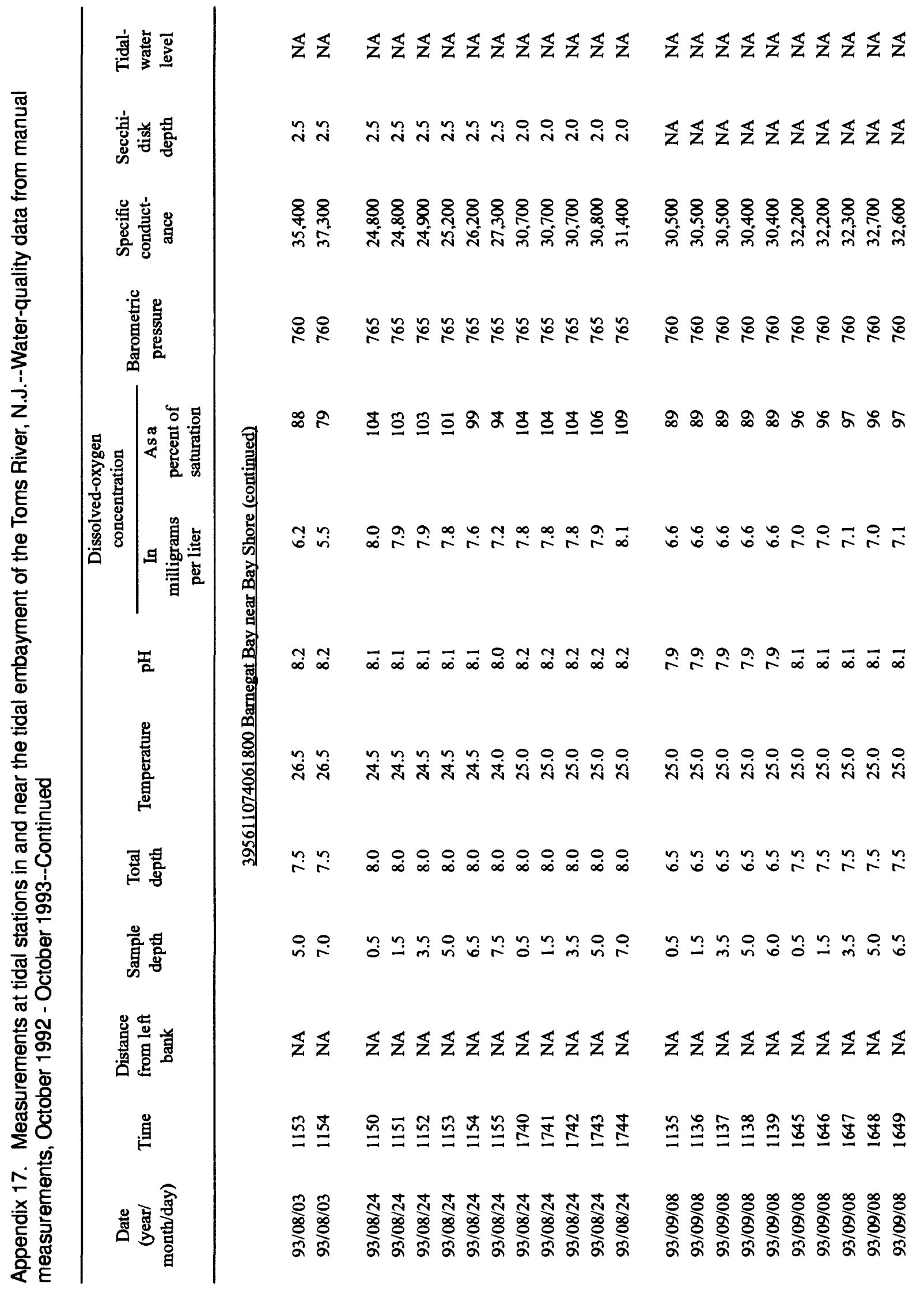




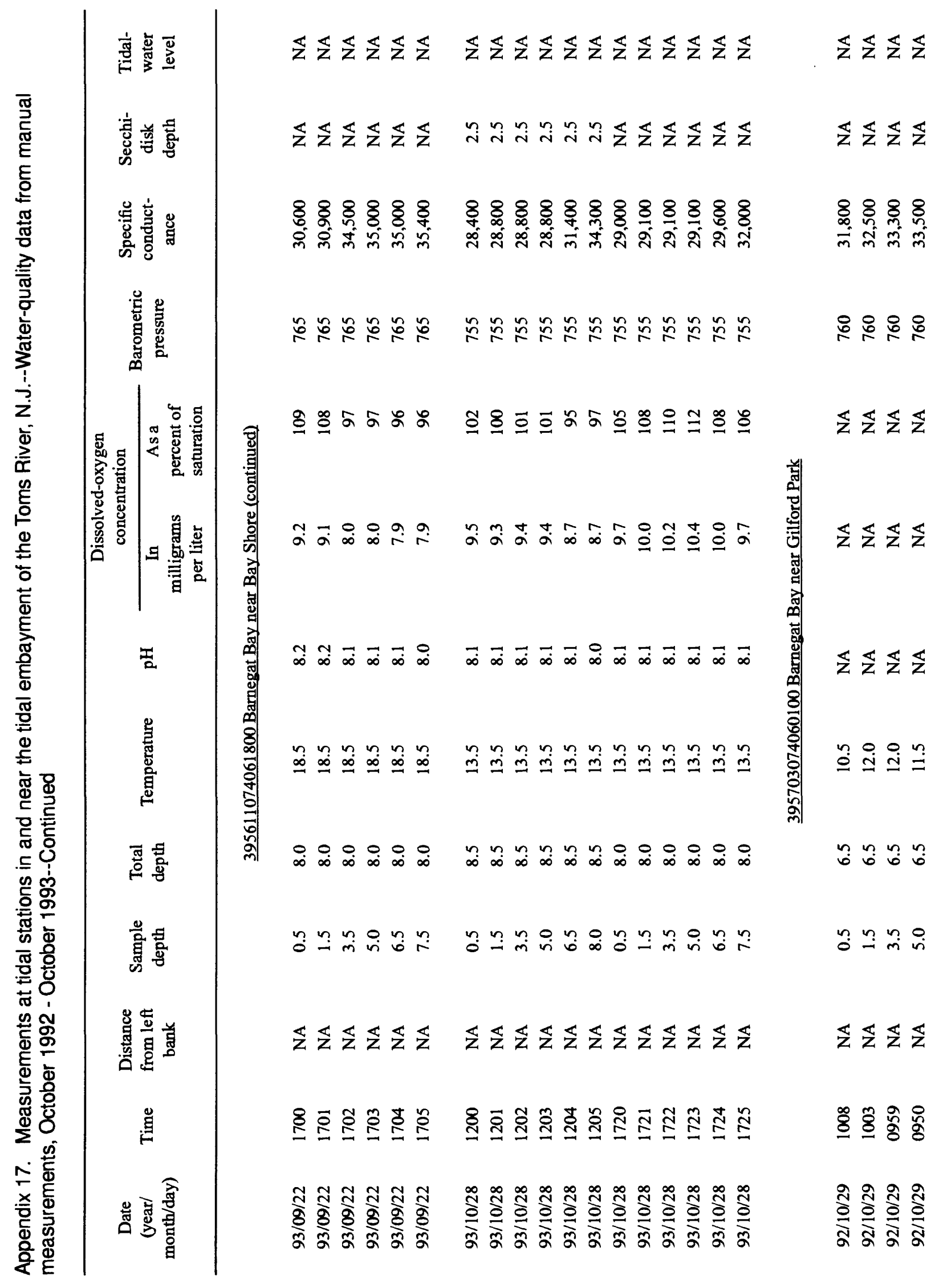




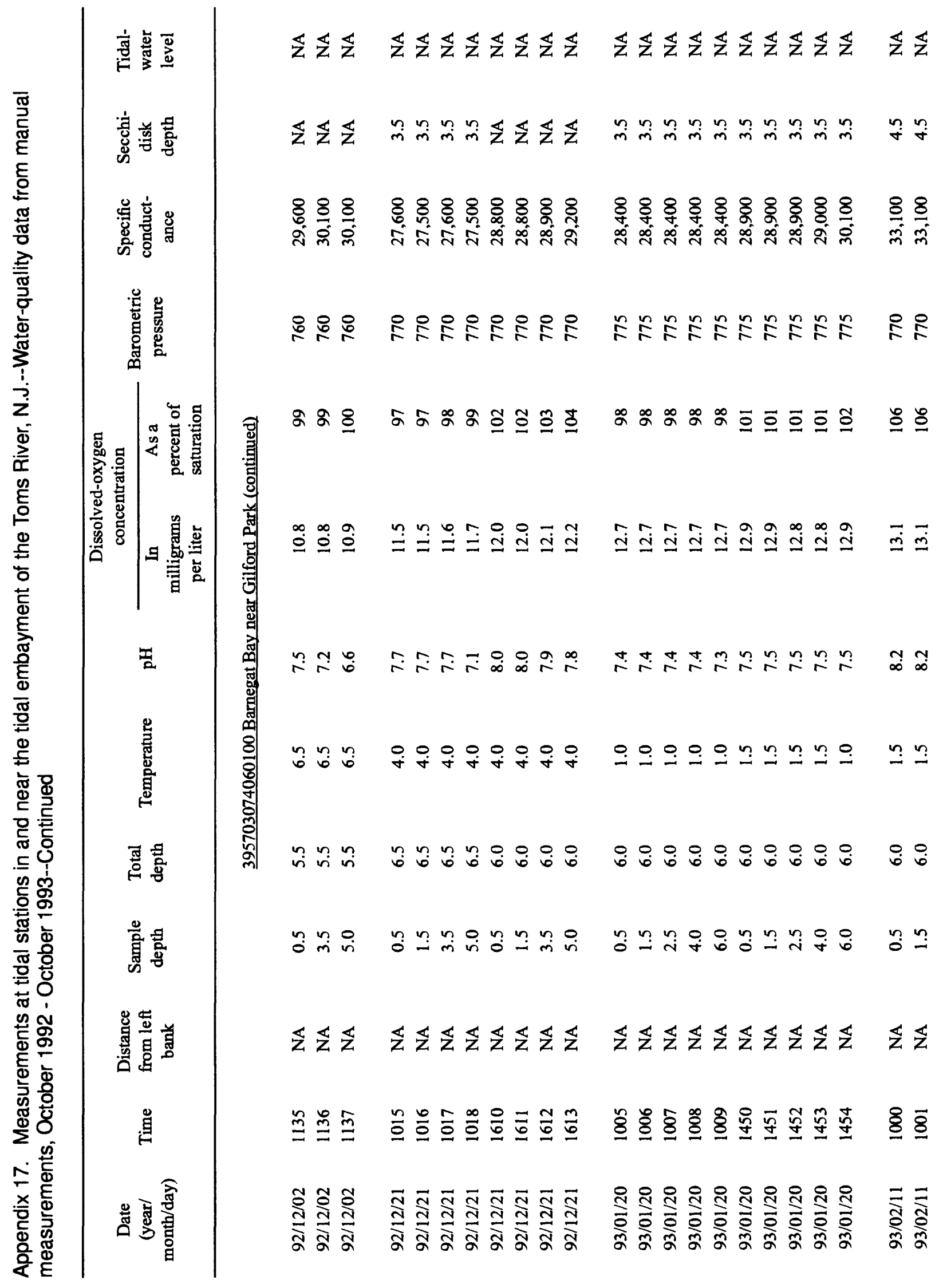




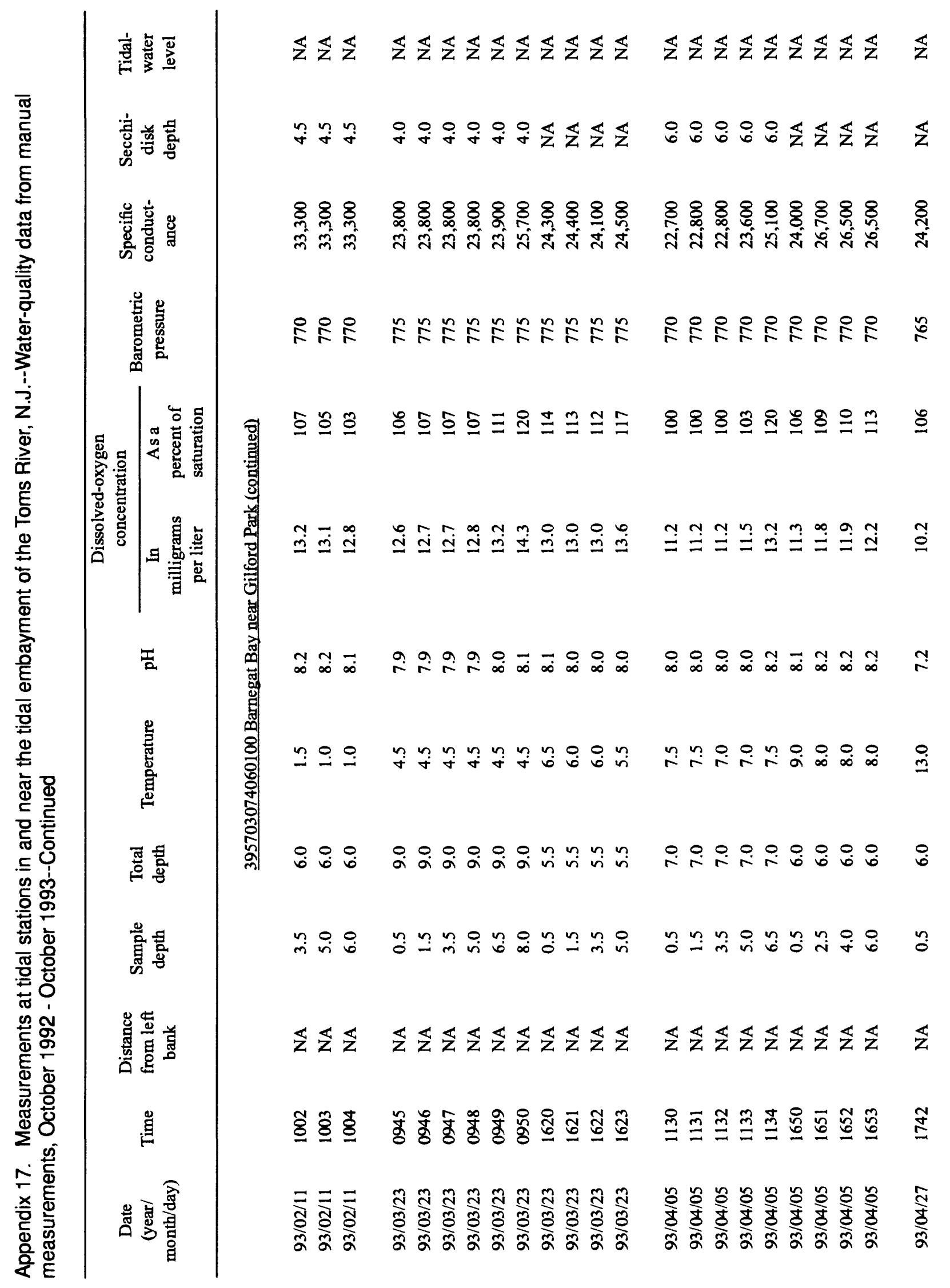




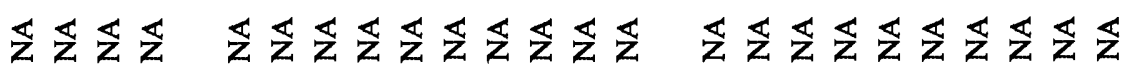

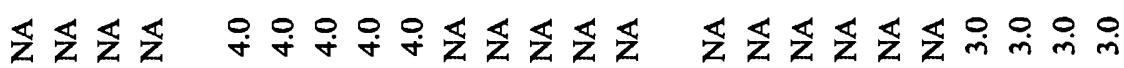

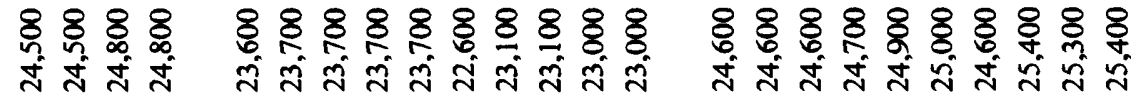

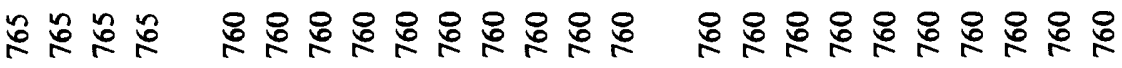

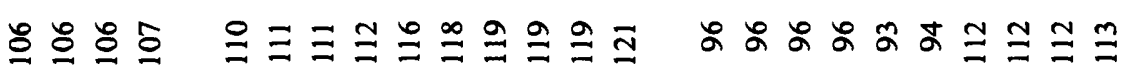

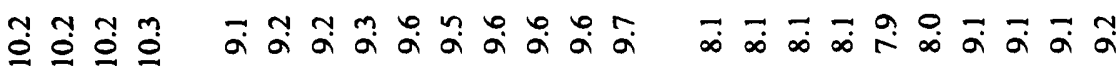

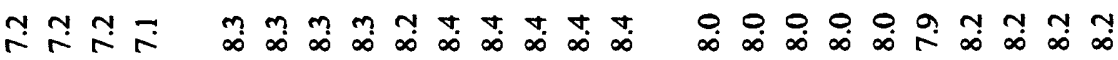

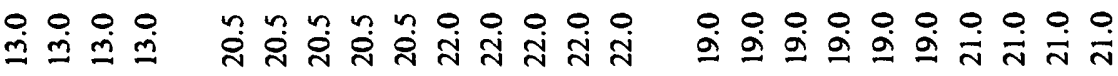

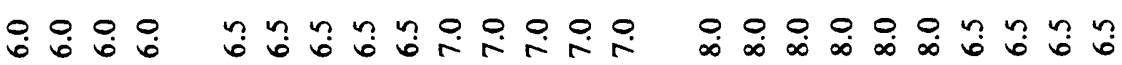

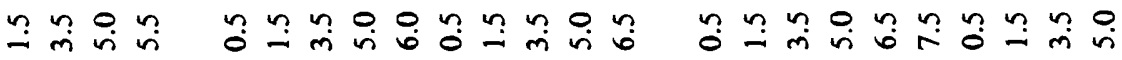

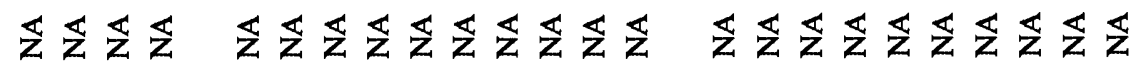

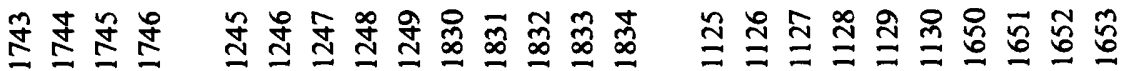

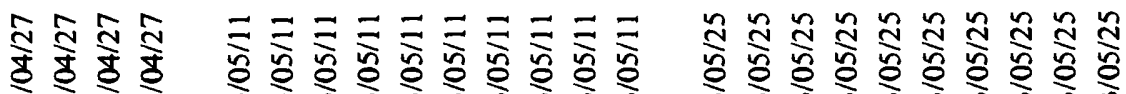

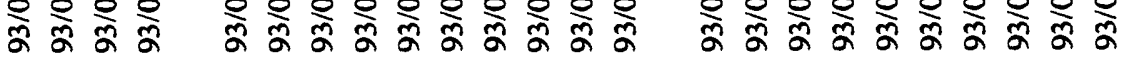




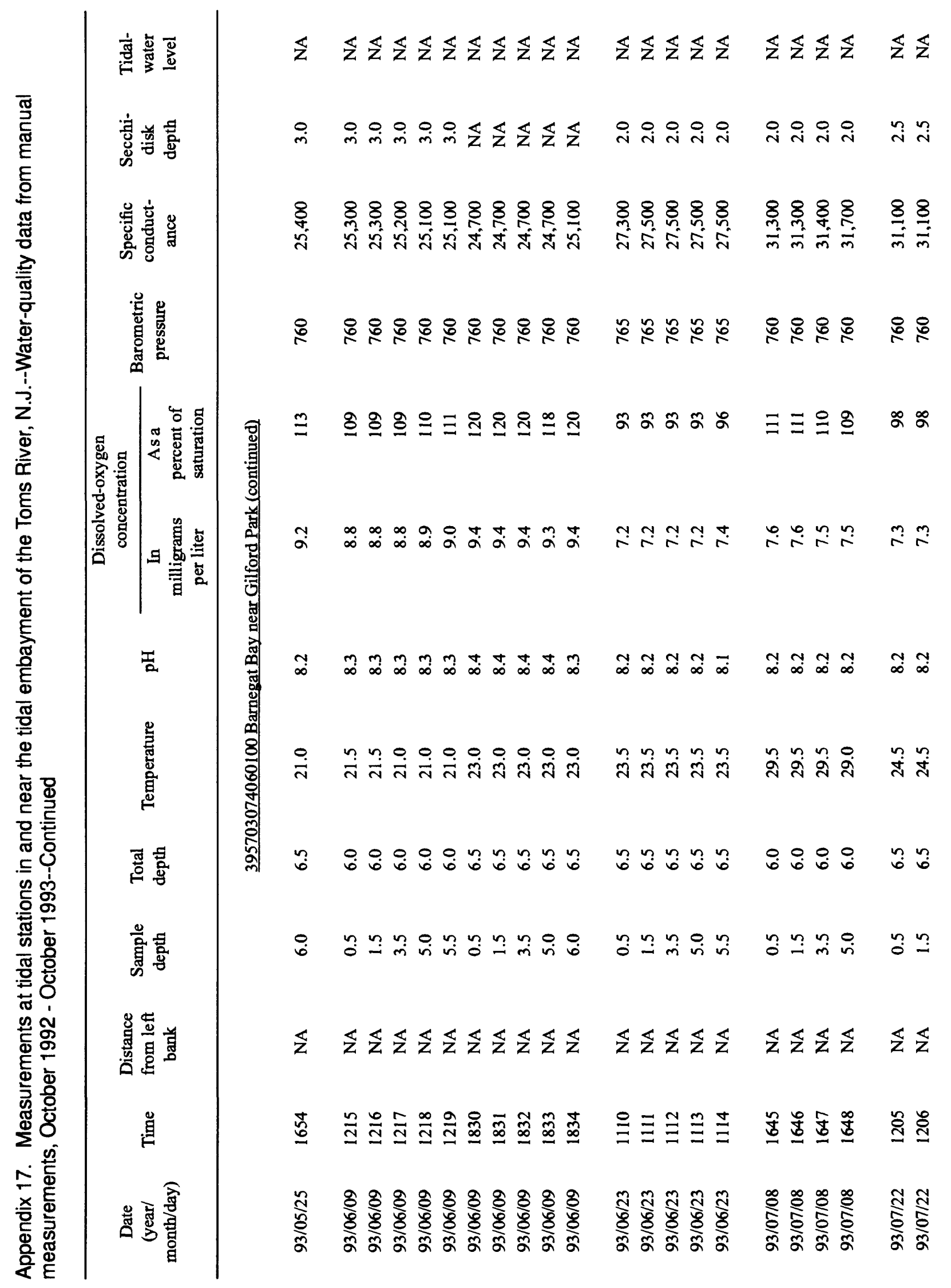




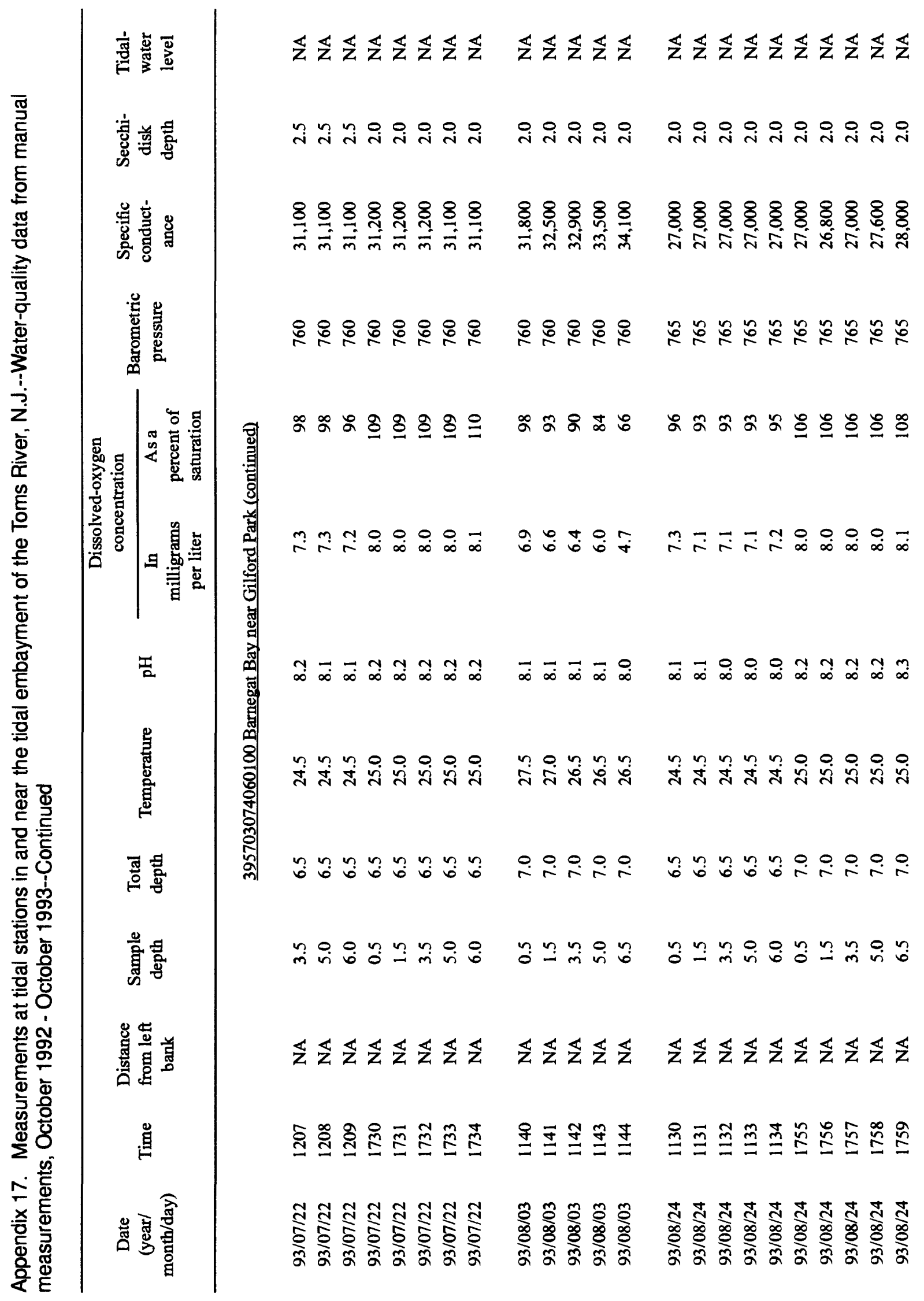




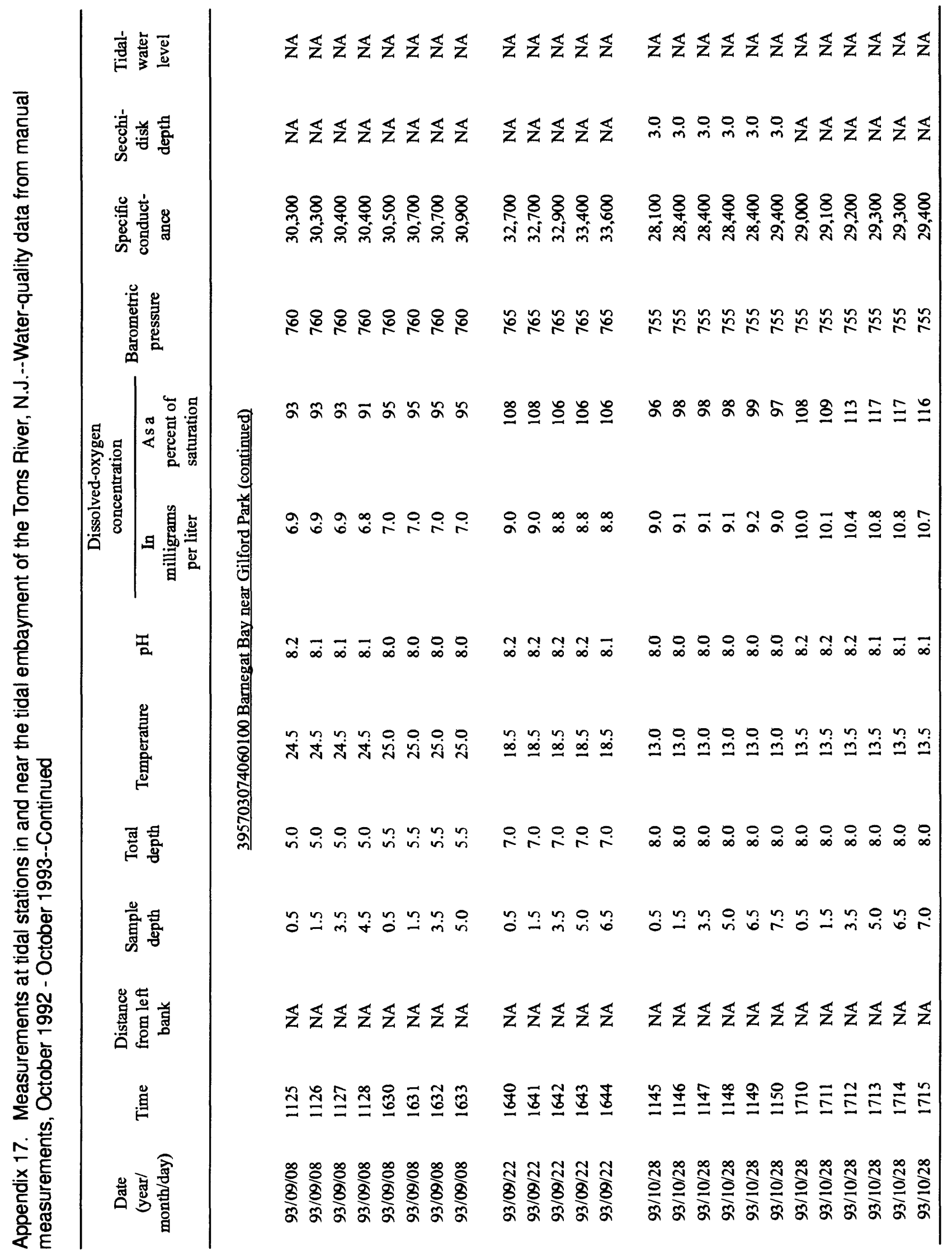


Appendix 18. Measurements at tidal stations in and near the tidal embayment of the Toms River, N.J.--Results of laboratory measurements of dissolved constituents, October 1992 - October 1993

[Laboratory analyses were conducted on water samples at the stations listed below. USGS, U.S. Geological Survey; NA, not applicable or not available]

\begin{tabular}{|c|c|c|c|}
\hline $\begin{array}{c}\text { Index } \\
\text { number } \\
\text { (fig. 3) }\end{array}$ & USGS station number & USGS station name & $\begin{array}{l}\text { Location of the } \\
\text { channel in the } \\
\text { cross section, in } \\
\text { percent distance } \\
\text { from left to right } \\
\text { bank }\end{array}$ \\
\hline 18 & 01408685 & Toms River at Garden State Parkway & 50 \\
\hline 19 & 01408690 & $\begin{array}{l}\text { Toms River } 0.2 \text { miles downstream from } \\
\text { Garden State Parkway }\end{array}$ & 10 \\
\hline 20 & 01408695 & Toms River near South Toms River & 50 \\
\hline 21 & 01408700 & Toms River at Toms River & $\begin{array}{l}25,75 \\
\text { (two channels) }\end{array}$ \\
\hline 22 & 01408719 & $\begin{array}{l}\text { Toms River at Cedar Point at South Toms } \\
\text { River }\end{array}$ & 50 \\
\hline 23 & 01408722 & Toms River near Toms River & 50 \\
\hline 24 & 01408730 & Toms River at Pine Beach & 50 \\
\hline 25 & 01408735 & $\begin{array}{l}\text { Toms River at Maple Avenue Pier at Island } \\
\text { Heights }\end{array}$ & 50 \\
\hline 26 & 01408740 & Toms River at Island Heights & 50 \\
\hline 27 & 395540074055400 & Barnegat Bay near Ocean Gate & NA \\
\hline 28 & 395611074061800 & Barnegat Bay near Bay Shore & NA \\
\hline 29 & 395703074060100 & Barnegat Bay near Gilford Park & NA \\
\hline
\end{tabular}


Appendix 18. Measurements at tidal stations in and near the tidal embayment of the Toms River, N.J.--Results of laboratory measurements of dissolved constituents, October 1992 - October 1993 --Continued

The following information is presented:

\begin{tabular}{lll}
\hline \multicolumn{1}{c}{ Characteristic } & \multicolumn{1}{c}{ Unit } & Rounding \\
\hline $\begin{array}{l}\text { Date and time of water } \\
\text { sample }\end{array}$ & NA & NA \\
$\begin{array}{lll}\text { Distance from left bank } & \text { Percent of distance from left to } \\
\text { right bank }\end{array}$ & 1 \\
$\begin{array}{l}\text { Sample depth } \\
\text { Specific conductance }\end{array}$ & $\begin{array}{c}\text { Meet } \\
\text { 25 degrees Celsius }\end{array}$ & 0.5 \\
$\begin{array}{c}\text { Salinity } \\
\text { Dissolved-chloride } \\
\text { concentration }\end{array}$ & Parts per thousand & 1 \\
$\begin{array}{c}\text { Dissolved-solids } \\
\text { concentration }\end{array}$ & Milligrams per liter as chloride & 1 \\
\hline
\end{tabular}


Appendix 18. Measurements at tidal stations in and near the tidal embayment of the Toms River, N.J.--Results of laboratory measurements of dissolved constituents, October 1992 - October 1993-Continued

\begin{tabular}{|c|c|c|c|c|c|c|c|}
\hline $\begin{array}{c}\text { Date } \\
\text { (year/month/ } \\
\text { day) }\end{array}$ & Time & $\begin{array}{c}\text { Distance } \\
\text { from left } \\
\text { bank }\end{array}$ & $\begin{array}{l}\text { Sample } \\
\text { depth }\end{array}$ & $\begin{array}{c}\text { Specific } \\
\text { conductance }\end{array}$ & Salinity & $\begin{array}{l}\text { Dissolved- } \\
\text { chloride } \\
\text { concentration }\end{array}$ & $\begin{array}{l}\text { Dissolved- } \\
\text { solids } \\
\text { concentration }\end{array}$ \\
\hline
\end{tabular}

01408685 Toms River at Garden State Parkway at Toms River

$\begin{array}{rrrrrrrr}92 / 10 / 29 & 1200 & 50 & 0.5 & 76 & 0.05 & 11 & 176 \\ 92 / 10 / 29 & 1155 & 50 & 6.5 & 81 & 0.04 & 12 & 75\end{array}$

01408690 Toms River 0.2 miles downstream from Garden State Parkway at Toms River

$\begin{array}{rrrrrrrr}92 / 10 / 29 & 1145 & 20 & 0.5 & 85 & 0.06 & 11 & 75 \\ 92 / 10 / 29 & 1135 & 20 & 5.0 & 163 & 0.06 & 14 & 56\end{array}$

01408695 Toms River near South Toms River

$\begin{array}{rrrrrrrr}92 / 10 / 29 & 1120 & 50 & 0.5 & 172 & 0.05 & 12 & 120 \\ 92 / 10 / 29 & 1100 & 50 & 7.0 & 26,100 & 17.20 & 10,100 & 17,600\end{array}$

01408700 Toms River at Toms River

$\begin{array}{rrrrrrrr}92 / 10 / 29 & 1025 & 75 & 0.5 & 613 & 0.22 & 154 & 328 \\ 92 / 10 / 29 & 1015 & 75 & 4.0 & 29,200 & 19.90 & 11,100 & 19,200 \\ 92 / 12 / 02 & 1000 & 75 & 0.5 & 191 . & 0.12 & 50 & 35 \\ 93 / 02 / 11 & 0830 & 75 & 0.5 & 84 . & 0.04 & 19 & 20 \\ 93 / 03 / 23 & 0820 & 75 & 0.5 & 75 & 0.04 & 10 & 30 \\ 93 / 04 / 05 & 0920 & 75 & 0.5 & 67 & 0.04 & 8 & 50 \\ 93 / 07 / 08 & 1510 & 25 & 0.5 & 695 & 0.40 & 217 & 440 \\ 93 / 08 / 03 & 1010 & 25 & 0.5 & 235 & 0.13 & 57 & 133 \\ 93 / 09 / 22 & 1550 & 25 & 0.5 & 2,100 & 1.01 & 562 & 957 \\ 93 / 10 / 28 & 1015 & 25 & 0.5 & 1,200 & 0.59 & 330 & 585\end{array}$

01408719 Toms River at Cedar Point at South Toms River

$\begin{array}{rrrrrrrr}92 / 10 / 29 & 1338 & 50 & 0.5 & 11,800 & 6.81 & 13,800 & 7,080 \\ 92 / 10 / 29 & 1320 & 50 & 5.0 & 30,400 & 19.50 & 11,400 & 20,600 \\ 93 / 01 / 20 & 0920 & 50 & 0.5 & 11,700 & 5.64 & 2,690 & 5,240 \\ 93 / 04 / 27 & 1106 & 50 & 0.5 & 2,740 & 1.63 & 808 & 1,520 \\ 93 / 05 / 11 & 1115 & 50 & 0.5 & 7,010 & 4.44 & 2,160 & 3,880 \\ 93 / 05 / 25 & 1045 & 50 & 0.5 & 6,570 & 4.18 & 2,070 & 3,740 \\ 93 / 06 / 09 & 1115 & 50 & 0.5 & 78 & 0.05 & 14 & 20 \\ 93 / 06 / 23 & 1010 & 50 & 0.5 & 13,300 & 8.98 & 4,130 & 8,330 \\ 93 / 07 / 22 & 1020 & 50 & 0.5 & 6,870 & 4.42 & 2,270 & 3,980 \\ 93 / 08 / 24 & 1025 & 50 & 0.5 & 4,960 & 3.01 & 1,460 & 2,630 \\ 93 / 09 / 08 & 1020 & 50 & 0.5 & 7,360 & 3.83 & 1,960 & 3,540\end{array}$


Appendix 18. Measurements at tidal stations in and near the tidal embayment of the Toms River, N.J.--Results of laboratory measurements of dissolved constituents, October 1992 - October 1993-Continued

\begin{tabular}{|c|c|c|c|c|c|c|c|}
\hline $\begin{array}{c}\text { Date } \\
\text { (year/month/ } \\
\text { day) }\end{array}$ & Time & $\begin{array}{c}\text { Distance } \\
\text { from left } \\
\text { bank }\end{array}$ & $\begin{array}{l}\text { Sample } \\
\text { depth }\end{array}$ & $\begin{array}{c}\text { Specific } \\
\text { conductance }\end{array}$ & Salinity & $\begin{array}{l}\text { Dissolved- } \\
\text { chloride } \\
\text { concentration }\end{array}$ & $\begin{array}{l}\text { Dissolved- } \\
\text { solids } \\
\text { concentration }\end{array}$ \\
\hline
\end{tabular}

01408722 Toms River near Toms River

$\begin{array}{rrrrrrrr}92 / 10 / 29 & 1305 & 50 & 0.5 & 23,200 & 13.90 & 8,100 & 14,400 \\ 92 / 10 / 29 & 1245 & 50 & 5.0 & 30,400 & 19.60 & 11,700 & 20,400\end{array}$

01408735 Toms River at Maple Avenue Pier at Island Heights

$\begin{array}{llllllll}92 / 10 / 29 & 1230 & 50 & 0.5 & 21,700 & 13.80 & 10,600 & 15,000 \\ 92 / 10 / 29 & 1210 & 50 & 5.0 & 32,200 & 20.10 & 12,700 & 21,400\end{array}$

01408740 Toms River at Island Heights

$\begin{array}{llllllll}92 / 10 / 29 & 1152 & 50 & 1.0 & 29,800 & 18.90 & 10,400 & 18,900 \\ 92 / 10 / 29 & 1135 & 50 & 5.0 & 29,700 & 20.20 & 11,100 & 31,900\end{array}$

395540074055400 Barnegat Bay near Ocean Gate

$\begin{array}{rrrrrrrr}92 / 10 / 29 & 1115 & \text { NA } & 1.0 & 28,500 & 18.40 & 9,300 & 18,900 \\ 92 / 10 / 29 & 1100 & \text { NA } & 5.0 & 35,600 & 22.40 & 12,700 & 12,600\end{array}$

395611074061800 Barnegat Bay near Bay Shore

$\begin{array}{llllllll}92 / 10 / 29 & 1045 & \text { NA } & 0.5 & 29,300 & 19.80 & 10,800 & 20,900 \\ 92 / 10 / 29 & 1030 & \text { NA } & 4.0 & 31,300 & 21.70 & 11,800 & 22,000\end{array}$

395703074060100 Barnegat Bay near Gilford Park

$\begin{array}{llllllll}92 / 10 / 29 & 1008 & \text { NA } & 0.5 & 30,900 & 19.40 & 10,900 & 20,600 \\ 92 / 10 / 29 & 0950 & \text { NA } & 5.0 & 29,000 & 19.80 & 10,100 & 20,400\end{array}$

